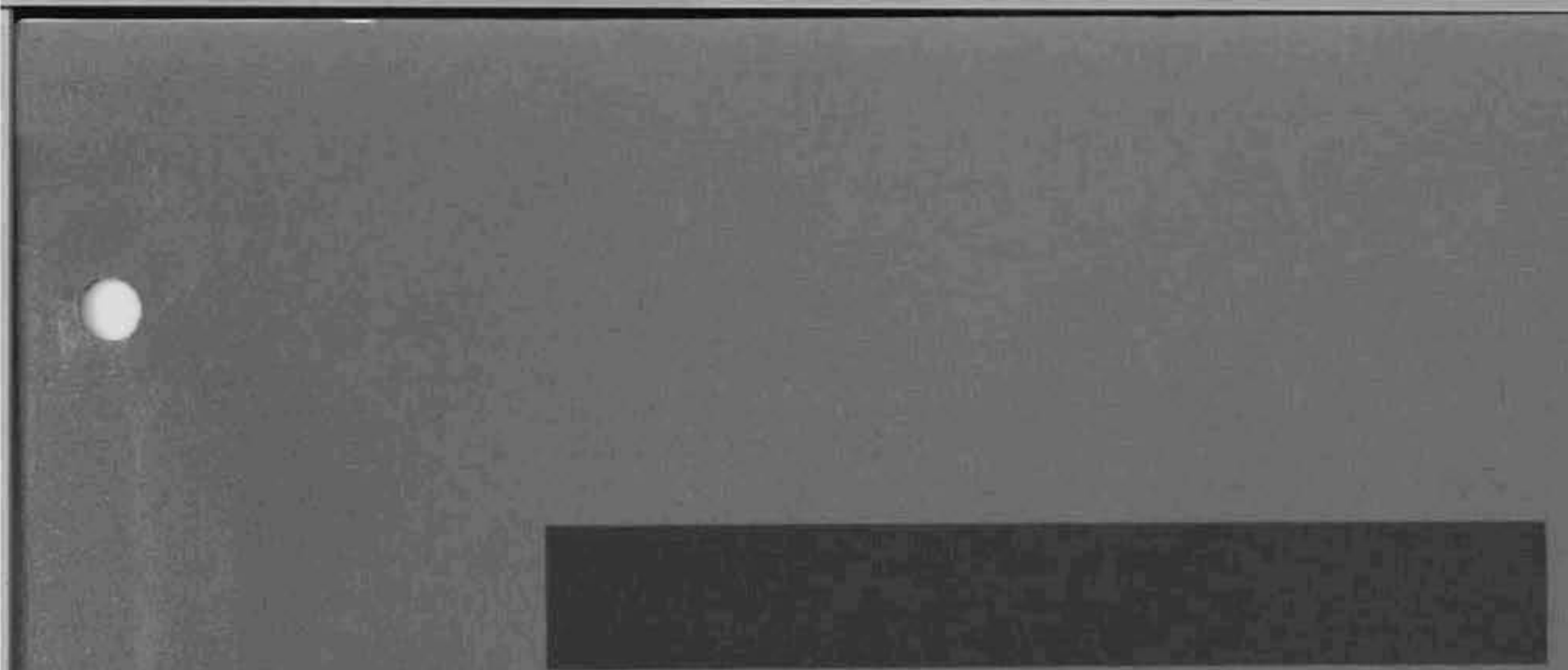

$+$

POWER REACTOR DESIGNS

\title{
AN ANNOTATED BIBLIOGRAPHY
}

VOLUME 2

SECTIONS FD-JF, INDEX

JANUARY, 1969

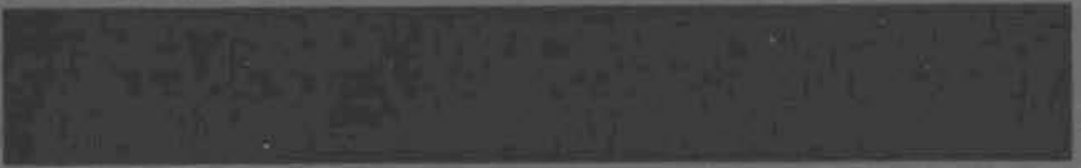

\section{AEC RESEARCH \& DEVELOPMENT REPORT}




\section{LEGAL NOTICE}

This report was prepared as an acsount of Government sponsored work. Neither the United States, nor the Commission, nor any person acting on behalf of the Commission:

A. Makes any warranty or tepresentation, expressed or implied, with respect to the accuracy, com. pleteness, or usefulness of the information contained in this report. of that the use of any information, apparctus, method, or process disclosed in this report may not infringe privately owned rights; or

B. Assumes any liabilities with respect to the use of, of for damages resulting from the use of any information, apporatus, method, or process disclosed in this report.

As used in the above, "person acting on behalf of the Commission" includes any employee or contractor of the Commission, or employee of such contractor, to the extent that such employee or con. fractor of the Commission, or employee of such contractor prepares, disseminates, or provides occess to, any information pursuant to his employment or contract with the Commission, or his employment with such contractor.

PACIFIC NORTHWEST LABORATORY

RICHLAND, WASHINGTON

operated by

BATTELLE MEMORIAL INSTITUTE

for the

UNITED STATES ATOMIC ENERGY COMMISSION UNDER CONTRACT AT(45-1)-1830 
BNWL-936 VOL2

UC-80, Reactor

Technology

\author{
POWER REACTOR DESIGNS \\ An Annotated Bibliography \\ Compiled by \\ E. R. Appleby \\ Technical Information
}

January 1969 
Printed in the United States of America Available from

Clearinghouse for Federal Scientific and Technical Information National Bureau of Standards, U.S. Department of Commerce Springfield, Virginia 22151

Price: Printed Copy $\$ 3.00 ;$ Microfiche $\$ 0.65$ 
NOTE: This document is three-hole punched so that it may be placed in a three-ring binder. 


\section{TABLE OF CONTENTS}

Text

\begin{tabular}{|c|c|}
\hline ETION & \\
\hline $\mathrm{FD}$ & LIQUID METAL REACTORS, DOMESTIC \\
\hline $\mathrm{FF}$ & LIQUID METAL REACTORS, FOREIGN \\
\hline $\mathrm{GD}$ & ORGANIC COOLED REACTORS, DOMESTIC \\
\hline GF & ORGANIC COOLED REACTORS, FOREIGN \\
\hline $\mathrm{HD}$ & PRESSURIZED LIGHT WATER REACTORS, DOMESTIC \\
\hline $\mathrm{HF}$ & PRESSURIZED LIGHT WATER REACTORS, FOREIGN \\
\hline ID & STEAM OR SUPERCRITICAL WATER COOLED REACTORS, DOMESTIC \\
\hline IF & STEAM OR SUPERCRITICAL WATER COOLED REACTORS, FOREIGN \\
\hline $\mathrm{JD}$ & NUCLEAR AUXILIARY POWER SYSTEMS, DOMESTIC \\
\hline $\mathrm{JF}$ & $\begin{array}{l}\text { NUCLEAR AUXILIARY POWER SYSTEMS, FOREIGN } \\
\text { INDEX }\end{array}$ \\
\hline
\end{tabular}



LIQUID METAL COOLED REACTORS

DOMESTIC 


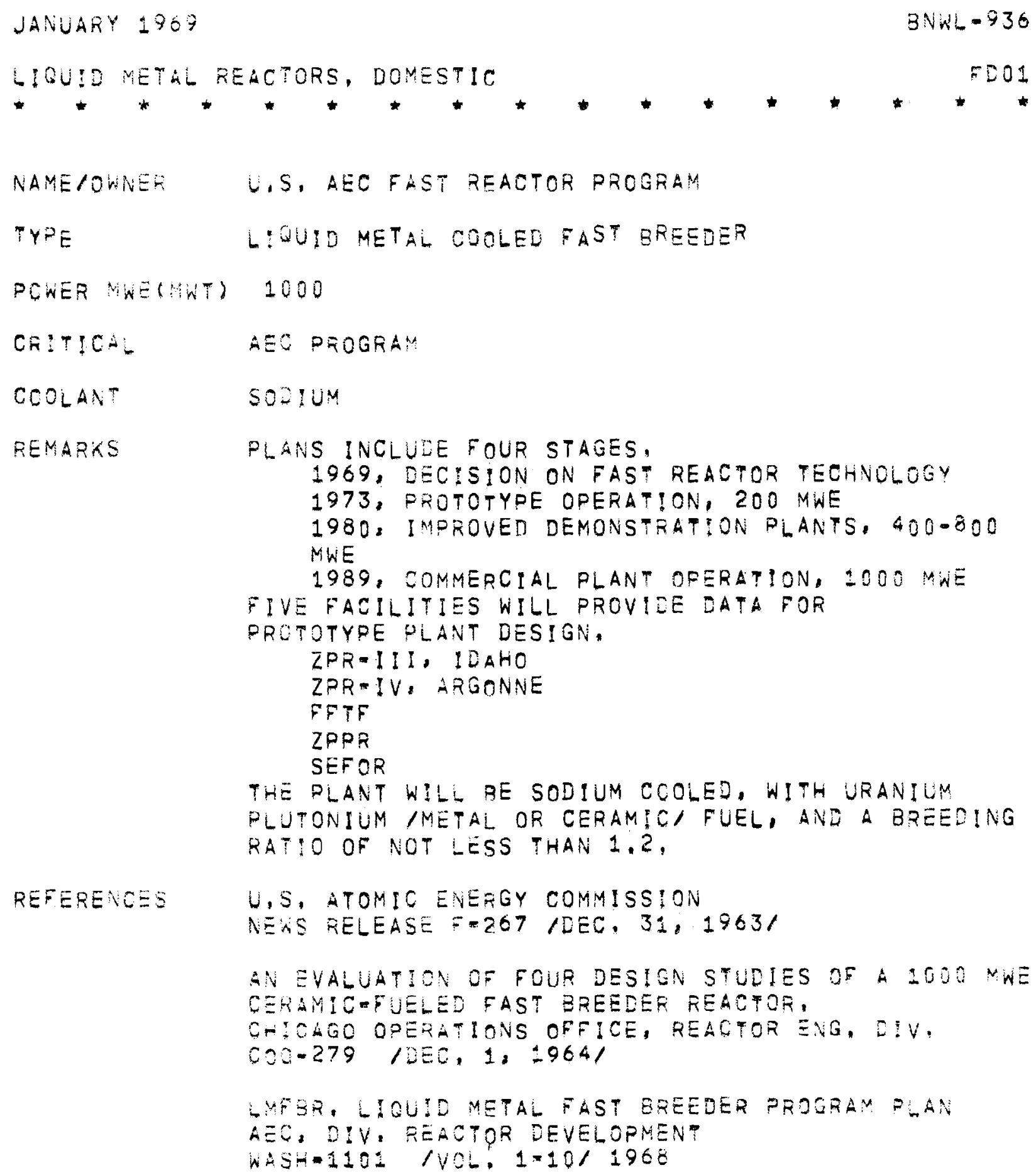


JANUARY 1969

$B N W L=936$

LIOUID METAL REACTORS, DOMESTIC

F002

NAME/OWNER LARGE FAST REACTOR STUDY/AEC

DESIGNER ALLISICHALMERS MANUFACTURING CO.

TYPE LIOUID METAL COOLED, ANNULAR CORE, FAST BREEDER

POWER MWE(MWT) $1000 \quad 2500$

CRITICAL DESIGN STUDY

COOLANT SODIUM

FUEL MATERIAL PLUTONIUMIURANIUM DIOXIDE PELLETS 0.241 IN, OD\%. $0: 1$ IN. ID. BLANKET DEPLETED URANIUM DIOXIDE 0,607 IN.DIA,

FUEL GEOMETRY PINS 48 IN. ACTIVE LENGTH + 6 IN. LOWER AXIAL BLANKET

FUEL CLADDING SS 0,028 IN, THICK. HELIUM BOND

FUEL ENRICH, AVE, 20,8 PER CENT U.235

FUEL ASSEMBLY 123-PIN ASSEMBLY, HEXAGONAL SUPPORT CAN /CORE/ 37 II IN ASSEMBLY /BLANKETY

498 FUEL ASSEMBLIES

858 BLANKET ASSEMBLIES

F.UEL CHARGE $3690 \mathrm{KG}$. FISSILE METAL. BLANKET 6 KG, SPECIFIC POWER $640 \mathrm{KW/KG} \mathrm{FISSILE}$

BURNUP (REFUEL) 100,000 MWD/TON SCATTER, 1,3 BATCH CORE AND 1,4 BATCH BLANKET

CONTROL RODS, BORON CARBIDE AND DEPLETED URANIUM DIOXIDE IN SS

COQLANT TEMP, INLET $950 \mathrm{~F}$ OUTLET $1200 \mathrm{~F}$

REACTOR VESSEL STAINLESS STEEL VESSEL $20 \mathrm{FT}, D D, 125$ FT, HIGH CONTAINMENT STEEL BUILDING

REMARKS, STUDY FOR AEC FAST REACTOR DEVELOPMENT PROGRAM.

REFERENCES LARGE FAST BREEDER DESIGN STUDY, FINAL REPORT, ALLIS-CHALMERS AND BABCOCK + WILCOX CO. $A C N P=64503$ /JAN: 1964/ 
JANUARY 1969

BNWL $=936$

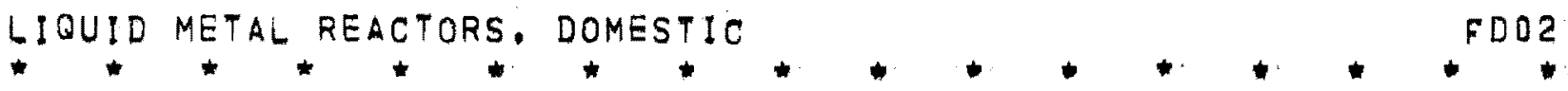

THE DESIGN OF A 1000 MWE FAST BREEDER REACTOR, CE KLOTZ, OTHERS

TRANS, ANS, JUNE 1964:

AN EVALUATION OF FOUR DESIGN STUDIES OF A 1000 MWE CERAMIC FUELED FAST BREEDER REACTOR,

REACTOR ENG, DIVIUS AEC

COO-279/DEC. 1, 1964 , 


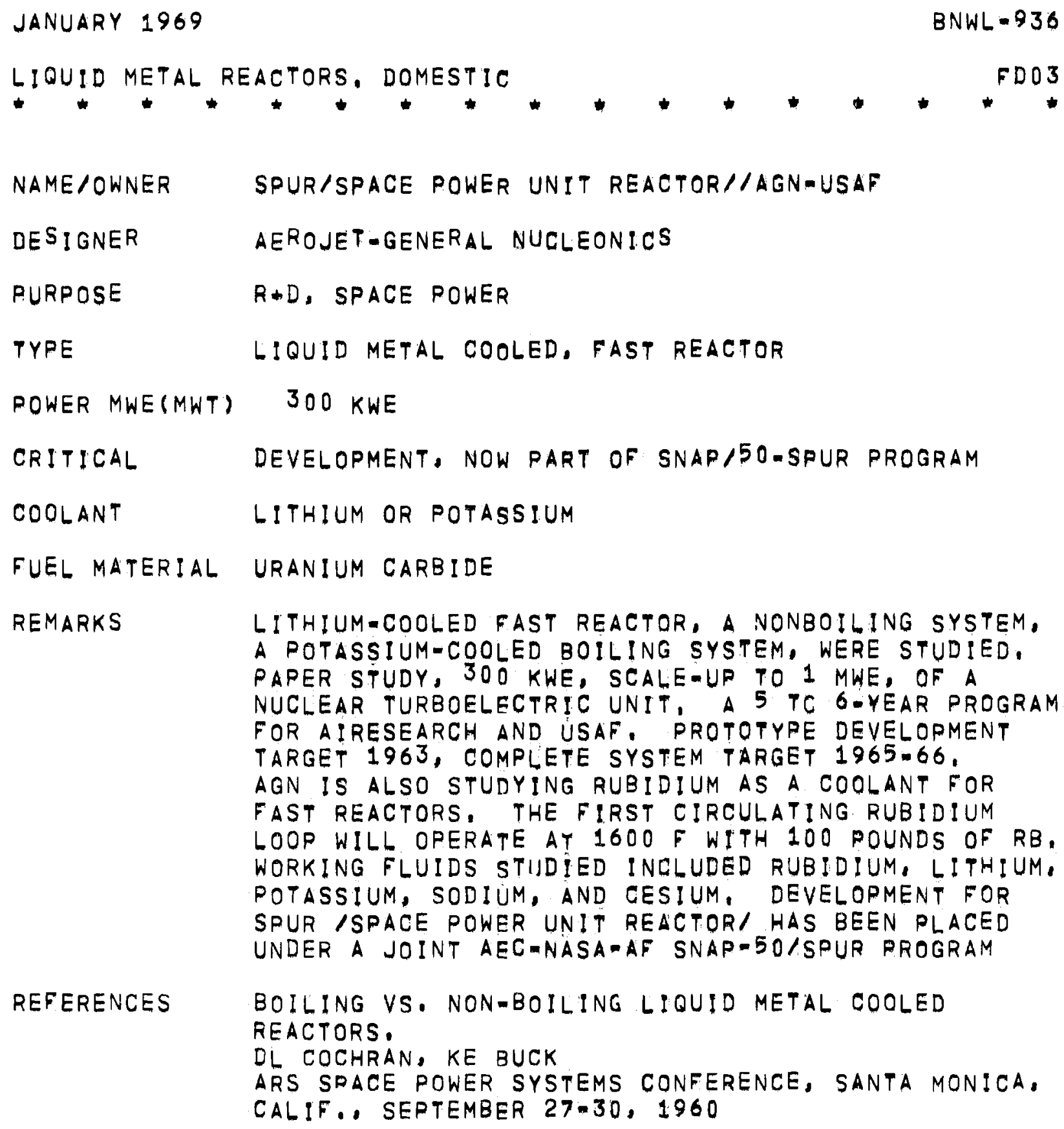


JANUARY 1969

$B N W L-936$

LIOUID METAL REACTORS. DOMESTIC

NAME/OWNER SRE/SODIUM REACTOR EXPERIMENT//AEC,AI,S,CAL, ED.

DESIGNER ATOMICS INTERNATIONAL

OPERATOR ATOMICS INTERNATIONAL, S. CALIF, EDISON

LOCATION SANTA SUSANA, CALIF:

PURPOSE POWER EXPERIMENT.

TYPE SODIUM=GRAPHITE THERMAL BREEDER

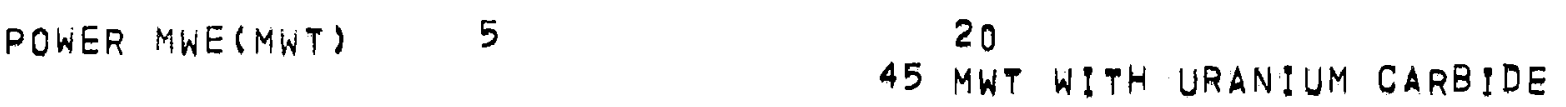

CRITICAL $\quad 1957$, CORE 3 IN 1963

SHUT DOWN 1964

COQLANT SODIUM

MODERATOR GRAPHITE, ZIRCONIUM ALLOY CANS

REFLECTOR, GRAPHITE

FUEL MATERIAL CORE I URANIUM SLUGS 0.75 IN, DIA./6 IN. LONG CORE 2 CONTAINED THORIUM/URANIUM ALLOY

CORE 3, URANIUM CARBIDE FUEL

FUEL GEOMETRY RODS

FUEL CLADDING SS 0.01 IN. THICK. SODIUM=POTASSIUM BOND

FUEL ENRICH, CORE $1,2.8$ PER CENT U-235

CORE 2, 93.1 PER CENT

CORE 3, 5.2 PER CENT

FUEL ASSEMBLY CORE 1, 7-ROD CLUSTER, 43 ASSEMBLIES

CORE 2, 5-ROD CLUSTER

CORE 3, 9-ROD CLUSTER, 33 ASSEMBLIES

FUEL CHARGE CORE 1, $3000 \mathrm{KG}$, URANIUM

SPECIFIC POWER $240 \mathrm{KW/KG} \mathrm{U-235}$

BURNUP(REFUEL) 2500 MWD/T 300 FULL-POWER DAYS

NEUTRON FLUX THERMAL AVE, $2 \times 10 E+13$

WITH URANIUM CARBIDE ELEMENTS 10 E $\$ 14$

CONTROL BORON=NICKEL ALLOY RODS

COOLANT TEMP, INLET $650 \mathrm{~F}$ OUTLET $1200 \mathrm{~F} / \mathrm{CORE} 3 /$ 
JANUARY 1969

LIOUID METAL REACTORS, DOMESTIC
$B N W L=936$

$F D 04$

\section{COOLANT PRESS, INLET 6 PSIG OUTLET 3 PSIG}

REACTOR VESSEL FLAT-BOTTOMED OPEN TOP CYLINDER, SS, 1,5 IN, THICK $11 \mathrm{FT}$, ID, $118,87 \mathrm{FT}$ : HIGH, OUTER TANK CARBON STEEL

REMARKS

REACTOR INSTABILITY WAS NOTED AT 2 MWT OPERATION

DUE TO THERMAL BOWING OF THE FUEL ELEMENTS. CONSTRAINT WAS IMPOSED BY MEANS OF A HELICAL WIRE WRAP AROUND THE FUEL CLUSTERS, RESULTING IN A REDUCED POWER COEFFICIENT, FUEL ELEMENT MODIFICATIONS WERE COMPLETED IN JANUARY 1962!, IN WULY 1963 THE SRE CRITICAL ASSEMBLY ACHIEVED CRITICALITY WITH A FULL CORE LOADING OF 24 UE ELEMENTS, AND THE SRE PRODUCED POWER, ALSO IN JULY 1963, WITH A FULL-SCALEE UC ELEMENT IN THE CORE. THE AEC HAS ANNOUNCED THAT IT WILL DEACTIVATE THE REACTOR RATHER THAN CONVERT IT TO HIGHE. TEMPERATURE TESTING OF BREEDER FUELS AND MATERIALS; MODIFICATIONS TO RAISE THE POWER FROM 15 MWT TO 30 MWT HAVE BEEN COMPLETED, BUT THE REACTOR WAS NOT OPERATED AFTER SHUT=DOWN IN 1964 ,

REFERENCES OPERATING EXPERIENCE WITH THE SODIUM REACTOR EXPERIMENT, FE FARIS, OTHERS

SECOND U. N, INTL: CONF, ON THE PEACEFUL USES OF ATOMIC ENEROYY 9, 493-509/1958/

SRE OPERATES AGAIN WITH CORE 2 BUT WITH 5 MWT PONER LIMIT,

NUCLEONICS 20, 12 /JANUARY 1962 ,

PROCEEDINGS OF THE SYMPOSIUM ON SOLIUM REACTORS TECHNOLOGY, MAY 24.25; 196:, LINCOLN, NEBRASKA. TID.7623/JUNE 1962 /

A 200 MWE PROTOTYPE SODIUM COOLED REACTOR HIGH EFFICIENCY POWER PLANT

JA FALCON, PR KEEP

CONF-51-4 /AMERICAN PUBLIC POWER ASSOCIATION

ANVUAL CONVENTION, CLEVELAND, APR:L-MAY 1963,

NUCLEONICS WEEK DEC: 6, $1966 \%, 2$ NEWS RELEASE 


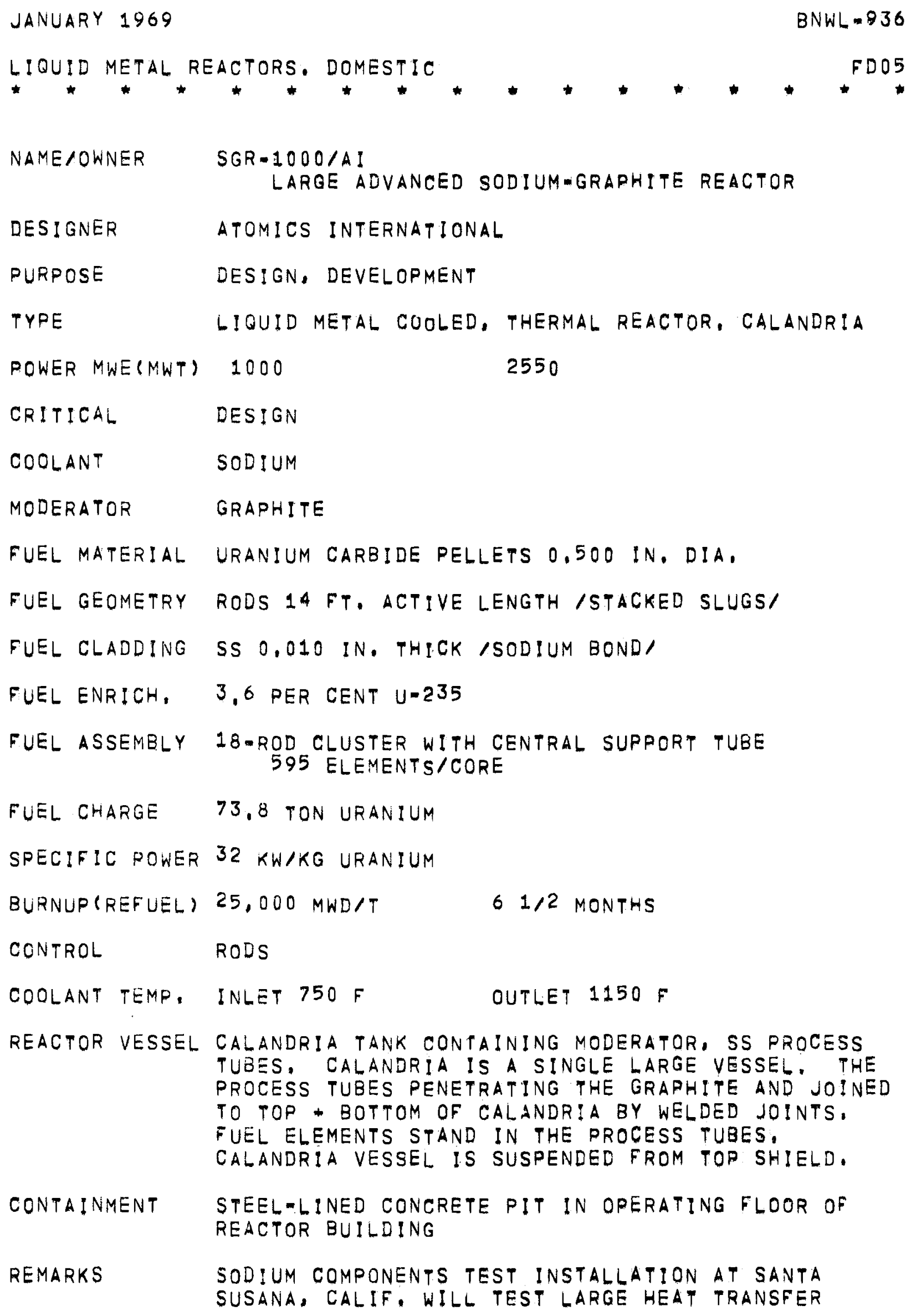




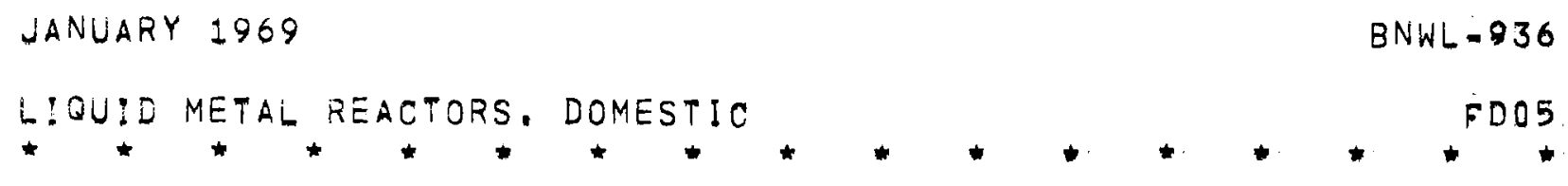

SYSTEM COMPONENTS; THE STEAM GENERATORS ARE MODULAR UNITS. DESIGN FOR A SINGLE-LOOP, 200 MWE SGR HAS BEEN COMPLETED.

REFERENCES DESIGN, PERFORMANCE: AND ECONOMIC EVALUATION OF SOD!UM HEATED, MODULAR SUPERCRITICAL STEAM GENERATORS, DF CASEY: OTHERS NAA-SR= 8508

SOD:UM-COOLED THERMAL REACTORS, C STARR, OTHERS THIRD U,N: INTL: CONF: ON THE PEACEFUL USES OF ATOMIC ENERGY, GENEVA, 1964, A/CONF,28/P/206

AN OPTIMIZED CORE DESIGN FOR A 1000 MWE SODIUM:GRAPHITE REACTOR. iH ROOD

TRANS. ANS, /JUNE $1964 /$

A 200 MWE PROTOTYPE SODIUM COOLED REACTOR HI UHH EFFICIENCY POWER PLANT: SA FALCON, PR KEEP CONF-51-A /AMER: PUB, POWER ASSOC, ANNUAL CONVENTION, CLEVELAND, APRIL 1963,

200 MWE PROTOTYPE LARGE SGR REACTOR, STRUCTURE DESIGN AND EVALUATION

DR LNDERSON: KW FOSTER - EDS. $N A A-S R=11314 / 1965 /$

1000 MWE SGR AND PROTOTYPE EVALUATION STUDY. ATOMICS INTERNATIONAL

$N A A=S R=9213$

FEASIBILITY STUDY OF A 1000-MWE SODIUM-COOLED FAST REACTOR DT EGGEN, HA MOREWITZ

NAA-SR-11378 /VOL, 3, METHODS DEVELOPMENT/ 


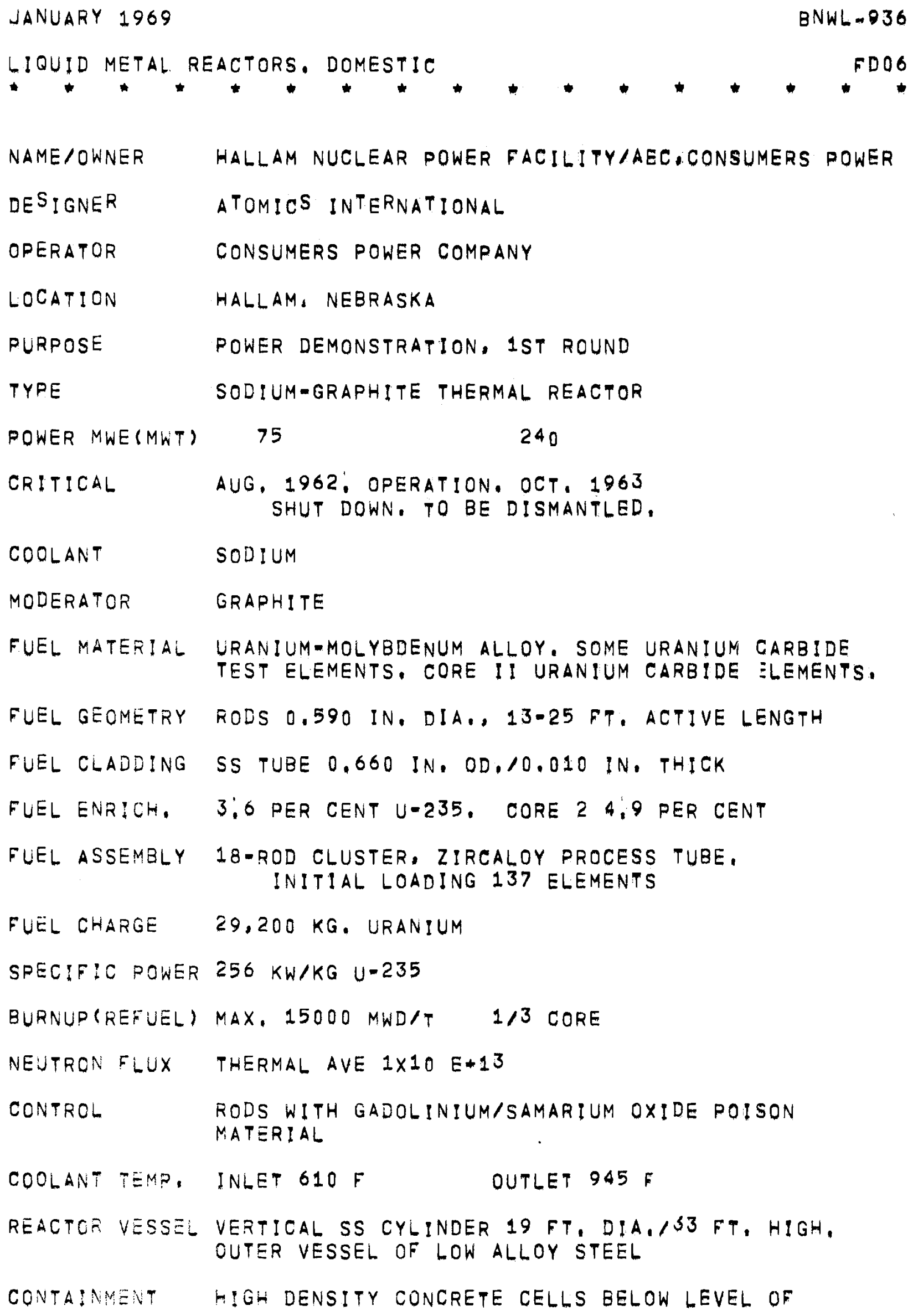




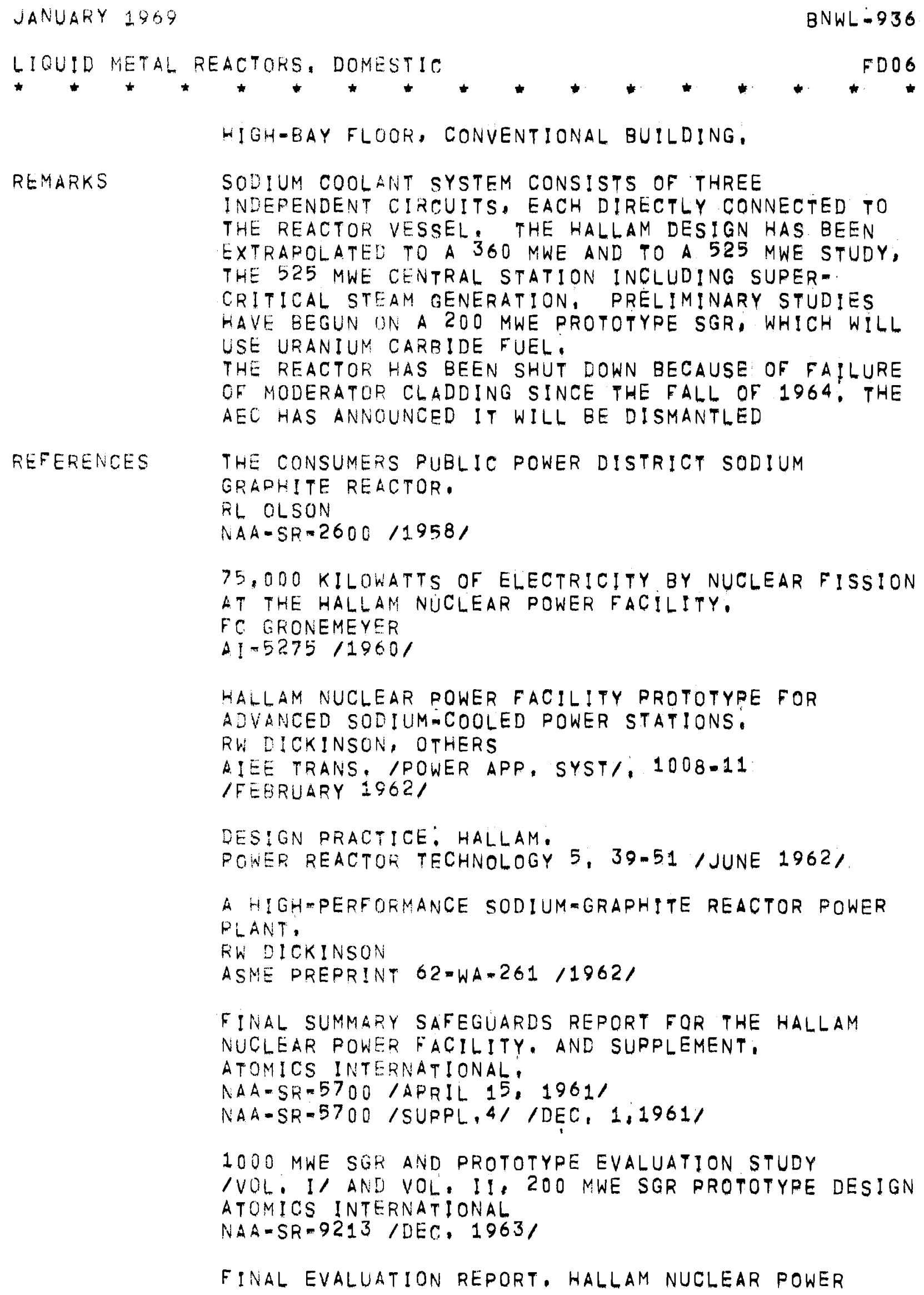




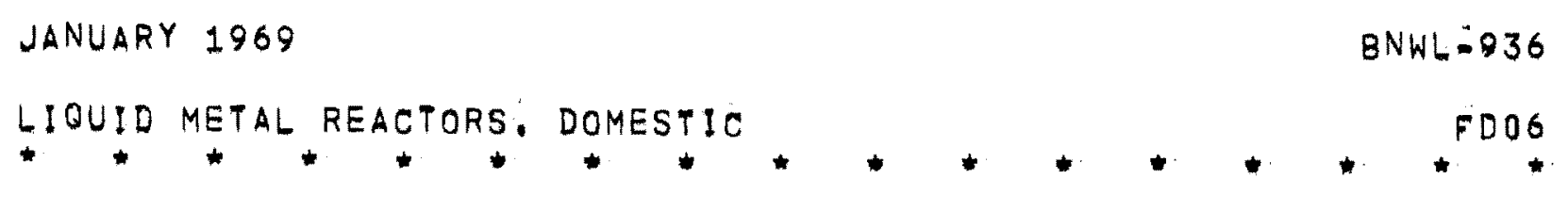

FACILITY.

JE PRICE, COMP,

NAA-SR=9777 /VOLS: 1-6/ /SEPT: 29; 1964/ 


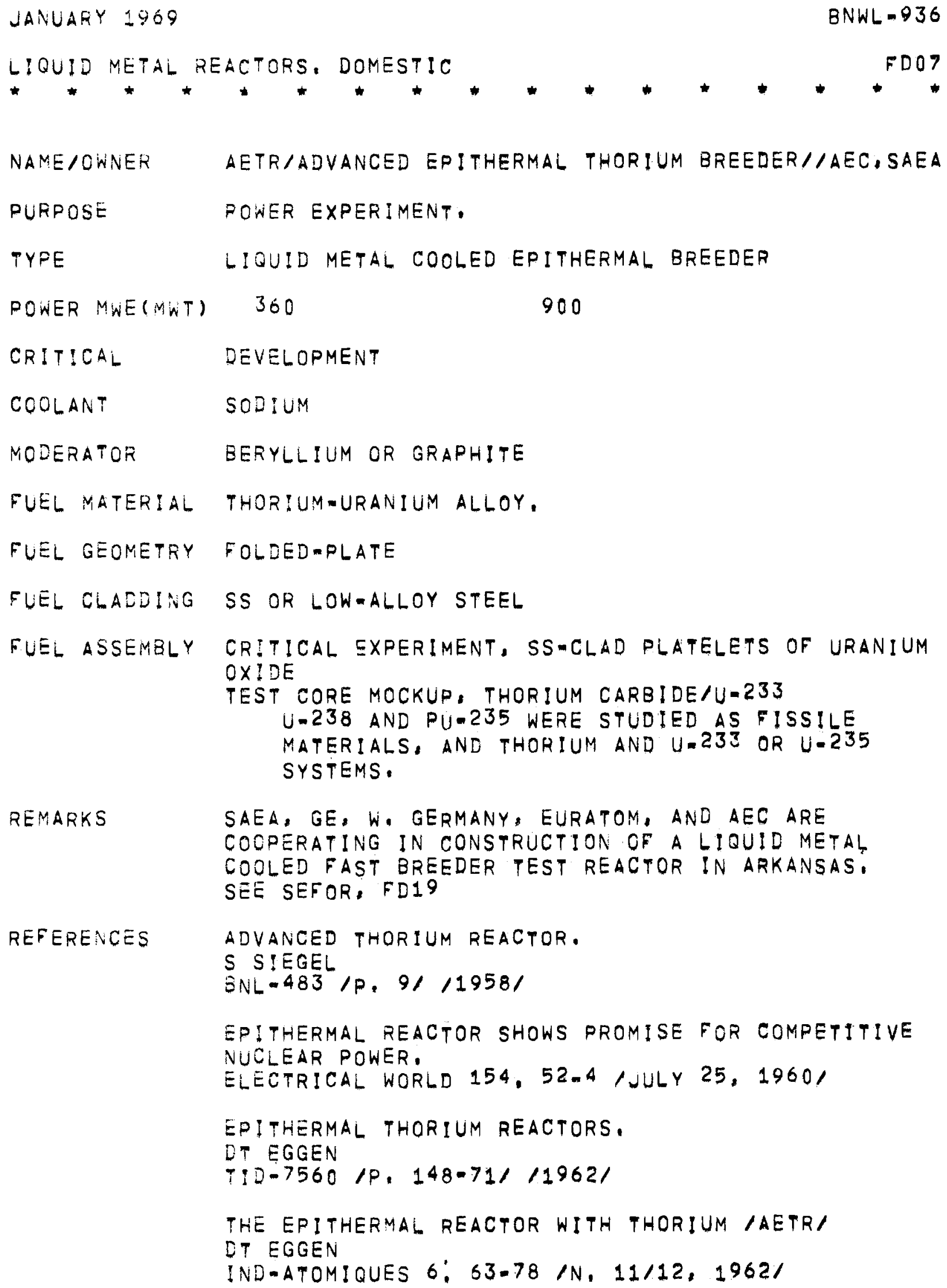




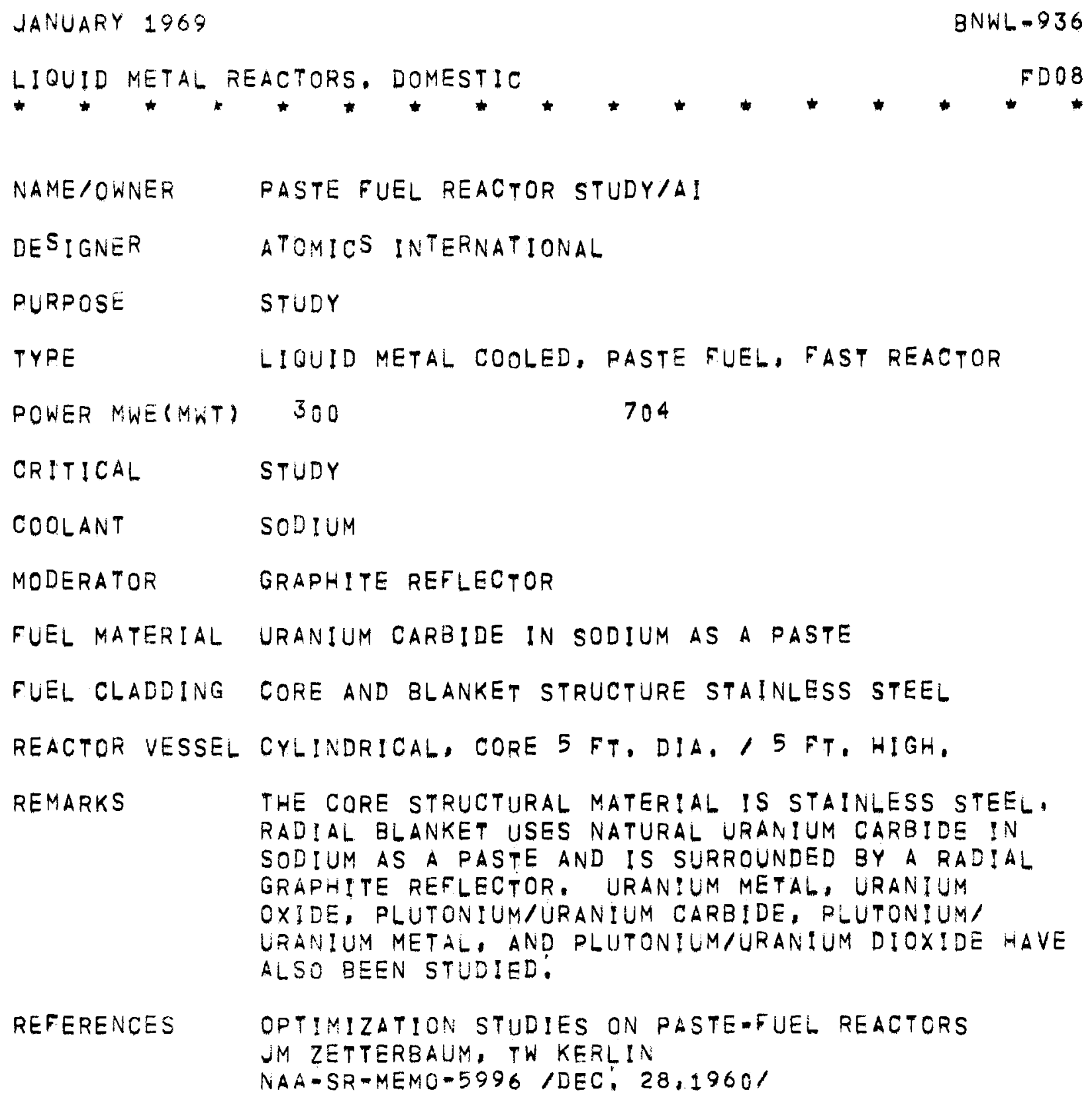


JANUARY 1969

BNWL: :036

LIOUID METAL REACTORS: DOMESTIC

NAME / OWNER

EBR-1 /EXP. BREEDER REACTOR-1//AEC

DES \GNER

ARGONNE NATIONAL LABORATORY

OPERATOR

ARGONNE NATIONAL' LABORATORY

LOCATION

NATIONAL REACTOR TEST STATION: IDAHO

PURPOSE

POWER EXPERIMENT

TYPE

LIQUID METAL COOLED; FAST BREEDER

POWER MWE(MWT)

0,2

$1 ; 4$

CRITICAL

MARK 1; 1951.

MARK 4, 1962

RETIRED DEC, 1963

COOLANT

SODIUM-POTASSIUM NAK

MODERATOR

URANIUM REFLECTOR

FUEL MATERIAL URANIUM-ZIRCONIUM SLUGS: SEE REMARKS

FUEL GEOMETRY ROD:' SEE REMARKS

FUEL CLADDING SS, 0,022 IN, THICK: SEE REMARKS

FUEL ENRICH, 93,2 PER CENT U:235: SEE REMARKS

FUEL ASSEMBLY SINGLE ROD ELEMENT, 217 TO CORE: SEE REMARKS

SPECIFIC POWER $23 \mathrm{KW} / K G \quad U-235$

BURNUP (REFUEL) $0: 3$ PER CENT

NEUTRON FLUX FAST AVE, $0.8 \times 10^{\circ}$ E+14

CONTROL MOVABLE URANIUM REFLECTOR

COOLANT TEMP: INLET $442 \mathrm{~F}$ OUTLET $601: \mathrm{F}$

COOLANT PRESS: 13 PSIG

REACTOR VESSEL SS VESSEL; $15: 87$ IN: ID:

CONTAINMENT CONCRETE SHIELDING, CONVENTIONAL BUILDING:

REMARKS

THE MARK-3 CORE HAD FUEL RODS ASSEMBLED INTO 


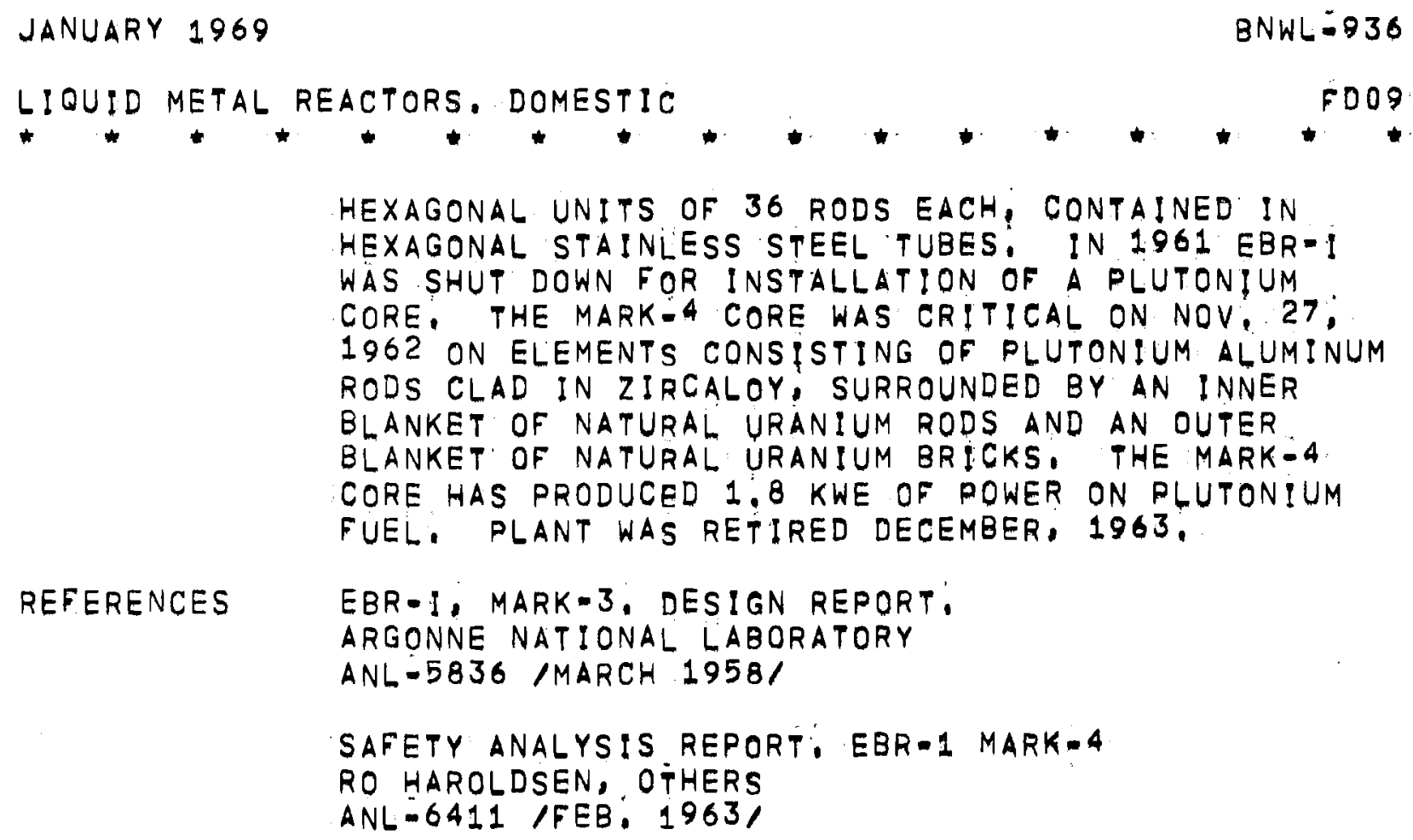




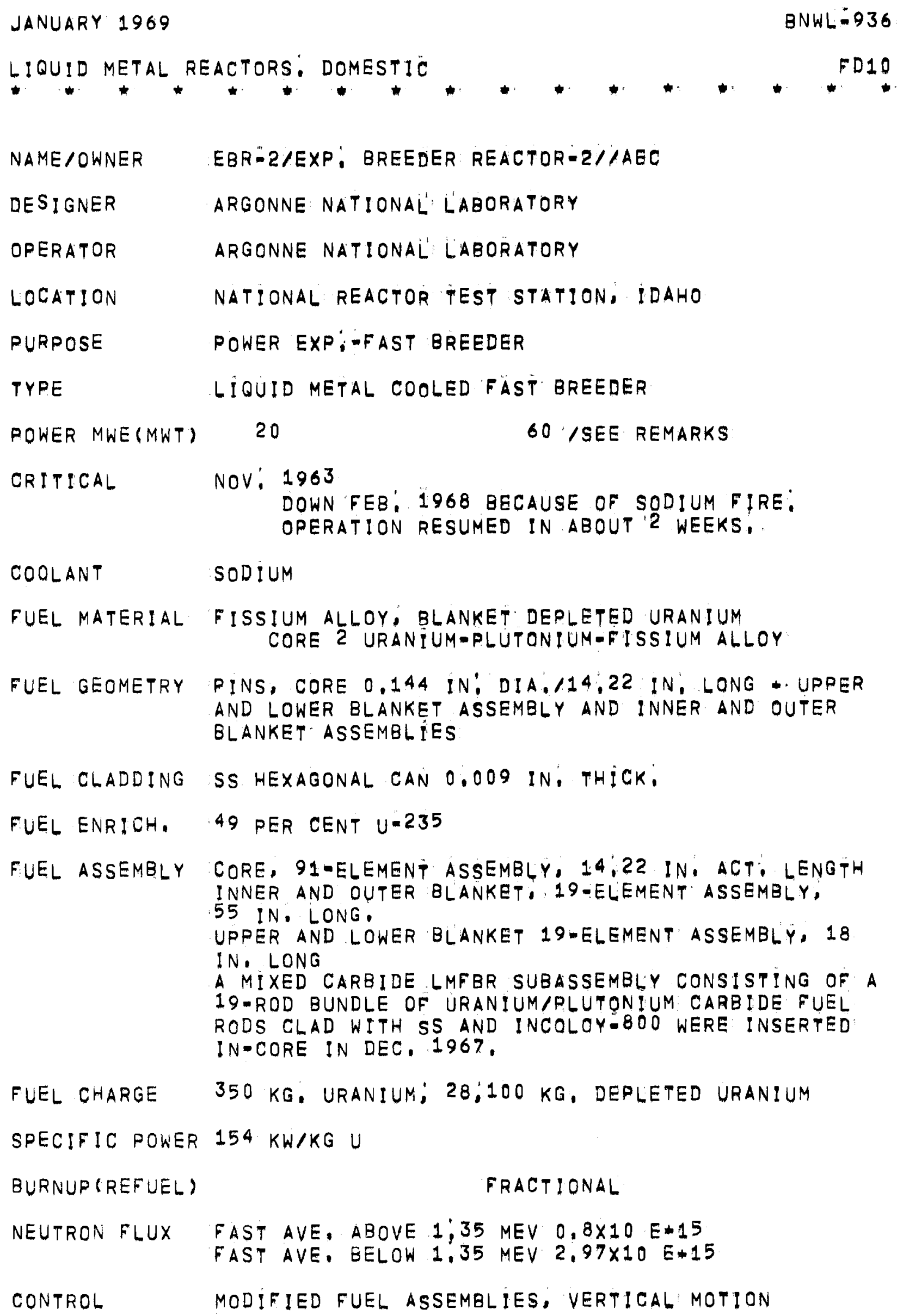




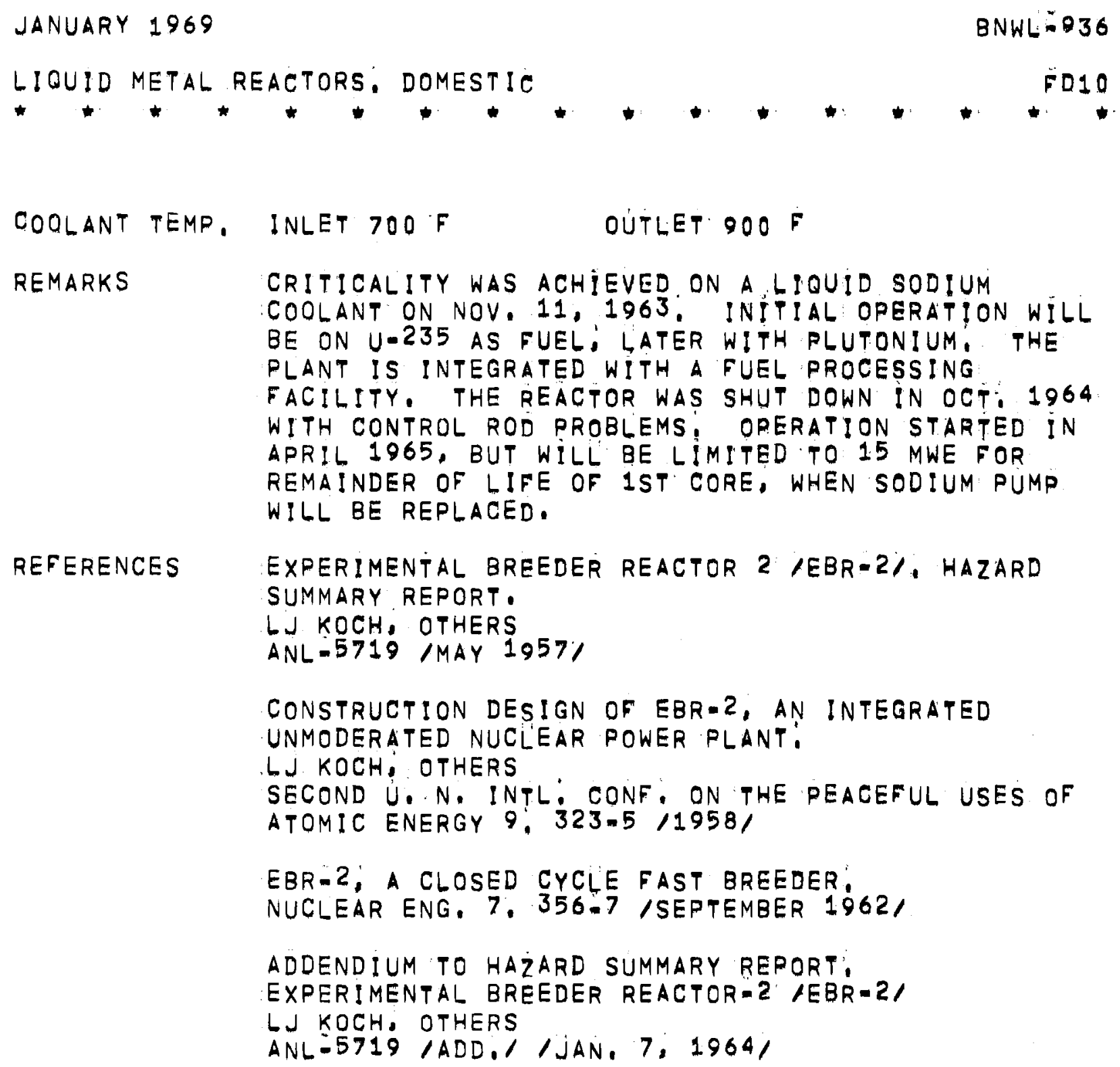




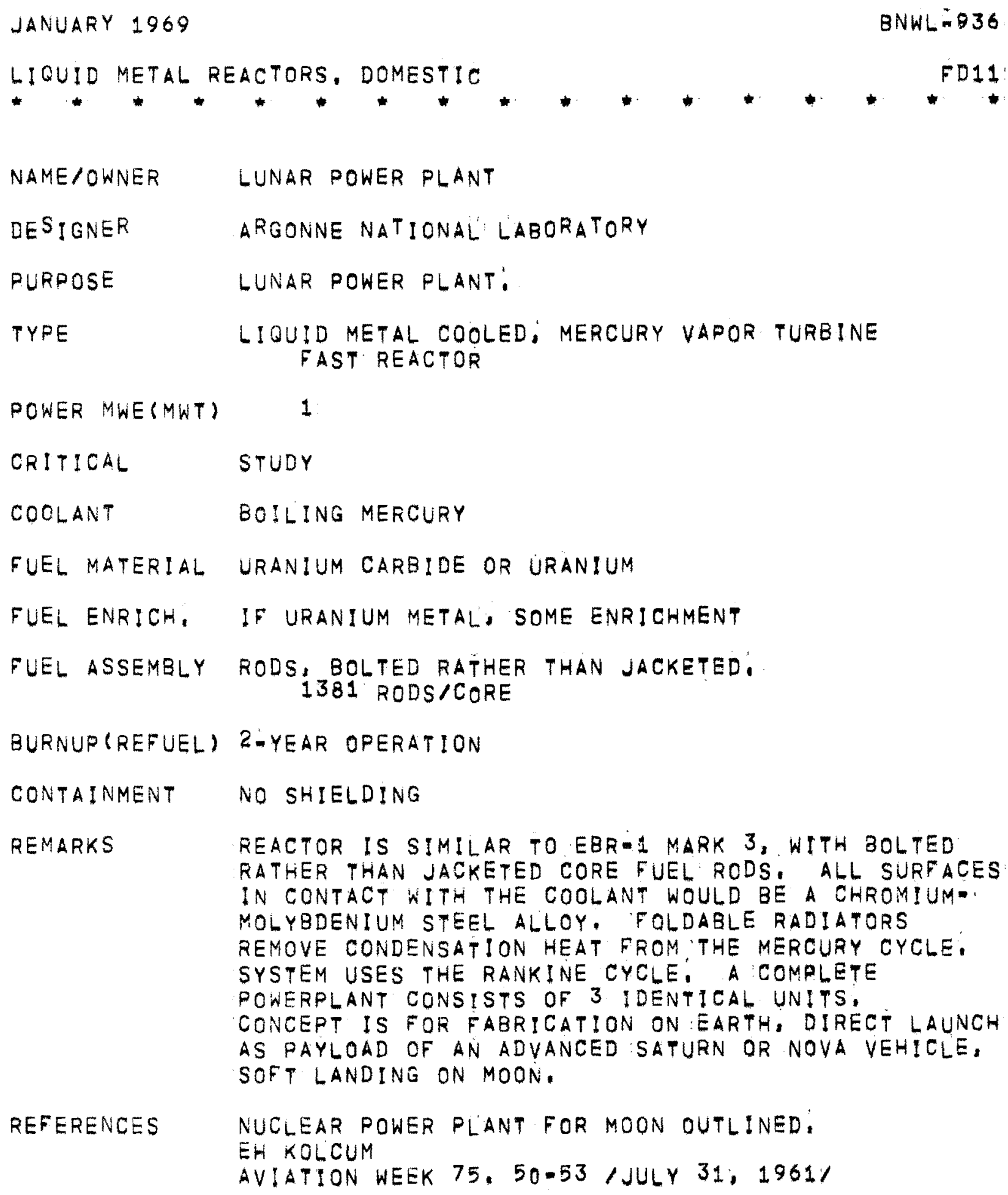


BANUARY 1969
LIOUID METAL REACTORS. DOMESTIC $+\cdots+\cdots+\cdots+\cdots$

NAME/OWNER

$D E S$ I $N E R$

OPERATQR

LOCATION

PURPOSE

TYPE

POWER MWE (MWT)

CRITICAL

COOLANT

FUEL MATERIAL

FUEL GEOMETRY RODS

FUEL ASSEMBLY CERAMiC FUEL, 37-ELEMENT ASSEMBLy

24 ASSEMALIES

ALLOY FUEL, G1:ELEMENT ASSEMBLY

8 ASSEMBLIES

CARBIDE FUEL, 16 ASSEMBLIES

COOLANT TEMP, INLET 600-960 F OUTLET 840-1200 F

REACTOR VESSEL DOUBLE-WALLED OUTER VESSEL AS AN INTEGRAL PART OF THE HEAT TRANSFER SYSTEM, AN INNER CORE-SUPPORT STRUCTURE AND REMOVABLE COVER,

CONTAINMENT

REMARKS

REFERENCES
REINFORCED-CONCRETE STEEL-LINED CELL, NORMALLY

FILLED WITH HIGH PURITY INERT GAS,

FARET HAS GEEN DROPPED IN FAVOR OF THE LARGER FFTF FAST FLUX TEST FACILITY. SEE FD 30

FARETIA FAST-REACTOR TEST FACILITY

A SMAARDYK, OTHERS

TRANS: ANS 5,435.6 /NOV, $1962 /$ /ABSTRACT/

NUCLEONICS WEEK DEC: 2, 1965 P, 1

NEWS RELEASE 
JANUARY $\$ 969$

BNWL:-936

LIOUID METAL REACTORS, DOMESTIC $* * * * * * * * * * *+*$

FAST REACTOR TEST FACILITY FARET

VOL, 1 ; DESCRIPTION AND PROQRAM

ARGONNE NATIONAL LAB.

ANL-7168, VOL.1 /MARCH 1966/

FARET CORE-1 REFERENCE DESIGN:

PJ PERSIANI, OTHERS

ANL-7120 P. 766-75/1966/ 
JANUARY 1969

$B N W L=936$

LIOUID METAL REACTORS, DOMESTIC

NAME /OWNER

ENRICO FERMI ATOMIC POWER PLANTIPRDC

DES ! GNER

ATOMIC POWER DEVELOPMENT ASSOCIATES, INC:

OPERATOR

POWER REACTOR DEVELOPMENT COMPANY

LOCATION

MONROE; MICHIGAN

PURPOSE

POWER DEMONSTRATION

TYPE

LIOUID METAL COOLED FAST BREEDER

POWER MWE (MWT)

60

200

INITIAL 110 MWT

CRITICAL

AUG: 1963: SEE REMARKS

COOLANT

SODIUM

FUEL MATERIAL URANIUM-MOLYBDEÑUM ALLOY

FUEL GEOMETRY ROD: CORE, ACT: LENGTH 30.5 IN', UPPER AND LOWER AXIAL BLANKET 14:0 IN LONG RADIAL BLANKET O1,75 IN, LONG

FUEL CLADDING CORE ZIRCONIUM 0,005 INI THICK BLANKET SS 0.005 IN: THICK

FUEL ENRICH, 25,6 PER CENT U:-235

BLANKET DEPLETED URANIUM

FUEL ASSEMBLY CORE 1

105-PIN ASSEMBLY, CORE AND BLANKET ASSEMBLIES ENCLOSED IN SS TUBE, PLUS RADIALI BLANKET ASSEMBLIES.

FUEL CHARGE $2000 \mathrm{KG}$ : URANIUM

SPECIFIC POWER $675 \mathrm{KW} / \mathrm{KG} \mathrm{U-235}$

BURNUP(REFUEL) 48,800 MWD/T

NEUTRON FLUX $1: 6 \times 10 \quad E+15$

CONTROL RODS

COOLANT TEMP, INLET $550 \mathrm{~F}$ OUTLET $800 \mathrm{~F}$

COOLANT PRESS, INLET 82 PSIG

REACTOR VESSEL SS VESSEL 14 FT: 2 IN: ID, ISODIUM POOL/. 
JANUARY 1969

BNWL-936

LIQUID METAL REACTORS. DOMESTIC

FD13

VESSEL IS IN TWO PARTS, THE LOWER CONTAINING THE

CORE, VESSEL IS ENCLOSED IN A CARBON STEEL

LEAK-TIGHT VESSEL:

CONTAINMENT

WELDED STEEL BUILDING:

REMARKS

INITIAL CRITICALITY IN AUG, 1963 WAS FOLLONED BY A PROGRAM OF LOW-POWER TESTS LEADING UP TO $200 \mathrm{MWT}$ THE FERMI REACTOR WILL BE OPERATED AT A SUSTAINED 110 MWE WITH CORE A: TEST FUELS AND MATERIAL SPECIMENS WILL BE TESTED. FERMI WAS SHUT DOWN OCT, 5, 1966 FOLLOWING A MALFUNCTION PROBABLY INVOLVING FUEL MELTING IN ONE OR MORE SUBASSEMBLIES, SIGNIFICANT DAMAGE IS THOUGHT TO HAVE OCCURED, STARTUP IS EXPECTED AGAIN IN 1968-1969,

REFERENCES DESCRIPTION OF DEVELOPMENTAL FAST NEUTRON BREEDER PONER REACTOR PLANT APDA-108/SEPTEMBER 1955/

ENRICO FERMI ATOMIC POWER PLANT: A,P, DONNELL, OTHERS

SECOND U. N. INTL: CONF; ON THE PEACEFUL USES OF ATOMIC ENERGY 8;,535-42/1958;

ENRICO FERM! POWER PLANT: APDA-124/JANUARY 1959/

CIVILIAN POWER REACTOR PROGRAM, PART 3 , STATUS REPQRT ON FAST REACTORS AS OF 1959,

$T ! D-8518 / 1 /$ BOOK I

ENRICO FERMI ATOMIC POWER PLANT REVISED LICENSE APPLICATION, PART B, TECHNICAL INFORMATION AND HAZARDS SUMMARY REPORT: POWER REACTOR DEVELOPMENT COMRANY NP-10458/JULY 1961 ,

A REPORT ON FERMI:

OJ DU TEMPLE

NUCLEAR NEWS /ANS/ 7, 28-33 /JAN: $1964 /$

THE ENRCO FERMI FAST NEUTRON BREEDER, DESCRIPTION AND OPERATION

$J: F$, ANDERSON /PRDC/

CONF-631201/P, $9-16 /$

PROC, FAST NEUTRON BREEDER REACTOR CONF, DEC: 1963

ENRICO FERMI POWER PLANT:IRRADIATION TEST 


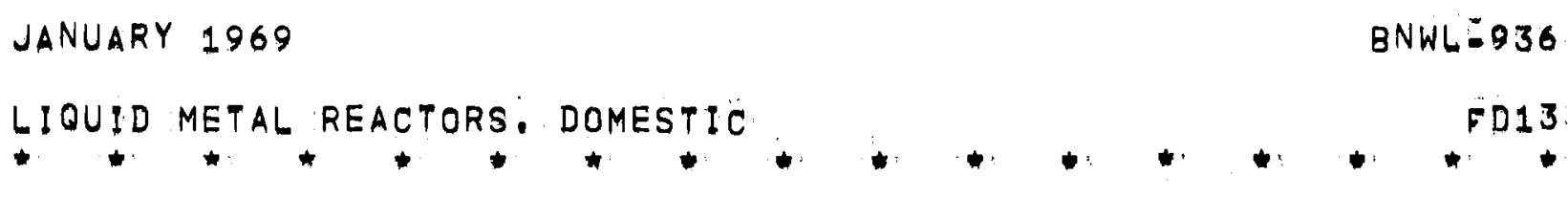

FACILITY

PR HUEBOTTER, WH JENS

ASME $65-W A / N E-5$

NUCLEONICS WEEK OCT; 13; 1966 P: 1:

NEWS RELEASE 
BNANUARY 1969
LIOUID METAL REACTORS, DOMESTIC

NAME/OWNER PASTE FUEL REACTOR/APDA

DESIGNER ATOMIC POWER DEVELOPMENT ASSOCIATES, INC.

PURPOSE POWER EXP: STUDY

TYPE LIQUID METAL COOLED; FAST BREEDER

POWER MWE(MWT) 300

CRITICAL DESIGN

COOLANT SODIUM

FLEL MATERIAL PLUTONIUM-URANIUM-FISSIUM EQUILIBRIUM MIXTURE IN SODIUM

BLANKET, URANIUM-238/MOLYBDENUM

FUEL GEOMETRY PASTE

FUEL ASSEMBLY CORE AND BLANKET SHELL-ANB-TUBE WITH FUEL PASTE

ON SHELL SIDE AND COOLANT ON TUBE SIDE,

DIVISION IS INTO 19 CORE AND IR BLANKET

SUBASSEMBLIES

SPECIFIC POWER $2000 \mathrm{KW} / \mathrm{KG}$

COOLANT TEMP, INLET $600 \mathrm{~F}$ OUTLET $900 \mathrm{~F}$

REACTOR VESSEL SS VESSEL $133 / 4$ FT. HIGH, 5:5 FT, DIA.

REFERENCES SUMMARY OF THE APDA FUEL DEVELOPMENT PROGRAMS. WG BLESSING, OTHERS

APDA-143 /APRIL $1961 /$

CONCEPTUAL DESIGN OF A 300 MWE PASTE FUELED FAST

BREEDER POWER REACTOR

ATOMIC POWER DEV. ASSOC:

APDA.146 /OCT, 1961/

MOBILE-FUELED FAST REACTORS

EC KOVACIC

FAST REACTOR TECHNOLOGY, NATIONAL TOPIOAL MEETING APRIL 26-28, 1965: AMERICAN NUCLEAR SOC. ANS-100 /P. $75.81 /$ 


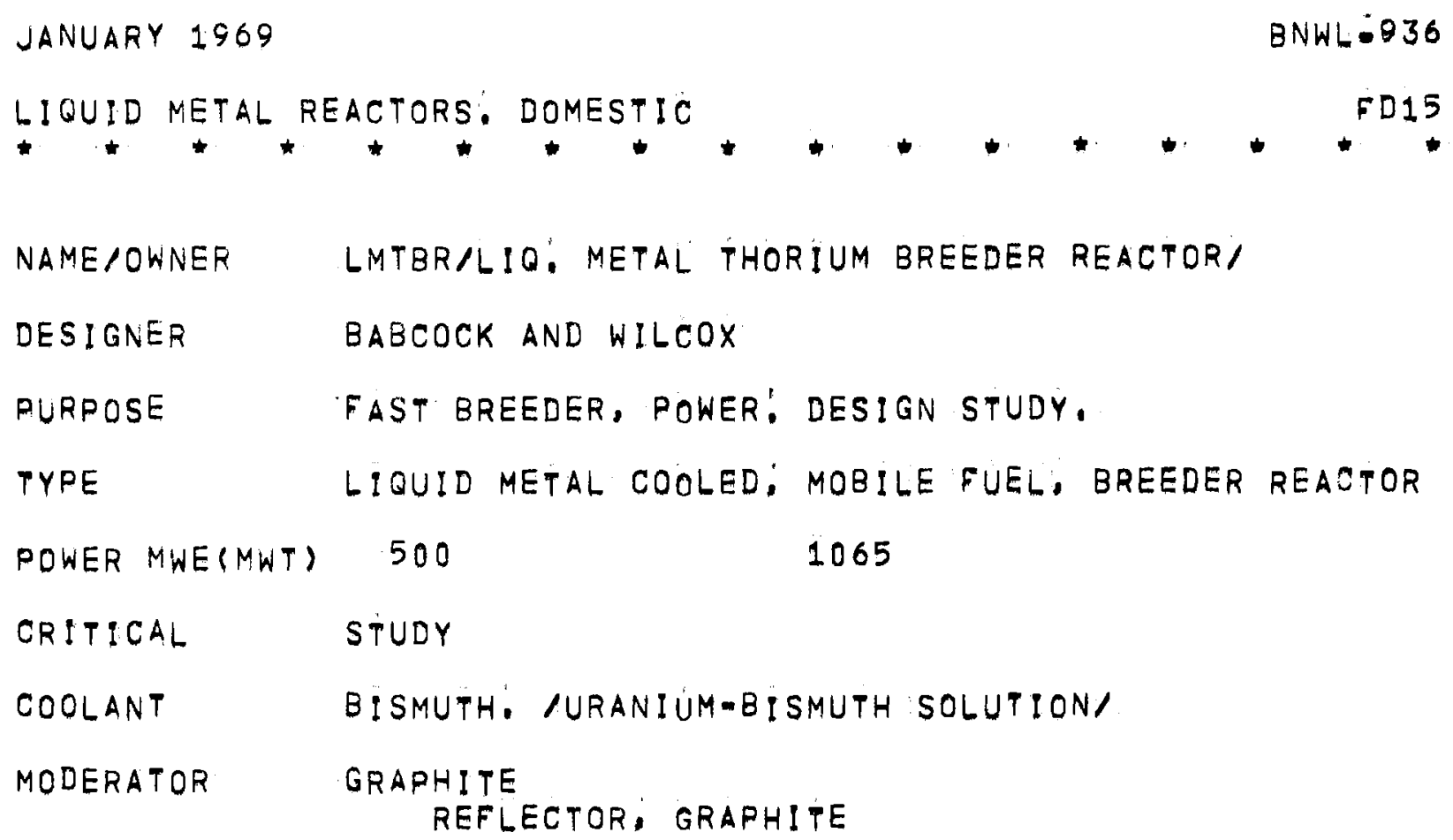


JANUARY 1969

BNWL $=936$

LIOUID METAL FEACTORS. DOMESTIC FD15

CORE FLUID HEAT EXCHANGER /TUBE-SIDE/: BLANKET FLUID FLOWS UPWARD FROM THE INNER BOTTOM PLENUM, THROUGH CRUCIFORM CHANNELS IN THE CORE AND THE

INNER SIDE-BLANKET CHANNELS, UP THROUGH TOP END-BLANKET AND HORIZONTAL PASSAGES TO TOP INNER PLENUMS. REFERENCE DESIGN IS FOR A TWO-REACTOR 11000 MWE/ PLANT. CORE FLUID HEAT EXCHANGER IS A BAYONET-TYPE TANTALUM UNIT, BLANKET FLUID EXCHANGER HASTELLOY-X AND TANTALUM,

REFERENCES THE LIQUID METAL THORIUM BREEDER REACTOR: BABCOCK W WILCOX CO: $B A N=1171 / 1960 /$ 


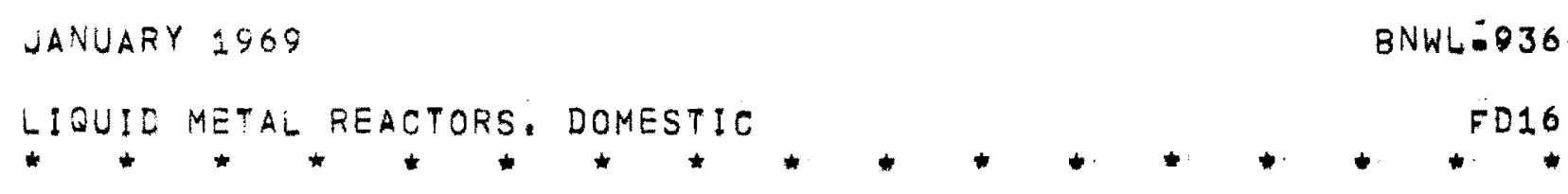

NAME/OWNER SETTLED BED FAST REACTOR/BNL SBFR

DESIGNER GROOKHAVEN NATIONAL LABORATORY

PURPOSE PRELIMINARY STUDY, FAST BREEDER POWER REACTOR,

TYPE LIOUID METAL COOLED; SETTLED BED, FAST BREEDER

POWER NWE(MNT) $1000 \quad 2500$

CRITICAL REFERENCE DESIGN

COOLANT SODUUM

MODERATOR NONE

FUEL MATERISL URANIUMIPLUTONIUM CARBIDE, HYPOSTOICHIOMETRIC

FUEL GEChETAY SPHERES 0.120 in: DIA.

FUEL CLADE:NG NONE

FUEL ENRICH, URANIUM/PLUTONIUM 7/1, DEPLETED URANIUM

FUEL ASSEMBLY CORE AND BLANKET AS BEDS OF SMALL, RANDOMLY-PACKED

TUEL SPHERES, COOLANT FLOW IS DOWNWARD, AESTRAINING THE BED: THE BED IS SUPPORTED BY PERFORATED PLATE.

ANNULAR CORE 6 FT. 9 IN, ODI, 4 FT, 9 IN: ID., IO FT, 6 IN. HIGH, IS SURROUNDED ON ALL SIDES BY BLANKET MATERIAL, CENTRAL BLANKET IS $4 . F T$. DIA.

FUEL GHARGE TOTAL WEIGHT OF FUEL IS $21.154 \mathrm{KG}$,

SPECIFIC FCKER $1100 \mathrm{KW} / \mathrm{KG}$ PLUTONIUM CARBIDE

BURNURLREFUEL) 100,0000 MWD/T

CONTROL RODS, WITHIN CORE RING

COOLANT TEKP, INLET $550 \mathrm{~F}$ OUTLET $1200 \mathrm{~F}$

COOLAN PRESS. INLET 316 PSIG OUTLET 20 PSIG

REACTOR VESSEL EYLINDRICAL, SS VESSEL

:6 FT, 10 IN. ID.. $18 \mathrm{FT}, \mathrm{HIGH}, 5 \mathrm{IN}$, WALL PRESSURE VESSEL

PERFORATED PLATES FORM THE FUEL BEDS

CCNTAIMENT VERT:CAL STEEL CYLINDER $115 \mathrm{FT}, 10,168 \mathrm{FT}$, HIGH 
LIQUID METAL REACTORS: DOMESTIC

65 FT: BELOW-GRADE.

PRESSURE VESSEL:

REACTOR, RADIOACTIVE COOLANT, AND FUEL

HANDLING SYSTEMS ARE BELOW OPERATING FLOOR,

REMARKS

TWO GENERAL REACTOR CONFIGURATIONS WERE STUDIED, AXIAL AND RADIAL FLOW DESIGNS, A ZERO-POWERED, COLD CRITICAL EXPERIMENT CONSISTING OF A TWO-REGION WATERMMODERATED FACILITY WITH A CENTRALLY LOCATED SETTLED BED IS PLANNED, TO BE FOLLOWED BY A LOW POWER, HOT CRITICAL EXPERIMENT USING CERAMIC FUEL LUMPS AND SODIUM COOLANT, A SIMILAR STUDY IS CONCERNED WITH URANIUM/RLUTONIUM DIOXIDE AND URANIUM DIOXIDE SPHERES AS: BLANKET. POWER IS 359 MWE AND 881 MWE, DESIGN CONCEPTS FOR A 1000 MWE VERSION HAVE BEEN DONE, AXIAL TYPE WAS SELECTED FOR REFERENCE DES!GN SEE ALSO OFBR, FD36

REFERENCES CHARACTERISTICS OF FAST SETTLED BED REACTORS: $\checkmark$ CHERNICK

BNL-7592/OCT; 1963 ,

NUCLEAR PARAMETER STUDIES OF THE FAST SETTLED BED REACTOR.

MM LEVINE, A ARONSON

BNL-7593/OCT. $1963 /$

SETTLED-BED FUEL REACTORS:

LP HATCH, OTHERS

$B N L=5830 / N O V \cdot 1961 /$

PROC, CONFERENCE IN BREEDING, ECONOMICS, AND

SAFETY IN LARGE FAST POWER REACTORS, OCTOBER 7:10, 1963 . ARGONNE NATIONAL LAB. $A N L=6792$ /DEC. 1963 ,

NUCLEAR PARAMETER STUDIES OF THE FAST SETTLED BED REACTOR, $\checkmark$ CHERNICK, OTHERS.

1000 MWE SETTLEDIBED FAST REACTOR PHYSICS, A ARONSON, MM LEVINE AMERICAN NUCLEAR SOC. TRANS: 7, 240-1/NOV: 1964/

THE SETTLED BED FAST REACTOR CONCEPT, L: GREEN, OTHERS /BNL! NUCLEAR APPLICATIONS 1, 107.130/APR!L 1965/ 
JANUARY 1969 BNWL: 936

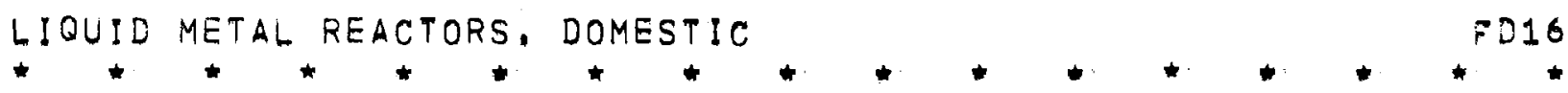
SETTLED-BED FAST REACTOR CONCEPT - STATUS REPORT, C ROSEMAN, OTHERS FAST REACTOR TECHNOLOGY, NATIONAL TOPICAL MEETING APRIL 26-8, 1965, AMERICAN NUCLEAR SOCIETY: ANS-100P, $68-74$

THE RANDOMLY $=$ PACKED SETTLED-BED FAST REACTOR CONCEPT, 1000 MWE REACTOR DESIGN

$L$ GREEN, MM LEVINE, EDS. BNL-887/JUNE 1; 1965 / 
JANUAR 1969

LIOUIE YETAL REACTORS, DONESTIC

FD17

NAME/ONNE WMFR /LIQ, METAL FAST BREEDER REACTOR/CE

DESIGNER COMBUSTION ENGPNEERING, INC,

PURPUSE DESIGN STUEY, FAST BREEDER POWER REACTOR,

FYOE WOUIE METAL COOLED FAST BREEDER

PONER WE(W, $\$ 000$

OAITICHL ZESEON STUDY

COOLANT SOEIUM

FUEL MATEFAL CORE, URANIUMAPLUTCNIUM CARBIDE PELLETS Q,26:N, DIA. ELANKET, AXIAL AND RADIAL, O.4 IN, DIA, PELLETS

FUEL GEOMETFY ROZS 30 IN, ACTIVE LENGTH - TOP AND BOTTOM SLANKET 18 IN.

RAD?AL BLANKET 54 IN. ACTIVE LENGTH

FLEL CIAEDING SS 0.011 IN, THICK, SODIUM BOND

FUEL ENG:CH, AVERAGE 13 PER CENT

PUEL ASSEMELY COAE, 169-PIN ASSEMBLY, INGLUDING AXIAL BLANKET. RAE:AL BLANKET: 127 -ROD ASSEMELY

157 CORE ASSEMELIES

156 RADIAL BLANKET ASSEVBLIES

PLEL CHAREE :-155 KG, FISSIEE METAL. ELANKET $106 \mathrm{KG.}$

SEECIFIC FONER -600 KW/KG FISSILE

BURNUP(REFUELI T00,000 MWETT 1/6 CORE ANO 1/ZA BLANKET URAN!UM + OUTON!UM

CONTROL RONS, BCRON CARBIDE AND DERLETED URANIUM CARB!DE

COOLANT TEME, NVET OEOF OUTLET I:20 F

REACTOR VESSEL STGUCTURAL MATERIALS STAINLESS STEEL REACTOR VESSEL CONTAINS CORE AND BLANKET ASSEMBLIES.

REFERENCES LIOUTD METAL FAST BREEDER REACTOR DESIGN STUDY: VUL. I AND 2 . COMBUSTION ENGINEERING, NUCLEAR DIVI

CENDE200 /VOL, 1 AND $2 /$ /JANUARY 1964/ 
JANUARY 1969

BNWL: $: 936$

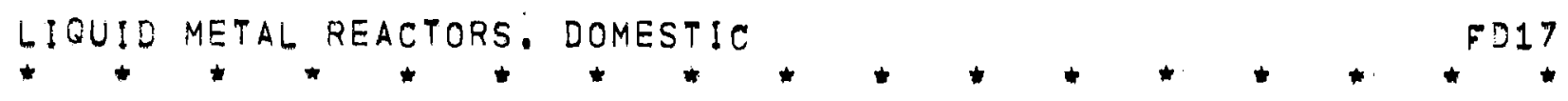

AN EVALUATION OF FOUR DESIGN STUDIES OF A 1000 MWE CERAMIC FUELED FAST BREEDER REACTOR,

REACTOR ENG, DIV. CHICAGO OPERATIONS OFFICE. COO-279/DEC. 1; 1964/ 
JANUARY 1969

$B N+=936$

LIOUIO METAL REACTORS, DOMESTIC

Fח18

NAME/OWNER LIME /LIQUID METAL BREEDER//DOUGLAS AJRCRAFT

DESIGNER DOUGLAS AIRORAFT

PURPOSE DESIGN EVALUATION

TYPE LIOUTO METAL THERMAL BREEJER, INTERNALIY CODLED

POWER WEMMT 200

COOLANT MOLTEN LEAD

MODERATQR GRAPHITE

FUEL MATERIAL URANIUMMISMUTH SOLUTION, GLANKET SOLUTDON A PISFEASION OF THORIUMMEISMUTHIDE IN MOLTEN LEAOE BISMUTH,

FUEL ENRYCH, NATURAL

FUEL ASSEMBLY GRAPHITE FUEL ELEMENT IS HEXAGONAL, A CENTPAL PLUNGER, MOVING DOWNWARD, DISPLACES THE FUEL FIUID COLLECTED IN THE BOTTOM OF THE OUTER GRARHITE TUBE UPWAFD INTO THE CORE ZONE, AT CRITICALITY A RESERVOIR IS FORMED ABOVE TLE UPFER BLANKET REQ:ON, BLANKET FLUIE CIROULATES FROM TOR TO BOTTOM ZY MEANS OF CHANNELS IN THE PLUNGER, CORE, UPPEP AND LONER BLANKETS ARE FORMED BY A CLUSTER OF FHE GRAPH!TE ELEMENTS.

SPECIFIO POAER COOO KW/KG

NEUTRON FIUN THERMAL AVE, $2.10 \times 10$ E=14

EPT THERMAL AVE, $5,43 \times 10$ EN 14

CODLANT TEMD, $\quad 200 \mathrm{C}$

FEACTOR VESSEL STEEL VESSEL CONTAINING A GRARATE STRUCTURE TABRICATED TO CONDUCT TAE COOLANT, FUEL, AND ELANKET FLUIDS THROUGH THE REACTOR,

REMARKS

TWO SIZES WERE SELECTED FOR DETAILED EVALUATION, THE 200 MWT OANT AND A 1000 MWT PLANT, S:NGLE WNET CONSTRUCTION FOR ALL REACTOR FUNCTIONS. INCLUDING PHCCESSING BY MOLTEN SALF PROCESS, IS PROPOSED.

REFERENCES LOUID METAL EREEDER REACFOR IIIMB/

RJ TEITEL /ROUGIAS AIRCRAFTI

PROC. THORIUN FUEL CYCLE SYMPOS:UM, P, 210.43 


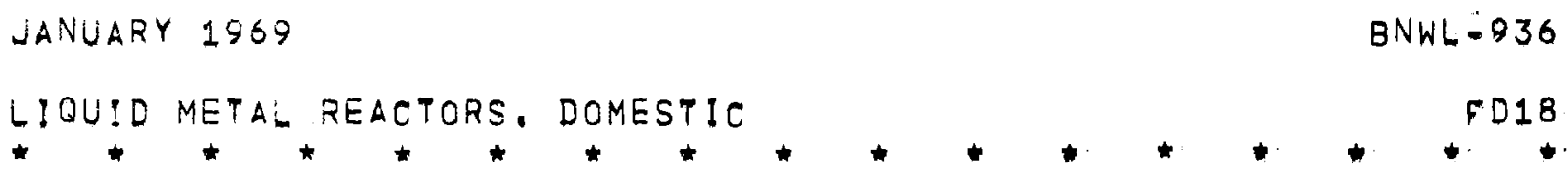

TID-7650/1962/

LIQUID METAL BREEDER REACTOR /LIMB/ NUCLEAR EVALUATION.

RJ TE!TEL, JB BROWN /DOUGLAS AIRCRAFT!

NUCLEAR APPLICATIONS 1/1/, 13:24/FEB, 1965/ 
BANUARY 1969
LIOUID METAL REACTORS. DOMESTIC

NAME IOWNER SEFOR /SOUTHWEST EXPERIMENTAL FAST OXIDE REACTOR/. GE, SAEA, W: GERMANY; EURATOM, AEC

DESIGNER GENERAL ELECTRIC COMPANY

LOCATION FAYETTEVILLE, ARKANSAS

PURPOSE FAST BREEDER EXP.TTEST REACTOR

TYPE LIQUID METAL COOLED; FAST BREEDER EXPERIMENT'

POWER MWE(MWT) 0

CRITICAL FUEL LOADING TARGET 1968

COOLANT SODIUM. TWO PARALLEL LOOPS

MODERATOR NICKEL REFL'ECTOR SLABS

FUEL MATERIAL PLUTONIUMAURANIUM DIOXIDE PELLETSi URANIUM DIOXIDE PELLETS, BLANKET

FUEL GEOMETRY RODS 1,00 IN, DIA:, ACTIVE LENGTH 33,8 IN:

FUEL CLADDING SS 0,055 IN. THICK

FUEL ENRICH, 19 PER CENT PLUTONIUMM

FUEL ASSEMBLY 6 RODS/CHANNEL, TOTAL 630 RODS

NEUTRON FLLUX AVE: $3.4 \times 10$ E+14

CONTROL MOVABLE REFLECTOR CONTROL RODS, SURROUNDING CORE OUTSIDE REACTOR VESSEL.

COQLANT TEMP, INLET $700 \mathrm{~F}$ OUTLET $820 \mathrm{~F}$

REACTOR VESSEL LOWER -CORE- SECTION SMALL DIA., UPPER SECTION LARGE DIA: SHIELDING.

CONTAINMENT OUTER ALL-WELDED STEEL CYLINDER, 5O FT, DIA,, 114 FT. HIGH, BOTTOM LEVEL 60 FT, BELOW GRADE. TWO BARRIERS IN SERIES, INNER CONTAINMENT STEEL : REINFORCED CONCRETE WITH AN INERT GAS ATMOSPHERE:

REMARKS PROGRAM HAS BEEN UNDER AEC SPONSORSHIP SINCE 1959, PROGRAM CALLS FOR A $100-300$ MWE PROTOTYPE FOR OPERATION BY 1970; AND A 500 MWE REACTDR ON-LINE BY 1975:

CRITICAL ASSEMBLY MOCK-UP REACHED CRITICALITY IN 
LIOUID METAL REACTORS, DOMESTIC

ZPR-3 IN APRIL 1966:

SEFOR WILL DEVELOP DESIGN INFORMATION FOR THE

300 MWE DEMONSTRATION PLANT:

REFERENCES

FAST OXIDE BREEDERIREACTOR PHYSICS, PART I

PARAMETRIC STUDY OF 300 MWE REACTOR CORE:

P GREEBLER, OTHERS

GEAP-3721 /DECEMBER 1961/

EXPERIMENTAL FAST OXIDE REACTOR:

MJ MC NELLY, OTHERS

POWER REACTOR EXPERIMENTS: VOL, I, F; 317-43

INTERNATIONAL ATOMIC ENERGY AGENCY, VIENNA: 1962:

EXPERIMENTAL FAST CERAMIC REACTOR DESIGN, STATUS

REPORT AS OF OCTOVER 31; 1961:

KM HORST; ED.

GEAP-3885/APRIL' 1962 /

DEVELOPMENT OF THE FAST CERAMIC REACTOR,

$K$ COHEN, B WOLFE

NUCLEAR ENG, 7.358-9/SEPTEMBER $1962 /$

DEVELOPMENT OF THE FAST CERAMIC REACTOR, K COHEN; B WOLFE

NUCLEAR NEWS 6, 11-15 /FEBRUARY 1963/

CONCEPTUAL DESIGN OF A 565 MWE FAST CERAMIC

REACTOR.

HE DODGE, OTHERS

GEAP-4226/APRIL 1963/

THE SOUTHWEST EXPERIMENTAL FAST OXIDE REACTOR, $K$ COHEN, OTHERS:

EIGHTH NUCLEAR CONGRESSS, ROME, ITALY, APED-4281/JUNE 17-20,1963/

SOUTHWEST EXPERIMENTAL FAST OXIDE REACTOR

DEVELOPMENT PROGRAM: FIRST OUARTERLY REPORT,

APRIL-JUNE 1964:

GEAP $=4594$ /JULY 1964 /

PRELIMINARY SAFEGUARDS SUMMARY REPORT: PART 1 . SOUTWEST EXPERIMENTAL FAST OXIDE REACTOR

UNDATED

NP-15077/MAY $1965 /$

SEFOR - A STATUS REPORT

$B$ WOLFE, OTHERS

ANS $=100$ P. $247: 67$ 
FD19

JANUARY 1969

LIQUID METAL REACTORS, DOMESTIC
$B N W L-936$

FD 19

SEFOR READY SOON FOR FAST BREEDER EXPERIMENTS'.

HT HOLMES

ELECTRICAL WORLD 169; 32:33 /MAY 13, 1968/

THE SEFOR PROJECT - ITS SIGNIFICANCE AND PROGRESS,

$B$ WOLFE

AMERICAN POWER CONF! PROC: 29, 189-201/1967/

SEFOR PLANT DIS IGN

ANS TOPICAL MEETING ON FAST REACTORS, 1967,

ANS -101 P. 5.31; 5-54; 
JANUARY 1969

BNWL: $=936$

LIQUID METAL REACTORS, DOMESTIC

FD20

NAME/OWNER

DES IGNER

PURPOSE

TYPE

POWER MWE (MWT)

CRITICAL

COQLANT

FUEL MATERIAL
LMFBR /L1Q. METAL FAST BREEDER REACTOR/GE

GENERAL ELECTRIC COMPANY

DESIGN STUDY FOR AEC

LIQUID METAL COOLED FAST BREEDER, PANCAKE CORE? 2500

STUDY

SODIUM

CORE, URANIUMIPLUTONIUM DIOXIDE CQRED PELLETS 0.22 IN. OD: 0:01: IN, ID: BLANKET, AXIAL AND RADIAL, DEPLETED URANIUM OXIDE PELLETS 0.058 AND 0.45 IN. DIA.

FUEL GEOMETRY CORE, PINS 2 FT. ACT: LENGTH * $2 \times 18$ IN. UPPER AND LOWER AXIAL BLANKET

RADIAL BLANKET, 30 IN: ACT, LENGTH

FUEL CLADDING SS

CORE, 15 MILS THICK

RADIAL BLANKET, 20. MILS THICK

FUEL ENRICH, AVERAGE 18 PER CENT

FUEL ASSEMBLY CORE AND AXIAL BLANKET

470 FUEL PINS

138 BERYLLIA PINS

225 ASSEMBLIES

RADIAL BLANKET

208 PINS

108 ASSEMBLIES

HEXAGONAL SS FUEL CHANNELS, CORE IS 2 FT, HIGH AND $11.65 \mathrm{FT}$. DIA.

FUEL CHARGE $2304 \mathrm{KG}$. FISSILE METAL, BLANKET $58 . \mathrm{KG}$

SPECIFIC PONER $900 \mathrm{KW/KG} \mathrm{PLUTONIUM}$

BURNUP(REFUEL) 100,000 MWD/T

CONTROL RODS, BORON CARBIDE; SODIUM FOLLOWER

COOLANT TEMP, INLET $800 \mathrm{~F}$ OUTLET $1100 \mathrm{~F}$

COQLANT PRESS, INLET 50 PSIA OUTLET 25 PSIA 
LIOUID METAL REACTORS; DOMESTIC

FD20

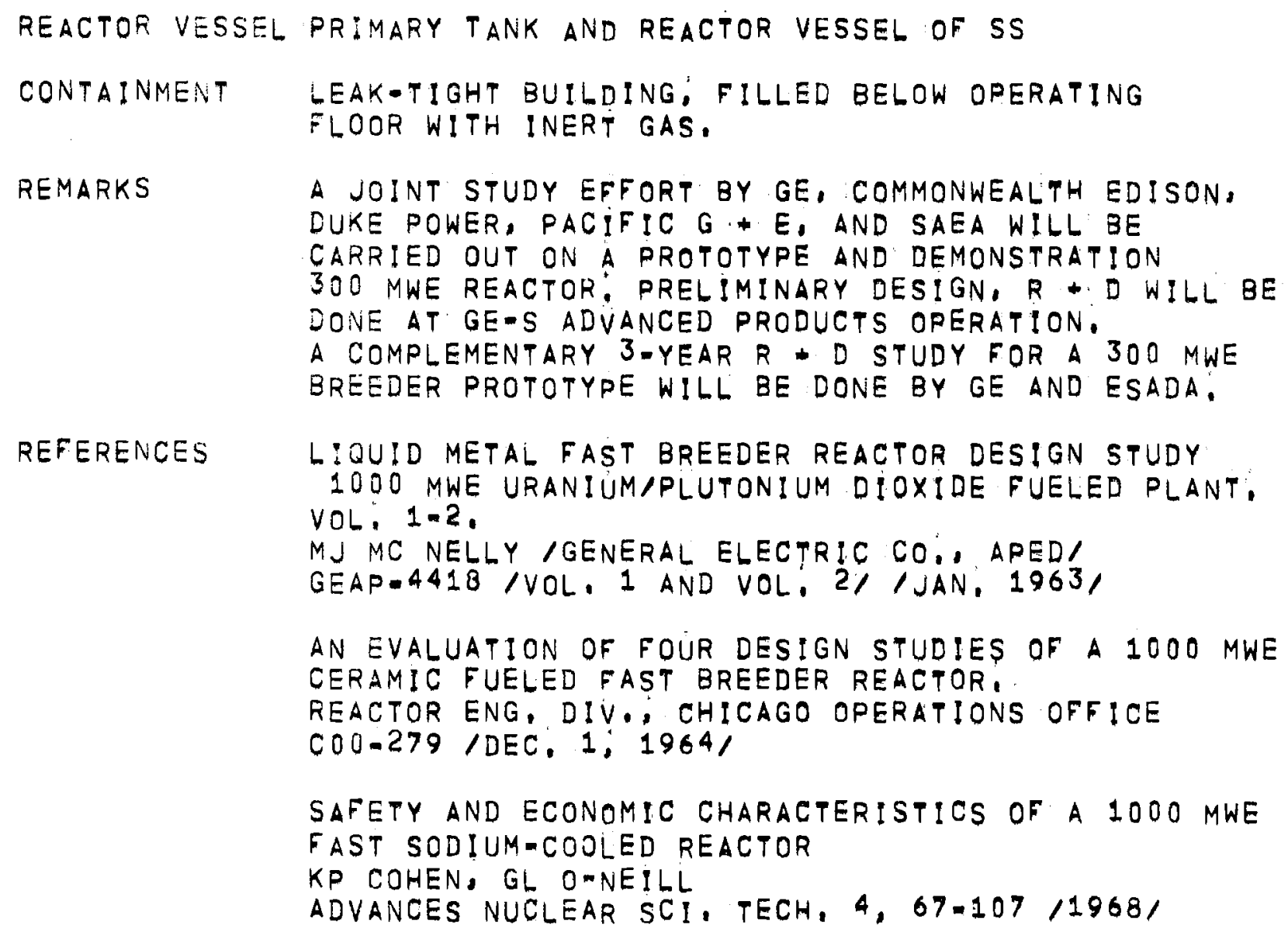




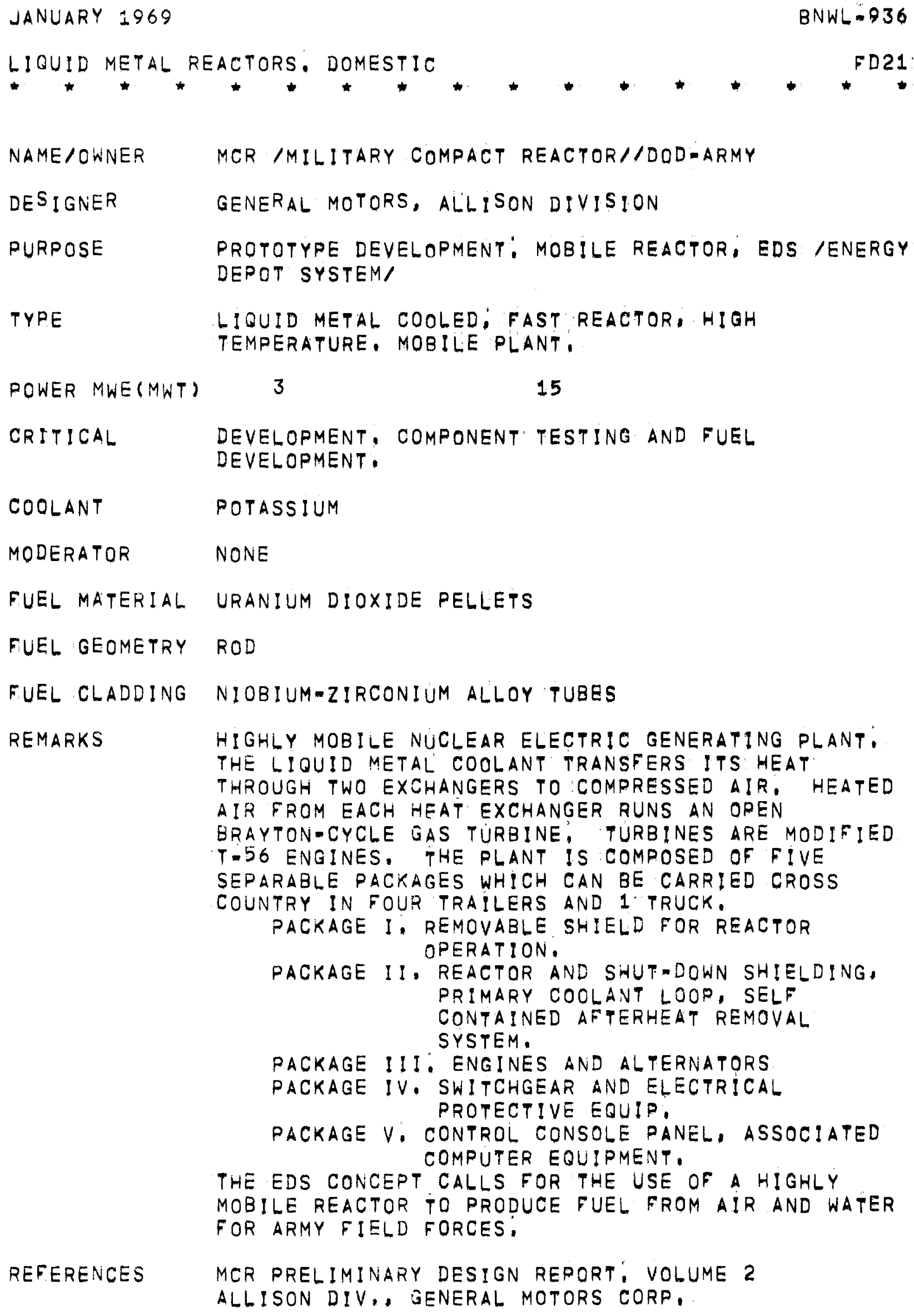


JANUARY 1969

BNWL:936

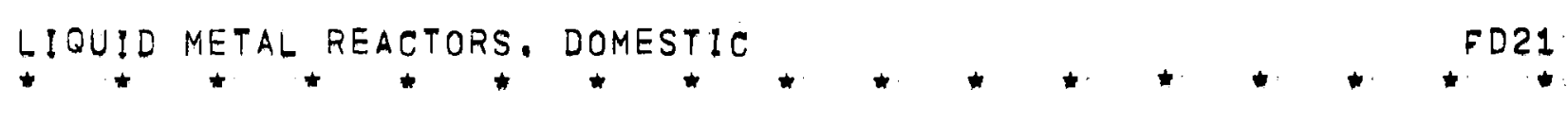
GMAD-3078-8, VOL, 2 IDECLASSIFIEDI 
LIQUID METAL REACTORS: DOMESTIC FDZ2

NAME/OWNER LAMPRE /LOS ALAMOS MOLTEN PLUTONIUM REACTOR/IAEC

DESIGNER LOS ALAMOS SCIENTIFIC LABORATORY.

OPERATOR LOS ALAMOS SCIENTIFIC LABORATORY

LOCATION LOS ALAMOS, N: M.

RURPOSE POWER EXPERIMENT

TYPE LIQUID METAL COOLED: FAST REACTOR, LIQ: METAL FUEL POWER MWE(MWT) 0

CRITICAL 1961. CORE REMOVED IN 1964
SEE REMARKS

COOLANT SODIUM

FUEL MATERIAL PLUTONIUM-IRON ALLOŸ /MOLTEN/ /SEE REMARKS/

FUEL GEOMETRY MOLTEN ALLOY

FUEL CLADDING TANTALUM-TUNGSTEN ALLLOY CAPSULES

$$
0,376 \text { IN. ID. } 8 \text { IN. LONG }
$$

FUEL ENRICH, 90 PER CENT

FUEL ASSEMBLY CORE CONSISTS OF FOOT-LONG TANTALUM-TUNGSTEN

THIMBLES CONTAINING THE PLUTONIUM-IRON FUEL,

PLACED IN THE SODIUM COOLANT, THERE ARE 199

CAPSULES, SOME OCCUPIED BY UNFUELED REFLECTOR PINS

SPECIFIC POWER 40 W/G FUEL

COOLANT TEMP, INLET $450 \mathrm{C}$ OUTLET $563 \mathrm{C}$

REACTOR VESSEL INNER CONTAINMENT VESSEL OF SS, OUTER VESSEL SS

REMARKS A NEW FUEL ALLOY UNDER DEVELORMENT WILL BE A TERNARY PLUTONIUM-COBALT-OESIUM ALLOY IN WHICH THE PLUTONIUM CONTENT CAN BE VARIED OVER A WIDE RANGE; A PROJECT ORIGINALLY DESIGNATED AS LAMPRE-2 IS NOW KNOWN AS FRCTF /FAST REACTOR CORE TEST FACILITYI SEE FD23

REFERENCES LAMPRE, A MOLTEN PLUTONIUM FUELED REACTOR CONCEPT: RM KIEHN, OTHERS 
JANUARY 1969

BNWL $=936$

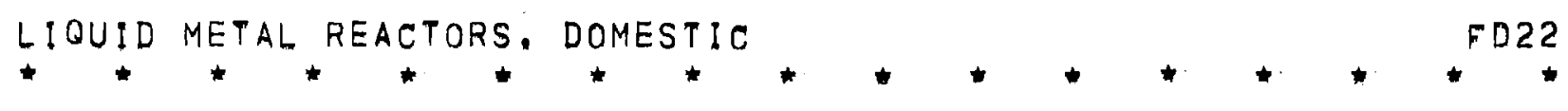

LOS ALAMOS MOLTEN PLUTONIUM REACTOR EXPERIMENT:

/LAMPRE/ HAZARD REPORT,

EO SWICKARD

LA-2327/JUNE $1959 /$ 


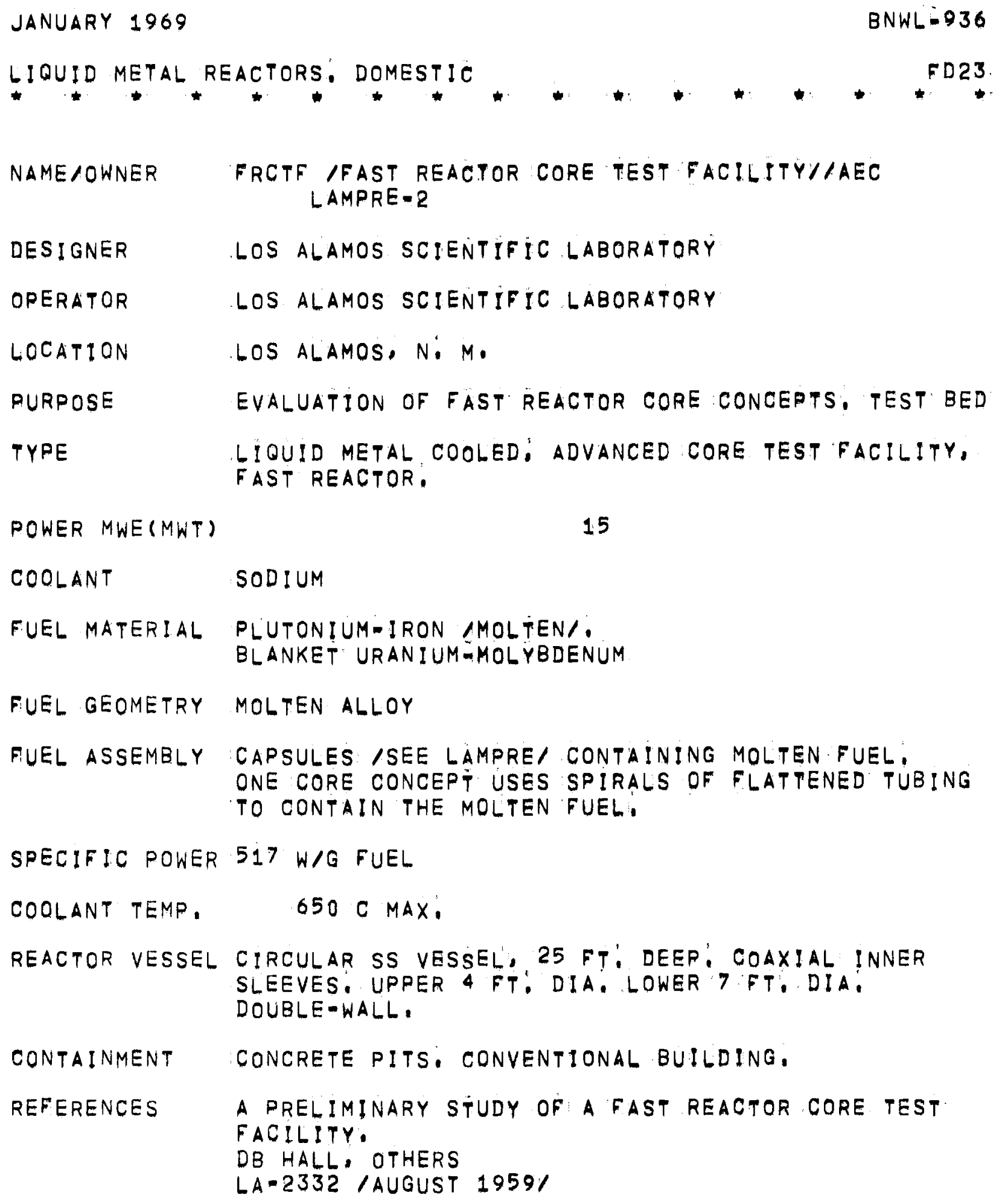


JANUARY 1969

$B N W L=936$

LIOUID METAL REACTORS. DOMESTIC

NAME/OWNER DCR/DIRECT CONTACT REACTOR//LASL

DESIGNER LOS ALAMOS SCIENTIFIC LABORATORY

PURPOSE FAST EREEDER CONCEPT; STUDY

TYPE LIOUID METAL COOLED; FAST BREEDER:

POWER MWE (MWT)

227

CRITICAL DESIGN STUDY

COOLANT SODIUM

FUEL MATERIAL PLUTONIUM-CERIUM-COBALT COMPOSITION, MOLTEN URANIUM DIOXIDE ISODIUM PASTE BLANKET

FUEL GEOMETRY MOLTEN ALLOY, PASTE BLANKET

FUEL CLADJING TANTALUM-TUNGSTEN ALLOY STRUCTURAL MATERIAL

FUEL ASSEMELY VESSEL IS FILLED WITH THE LIOUID FUEL: AN EXTERNAL JET OF LIQUID SODIUM CIRCULATES THE FUEL AND SEPARATES THE LIQUIDS CENTRIFUGALLY, AFTER SEPARATION FUEL RETURNS TO THE CORE, CORE DIAMETER IS $70 \mathrm{CM}$.

FUEL CHARGE 350 LITERS OF FUEL 11 KG PLUTONIUM/LITER/

SPECIFIC POWER 0:65 KW/G PLUTONIUM /EXCLUDING BLANKETI

COOLANT TEMP. OUTLET $850 \mathrm{C}$

REACTOR VESSEL STRUCTURELESS VESSEL'

REMARKS THE SODIUM COOLANT, IMMISCIBLE WITH THE MOLTEN

FUEL ALLOY, IS IN DIRECT CONTACT WITH IT:

SEPARATION IS DONE CENTRIFUGALLY BY THE ENERGY

FROM AN EXTERNAL JET OF SODIUM.

REFERENCES MOBILE FUEL PLUTONIUM BREEDERS A STUDY OF ECONOMIC POTENTIAL.

RP HAMMOND. OTHERS

LA-2644 /NOV, 30, $1961 /$

DIRECT CONTACT CORE SYSTEMS: RP HAMMOND, JR HUMPHREYS JR,

NUCLEAR SCIENCE AND ENGINEERING 18, 421.425

/APRIL $1964 /$ 


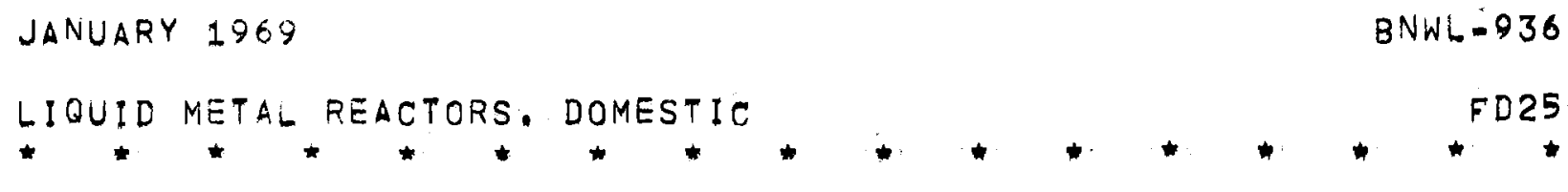

NAME/OWNER FAST OXIDE REACTOR STUDY/LASL

DESIGNER LOS ALAMOS SCIENTIFIC LABORATORY

PURPOSE WATER DESALINATION

TYPE LIOUID METAL COOLED FAST BREEDER

POWER MWE (MWT)

25000

CRITICAL

STUDY

COOLANT SODIUM

FUEL MATERIAL URANIUMAPLUTONIUM DIOXIDE PELLETS 0.260 IN: DIA. URANIUM DIOXIDE AXIAL AND RADIAL BLANKET

FUEL GEOMETRY RODS, ACT, LENGTH 4 FT, +2X3 FT: UPPER AND LOWER AXIAL BLANKET

FUEL CLADDING SS 0.020 IN. THICK

FUEL ENRICH, 15 PER CENT

FUEL ASSEMBLY 400 RODS/ASSEMBLY 1016 ASSEMBLIES/CORE

SPECIFIC POWER 1:1 MH/KE PLUTONIUM

BURNUP (REFUEL) 100,000 MWD/MT BATCH, 3 MONTHS

COOLANT TEMP, INLET 593 F

REMARKS THE ENERGY IS USED ENTIRELY FOR THE PRODUCTION OF DISTILLED WATER, WATER CONVERSION PLANT IS A MULTISTAGE FLASH EVAPORATION UNIT CONSISTING OF 30 STAGES.

REFERENCES A PRELIMINARY EVALUATION OF FAST OXIDE BREEDERS FOR SEA-WATER CONVERSION:

A SESONSKE, RP HAMMOND LA-2733/AUG, 1962/ 
BNWLË:936
JIOUID METAL REACTORS, DOMESTIC 1969

NAME/OWNER PWAR=6 /PRATT - WHITNEY AIRCRAFT REACTOR=6//PWAC

DESIGNER PRATT AND WHITNEY AIRCRAFT'

PURPOSE NUCLEAR AIRCRAFT HEAT SOURCE

type LiQUid metal CoOLed: circulating fuel

POWER MWE (MWT)

CRITICAL CONCEPTUAL DESIGN STUDY

COOLANT SODIUMAPOTASSIUM NAK

MODERATOR BERYLLIUM REFLECTOR-MODERATOR: HOLLOW SPHERE SURROUNDING CORE.

FUEL Material fluORIDE SALTS /SODIUM, ziRCONIUM AND URANIUM/

FUEL CLADDING INCONEL MATERIAL OF CONSTRUCTION

FUEL ASSEMBLY FUEL FLOWS OUTSIDE THE HEAT EXCHANGER TUBES

COUNTER CURRENT TO NAK FLOWING INSIDE THE TUBES:

NEUTRON FLUX THERMAL AVE, $1 \times 10$ E +14

FAST AVE, $3 \times 10 E+14$

CONTROL CONTROL ROD

COOLANT TEMP, INLET $200 \mathrm{~F}$ OUTLET $600 \mathrm{~F}$

REACTOR VESSEL HASTELLOY=X PRESSURE SHELL 70 IN: DIAIII: IN. THICK. INNER SHELLS CONTAINING BORON-10, AND AN INCONEL SHELL JUST INSIDE THE PRESSURE SHELL,

REMARKS EVOLUTION OF ORNL FIREBALL REACTOR CONCERT IART DESIGN MODIFICATION/.

REFERENCES COREMMODERATED CIRCULATING FUEL REACTOR STUDY: PRATT - WHITNEY AIRCRAFT, DIVI, UNITED AIRCRAFT CORP:

PWAC-186/JULY 15; 1957 /

THE $P$ - W CIRCULATING FUEL REFLECTORMMODERATED

REACTOR,

CC BIGELOW, ME GREENSTREET.

PWAC.189 /VOL. 1/ /NOVEMBER 15, 1957/

VOLUME 2, APPENDIX A, DESIGN SPECIFICATIONS AND

REFERENCE INFORMATION FOR THE PWAR=6. FLYING TEST 


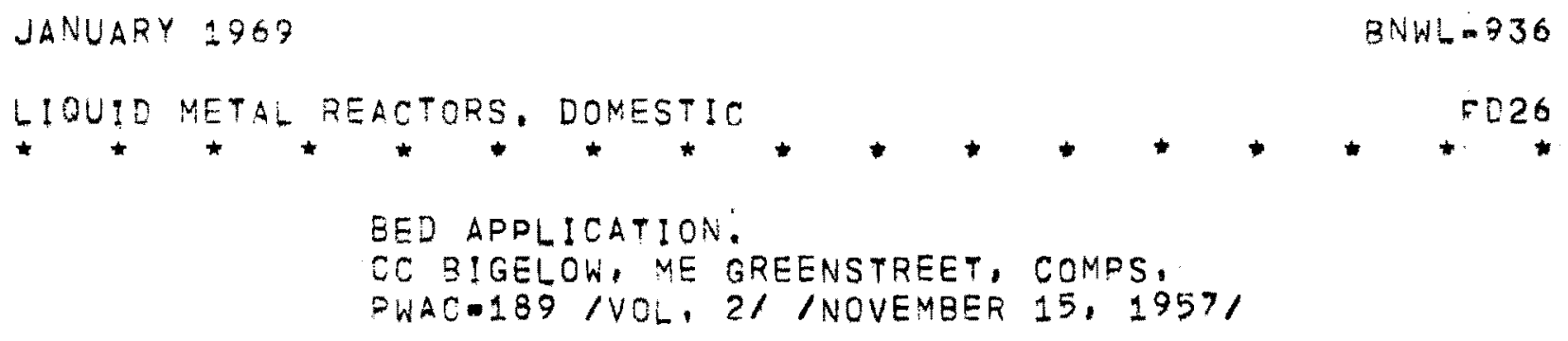




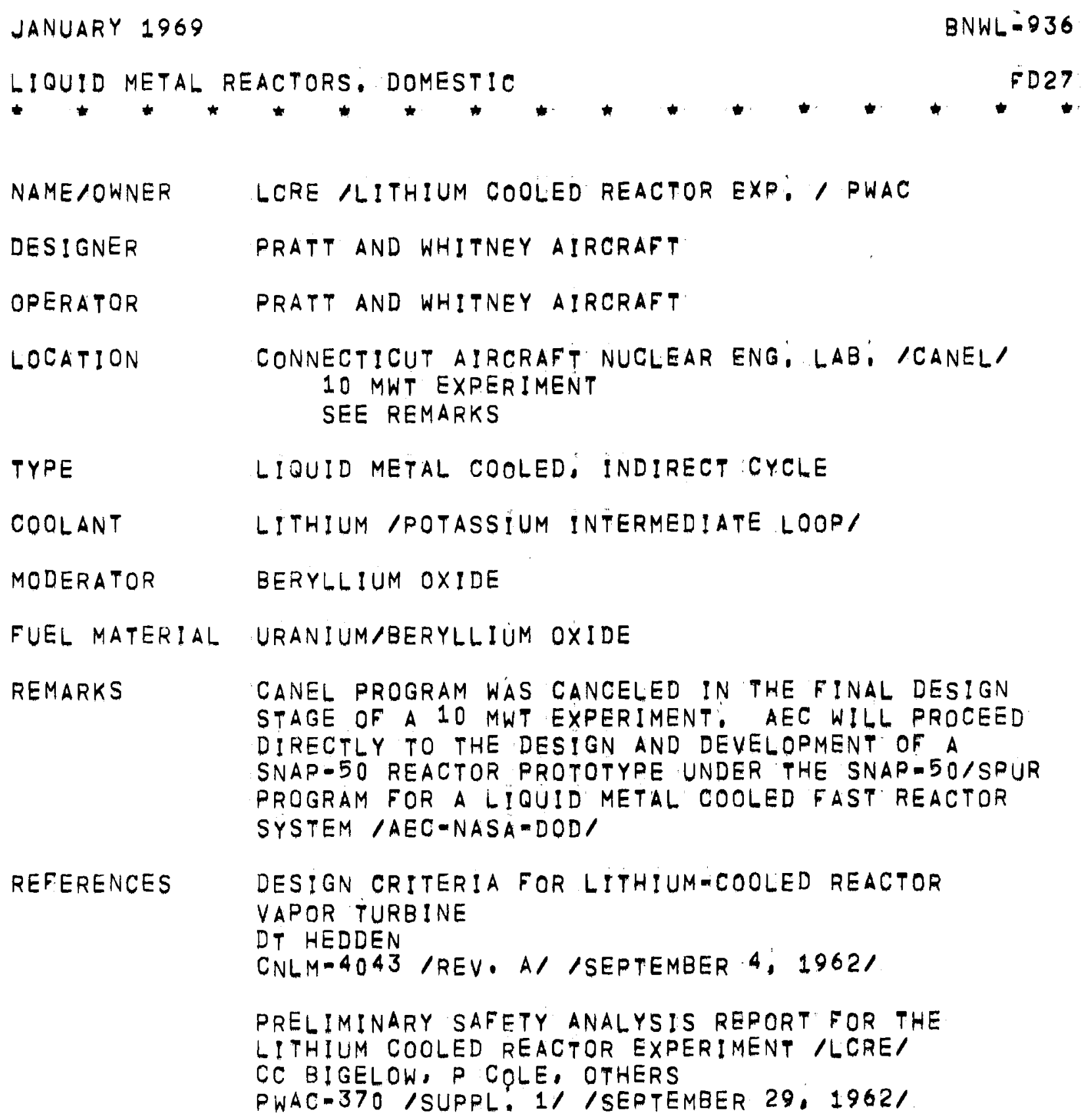




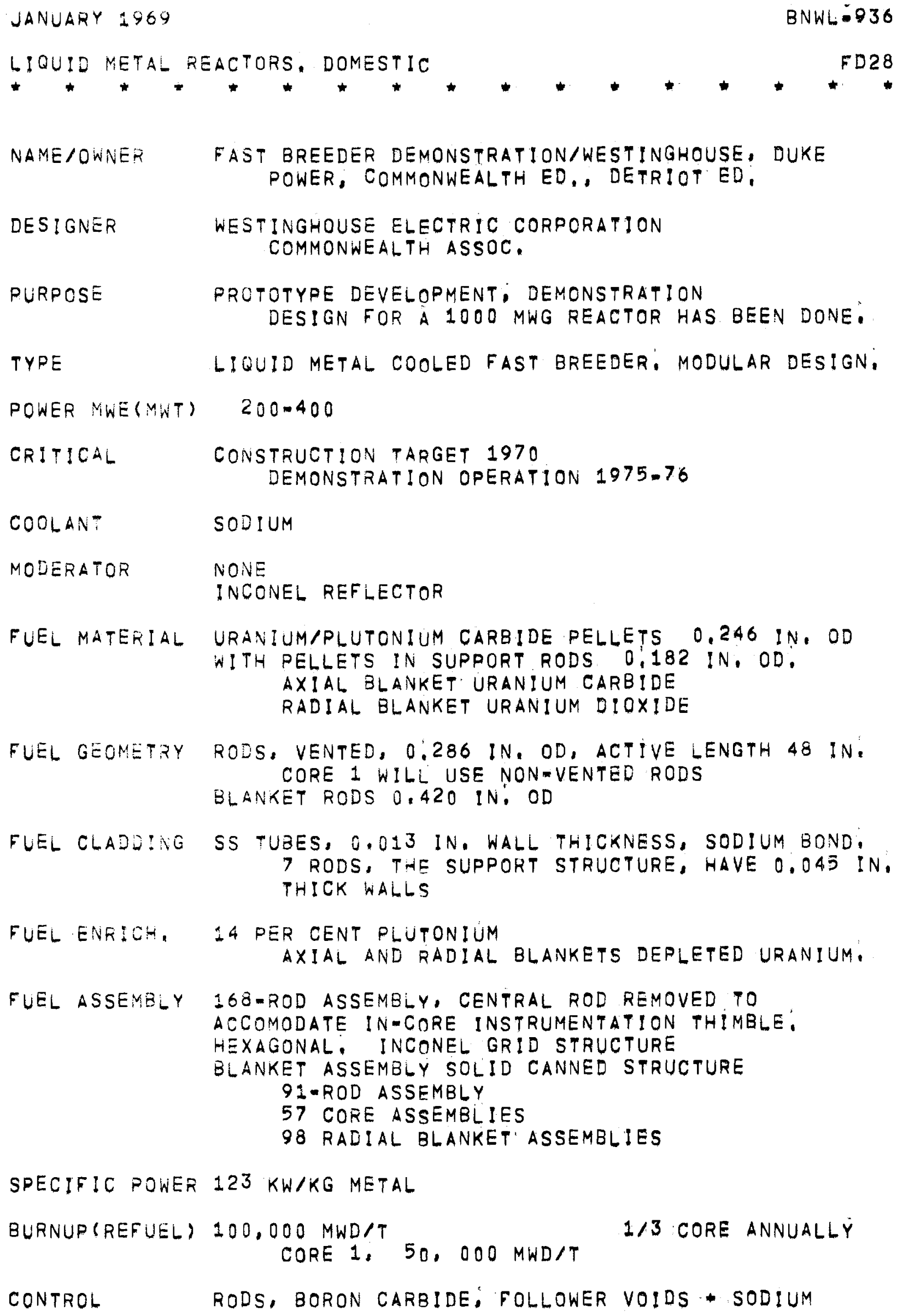




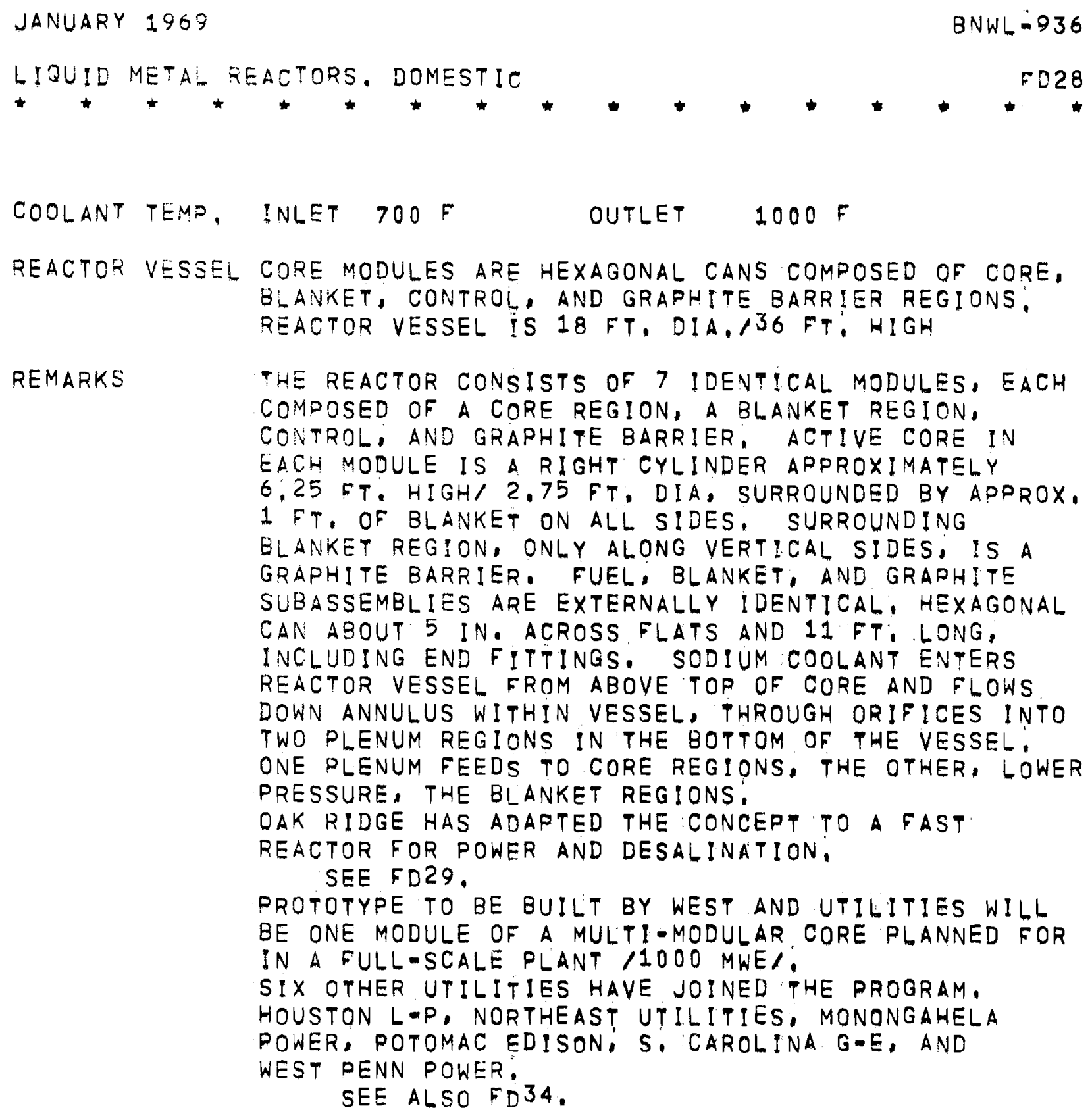

REFERENCES LIQUID METAL FAST BREEDER REACTOR DESIGN STUDY, FINAL REPORT. RB STECH, COMP, WESTINGHOUSE ELECTRIC CORP, ATOMIC POWER DIV: WCAP 3251-1/JANUARY 1964/

AN EVALUATION OF FOUR DESIGN STUDIES OF A 1000 MWE CERAMIC FUELED FAST BREEDER REACTOR

REACTOR ENG. DIV.: CHICAGO OPERATIONS OFFICE COO-279/DEC, 1;1964/

CONCEPTUAL DESIGN AND PRELIMINARY ACCIDENT ANALYSIS OF A SODIUM-COOLED, CARBIDE-FUELED, LARGE MODULAR FAST REACTOR, JH WRIGHT, OTHERS 
JANUARY 1969

$B N W L=936$

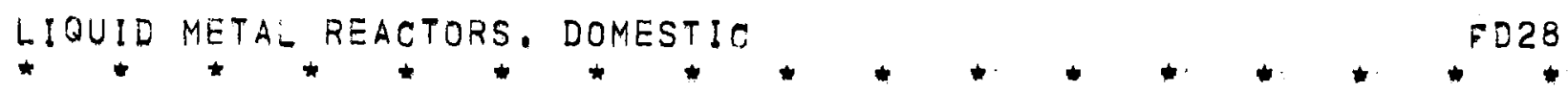
ANL-7120, P.205.29, 11966/

A PROTOTYPE PLANT WILL PROVE THE FAST BREEDER CONCEPT,

LCR KELLY JR, , PG DEHUFF WESTINGHOUSE ENG.

DESIGN PHILOSOPHY FOR WESTINGHOUSE, PROTOTYPE FAST BREEDER REACTOR:'

JH WRIGHT, OTHERS

ANS-101, P, 1-20 
JANUARY 1969

BNWL: $=936$

LIQUID METAL REACTORS. DOMESTIC FD29

NAME/OWNER LIQUID METAL COOLED FAST BREEDER/ORNL

DES \GNER

OAK RIDGE NATIONAL: LABORATORY

PURPOSE

DESALINATION AND/OR POWER

APPLICATIONS CONSIDERED ARE CENTRAL STATION

POWER, DUAL PURPOSE SELF-CONTAINED STATION;

AND PROCESS HEAT PLANT,

TYPE

LIQUID METAL COOLED FAST BREEDER,

LOW TEMPERATURE: MODULAR CONCEPT

POWER MWE (MWT)

420

3500

DUAL PURPOSE

CRITICAL REFERENCE DESIGN

COOLANT SODIUM

FUEL MATERIAL CORE, URANIUM/PLUTONIUM DIOXIDE, VIBRATORY COMPACT AXIAL BLANKET; URANIUM DIOXIDE

RAD!AL BLANKET, URANIUM METAL

FUEL GEOMETRY CORE, PIN 0.246 IN: OD., 9 FT: ACT: LENGTH PLUS $2 \times 1 \mathrm{FT}$ : AXIAL BLANKET RADIAL BLANKET, ROD 0.731 IN, OD.

FUEL CLADDING SS

CORE 0.012 IN: THICK

RADIAL BLANKET: 0.020 IN: THICK

FUEL ASSEMELY 91-PIN BUNDLE, HEXAGONAL CONTAINER, SPACING BY GRIDMTYPE SPACERS FOR ICORE MODULES AND BY HEL ICAL WIRE WRAP FOR RADIAL BLANKET RODS. CORE MODULE SEPARATED BY 5 ROWS OF RADIAL BLANKET ASSEMBLIES

12 REACTOR MODULES. EACH SURROUNDED BY RADIAL

BLANKET AND CONTAINING 4 CONTROL RODS: 60 FUEL POSITIONS PER MODULE:

FUEL CHARGE CORE, 31,457 KG: URANIUM/PLUTONIUM OXIDE AXIAL BLANKET, $12: 071 \mathrm{KG}$, URANIUM OXIDE RADIAL BLANKET, 226;123 KG, URANIUM

SPECIFIC POWER CORE, $88.7 \mathrm{KW} / \mathrm{KG}$

BLANKET, $2.96 \mathrm{KW} / \mathrm{KG}$

BURNUP(REFUEL) CORE, 100,000 MWD/T SHUT - DOWN

BLANKET, 41,000 MWD/T

6 MO:-1 YEAR

CONTROL

ABSORBER RODS, 4 PER MODULE 
JANUARY 1969

$B N W L=936$

LIQUID METAL REACTORS, DOMESTIC FDZ9

COQLANT TEMP, INLET $500 \mathrm{~F}$ OUTLET $800 \mathrm{~F}$

REACTOR VESSEL MODULE CONTAINER OF SS, BIOLOGICAL SHIELD: GRID STRUCTURE LOCATES AND SUPPORTS THE FUEL ASSEMBLIES.

CONTAINMENT STEELIIINED CELL BELOW OPERATING FLOOR OF MILL: TYPE REACTOR BUILDING:

REMARKS

DESIGN IS AN ADAPTATION OF THE WESTINGHOUSE

MODULAR CONCEPT FOR A HIGH TEMPERATURE FAST BREEDER:

SEE FD28

CORE STRUCTURAL MATERIAL IS STAINLESS STEEL.

COOLANT FLOW IS UPWARD THROUGH REACTOR.

THE REFERENCE DESIGN ABSTRACTED REFERS TO A

DUAL PURPOSE SYSTEM:

REFERENCES A 3500 MWT LOW-TEMPERATURE SODIUM-COOLED FAST BREEDER REACTOR FOR DESALINATION

RC OLSON, OTHERS

CRNL-TM-1143 /JUNE 10,1965/

COST STUDY OF A LARGE SODIUM-COOLED FAST BREEDER

REACTOR FOR A SEA W WATER DESALINATION PLANT. WR GALL

ORNL-TM-784, /MARCH $1964 /$ 
JANUARY 1969

$B N W L=936$

LIQUID METAL REACTORS. DOMESTIC

FD30

NAME/OWNER

DES!GNER

TYPE

POWER MWE (MWT)

CRITICAL

COOLANT

FUEL MATER!AL

FUEL GEOMETRY

FUEL CLADDING

FUEL ASSEMBLY

FUEL CHARGE

NEUTRON FLUX

COOLANT TEMP.

REMARKS

REFERENCES
FFTF /FAST FLUX TEST FACILITYIAEC

PRELIMINARY DESIGN PACIFIC NORTHWEST LAB I,

BATTELLE MEMORIAL INSTITUTE,

FINAL DESIGNER SELECTION IN 1968

LIQUID METAL COOLED FAST TEST REACTOR. PRESSURE-TUBE; SPLIT CONICAL CORE,

FIRST DESIGN HAD A SKEWEDITUBE CORE.

CONCEPTUAL DESIGN:

DETAILED DESIGN 1968

CRITICALITY TARGET 1973; OPERATION 1974

FIRST OPERATION 300 MWT

SODIUM

URANIUM-PLUTONIUM OXIDE

PINS, ACTIVE LENGTH ABOUT 3 'FT,

SS, THINMWALL

DRIVER ASSEMBLY 217-PIN BUNDLE

ASSEMBLIES WILL BE ARRANGED IN HEXAGONAL RINGS INCLINED SLIGHTLY TO THE VERTICAL.

APPROX. 75 ASSEMBLIES.

600 KG PLUTONIUM

INITIAL FAST FLUX $0: 7$ E+16 AT $2 \mathrm{KW/GM} \mathrm{PLUTONIUM}$

BULK SODIUM COOLANT HEATED TO $1200 \mathrm{~F}$

TEST LOOP COOLANT TO $1400 \mathrm{~F}$

DISPERSED CORE REACTOR, WITH PIPED HEAT REMOVAL SYSTEM AND A HOT CELL: SHIELDED FUEL HANDLING MACHINE, DISPERSED CORE WILL ACCOMODATE A RANGE OF BOTH VERTICAL AND INCLINED FUEL ASSEMBLIES AND TEST LOOPS.

INITIAL CORE VOLUME 800-900 LITERS WITH MIXED

CXIDE AS DRIVER FUEL,

WESTINGHOUSE HAS BEEN SELECTED AS PRIME CONTRACTOR AND WILL AWARD BATTELLE-NORTHWEST CONTRACT ON BEHALF OF AEC, CORE DESIGN CHOICE IS BETWEEN THE SKEWED CORE AND WEST-S MODIFIED VERTICAL CORE,

THE FAST FLUX TEST FACILITY DESIGN CONCEPT, 
LIQUID METAL REACTORS. DOMESTIC FD3O

ER ASTLEY; LM FINCH; RJ. HENNIG

ANS TRANS, 8, 607 INOV. 1965,

PHYSICS ASPECTS OF THE FAST FLUX TEST REACTOR

WW LITTLE, PL HOFMANN

ANS TRANS, 8, 608 INOV, 1965 ,

FFTF DRIVER FUEL. PLUTONIUM DIOXIDE-STAINLESS

STEEL CERMET:

WI BAILEY, OTHERS

BNWL-SA-251 /NOV. 3; 1965 /

FAST FLUX TEST FACILITY

A AMOROSI; OTHERS

$A P D A=178$ P. $67: 70$

THE CONCEPTUAL EVALUATION OF A FAST FLUUX TEST

FACILITY.

ER ASTLEY

FAST REACTORS, ANS NATIONAL TOPICAL MEETING, APRIL 1967.

ANS-101/BNWL-SA-978/ 
BNWL-936
LIQUID METAL REACTORS. DOMESTIC 1909

NAME/ONNER GPU-AI FAST BREEDER STUDY

DESIGNER GENERAL PUBLIC UTILITIES IGPU/ AND ATOMICS

INTERNATIONAL /AII:

LOCATION PENNSYLVANIA ISUSQUEHANNA RIVERI

PURPOSE POWER

TYPE LIQUID METAL COOLED FAST REACTOR

PONER MWE(MWT) $500 \quad 1250$

CRITICAL

DEMOSTRATION PLANT PLANNED

AI-S FEASIBILITY STUDY FOR A 1000 MWE REACTOR WAS FOR A MODULAR FLUX TRAP SYSTEM, A VERSION OF THE SSCR /SEE EDOI/

THE FOLLOWING DATA REFERS TO THIS DESIGN:

COOLANT SODIUM

FUEL MATERIAL URANIUM-PLUTONIUM OXIDE PELLETS

OR URANIUM-PLUTONIUM CARB!DE, CORED.

BLANKET DEPLETED URANIUM DIOXIDE

FUEL GEOMETBY FOD

FUEL CLADE:NG STAINLESS STEEL

FUEL ENR!CM.

BLANKET DEPLETED URANIUM

FUEL ASSEMBLY EUNDLE

CORE

AXIAL BLANKET

RADIAL ELANKET

CONTROL TANTALUM AESOREER; RODS

COOLANT TEMP, INLET $760 \mathrm{~F}$ OUTLET $1060 \mathrm{~F}$

REACTOR VESSEL VESSEL SUSFENDED IN STEEL-LINED CONCRETE CAVITY,

REMARKS THE CONCEPTUAL DESIGN STUDY BEGAN EARLY IN 1967, BECHTEL SELECTED AS ARCHITECT-ENGINEER,

A DECISION IS EXPECTED BY 1970 ON CONSTRUCTION OF

A 300.500 MWE PROTOTYPE, PROBABLY ON PENELEC

SERVICE AREA.

DUKE POWER, BALTIMORE G=E, AND SOUTH CAROLINA G=E HAVE JOINED THE PROGRAM. GPU MEMBERS ARE

PENNSYLVANIA ELECTRIC; METROPOLITAN ED,. NEW 
JANUARY 1969

LIQUID METAL REACTORS. DOMESTIC

JERSEY $P=L$, AND JERSEY CENTRAL $P=L$.
BNWL -936

FD31

FEASIBILITY STUDY OF A 1000 MWE SODIUM=COOLED FAST REACTOR,

VOL, 1, TECH, AND ECONOMIC POTENTIAL,

NAA-SR-11378 /VOL. $1 /$ JULY 1966

THE GPU-AI DEMONSTRATION FAST BREEDER REACTOR.'

HM DIECKAMP, OTHERS

NUCLEAR NEWS JULY 1968, P, 41-50

ATOMICS INTERNATIONAL DEMONSTRATION FAST BREEDER

REACTOR.

HM DIECKAMP, OTHERS

ANS TOPICAL MEETING ON FAST REACTORS, 1967

ANS-101/1967/ 


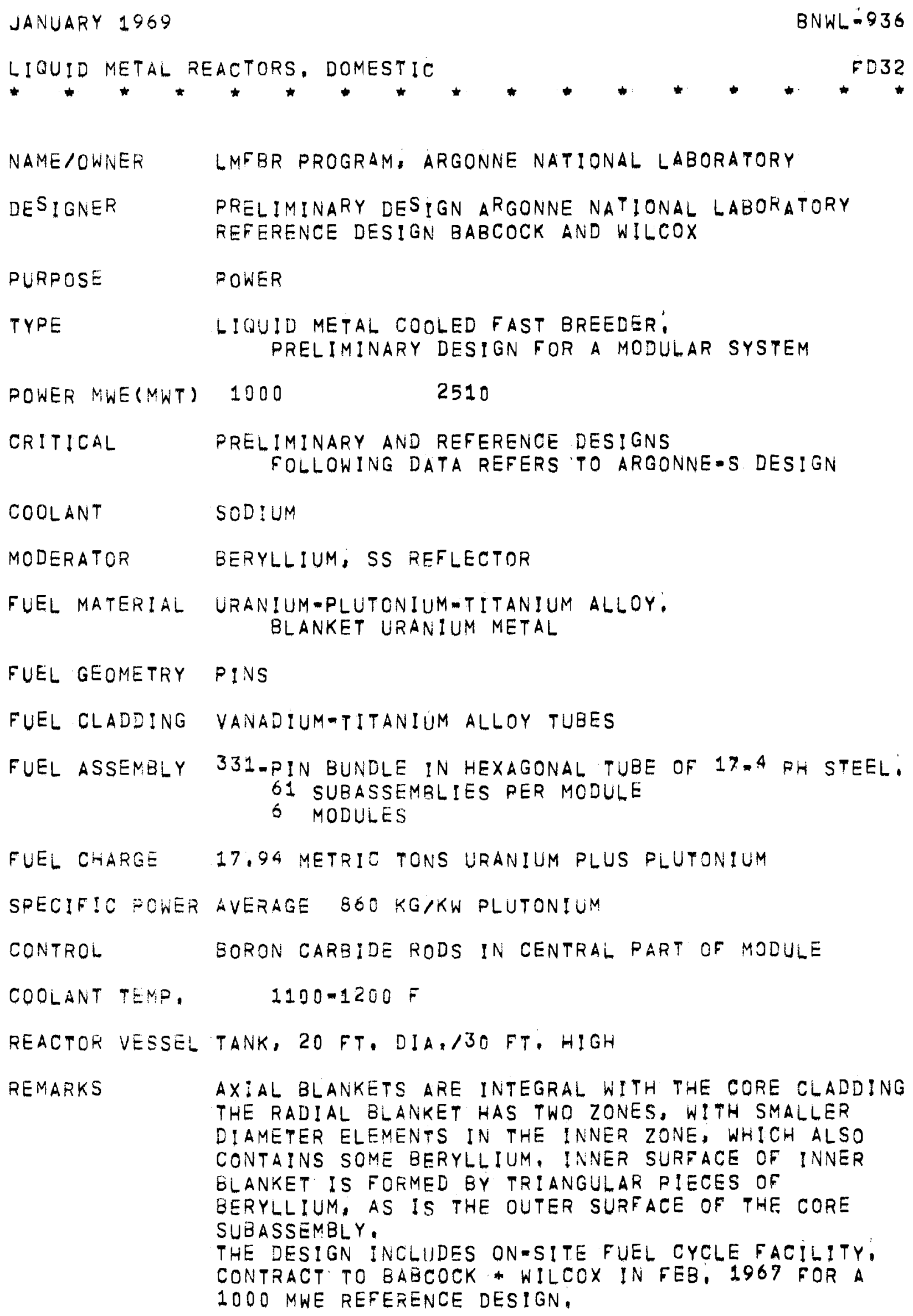


JANUARY 1969

BNWL $=036$

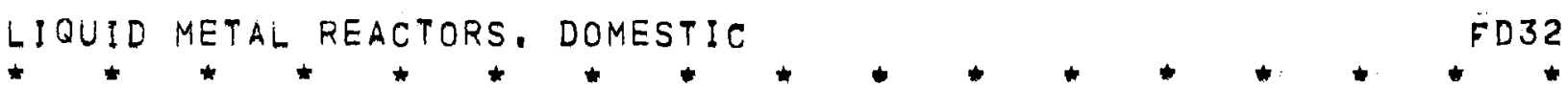

ANL WILL STUDY FIVE DESIGN CONCEPTS WITH

ATOMICS INTERNATIONAL, BABCOCK + WILCOX, COMBUSTION ENGINEERING, GENERAL ELECTRIC, AND WESTINGHOUSE.

REFERENCES

1000 MWE METAL-FUELED FAST BREEDER REACTOR: LE LINK; OTHERS

ANL-7001 /JUNE 1966/

APPLIED ATOMICS FEB: 22, $1967: 7$

NEWS RELEASE 


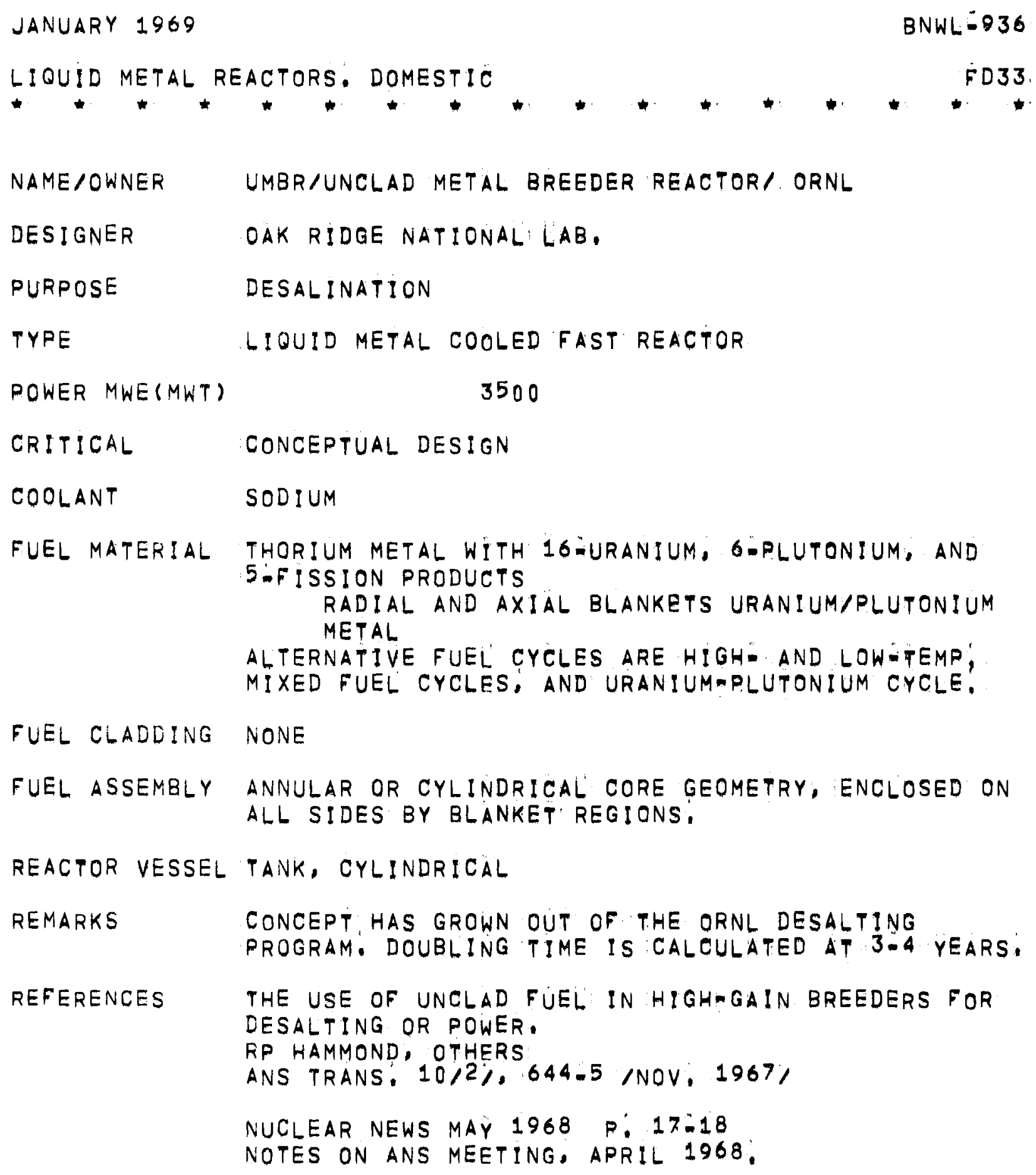


JANUARY 1969

$B N W L=936$

LIQUID METAL REACTORS, DOMESTIC

FD34

NAME/OWNER LARGE FAST BREEDER/ÄL: WESTINGHOUSE
TYAE
$\begin{aligned} & \text { LIQUID METAL COOLED FAST BREEDER } \\ & \text { ANNULAR DESIGN }\end{aligned}$

POWER MWE(MWT) 3880

CRITICAL CONCEPTUAL DESIGN

COOLANT SODIUM

MODERATOR BERYLLIUM OXIDE REFLECTOR

FUEL MATERIAL URANIUYMIPLUTONIUM CAREIDE VIBRATORY COMPACTED INTO TUBES: BLANKET URANIUM-ZIRCONIUM ALLOY

FUEL GEOMETRY ROD 0,275 IN, OD

BLANKET PINS $0: 385$ IN, OD AND 0.515 IN, OD

FUEL CLADDING CORE SS TUBES, WALL THICKNESS 0:015 IN, RADIAL BLANKETS A AND C, SS CLAD O,385 IN: OD AND 0.022 IN: THICK

RADIAL BLANKETS B AN D, SS CLAD O:515 IN, OD HELIUM BOND, AND 0.025 IN: THICK

FUEL ENRICH, AXIAL BLANKET DEPLETED URANIUM

FUEL ASSEMBLY CORE, 331-ELEMENT ASSEMBLY, HEXAGONAL 456 CORE ASSEMBLIES

RADIAL BLANKETS A AND $C$; 217-RIN ASSEMBLY 132 ASSEMBLIES

RADIAL BLANKETS B AND; 169-PIN ASSEMBLY

B. 96 ASSEMBLIES

D. 138 ASSEMBLIES

FUEL CHARGE 10.500 KG PLUTONIUM

BURNUP (REFUEL) CORE, 110,000 MWD/T

REACTOR: 35,000 MWD/T

$1 / 2$ YEAR INTERVALS

CONTROL POISON RODS

COOLANT TEMP, INLET $720 \mathrm{~F}$ OUTLET $1050 \mathrm{~F}$

REACTOR VESSEL CYLINDRICAL STEEL VESSEL

REFERENCES FEASIBILITY STUDY OF NUCLEAR STEAM SUPPLY SYSTEM

USING 10,000 MW SODIUM COOLED BREEDER REACTOR. 
JANUARY 1969

BNWL-936

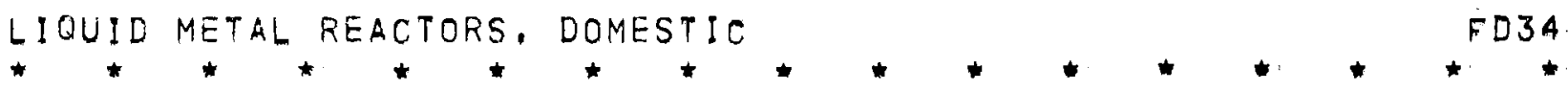

KA HUB, OTHERS

ANL-7183 /SEPT: 1966/ 


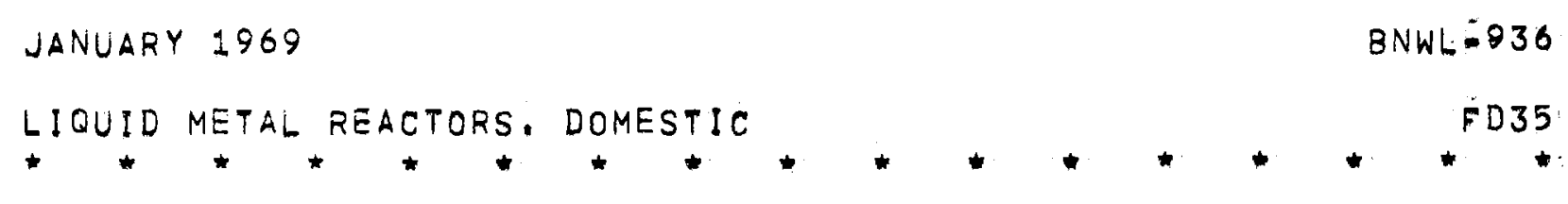

NAME/OWNER LMFBR/LIQ: METAL FAST BREEDER REACTOR/BAW; AEC

DESIGNER BABCOCK + WILCOX

TYPE LIQUID METAL COOLED FAST BREEDER

POWER MWE(MWT) 1000

CRITICAL DESIGN

COOLANT SODIUM

FUEL MATERIAL URANIUM/PLUTONIUM DIOXIDE, VIBRATORY COMPACTED

BLANKET URANIUM DIOXIDE

FUEL GEOMETRY PIN, VENTED. ACTIVE LENGTH 37 IN,

FUEL CLADDING SS

FUEL ENRICH. BLANKET DEPLETED URANIUM

FUEL ASSEMBLY HEXAGONAL ASSEMBLIES, UNCANNED

177 ASSEMBLIES/CORE

FIRST CORE WOULD BE NON-VENTED, EQUILIBRIUM

CORE VENTED TO SODIUM.

CONTROL RODS, SSMLLAD BORON CARBIDE

REACTOR VESSEL CORE VESSEL $171 / 2$ IN: DIAM: WELDED SS PLATE; 2 IN, THICK. DEPTH 26 FT. LARGER VESSEL ACCOMODATES ALL PRIMARY SYSTEM:

CONTAINMENT CONTROLLED LEAKAGE BUILDING; CYYINDRICAL CONTAINMENT VESSEL $110 \mathrm{FT}$, ID:

REMARKS

CONCEPT FOR A NUCLEAR ISLAND AND TURBINE ISLAND COMPLEX.

REFERENCES DESIGN OF A $1000 \mathrm{mWE}$ LIOUID-METAL-COOLED FAST BREEDER POWER REACTOR:

RL BOYER, FX MASSETH

ASME PREPRINT $67=$ WA/NE-10 /1967/ 
JANUARY 1969

$B N W L=936$

LIOUID METAL REACTORS, DOMESTIC

FD 36

NAME/ONNER OBFR /ORDERED BED FAST REACTOR/BNL

DESIGNER BROOKHAVEN NATIONAL LABORATORY

PURPOSE POWER

TYPE LIQUID METAL COOLED FAST BREEDER

POWER MWE(MWT) 1000

CRITICAL DESIGN

COOLANT SODIUM

FUEL MATERIAL UNCLAD URANIUM/PLUTONIUM CARBIDE SPHERES

D:410 IN. DIAM.

FUEL GEOMETRY ORDERED BED OF FUELED SPHERES IN SHAPED CONTAINERS

FUEL CLADDING FUEL CONTAINERS WALL THICKNESS I/4, IN, FILLED HEIGHT /ACTIVE/ 48 IN:

FUEL ASSEMBLY SQUARE CONTAINERS $12 \times 12 \times 59$ IN.

156 CONTAINERS/CORE

CENTRAL BLANKET, 4

OUTER BLANKET, 36

CORE AND AXIAL BLANKET 116

SPECIFIC POWER 383 KWT/KG

BURNUP (REFUEL) 100,000 MWD/T

EMPTYING AND REFILLING CONTAINERS

CONTROL RODS

COOLANT TEMP, INLET $700 \mathrm{~F}$ OUTLET $1200 \mathrm{~F}$

COOLANT PRESS, INLET 320 PSIG OUTLET 20 PSIG

REACTOR VESSEL SS CYLINDER 241:5 IN, OD, $251: 5$ IN, H:GH WALL THICKNESS 7 IN:

CONTAINMENT VERTICAL STEEL CYLINDER $124 \mathrm{FT}, 1 \mathrm{D}, 184 \mathrm{FT}$, HIGH W! TH 72 FT. BELOWIGRADE, HOUSES REACTOR, PR:MARY SYSTEM, AND FUEL HANDLING SYSTEM,

REMARKS

OMFBR IS AN OUTGROWTH OF SBDR, SEE FD 16

REFERENCES THE ORDERED-BED FAST REACTOR CONCEPT 


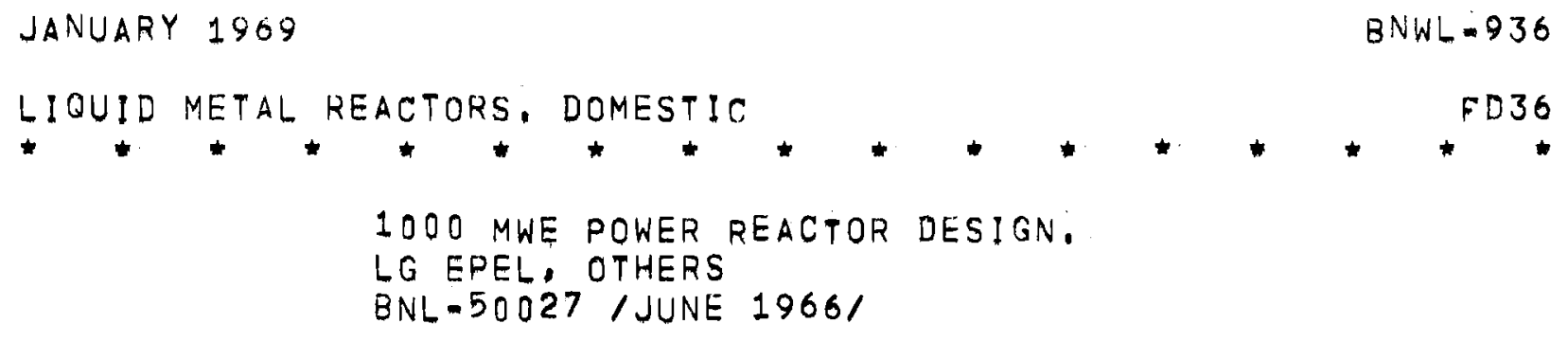


LIQUID METAL COOLED REACTORS

FOREIGN 
JANUARY 1969

BNWL:-936

LIQUID METAL REACTORS; FOREIGN

$F F 01$

NAME/OWNER RAPSODIE/CEA, FRANCE

DESIGNER CEA, FRANCE

OPERATOR CEA; FRANCE

LOCATION CADARACHE RESEARCH CENTER, FRANCE

PURPOSE POWER EXPERIMENT

TYPE FAST BREEDER EXPERIMENT

POWER MWE (MWT)

CRITICAL

FULL POWER MARCH 1967

COOLANT

SODIUM. TWO PRIMARY SYSTEMS: CENTRAL AREA HIGH

PRESSURE AND PERIPHERAL AREA LOW PRESSURE,

FUEL MATERIAL CORE, URANIUMIPLUTONIUM DIOXIDE PELLETS

$$
5: 8 \text { MM. DIA., 11:5 MM. LONG }
$$

BLANKET, URANIUM DIOXIDE

FUEL GEOMETRY PENCILS: PELLET STACK 340 MM. HIGH

FUEL CLADDING SS 0.45 MM. WALL THICKNESS

FUEL ENR!CH,

$$
\begin{aligned}
& \text { PLUTONIUM-240 AND } 24110: 2 \text { PER CENT } \\
& 60 \text { PER CENT U-235 }
\end{aligned}
$$

BLANKET, DEPLETED URANIUM

FUEL ASSEMBLY 37 PENCILS/ASSEMBLY IN HEXAGONAL CASING,

CORE PENCILS INCLUDE AXIAL: BLANKET:

53 ASSEMBLIES + 500 PERIPHERAL

FERTILE ASSEMBLIES

AX: AL BLANKET 14 PINS/ASSEMBLY

RADIAL BLANKET 7 PINS/ASSEMBLY

FUEL CHARGE 8 TONS URANIUM DIOXIDE

SPECIFIC POWER $1750 \mathrm{~W} / \mathrm{CC}, 11.52 \mathrm{KW/CC,} \mathrm{FUEL/}$

BURNUP(REFUEL) 30,000 MWD/TON FUEL

NEUTRON FLUX HIGH $2 \times 10 \quad E+15$

CONTROL RODS IN PERIPHERY OF CORE, BORON CARBIDE

COOLANT TEMP, INLET $450 \mathrm{C}$ OUTLET $540 \mathrm{C}$

REACTOR VESSEL SS SHELL 7 FT, 8 IN: DIA, 0,6 IN, THICK, 


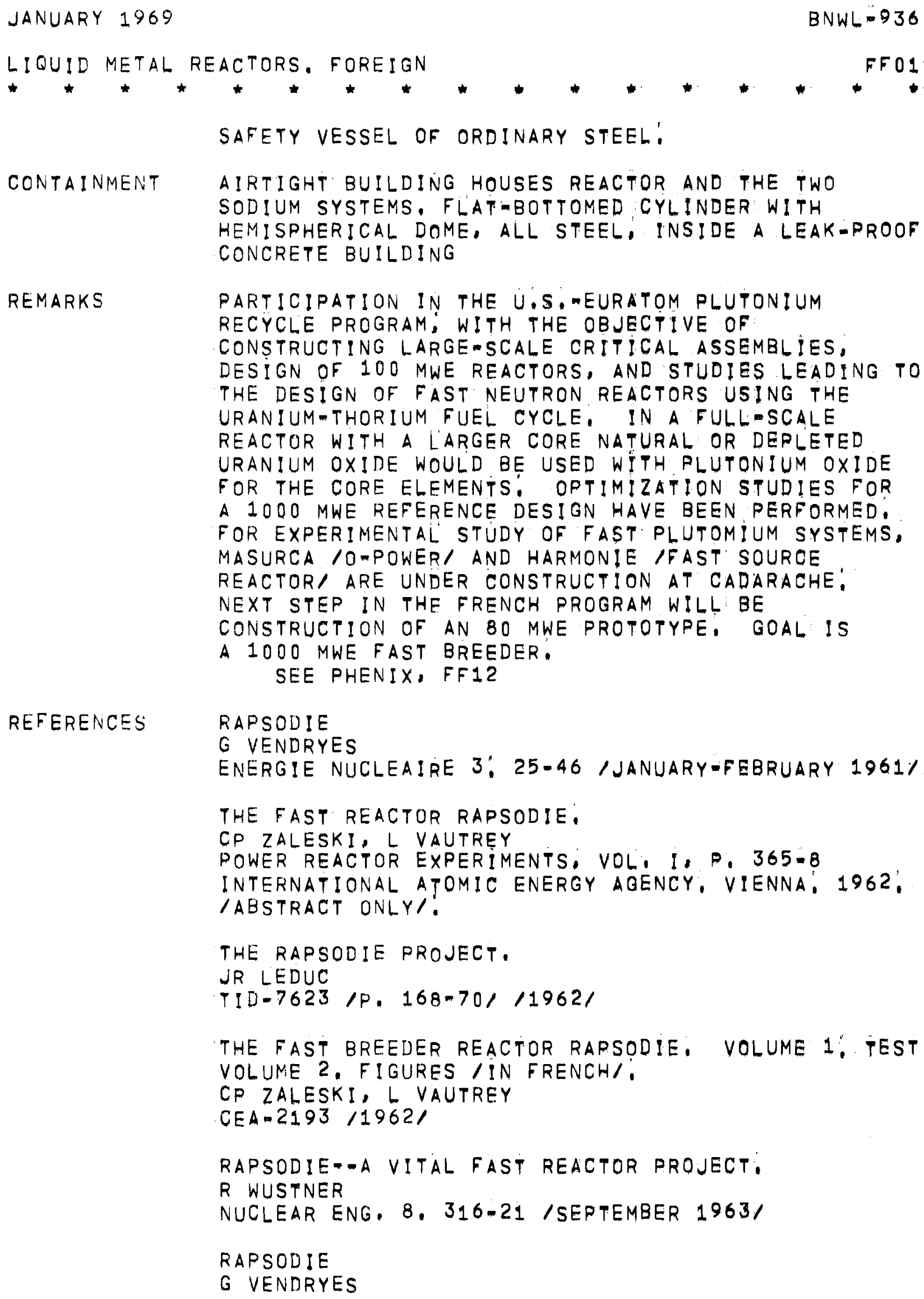

THE FAST BREEDER REACTOR RAPSODIE; VOLUME 1: TEST VOLUME 2, FIGURES /IN FRENCH/: 
JANUARY 1969

BNWL-936

LIOUID METAL REACTORS, FOREIGN

3RD UN, INTL, CONF, ON THE PEACEFUL USES OF ATOMIC ENERGY, GENEVA, 1964

$A / C O N F, 28 / P / 42$ 


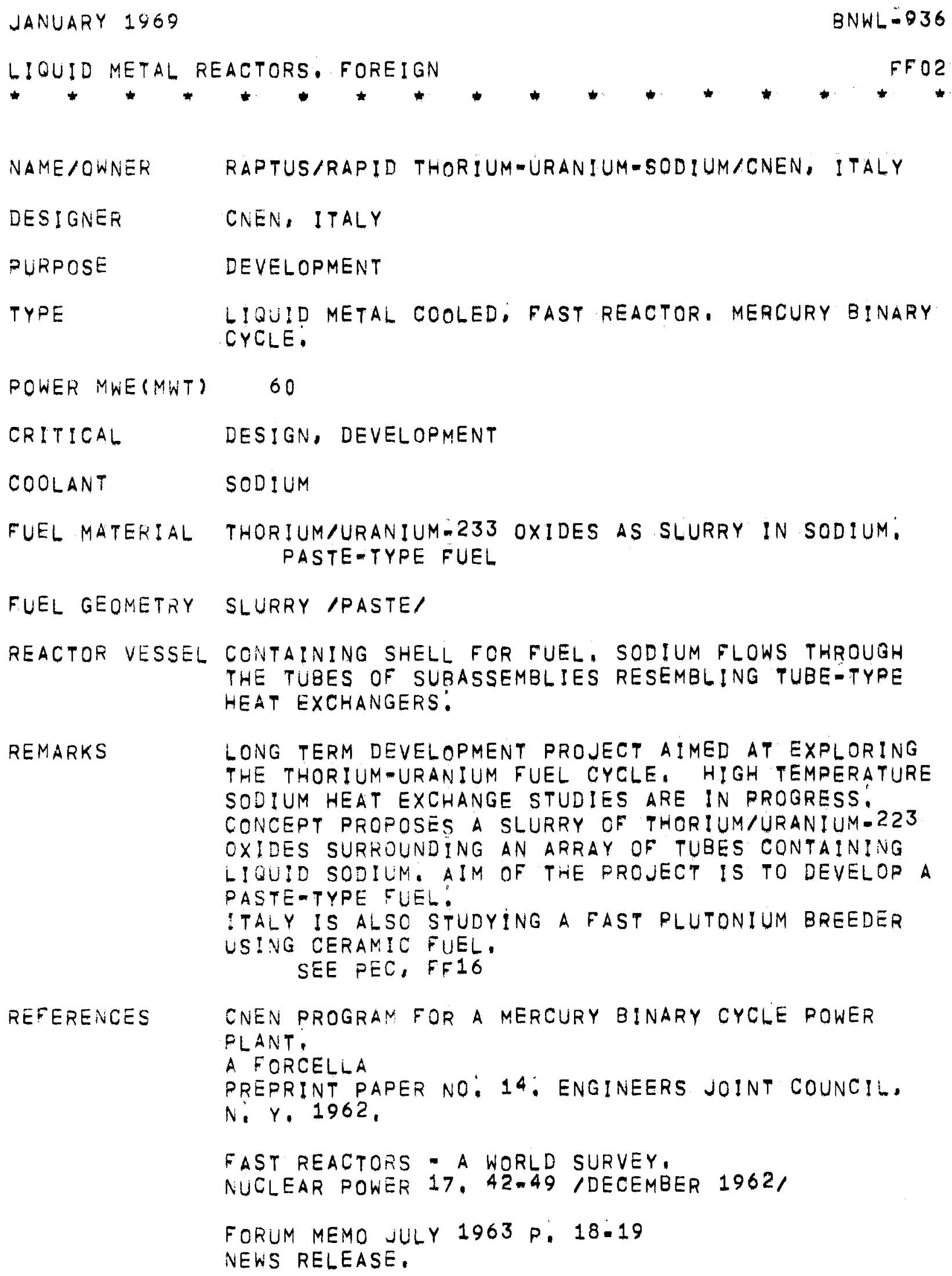


JANUARY 1969
LIOUID METAL REACTORS, FOREIGN
$* \star *$ BNWL $=936$

NAME/OWNER DFR IDOUNREAY FAST REACTOR/ AEA; UNITED KINGDOM

DESIGNER AEA, UNITED KINGDOM

OPERATOR AEA, UNITED KINGDOM

LOCATION DOLNREAY, CAITHNESS; SCOTLAND

PURPOSE POWER EXPERIMENT

TYPE LIQUID METAL COOLED FAST BREEDER

POWER MWE(MWT) $15 \quad 60$

CRITICAL NOV: 1959

COOLANT SODIUM-POTASSIUM NAK, LATER SODIUM.

FUEL MATERIAL URANIUM-MOLYEDENUM ALLOY

NATURAL URANIUM BLANKET

FUEL GEOMETRY HOLLOW RODS,

BLANKET SOLID RODS,

FUEL CLADDING NIOBIUM /OUTER/ AND VANADIUM IINNER/.

BLANKET SS.

FUEL ENRICH, 40 FER CENT U-235

FUEL ASSEPBLY ANNULAR TUEE 525 MM: LONG/18,75 MM. DIA, UPPER

PART OF ELEMENT-THE GREEDER END-PIECE- IS $150 \mathrm{MM}$. LENGTH OF NATURAL URANIUM, BREEDER RODS SURROUND

THE CORE, SEE REMARKS. 345 ELEMENTS/CORE

1872 BREEDER ELEMENTS

SPECIF!C FONER $286 \mathrm{KW} / \mathrm{KG} \mathrm{U}-235$

BURNUP(REFLEL) APPROX, 2 PER CENT

CONTROL RODS, REACTIVITY CONTROL BY MOVEMENT OF FUEL:

COOLANT TEMP. INLET $200 \mathrm{C}$ OUTLET $400 \mathrm{C}$

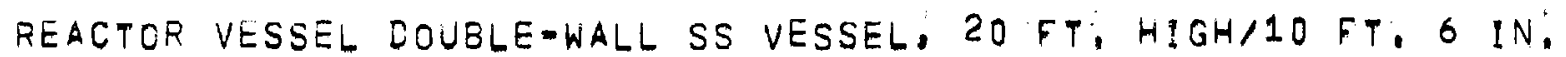
$O D$.

REMARKS DOUNREAY WAS IN OPERATION IN 1960, SHUT DOWN FOR CORE MODIFICATIONS, WITH RESUMED OPERATION IN AUGUST 1960. LATTICE IS STAR-SHARED. THE FORM 


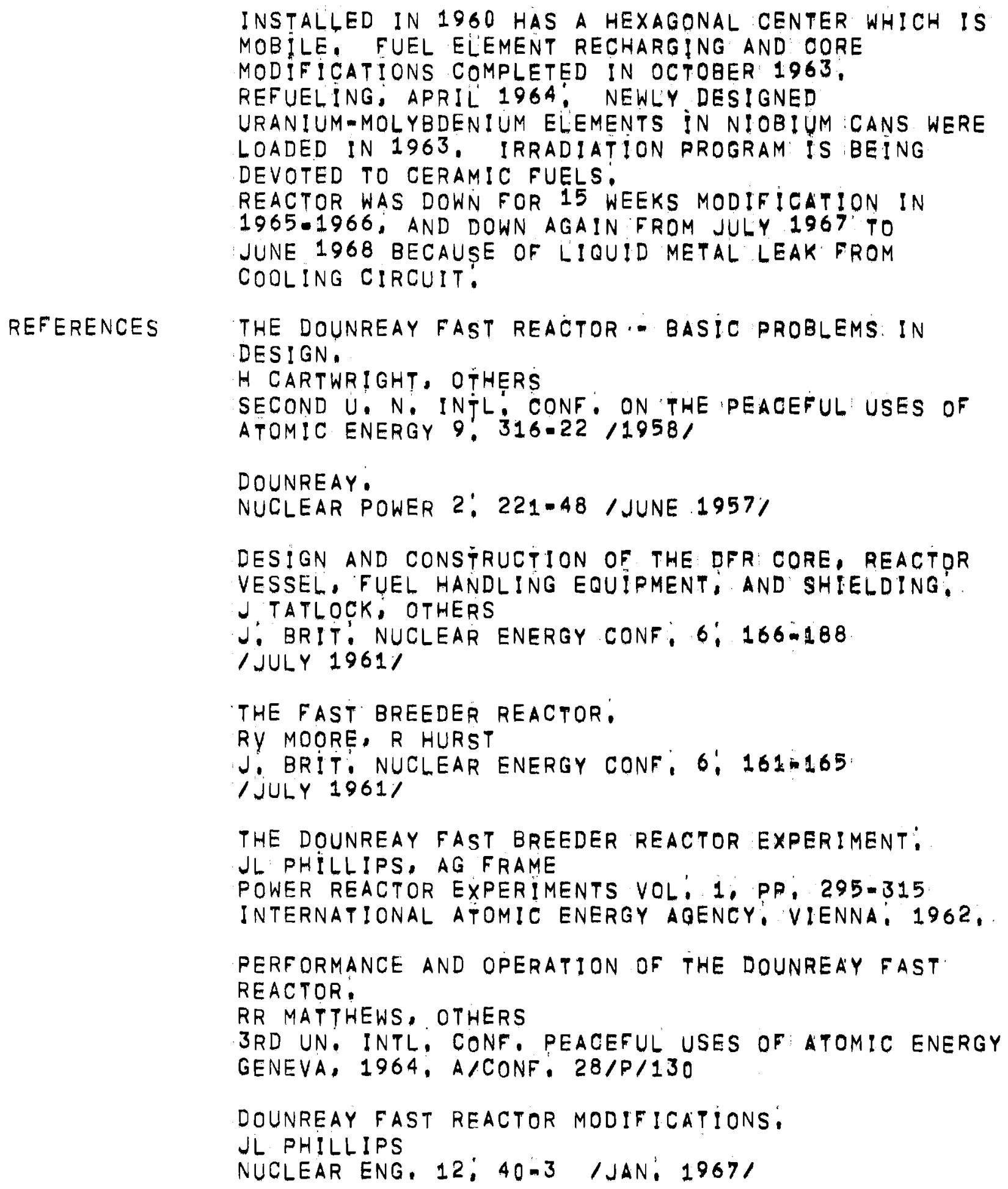


JANUARY 1969
LIOUID METAL REACTORS. FOREIGN BNWL=036

NAME/OWNER

PFR /PROTOTYPE FAST REACTOR/ UK ATOMIC ENERGY AUTH

DESIGNER

AEA; UNITED KINGDOM

LOCATION

DOUNREAY, SCOTLAND

PURPOSE

POWER PROTOTYPE; INTERMEDIATE TO 1000 MWE PLANT

TYPE

LIOUID METAL COOLED FAST BREEDER

POWER MWE(MWT) 250

600

CRITICAL CONSTRUCTION. TARGET 1970:

COOLANT SODIUM, PRIMARY AND SECONDARY CIRCUITS

MODERATOR RADIAL GRAPHITE REFLECTOR

FUEL MATERIAL PLUTONIUMIURANIUM OXIDE

BREEDER MATERIAL U-238 OXIDE

FUEL GEOMETRY PIN 0.23 IN. OD:36 IN. LONG UPPER AND LOWER AXIAL BREEDER PINS 18 IN.LONG UPPER AXIAL PIN 0.75 IN! OD:

FUEL CLADDING SS 0.015 IN. WALL THICKNESS

FUEL ENRICH. TWO-ZONE, PLUTONIUMAURANIUM $1 / 3$

FUEL ASSEMBLY 325-PIN ASSEMBLY, HEXAGONAL 78 ASSEMBLIES/CORE

UPPER AXIAL BREEDER ELEMENTS 19MP!N ASSEMBLIES. SEE REMARKS

BURNUP(REFUEL) 7:5 PER CENTT OFFMLOAD

CONTROL CONTROL AND SHUTMOFF RODS

COOLANT TEMP, INLET $400.420 \mathrm{C}$ OUTLET $560-585 \mathrm{C}$

REACTOR VESSEL CYLINDRICAL SS TANK: $40 \mathrm{FT}$, DIAM: $50 \mathrm{FT}$, DEEP, WALL THICKNESS $1 / 2$ IN: SURROUNDED BY ALLOY STEEL LEAK JACKET.

CONTAINMENT REINFORCED CONCRETE VAULT BELOW GROUND LEVEL;' LEAK-TIGHT BUILDING

REMARKS INNER ZONE OF CORE CONTAINS 30 ELEMENTS, OUTER ZONE SOME 48, CORE IS SURROUNDED BY RADIAL CORE REFLECTOR; FOLLOWED BY SINGLE ROW OF RADIAL 
JANUARY 1969

$B N W L=936$

LIQUID METAL REACTORS. FOREIGN

BREEDER ASSEMBLIES, OUTSIDE THERE IS A BREEDER REFLECTOR:

THE PROJECT INCLUDES FUEL FABRICATION FACILITIES

AT WINDSCALE.

REFERENCES

APPLIED ATOMICS FEB; 16, $1966 \mathrm{P} ; 1=\mathrm{A}$

NUCLEONICS WEEK FEB，17, 1966 P, $1=2$

NEWS RELEASES

POWER FROM PLUTONIUM

W PENNEY

NEW SCIENTIST 29, 484:86,/FE8; 24; $1966 /$

FAST REACTORS ON THE LINE BY 1971

AG FRAME, RR MATTHEWS

NUCLEONICS 24, 54-59 /SEPT; $1966 /$

DESIGN OF THE PROTOTYPE FAST REACTOR,

AG FRAME, OTHERS

FAST BREEDER REACTORS: PROC; LONDON CONF, BRITISH

NUCLEAR ENERGY SOC.: MAY 1966, P, 291-315

PERGAMON, 1967

STATUS OF UKAEA-S PROTOTYPE FAST REACTOR, AG FRAME

FAST REACTORS; ANS NATIONAL TOPICAL MEETING 1967. ANS-101; $P, 6-15$ TO 6-35 


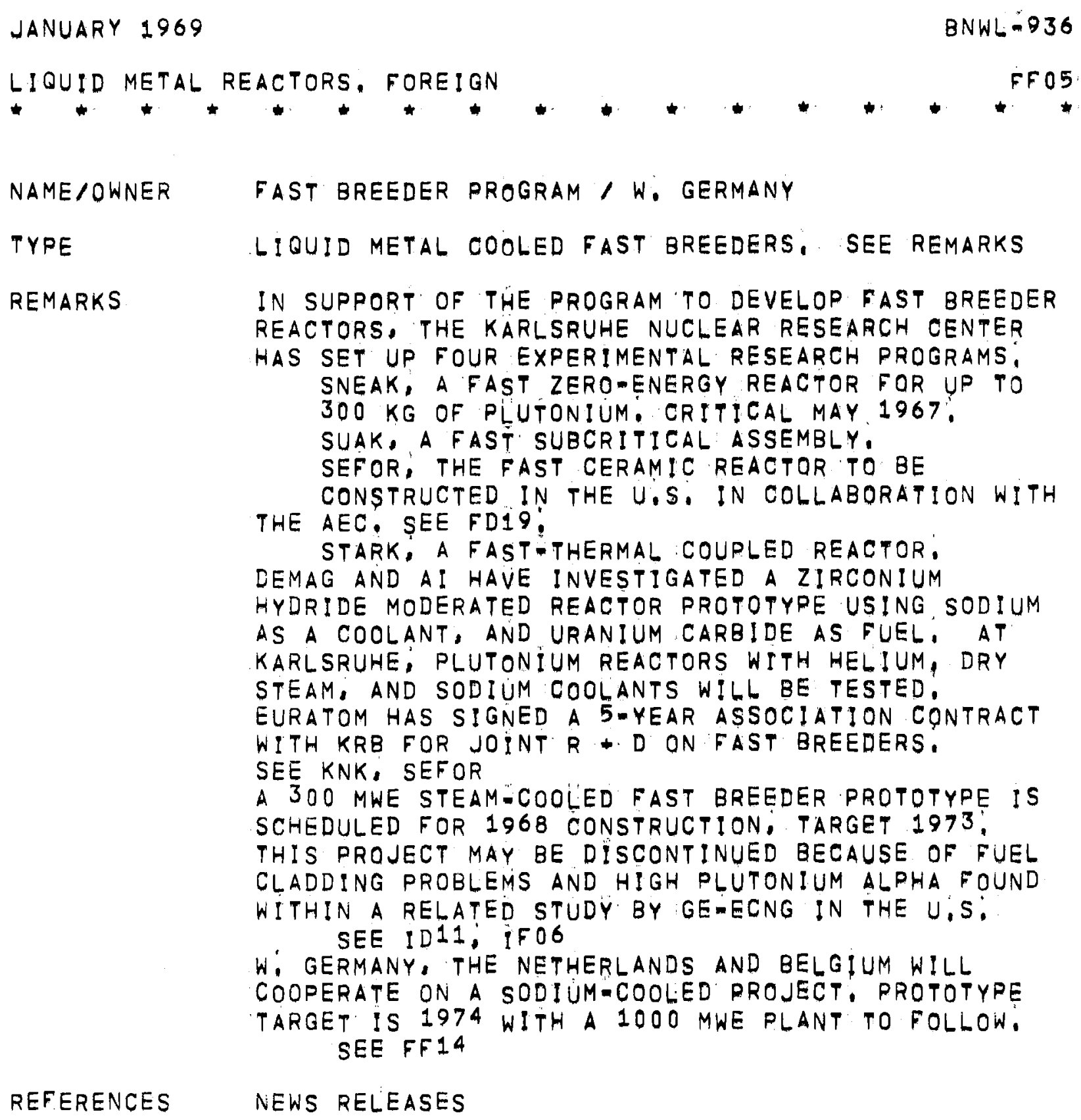


JANUARY 1969

BNWL:936

LOUID METAL REACTORS, FOREIGN

NAME / OWNER

KNK /KOMPAKTES NATRIUM KERNKRAFTWERK//W, GERMANY

DES IGNER

INTERATOM

LOCATION

GROSSWELZHEIN/KAHLIMAIN/, W: GERMANY

KARLSRUHE

PURPOSE

EUROPEAN FAST BREEDER PROGRAM

TYPE

LIOUID METAL COOLED: ZIRCONIUM HYDRIDE MOD, FAST GREEDER, SEE REMARKS

POWER MWE(MWT)

21

58

CRITICAL

CONSTRUCTION. TARGET 1970

COOLANT

SODIUM

MODERATOR

ZIRCONIUM HYDRIDE

FUEL MATER!AL

URANIUM DIOXIDE PELLETS

FUEL GEOMETRY ROD

FUEL ENRICH. 6:8 PER CENT U-235

FUEL ASSEMBLY

RING-SHAPED ASSEMBLY, ENCLOSED BETWEEN CENTRAL

CYLINDRICAL AND OUTER RING=SHAPED MODERATOR PART 66 FUEL ASSEMBLIES/CORE CORE DIAM. 1175 MM. HEIGHT $1050 \mathrm{MM}$

FUEL CHARGE 1,68 TONS FUEL

BURNUP(REFUEL) $10,000 \mathrm{MWD} / \mathrm{T}$

CONTROL RODS, BUILT-IN ABSORBING ELEMENTS

COOLANT TEMP, INLET $410 \mathrm{C}$ OUTLET $560 \mathrm{C}$

REACTOR VESSEL DOUBLE TANK. INNER 1,9 M. DIA:, 10,5 M. HIGH: SEPARATED FROM SECOND TANK BY NITROGEN ATMOSPHERE,

CONTAINMENT $17 \mathrm{M}$, DIA, STRUCTURE INCORPORATING THE REACTOR, HANDLING EQUIPMENT, PRIMARY SODIUM PLANT,

REMARKS

A 300 MWE PROTOTYPE FAST REACTOR IS SCHEDULED FOR $\$ 968$ CONSTRUCTION: PROGRAM INVOLVES SIEMENS, INTERATOM. AND DUTCH COMPANIES.

REFERENCE DESIGN FOR A 1000 MWE FAST REACTOR HAS BEEN COMPLETED. 
JANUARY 1969

BNWL -936

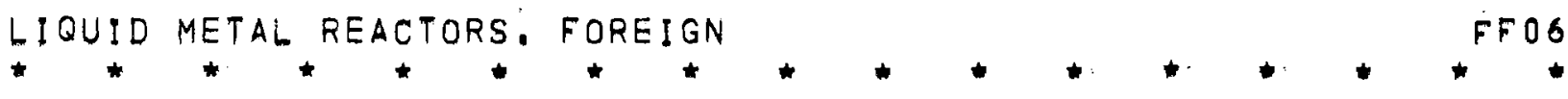

SEE FF14

AFTER TECHNICAL TESTING, A FAST CORE WILL BE INSTALLED: REACTOR WILL BE AVAILABLE AS RADIATION SOURCE FOR FAST BREEDER FUELS,

REFERENCES KARLSRUHE NUCLEAR, RESEARCH CENTER,

EURONUCLEAR 2: 41:8/JAN. 1965/

SAFETY ASPECTS OF THE EXPERIMENTAL POWER STATION KNK

NUCLEAR ENGINEERING 10, 335-37 /SEPT: 1965/

COMPACT SODIUM=COOLED NUCLEAR REACTOR INSTALLATION AT KARLSRUHE /KNK/

H TEBBERTS, OTHERS

ATOM U STROM 13; 117-29 /SEPT-OCT, $1967 /$ 


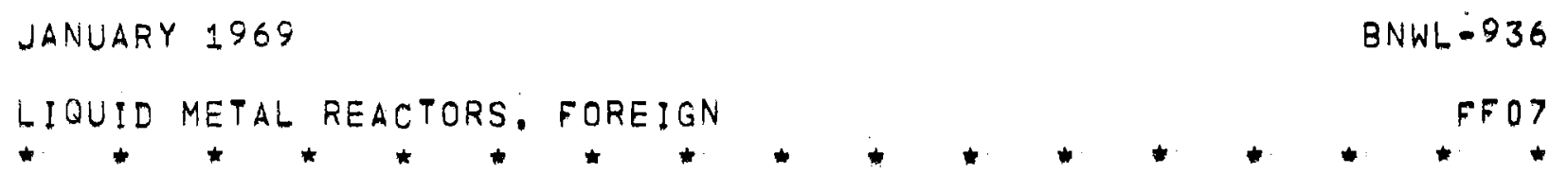

NAME/OWNER BR=5 /SOVIET BREEDER REACTOR//USSR

DESIGNER USSR

OFERATOR LUSS

LOCATION OBN!NSK, USSR

INST, PHYSICS AND POWER ENG: IPHYSITECH, INSTI

PURPOSE FAST REACTOR $R+D$

TYPE LIQUID METAL COOLED: FAST BREEDER

POWER MWE(MWT) 5 KWE 5

CFITICAL DPERATION SUMMER 1959

COOLANT SODIUMMPOTASSIUM NAK

MODERATOR NICKEL REFLECTOR, INTERNAL URANIUM REFLECTOR

FUEL MATERIAL PLUTONIUM DIOXIDE PELLETS 0.4 CM: DIA:

$$
\text { BLANKET, URANIUM }
$$

FUEL GEOMETRY FIN: 0.5 CM. OD:, ACTIVE LENGTH 28 CM: BLANKET, ROD $0.9 \mathrm{CM}, \mathrm{DIA}$.

FUEL CLADDING SS TUBES 0.04 CM, WALL THICKNESS

FUEL ASSEMBLY CORE, 19-PIN ELEMENT 81 ASSEMBLIES/CORF BLANKET, 7-ROD ELEMENT $4 O$ ASSEMBLIES/CORE WITH UC FUEL THERE WOULD BE 1007 MIN ELEMENTS IN THE CORE.

NEUTRON FLUX FAST MAX. $1 \times 10$ E+15

CONTROL MOVEMENT OF NICKEL REFLECTOR

COOLANT TEMP, INLET $806 \mathrm{~F}$ OUTLET $932 \mathrm{~F}$

REACTOR VESSEL SODIUM COOLANT CONTAINER TUBE IENCLOSES FUEL ASSEMBLIES/, WATER TANK, CAST IRON SHIELD.

CONTAINMENT CAST IRON AND WATER SHIELDING, HIGH DENSITY CONCRETE STRUCTURE

REMARKS

BR=1, BUILT IN 1955; WAS A 100-WATT PLUTONIUM FUELED REACTOR, 
LIQUID METAL REACTORS, FOREIGN

BR-2, BUILT IN 1956; WAS A 100-KWT MERCURY-COOLED. PLUTONIUM FUELED SYSTEM, BR-2 WAS REDESIGNED TO THE BR-5 FAST BREEDER: 50 ELEMENTS CONTAINING URANIUM DIOXIDE REPLACED PLUTONIUM ELEMENTS IN THE CORE ASSEMBLY, AND LATER 8 OF THESE WERE REPLACED BY URANIUM CARBIDE ELEMENTS, BY JANUARY 1965 THE CORE WAS TO BE COMPLETELY URANIUM CARBIDE;

REFERENCES

THE SOVIET FAST REACTOR BR=5, RR MATTHEWS

NUCLEAR ENG.4. 359-60 /OCTOBER 1959/

SOME PROBLEMS IN THE OPERATION OF THE BR-5 FAST NEUTRON REACTOR: MS PINKHASIK, OTHERS AEC-TR- 5266

TRANSLATION FROM A PUBLICATION OF THE STATE COMMITTEE OF THE COUNCIL OF MINISTERS OF THE USSR ON THE USE OF ATOMIC ENERGY, MOSCOW, 1961

OPERATIONAL EXPERIENCE WITH THE BR-5 REACTOR: AI LEIPINSKII, OTHERS

I AEA PREPRINT NO. CN-15/40/CONFERENCE ON OPERATIONAL EXPERIENCE WITH ATOMIC POWER REACTORS, VIENNA, JUNE 4-10;1963/ AEC-TR-5890/TRANSLATION/

EXPERIMENTAL FAST REACTORS IN THE SOVIET'UNION, AI LEIPINSKY, OTHERS

PROC, 2ND, UN, INTL! CONF, PEACEFUL USES OF ATOMIC ENERGY, GENEVA, 1958, VOL, 9, P: 348.357

NUCLEAR REACTORS IN THE SOVIET UNION, REPORT OF A RECIPROCAL EXCHANGE TRIP BY US SCIENTISTS, DEC: 11-22, 1964, WASH=1060 
JANUARY 1969

BNWL:D\$36

LIOUID METAL REACTORS, FOREIGN

FFO8

NAME/OWNER

$B N-50 /$ USSR

DES \GNER

USSR

PURPOSE

FEASIBILITY STUDY:

TYPE

LIOUID METAL COOLED FAST BREEDER

POWER MWE (MWT)

50

200

CRITICAL

DESIGN AND FEASIBILITŸ STUDY

COOLANT

SODIUM

FUEL MATERIAL

URANIUM-MOLYBDENUM ALLOY: URANIUM DIOXIDE IN

NICKEL MATRIX, OR SINTERED URANIUM DIOXIDE?

FUEL GEOMETRY RODS

COOLANT TEMP, INLET $315 \mathrm{C}$ OUTLET $450 \mathrm{C}$

REMARKS

PROPOSED PLANT ḦAS BEEN DISCONTINUED IN FAVOR OF A 350 MWE STATION: REPORTEDLY UNDER CONSTRUCTION:

REFERENCES

SODIUM COOLED FAST REACTORS

AI LEIPINSKII, OTHERS

THIRD UN, INTL, CONF, ON THE PEACEFUL USES OF ATOMIC ENERGY, QENEVA; 1964; A/CONF, 28/P/311: 
JANUARY 1969

BNWL $=036$

LIQUID METAL REACTORS. FOREIGN

NAME /OWNER

$B N=350, U S S R$

SHEVCHENKO STATION

DES IGNER

USSR

LOCATION

SCHEVCHENKO, USSR

EASTERN SHORE OF CASPIAN SEA; MANGYSHLAK

PENINSULA

PURPOSE

POWER AND WATER DESALINATION

TYPE

LIQUID METAL COOLED FAST BREEDER

POWER MWE (MWT)

150

1000

PRODUCTION OF 12;000 TONS FRESH WATERIDAY DESIGN POWER 350 MWE

CRITICAL

CONSTRUCTION, TARGET 1969

COQLANT

SODIUM

FUEL MATERIAL URANIUMIPLUTONIUMM DIOXIDE OR URANIUM CARBIDE

BLANKET, AXIAL AND RADIAL, URANIUM DIOXIDE

FUEL GEOMETRY PIN, 0.6 CM, DIA.; 1060 MM, ACTIVE LENGTH BLANKET, ROD 1,4 CM, OD:

FUEL CLADDING SS

CORE, O,O4 CM: THICK

BLANKET, $0.05 \mathrm{CM}$.

FUEL ENRICH, OXIDE FUEL, ZONED; 15 AND 20 RER CENT PLUTONIUM BLANKET, DEPLETED URANIUM

CARBIDE FUEL, 23 PER CENT U 235

FUEL ASSEMBLY CORE, 169-PIN HEXAGONAL ASSEMELY 211 ASSEMBLIES/CORE

BLANKET, 37-ROD ASSEMELY

440 ASSEMBLIES

RADIAL AND AXIAL BLANKETS; THE CORE ASSEMBLY

INCLUDES DEPLETED URANIUM DIOXIDE RODS AT EACH END

THE SIDE BLANKET HAS 440 HEXAGONAL ASSEMBLIES IN

SHROUD CANS. ACTIVE LENGTH OF RADIAL BLANKET ROD

IS $1100 \mathrm{MM}$.

FUEL CHARGE $850 \mathrm{KG}$. PLUTONIUM-239

BURNUPSREFUEL) MAX: 5 PER CENT 


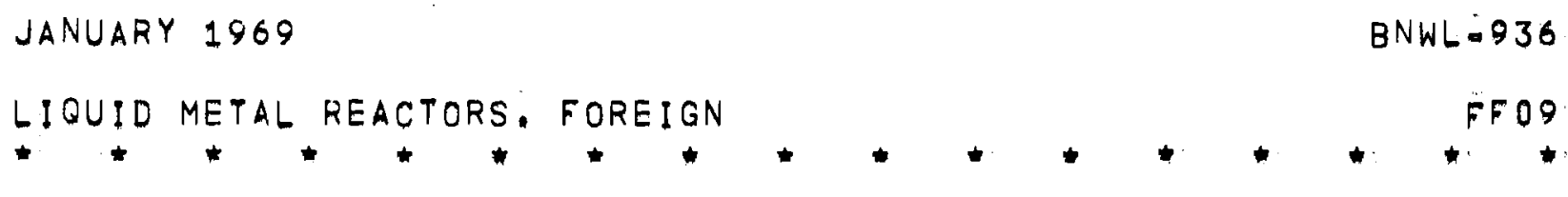

NEUTRON FLUX MAX, $8 \times 10 \quad E+15$

CONTROL BORON CARBIDE RODS, MOTION OF CENTRAL FUEL ASSEMBLIES. AUTOMATIC SYSTEM

COOLANT TEMP, INLET 572 F OUTLET $932 \mathrm{~F}$

REACTOR VESSEL VARIABLE DIAMETER SS VESSEL, 6000-2200 MM, DIA:. 1300 MM. HIGH WALL THICKNESS 30 MM.

CONTAINMENT REACTOR IS IN A CENTRAL PIT IN THE REACTOR BUILDING.

REMARKS

THE FIRST LOADING MAY BE ENRICHED URANIUM DIOXIDE

FUEL. THE 350 MWE REACTOR IS A STEP IN THE

DEVELOPMENT OF POWER REACTORS, THE BN-10OD IS

UNDER DEVELOPMENT, FUEL WILL BE A MIXTURE, OF. URANIUM CARBIDE WITH A BREEDING RATIO OF 1:75; A FAST PILOT REACTOR BOR IS ALSO UNDER DEVELOPMENT IT WILL BE A $40=60$ MWT FACILITY WITH SODIUM COOLANT TEMPERATURE OUTLET OF $630-650 \mathrm{C}$

CONDENSER COOLING WATER IS DRAWN FROM THE SEA,

DISCHARGED TO A DIKE-ENCLOSED DEPRESSION AND THEN

BY CHANNEL TO THE OPEN SEA.

REFERENCES SODIUM COOLED FAST REACTORS, A LEIPUNSKI

$3 R D$ UN, INTL, CONF, PEACEFUL USES OF ATOMIC ENERGY GENEVA, 1964. A/CONF:28/P/311

NUCLEAR REACTORS IN THE SOVIET UNION.

REPORT OF A RECIPROCAL EXCHANGE TRIP TO USSR BY US SCIENTISTS, DEC, 11-22, 1964:

WASH-1060

NUCLEAR POWER PLANT BN=350

AI LEIPUNSKY. OTHERS

ANS-100/SUPPLEMENT' P, 15.26

SEE ALSO JPRS-31193

THE BN-350 AND BOR FAST REACTORS:

A! LEIPUNSKII, OTHERS

FAST BREEDER REACTORS, PROC, LONDON CONF, BRITISH NUCLEAR ENERGY SOC, MAY $1966 \mathrm{P}, 243.74$

PERGAMON, $\$ 967$

CONSTRUCTION OF AN ATOMIC ELECTRIC STATION BASED 


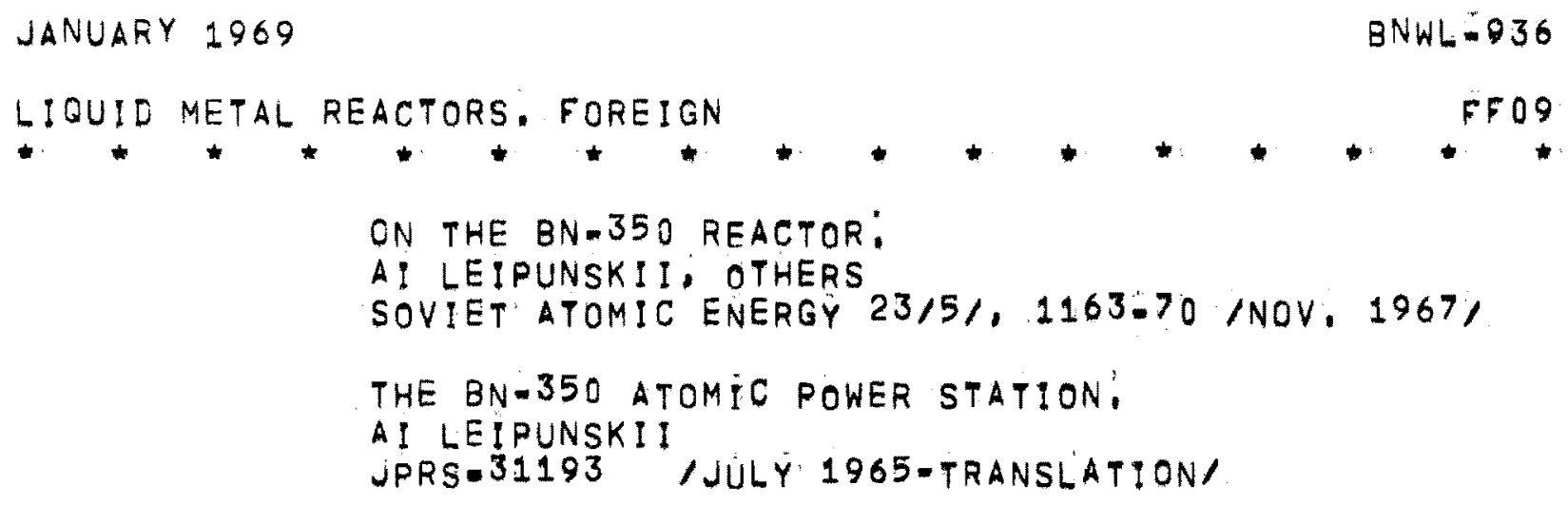


JANUARY 1969

BNWL=936

LIQUID METAL REACTORS; FOREIGN

$F F 10$

NAME/OWNER

$B R=60 / U S S R$

BOR

DES IGNER

USSR

OPERATOR

USSR

LOCATION

MELEKESS, USSR

NUCLEAR REACTOR SCI, RES, INSTITUTE

PURPOSE

FAST REACTOR EXPERIMENT,

TYPE

LIOUID METAL COOLED FAST REACTOR, HIGH TEMPERATURE

POWER MWE(MWT)

10

60

CRITICAL

CONSTRUCTION 1965: TARGET 1968

COOLANT

SOD!UM

MODERATOR

ANNULAR STEEL REFLECTOR

FUEL MATERIAL COMPOSITIONS STUDIED INCLUDE ENRICHED URANIUM

OXIDE, PLUTONIUM OXIDE, CARBIDES, BLANKET DEPLETED URANIUM DIOXIDE

FUEL GEOMETRY ROD 6.1 MM, DIAM. 400 MM. ACTIVE LENGTH RADIAL BLANKET ROD 14,5 MM: DIAM, 900 MMILONG

FUEL CLADDING SS TUBES, WIRE WRAPPED, SEALED OR VENTED DESIGN, WALL THICKNESS FOR SEALED ELEMENTS $0.4 \mathrm{MM}$ : WALL THICKNESS FOR VENTED ELEMENTS 0.25 MM.

FUEL ENRICH, WITH URANIUM DIOXIDE FUEL; 90 PER CENT U $=235$

FUEL ASSEMBLY CORE, HEXAGONAL ASSEMBLIES VARYING ACCORDING TO OPERATING CONDITIONS, BURNUP, AND TYPE OF FUEL. WITH 90 PER CENT ENRICHNMENT THERE ARE $70=80$ ASSEMBLIES MADE UP OF 37-ELEMENT BUNDLES, RADIAL ELANKET, 7-ROD BUNDLE ASSEMBLIES:

FUEL CHARGE 150-160 KG U-235 WITH URANIUM DIOXIDE FUEL SPECIFIC POWER $900 \mathrm{KW/L}$

BURNUP (REFUEL) 440-DAY FUEL LIFE

CONTROL BORON CARBIDE RODS, ONE MOVABLE FUEL ELEMENT

COQLANT TEMP, INLET 300-500 C

OUTLET 650 C MAX: 


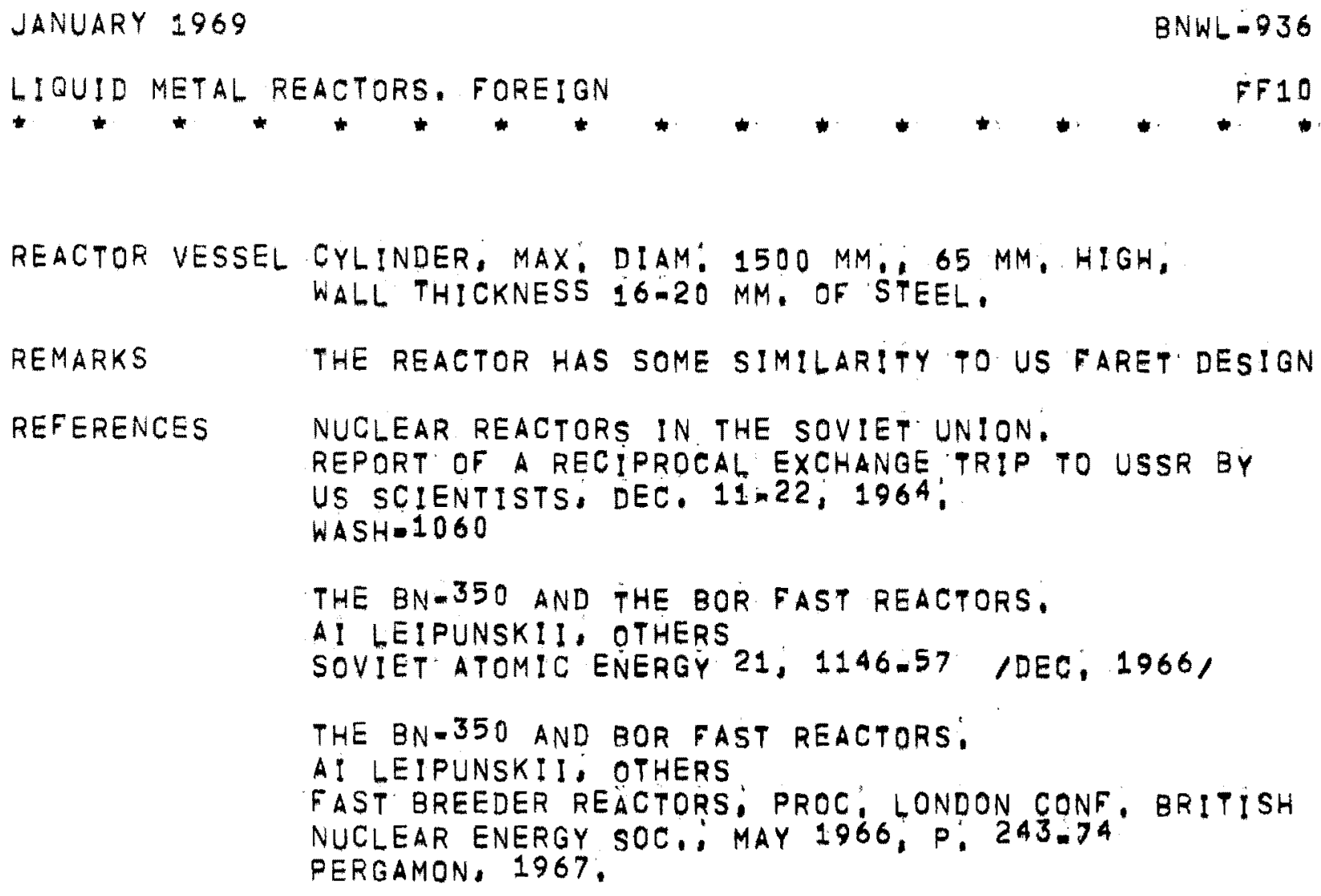


JANUARY 1960

BNWL- -936

LIQUID METAL REACTORS, FOREIGN

$F F 11$

NAME /OWNER BN-1000/USSR

DES】GNER USSR

OPERATOR USSR

POWER MWE(MWT) 1000

CRITICAL PROPOSED, DESIGN AND DEVELOPMENT

FUEL MATERIAL URANIUM/PLUTONIUM CARBIDE

OR URANIUM CARBIDE

FUEL ASSEMBLY CORE WILL BE FLATTER THAN BN-350 CORE

REFERENCES NUCLEAR REACTORS IN THE SOVIET UNION. REPORT OF A RECIPROCAL EXCHANGE TRIP TO USSR BY US SCIENTISTS, DEC, 11-22, 1964, $W A S H=1060$ 
JANUARY 1969

BNWL:036

LIQUID METAL REACTORS: FOREIGN

$F F 12$

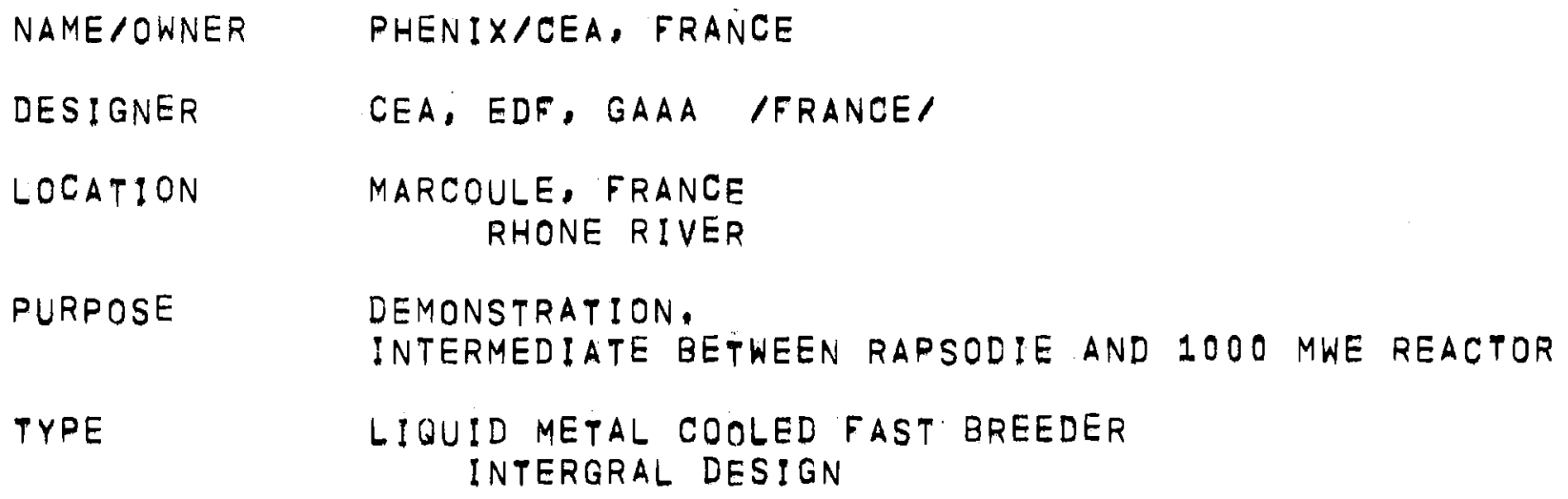




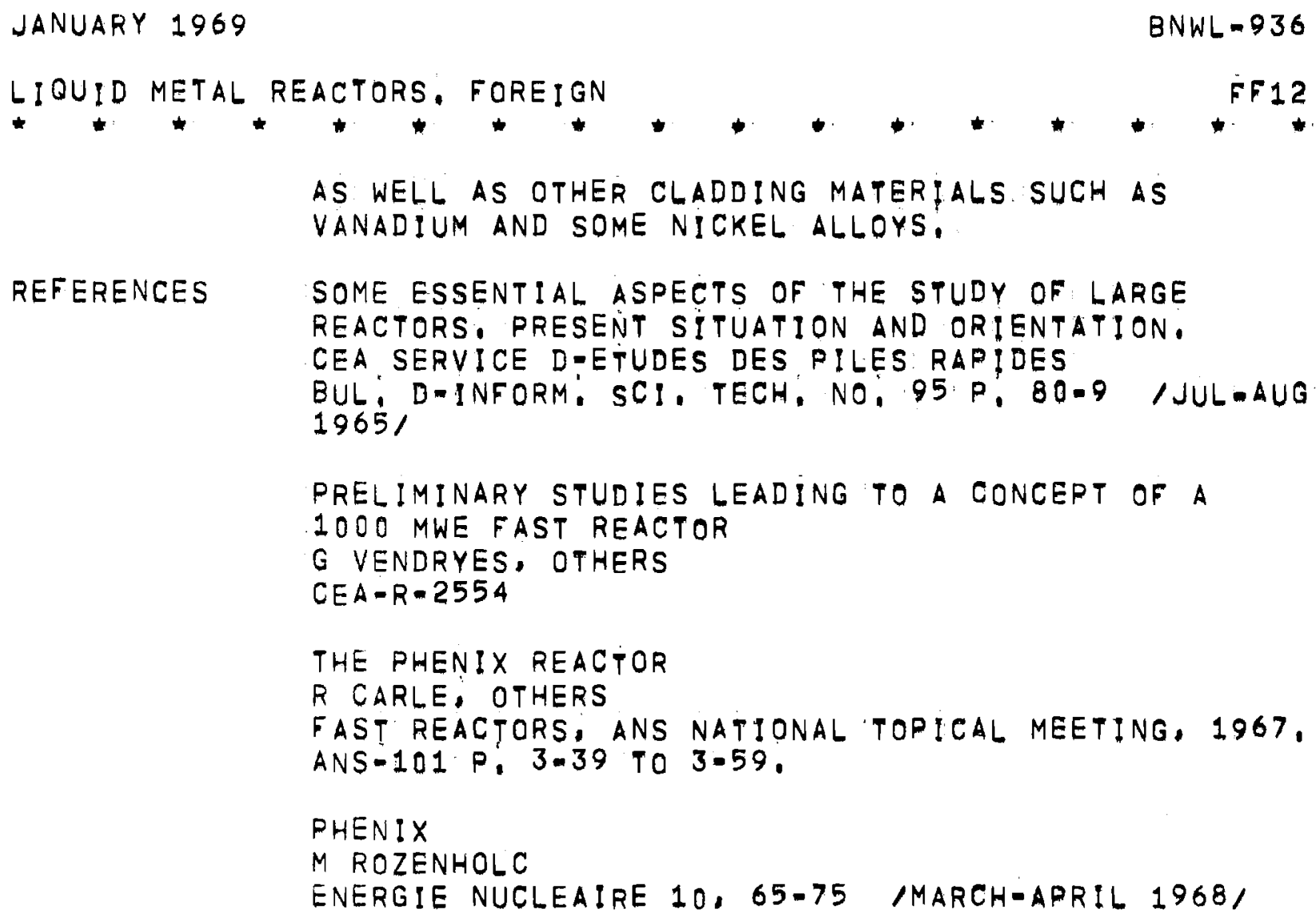


JANUARY 2909

BNWL-936

LIOUID NETAL REACTORS, FOREIGN $* * * * * * * * * * *$

NAME/OWNEA FAST BREEDER REACTOR DESIGN/JAERI, JARAN

DESIGNER

JAPAN ATOMIC ENERGY RESEARCH INST, /JAERI/

PURPOSE

EXPERIMENTAL REACTOR

TYPE

LIQUID METAL COOLED FAST BREEDER

PCWER MWE(MNT)

100

CR:TICAL

DESIGN, CONSTRUCTION 1968, TARGET 1971

COOLANT

SOD!UM

FLEL MATER:AL

URANIUM DIOXIDE

FLEL GEOMETRY CORE

$$
\begin{aligned}
& \text { PIN. } 5.4 \text { MM: DIA.' } \\
& \text { ACT. LENGTH } 600 \text { MM: *2X300 MM, AXIAL } \\
& \text { BLANKET }
\end{aligned}
$$

RADIAL BLANKET

ROD, 13.5 MM, DIA:, ACT, LENGTH $1200 \mathrm{MM}$.

FUEL CLADDING CLAD WILL EE 0.25 MM. THICK ON CORE ELEMENTS AND

0.60 MM. THICK FOR RADIAL BLANKET ELEMENTS

FUEL ENRICH, 50 PER CENT U.235

BLANKET DEPLETED URAN!UM

FUEL ASSENBLY CORE, 88-PIN-CORE + AXIAL BLANKET- BUNDLE

RADIAL BLANKET, I9 ROD ASSEMELY
SS ASSEMBLIES

NEUTRON FLUX AVE, $2.8 \times 10$ E\$15

COOLANT TEMP. INLET $250 \mathrm{C}$ OUTLET $400 \mathrm{C}$

REMARKS

A LOINT PROGRAM BY APDA IU,S./, THE CENTRAL FESEARCH INST, ELECTRIC POWER IND, /JARAN/, AND DETROIT ED /U.S./ WILL BE CONCERNED WITH THE

EEVELOPMENT OF A 350 MWE FAST BREEDER.

REFERENCES PRELIMINARY DESIGN WORK OF THE 100 MWT

EXPERIMENTAL FAST REACTOR

T TETSUO, OTHERS

AP. 15572 /SEPT. 1, 1965/

UAPAN FAST CRITICAL EXPERIMENT PROGRAM

JAPAN ATOMIC ENERGY RESEARCH INSTITUTE 
JANUARY 1969

LIOUID METAL REACTORS, FOREIGN

$$
N P=15573 \text { /SEPT, 25, } 1965 /
$$

$3 N W L=336$

FF 13 


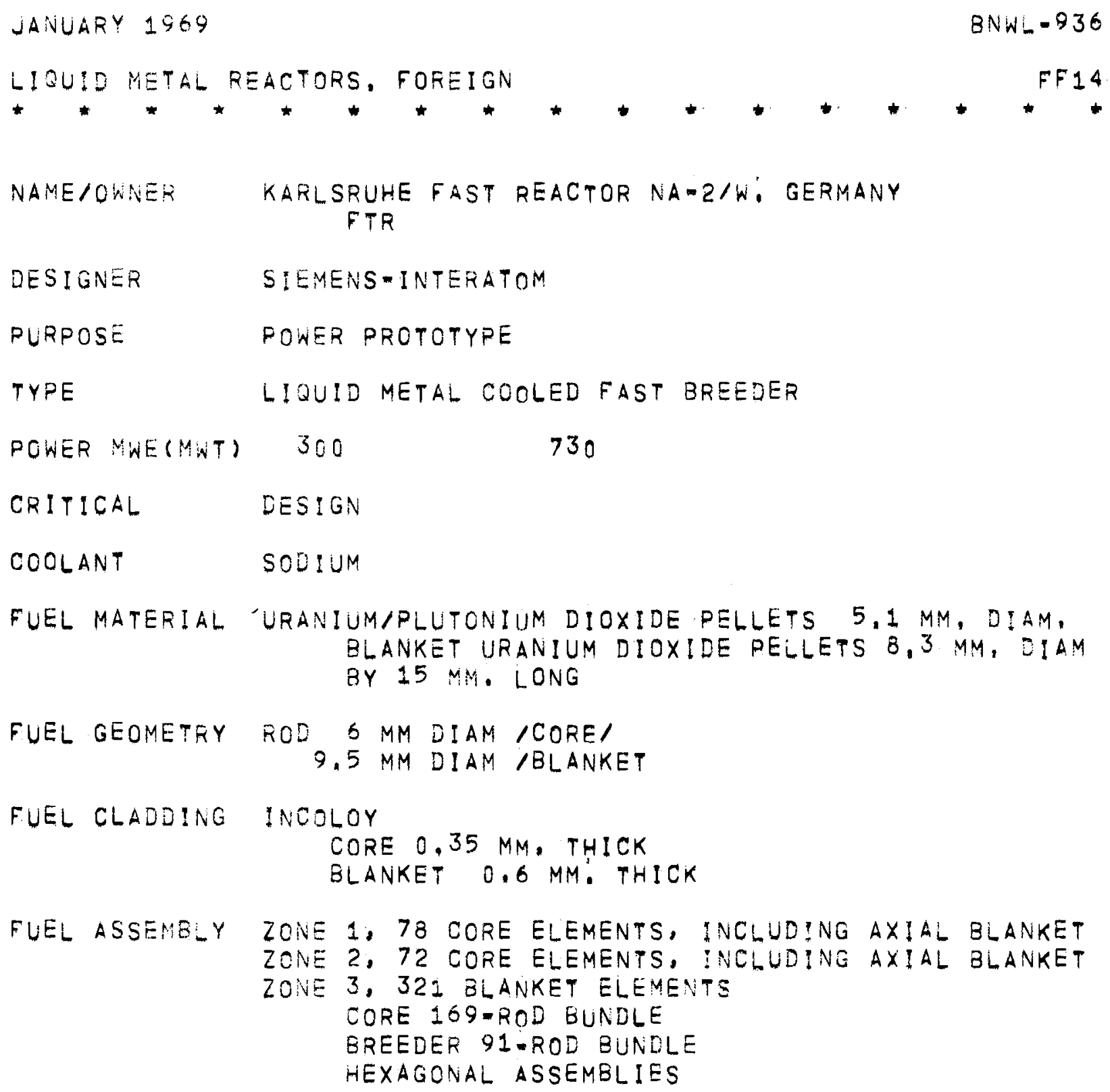

FUEL CLADDING INCOLOY

CORE 0.35 MM. THICK

BLANKET 0.6 MM: THICK

FUEL ASSEMB:Y ZONE 1; 78 CORE ELEMENTS, INCLUD!NG AXIAL BLANKET

ZONE 2, 72 CORE ELEMENTS, INCLUDING AXIAL BLANKET

ZONE 3, 321 BLANKET ELEMENTS

CORE 169-ROD BUNDLE

BREEDER 91-ROD BUNDLE

HEXAGONAL ASSEMBLIES

BURNUP(REFUEL) 30.000 MWD/T

NEUTRON FLUX TOTAL, CORE CENTER 10 E*\$6

CONTROL RODS

COOLANT TEMP, INLET $380 \mathrm{C}$ OUTLET $560 \mathrm{C}$

REACTOR VESSEL SS VESSEL 16,900 MM: HIGH, 7900 MM, OD, WALL THICKNESS $30 \mathrm{MM}$.

CONTAINMENT STEEL SHELL $35 \mathrm{M}$, DIAM/55,75 M, HIGH

INNER CONCRETE SHELL $27 \mathrm{M}$, DIAM/26 M, H!GH

REMARKS THE REFERENCE DESIGN FOR A 1000 MWT REACTOR IS DES?GNATED NAM1:

REFERENCES THE KARLSRUHE REFERENCE DESIGN OF A $1000 \mathrm{MWE}$ 
JANUARY 1969

BNWL -936

LIOUID METAL REACTORS, FOREIGN

FF 14

SODIUM COOLED FAST BREEDER REACTOR /FTRI

W HAFELE, OTHERS

$K F K=369$ /OCT. 1965/

FAST SODIUM-COOLED REACTOR NAm2,

$K$ GAST, EG SCHLECHTENDAHL

EURFNR-462/KFK-660, 1967

NA 2 DESIGN, 300 MWE GERMAN FCR,

$K$ CAST, OTHERS

ANS NATIONAL TOPICAL MEET!NG, FAST REACTORS,

SAN FRANCISCO, APRIL 1967.

ANS-101 P, 3-23 TO $3-28$ 
JANUARY 1969
LIOUID METAL REACTORS. FOREIGN
$* * 936$.

NAME/OWNER $\quad 8 N=600 /$ SSR

DESIGNER USSR

OPERATOR USSR

LOCATION URAL MOUNTAINS, USSR

PURPOSE POWER

TYPE LIOUID METAL COOLED FAST REACTOR

POWER MWE(MWT) $600 \quad 1430$

CRITICAL TARGET $1972-1973$

COOLANT SODIUM

MODERATOR NONE

FUEL MATERIAL DRIVER ZONE, URANIUM DIOXIDE OR URANIUM/PLUTONIUM BLANKET; URANIUM

FUEL GEOMETRY DRIVER ZONE, PIN 6.7 MM OD. BLANKET, ROD

FUEL CLADDING DRIVER ZONE, SS D:4 MM THICK

BLANKET, SS 0.4 MM THICK, RIBBED

FUEL ENRICH. DRIVER ZONE, LOW AND HIGH ENRICHMENT. BLANKET, DEPLETED URANIUM

FUEL ASSEMBLY DRIVER ZONE, 127-PIN BUNDLE, TRIANGULAR 397 ELEMENTS

BLANKET, 37 MOD BUNDLE, TRI ANGULAR

BURNUP(REFUEL) TEN PER CENT, PARTIAL EVERY 147 DAYS

COOLANT TEMP, INLET $410 \mathrm{C}$ OUTLET $580 \mathrm{C}$

REFERENCES NUCLEAR INDUSTRY SEPT: $1968 \mathrm{P} ; 31$ NEWS RELEASE 
JANUARY 1969

BNWLing 936

LIQUID METAL REACTORS. FOREIGN

516

NAME/OWNER

PEC /PROVA ELEMENTI DI COMBUSTIBILI/CNEN, ITALY ITALIAN FAST BREEDER PROGRAM

DESIGNER

CONTRACT TO SNAM PROGETTI IENI GROUPI

LOCATION

BRASIMONE; IN APPENINES INEAR TUSCANY/, ITALY

PURPOSE

TEST BED IN ITALIAN FAST REACTOR DEVELOPMENT PROGRAM

TYPE

LIQUID METAL COOLED FAST REACTOR, TWO-ZONE

POWER MWE (MWT)

116

CRITICAL

CONCEPTUAL DESIGN COMPLETED: TARGET:1971

COOLANT

SODIUM

MODERATOR

NICKEL OR SS REFLECTORS

FUEL MATERIAL

URANIUM DIOXIDE-SS CERMET

BLANKET URANIUM DIOXIDE-BORON CARBIDE RODS

LATER CORES, PLUTONIUM DIOXIDE-SS CERMET

FUEL GEOMETRY PINS 8 MM, OD: ACTIVE LENGTH 900 MM.

FUEL CLADDING SS 0,4 MM. THICKNESS

FUEL ASSEMBLY 61-PIN ASSEMBLY: HEXAGONAL, CANNED

NEUTRON FLUX $3: 5 \quad E+15$

CONTROL RODS, BORON CARBIDE

COOLANT TEMP, INLET $375 \mathrm{C}$ OUTLET $525 \mathrm{C}$

REACTOR VESSEL CYLINDRICAL DOUBLE=WALLED TANK,

CONTAINMENT REACTOR TANK SURROUNDED BY GRAPHITE SHIELD AND CONCRETE SHIELD: REACTOR BUILDING.

REMARKS

PEC WILL BE USED FOR TESTING FUEL, ALSO HEAT EXCHANGERS AND STEAM GENERATORS: URANIUM DIOXIDE OR URANIUM/BERYLLIUM OXIDE CERAMIC FUEL CONSIDERED AS ALTERNATE,

REFERENCES ITALY $=5$ FAST BREEDER PROGRAM

NUCLEAR INDUSTRY MARCH 1967 P, 41 
JANUARY $\$ 969$ $8 N W L=936$

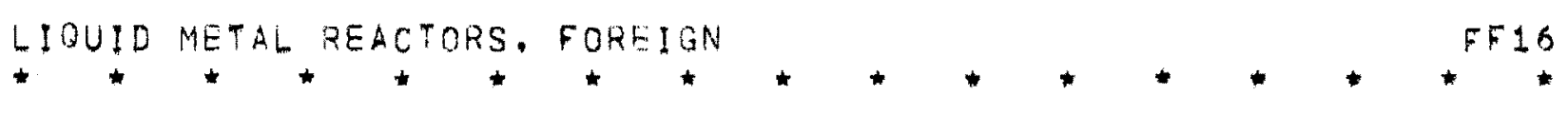

THE PEC REACTOR; A FUEL TEST FAST REACTOR.

F: PIERANTONI, OTHERS

ANS-101/SUPPLEMENT/, 1967 
$F F 17$

JANUARY 1969

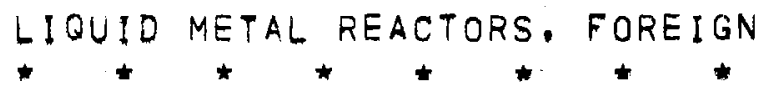

BNWL:0036

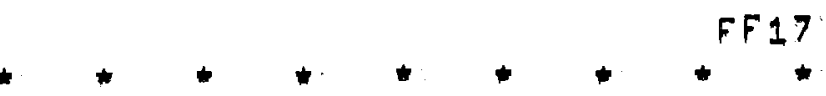

NAME/OWNER

CORAL-1/SPAIN

LOCATION

JUAN TIGON NATIONAL NUCLEAR CENTER, SPAIN

PURPOSE

EXPERIMENT

TYPE

FAST BREEDER EXPERIMENTAL FACILITY

POWER MWE(MWT)

0

CRITICAL MARCH 1968

FUEL MATERIAL URANIUM

FUEL ENRICH. 90 PER CENT U-235

FUEL CHARGE 22 KG: URANIUM

REFERENCES NEWS RELEASE 


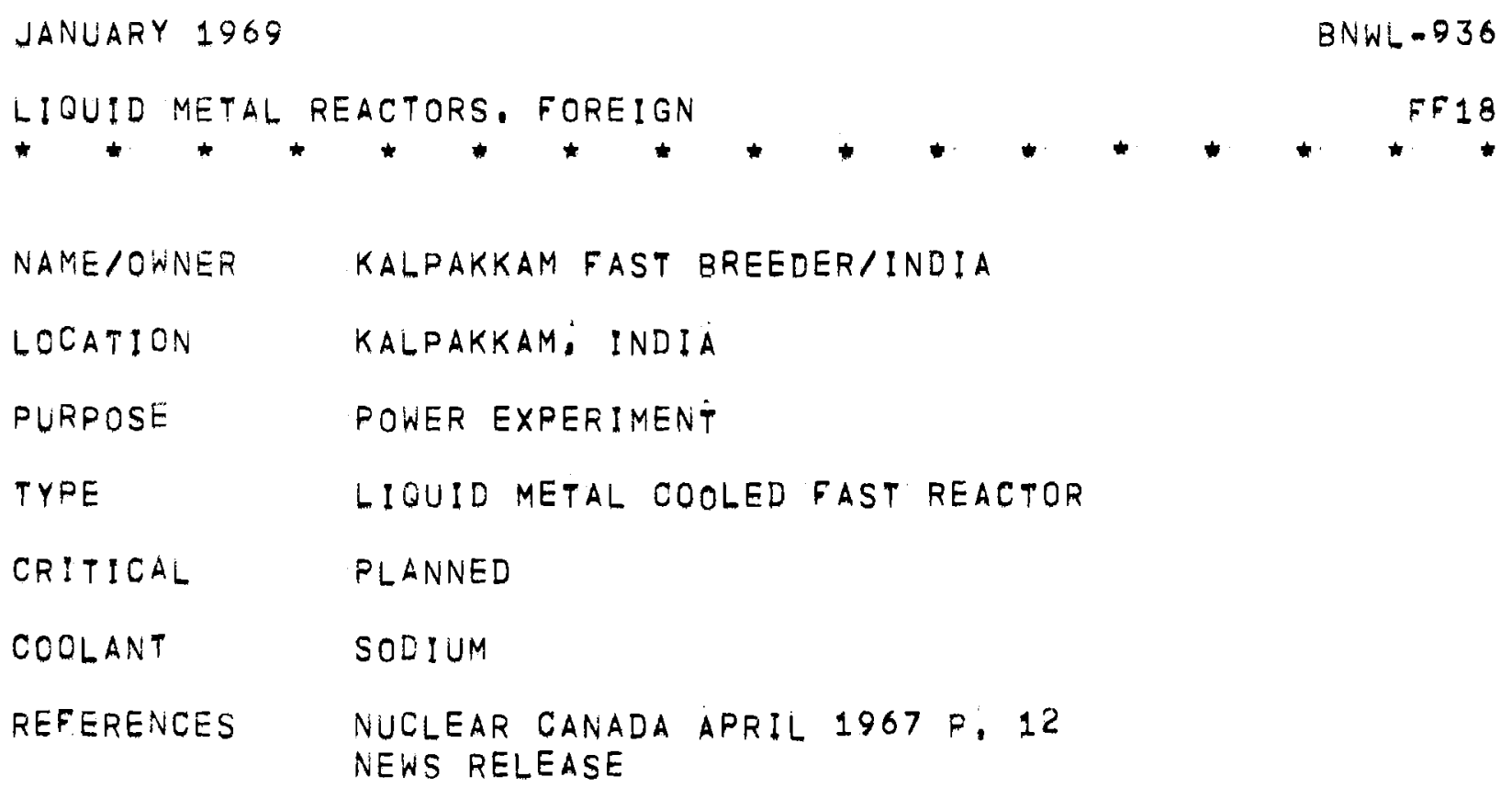


JANUARY 1969
LIQUID METAL REACTORS: FOREIGN BNWL-936

NAME IOWNER LIQUID METAL COQLED REACTOR/S: AFRICA

LOCATION DESIGN STUDY

PURPOSE POWER

TYPE LIO: METAL COOLED; HEAVY WATER MODERATED REACTOR, PEBBLE-BED

POWER MWE(MWT) 1000

COOLANT SODIUM

MODERATOR HEAVY WATER

FUEL MATERIAL URANIUM SPHERES

FUEL GEOMETRY SPHERES, UNCLAD

FUEL CLADDING NONE

FUEL ENRICH, NATURAL

FUEL ASSEMBLY UNCLAD FUEL SPHERES IN DIRECT CONTACT WITH SODIUM COOLANT.

SPECIFIC POWER $40 \mathrm{~W} / \mathrm{GM}$

CONTROL DISPLACEMENT OF MODERATOR BY HELIUM GAS

REFERENCES S, A, NUCLEAR DESIGN, WOULD PRODUCE TOO MUCH POWER,

S. AFRICA MINING ENG: J. 78, 2491 /OCT, 6, 1967/ 
ORGANIC COOLED REACTORS

DOMESTIC 
JANUARY 1969

BNWL- -936

ORGANIC COOLED REACTORS, DOMESTIC

GOO

NAME/OWNER

POPR /PROTOTYPE ORGANIC POWER REACTOR//AEC

DES I GNER

ATOMICS INTERNATIONAL

PURPOSE

POWER PROTOTYPE

TYPE

ORGANIC MODERATED AND COOLED REACTOR

POWER MWE (MWT)

50

160

CRITICAL

PROPOSAL

COOLANT

LIQUID HYDROCARBON

MODERATOR

LIQUID HYDROCARBON

FUEL MATERIAL

URANIUM DIOXIDE PELLETS

FUEL GEOMETRY PIN

FUEL CLADDING APM-ALUMINUM FOWDERED METAL INNED TUBING

FUEL ASSEMBLY

54 PINS/ASSEMBLŸ /HEXAGONAL APM BOX WITH CENTRAL TUBE/

121 ASSEMBLIES/CORE

BURNUP(REFUEL) 9000 MWD/TON

NEUTRON FLUX THERMAL $3 \times 10$ E +13

CONTROL

RODS, SS TUBES WITH BORON CARBIDE AT CENTER OF ELEMENTS,

COOLANT TEMP, INLET $644 \mathrm{~F}$ OUTLET $700 \mathrm{~F}$

REACTOR VESSEL CYLINDRICAL, $11 \mathrm{FT}, 6$ IN, ID, AT: UPPER PLENUM, 8 FT, 6 IN. AT CORE REGION, 6 FT. AT BOTTOM SECTION: CYLINDRICAL INNER TANK CONTAINS CORE:

CONTAINMENT REACTOR BUILDING /PRESSURE VESSEL/ 85 FT, DIA.1160 FT, HIGH

REMARKS

AEC PROGRAM INITIATED IN JANUARY 1961. PROPOSAL BY GRAND RIVER DAM AUTHORITY REFUSED BY AEC, A PROPOSAL WAS SUBMITTED TO AEC FOR A 30 MWE 160 MWT PROCESS HEAT REACTOR FOR PACKAGING CORPORATION OF AMERICA-S PLANT AT FILEK CITY, MICHIGAN.

REFERENCES THE 50 MWE PROTOTYPE ORGANIC NUCLEAR PLANT: 
GDO1

JANUARY 1969

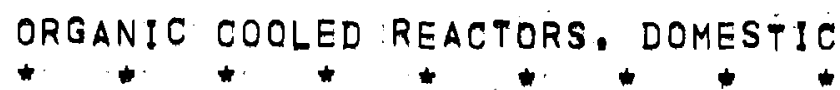

$B N W L=936$

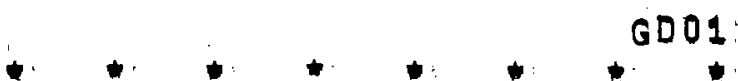

CW WHEELOCK, GS BUDNEY

ASME PREPRINT 61:WA:226/1961/

PRELIMINARY POPR CONCEPTUAL DESIGN EXCURSION STUDY

FJ HALFEN

NAA-SR-MEMO-6448/JUNE 1961/

PROTOTYPE ORGANIC POWER REACTOR.

ATOMICS INTERNATIONAL.

NAA-SR-7400 /SECT! IVI /NOVEMBER 15, 1962, 
JANUARY 1969

BNWL $=936$

ORGANIC COOLED REACTORS, DOMESTIC

NAME/ONNER

OMRE /ORGANIC MODERATED REACTOR EXP,//AEC-AI

DES!GNER

ATOMICS INTERNATIONAL

OPERATOR

ATOMICS INTERNATIONAL

LOCATION

NATIONAL REACTOR TEST: STATION, IDAHO

PURPOSE

POWER EXPERIMENT, DEMONSTRATION

TYPE

ORGANIC MODERATED AND COOLED, CIRCULATING COOLANT MODERATOR

POWER MWE(MWT)

$5-15$

CRITICAL

SEPT, 1957

PHASED OUT IN 1963

COOLANT

ORGANIC LIQUID /TERPHENYL/

MODERATOR

ORGANIC LIQUID /TERPHENYL'

FUEL MATERIAL URANIUM DIOXIDE IN SS MATRIX, 20 MILS THICK

FUEL GEOMETRY PLATES ACTIVE AREA 36 IN, $/ 21 / 2$ IN.

FUEL CLADDING SS 5 MILS THICK

FUEL ENFICH, HIGH

FUEL ASSEMBLY 16 PLATES PER, BOX $/ 2,9 / 2.8 / 60$ IN.'
31 BOXES, CORE

FUEL CHARGE $25.5 \mathrm{KG}, U=235$

SPECIFIC POWER $470 \mathrm{KW} / K G$ U-235

NEUTRON FLUX THERMAL AVE, $4.1 \times 10 . E+13$

FAST AVE. $1,8 \times 10 E+14$

CONTROL CYLINDRICAL RODS

COOLANT TEMP, INLET 500.700 F OUTLET 520-710 F

COQLANT PRESS, 200 PSIG

REACTOR VESSEL LOW ALLOY STEEL: $4.5 \mathrm{FT}$. ID:

CONTAINMENT NONE EXCEPT FOR REACTOR VESSEL AND PRIMARY COOLANT PIPING 


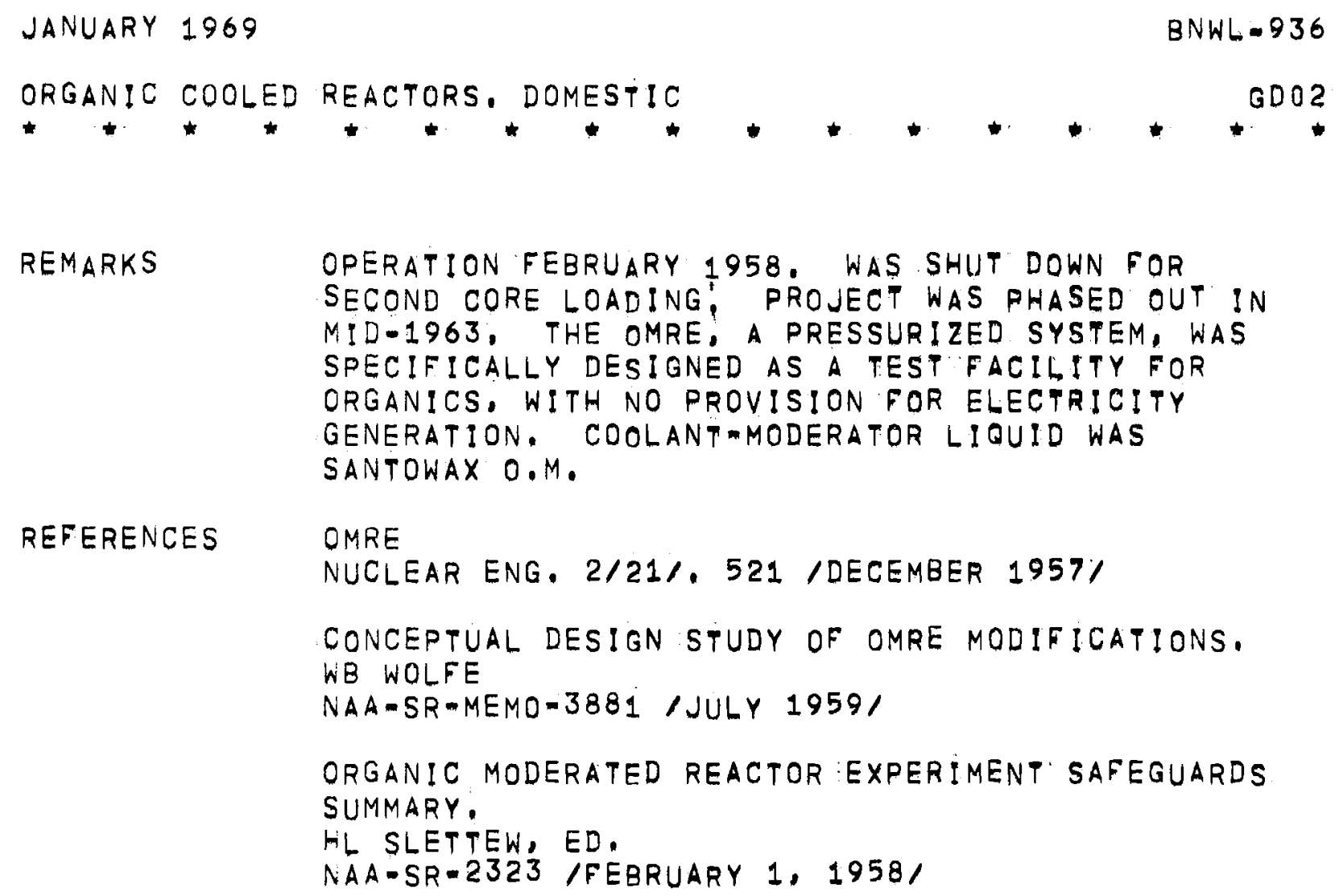


JANUARY 1969

BNWL $=936$

ORGANIC COOLED REACTORS, DOMESTIC

NAME /OWNER

PIQUA NUCLEAR POWER FACILITY/AEO-CITY OF PIQUA

DES】GNER

ATOMICS INTERNATIONAL

OPERATOR

CITY OF PIOUA

LOCATION

PIQUA; OHIO GREAT MIAMI RIVER

PURPOSE

POWER DEMONSTRATION

TYPE

ORGANIC MODERATED AND COOLED

POWER MWE (MWT)

11.4

45,5

CRITICAL

1903, SHUT DOWN JANUARY 1966

SEE REMARKS

CQOLANT

TERPHENYL /SINGLE COOLANT LOOP/

MODERATOR

TERPHENYL

FUEL MATERIAL URANIUM-MOLYBDENUM-ALUMINUM ALLOY

FUEL GEOMETRY DOUBLE ANNULAR TUBES,

INNER ANNULUS 3:6 IN. DIA:10,208 IN, THICK

OUTER 4,63 IN. DIA:10,208.IN. THICK

TOTAL ACTIVE LENGTH 54 IN.

FUEL CLADDING ALUMINUM TUBES, CONCENTRIC, FINNED

FUEL ENRICH, 1:9 PER CENT U $=235$

FUEL ASSEMBLY NESTED FUEL ASSEMBLIES, 4 SETS OF COAXIAL TUBULAR

ELEMENTS-STACKED /FULL CORE LENGTH/ IN THIN WALLED SS COOLANT DUCT?

85 ASSEMBLIES/CORE /13CONTAIN CONTROL RODS/

72 REGULAR FUEL ASSEMBLIES

FUEL CHARGE $6550 \mathrm{KG}$. URANIUM

BURNUP(REFUEL) 1000 MWD/TON 1/4 CORE BATCHES

NEUTRON FLUX THERMAL AVE, $1.9 \times 10$ E\$13

FAST AVE, $4 \times 10 \quad E+13$

CONTROL RODS, BORON EARBIDE IN SMALL SS TUBES BRAZED

TOGETHER TO FORM DOUBLE RING 


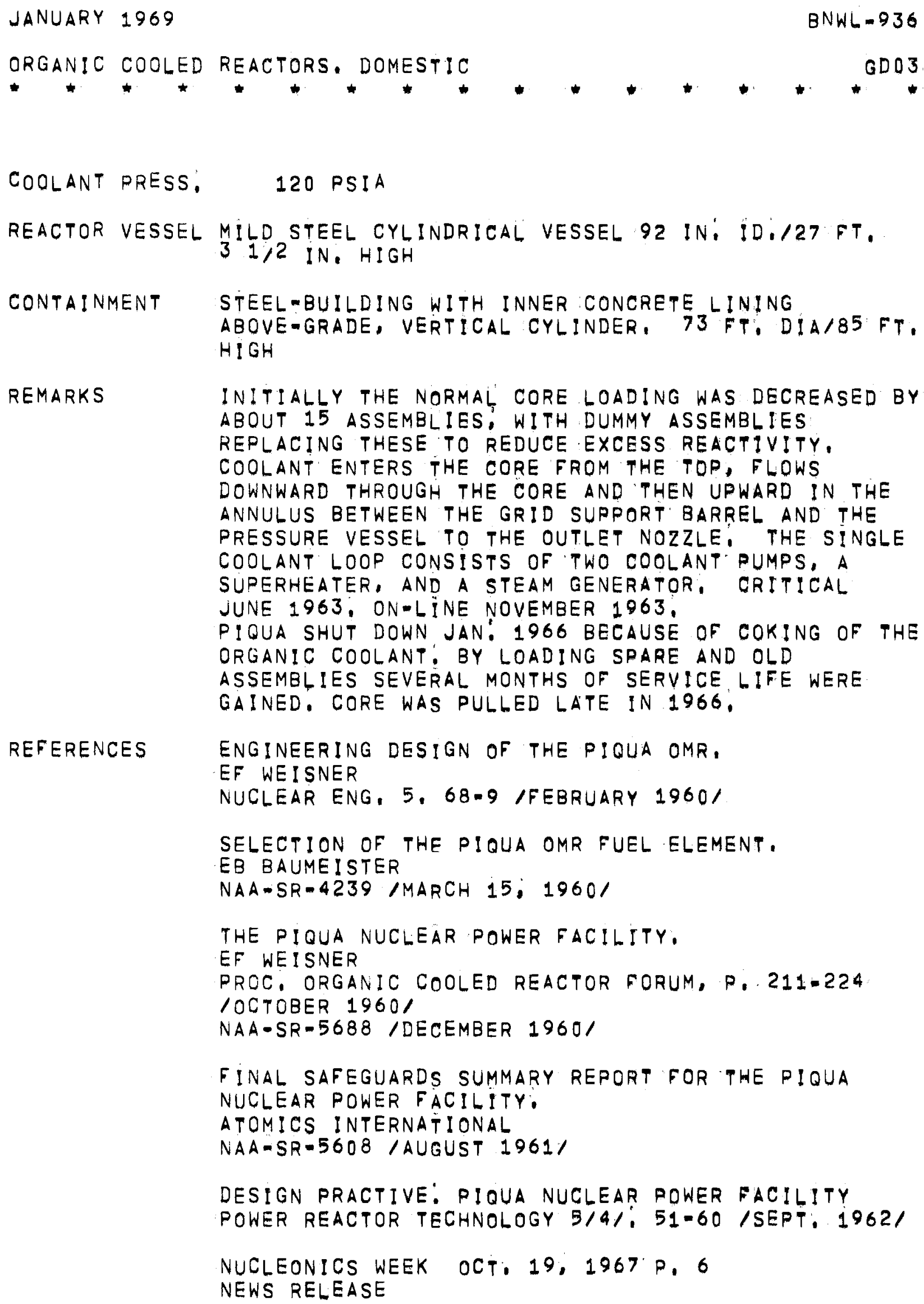

REFERENCES ENGINEERING DESIGN OF THE PIQUA OMR, EF WEISNER

NUCLEAR ENG, 5, 68.9/FEBRUARY $1960 /$

SELECTION OF THE PIQUA OMR FUEL ELEMENT. EB BAUMEISTER

$N A A-S R-4239 / M A R C H$ 15; $1960 /$

THE PIQUA NUCLEAR POWER FACILITY. EF WEISNER

PROC, ORGANIC COOLED REACTOR FORUM, $P, 211.224$

IOCTOBER $1960 /$

NAA-SR-5688/DECEMBER $1960 /$

FINAL SAFEGUARDS SUMMARY REPORT FOR THE PIQUA

NUCLEAR POWER FACILITY:

ATOMICS INTERNATIONAL

$N A A=S R-5608$ /AUGUST $1961 /$

DESIGN PRACTIVE: PIQUA NUCLEAR POWER FACILITY

POWER REACTOR TECHNOLOGY 5/4/:51-60//SEPT, $1962 /$

NUCLEONICS WEEK OCT, 19, 1967 P, 6

NEWS RELEASE 
JANUARY 1969

BNWL-936

ORGANIC COQLED REACTORS. DOMESTIC

QDO4.

NAME/OWNER EOCR /EXP: ORGANIC COOLED REACTOR/IAEC

DES IGNER

ATOMIOS INTERNATIONAL CONCEPTUAL DESIGN; PHILLIAS PETROLEUM

OPERATOR

ATOMICS INTERNATIONAL

LOCATION

NATIONAL REACTOR TEST, STATION, IDAHO

PURPOSE

TEST BED FOR FUEL ELEMENTS, ORGANIC COOLANTS'.

TYPE

ORGANIC MODERATED AND COOLED

POWER MWE (MWT)

40

CRITICAL

POSTPONED

COOLANT

TERPHENYL

MODERATOR

TERPHENYL

FUEL MATERIAL URANIUM DIOXIDE IN SS MATRIX 0,020 IN: THICK

FUEL GEOMETRY PLATES

FUEL CLADDING SS 0,005 IN. THİCK

FUEL ENRICH. HIGH

FUEL ASSEMBLY 28 PLATES + 1 DUMMY SPACER PLATE TO BOX /4/6 1/2 IN II

32 FUEL UNITS 120 DRIVER FUEL ELEMENTS AND 12 CONTROL=ROD FOLLOWERS/

CONTROL RODSOFUEL FOLLOWER TYPE

COQLANT TEMP: INLET $500 \mathrm{~F}$ OUTLET.525 F

COOLANT PRESS.

OUTLET 157'RS!

REACTOR VESSEL CARBON STEEL, 95 IN: ID:/32 FT: LONG, CORE IS ENCLOSED IN AN INNER TANK

REMARKS

THIS PROJECT HAS BEEN INDEFINITELY DEFERRED WITH THE PLANT 99 PER CENT COMPLETE, CORE DESIGN CONSISTS OF RECTANGULAR FUEL ASSEMBLIES COMPOSED OF TWO SUBASSEMBLIES. SUBASSEMBLY CONTAINS 28 ACTIVE PLATES AND ONE DUMMY PLATE, THE ACTIVE PLATES BEING OF HIGHLY ENRIOHED URANIUM DIOXIDE-SS WITH STAINLESS STEEL CLADDING; CONGEPTUAL DESIGN 
JANUARY 1969

$B N W L=936$

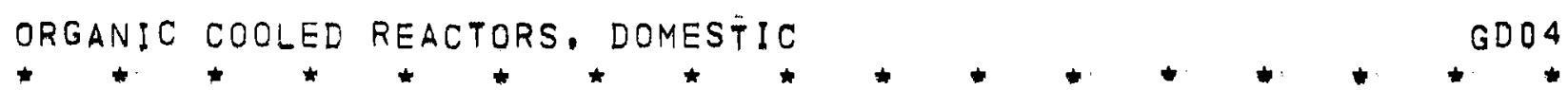

WAS BY PHILLIPS:

REFERENCES EXPERIMENTAL ORGANIC COOLED REACTOR CONCEPTUAL DESIGN.

WE NYER: JH RAINWATER

IDO-16570 /DECEMBER 1; 1959 /

EXPERIMENTAL ORGANIC COOLED REACTOR, MR DUSBABEK

NAA-SR $-5688 / P, 189: 210 / / D E C E M B E R$ 1960/

EXPERIMENTAL ORGANIC̈ COOLED REACTOR, PRELIMINARY HAZARDS REPORT. ADDENDUM.

FLUOR CORP.

IDO- 24034

IDO-24034 /ADD./ /NOVEMBER $1960 /$

IDO-24034 /ADD, 2/ /APRIL 1961/

THE EXPERIMENTAL' ORGANIC COOLED REACTOR.

POWER REACTOR TECHNOLOGY 5/1\%; 88-91: /DEC, 1961\% 
JANUARY 1969

BNWL-936

ORGANIC COOLED REACTORS: DOMESTIC

GDO5

NAME / OWNER

OMRISHIP PROPULSION STUDY, AI

DES IGNER

ATOMICS INTERNATIONAL

PURPOSE

SHIP PROPULSION

TYPE

ORGANIC MODERATED AND COOLED

POWER MWE(MWT) 10,000 SHP

$30-36$

QRITICAL

DES IGN

COOLANT

ORGANIC LIOUID

MODERATOR

ORGANIC LIQUID

FUEL MATERIAL URANIUM DIOXIDE PELLETS OR URANIUMIMOLYBDENUM ALLOY

FUEL GEOMETRY RODS /TUBES/

FUEL CLADDING ISS TUBING OR ALUMINUM TUBING WITH URANIUM-MOLYBDENUM ALLOY FUEL

FUEL ENRICH. APPROX. 3:7 PER CENT U-235

FUEL ASSEMBLY 100-ROD ASSEMBLY /SS BOXY

CONTROL RODS

COOLANT TEMP, INLET $300 \mathrm{C}$ OUTLET $325 \mathrm{C}$

CONTAINMENT

CONTAINMENT SHELLL WITHIN GAS-TIGHT REACTOR COMPARTMENT.

REMARKS

REFERENCE DESIGN FOR AN OMRAPOWERED TANKER, WAS CONSIDERED UNDER AN INTERATOM DESIGN PROPOSAL FOR THE GKSS /GERMAN/ SHIP PROGRAM,

REFERENCES

REFERENCE DESIGN FOR AN OMR NPOWERED 38,000 DWT

TANKER

RJ GIMERA; RE STANBRIDGE

NAA-SR-1851 AND SUPPLEMENT /MARCH 1957\%

MARITIME ORGANIC MODERATED AND COQLED REACTOR. $N A A-S R=3859$

THE INTERATOM MARINE REACTOR,

FE FARIS

NUCLEAR POWER 6:75:6/MARCH $1961 /$ 
JANUARY 1969

BNWL $=936$

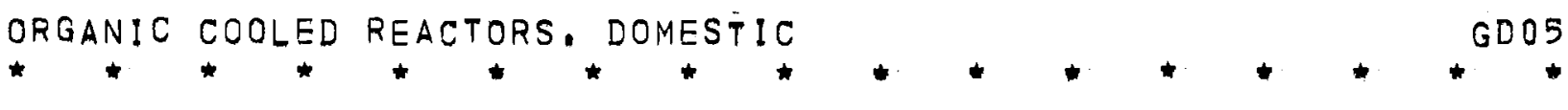

SKETCH OF A SHIP REACTOR COOLED AND MODERATED

WITH ORGANIC SUBSTANCES.

KERNREAKTOREN FUR SCHIFFSANTRIEBE, $P, 33-6$

VERLAG KARL THIEMIG AG, MUNICH, 1961 


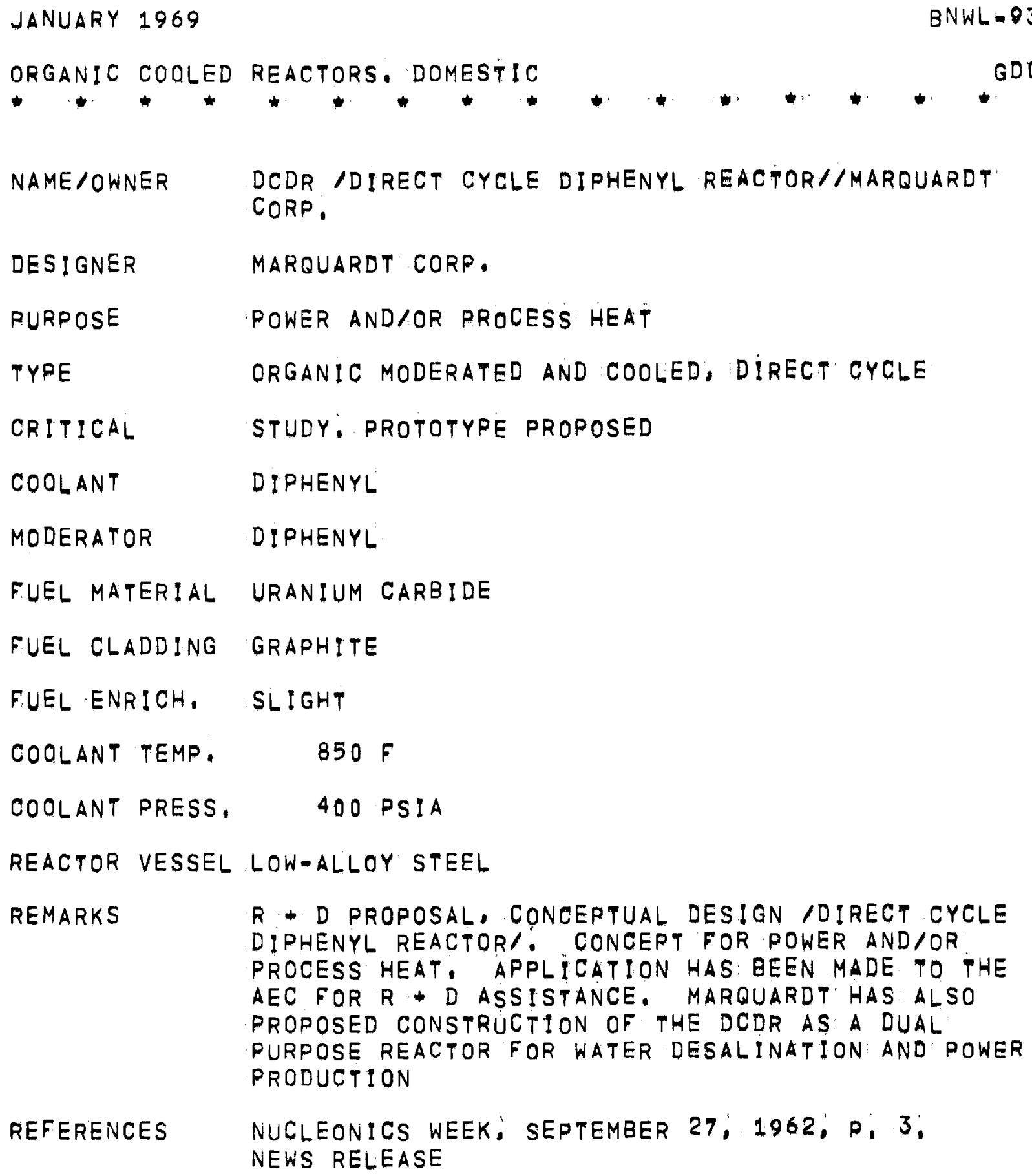


JANUARY 1969

BNWL -936

ORGANIC COOLED REACTORS. DOMESTIC

GDO 7

NAME /OWNER

OMFBR /ORGANIC MOD. FLUIDIZED BED REACTOR//WEST

DES IGNER

WESTINGHOUSE ELECTRIC CORPORATION

PURPOSE

POWER

TYPE

ORGANIC MODERATED AND COOLED, FLUIDIZED BED,

POWER MWE (MWT)

50

150

CRITICAL

CONCEPTUAL DESIGN: DEVELOPMENT PROPOSED.

COOLANT

POLYPHENYL

MODERATOR

POLYPHENYL

FUEL MATERIAL URANIUM

FUEL GEOMETRY SPHERES

FUEL ENRICH, APPROX, 2 PER CENT U-235

FUEL ASSEMBLY FLUIDIZED BED OF URANIUM PELLETS. COOLANT IS HEATED ON PASSING THROUGH THE FUEL BED.

FUEL CHARGE

15 TONS URANIUM

CONTROL

RATE OF COOLANT CIRCULATION.

COOLANT TEMP, INLET $610 \mathrm{~F}$

OUTLET $690 \%$

REACTOR VESSEL STRUCTURAL STEEL

REMARKS

PROPOSAL FOR DEVELOPMENT /BURLINGTON, VT, LIGHT DEPT:,. CURRENT STATUS UNKNOWN:

REFERENCES

ATOMIC DEYELOPMENT AT WESTINGHOUSE,

POWER ENG, 62, 44-50 /DECEMBER 1958/

ORGANIC MODERATED FLUIDIZED BED REACTOR CONCERT: JH WRIGHT

TRANS: ANS $1 / 2 /: 126=7$ /DEC: $1958 /$ 
ORGANIC COOLED REACTORS

FOREIGN 


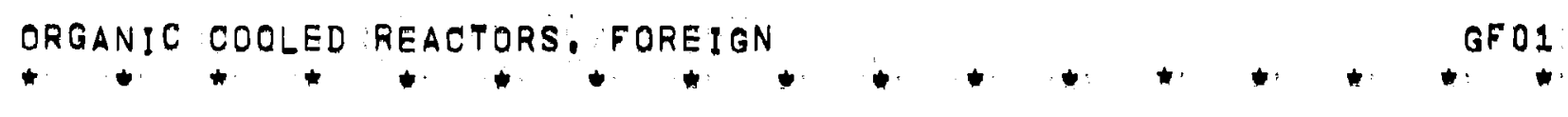

NAME /OWNER

PRO /PROGRAMMA REATTORE ORGANICO/ CNEN, ITALY

DES IGNER

CNEN, ITALY

OPERATOR

SORIN-AGIP

LOCATION

BOLOGNA; N. ITALY

PURPOSE

POWER PROTOTYPE

TYPE

ORGANIC MODERATED AND COOLED

POWER MWE (MWT)

$30 / 2 N D$ CORE 60 MHTY

CRITICAL

A ZERO-POWER EXPERIMENT ROSPO /REATTORE ORGANICO SPER!MENTALE A POTENZA O/ CRITICAL JUNE, 1963 AT THE CASACCIA CENTER: NO RECENT INFORMATION,

COOLANT

ORGANIC LIOUID

MODERATOR

ORGANIC LIOUID

FUEL MATERIAL

URANIUM DIOXIDE IN SS MATRIX, FIRST CORE, THORIUM WILL ALSO BE USED IN A THORIUM/URANIUM-233 CYCLE.

FUEL GEOMETRY PLATE /APPR-TYPE/

FUEL CLADDING SS ILATER SAP/

FUEL ENRICH. HIGH

COQLANT TEMP,

$315-330 \mathrm{C}$

COOLANT PRESS:

$30 K G / S Q . C M$.

REMARKS

A NUMBER OF CORES HAVE BEEN EXAMINED FOR THE PURPOSE OF GIVING THE PLANT AN INCREASED.

FLEXIBILITY FOR SECOND CORES, TWO MAJOR R+D PROGRAMS ARE IN PROGRESS, PRD, THE ORGANIC REACTOR PROGRAM FOR A 30 MWT REACTOR, AND PCUT, THE URANIUM THORIUM CYCLE PROQRAM WHICH VISUALIZES A URANIUM-THORIUM REPROCESSING PILOT PLANT, AIM IS TO STUDY THE URANIUM-THORIUM CYCLE WITH A VIEW TO A POWER REACTOR IREPROCESSING PLANT INTEGRATED COMPLEX: A CONSORTIUM INCLUDING CNEN IS INVESTIGATING AN ORGANIC REACTOR FOR SMALL DESALINATION SYSTEMS: SEE ROVI PROJECT, GFO4, 


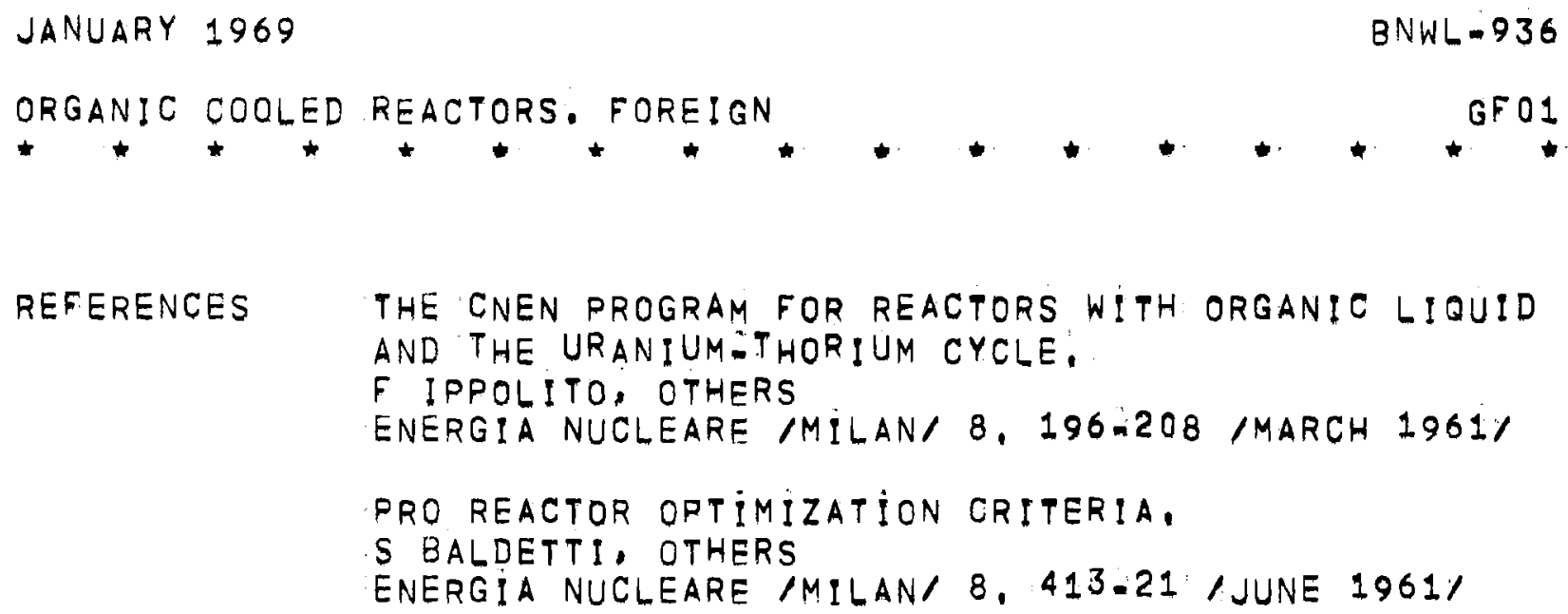


JANUARY 1969

BNWL $=936$

ORGANIC COQLED REACTORS, FOREIGN

NAME/OWNER

ARBUS /ARCTIC MODULAR PLANT//USSR

DESIGNER

USSR

OPERATQR

USSR

LOCATION

MELEKESS, ULYANOVSK DISTRICT; RUSSIA

PURPOSE

PORTABLE POWER REACTOR,

TYPE

ORGANIC MODERATED

AND COOLED, TRANSPORTABLE PLANT.

POWER MWE(MWT)

750 KWE

5

CRITICAL

OCTOBER 1963. PILOT OPERATION JUNE 1963

COOLANT

ORGANIC LIQUID

MODERATOR

ORGANIC LIOUID

FUEL MATERIAL

URANIUM-ALUMINUM ALLOY OR ALUMINUM-URANIUM DIOXIDE CERMET.

FUEL GEOMETRY PLATES OR hEXAGONAL TUBES

FUEL CLADDING ALUMINUM

FUEL ENRICH.

36 PER CENT U -235

FUEL ASSEMBLY HEXAGONAL PLATES /ASSEMBLIES OF 3 HEX. TUBES/ 69 PLATES/CORE

FUEL CHARGE 02 KG: URANIUM

BURNUP(REFUEL) APPROX. 2 YEARS

CONTROL BORON STEEL RODS AND BORON CARBIDE RODS

COOLANT TEMP, INLET $230 \mathrm{C}$ OUTLET $243 \mathrm{C}$

COQLANT PRESS. 10 ATM.

REACTOR VESSEL WELDED CYLINDER 4365 MM, HIOH, $1340 \mathrm{MM}$, DIA, 20 MM. WALL THICKNESS. INNER VESSEL TO FORM COOLANT

FLOW AND AS CORE SUPPORT STRUCTURE, EQUIPMENT: FITTINGS AND PIPES ARE CARBON STEEL /PRIMARY CIRCUIT\%,

CONTAINMENT PLANT CONSISTS OF 19 UNITS, NO GREATER THAN 20 TONS EACH: ARBUS OCCUPIES A $12,36,28,5 \mathrm{M}$. 
ORGANIC COOLED

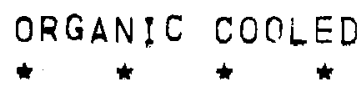

BUILDING $6.36 \mathrm{M}$ : HIGH:

REMARKS

COOLANT WILL BE CONTINUOUSLY CLEANED BY FILTRATION THROUGH METAL=CERAMIC FILTERS, CHEMICIL PURIFICATION WILL BE BY DISTILLATION, STANDARD INDUSTRIAL AND REFINERY EQUIPMENT OF LOW CARBON STEEL IS USED THROUGHOUT,

REFERENCES

ATOMIC ENERGY IN THE SOVIET UNION, TRID REPORT OF THE U. S. ATOMIC ENERGY DELEGATION. MAY 1963.

ARBUS-SOVIET MODULAR NUCLEAR POWER PLANT N SINEV

NUCLEAR ENGINEERING 8:429 /DEC, 1963/

STARTUP OF THE WORLDOS FIRST ATOMIC POWER STATION WITH AN ORGANIC-COOLED, ORGANIC MODERATED REACTOR, YU ARKHANGEL-SKII: I KOVALEV

AT, ENERG: /USSR/ 15,443 /NOV' 1963/

THE ARBUS ORGANIC COOLED AND MODERATED NUCLEAR POWER STATION: KK POLUSHKIN, OTHERS

THIRD U.N: INTL: CONF: ON THE PEACEFUL USES OF ATOMIC ENERGY, MAY 1964, A/CONF, 28/P/307 


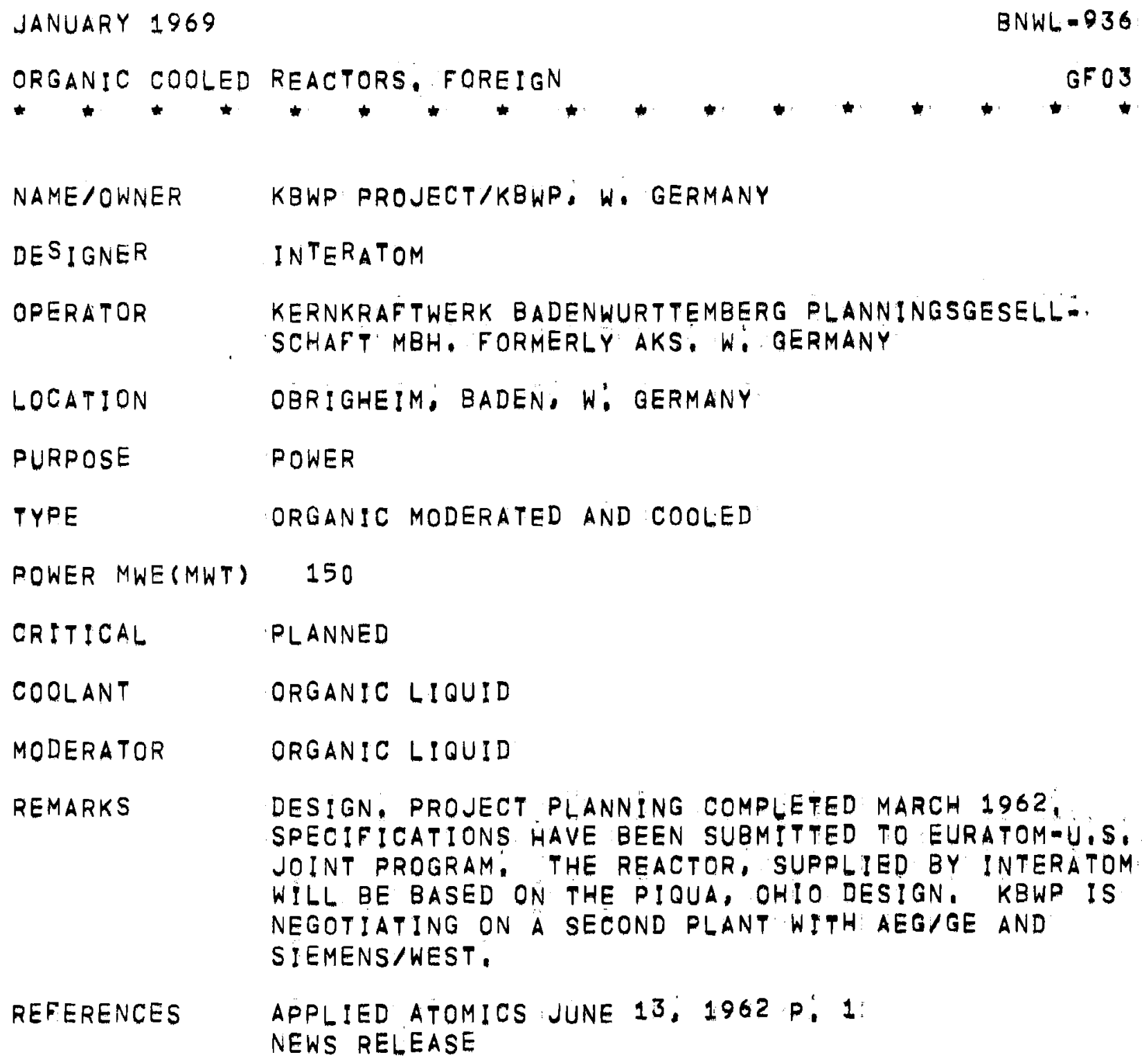




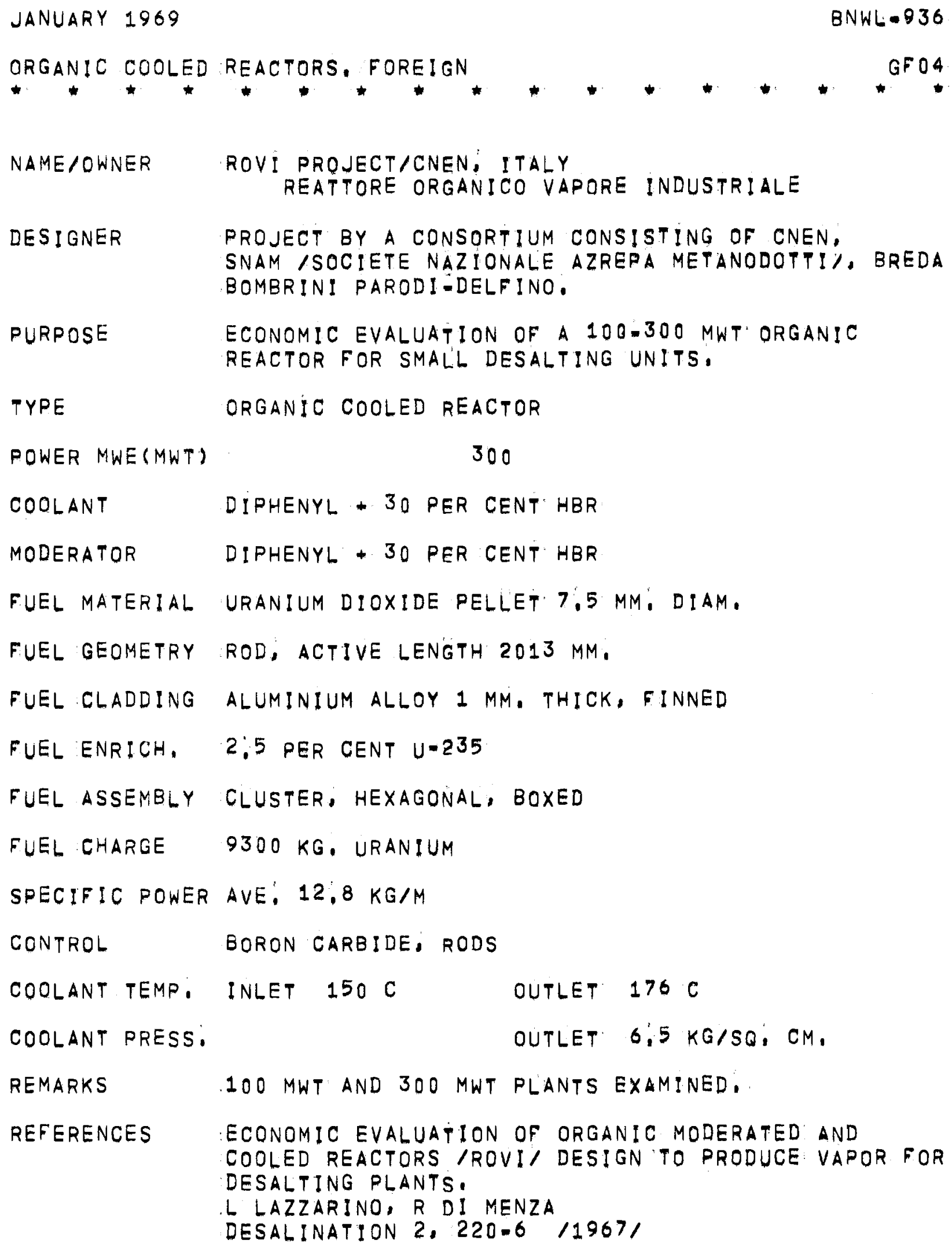




\section{PRESSURIZED LIGHT WATER REACTORS \\ DOMESTIC}


JANUARY 1969

BNWL- -936

PRESSURIZED LIGHT WATER REACTORS: DOMESTIC

NAME / OWNER

DESIGNER

OPERATOR

LOCATION

PURPOSE

TYPE

POWER MWE(MWT)

CRI TICAL

COOLANT

MODERATOR

FUEL MATERIAL

FUEL GEOMETRY

FUEL CLADDING

FUEL ENRICH.

FUEL ASSEMBLY
SM-1: /STATIONARY MEDIUM POWER:1/ DOD APPR $=1$

ALCO PRODUCTS INC:

US ARMY

FT, BELVOIR, VIRGINIA

PROTOTYPE PACKAGE POWER FOR REMOTE LOCATIONS: PWR: PACKAGE POWER

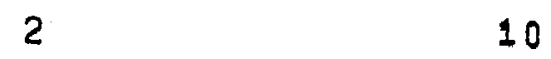

1957 CORE 2, 1960, CORE 3,1964

LIGHT WATER

LIGHT WATER

URANIUM DIOXIDEISS CERMET PLUS BORON COMPOUND

PLATE 0.03 IN. THIEK

CORE $3,0.040$ IN.

SS 0.005 IN. THICK

93 PER CENT U-235

SANDWICH, BRAZED. CORE 3 WELDED:'

ACTIVE CORE HEIGHT 22 IN,

38 STATIONARY, 7 MOVABLE RLATES, $7 \times 7$ WITH CORNERS MISSING:

CORE 3, 37 ELEMENTS, 8 DUMMY ELEMENTS; WITH

INCREASED FUEL AND BORON LOADINGS?

FUEL CHARGE $24 \mathrm{KG}$, URANIUM

SPECIFIC POWER $444 \mathrm{KW} / \mathrm{KG}$ U-235

BURNUP(REFUEL) AVE: 33 PER CENT 78 WEEKS

NEUTRON FLUX THERMAL AVE, $2,7 \times 10 \quad E+13$

FAST AVE, $1 \times 10 E+14$

CONTROL

CONTROL = ROD=FUEL-ELEMENT AND MTR TYPE RODS

COOLANT TEMP,

OUTLET 450 F

COOLANT PRESS,

1200 PSIA 
JANUARY 1969

BNWL -936

PRESSURIZED LIGHT WATER REACTORS, DOMESTIC

REACTOR VESSEL CARBON STEEL 48 IN, ID,164 IN: LONG; SS-CLAD: CONTAINMENT

VAPOR CONTAINER A COMBINATION STEEL AND CONCRETE STRUCTURE 32 FT: ID:160 FT: HIGH: 718 IN. THICK STEEL CYLINDRICAL OUTER SHELL, INNER OF 2 FT: THICK REINFORCED́ CONCRETE LINED WITH LIGHT STEEL PLATE',

REMARKS

REFERENCES
REACTOR WILL BE OPERATED FOR THE NEXT FEW YEARS FOR RESEARCH AND TRÄINING,

HAZARDS SUMMARY REPORT' FOR THE ARMY PACKAGE POWER REACTOR SM=1 SS ROSEN, ED. APAE-2 /1955/

ORNL-S DESIGN FOR A POWER REACTOR PACKAGE, RS LIVINGSTON, AL BLOCH NUCLEONICS 13, 24.7/MAY 1955/

A PACKAGE POWER REACTOR FOR REMOTE LOCATIONS, $A L$ BLOCH

$A E C U=3170 / 1955 /$

APPR-1. DESIGN, CONSTRUCTION, AND DPERATION. APAE-23 /NOVEMBER $1957 \%$

REACTOR ANALYSIS APPRII, CORE 2 APAE $32 / 1958 /$

PORTABLE NUCLEAR POWER PLANT OFFERS OPERATING FLEXIBILITY, EASY MAINTENANCE, AND LONG LIFE FOR REMOTE INDUSTRIAL; MINIMG, AND MILITARY SITES. POWER 103: 61-3/JANUARY 1999/

DESIGN ANALYSIS OF A PREPACKAGED NUCLEAR POWER PLANT 11000 EKW/. VOLUME 1; PRIMARY AND SECONDARY SYSTEM DESIGN. VOLUME 2, REACTOR, DESIGN ANALYSIS.

ALCO PRODUCTS INC:

CORE CHARACTERISTICS OF FOUR ARMY PACKAGE POWER REACTORS. JG GALLAGHER, DTHERS NUCLEAR SCI. *ENG, 2 /SUPPLEMENT/. 15-16/JUNE $1959 /$

HAZARDS REPORT FOR SM:1, CORE 3 FG MOOTE 
JANUARY 1969

BNWL-936

PRESSURIZED LIGHT WATER REACTORS: DOMESTIC ACNP:64516/JAN: 1964/ 


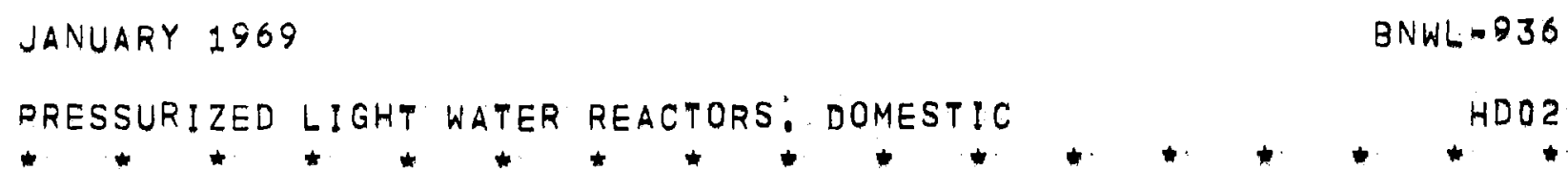




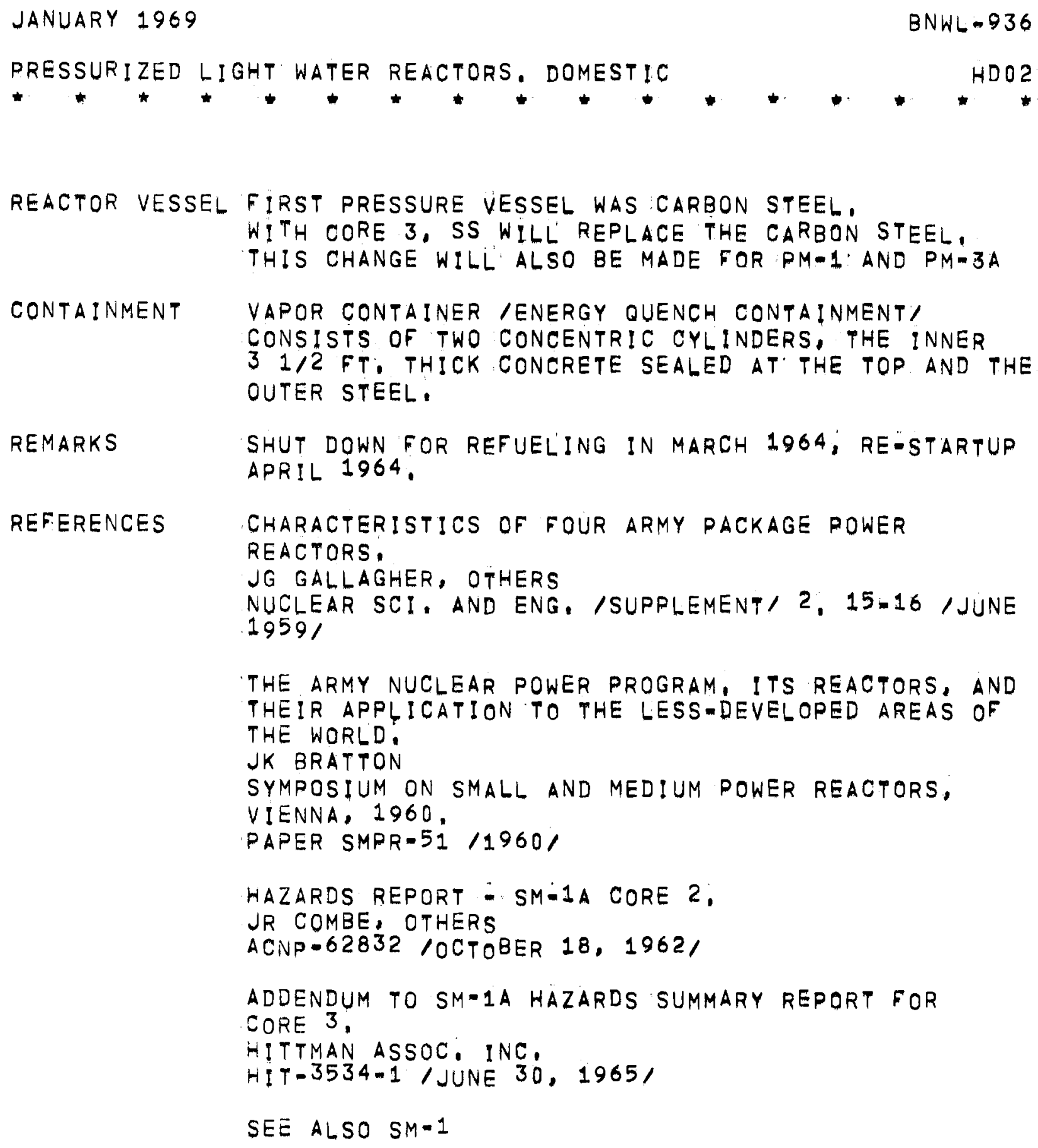


JANUARY 1969

BNWL-936

PRESSURIZED LIGHT WATER REACTORS: DOMESTIC

HDO3

NAME /OWNER

PM-2A /PORTABLE MEDIUM POWER 2A/ DOD

SKID-MOUNTED APPR

DESIGNER

ALCO PRODUCTS INC:

OPERATOR

US ARMY

LOCATION

CAMP CENTURY, GREENLAND /IN 1965 NRTS, IDAHO/

PURPOSE

POWER AND SPACE HEAT

TYPE

PWR, PACKAGED POWER

POWER MWE (MWT)

1.5

CRITICAL

1960, TRANSFERRED TO NRTS, RE-STARTUP 1965

COOLANT

LIGHT WATER

MODERATOR

LIGHT WATER

FUEL MATERIAL URANIUM DIOXIDE-SS CERMET, BORON ADDED

FUEL GEOMETRY PLATE 30 MILS THICK

FUEL CLADDING SS 5 MILS THICK

FUEL ENRICH, 93 PERCENT U-235

FUEL ASSEMELY ASSEMBLY OF FUELED PLATES

FUEL CHARGE $18,49 \mathrm{KG}, U=235$

BURNUP(REFUEL) CORE LIFE 1OMW-YR

COOLANT TEMP,

OUTLET 518 F

COOLANT PRESS:

1750 PSIA

CONTAINMENT

UNDER-SNOW CONSTRUCTION, VAPOR CONTAINER,

REMARKS

THE PLANT WAS DISMANTLED IN JANUARY: 1964 ; FOR

TRANSFER TO NRTS, IDAHO TO TAKE PART IN AEC=S

REACTOR SAFETY R+D PROGRAM.

REFERENCES

HAZARDS SUMMARY REPORT, PREPACKAGED NUCLEAR POWER PLANT FOR AN ICE-CAP STATION /PM-2A/ EM RIEBACK, OTHERS

APAE-49/JULY 1959/ AND SUPPLEMENT /ARRIL 1960 / 
JANUARY 1969

BNWL-936

PRESSURIZED LIGHT WATER REACTORS: DOMESTIC

HDO3

$+$

SEE ALSO SM-1 
JANUARY 1969

BNWL: $=936$

PRESSURIZED LIGHT WATER REACTORS: DOMESTIC

NAME/OWNER

SM=2 ISTATIONARY MEDIUM POWER-2/ AEO-DOD $A P P R=1 B$

DESIGNER

ALCO PRODUCTS INC:

RURPOSE

POWER FOR NIKE=ZEUS INSTALLATIONS, REMOTE LOCATIONS

TYPE

PWR, PACKAGED POWER

POWER MWE(MWT)

6

28

CRITICAL

DES IGN

COQLANT

LIGHT WATER

MODERATOR

LIGHT WATER

FUEL MATERIAL

URANIUM DIOXIDE-SS CERMET, BORON ADDED

FUEL GEOMETRY PLATE

FUEL CLADDING SS

FUEL ENRICH, 93 PER CENT U.235

Fuel assembly assembly of fueled plates

FUEL CHARGE $36.2 \mathrm{KG}$. U-235

BURNUP(REFUEL)

2 CORESIYEAR

CONTROL

FUEL ELEMENTS WITH ABSORBER FOLLOWER AND MTR-TYPE CONTROL RODS

COOLANT TEMP. OUTLET 525 F

COOLANT PRESS.

\section{PSIA}

CONTAINMENT

SEPARATE VAPOR CONTAINMENT FOR EACH REACTOR IN A MULTIPLE-REACTOR COMPLEX. 20 FT? VERTICAL

CYLINDER, PARTLY BELOW-GRADE,

REMARKS

COMPLEX OF TWO SEPARATE REACTORS TO PROVIDE 12 MWE FOR SITE POWER, CONSTRUCTION DEPENDS ON DOD REQUIREMENTS,

REFERENCES

CONCEPTUAL DESIGN OF SM=2 12000 EKW POWER COMPLEX. ALCO PRODUCTS

APAE-68 /MAY $1960 /$ 
JANUARY 1969

$B N W L=936$

PRESSURIZED LIGHT WATER REACTORS: DOMESTIC ${ }^{*} \ldots \ldots$

SM-2 CORE AND VESSEL DESIGN ANALYSIS. ALCO PRODUCTS

APAE-69/VOL. 1;2; AND 3/ /MARCH 1961/

SEE ALSO SM-1 
PRESSURIZED LIGHT WATER REACTORS: DOMESTIC

NAME / OWNER

PL-3 /PORTABLE LOW-POWER-3//AECIDOD

DES IGNER

ALCO PRODUCTS IÑC:

LOCATION

PROPOSED FOR BYRD STATION, ANTARCTICA

RURPOSE

POWER AND SPACE HEAT FOR ICE-CAP INSTALLATION.

TYPE

PWR; PACKAGED POWER

POWER MWE (MWT)

1

9.3

CRITICAL

DESIGN. FOR REDESIGN BY AC SEE RM=3B

COOLANT

LIGHT WATER

MODERATOR

LIGHT WATER

FUEL MATERIAL

URANIUM DIOXIDE-SS CERMET, ZIRCONIUM DIBORIDE ADDED.

FUEL GEOMETRY PLATE 21:75 IN, ACT: LENGTH: 2,778 IN. WIDE, 0,030 IN. THICK

FUEL CLADDING SS 0:005 IN: THICK

FUEL ENRICH, 93 PER CENT U $=235$

FUEL ASSEMBLy 18-PLATE RECTANGULAR ASSEMBLY PLUS MOVABLE PLATES, FUEL :CHARGE 22,48 KG U-235

BURNUP(REFUEL) AVE; 35 PER CENT, 2 YEARS

NEUTRON FLUX THERMAL AVE $1 \times 10 \mathrm{E}+13$

CONTROL MTRITYPE ROD, FUEL ELEMENT ABSORBER FOLLOWER

COOLANT TEMP, INLET $454 \mathrm{~F}$ OUTLET $487 \mathrm{~F}$

COOLANT PRESS. 1200 PSIA

REACTOR VESSEL SS-LINED STEEL VESSEL 40 IN, ID:111 FT: 6 IN, LONG CONTAINMENT SNOW TUNNEL, DOUBLE VAPOR CONTAINER COMPOSED OF VERTICAL CYLINDERS, ONE CONTAINING THE REACTOR VESSEL AND THE OTHER THE STEAM GENERATOR, PRESSURIZER, AND PRIMARY PUMP: VESSELS CONNECTED BY FLEXIBLE DUCTS: 
JANUARY 1969

BNWL -936

PRESSURIZED LIGHT WATER REACTORS: DOMESTIC HDOS

DESIGN RECOMMENDATION HAS BEEN COMPLETED, AC HAS

DONE A RE-EVALUATION OF ALL WORK DONE ON PM-JB

WITH THE OBJECTIVE OF A NEW DESIGN CONCEP F FOR A

LIGHTER, MORE COMPACT PORTABLE REACTOR OF AT

LEAST 1 MWE POWER RATING.

REFERENCES

PWR PRELIMINARY DESIGN DATA FOR PLIS3. ALCO PRODUCTS

APAE-115 /VOL: $2 /$ /FEBRUARY $1962 /$ 


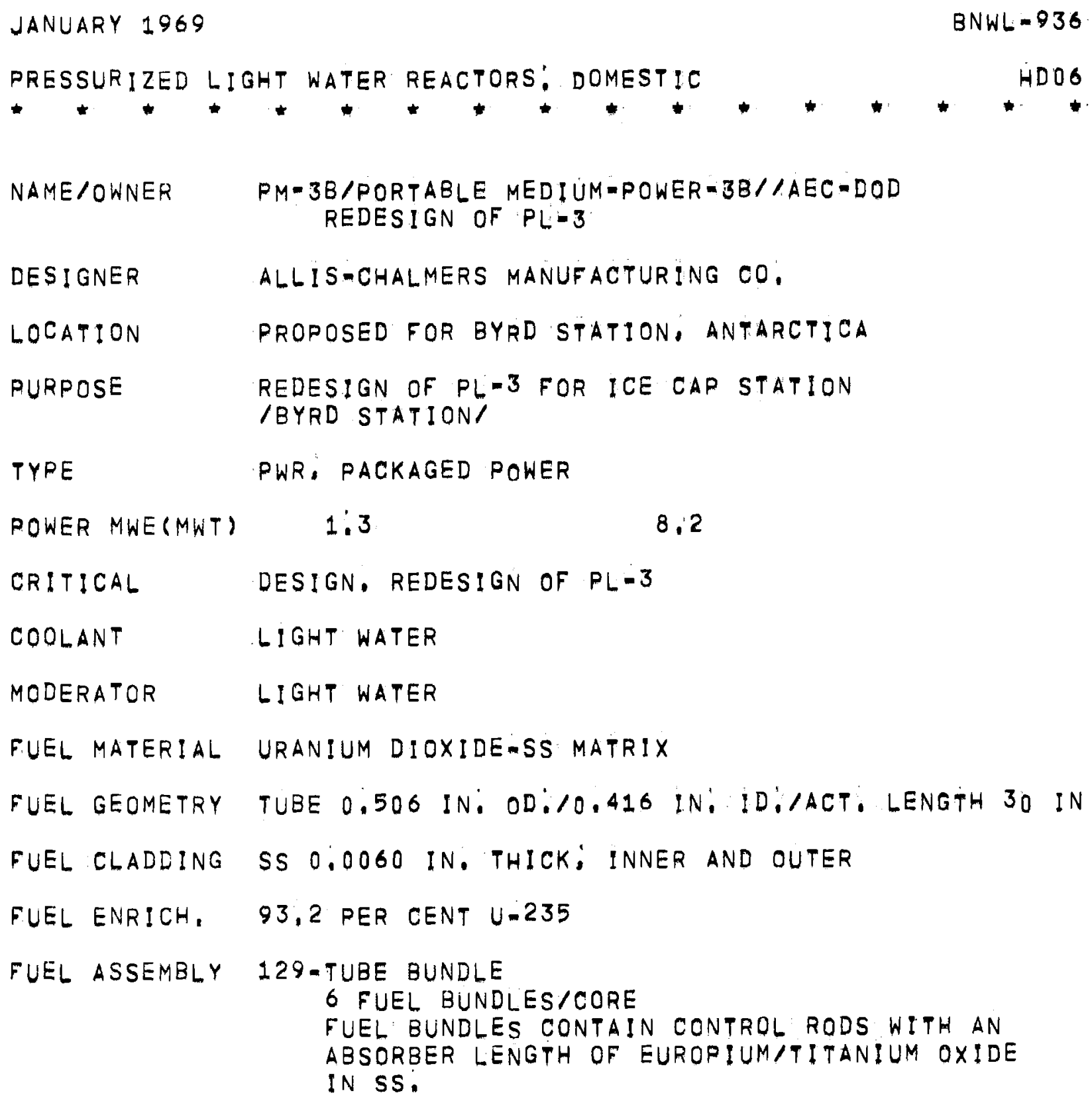


JANUARY 1969

$B N W L-936$

PRESSURIZED LIGHT WATER REACTORS: DOMESTIC

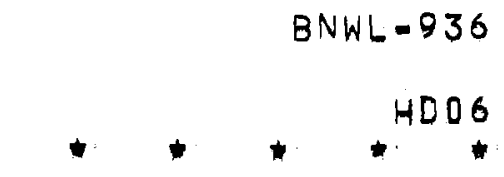

REFERENCES

BYRD STATION STUDY, FINAL REPDRT, INCLUDES ADDENDUM 1.

ALLIS=CHALMERS MANUFACTURING CO.

ACNP-62848 AND ADD, 1 /FEBRUARY 1, 1963/

INLAND ANTARCTIC STATION NUGLEAR POWER PLANT

SUMMARY DESIGN REPORT.

VOL: 2: PRELIMINARY SAFETY ANALYSIS:

ALLIS-CHALMERS MANUFACTURING CO,

ACNP-63809/VOL: 1-2/ /JULY 1963/ 


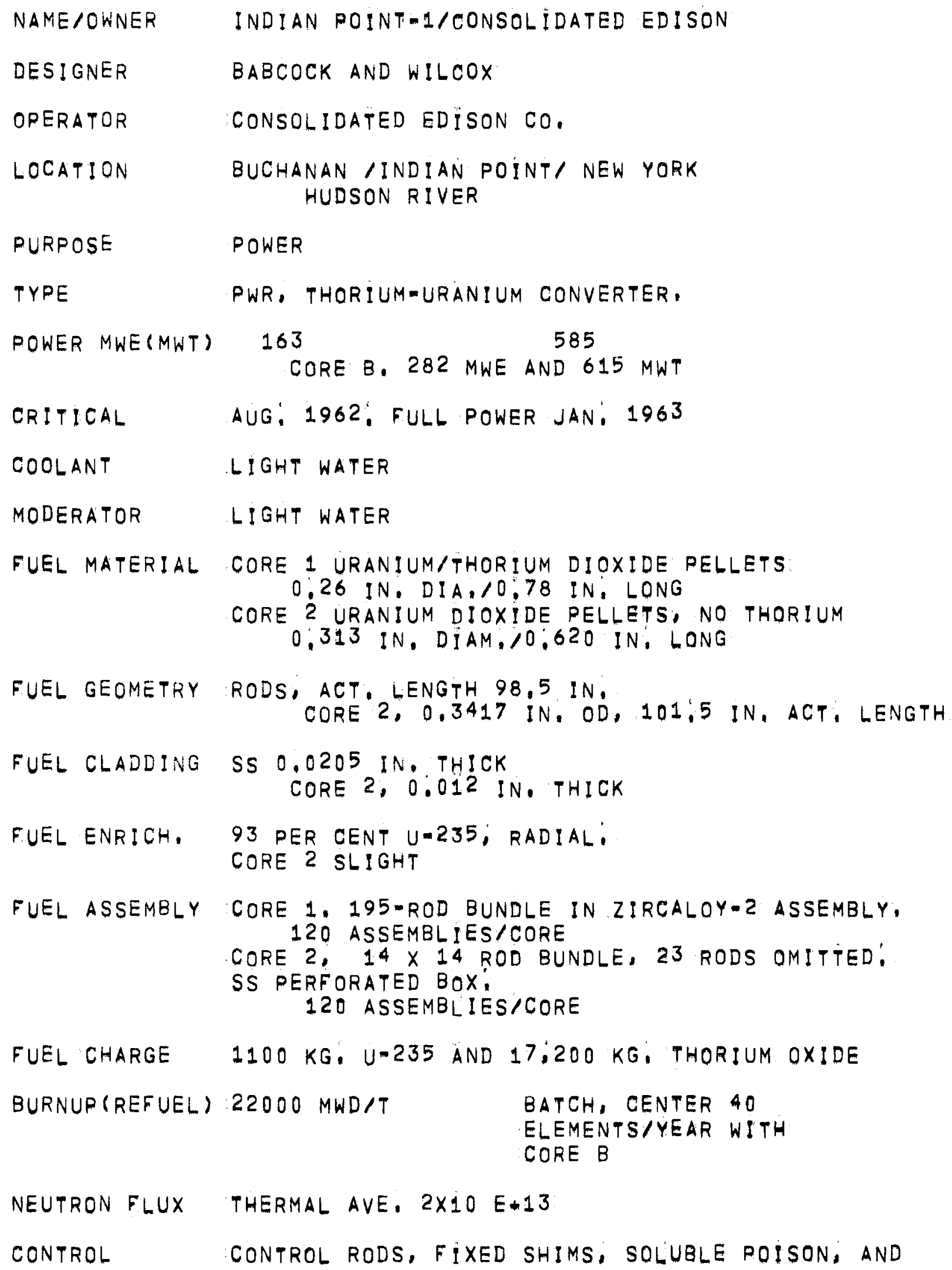


JANUARY 1969

BNWL -936

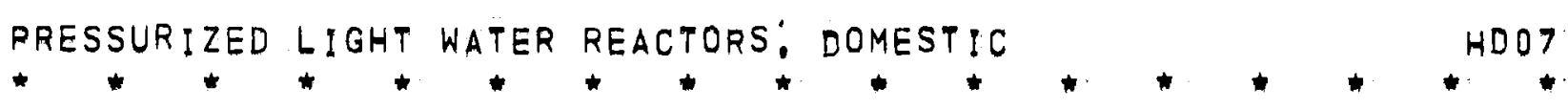

BURNABLE POSION IN THE FUEL CLADDING,

COOLANT TEMP, INLET $486 \mathrm{~F}$ OUTLET $570 \mathrm{~F}$

COOLANT PRESS: 1500 PSIA

REACTOR VESSEL CARBỌ STEEL CYLINDER, 36 FT: 9 IN, HIGH / 9 FT,

9 IN. ID. AND 6:95 IN: WALL THICKNESS:

CONTAINMENT STEEL SPHERE $160 \mathrm{FT}$ : ID: SURROUNDED BY CONCRETE

STRUCTURE WITH $51 / 2$ FT. THICK WALLS AND $23,4 . F T$, THICK DOMED ROOF,

REMARKS

SECOND CORE IS AN ADVANCED 3-REGION DESIGN, REFUELING COMPLETED MARCH 16, 1966:

CRITICAL APRIL 1, FULL POWER END OF APRIL 1966.

REFERENCES

REACTOR STUDIES, FINAL REPORT, PART I',
TID-10117/1955! DECLASSIFIED MARCH 1957,

THE CONSOLIDATED EDISON THORIUM REACTOR

$F$ WARD

BNL=483/JANUARY 1958/

CORE DESIGN AND CHARACTERISTICS OF THE

CONSOLIDATED EDISON REACTOR:

BAW:9/REV,3/ /AUUGST 1958/

THE CONSOLIDATED EDISON COMPANY OF NEW YORK

NUCLEAR ELECTRIC GENERATING STATION,

GR MILNE, OTHERS

SECOND U. N. INTL: CONF, ON THE PEACEFUL USES OF

ATOMIC ENERGY 8: $483=9$ /1958\%

HAZARDS SUMMARY REPORT, CONSOLIDATED EDISON

THORIUM REACTOR:

USAEC DOCKET 50-3, EXHIBIT K-5 /REV, 1/

BABCOCK AND WILCOX CO: IJANUARY $1960 /$

CONSOLIDATED EDISON THORIUM REACTOR PHYSICS

DES!GN.

HS BARRINGER, OTHERS

BAW-120/REV.1/ Y JULY $1960 /$

INDIAN POINT -CONSOLIDATED EDISON-S THORIUM

CONVERTER:

NUCLEAR ENG: 6.413:23/OCTOBER 1961/

INDIAN POINT ON-THE-LINE.

NUCLEONICS REACTOR FILE NO, 14 
JANUARY 1969

BNWL-036

PRESSURIZED LIGHT WATER REACTORS: DOMESTIC

NUCLEONICS $21,45.52$ /APRIL 1963\%

CONSOLIDATED EDISON NUCLEAR STEAM GENERATING STATION.

POWER REACTOR TECHNOLOGY: $6 / 3 \% 28: 40 / J U N E 1963 /$

PRELIMINARY HAZARDS SUMMARY REPORT FOR THE

CONSOLIDATED EDISON INDIAN ROINT REACTOR, CORE B, CONSOL IDATED EDISON INIY,I AND WESTINGHOUSE

ELECTRIC CORPORATION.

$N P-12869$ /ND/

CONCEPTUAL DESIGN OF CORE B; CONSOLIDATED EDISON

COMPANY.

INDIAN POINT NUCLEAR FACILITY. WESTINGHOUSE ELECTRIC CORP.

TID-20964. 
JANUARY 1969

BNWL-936

PRESSURIZED LIOHT WATER REACTORS: DOMESTIC
$*+\cdots+\cdots+\cdots$

NAME/OWNER MSR /MERCHANT SHIP REACTOR//AEC-MA
N,S: SAVANNAH: R

DESIGNER BABCOCK AND WILCOX

OPERATOR MARITIME ADMINISTRATION: U. S. A.

LOCATION LANDEBASED PROTOTYPE AT LYNCHBURG, VA.

RURPOSE SHIP PROPULSION: SURFACE:

TYPE PWR

POWER MWE(MWT) 20,000 SHP 70

CRITICAL FEB', 1960
SAVANNAH WILL OPERATE THROUGH 1968
REFUELING SCHEDULED FOR AUG: 1968,

COOLANT LIGHT WATER

MODERATOR LIGHT WATER

FUEL MATERIAL URANIUM DIOXIDE PELLETS 0:4245 IN, DIA,10:5: IN. LONG

FIEL GEOMETRY ROD 66 IN, ACTIVE LENGTH

FUEL CLADDING SS TUBES 0.035 IN: WALL THICKNESS

FUEL ENRICH, $4: 2$ AND $4: 6$ PER CENT U-235

FUEL ASSEMBLY 164"TUBE ELEMENT. TUBES ARE HELD IN A RIGID BUNDLE OF 41 ELEMENTS BY BRAZED SS FERRULES /EGG-CRATE STRUCTURE/. 32 ASSEMBLIES/CORE

FUEL CHARGE 312:4 KG U-235 SEE REMARKS

BURNUP(REFUEL) 7352 MWD/MTU SEE REMARKS

CONTROL CRUCIFORM RODS, BORONISS: TORWDRIVEN,

COQLANT TEMP, INLET $463 \mathrm{~F}$ OUTLET $519 \mathrm{~F}$

COOLANT PRESS, 1750 PSIA

REACTOR VESSEL SSMLINED CARBON STEEL CYLINDER 98 IN, ID,126 FT. 10 IN. HIGH.

CONTAINMENT SHELL LYING LENGTHIWISE OF SHIP IS A OYLINDER WITH 


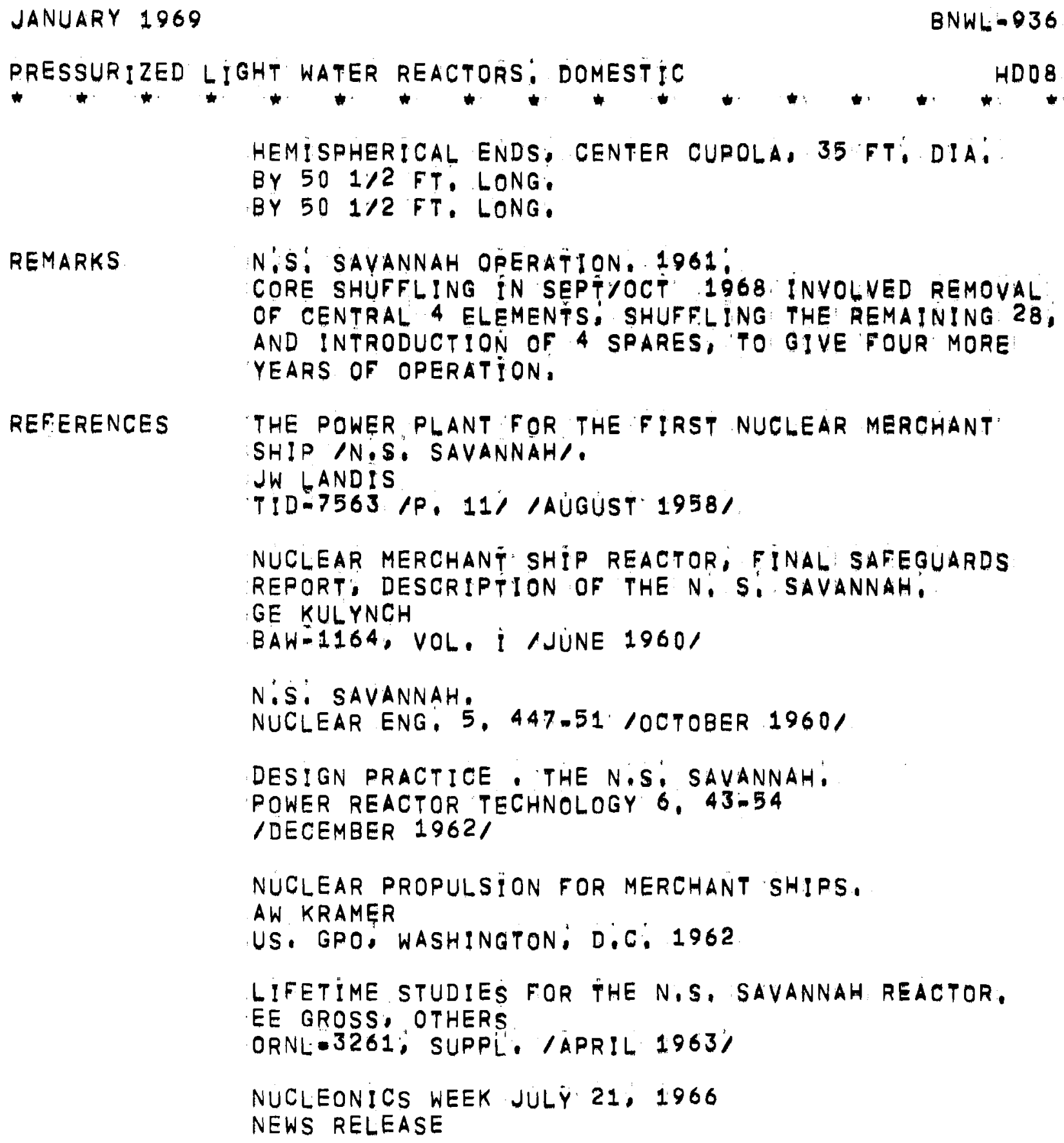


JANUARY 1969

BNWL-936

PRESSURIZED LIGHT WATER REACTORS: DOMESTIC

NAME/OWNER

CNSG /CONSOLIDATED NUCLEAR STEAM GENERATOR//BAW

DESIGNER

BABCOCK AND WILCOX

PURPOSE

SHIP PROPULSION

TYPE

PWR, FORCED CIRCULATION, CLOSED CYCLE

POWER MWE (MWT)

27,500 SHP

$62: 4$

CRITICAL

REFERENCE DESIGN, SEE REMARKS,

COOLANT

LIGHT WATER

MODERATOR

LIGHT WATER

FUEL MATERIAL URANIUM DIOXIDE PELLETS, COMPACTED

FUEL GEOMETRY PIN, SWAGED, 0,460 IN: DIA, ACT: LENGTH 42 IN.

FUEL CLADDING ZIRCALOY-2 0.021: IN: THICK

FUEL ENRICH, ZONE 1,2 AND 3, 1:6;2,1, AND 3:1 PER CENT U-235

FUEL ASSEMBLY TYPE A: 108-PIN BUNDLE, TOTAL 13 ASSEMBLIES,

TYPE B, 121-ROD BUNDLE, TOTAL 24. ASSEMBLIES,

REGION 1; 1 TYPE A ELEMENT' /CENTRAL/

REGION 2; 6 TYPE B ELEMENTS /INNER RING/ PLUS

RING OF 12 TYPE A ELEMENTS

REGION 3, 18 TYPE B ELEMENTS /OUTER RING/

CORE CONTAINER IS A SEGMENTED SS STRUCTURE

FUEL CHARGE A100 KG, URANIUM DIOXIDE, 92.85 KG, U-235

BURNUP(REFUEL) 15,000 MWD/T FUEL SHUFFLING

URANIUM DIOXIDE

CONTROL CHEMICAL SHIM: BORIC ACID: CONTROL RODS

COOLANT TEMP, INLET $490.4 \mathrm{~F}$ OUTLET $520 \mathrm{~F}$

COOLANT PRESS, 816 PSIA

REACTOR VESSEL STEEL CYLINDER 10FT, DIA,/27 FT: HIGH, OUTER

8 IN. THICK LEAD CLADDING BONDED TO SHELL, VESSEL COMPLETELY SUBMERGED IN WATER; SEE REMARKS.

CONTAINMENT WATER-FILLED STEEL CYLINDRICAL VESSEL 19 FT, DIA.I

38 FT HIGH, PRESSURE-SUPPRESSION, SEE REMARKS,

REMARKS

THE CONCEPT IS BASED ON MSR AND IBR DESIGNS, IN 
PRESSURIZED LIGHT WATER REACTORS: DOMESTIC HDO9

\begin{tabular}{|c|c|}
\hline & 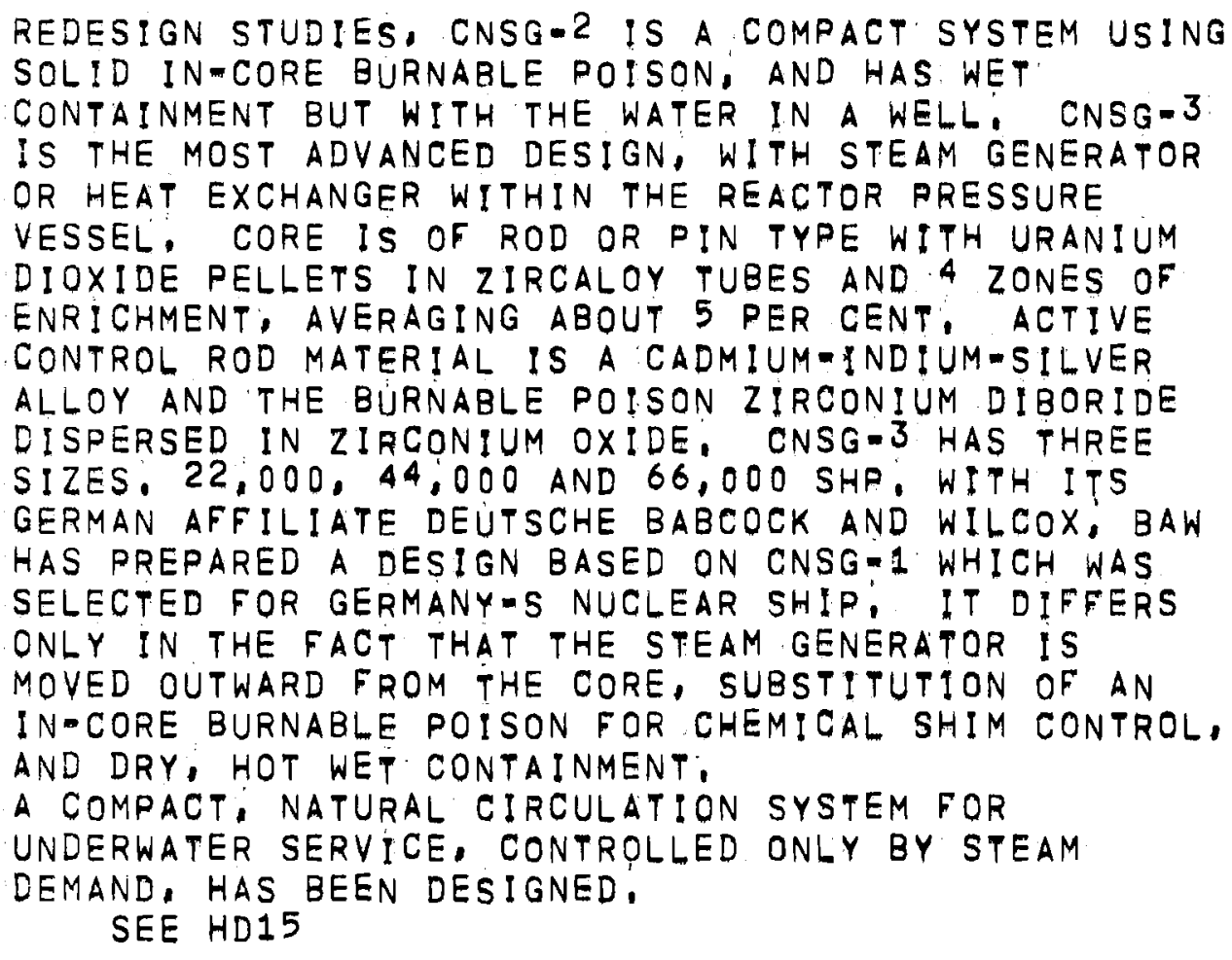 \\
\hline \multirow[t]{3}{*}{ REFERENCES } & $\begin{array}{l}\text { CONSOLIDATED NUCLEAR STEAM GENERATOR FOR MERCHANT } \\
\text { SHIP APPLICATION. A CONCEPTUAL DESIGN, } \\
\text { BABCOCK + WILCOX CO: } \\
\text { BAW-1243/AUGUST } 19621\end{array}$ \\
\hline & $\begin{array}{l}\text { CONSOLIDATED NUCLEAR STEAM GENERATOR FOR MARINE } \\
\text { APPLICATION. } \\
\text { ENGINEER } 214,789.90 \text { /NOVEMBER } 2,1962 \text { / }\end{array}$ \\
\hline & $\begin{array}{l}\text { COMPACT MARINE REACTOR, } \\
\text { NUCLEAR ENERGY, DECEMBER 1962, R, } 465,\end{array}$ \\
\hline
\end{tabular}


JANUARY 1969

BNWL:036

PRESSURIZED LIGHT WATER REACTORS: DOMESTIC

$*$

NAME /OWNER

DESIGNER

OPERATOR

LOCATION

PURPOSE

TYPE

POWER MWE (MWT)

CRITICAL

COOLANT

MODERATOR

FUEL MATERIAL

FUEL GEOMETRY

FUEL CLADDING

FUEL ENRICH,
PALISADES PARK NUCLEAR STATION/CONSUMERS POWER

COMBUSTION ENG INEERING

CONSUMERS POWER CO.

PALISADES PARK, MICHIGAN ILAKE MICHIGAN/

POWER

PWR, 2-LOOP SYSTEM

770

2200

TARGET NOV. 1969

LIGHT WATER

LIGHT WATER 


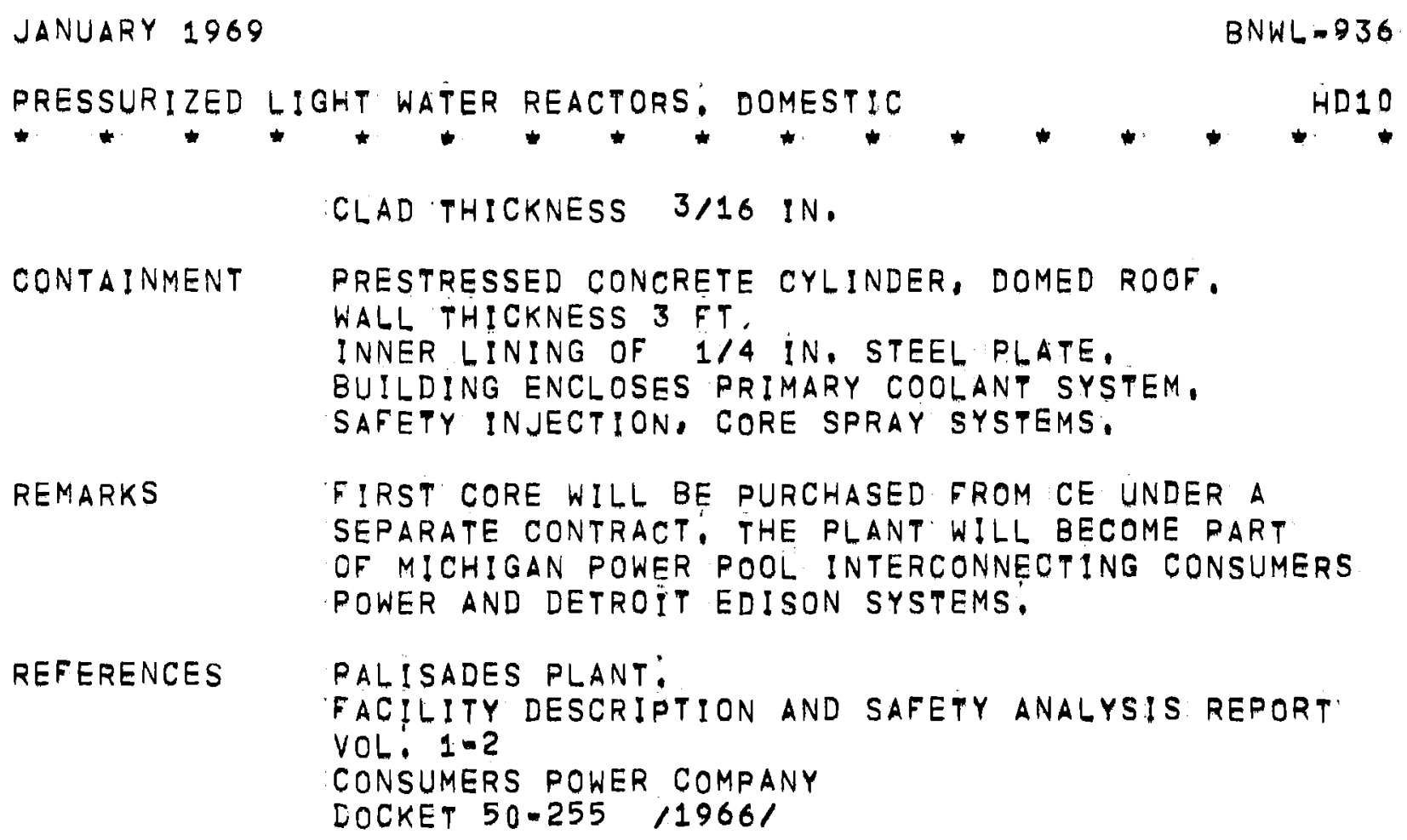


JANUARY 1969

BNWL $=936$

PRESSURIZED LIGHT WATER REACTORS: DOMESTIC

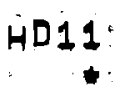

\author{
NAME/OWNER NUCLEAR TANKER DESIGN STUDY/CE-AEC \\ DESIGNER COMBUSTION ENGINEERING, INC, \\ PURPOSE SHIP PROPULSION \\ TYPE PWR, FORCED CIRCULATION
}

POWER MWE(MWT) 30.000 SHP 82

CRITICAL REFERENCE DESIGN

COOLANT LIGHT WATER

MODERATOR .LIGHT WATER

FUEL MATERIAL URANIUM DIOXIDE PELLETS $0: 312$ IN: DIA:10:468 IN. LONG

FUEL GEOMETRY RODS, ACTIVE LENGTH 52 IN,

FUEL CLADDING SS TUBES 0.023 IN: WALL THICKNESS

FUEL ENRICH, 5:4 PER CENT U-235, ZONE ENRICHMENT

FUEL ASSEMBLY 91-ROD CLUSTER

87 CONTAIN FUEL AND 3 BURNABLE POISON

91 CLUSTERS/CORE, 19 ARE MOVABLE FOR CONTROL.

FUEL :CHARGE $\quad 4: 424$ METRIC TONS URANIUM

BURNUP (REFUEL) $\quad 890$ DAYS

NEUTRON FLUX THERMAL AVE, $1.7 \times 10$ E $\$ 13$

CONTROL NEUTRON RECTIFIER ASSEMBLIES, BURNABLE POISON

COOLANT TEMP, INLET 589 F OUTLET $620 \mathrm{~F}$

COQLANT PRESS: 2000 PSIA

REACTOR VESSEL CARBON, STEEL, SS LINED, $75:$ IN, ODI/228 IN, LONG. WALL 4.75 IN. THICK:

CONTAINMENT VAPOR CONTAINER: STEEL, ENCLOSES ENTIRE MAIN COOLANT SYSTEM. CYLINDRICAL SHELL, HEMISPHERICAL ENDS, 34 FT, DIA. /52 FT, HIGH,

REMARKS DEVELOPMENT OF A REFERENCE CONCEPTUAL DESIGN FOR NUCLEAR CARGO OR TANKER, IN CONJUNCTION WITH 
JANUARY 1969

BNWL: $=936$

PRESSURIZED LIGHT WATER REACTORS, DOMESTIC GEORGE C. SHARP INC, FOR THE AEC,

REFERENCES NUCLEAR POWERED TANKER DESIGN AND ECONOMIC ANALYSIS PRESSUR!ZED WATER REACTOR: COMBUSTION ENG. INC: CEND-62/1960/ NYO $-2860 / 1960 /$ 
JANUARY 1969

BNWL: 936

PRESSURIZED LIGHT WATER REACTORS: DOMESTIC

HD12

NAME/OWNER

DES IGNER

PURPOSE

TYPE

POWER MWE (MWT)

CRITICAL

COQLANT

MODERATOR

FUEL MATERIAL

FUEL GEOMETRY

FUEL CLADDING

FUEL ENRICH.

FUEL ASSEMBLY

\section{8-ROD CLUSTER /FUEL'}

61-ROD CLUSTER /MOVABLE CONTROL/

61 FUEL CLUSTERS. INCLUDING 12 MOVABLE

CONTROL CLUSTERS, IN CORE:

FUEL CHARGE $6: 67$ METRIC TONS URANIUM

BURNUP(REFUEL) 14,100 MWD/MTU

CONTROL NEUTRON RECTIFIER ASSEMBLIES, SILVER-INDIUM CADMIUM IN SS

COOLANT TEMP, INLET $600 \mathrm{~F}$ OUTLET $642,7 \mathrm{~F}$

COOLANT PRESS, 2100 PSIA

REACTDR VESSEL STEEL CYLINDER, SS INNER GLADDING, 90.8 IN: OD. AND 23,4 FT. LONG:

CONTAINMENT STEEL CYLINDER, HEMISPHERICAL HEADS, 3A FT: DIA, BY 50 FT. HIGH, CONTAINING ALLIMAJOR COMPONENTS OF THE REACTOR SYSTEM. THE VESSEL IS SEALED.

REMARKS

UP-DATING OF THE NUCLEAR TANKER REFERENCE DESIGN STUDY. A LOW-PRESSÜR 1300 PSI SYSTEM WOULD UTILIZE THINNER CLADDING AND LOWER ENRICHMENT FUEL 
JANUARY 1969
PRESSURIZED LIGHT WATER REACTORS: DOMESTIC
$+\cdots 36$

REFERENCES ADVANCED INDIRECT CYCLE WATER REACTOR STUDIES FOR MARITIME APPLICATIONS: PART 2, PLANT CONGEPTUAL STUDIES,

COMBUSTION ENG. INC:

CEND-150 /PT.2/ /1962/ 
JANUARY 1969

$B N W L=936$

PRESSURIZED LIGHT WATER REACTORS: DOMESTIC

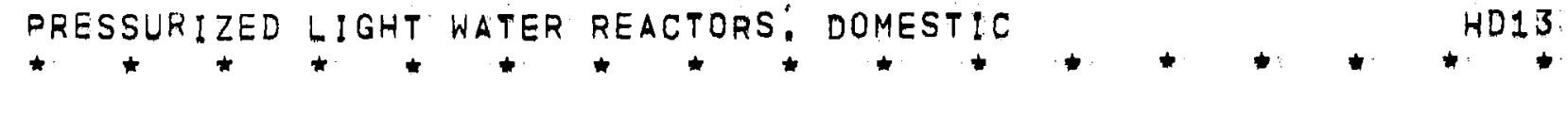

NAME/OWNER UMP /UNIFIED MODULAR PLANT//CE

DESIGNER COMBUSTION ENGINEERING, INC.

PURPOSE SHIP PROPULSION

TYPE PWR, CLOSED CYCLE; MODULAR

POWER MWE(MWT) 30,000 SHP 80

CRITICAL DESIGN

COOLANT LIGHT WATER

MODERATOR LIGHT WATER

FUEL MATERIAL URANIUM DIOXIDE

FUEL GEOMETRY ROD $0: 328$ IN, OD, OÚTSIDE CLADDING, ACT. LENGTH 50 IN:

FUEL CLADDING SS TUBES 0.015 IN: WALL THICKNESS

FUEL ENRICH, 5:9 PER CENT U-235, ZONE ENRICHMENT

$\begin{aligned} \text { FUEL ASSEMBLY 127-ROD BUNDLE, } & 12 \text { MOVABLE BUNDLES INCLUDE A HOLLOW }\end{aligned}$

WATER - FILLED NEUTRON ABSORBING SECTION FOR

COLD SHUT-DOWN. ALL ABSORBER SECTIONS ARE OUT OF CORE DURING OPERATION.

61 BUNDLES/CORE

36 ARE IN FIRST COOLANT PASS AND 25 IN SECOND,

OR INNER, COOLANT PASS.

FUEL CHARGE $3: 93$ METRIC TONS URANIUM

BURNUP (REFUEL) 20,000 MWD/MTU

CONTROL SELF-REGULATED CHANGES IN COOLANT DENSITY, AND MOVABLE ELEMENTS FOR COLD SHUT-DOWN.

COOLANT TEMP, INLET $630 / 590 \mathrm{~F}$ OUTLET $668 / 636 \mathrm{~F}$

COOLANT PRESS, $2500 / 2000$ PSI

REACTOR VESSEL STEEL VESSEL 71 IN, ID,/22 FT, 4 IN, HIGH, HEAT EXCHANGER IS WITHIN THE PRESSURE VESSEL. WATER TIGHT CANNING SURROUNDS THE VESSEL, CIRCULATING PUMPS, AND COLD SHUT-DOWN MECHAN!SMS,

CONTAINMENT VAPOR SUPPRESSION: VESSEL $16 \mathrm{FT}$, ID./34FT, LONG 


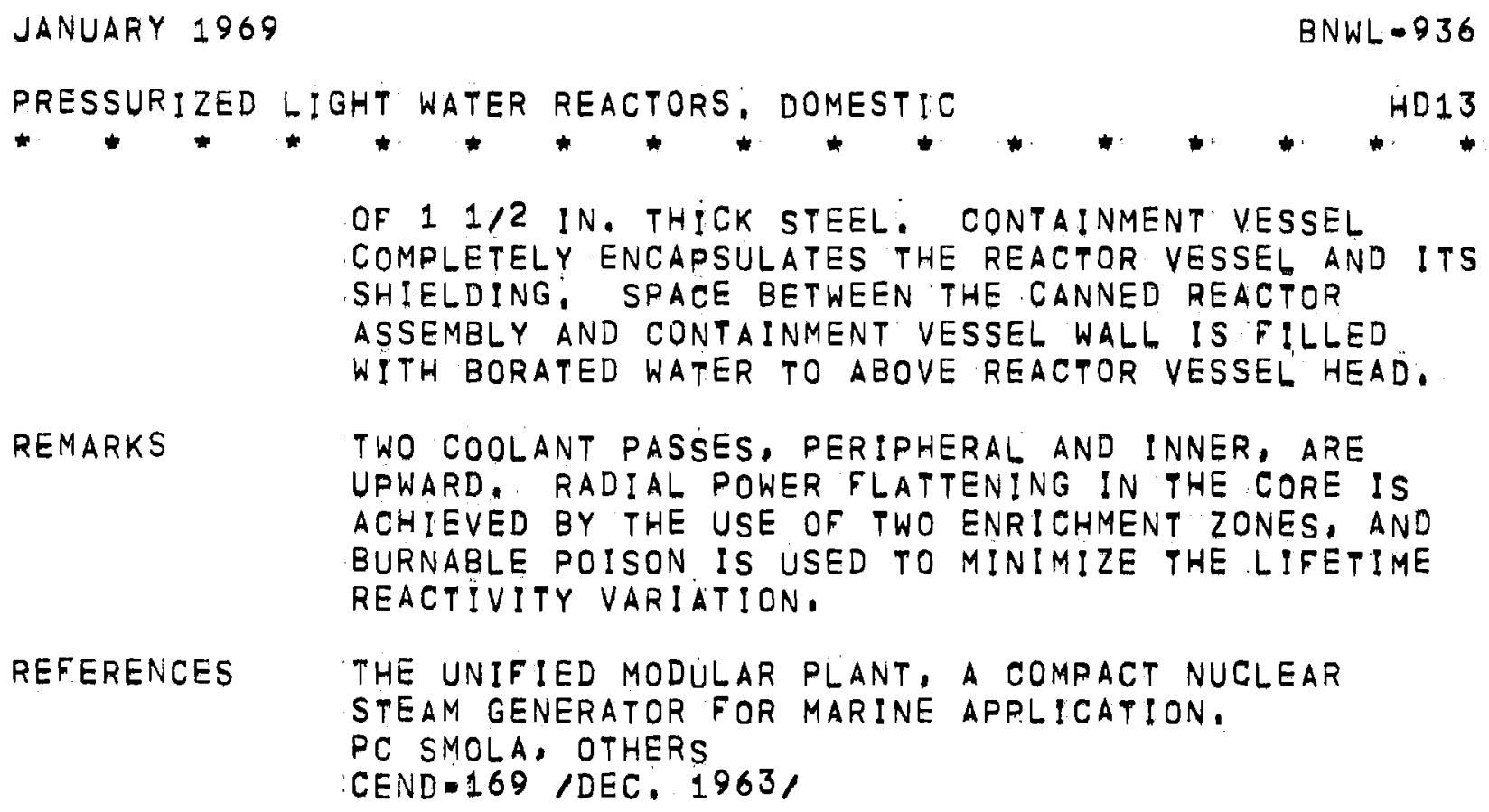

REMARKS

TWO COOLANT PASSES, PERIPHERAL AND INNER, ARE UPWARD. RADIAL POWER FLATTENING IN THE CORE IS ACHIEVED BY THE USE OF TWO ENRICHMENT ZONES, AND BURNABLE POISON. IS USED TO MINIMIZE THE LIFETIME REACTIVITY VARIATION.

REFERENCES THE UNIFIED MODULAR PLANT, A COMPACT NUCLEAR STEAM GENERATOR FOR MARINE APPLICATION, PC SMOLA, OTHERS CEND-169/DEC. 1963/ 


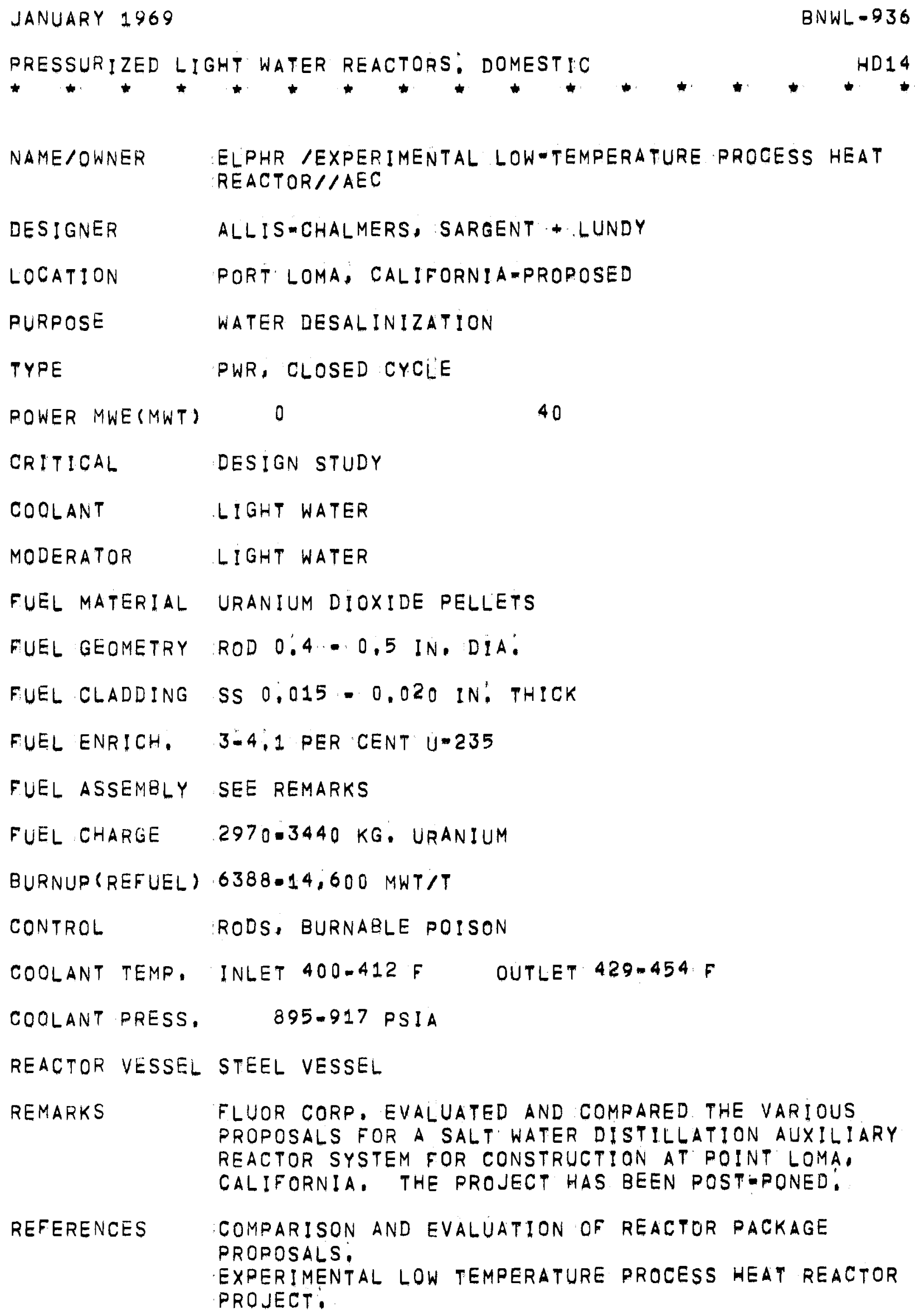


JANUARY 1969

BNWL -936

PRESSURIZED LIGHT WATER REACTORS: DOMESTIC HDI4.

EH HYKAN, RA JOHNSON

SL-1767/DECEMBER 1959/

EXPERIMENTAL LOW-TEMPERATURE PROCESS HEAT REACTOR PROJECT:

CONTRACT TERMINATION REPORT.

SARGENT * LINDY: ALLIS-CHALMERS MNFG, CO. TID-12385 /FEB, 24, 1961\% 
JANUARY 1969

$B N W L=936$

PRESSURIZED LIGHT WATER REACTORS: DOMESTIC

HD15

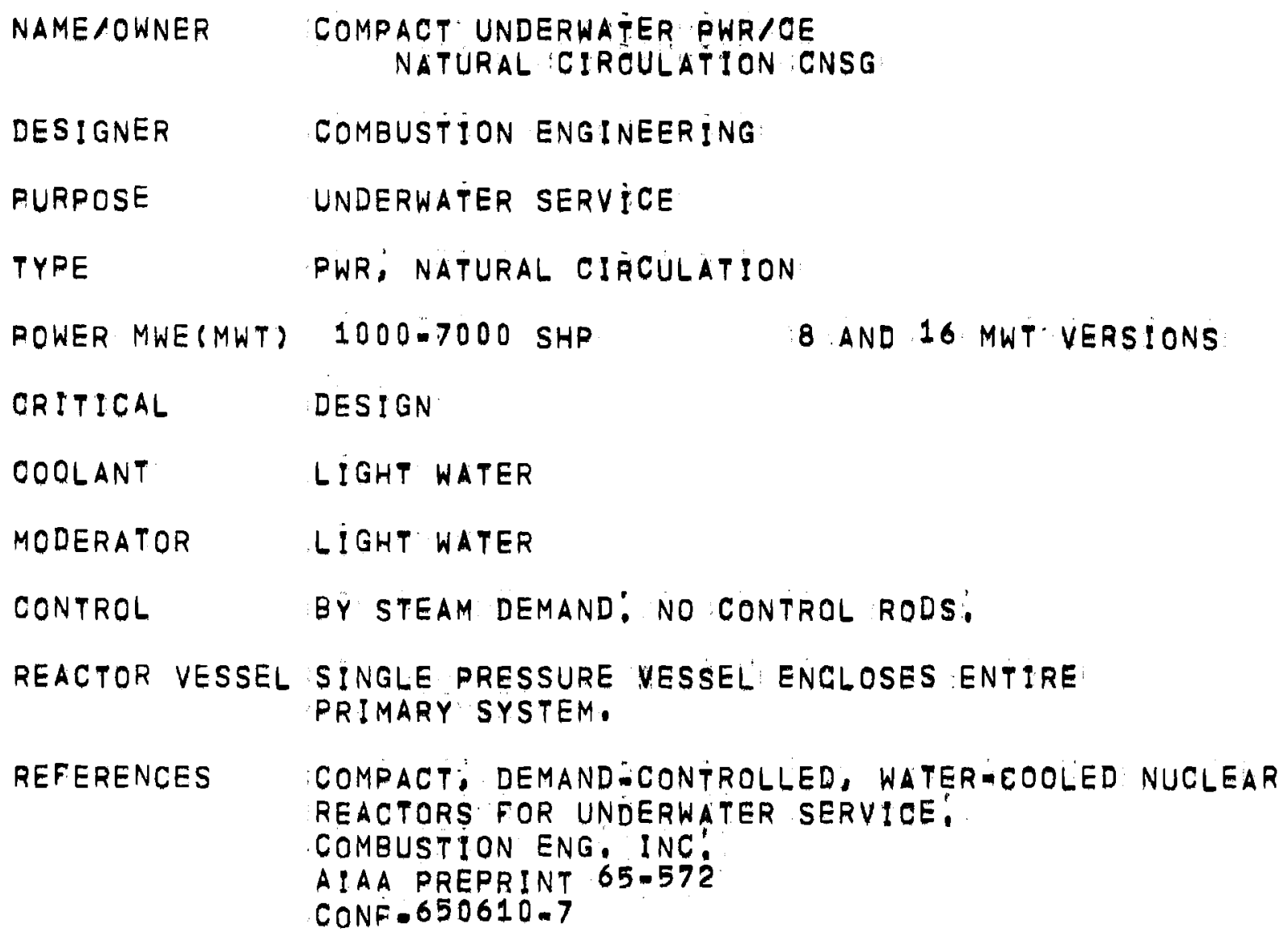




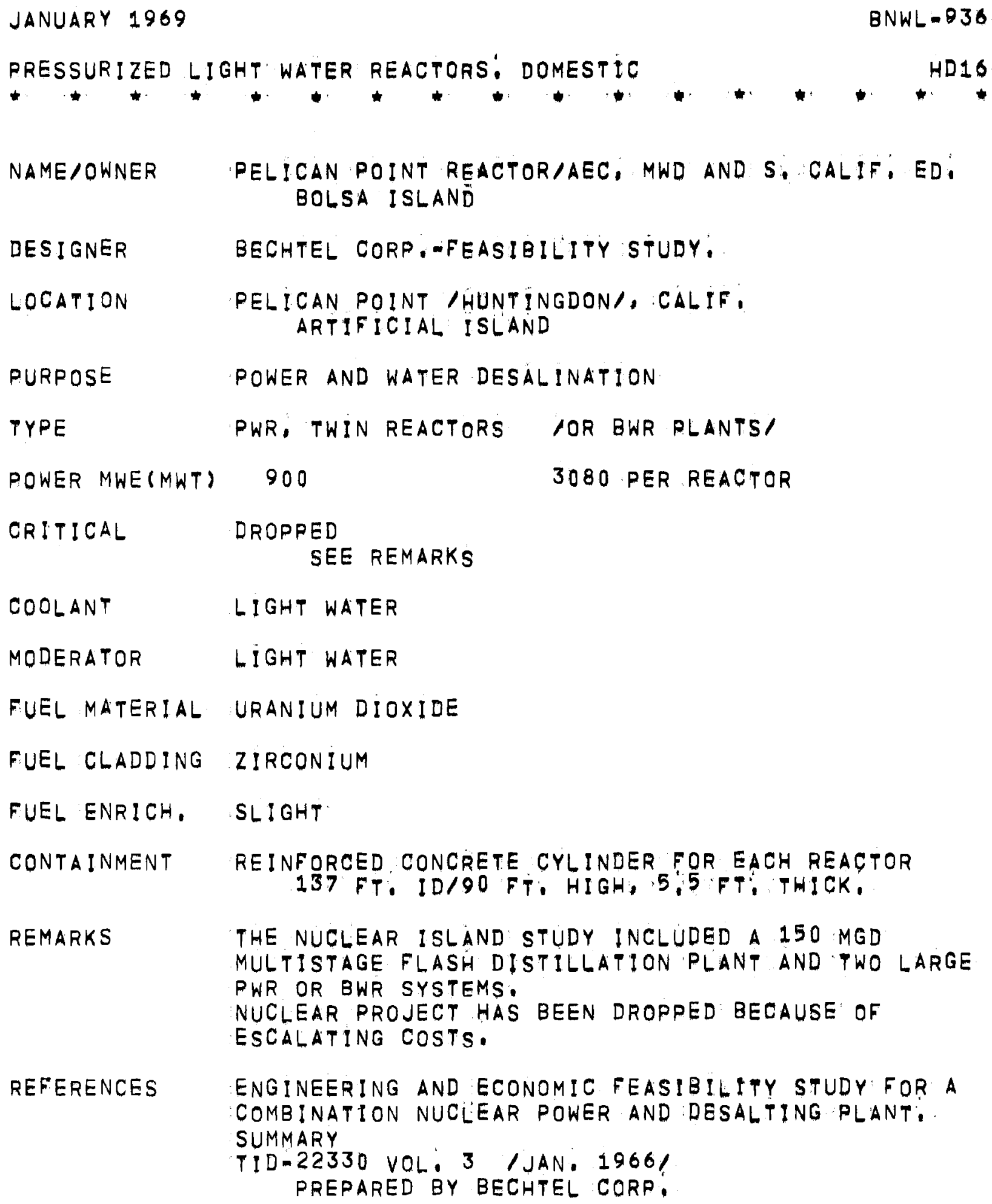


JANUARY 1969

$B N W L=936$

PRESSURIZED LIGHT WATER REACTORS: DOMESTIC

NAME/OWNER ONCE-THROUGH PWR/BAW

DESIGNER BABCOCK AND WILCOX

PURPOSE POWER

TYPE

PWR, ONCE-THROUGH STEAM=GENERATOR, TWO-LOOP SYSTEM

POWER MWE(MWT) 750 2300

CRITICAL

DESIGN

COQLANT

LIGHT WATER

MODERATOR

LIGHT WATER

FUEL MATERIAL

URANIUM DIOXIDE

FUEL GEOMETRY PINS

FUEL CLADDING

ZIRCALOY 4

FUEL ENRICH

2:55 PER CENT U-235; AVE:

FUEL ASSEMBLY

177 ASSEMBLIES/CORE

CONTROL

SILVER-INDIUM-CADMIUM RODS

COOLANT TEMP; $565,5 \%$

COOLANT PRESS: 2185,0 PSIG

REMARKS PRELIMINARY SAFETY REVIEW BY AEC REQUESTED

REFERENCES

NUCLEAR INDUSTRŸ JAN, 1966 P, 9

NEWS RELEASE 


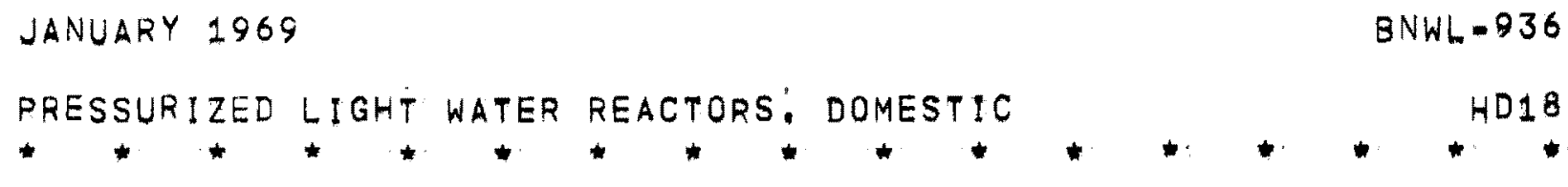

NAME/OWNER ISRAELI PLANT STUDYIUS-ISRAEL JOINT BOARD PWR

DESIGNER

LOCATION

PURPOSE

TYPE

POWER MWE (MWT)

CRITICAL

COOLANT

MODERATOR

FUEL MATERIAL

FUEL GEOMETRY

FUEL CLADDING

FUEL ASSEMBLY

CONTAINMENT

REMARKS

REFERENCES
KAISER ENG./CATALYTIC CONSTRUCTION

NITZANIM OR NAHAL SOREO INEVE RUB!N/, ISRAEL PREFERRED SITE NITZAMIN. NEAR ASHDOD.

POWER AND DESALINATION

PWR, INDIRECT CYCLE; 2-LOOP SYSTEM, 1500

ENGINEERING, ECONOMIC FEASIBILITY STUDY, LIGHT WATER

LIGHT WATER

URANIUM DIOXIDE PELLETS

RODS

\section{ZIRCALOY TUBES /THIN/}

3 CONCENTRIC CORE REGIONS

STEEL SPHERE OR CONCRETE CYLINDER, PRESSURE=TIGHT REINFORCED CONCRETE; INNER STEEL LINER, PRESTRESSED CONCRETE, STEEL LINER,

DESALTING PLANT CAPACITY IS 100 MGD. PLANT CONSISTS OF THE REACTOR, TURBINE GENERATOR, FOUR MULTISTAGE FLASH EVAPORATOR UNITS. A REFERENCE BWR PLANT IS ALSO CONSIDERED; FUEL IS MODULAR: $7 \times 7$ ROD BUNDLE IN ZIRCALOY-4 CHANNEL. FUEL PELLETS OF URANIUM DIOXIDE IN ZIRCALOY-2 CLAD RODS.

ENGINEERING FEASIBILITY AND ECONOMIC STUDY FOR DUAL-PURPOSE POWER-WATER DESALTING PLANT, KAISER ENG., CATALYTIC CONSTRUCTION CO. REPORT NO, 66-1-RE /JAN, 1966/ 
JANUARY 1969

BNWL: $=936$

PRESSURIZED LIGHT WATER REACTORS: DOMESTIC

NAME /OWNER

DES IGNER

OPERATOR

LOCATION

PURPOSE

TYPE

POWER MWE(MWT)

CRITICAL

COOLANT

MODERATOR

FUEL MATER!AL

FUEL GEOMETRY

FUEL CLADDING

COQLANT TEMP,

CONTAINMENT

REMARKS

REFERENCES
N-REACTOR/AEC; WPPSS

GENERAL ELECTRIC COMPANY

DOUGLAS UNITED NUCLEAR /DUN/

RICHLAND, WASHINGTON COLUMBIA RIVER

PLUTONIUM AND POWER PRODUCTION,

PWR, PRESSURE TUBE

$\begin{array}{ll}800 & 4000 \\ \text { POWER } & \text { ONLY OPERATION }\end{array}$

APRIL 1966. FULL' POWER DEC, $1966^{\circ}$

LIGHT WATER

GRAPHITE

URANIUM METAL: FOR PHASE 3, URANIUM DIOXIDE

RODS, COMEXTRUDED

ZIRCALOY $=2$

INLET $469 \mathrm{~F}$

OUTLET $572 \mathrm{~F}$

SERARATE BUILDINGS FOR REACTOR AND HEAT EXCHANGERS CONFINEMENT-VOLUME WALLS ARE REINFORCED CONCRETE COATED WITH AN ELASTOMER. FOG-SPRAY SYSTEM. AND VENT STACKS /PRESSURE-RELEASE/.

ELECTRIC GENERATING EQUIPMENT BUILT BY THE WASHINGTON PUBLIC POWER SUPPLY SYSTEM /WPPSS/ HAS CAPACITY OF 800 MWE: N-REACTOR PRODUCED ELECTRICITY IN APRIL 1966 WITH FIRST GENERATOR ON-LINE AT 200 MWE, FULL POWER 800 MWE DEC, 1966:

THE NEW PRODUCTION REACTOR, $H W=S A-1755 / 1959 /$

UTILITY PROPOSAL' FOR POWER PLANT ADDITION TO HANFORD NEW PRODUCTION REACTOR. UNITED STATES CONGRESS JOINT COMMITTEE ON ATOMIC ENERGY, /JULY 1962 / 
JANUARY 1969

BNWL $=936$

PRESSURIZED LIGHT WATER REACTORS: DOMESTIC

BASIS, OF DESIGN: HANFORD NEW PRODUCTION REACTOR INPR/:

WJ DOWIS

$H W=S A=2981 / A P R I L 19,1963 /$

NUCLEONICS WEEK APRIL 21: 1966

NEWS RELEASE

ANNUAL REPORT TO CONGRESS OF THE ATOMIC ENERGY

COMMISSION FOR 1965: /JAN, 1966/P, 76-77 
JANUARY 1969

BNWL $=936$

PRESSURIZED LIGHT WATER REACTORS; DOMESTIC

HD20

NAME /OWNER

PROCESS STEAM REACTOR/MARTIN

DES IGNER

MARTIN-MARIETTA CORPORATION

PURPOSE

PROCESS STEAM

TYPE

PWR

POWER MWE (MWT)

0

45

CRITICAL

CONCEPTUAL DESIGN

COOLANT

LIGHT WATER

MODERATOR

LIGHT WATER

FUEL MATER IAL

URANIUM DIOXIDE PELLET 0.252 IN: DIA.

FUEL GEOMETRY ROD $0: 280$ IN: OD.14:5 FT: LONG

FUEL CLADDING ALUMINUM ALLOY 0.012 IN. THICK /LEAD-BISMUTH BOND/

FUEL ENRICH, 2:5 PER CENT U-235

FUEL ASSEMELY 19 BUNDLES CONTAINING 7250 RODS

FUEL CHARGE $3437^{\circ} \mathrm{KG}$ : URANIUM DIOXIDE

COOLANT TEMP, INLET 376 F OUTLET $430 \mathrm{~F}$

COOLANT PRESS, 890 PSIA

REACTOR VESSEL SS-LINED CARBON STEEL VESSEL 14 FT, 5 IN, HIGH 52 IN. ID. CYLINDER WITH HEMISPHERICAL ENDS.

CONTAINMENT

REMARKS

REFERENCES
ALUMINUMMLINED CONCRETE PIT FILLED WITH WATER, STEEL CONTAINMENT VESSEL,

COOLANT ENTERS AT BOTTOM OF CORE, FLOWS UPWARD

THROUGH OUTER 12 FUEL-ROD BUNDLES, DOWNWARD

THROUGH ANNULUS BETWEEN PRESSURE VESSEL WALL AND

INNERMMOST THERMAL' SHIELD; ON SECOND PASS COOLANT

FLOW IS THROUGH CENTRAL OF FUEL-ROD BUNDLES AND

OUT OF PRESSURE VESSEL. MICHIGAN CHEMICAL CO.

HAD PLANNED AN INSTALLATION: NO RECENT

INFORMATION.

CONCEPTUAL DESIGN FOR A PROCESS STEAM REACTOR. 


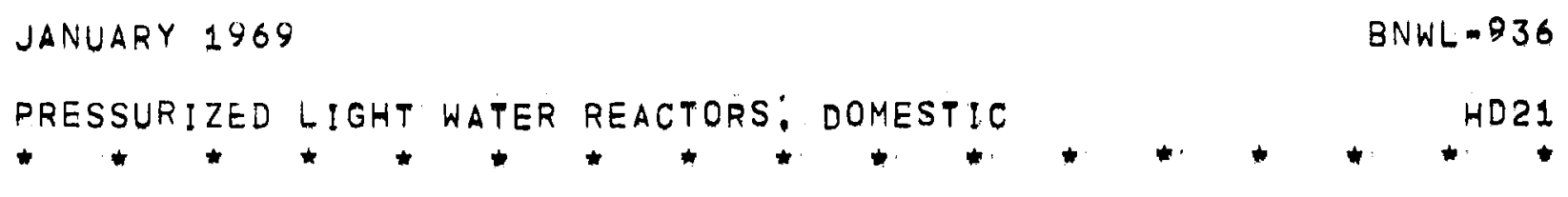

NAME/OWNER PM-1 /PORTABLE MEDIUM-POWER-1/DOD SUNDANCE

DESIGNER MARTIN-MARIETTA CORPORATION

OPERATOR MARTINMMARIETTA CORPORATION

LOCATION SUNDANCE, WYOMING

PURPOSE POWER AND SPACE HEAT

TYPE PWR, PACKAGED REACTOR

POWER MWE(MWT) 199

CRITICAL FEB: 1962 CLOSED DOWN IN APRIL 1968,

COQLANT LIGHT WATER

MODERATOR LIGHT WATER

FUEL MATERIAL URANIUM DIOXIDE-SS CERMET 0,0285 IN. MEAT THICKNESS

FUEL GEOMETRY TUBE 0.506 IN: OD:10.417 IN: ID./30 IN, ACT: LENGTH

FUEL CLADDING SS 0.0085 IN. THICK, INNER AND OUTER

FUEL ENRICH, 93,3 PER CENT U:235

FUEL ASSEMBLY 121-ROD BUNDLE /PERIPHERALI

15-ROD BUNDLE /CENTRAL,

BUNDLES INCLUDE FUEL, POISON, AND DUMMY ELEMENTS.

741 RODS/CORE

6 PERIPHERAL BUNDLES AND 1 CENTRAL

FUEL CHARGE $29.14 \mathrm{KG}, U=235$

BURNUP (REFUEL) 9 KG, U-235 2 YEARS

NEUTRON FLUX THERMAL AVE, 1 X10 E*13

CONTROL CONTROL RODS WITH EUROPIUM TITANATE AS ABSORBER MATERIAL. LUMPED BURNABLE POISON ELEMENTS,

PARTIAL AND FULL LENGTH 


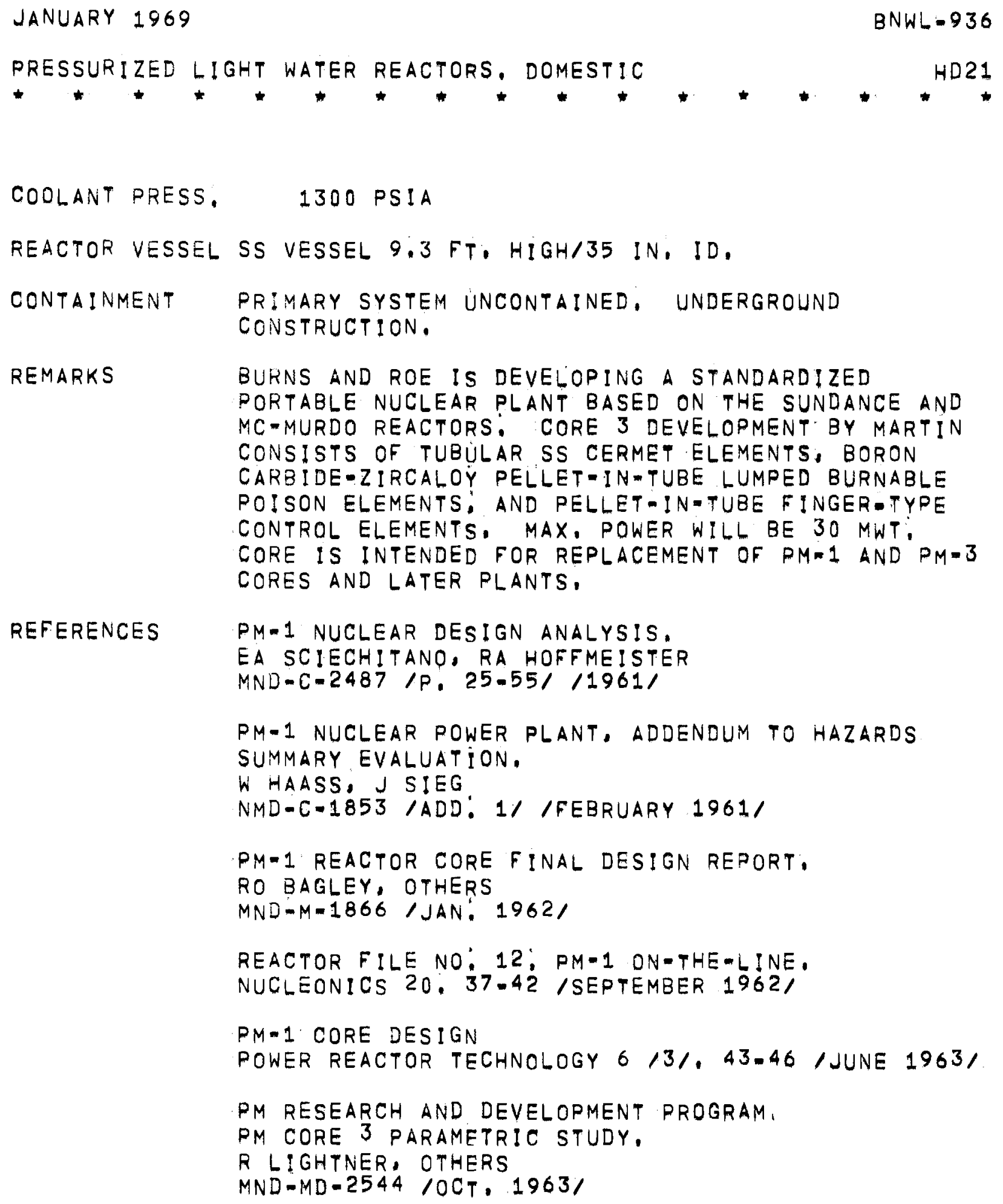


JANUARY 1969

BNWL -936

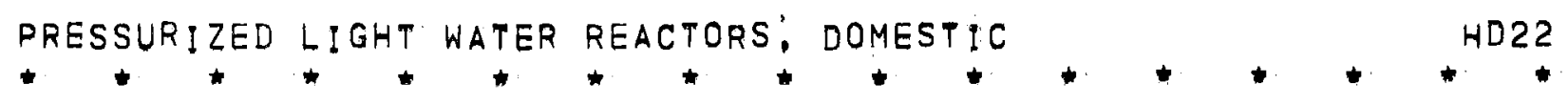

NAME/OWNER

PM-3A/US NAVY

MGMURDO SOUND; ANTARCTIC

DESIGNER MARTIN-MARIETTA CORPORATION

OPERATOR US NAVY

LOCATION MC MURDO SOUND. ANTARCTICA

PURPOSE POWER AND SPACE HEAT.

TYPE PWR, PACKAGED REACTOR

POWER MWE (MWT)

1,5

9,3

CRITICAL

MARCH 1962. REFUELING JANUARY 1965

COOLANT

LIGHT WATER

MODERATOR

LIGHT WATER

FUEL MATERIAL URANIUM DIOXIDE-SS CERMET

FUEL GEOMETRY TUBE 0,506 IN, OD:/0,417 IN: ID:130 IN, ACT. LENGTH

FUEL CLADDING SS 0,0085 IN, THICK: INNER AND OUTER

FUEL ENRICH, 93,3 PER CENT U-235

FUEL ASSEMBLY 121-ROD BUNDLE /PERIPHERAL/

15-ROD BUNDLE /CENTRAL/

1 CENTRAL BUNDLE

6 PERIPHERAL BUNDLES

BUNDLES. INCLUDE POISON AND DUMMY ELEMENTS.

FUEL CHARGE $29.14 \mathrm{KG}, \mathrm{K}=235$

NEUTRON FLUX THERMAL AVE, $1 \times 10 \cdot E+13$

CONTROL BURNABLE POISON ELEMENTS, UNCLAD BORON-SS

CYLINDERS, YMSHAPED SS CLAD CERMET OF EUROPIUM-SS CONTROL ELEMENT

COOLANT TEMP, $463 \mathrm{~F}$

COOLANT PRESS, $\quad 1300$ PSIA

REACTOR VESSEL SS CYLINDRICAL VESSEL

REMARKS INSTALLATION DECEMBER 1961 AT BASE, AND STARTUP 


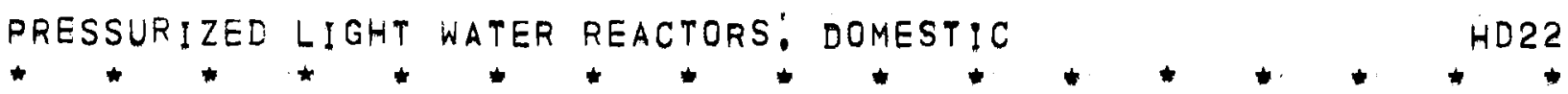

IN MARCH, 1962, CORE DESIGN FOR REPLACEMENT HAS BEEN DEVELOPED BY MARTIN, SEE PM-1, DISTILLATION PLANT OPERATED BY STEAM FROM THE REACTOR WAS TESTED SUCCESSFULLY IN FEB, 1966. THE PLANT BEGAN FULL-TIME OPERATION IN FALL OF 1966. EVAPORATOR PLANT CAN PRODUCE 11 GAL. FRESH WATER PER MINUTE AND STORE 55,000 GAL, SHUT-DOWN LATE SUMMER 1967 FOR REFUELING WITH ROD-TYPE CORE-3: LOW-ENRI CHMENT CORE-4, DUE AT MCMURDO DEC, 1968;, WILL SERVE AS BACK-UP CORE UNTIL NECESSARY REFUELING IN 1969-7D. REDESIGNED RADIOACTIVE WASTE DISPOSAL SYSTEM IS ALSO ON TAP. REACTOR MAY BE REPLACED BY A NEW 5.MWE PLANT IN 1975:

REFERENCES MARTIN DESIGN REPORT-ICE CAP NUCLEAR POWER PLANT, VOL: 1 AND 2 . RJ AKIN

MND-MPR-1581/VOL 1 AND 2//1959/

THE PM=3A ANTARCTIC REACTOR, U.S: AEC, U.S, NAVY BROCHURE, DISTRIBUTED 1961;

PM- 3 A NUCLEAR POWER PLANT HAZARDS SUMMARY REPQRT, SAFETY EVALUATION:

$T$ DOBRY

MND-M3A-2496-2 /ADD/ /NOVEMBER 1951/

DCEAN SCIENCE NEWS MARCH 4, 1960 \%, 3

NEWS RELEASE

PM=3A NUCLEAR POWER PLANT HAZARDS SUMMARY REPORT. DESCRIPTION OF PLANT EXPERIENCE AND CHANGES. CH FOX, OTHERS

MND-3315-1 /JAN. $1964 /$

NUCLEONICS WEEK APRIL 6, 1967 P:, 2

NEWS RELEASE 
PRESSURIZED LIGHT WATER REACTORS: DOMESTIC

NAME/OWNER

$D E S$ \GNER

OPERATOR

LOCATION

PURPOSE

TYPE

POWER MWE (MWT)

CRITICAL

COOLANT

MODERATOR

FUEL MATER!AL

FUEL GEOMETRY

FUEL CLADDING

FUEL ENRICH.

FUEL ASSEMBLY

COOLANT TEMP.

REACTOR VESSEL
FBR /FLUIDIZED BED REACTOR//AEC

MARTIN-MARIETTA CORPORATION

CRITICAL EXPERIMENT-MARTIN

CRITICAL' EXPERIMENT; MIDDLE RIVER, MD,

POWER EXPER IMENT

PWR, FLUIDIZED BED

$$
100
$$

400

FEASIBILITY STUDY: CRITICAL EXPERIMENT: SEE REMARKS:

LIGHT WATER

LIGHT WATER

URANIUM DIOXIDE CYLINDRICAL PELLETS 205 MILS DIA.

PEBBLES /CYLINDERS/

UNCLAD OR COATED. SEE REMARKS

$1: 6$ PER CENT U-235

FLUIDIZED BED OF FUEL PEBBLES.'

$550 F$

\section{EACH END.}

REMARKS
THE UNCLAD FUEL PELLETS ARE PLACED IN THE TANK AND WATER IS PUMPED IN FROM THE BOTTOM, VELOCITY OF THE FLOW SUSPENDS THE PELLETS, PELLETS WERE PREPARED BY COLD COMPACTING FOLLOWED BY SINTERING. OR BY THE CAPILLARY-DROP METHOD, EXPERIMENTAL THIN NICKEL CLADDING BY THE ELECTROLESS NICKEL PLATING METHOD WAS INVESTIGATED, CRITICAL EXPERIMENT FAILED TO GO CRITICAL AFTER FULL LOADING IN APRIL, 1962 BECAUSE OF ABRASION OF THE UNCLAD FUEL, 4 PER CENT ENRICHED PELLETS WERE ADDED TO THE REPLACEMENT FUEL: PROPOSAL FOR REDESIGNED FUEL ELEMENTS SPECIFIED URANIUM MOLYBDENUM ALLOY PELLETS ENRICHED TO 6 PER CENT' AND COATED WITH SS, NIOBIUM OR ALUMINUM, STUDIES 


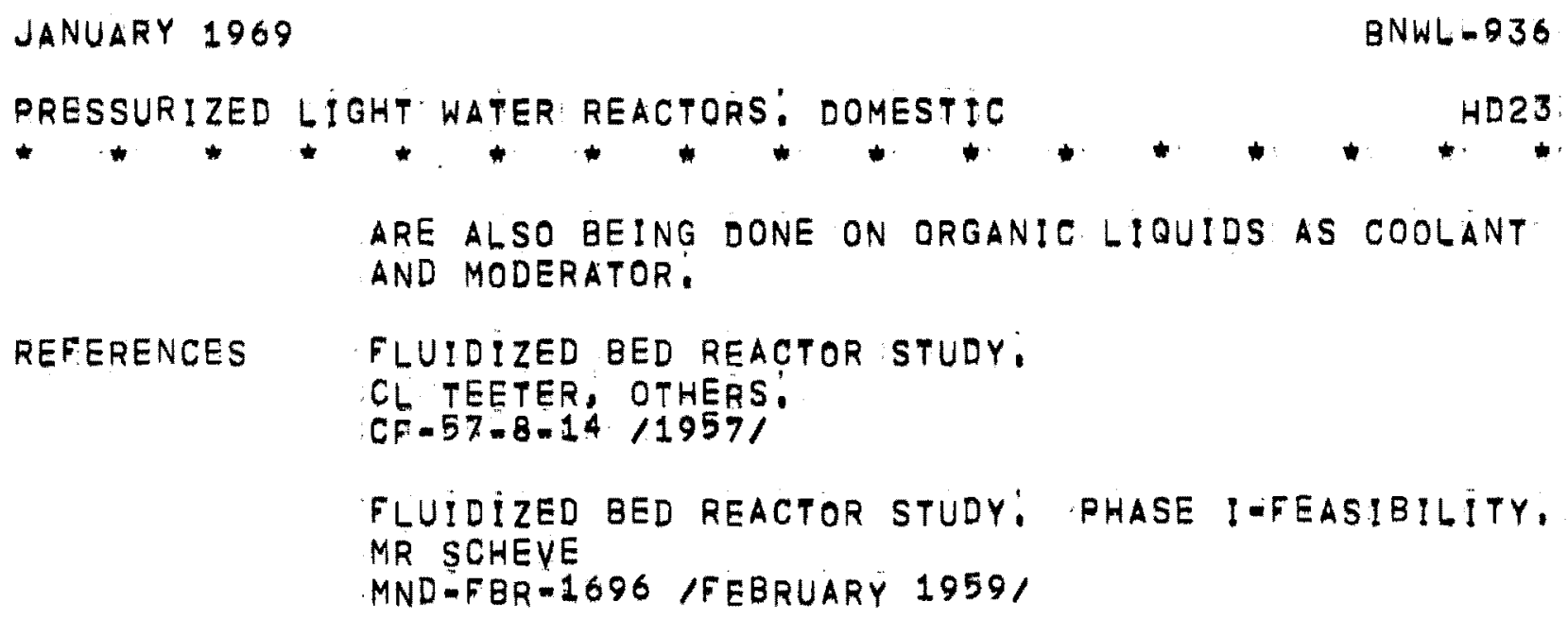


JANUARY 1969

$B N W L=936$

PRESSURIZED LIGHT WATER REACTORS: DOMESTIC

NAME/OWNER

DES IGNER

OPERATOR

LOCATION

PURPOSE

TYPE

POWER MWE (MWT)

CRITICAL

COOLANT

MODERATOR

FUEL MATERIAL

FUEL GEOMETRY

FUEL CLADDING
MH-1A /MOBILE HIGH=POWER IA//DOD

MARTIN-MARIETTA CORPORATION

US ARMY

TEST INSTALLATION FT', BELVOIR, VA:

EMERGENCY POWER FOR MILITARY INSTALLATIONS,

COASTAL CITIES. BARGEMMOUNTED.

PWR, BARGE-MOUNTED

10

45

SEE REMARKS

JANUARY 1967

FULL=POWER RUN APRIL-MAY 1967 , SEE REMARKS

LIGHT WATER

LIGHT WATER

URANIUM DIOXIDE PELLETS 0:4565 IN, DIA. ROD, ACTIVE LENGTH 36 IN.

SS TUBES WITH BORON ADDED, 0,023 IN, WALL THICKNESS

FUEL ENRICH, ZONE ENRICHMENT, OUTER 5,4 AND INNER 5,O PER CENT

FUEL ASSEMBLY 104:ROD BUNDLE

32 BUNDLES/CORE WITH 12 INTERSPACED CONTROL RODS

BURNUP (REFUEL) FUEL SHUFFLING

CONTROL

RODS, BORON-10 AND SS

COQLANT TEMP.

$490 \mathrm{~F}$

COQLANT PRESS,

1600 PSI

REACTOR VESSEL SS-LINED STEEL VESSEL 80 IN, ID.

REMARKS

THE REACTOR HOUSING IS A HULL SECTION THAT

REPLACED THE MIO-BODY OF A WORLD WAR-2 LIBERTY 
RRESSURIZED LIGHT WATER REACTORS: DOMESTIC * * * * * HD24.

SHIP CHARLES H. CUGLE: RENAMED STURGIS, THE PLANT WILL BE AVAILABLE TO BE TOWED TO ANY PORT FOR MILITARY OPERATIONS OR IN DISASTER. AREAS. REACTOR OUTPUT IS 10 MWE $\triangle T$ TO CYCLES; OR $8083 \mathrm{KW}$ AT 50 CYCLES. PROVISION FOR A DESALINATION PLANT' IS INCLUDED: STURGIS WAS MOVED TO THE CANAL ZONE IN AUG, 1968 WHERE IT WILL TEMPORARILY AUGMENT THE GATUN HYDRO STATION.

REFERENCES THE ARMY NUCLEAR POWER PROGRAM, ITS REACTORS; AND THEIR APPLICATION TO THE LESSIDEVELOPED AREAS OF THE WORLD: SYMPOSIUM ON SMALL AND MEDIUM POWER REACTORS. VIENNA, 1960 ,

PAPER SMPR-51/VIENNA: 1960 /

MECHANICAL DESIGN OF THE MHEIA REACTORS, R PICKERING AMERICAN NUGLEAR SOC, TRANS, 5/2\%, 439.4O INOV. 1962 /

DESCRIPTION: APPLICATION, AND SITING OF THE STURGIS /MH-1A/FLOATING NUCLEAR POWER PLANT: RM BUNKER

TRANS: AMERICAN NUCLEAR SDC; 8, 152 /JUNE 1965 /

NUCLEAR INDUSTY FEB: 1967 P: 38 NEWS RELEASE 
JANUARY 1969

$B N W L-936$

PRESSURIZED LIGHT WATER REACTORS: DOMESTIC

HD25

NAME /OWNER

BROOKWOOD NUCLEAR STATION/ROCHESTER GAS/ELECTRIC GINNA STATION

DESIGNER

WESTINGHOUSE ELECTRIC CORP:

OPERATOR

ROCHESTER GAS - ELECTRIC CORPORATION

LOCATION

BROOKWOOD; LAKE ONTARIO, N.Y,

NORTH OF ROCHESTER

PURPOSE

POWER

TYPE

PWR

POWER MWE(MWT) 450

1300

CRITICAL

CONSTRUCTION, TARGET 1969

COQLANT

LIGHT WATER

MODERATOR

LIGHT WATER

FUEL MATERIAL URANIUM DIOXIDE PELLETS 0,360 IN: DIAMI10:600 IN. FUEL GEOMETRY ROD 0.422 IN: OD:

FUEL CLADDING ZIRCALOY 0,0243 IN. THICK

FUEL ASSEMBLY 179-ROD BUNDLE, 14X14 ASSEMBLIES ARE CANILESS, WITH SPRING=CLIP GRID 120 ASSEMBLIES/CORE

CONTROL

ROD CLUSTER CONTROL

COOLANT TEMP, INLET 556 F

COOLANT PRESS:

2250 PSIA

CONTAINMENT

PRESTRESSED CONCRETE CYLINDER $105 \mathrm{FT}$, DIAM, HEMISPHERICAL DOME,HEIGHT 99 FT., WALL 3 FT, 6 IN. DOME 2 FT: 6 IN: THICK. STEEL REINFORCED CONCRETE SHELL, VESSEL LINED WITH $3 / 8$ IN, STEEL PLATE,

REMARKS

CONTRACT AWARD SEPT: 1965

CONSTRUCTION PERMIT APRIL 1966

REF.ERENCES

NEWS RELEASES

BROOKWOOD NUCLEAR STATION UNIT NO, 1

PRELIMINARY FACILITY DESCRIPTION AND SAFETY 
PRESSURIZED LIGHT WATER REACTORS: DOMESTIC $* * * * * * * * *$

ANALYSIS REPORT:

ROCHESTER GAS AND ELECTRIC CO.

NP=15908 VOL. 1 /ND/

ROBERT EMMETT GINNA NUCLEAR POWER PLANT, UNIT 1

FINAL FACILITY DESCRIPTION AND SAFETY ANALYSIS REPORT

ROCHESTER GAS - ELECTRIC CO

DOCKET 50.244 VOL. $1=2$ 
JANUARY 1969

$B N W L-936$

PRESSURIZED LIGHT WATER REACTORS: DOMESTIC

HD26

NAME /OWNER

INDIAN POINT 2 AND 3/CONSQLIDATED EDISON

DES I GNER

WESTINGHOUSE ELECTRIC CORP.

OPERATOR

CONSOLIDATED EDISON CO.

LOCATION

INDIAN POINT. N:Y:

HUDSON RIVER

PURPOSE

POWER

TYPE

PWR, A-LOOP SYSTEM

TWO-REACTOR ADDITION

POWER MWE (MWT)

No.2, 873

$\mathrm{NO}, 3, \quad 965$

2758

3025

CRITICAL

NO.2, 1969 /CONSTRUCTION/

No.3, 1971

COQLANT

LIGHT WATER

MODERATOR

LIGHT WATER

FUEL MATERIAL URANIUM DIOXIDE PELLETS 0.3669 IN, DIAM.10.600 IN.

FUEL GEOMETRY RODS 0.422 IN, OD.: ACT, LENGTH 144 IN.

FUEL CLADDING ZIRCALOY, 0.0243 IN. THICK

FUEL ENRICH, REGIONAL, LOW

FUEL ASSEMBLY 204-ROD BUNDLE, 15X15, 21 RODS OMITTED

FOR RCC ELEMENTS, IN=CORE INSTRUMENTATION, 193 ASSEMBLIES/CORE

FUEL CHARGE 215;319 POUNDS URANIUM DIOXIDE

BURNUP(REFUEL) 12,000 MWD/MTU

CONTROL ROD-CLUSTER CONTROL, UNCANNED ASSEMBLIES, CHEMICAL: SHIM

COOLANT TEMP, INLET $549.7 \mathrm{~F}$ OUTLET $607.7 \mathrm{~F}$

COQLANT PRESS; OPERATING PRESSURE 2235 PSIG;

REACTOR VESSEL 173:IN, ID:142 FT:4 IN: HIGH:CLAD SS CLAD $5 / 32$ IN: THICK

OONTAINMENT CONCRETE CYLINDER: HEMISPHERICAL DOME; WELDED 
JANUARY 1969

BNWL-936

PRESSURIZED LIOHT WATER REACTORS: DOMESTIC

STEEL LINER 1/4 IN, THICK, BUILDING 135 FT; ID, WALL THICKNESS 4 FT: 6 IN.

REMARKS

SITE ADJACENT TO INDIAN POINT-1 SELECTED

A CONTRACT HAS BEEN SIGNED WITH WESTINGHOUSE FOR

A THIRD,REACTOR AT SITE. REACTOR CAPACITY WILL BE 965. MWE; TARGET 1971 .

REFERENCES INDIAN POINT NUCLEAR GENERATING UNIT NO, 2, PRELIMINARY SAFETY ANALYSIS REPORT, PLANT DESIGN DESCRIPTION AND SAFETY ANALYSIS, CONSOLIDATED EDISON CO.

NP-16181 /EXHIBIT B/, VOL: 2, PARTS AmB /1965,

INDIAN POINT NUCLEAR GENERATING STATION NO: 3 -PRELIMINARY SAFETY ANALYSIS REPORT CONSOLIDATED EDISON CO. OF N,Y, INC, DOCKET 50.286 
JANUARY 1969

BNWL-836

PRESSURIZED LIGHT WATER REACTOAS: DOMESTIC

NAME NOWNER

DES I GNER

OPERATOR

LOCATION

PURPOSE

TYPE

POWER MWE (MWT)

CRITICAL

COOLANT

MODERATOR

FUEL MATERIAL

FUEL GEOMETRY

FUEL CLADDING

FUEL ENR ICH,

FUEL ASSEMBLY
SHIPPINGPORT 1/AEC, DUQUESNE

WESTINGHOUSE ELECTRIC CORAORATION

DUQUESNE LIGHT COMPANY

SHIPPINGPORT, PÁ.

'POWER DEMONSTRATION

PWR: SEED AND BLANKET

SEE REMARKS.

$$
\begin{array}{r}
60 \\
150
\end{array}
$$

231 /OORE 11

525 / CORE 21

1957: SEE REMARKS

LIGHT WATER

LIGHT WATER

CORE, URANIUM-ZIRCONIUM CORE 2; URANIUM/ZIRCONIUM OXIDE

BLANKET; URANIUM DIOXIDE PELLETS:

CORE, PLATES /SANDWICH/ CORE 2 , COMRARTMENTED PLATES

CORE, ZIRCALOY-2 0,015 IN, THICK CORE 2 ZIRCALOY:4

BLANKET, ZIRCALOY=2 TUBES 0:027 IN: WALL THICKNESS CORE 2, COMPARTMENTED PLATES

CORE 92:3 PER CENT U. 235

BLANKET' NATURAL

CORE, 15-PLATE ASSEMBLY, 4 ASSEMBLIES JOINED IN A CLUSTER.

BLANKET; $120-R O D$ BUNDLE, 7 BUNDLES STACKED

END-TO-END. CORE HEIGHT 6 FT: 32 SEED CLUSTERS 5,6 IN, 15,6 IN, 113 BLANKET ASSEMBLIES 5,5 IN, 15,5 IN CORE 2, 20 SEED CLUSTERS 7,376 IN, /7,376 IN, $77^{\circ}$ BLANKET ASSEMBLIES 7,376 IN, 17,40 IN. CORE HEIGHT 8 FT?

FUEL CHARGE NATURAL URANIUM FUEL $14: 16$ TONS, ENR, FUEL 165 LBS SPECIFIC POWER CORE $360 \mathrm{KW} / \mathrm{KG} \quad \mathrm{U}-235$ 
JANUARY 1969

BNWL: 936

PRESSURIZED LIGHT WATER REACTORS', DOMESTIC

HD27

BLANKET 2:75 KW/KG URANIUM

BURNUP(REFUEL) SEE REMARKS

NEUTRON FLUX THERMAL AVE, CORE $6 \times 10 E+13$

THERMAL AVE. BLANKET $4 \times 10=13$

FAST AVE, CORE $2 \times 10$ E-14

FAST AVE, BLANKET $1 \times 10 \quad E+14$

CONTROL RODS AND LUMPED BURNABLE POISON IN SEED

COOLANT TEMP: INLET $508 \mathrm{~F}$ OUTLET $538 \mathrm{~F}$

COOLANT PRESS: 2000 PSIA

REACTOR VESSEL CARBON STEEL CYIINDER: SS LINED; 31 FT:19 FT: ID: VESSEL WALLS 8 IN: THICK

CONTAINMENT INTERCONNECTING STEEL CONTAINERS 50 FT: DIA, BY $100-135 \mathrm{FT}$. LONG. CONTAINERS SURROUNDED BY $5.7 \mathrm{FT}$, THICK CONCRETE AND BURIED IN EARTH TO ABOUT $3 / 4$ OF HEIGHT.

REMARKS

SEED 2 WAS INSTALLED IN 1960 AFTER OPERATION OF

5806 FULL POWER HOURS: 32 ENRICHED SEED ELEMENTS AS PARTIAL SEED 3 WERE INSTALLED IN 1962, AND SEED 4 IN NOVEMBER 1962, REACTOR WAS SHUT DOWN IN NOV. 1964 FOR INSTALLLATION OF SECOND CORE AND MODIFICATIONS FOR 150 MWE OPERATION, CORE 2 SEED WILL BE ANNULAR WITH 20 HIGHLY-ENRICHED FUEL CLUSTERS AND 77 BLANKET CLUSTERS: OPERATIONAL TARGET OF JANUARY 1965 HAS BEEN DELAYED ABOUT 3 MONTHS FOR REDESIGN OF HEAT EXCHANGER SUPPORT STRUCTURES, FOUR, CONCEPTUAL DESIGNS HAVE EVOLVED FROM SHIPPINGPORT'.

1. URANIUM CONVERTER-BURNER CORE

2: URANIUM CONVERTER, RECYCLE CORE

3: THORIUM CONVERTER - BURNER CORE

4. THORIUM CONVERTER, RECYCLE CORE,

DUQUESNE HAS AWARDED A CONTRACT TO WESTINGHOUSE

FOR A SECOND REACTOR AT SITE, POWER WILL BE

800 MWE, TARGET 1973 SEE HD55

REFERENCES PROGRESS IN NUCLEAR ENERGY, SERIES II: REACTORS, RA CHARPIE, OTHERS

MC GRAW-HILL BOOK CO.: NEW YORK $/ 1956 /$

SHIPPINGPORT PRESSURIZED WATER REACTOR, AEC 
JANUARY 1969

BNWL-936

RRESSURIZED LIGHT WATER REACTORS: DOMESTIC

HD27

ADDİSONFWESLY. 1958:

SHIPPINGPORT STATION - A PIONEERING PROJECT IN

ATOMIC POWER.

WESTINGHOUSE ENG. ISSUE: MARCH 1958:

OPERATING EXPERIENCE, SHIPPINGPORT:

POWER REACTOR TECHNOLOGY 5, 61:72 /SEPTEMBER 1962/.

SHIPPINGPORT ATOMIC POWER STATION OPERATING

EXPERIENCE, DEVELOPMENTS, AND FUTURE PLANS,

JT STEIFEL, OTHERS

WAPD-TM-390 /APRIL: $1963 /$

SPECIFIC REACTOR TYPES, SEED AND BLANKET:

POWER REACTOR TECHNOLOGY $7,197-200$ /SPRING $1964 /$ 


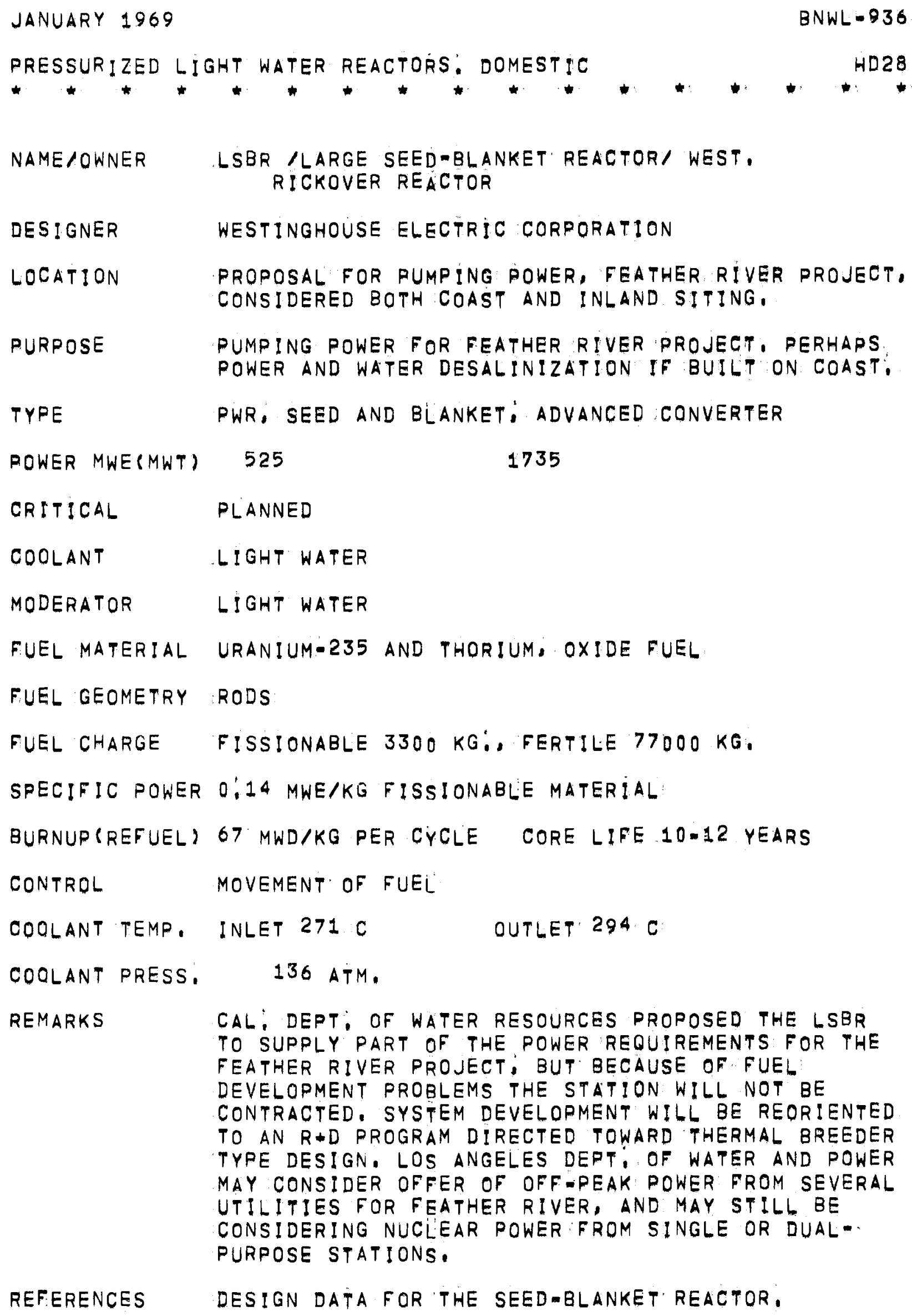

REFERENCES DESIGN DATA FOR THE SEEDMBLANET REACTOR. 
JANUARY 1969 $B N W L=936$

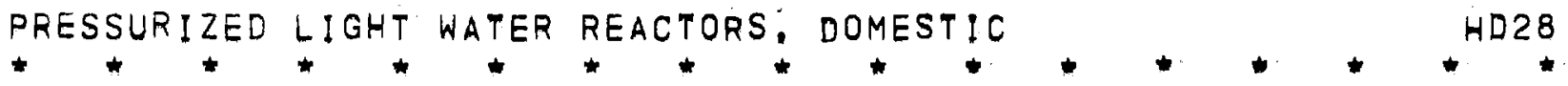
NUCLEAR INDUSTRY 11: 5 /OCT; 1964 /

REPORT ON LARGE POWER REACTOR /LPR/ PROGRAM. BETTIS ATOMIC POWER LAB, WESTINGHOUSE WAPD-LPR-181 /DEC, 1963/

PERFORMANCE POTENTIAL OF LARGE SEED-BLANKET BREEDER REACTORS. WAPD - LSBR-3DO /FEB: $1965 /$

AEC NEWS RELEASE H-285 /DEC, 27; 1965/

SEE ALSO SHIPPINGPORT, 
JANUARY 1969

$B N W L=936$

PRESSURIZED LIGHT WATER REACTORS: DOMESTIC

HD29

\begin{abstract}
NAME/OWNER YANKEE ATOMIC ELECTRIC PLANTIYAEC
DESIGNER WESTINGHOUSE ELECTRIC CORPORATION

OPERATOR YANKEE ATOMIC ELECTRIC COMPANY

LOCATION ROWE, MASSACHUSETTS

DEERF IELD RIVER

PURPOSE POWER DEMONSTRATION

TYPE PWR

$\begin{array}{lll}\text { POWER MWE(MWT) } & 110 & 392 / C O R E 1 / \\ & 134 & 482 / C O R E 2 /\end{array}$

CRITICAL 1960: FULL POWER JAN. 1961:

SEE REMARKS

COOLANT LIGHT WATER

MODERATOR LIGHT WATER

FUEL MATERIAL URANIUM DIOXIDE PELLETS 0,294 IN: DIA:10,60

IN. LONG
\end{abstract}

FUEL GEOMETRY ROD, ACTIVE LENGTH 90 IN:

FUEL CLADDING SS TUBE 0.021 IN. WALL THICKNESS

ZIRCALOY-CLAD FUEL BEING TESTED IN-CORE,

FUEL ENRICH, $3: 4$ PERGENT U:235

CORE 2 4:1 PERCENT

FUEL ASSEMBLY 36-ROD SUBASSEMBLY, $6 \times 6$

9 SUBASSEMBLIES/FUEL ASSEMBLY; OREN TYPE,

76 ASSEMBLIES/CORE

FUEL CHARGE $20,840 \mathrm{KG}$, URANIUM

SPECIFIC POWER 18,8 MWT/MTU

BURNUP(REFUEL) 7830 MWD/MTU SINGLE BATCH

SEE REMARKS

NEUTRON FLUX THERMAL AVE, 2,0X10, E\$13.

CONTROL CRUCIFORM RODS, 24 RODS AND 8 SHIMS

COQLANT TEMP, INLET $499 \mathrm{~F}$ OUTLET.603.F 


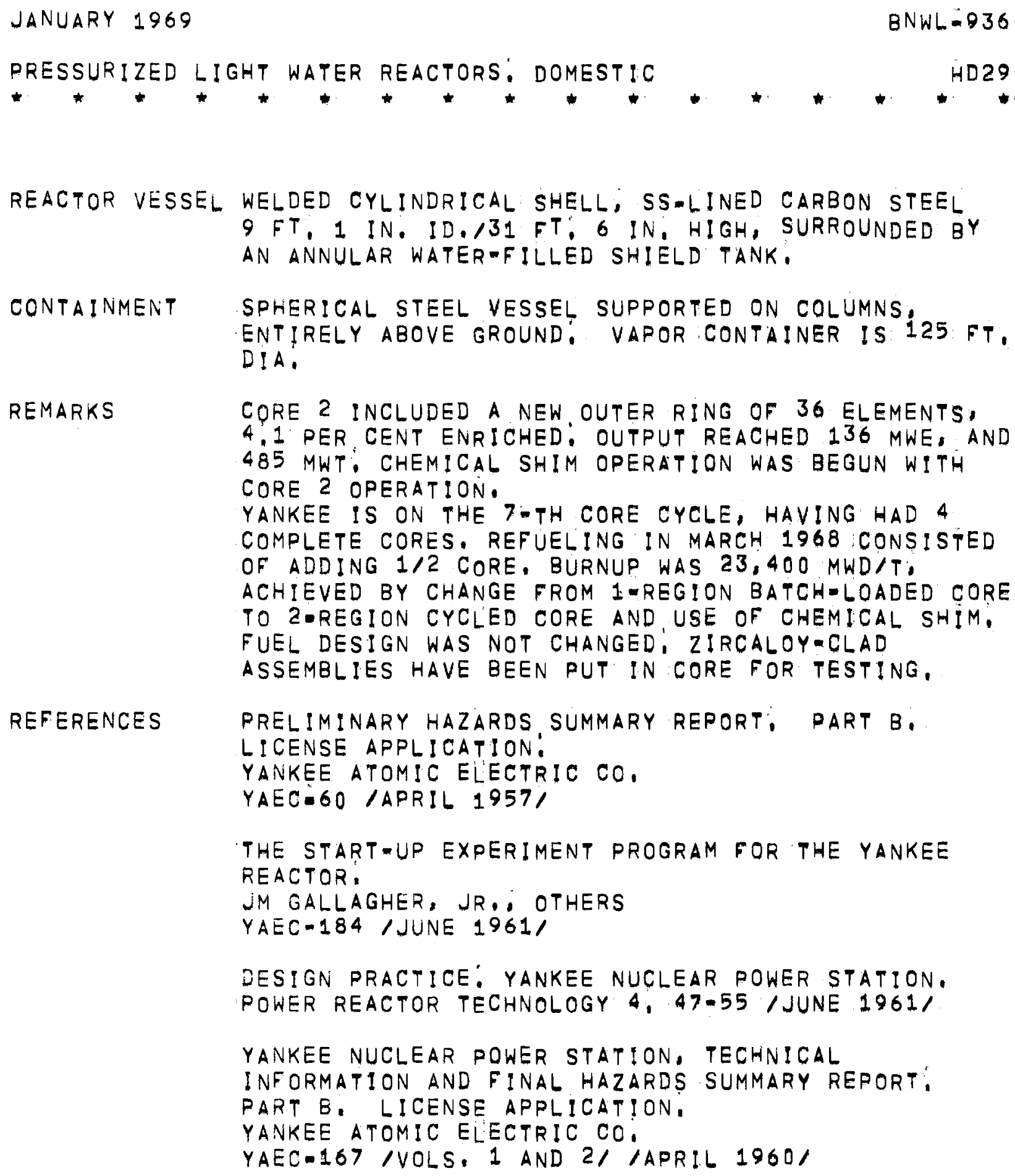


JANUARY 1969

BNWL -936

PRESSURIZED LIGHT WATER REACTORS: DOMESTIC

HD 30

NAME / OWNER

DES IGNER

OPERATOR

LOCATION

PURPOSE

TYPE

POWER MWE (MWT)

CRITICAL

COOLANT

MODERATOR

FUEL MATERIAL

FUEL GEOMETRY

FUEL CLADDING

FUEL ENRICH.

FUEL ASSEMBLY

72-ROD ASSEMBLY: INCORPORATED IN FUEL-FOLLOWER OR BOTTOM SECTION OF EACH CONTROL ROD ASSEMBLY,

21 ASSEMBLIES/CORE

FUEL CHARGE

$1270 \mathrm{KG}$ : URANIUM

SPECIFIC POWER 13,3 KW/FT.

BURNUP (REFUEL) $7300 \mathrm{MWD/T}$

CONTROL

CRUCIFORM RODS, SILVER=INDIUMACADMIUM, FUEL ROD FOLLOWER.

COOLANT TEMP, INLET $520 \mathrm{~F}$

OUTLET 540 F

COQLANT PRESS,

2000 PSI

REACTOR VESSEL MULTILAYER CONSTRUCTION, 20 LAYERS OF 1 IN, STEEL 
PRESSURIZED LIGHT WATER REACTOÄS: DOMESTIC

HD 30

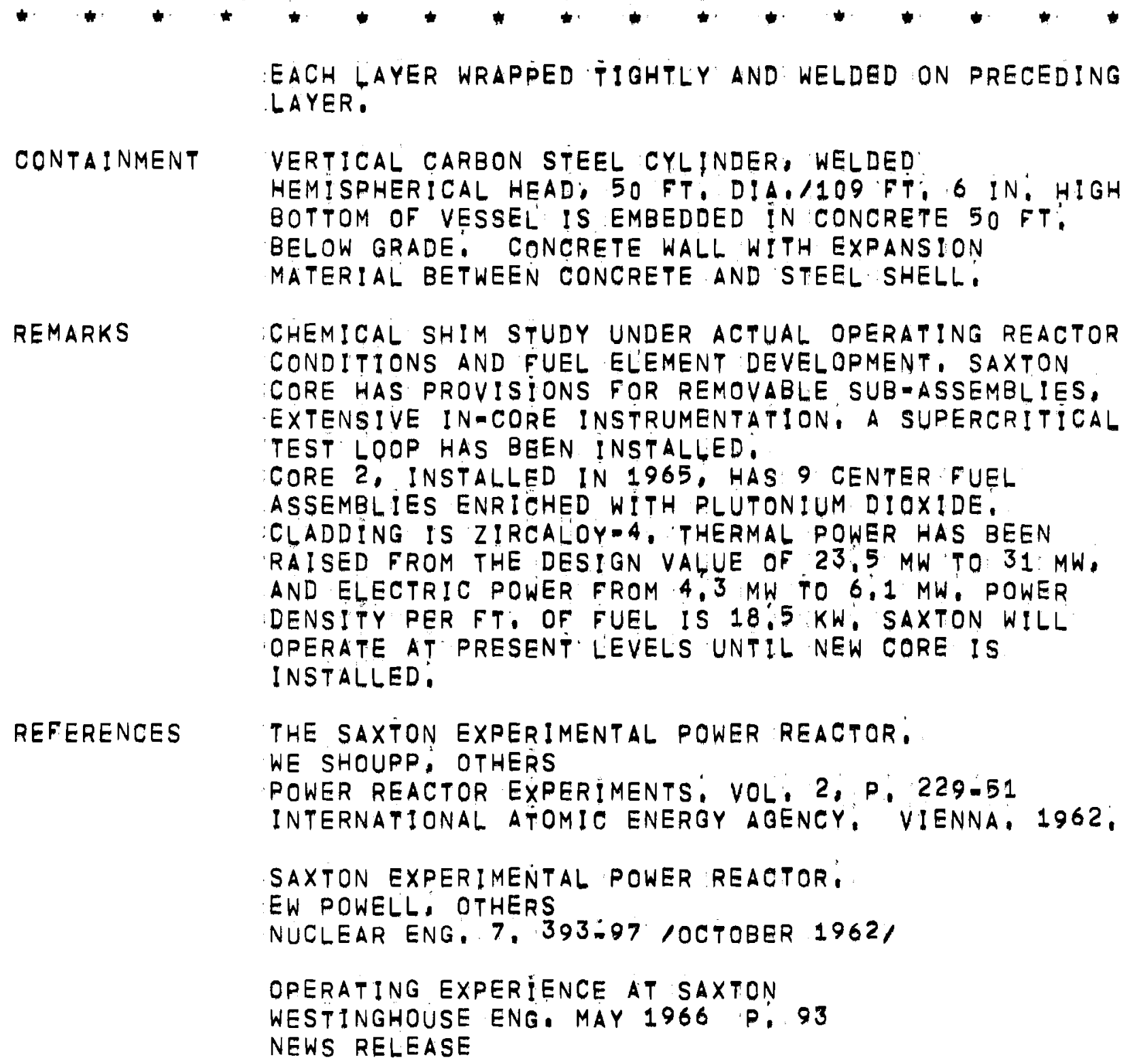


JANUARY 1969

BNWL -936

PRESSURIZED LIGHT' WATER REACTORS: DOMESTIC

HD 31.

$\begin{array}{ll}\text { NAMEIOWNER } & \text { CONNECTICUT YANKEE STATION/CYAPC } \\ \text { DESIGNER } & \text { WESTINGHOUSE ELECTRIC CORAORATION } \\ \text { OPERATOR } & \text { CONECTICUT YANKEE ATOMIC POWER CO } \\ \text { LOCATION } & \text { HADDAM NECK, CONNECTICUT } \\ & \text { CONNECTIOUT RIVER }\end{array}$

RURPOSE POWER: AEC 3RDEROUND ASSISTANCE:

TYPE PWR: 4-LOOP SYSTEM

POWER MWE(MWT) 463

1473

490 GROSS, STRETCH 590 MWE

CRITICAL JULY 1967:

POWER OPERATION JAN, 1968

COOLANT LIGHT WATER

MODERATOR LIGHT WATER

FUEL MATERIAL URANIUM DiOXIDE PELLETS

O:3825 IN: DIAM!, 0,600 IN, LONG

FUEL GEOMETRY ROD 0.422 IN: OD: 121,8 IN', ACTIVE LENGTH

FUEL CLADDING SS TUBES 0.016 IN: WALL THICKNESS

FUEL :ENRICH, 3;00,3,24,3,27 PER CENT U-235; ZONED

FUEL ASSEMBLY $15 \times 15$ ROD BUNDLE $/ 204$ RODS; WITH OMISSIONS/

157 ASSEMBLIE I / CORE

SPRING-CLIP ASSEMBLY, GRID

20 ROD-CLUSTER CONTROL ELEMENTS PER ASSEMBLY

FUEL CHARGE 75,000 KG URANIUM DIOXIDE

BURNUP(REFUEL) 20.000 MWD/MTU 12-14 MONTHS

FIRST CYCLE 14,100 MWD/MTU

NEUTRON FLUX THERMAL AVE, $2.3 \times 10$ E\$13

FAST AVE, $1.6 \times 10 \quad E+14$

CONTROL SS TUBE WITH SILVER-INDIUM-CADMIUM ALLOY, ROD= CLUSTER

SOLUBLE POISON, BORON

COQLANT TEMP, INLET $545 \mathrm{~F}$ OUTLET $583,5 \mathrm{~F}$ 
PRESSURIZED LIGHT WATER REACTORS: DOMESTIC

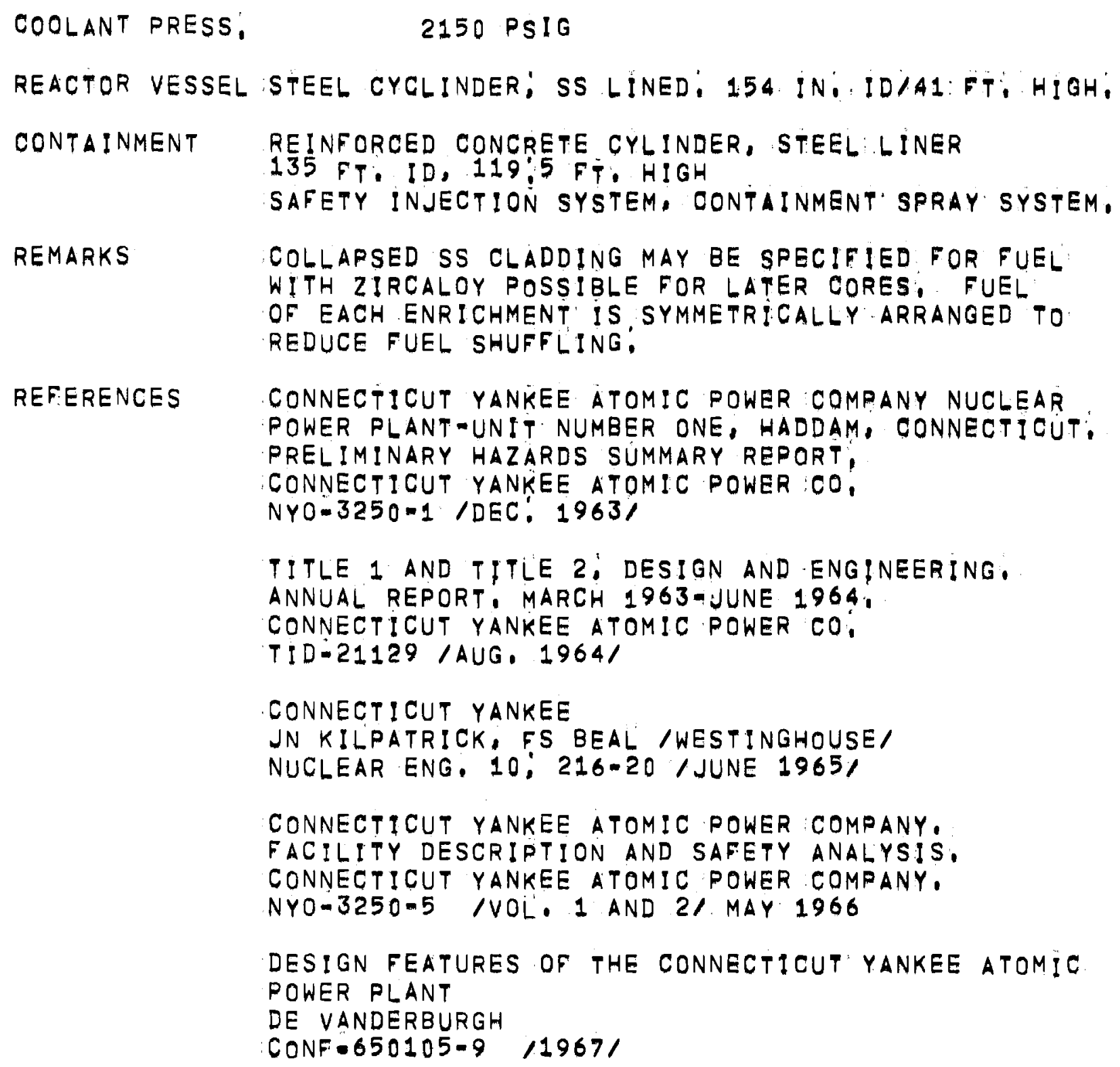


JANUARY 1969

BNWL -936

PRESSURIZED LIGHT WATER REACTORS: DOMESTIC

NAME/OWNER

MALIBU PLANT / LADWP

CORRAL CANYON

DES IGNER

WESTINGHOUSE ELECTRIC CORPORATION

OPERATOR

LOS ANGELES DEPT, WATER AND POWER

LOCATION

MALIBU BEACH, CALIF: ICORRAL CANYON/

PURPOSE

POWER:

TYPE

PWR, 4-LOOP SYSTEM

POWER MWE (MWT)

490

1473

CRITICAL

CONSTRUCTION. TARGET 1972

CONDITIONAL CONSTRUCTION PERMIT JULY 1966

COOLANT

LIGHT WATER

MODERATOR

LIGHT WATER

FUEL MATERIAL URANIUM DIOXIDE PELLETS 0.375 IN: DIAMI10:550 IN.

FUEL GEOMETRY ROD 0:4115 IN: OD: 120 IN: ACTIVE LENGTH

FUEL CLADDING REGIONS 1:2 AND 3 USE SS, REGION 4 /AND FUTURE/ USES ZIRCALOY

FUEL ENRICH, REGIONAL 3,8 PER CENT U-235

FUEL ASSEMBLY 217-ROD BUNDLE $15 \times 15$

156 ASSEMBLIES/CORE

CONTROL

ROD CLUSTER, CHEMICAL SHIM

CONTAINMENT

DOUBLE-BARRIER CONTAINMENT, LAYER OF POROUS

CONCRETE BETWEEN INNER AND OUTER SHELLS OF STEEL AND REINFORCED CONCRETE.

REMARKS

AEC HAS ACCEPTED PROPOSAL FROM LADWP FOR $R \rightarrow D$ AND DESIGN ASSISTANCE UNDER MODIFIED THIRD-ROUND RULES STATION DESIGN IS SIMILAR TO CONNECTICUT YANKEE,

REFERENCES

TECHNICAL INFORMATION IN SUPPORT OF APPLICATION FOR CONSTRUCTION PERMIT AND.LICENSE, MALIBU NUCLEAR PLANT UNIT NO: 1 . DEPT, WATER AND POWER; CALIF, , STONE + WEBSTER: NP $=14290$ /PT.B/ /NOV. 1963 / 
JANUARY 1969 BNWL-936

RRESSURIZED LIGHT WATER REACTORS: DOMESTIC

NUCLEONICS WEEK JULY 21; 1966

NEWS RELEASE

MALIBU NUCLEAR PLANT UNIT NO, 1 PRELIMINARY HAZARDS SUMMARY REPORT. DEPT. WATER AND POWER, LOS ANGELES NYO $=3266-1$ /MAY $1965 \%$ 
JANUARY 1969

BNWL-936

PRESSURIZED LIGHT WATER REACTORS: DOMESTIC * * * * HD33

NAME / OWNER

DES IGNER

OPERATOR

LOCATION

PURPOSE

TYPE

POWER MWE (MWT)

CRITICAL

COOLANT

MODERATOR

FUEL MATERIAL

FUEL GEOMETRY

FUEL CLADDING

FUEL ENRICH.
FUEL ASSEMBLY

SAN ONOFRE NUCLEAR PLANT/S,CAL, ED; S, DIEGO G+E CAMP PENDLETON

WESTINGHOUSE ELECTRIC CORPORATION

SOUTHERN CALIFORNIA EDISON COMPANY

SAN CLEMENTE, CALIF:

CAMP PENDLETON MARINE RESERVATION

POWER

PWR, 3-LOOP SYSTEM, CLOSED CYCLE. 450 1347

1967. 'COMMERCIAL OPERATION JAN, 1968

LIGHT WATER

LIGHT WATER

URANIUM DIOXIDE PELLETS 0,383 IN, DIAM/0,60 IN, ROD 0,422 IN, OD.: ACTIVE LENGTH 120 IN. ACTIVE LENGTH 10 FT.

SS TUBES, 0,0165 IN: WALL THICKNESS

0,0165 IN: THICK, 0,422 IN, OD.

$3: 5$ PER CENT U-235, ZONE ENRICHMENT

180-ROD BUNDLE, $14 \times 14$

RCC ELEMENTS /ROD CLUSTER CONTROL/

157 ASSEMBLIES/CORE

3-REGION CORE ARRANGEMENT

RODS ARE OMITTED FOR RCC $\triangle S$ SEMBLIES

FUEL CHARGE 150,000 POUNDS URANIUM DIOXIDE

$58,000 \mathrm{KG}$. URANIUM
BURNUP(REFUEL) 13,500 MWD FUEL SHUFFLING 14-MONTH INTERVALS
CONTROL ROD CLUSTER CONTROL /RCC/, A SERIES OF SMALL INDIVIDUAL CONTROL ELEMENTS ARRANGED IN A CLUSTER. CHEMICAL SHIM


PRESSURIZED LIGHT WATER REACTORS: DOMESTIC

COOLANT PRESS, 2065 PSIA

REACTOR VESSEL SS=LINED CARBON STEEL VESSEL A 1 FT. LONG 112 FY: ID.

CONTAINMENT STEEL SPHERE 100 FT: DIA: 40 FT; BELOW GRADE, AIR-TIGHT, WITH CONCRETE STRUCTURE INSIDE SPHERE FOR SUPPORT AND SHIELDING; THE 6-FT; SHIELD CONTAINS COOLING COILS TO PREVENT OVERHEATING OR DEHYDRATION OF THE CONCRETE:

REMARKS

BASIC THREE-LOOP REACTOR, VARYING PLANT SIZE IS ACHIEVED BY VARYING VESSEL AND CORE SIZE AND THE NUMBER OF LOOPS: LOOP COMPONENTS REMAIN THE SAME: SAN DIEGO GAS - ELECTRIC WILL CONTRIBUTE TO PLANT COSTS IN RETURN FOR SHARE OF POWER PRODUCED. CLADDING FOR SUBSEQUENT CORES WILL PROBABLY BE ZIRCALOY.

REFERENCES SOUTHERN CALIFORNIA EDISON REACTOR. CHARACTERISTICS NUCLEONICS 18, $18 /$ JUNE $1960 /$

PRELIMINARY HAZARDS SUMMARY REPORT ON SOUTHERN CALIFORNIA EDISON COMPANY NUCLLEAR STATION AT CAMP PENDLETON, CALIFORNIA, UNIT NO: 1. PART B. SOUTHERN CALIFORNIA EDISON CO: NP-12513/JAN. 1963/

DESIGN FEATURES OF THE SAN ONOFRE NUCLEAR STATION HJ VON HOLLEN, CF CURREY WESTINGHOUSE ENG, 25, 142-47 /SEPT. $1965 /$

SAN ONOFRE NUCLEAR GENERATING STATION: UNIT 1. FINAL ENGINEERING AND SAFETY ANALYSIS

VOL: $1,2,3 /$ S.CALIF. ED., SAN DIEGO/ $N Y O=3270-2$ /NDI

SAN ONOFRE, WEST-S NEWEST NUCLEAR STATION ELECTRICAL WEST 134; 33-35, 39 /MAY $1967 /$

UNIQUE ASPECTS OF THE SAN ONOFRE NUCLEAR: PLANT. KP BASKIN, OTHERS

ELECTRICAL WEST $135 / 2 /, 54 / 1968 /$

ABSTRACT OF PAPER 
PRESSURIZED LIGHT WATER REACTORS: DOMESTIC

NAME /OWNER

DESIGNER

RURPOSE

TYPE

POWER MWE (MWT

CRITICAL

COOLANT

MODERATOR

FUEL MATERIAL

FUEL GEOMETRY

FUEL CLADDING

FUEL - ENRICH,

FUEL ASSEMBLY

SPECIFIC POWER

BURNUP (REFUEL)

CONTROL

COQLANT TEMP, INLET $506^{\circ} \mathrm{F}$

COOLANT PRESS,

3750 PSIA

POWER

1000

LIGHT WATER
FUEL CHARGE

SCOTTER /SUPERCRIT ONCE THROUGH TUBE REACTOR/ WEST WESTINGHOUSE ELECTRIC CORPORATION

PWR; PRESSURE TUBE

2297

DESIGN DEVELOPMENT:

GRAPHITE; GRAPHITTE REFLECTOR

URANIUM DIOXIDE

RODS, CONCENTRIC̈ RINGS

SS 0.012 IN. THICK, TUBES:

$3: 03$ PER CENT U.235

ASSEMBLY CONSISTS OF 1 ROD AND 4 CONCENTRIC RINGS. 540 ASSEMBLIES IN 540 :PRESSURE TUBES,

121,900 KG URANIUM

$18,9 \mathrm{KWT} / K G$ URANIUM

16,500 MWD/MTU

BORON CARBIDE RODS

REACTOR VESSEL CARBON STEEL 42 FT. HIGH BY 35.5 FT:ID: PRESSURE TUBES ARE ZIRCONIUM ALLOY, INNER LINER OF ZIRCONIUM-TANTALUM-TUNGSTEN,

REMARKS

PRELIMINARY DESIGN OF THE 50 MWT SUPERHEAT POWER EXP: IS COMPLETED: TWO POWER CYCLES STUDIED WERE THE IN-CORE REHEAT, IN WHICH 600 PSIA STEAM EXHAUSTED FROM THE HIGH PRESSURE TURBINE IS REHEATED IN THE CORE TO 1000 F, AND CONDENSING STEAM REHEAT WHERE 182 RSIA STEAM IS REHEATED IN A HEAT EXCHANGER BY BLEED STEAM FROM THE: HIGH 
JANUARY 1969

$B N W L=936$

PRESSURIZED LIGHT WATER REACTORS: DOMESTIC HD34

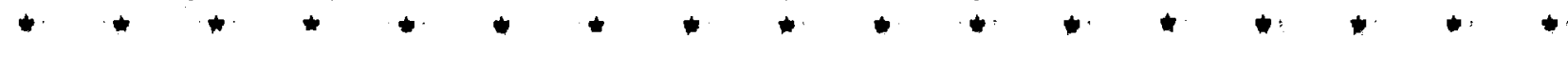

PRESSURE TURBINE, IN THE REFERENCE DESIGN, THE COOLANT FLOW MAKES 4 CONSECUTIYE PASSES, OUTSIDE TO CENTER OF THE FUEL ASSEMBLY, SCOTT-R: WAS SELECTED FOR FURTHER DEVELOPMENT'BY AEC;

REFERENCES

THE ROLE OF A SUPERHEAT POWER EXPERIMENT IN THE DEVELOPMENT OF SUPERCRITICAL STEAM NUCLEAR IFIRED POWER PLANTS. JH WRIGHT; OTHERS

ASME PREPRINT 62-WA-345 /DEGEMBER $1962 \%$

THE SCOTTIR DEVELOPMENT PROGRAM: JH WR!GHT. WESTINGHOUSE ENG, 23,50-53/MARCH 1963/

SUPERCRITICAL WATER REACTORS: POWER REACTOR TECHNOLOGY 6/3/; 73-8/JUNE 1963/

1000-MWE SCOTTAR CONCEPTUAL DESIGN, SN TOWER TID-7674/P. 65.6/ 
JANUARY 1969

$B N W L=936$

PRESSURIZED LIGHT WATER REACTORS: DOMESTIC HD 35:

DESIGNER

PURPOSE

TYPE

POWER MWE(MWT)

CRITICAL

OOQLANT

MODERATOR

FUEL MATERIAL.
NAME /OWNER

RÄVENSWOOD NUCLEAR UNITYCONSOLIDATED EDISON

WESTINGHOUSE ELECTRIC CORPORATION

POWER

PWR, CLOSED CYCLE; 5-LOOOP SYSTEM: OIL IFIRED SUPERHEAT

2850

PROPOSAL HAS BEEN WITHDRAWN

LIGHT WATER

LIGHT WATER

URANIUM DIOXIDE PELLETS; 0,375 IN: DIA:10,736 IN: LONG,

FUEL GEOMETRY RODS, 120 IN, ACTIVE LENGTH

FUEL CLADDING SS TUBE; 0,013 IN: WALL THICKNESS

FUEL ENRICH, ZONE ENRICHMENT 3:2:3:6 AND A PER OENT U-235

FUEL ASSEMBLY $280-R O D$ BUNDLES; $17 \times 17$ /9RODS OMITTED FOR CONTROL ROD PASSAGE/

156 ASSEMBLIES IN CORE, EACH ENRICHMENT ZONE HAVING 52 ASSEMBLIES.

FUEL CHARGE 103,000 KG. URANIUM DIOXIDE

BURNUP(REFUEL) 12000 MWD/MTU

CONTROL CHEMICAL SHIM, CONTROL RODS

COOLANT TEMP, INLET $543 \mathrm{~F}$ OUTLET $588 \mathrm{~F}$

COQLANT PRESS, 2035 PSIG

REACTOR VESSEL STEEL, 46 FT, $61 / 2$ IN: LONG

CONTAINMENT MULTI-WALL CONTAINMENT. TWO CARBON STEEL SHELLS WITH PERVIOUS CONCRETE BETWEEN THEM. AND NORMAL HIGH-DENSITY REINFORCED CONCRETE OUTER SHELL:

REMARKS

PROPOSAL FOR THE NUCLEAR PLANT WAS WITHDRAWN BY CONSOLIDATED EDISON IN JANUARY 1964, SITE WAS TO HAVE BEEN WITHIN NEW YORK :CITY, 
JANUARY 1969 $B N W L=936$ PRESSURIZED LIGHT WATER REACTORS: DOMESTIC * * * * * HD35 REFERENCES PRELIMINARY HAZÄRDS SUMMARY REPORT; DOCKET $50-204$. RAVENSWOOD NUCLEAR GENERATING UNIT'A; CONSOLIDATED EDISON CO:. OF N,YI: INC, 1962 ;, NP-12466 /VOL: 1,2, AND $3 /$. 
JANUARY 1969

BNWL-936

PRESSURIZED LIGHT WATER REACTORS: DOMESTIC

HD36

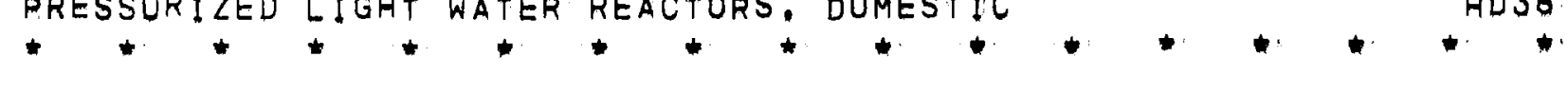

NAME /OWNER

POINT BEACH NUCLEAR STATION/WMPCO

DESIGNER

WESTINGHOUSE ELECTRIC CORP:

OPERATOR

WISCONSIN MICHIGAN POWER CO: IWMPCO/

WISCONSIN ELECTRIC POWER COMPANY

LOCATION

TWO CREEKS, MANITOWOC COUNTY, WISCONSIN NEAR GREEN BAY.

PURPOSE

POWER

TYPE

PWR, CLOSED CYCLE; TWO-LOOP SYSTEM. TWO-REACTOR STATION

POWER MWE (MWT)

500

1396

PER REACTOR

CRITICAL

TARGET NO:1, 1970

TARGET NO,2, 1971

COOLANT LIGHT WATER

MODERATOR LIGHT WATER

FUEL MATERIAL URANIUM DIOXIDE PELLETS 0,3669 IN, DIAM/0,600 IN. LONG

FUEL GEOMETRY ROD 0.422 IN: OD; ACTIVE LENGTH 144 IN.

FUEL CLADDING ZIRCALOY 0.0243 IN: THICK

FUEL ENRICH, ZONED: 2,35, 2,50 2:80 PER CENT:U-235

FUEL ASSEMBLY 179-ROD BUNDLE, $14 \times 14$, WITH RCC ELEMENTS UNCANNED. 121 ASSEMBLIES/CORE

FUEL CHARGE 118,506 LBS, URANIUM DIOXIDE

BURNUP(REFUEL) FIRST, CYCLE AVE: 14;000 MWD/MTU EQUIL. CORE AVE: 27,000 MHD/MTU

CONTROL ROD-CLUSTER CONTROL; 16-ROD CLUSTER

CADMIUM-INDIUM-SILVER IN SS GLADDING

COQLANT TEMP: INLET 551.8 F OUTLET 650 F

REACTOR VESSEL VERTICAL STEEL CYLINDER 132 IN, ID/37 FT, HIGH INNER SS CLADDING $0: 156$ IN, THICK,

CONTAINMENT PRESTRESSED CONCRETE CYLINDER; HALLS 3 FT, 6 IN. 


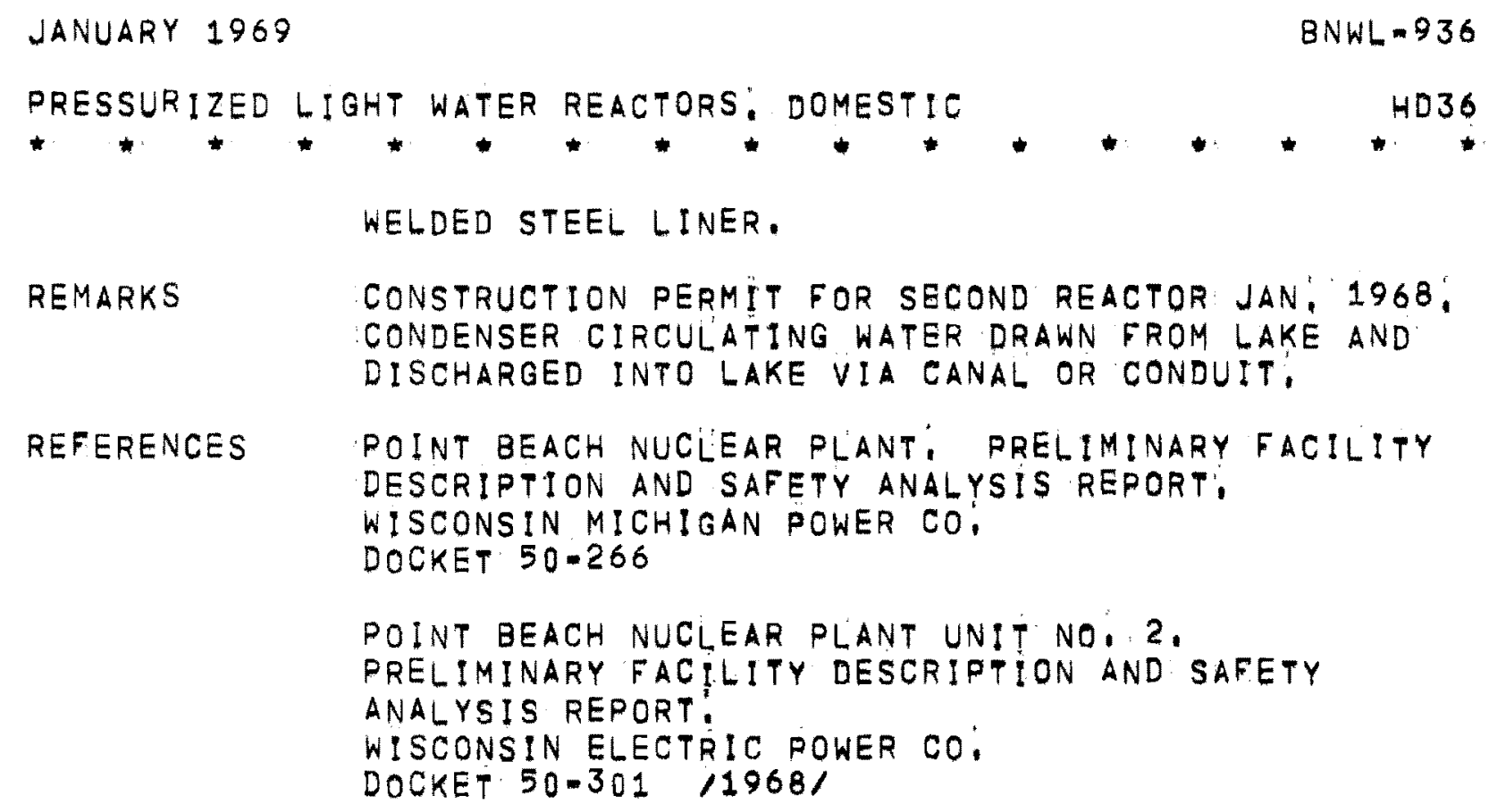




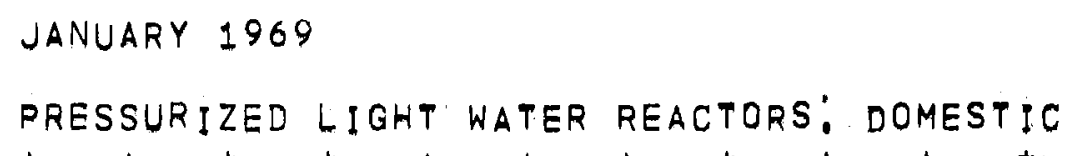


JANUARY 1969

BNWL -936

PRESSURIZED LIGHT WATER REACTORS: DOMESTIC

C STEEL: LINER,

REMARKS CONDENSER COOLING WATER DRAWN FROM LAKE ROBINSON, DISCHARGED TO LAKE VIA CANAL.

REFERENCES NUCLEAR UNIT WILL HAVE REMOTE DISPATCH, WB KINCAID

ELECTRICAL WORLD 170,28-9 /AUG: 19; 1968,

HB ROBINSON UNIT NO: 2

PRELIMINARY FACILITY DESCRIPTION AND SAFETY

ANALYSIS REPORT.

CAROLINA POWER AND LIGHT CO.

DOCKET $50=261 / 1966 /$ 
JANUARY 1969

$B N W L=036$

PRESSURIZED LIGHT' WATER REACTORS: DOMESTIC

HDSB

\author{
NAMEIOWNER TURKEY POINT 3 ALD A/FLORIDA ROWER * LIGHT \\ DESIGNER WESTINGHOUSE ELECTRIC CORP: \\ OPERATOR FLORIDA POWER AÑD LIGHT COMPANY \\ LOCATION TURKEY POINT, FLOORIDA \\ BISCAYNE BAYY \\ TYPE PWR: 3-LOOP SYSTEM \\ POWER MWE(MWT) 652 2300 \\ ULTIMATE 760 GROSS \\ CRITICAL TARGET́ NO': 1; 1971 \\ TARGET NO, $2 ; 1972$ \\ COQLANT LIGHT WATER \\ MODERATOR LIGHT WATER \\ FUEL MATERIAL URANIUM DIOXIDE PELLETSS 0,3690: IN, DIA10,000 IN. \\ FUEL GEOMETRY ROD O:422 IN: OD: ACT, LENGTH 144 IN, \\ FUEL CLADDING ZIRCALOY O.0243 IN'. THICK \\ FUEL ENRICH, ZONED; $2,73,2,43 ; 2,28$ PER CENT U:233 \\ FUEL ASSEMBLY 204 ROD ASSEMBLY, 15 X 15, WTTH RCC ELEMENTS \\ 157 ASSEMBL'IES/CORE \\ SPRING=CLIP GRID \\ FUEL CHARGE 179,000 POUNDS URANIUM DIOXIDE \\ BURNUP (REFUEL) 21,800 MWD/MTU \\ CONTROL ROD-CLUSTER CONTROL ASSEMBLIES \\ ABSORBER INDIUM-CADMIUM-SILVER IN SS TUQES \\ CHEMICAL SHIM \\ COOLANT TEMP, INLET 546:5 F OUTLET $600 \mathrm{~F}$ \\ COQLANT PRESS. 2236 FSIG \\ REACTOR VESSEL STEEL VESSEL 155,5 IN, ID:/24O IN OD: ACROSS \\ NOZZLES, HEIGHT 41 FT. \\ SS CLADDING $5 / 32$ IN: THICK
}

CONTAINMENT TWIN STEEL-LINED, PRESTRESSED CONCRETE CYLINDERS, 
HD 38

JANUARY 1969

PRESSURIZED LIGHT WATER REACTORS: DOMESTIC
BNWLE936

HD 38 .

EACH STRUCTURE COMPLETELY ENCLOSING A REACTOR AND ITS COOLANT SYSTEM, CYLINDERS ARE $116 \mathrm{FT}$ ' ID; 169 FT: INTERIOR HT: CYLINDRICAL WALL 3 FT, 9 IN. THICK: STEEL LINING OF 1\%4. IN: PLATE,

REMARKS

REFERENCES
BOTH NUCLEAR UNITS WILL BE IDENTICAL EXCEPT FOR CHANGES RESULTING FROM TECHNICAL ADVANCES:

TURKEY POINT NUCLEAR GENERATING UNITS ND, 3 AND: 4, PRELIMINARY SAFETY ANALYSIS REPORT"

FLORIDA POWER AND LIGHT CO. $N P=16146 / 1966 /$

TWO $760 \mathrm{MW}$ UNITS TO BRING NUCLEAR POWER TO FLORIDA WT PAYNE, RE STALE ELECTRICAL WORLD JAN, 15; 1968 P: 29; 32 
JANUARY 1969

BNWL: $=36$

RRESSURIZED LIGHT WATER REACTOAS: DOMESTIC

HD39

NAME IOWNER OCONEE STATION/DUKE POWER

DES I GNER

BABCOCK AND WILÖOX

OPERATQR

DUKE POWER COMPANY

LOCATION

KEOWEE LAKE: S. CAROLINA

RURPOSE

POWER

TYPE

PWR: 3IREACTOR STATION

POWER MWE(MWT)

822

2460

PER REACTOR

CRITICAL

TARGET NO,1, 1970

NO,2, 1971:

No.3, 1972

COOLANT

LIGHT WATER

MODERATOR

LIGHT WATER

FUEL MATERIAL URANIUM DIOXIDE PELLETS 0.362 IN: DIAMV0.8 IN.

FUEL GEOMETRY PIN 0,420 IN: OD: ACTIVE LEEGTH 144 IN.

FUEL CLADDING ZIRCALOYMA, 0,026 IN: THICK

FUEL ENRICH. ZONE, 2,24-2:77 PER CENT U-235

FUEL ASSEMBLY $208 \%$ IN ASSEMBLŸ, $15 \times 15$

177 ASSEMBLIES/CORE

FUEL CHARGE 201,520 LBS, URANIUM DIOXIDE

BURNUP (REFUEL) 28,200 MWD/T

CONTROL

RODS, SILVER-INDIUMACADMIUM IN SS CLADDING, RCC CHEMICAL SHIM

COQLANT TEMP, INLET $555 \mathrm{~F}$ OUTLET $603 \%$

COQLANT PRESS, 2200 FSIA

REACTOR VESSEL SS-CLAD STEEL VESSEL 171 IN: $10 / 42: F T: H ! G H$

CONTAINMENT STEEL-LINED PRESTRESSED CONCRETE VERT ICAL CYLINDER WITH SHALLOW DOMED ROOF, 116 FT: ID/206\%T: HIGH, VERTICAL WALL THICKNESS 3 3/4 FT: 
JANUARY 1969 BNWL: $=936$

PRESSURIZED LIGHT WATER REACTORS: DOMESTIC RETURNED.

REFERENCES OCONEE, NUCLEAR STATION TO COST'10O DOLLLARS PER NET KW. WS LEE ELECTRICAL WORLD 169;27-20;70 /APRIL 15; 1968 / 
JANUARY 1969

BNWL. 936

PRESSURIZED LIGHT WATER REACTORS, DOMESTIC

HD 40

NAME / OWNER

SALEM NUCLEAR STATION/DELAWARE VALLEY UTILITIES

DES I GNER WESTINGHOUSE ELECTRIC CORP.

OPERATQR PUBLIC SERVICE ELECTRIC - GAS, NEW JERSEY

LOCATION

ARTIFICIAL ISLAND SITE IN NEW JERSEY DELAWARE RIVER

RURPOSE

POWER

TYPE

PWR, CLOSED CYCLE; A LLOOP SYSTEM, TWO-REACTOR STATION

POWER MWE(MWT) 993 PER REACTOR

CRITICAL TARGET NO:1, 1972

COOLANT LIGHT WATER

MODERATOR LIGHT WATER

FUEL MATERIAL URANIUM DIOXIDE PELLETS 0:3669 IN, DIAM/0:6 IN.

FUEL GEOMETRY ROD; 0:422 IN: OD: ACTIVE LENGTH 1.44. IN.

FUEL CLADDING ZIRCALOY-4, 0.0243 IN. THICK

FUEL ENRICH, ZONE, 2:2;2,7 3:2 PER CENT U.235

FUEL ASSEMBLY 204-ROD BUNDLE, $15 \times 15$; WITH RCC ELEMENTS: UNCANNED

193: ASSEMBLIES/CORE

FUEL CHARGE $218 ; 530$ LBS, URANIUM DIOXIDE

SPECIFIC POWER 18,9 KW/FT:

BURNUP(REFUEL) 12,000 MWD/MTU

CONTROL

ROD CLUSTER CONTROL; 23-ROD CLUSTER

CADMIUM-INDIUM=SILVER IN SS GLADDING

CONTAINMENT

STEEL -LINED REINFORCED CONCRETE POMED BUILDING

FOR EACH REACTOR AND ITS SYSTEMS?

REFERENCES SALEM NUCLEAR GENERATING STATION /FORMERLY BURLINGTON NUCLEAR GENERATING STATION/

PRELIMINARY FACILITY DESCRIPTION AND SAFETY 


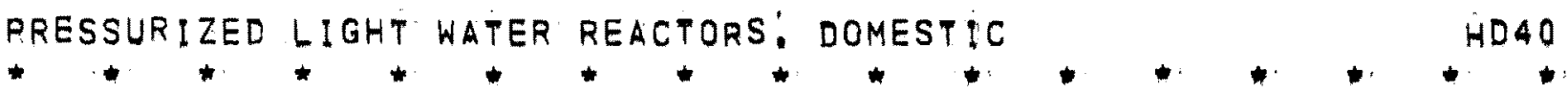
ANALYSIS REPORT: PUBLIC SERVICE ELECTRIC - GAS CO: NIJ, DOCKET 50.272 AND 50.311 
JANUARY 1969

$B N W L=936$

PRESSURIZED LIGHT WATER REACTORS: DOMESTIC

HD 41

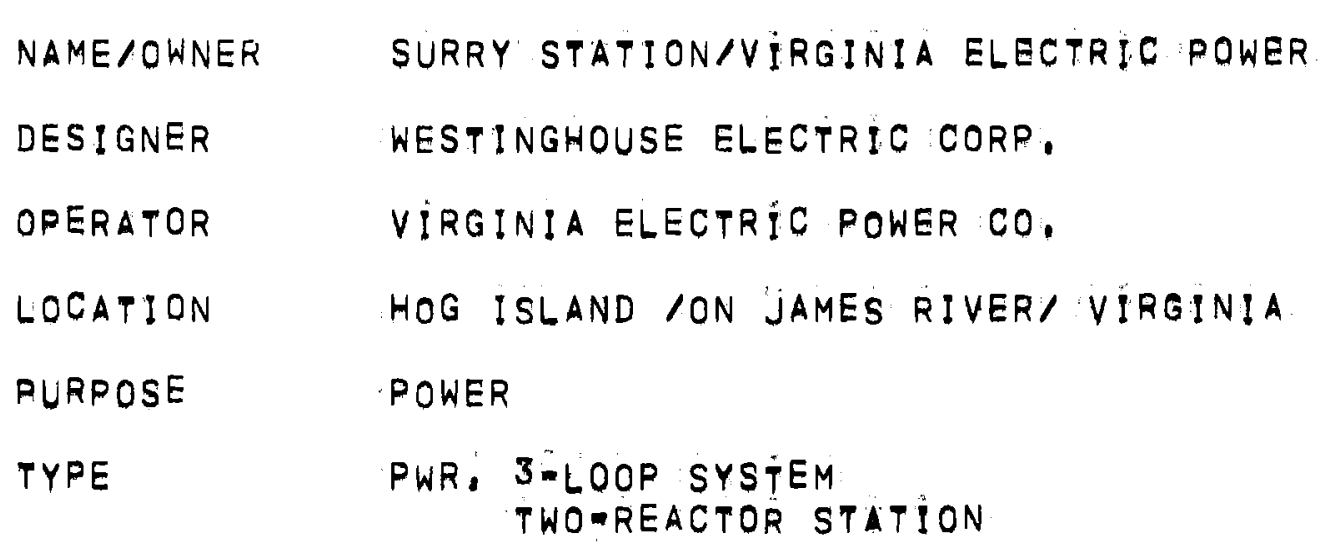

NAME/OWNER

SURRY STATION/VIRGINIA ELECTRIC PQWER

DES IGNER

WESTINGHOUSE ELECTRIC CORP.

OPERATOR

VIRGINIA ELECTRIC POWER CO,

LOCATION

HOG ISLAND ION JAMES RIVER/ VIRGINIA.

RURPOSE

POWER

TYPE

PWR, 3-LOOP SYSTEM

TWO-REACTOR STATION

POWER MWE (MWT)

800

2A41 PER REACTOR

STRETCH 847.5 MWT

GRITICAL NO.1 REACTOR 1971
NO.2 REACTOR 1972

COOLANT LIGHT WATER

MODERATOR LIGHT WATER

FUEL MATERIAL URANIUM DIOXIDE PELLETS 0.3669 IN. DIAM/0.600 IN.

FUEL GEOMETRY ROD; 0,422 IN, OD; ACTIVE LENGTH 144. IN:

FUEL CLADDING ZIREALOY-4 0,0243 IN: THICK

FUEL ENRICH, ZONE, 2,44, 2,542,94 PER CENT U.235:

FUEL ASSEMBLY 204-ROD BUNDLE, $15 \dot{x}$ 15, WITH RCC ELEMENTS. UNCANNED

157 ASSEMBLIIES/CORE

FUEL CHARGE 177,000 LBS. URANIUM DIOXIDE

BURNUP(REFUEL) FIRST CORE AVE, 21;800 MWD/MTU

CONTROL ROD CLUSTER CONTROL; INDIUM-CADMIUMASILVER IN SS, CHEMICAL: SHIM

COQLANT TEMP, INLET $542.9 \mathrm{~F}$ OUTLET 605.5:F

COQLANT PRESS: 2235 PSIG

REACTOR VESSEL STEEL VESSEL 157 IN: ID/42 FT: 4 IN: HIGH: SS LINING

CONTAINMENT STEEL-LINED REINFORCED CONCRETE CYLINDRICAL 
JANUARY 1969

$B N W L=936$

PRESSURIZED LIGHT WATER REACTORS: DOMESTIC

BUILDING, DOMED: REDUCED PRESSURE CONTAINMENT, EACH REACTOR HAS ITS OWN CONTAINMENT.

REFERENCES

SURREY POWER STATION UNITS 1 AND 2 , PRELIMINARY SAFETY ANALYSIS REPORT? VIRGINIA ELECTRIC AND POWER CO. DOCKET 50-280,50:281/1967/ 
JANUARY 1969

BNWL: $=036$

RRESSURIZED LIGHT WATER REACTORS: DOMESTIC

NAME IOWNER FT, CALHOUN STATIONMOMAHA PUBLIC POWER

DESIGNER COMBUSTION ENGINEERING

OPERATOR OMAHA PUBLIC POWER DISTRICT

LOCATION FT: CALHOUN, NEBRASKA: NEAR OMAHA

MISSOURI RIVER

PURPOSE IPOWER

TYPE PWR: TWOIREACTOR STATTION

POWER MWE(MWT) 450 1420 PER REACTOR

CRITICAL TARGET NO; 1,1971

COQLANT LIGHT WATER

MODERATOR LIGHT WATER

FUEL MATERIAL URANIUM DIOXIDE PELLETS 0,382 IN: OIAM10:0 IN:

FUEL GEOMETRY ROD; 0.440 IN: OD: ACTIVE LENGTH 128 IN.

FUEL CLADDING ZIRCALOYM 0;026 IN. THICK

FUEL ENRICH: ZONE, $1: 60,2,10,2: 95$ PER :CENT U:235

FUEL ASSEMBLY 176:ROD BUNDLE, $14 \times 14$ :

ZIRCALOY SPACERS; OPEN LATTICE, GUIDE TUBES

FOR CONTROL ELEMENTS:

133: ASSEMBLIES/CORE

FUEL CHARGE 50,000 KG URANIUUM

BURNUP(REFUEL) FIRST CORE AVE, 18,240 MWD/MTU 3EBATCH

MIXED CORE

CONTROL ROD, BORON CARBIDE IN INCONEL TUBE CHEMICAL SHIM

COOLANT TEMP, INLET $547 \mathrm{~F}$ OUTLET $598 \%$

COQLANT PRESS: 2100 PSIA

REACTOR VESSEL VERTICAL STEEL CYLINDER 140\%T, ID/38 FT, 2 IN:

REMARKS CONTRACT AWARD OCTOBER 1966 


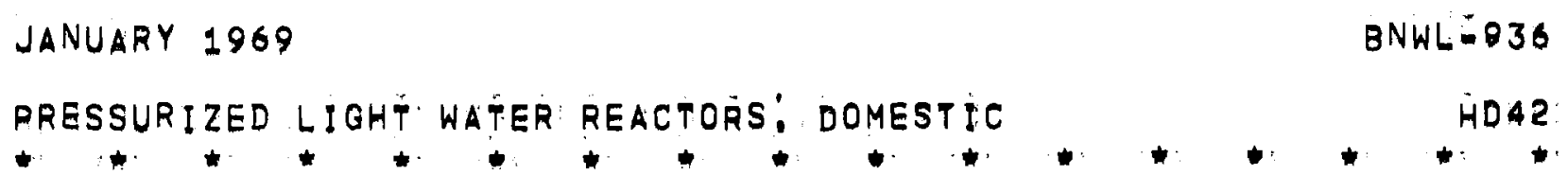

REFERENCES FORT CALHOUN STATION NO: 1 , VOL: I:2 OMAHA PUBLIC ROWER DISTRICT

DOCKET $50 \div 285 \quad 11967 \%$ 
JANUARY 1969

BNWL:D036

RRESSURIZED LIGHT' WATER REACTORS: DOMESTIIC

HD 43

NAMENOWNER

THREE MILE ISLAND STATIONAMETROPOLITAN EDISON

DES I GNER

BABCOCK WILCOX

OPERATQR

METROPOLITAN EDISON CO: IPENNSYLVANIA/

LOCATION

GOLDSBORO /NEAR HARRISBURG/; RENNSYLVANIA SUSOUEHANNA RIVER

PURPOSE

POWER

TYPE

840

2452

TWO REACTOAS PLANNED

POWER MWE (MWT) 840

PER REACTOR

CRITICAL

TARGET' 1971

OOOLANT

LIGHT WATER

MODERATOR

LIGHT WATER

FUEL MATERIAL URANIUM DIOXIDE PELLETS 0:362 IN, DIAM,

FUEL GEOMETRY ROD: 0:420 IN: OD: ACTIVE LENGTH 144 IN:

FUEL :CLADDING ZIRCALOY-4, 0.026 IN: THICK

FUEL ENRICH, ZONE, 2,29,2,64:2,90 PER CENT U-235:

FUEL ASSEMBLY $208-R O D$ BUNDLE, $15 \times 15 ;$ CANNED

177 ASSEMBLIES/CORE

FUEL CHARGE 91:61.TONS URANIUM DIOXIDE

BURNUP (REFUEL) DESIGN 28,200 MWD/MTU

CONTROL

CADMIUM-INDIUM-SILVER RODS, SS-CLAD

CHEMICAL SHIM

OOQLANT TEMP,

OUTLET OO4:3:F

COQLANT PRESS.

2185 PIG

REACTOR VESSEL STEEL VESSEL $37 \mathrm{FT}, 4$ IN, HIGH, WALL 8 7/16 IN. CONTAINMENT

RE INFORCED CONCRETE CYLINDER 130 FT: ID/57:FT: HIGH, WALL THICKNESS 3 FT, 6 IN! WALLS AND DOME ARE PRESTRESSED: CARBON STEEL LINER IS $3 / 8$ IN. THICK: 


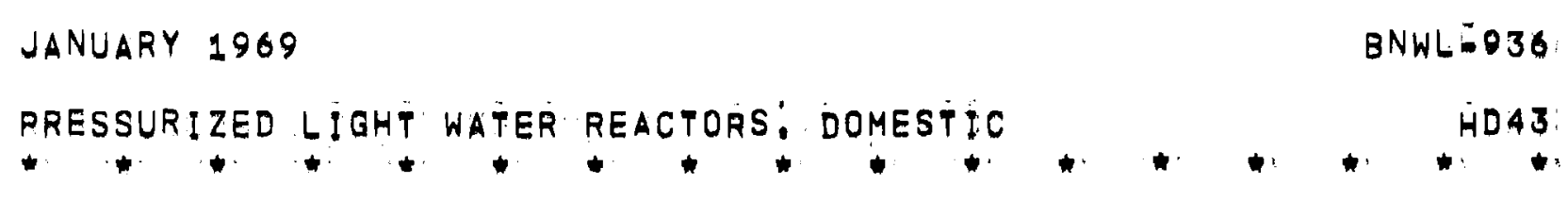

REMARKS :CONTRACT AWARD NOVEMBER 1966

REFERENCES METROPOLITAN EDISON COMPANY TLREE MILE ISLAND STATION UNIT 1 . PRELIMINARY SAFETY ANALYS IS REPQRT VOLIO 1 : DOCKET' 50.289 
JANUARY 1969
PRESSURIZED LIGHT WATER REACTORS: DOMESTIC BNWLE936

NAME/OWNER DIABLO CANYON/PACIFIC G AND:E

DESIGNER WESTINGHOUSE ELECTRIC COPR:

OPERATOR PACIFIC GAS AND ELECTRIC CO.

LOCATION DIABLO CANYON, NEAR SAN LUIS OBISPO, CALIF, ON PACIFIC OCEAN

FURPOSE POWER

TYPE PWR' TWO-REACTOR STATION

POWER MWE(MWT) NO, $1 ;$
NO, $2 ; 1070$

CRITICAL TARGET NO; $1 ; 1972$

COQLANT LIGHT WATER

MODERATOR LIGHT WATER

FUEL MATERIAL URANIUM DIOXIDE PELLETS 0,3669 IN: DIAM/0,600 IN.

FUEL GEOMETRY ROD; 0.422 IN; OD; ACTIVE LENGTH 144: IN.

FUEL CLADDING ZIRCALOY 0.0243 IN'. THICK

FUEL ENRICH, ZONE, 2,17, 2.27; 2,67 PER CENT U.235

FUEL ASSEMBLY 204\%ROD BUNDLE, $15 \times 15$ WITH RCC ELEMENTS UNCANNED; SPRING-CLIP ASSEMBLY

193 ASSEMBLIES/CORE

FUEL CHARGE 220,217 LBS, URANIUM DIOXIDE

BURNUP(REFUEL) FIRST CYCLE AVE', 12; 000 MWD/MTU

EQUIL, CORE 33,000 MWD/MTU

CONTROL ROD-GLUSTER, 20:ROD ASSEMBLY

CADMIUM-INDIUM-SILVER IN SS CLADDING,

CHEM!CAL SHIM

COQLANT TEMP, INLET $539 \mathrm{~F}$ OUTLET $646 \mathrm{~F}$

REACTOR VESSEL STEEL SHELL 173 IN: ID/A2 FT:.HIGH

CONTAINMENT VERTICAL REINFORCED CONCRETE CYLINDER, DOME, 14OFT. ID, SIDE WALLS 4 FT, 6 IN, THICK, 


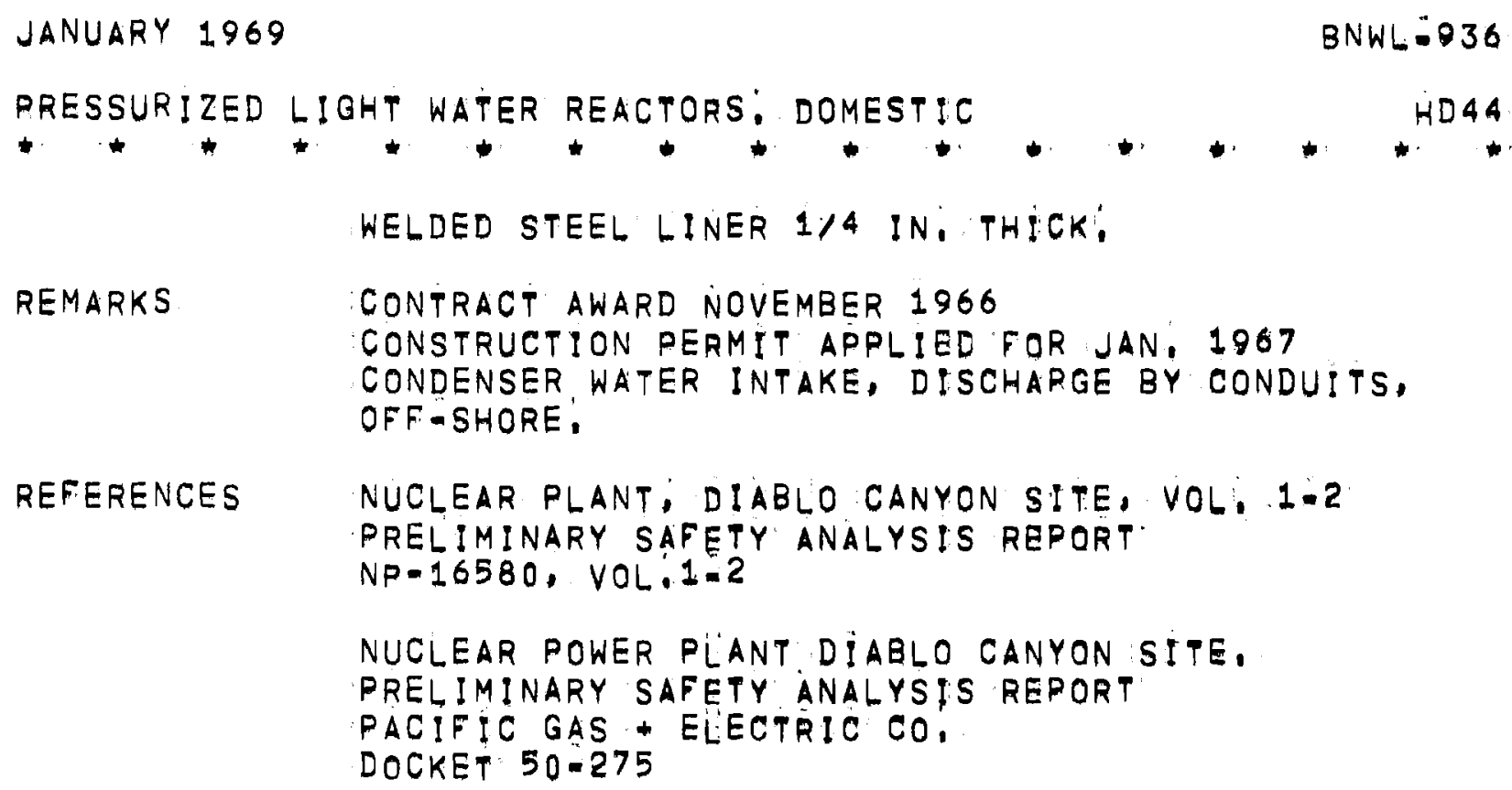




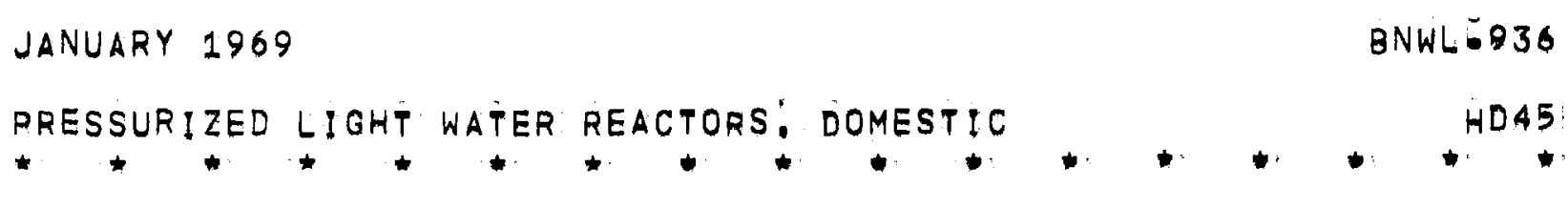

NAME/OWNER CRYSTAL RIVER STATION/FLORIDA POWER

DESIGNER BÄBCOCK AND WILCOX

OPERATOR FLORIDA POWER COMPANY

LOCATION CRYSTAL RIVER, FLORIDA

ON GULF OF MEXICO

PURPOSE POWER

TYRE TWO-REACTOR STATION
SECOND REACTOR CANCELED

POWER MWE(MWT) $850 \quad 2452$

CRITICAL TARGET 1972

COOLANT LIGHT WATER

MODERATOR LIGHT WATER

FUEL MATERIAL URANIUM DIOXIDE PELLETS 0,362 IN: DIAM/0.8 INILONG

FUEL GEOMETRY ROD; 0.420 IN: OD; ACTIVE LENGTH 144 IN:

FUEL CLADDING ZIRCALOYAA, 0,026 INं, THICK

FUEL ENRICH, ZONE, 2:29, 2,64, 2:90 PER GENT U-235

FUEL ASSEMBLY 208-ROD ASSEMBLY, CANNED

177. ASSEMBLIES/CORE

FUEL CHARGE 201,520 LBS, URANIUM DIOXIDE

BURNUP (REFUEL) AVE; 28;200 MWDIMTU

CONTROL CADMIUM-INDIUMISILVER RODS, SS CLADDING

CHEMICAL SHIM

COOLANT TEMP, INLET $555 \mathrm{~F}$ OUTLET $604 \mathrm{~F}$

COOLANT PRESS, 2185 FSIG

REACTOR VESSEL STEEL VESSEL 171 IN; ID/37 FT: HIOH: SHELL: THICKNESS 8 7/16 IN.

SS INNER CLADDING PLUUS 3 IN: INSULATION

CONTAINMENT

PRESTRESSED CONCRETE VERTICAL CYLINDER 130 FT: ID: 187 FT. HIGH, 3 1/2 FT. THICK VERTICAL WALLS: 
JANUARY 1969

$B N W L=936$

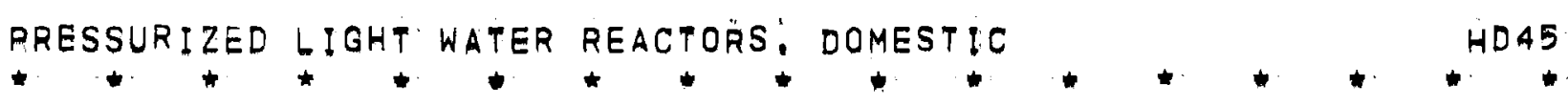

SHALLOW DOMED ROOF. STEEL LINER $3 / 8$ IN: THICK

REMARKS CONTRACT AWARD MARCH 1967

INTAKE AND DISCHARGE CANALS FOR SERVICE WATER.

REFERENCES :CRYSTAL RIVER UNITS 3 AND 4 NUCLEAR GENERATING PLANT, PRELIMINARY SAFETY ANALYSIS: REPORT?

FLORIDA POWER CO.

DOCKET $50-302$; VOL. $1-4$ 
JANUARY 1969

BNWL:-O36

RRESSURIZED LIGHT WATER REACTORS: DOMEST'DC

NAME /OWNER

MAINE YANKEE/MAINE YANKEE ATOMIC ROWER 00.

DESIGNER COMBUSTION ENGINEERING

OPERATOR

MAINE YANKEE LTOMIC POWER COMPANY

LOCATION

BAILEY POINT IWISCASSET/: IMAINE BACK RIVER

PURPOSE

POWER

TYPE

PWR:' JiLOOP SYSTEM

ROWER MWE (MWT

800

2440

CRITICAL

TARGET' 1972

COOLANT

LIGHT WATER

MODERATOR

LIGHT WATER

FUEL MATERIAL URANIUM DIOXIDE PELLETS 0,382 IN: DIAM/0,600 IN: FUEL GEOMETRY ROD: 0.44. IN: OD, ACTIVE LENGTH 137 IN. FUEL CLADDING ZIRCALOY 0.026 IN. THIEK

FUEL ENRICH, ZONE, 1.80;2.48:3:01: PER CENT UTZ35

FUEL ASSEMBLY 176:ROD ASSEMBLY

217 ASSEMBLLIES/CORE

FUEL CHARGE 218,000 LBS, URÃNIUM DIOXIDE

CONTROL ROD-CLUSTER, BORON CARBIDE IN INCONEL CLADDING,
S-ROD BUNDLE COOLANT TEMP, INLET $550 \mathrm{~F}$ OUTLET $601 \mathrm{~F}$ COQLANT PRESS, 2235 PS:G

REACTOR VESSEL STEEL VESSEL 172 IN: ID/A1 FT: HIGH SS INNER CLADDING S/32 IN. THICK

CONTAINMENT

PRESTRESSED CONCRETE CYLINDER, 135:FT, ID/172 FT: HIGH. WALL THICKNESS 4 1/2 FT: STEEL LINER,

REMARKS CONTRACT AWARD FEB, 1967

REFERENCES

NUGLEAR CANADA OCT: 1967 R: 22 
JANUARY 1969

BNWL-936

PRESSUR IZED LIGHT WATER REACTORS: DOMESTIC \# NEWS RELEASE 


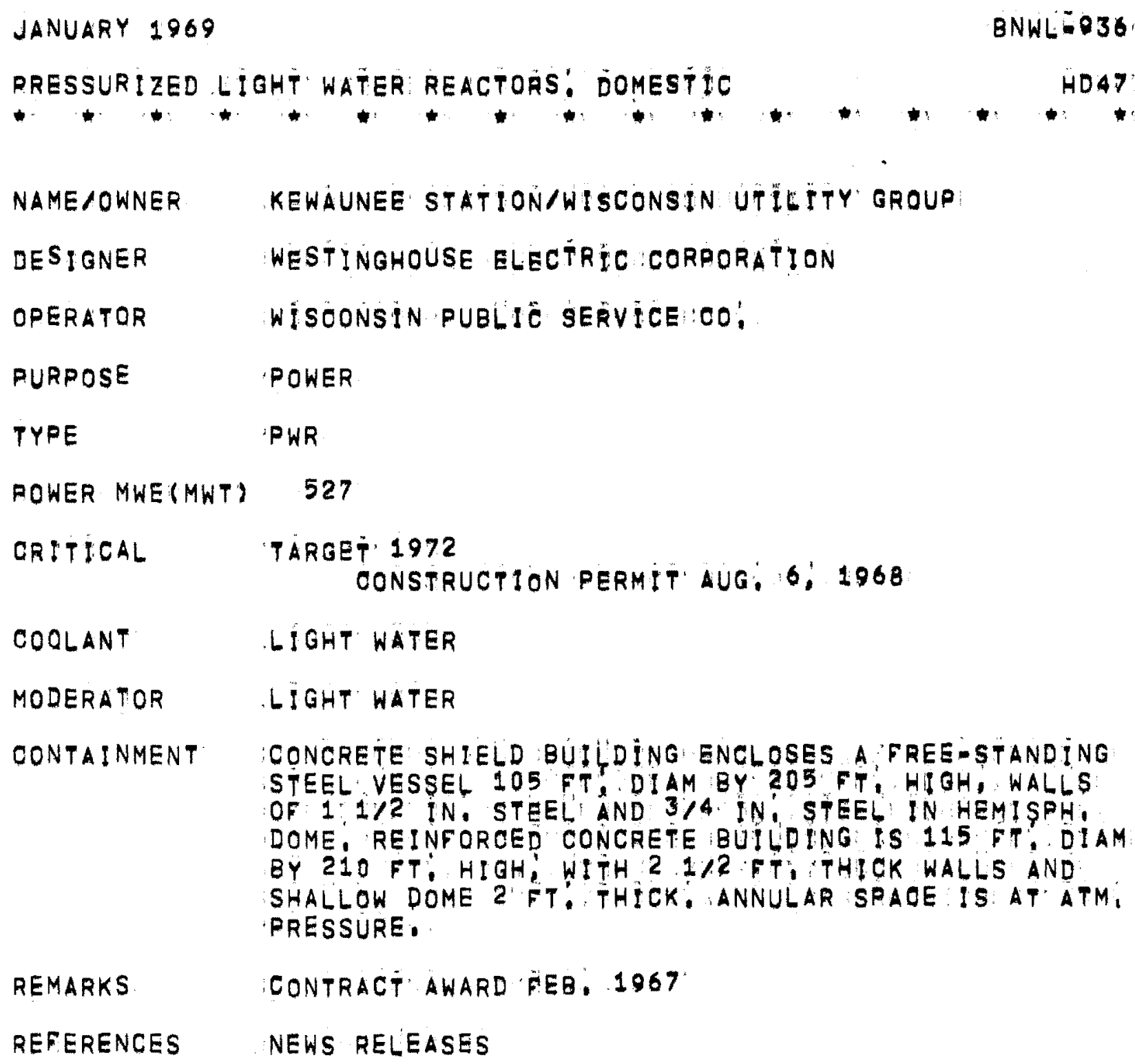




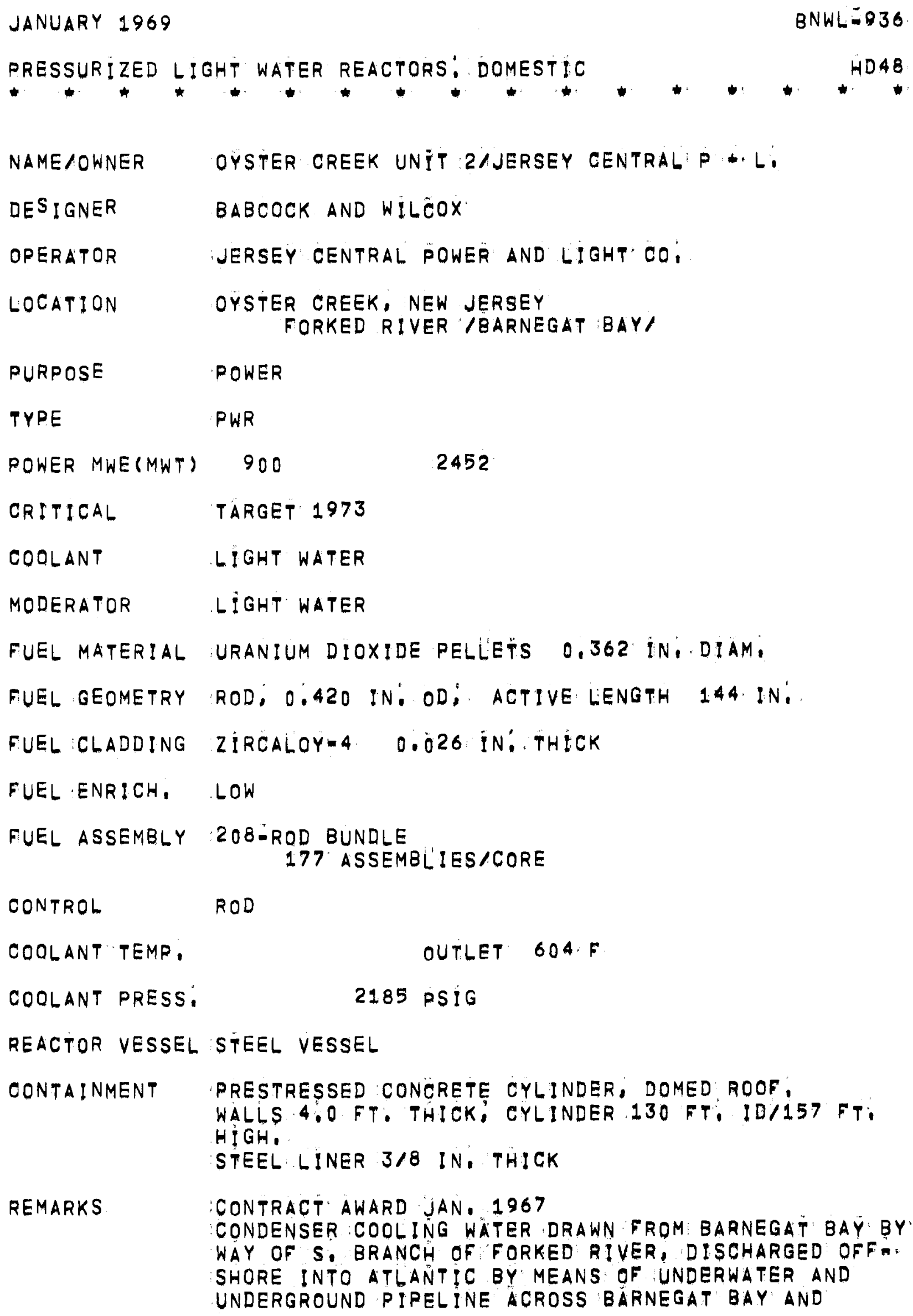




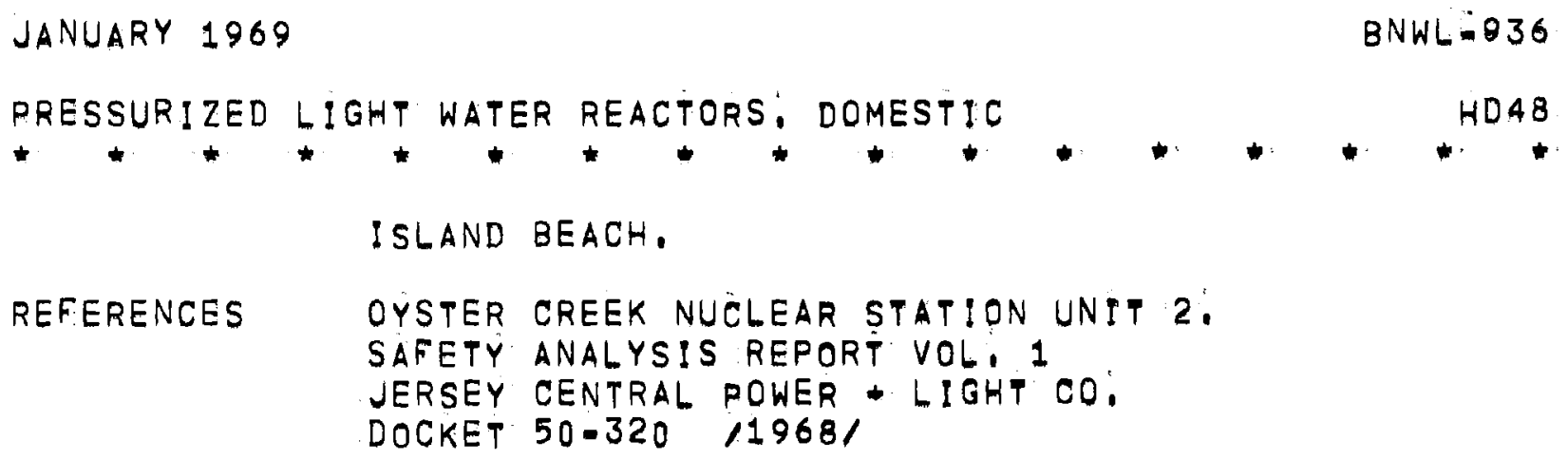




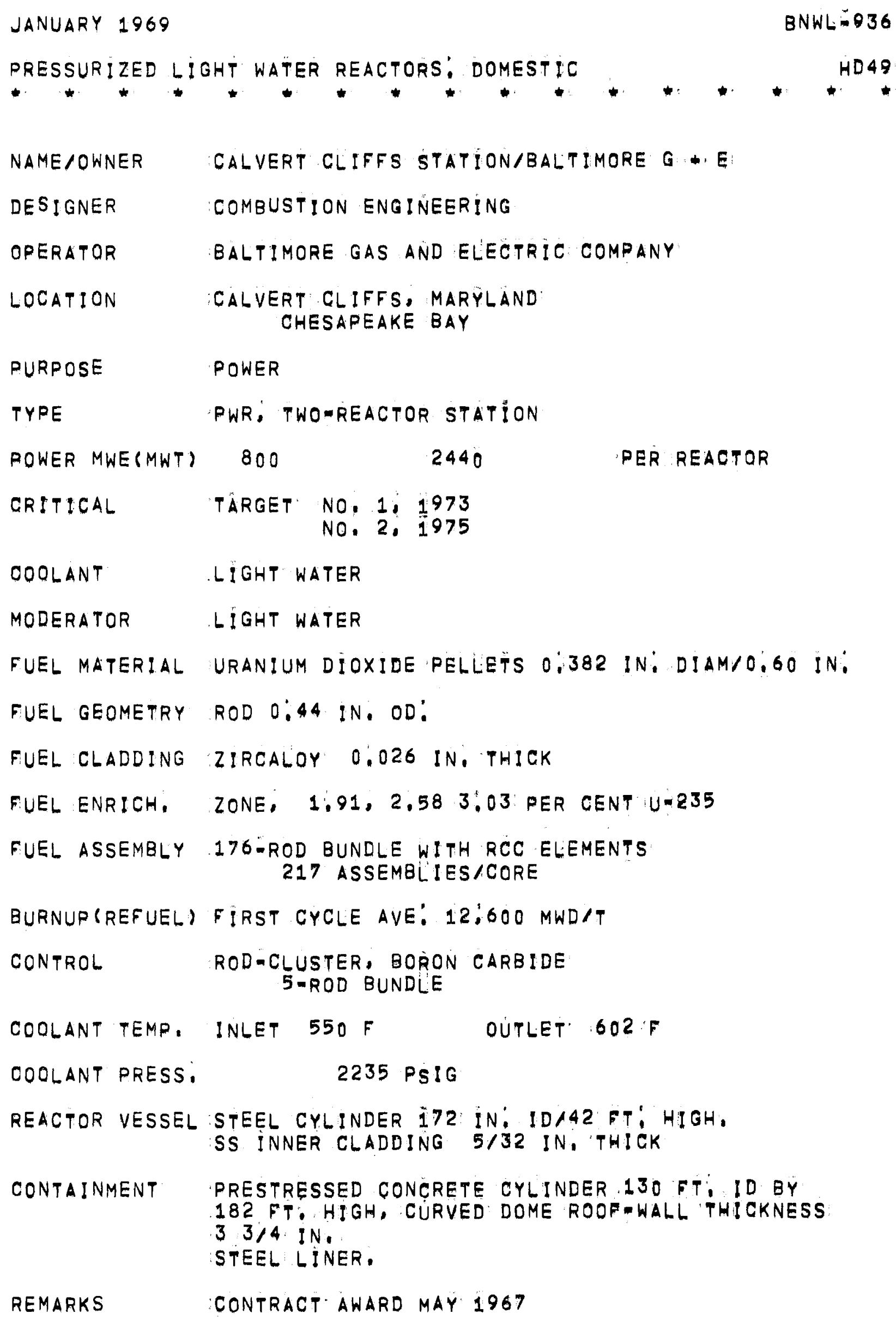


JANUARY 1969

BNWL $=936$

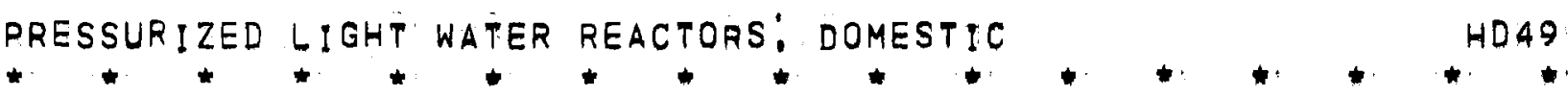

REFERENCES

CALVERT CLIFFS NUCLEAR POWER RLANT UNITS $1-2$

PRELIMINARY SAFETY ANALYSIS REPORT; VOL, 1

BALTIMORE GAS * ELECTRIC CO.

DOCKET 50-317; 50-318 /JAN, 1968/ 
JANUARY 1909
PRESSURIZED LIGHT WATER REACTOAS:. DOMESTIC HWEO36

NAMEIOWNER PRAIRIE ISLAND NUCLEAR STATION/NSP

DESIGNER WESTINGHOUSE ELECTRIC CORPORATION

OPERATOR NORTHERN STATES POWER

LOCATION REDWING MINNESOTA

MISSISSIPPI'RIVER

PURPOSE $560 \quad 1650^{\circ}$ PER REACTOR

TYPE TWR: TWO-LOOP SYYSTEM

TWO-REACTOR STATION

POWER MWE (MWT) 550

PER REACTOR

ORITICAL

TARGET NO: $1: 1972$

NO: $2 ; 1974$

OOQLANT LIGHT WATER

MODERATOR LIGHT WATER

CONTAINMENT PRIMARY STEEL SHELL ENCLOSES EAOH REACTOR: SHELL WALLS $1,1 / 2$ IN. THICK, VESSEL ID, 105 FT:", HEIGHT 205 FT. HEMISPHERICAL DOME 3/A IN, THICK. SECONDARY CONCRETE YERTICAL SHIELD CYLINDER 115 FT. DIAM/210 FT? HIGH WALL THICKNESS $2112 \mathrm{FT}$ ANNULAR SPACE AT ATM. PRESSURE,

REMARKS COOLING TOWERS WILLL BE PROVIOED:

REFERENCES NEWS RELEASES 
JANUARY 1969

BNWL: 936

RRESSURIZED LIGHT WATER REACTORS: DOMESTIC HDS1:

NAMEIOWNER

DONALD C. COOK NUELEAR PLANTIINDIANA-MIOHIGAN AEP STATION

DES IGNER

WESTINGHOUSE ELECTRIC CORRORATION

OPERATQR

INDIANA AND MIOHIGAÑ ELECTRIC COMPANY

/AMERICAN ELECTRIC POWER COMPANY SYSTEMI

LOCATION

BRIDGMAN, MICHIGAN

ON LAKE MIOHIGAN

RURPOSE POWER

PYPE

PWR; 4-LOOP SYSTEM

TWO-REACTOR STATTION

POWER MWE (MWT)

1100

3250

PER REACTOR

CRITICAL

TAROET NO; $1 ; 1972$

NO, 2,1973

OOQLANT LIGHT WATER

MODERATOR. LIGHT WATER

FUEL MATERIAL URANIUM DIOXIDE PELLETS 0.3669 IN. DIAM/0.600 IN:

FUEL GEOMETRY ROD O.422 IN: OD

FUEL CLADDING ZIRCALOY 0.0243 IN: THICK

FUEL ENRICH. ZONE, $2.20,2.70 ; 3,20$ PER CEENT U-835

FUEL ASSEMBLY :204-ROD ASSEMBLY, WITH RCC ELEMENTS UNCANNED

193 ASSEMBLL'IES/CORE

FUEL CHARGE 218,530 LBS, URÄNIUM DIOXIDE

BURNUP (REFUEL) FIRST CYCLE AVE: 12;00O MWD/MTU

FIRST CORE AVE, 21,800 MWD/MTU

CONTROL ROD-CLUSTER, INDIUM-CÄDMIUMASILVER, IN SS CLADDING,
2O-ROD CLUSTER

COOLANT TEMP, INLET $539 \mathrm{~F}$ OUTLET $602 \mathrm{~F}$

COQLANT PRESS: 2235 PSIA

REACTOR VESSEL STEEL SHELL 173 IN, ID/42 FT, HIGH 


\author{
$\begin{array}{ll}\text { JANUARY } 1969 & \text { BNWL-936 }\end{array}$ \\ PRESSURIZED LIGHT WATER REACTORS: DOMESTIC \\ SS INNER CLAD 5/32 IN. THICK \\ CONTA INMENT ICE-CONDENSER CONTA INMENT WHIOH MAKES USE OF A LOW \\ TEMPERATURE HEAT S!NK: LOCATED WITHIN THE CONTAIN- \\ MENT, CONSISTING OF A QUANTITY OF BORATED ICE IN A \\ COLD-STORAGE COMPARTMENT, THE CONTAINMENT \\ STRUCTURE IS A REINFORCED CONCRETE VERTICAL \\ CYLINDER WITH A DOMED ROOF, THE INTERIOR IS \\ DIVIDED INTO 3 VOLUMES, THE LOWER VOLUME HOUSING \\ THE REACTOR AND REACTOR COOLING SYSTEM, THE MIDDLE \\ VOLUME HOUSING THE ENERGY-ABSORBING ICE BED, AND \\ AN UPPER VOLUME TO ACCOMODATE AIR DISPLACED FROM \\ THE OTHER TWO DURING A LOSS-OF-COQLANT ACCIDENT. \\ THE CONTAINMENT HAS A WELDED STEEL: LINER $3 / 8$ IN. \\ THICK. THE ICE BED IS A COMPLETELY ENCLOSED \\ ANNULAR COMPARTMENT LOCATED AROUND THE UPPER \\ PERIMETER OF THE BUILDING AND PENETRATING THE \\ OPERATING DECK, EXTENDING INTO THE LOWER VOLUME: \\ A REFRIGERATING SYSTEM AND INSULATION ARE PROVIDED \\ REMARKS \\ CONTRACT FOR FIRST STATION AWARDED IN JULY 1967 \\ REFERENCES DONALD C, COOK NUCLEAR PLANT. \\ PRELIMINARY SAFETY ANALYSIS REPORT VOL: 1 \\ INDIANA - MICHIGAN ELECTRIC CO. \\ DOCKET $50.316,1967 /$
}




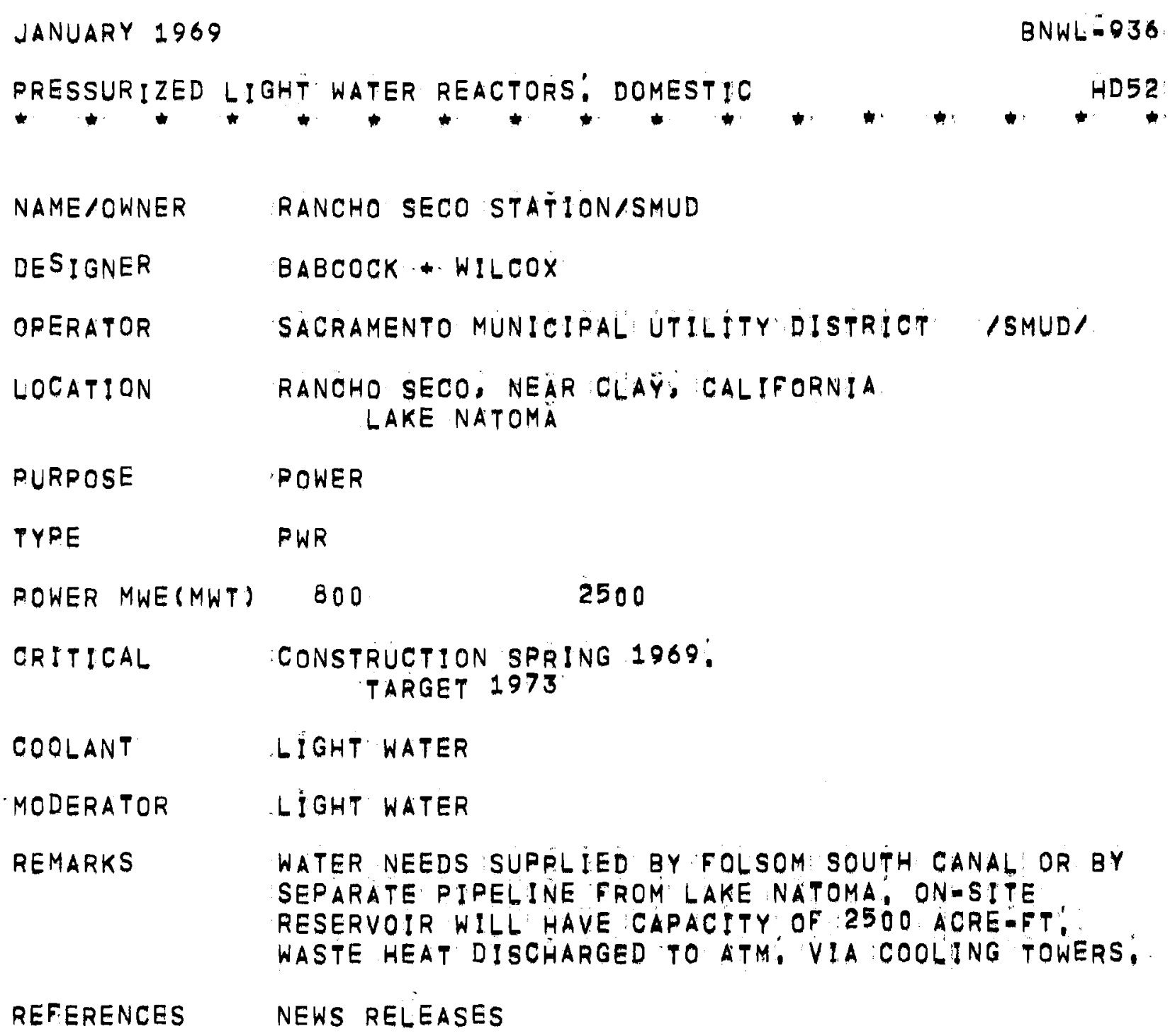


JANUARY 1969

BNWL: $=936$

PRESSURIZED LIGHT WATER REACTORS: DOMESTIC

HD53:

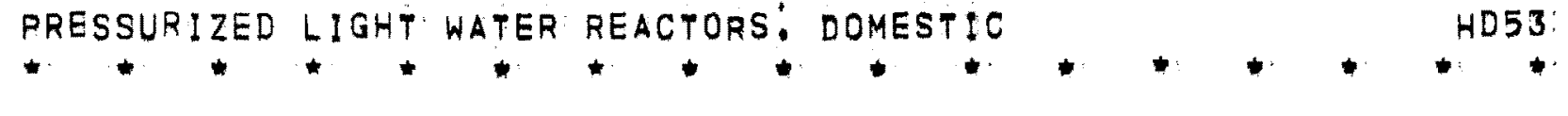

NAME IOWNER

ZION STATION/COMMONWEALTH EDISON

DESIGNER

WESTINGHOUSE ELECTRIC CORPORATION

OPERATOR

COMMONWEALTH EDISON

LOCATION

ZION, ILLINOIS

LAKE MICHIGAN

RURPOSE POWER

TYPE

PWR

TWO-REACTOR STATION

POWER MWE(MWT) 1050 3250

PER REACTOR

CRITICAL

TARQET NO: 1, 1972

COOLANT

LIGHT WATER

MODERATOR

LIGHT WATER

FUEL MATERIAL URANIUM DIOXIDE PELLETS 0,3669 INI DIAM/0,600 IN:

FUEL GEOMETRY ROD 0.422 IN: OD; ACTIVE LENGTH 144: IN,

FUEL CLADDING ZIRCALOY TUBES 0,0243: IN, WALL THICKNESS

FUEL ENRICH, ZONE, 2,2, 2:7; 3,2 PER GENTU-235

FUEL ASSEMBLY 2OA-ROD ASSEMBLY, $15 x$ 15 WITH RCC ASSEMBLIES SPRING-CLIP GRID

193: ASSEMBL'IES/CORE

FUEL CHARGE 218,530 LBS, URANIUM DIOXIDE

BURNUP(REFUEL) FIRST, CORE AVE, 21,800 MWD/MTU EQUIL: CORE AVE: 33;000

BATCH, 64 ASSEMBLIES IN-OUT:

CONTROL ROD-CLUSTER CONFROL: 2O-ROD BUNDLE CADMIUM-INDIUM-SILVER RODS, SS-CLAD CHEMICAL SHIM.

DOQLANT TEMP, INLET $539 \mathrm{~F}$ OUTLET $646: F$

COOLANT' PRESS: 2250 PSIA

REACTOR VESSEL STEEL CYLINDRICAL VESSEL. 


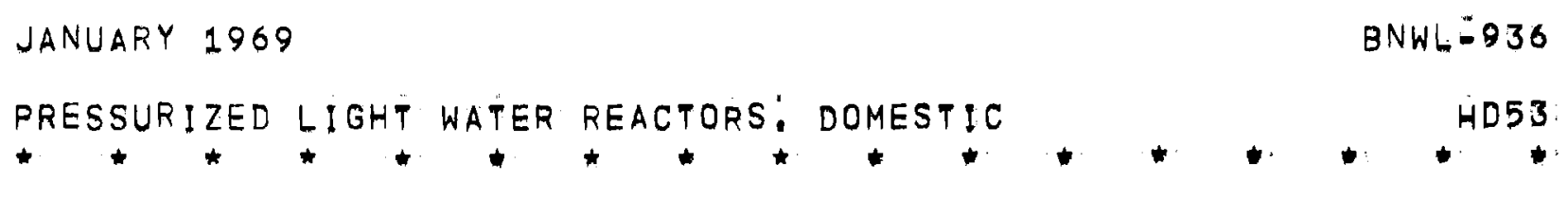

CONTAINMENT STEEL-LINED PRESTRESSED CONCRETE CYLINDER; DOMED ROOF:

REMARKS SERVICE WATER DRAWN FROM LAKE MICHIGAN AND DISCHARGED BACK INTO LAKE!'

REFERENCES ZION STATION UNITS 1 AND 2

PRELIMINARY SAFETY ANALYSIS REPORT VOL. 1 COMMONWEALTH EDISSON CO.

DOCKET $50-295-1$ 
JANUARY 1969

BNWL: $=036$

PRESSURIZED LIGHT WATER REACTORS: DOMESTIC

NAMENOWNER RUSSELLUILLE STATIONAARKANSAS P L L

DESIGNER BABCOCK + WILOOX

OPERATOR ARKANSAS POWER * LIGHT.

LOCATION RUSSELLIVILLE, ARKÄNSAS

ARKANSAS RIVER

PURPOSE POWER

TYPE PWR: 2-LOOP SYSTEM

POWER MWE(MWT) $800 \quad 2452$

CRITICAL CONSTRUCTION SEPT: 1968

TARGET 1972

COQLANT LIGHT WATER

MODERATOR LIGHT WATER

FUEL MATERIAL URANIUM DIOXIDE PELLETSS 0:302 IN: DIAM/DIB IN.

FUEL GEOMETRY ROD 0.420 IN: OD: ACTIVE LENGTH 1.44 IN.

FUEL CLADDING ZIRCALOY-4, 0.026 IN: THICK

FUEL ENRICH， ZONE, 2.29;2.64;2.90 PER CENT U:235:

FUEL ASSEMBLY 208-ROD BUNDLE, CANNED

177 ASSEMBLIIES/CORE

FUEL CHARGE 91:61 TONS URANIUM DIOXIDE

BURNUP (REFUEL) 28,200 MWD/MTU

CONTROL RODS, CADMIUMEINDIUMASILVER, SSACLAD' CHEMICAL SHIM:

COOLANT TEMF, INLET $555 \mathrm{~F}$ OUTLET $603 \%$

COQLANT PRESS, 2185 PSIG

REACTOR VESSEL CARBON STEEL VESSEL 171 IN: ID, 42 FT: HIGH SS-LINED

CONTAINMENT CONCRETE CYLINDER 116 FT: ID, 206 FT: INNER HEIGHT' WALL THICKNESS $33 / 4$ FT.

WELDED STEEL LINER. 


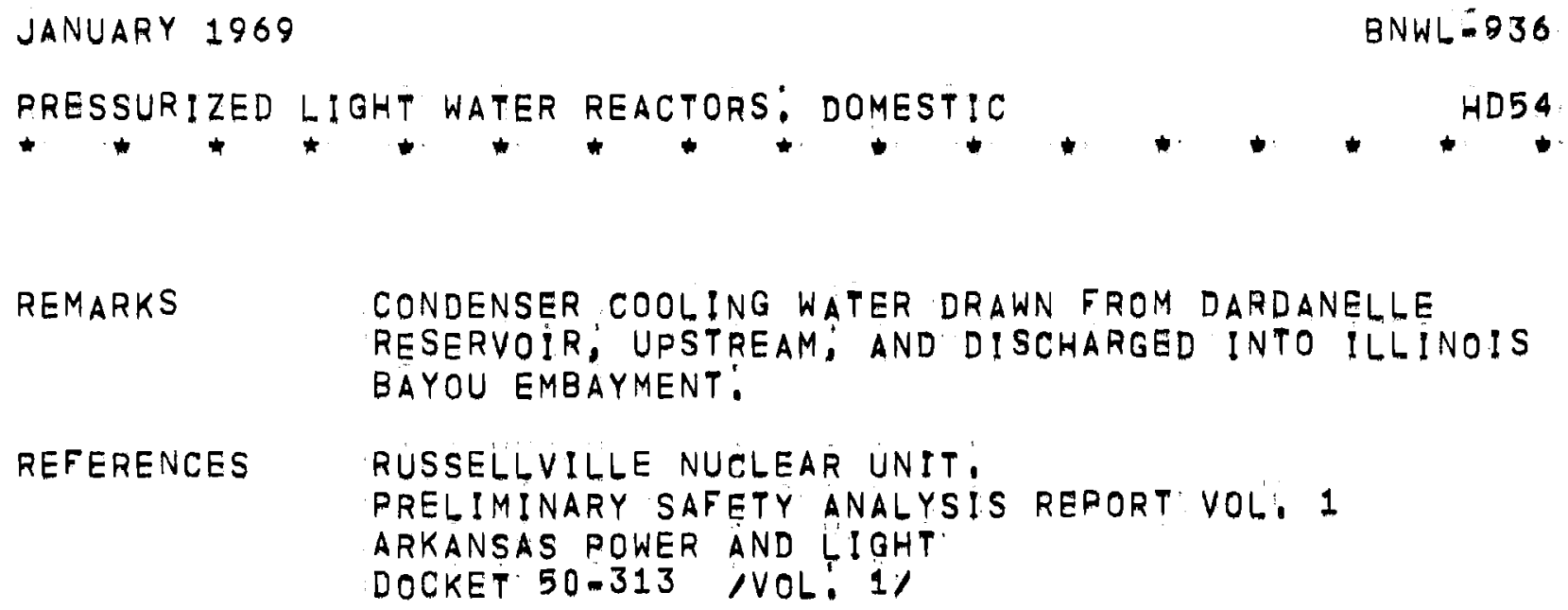




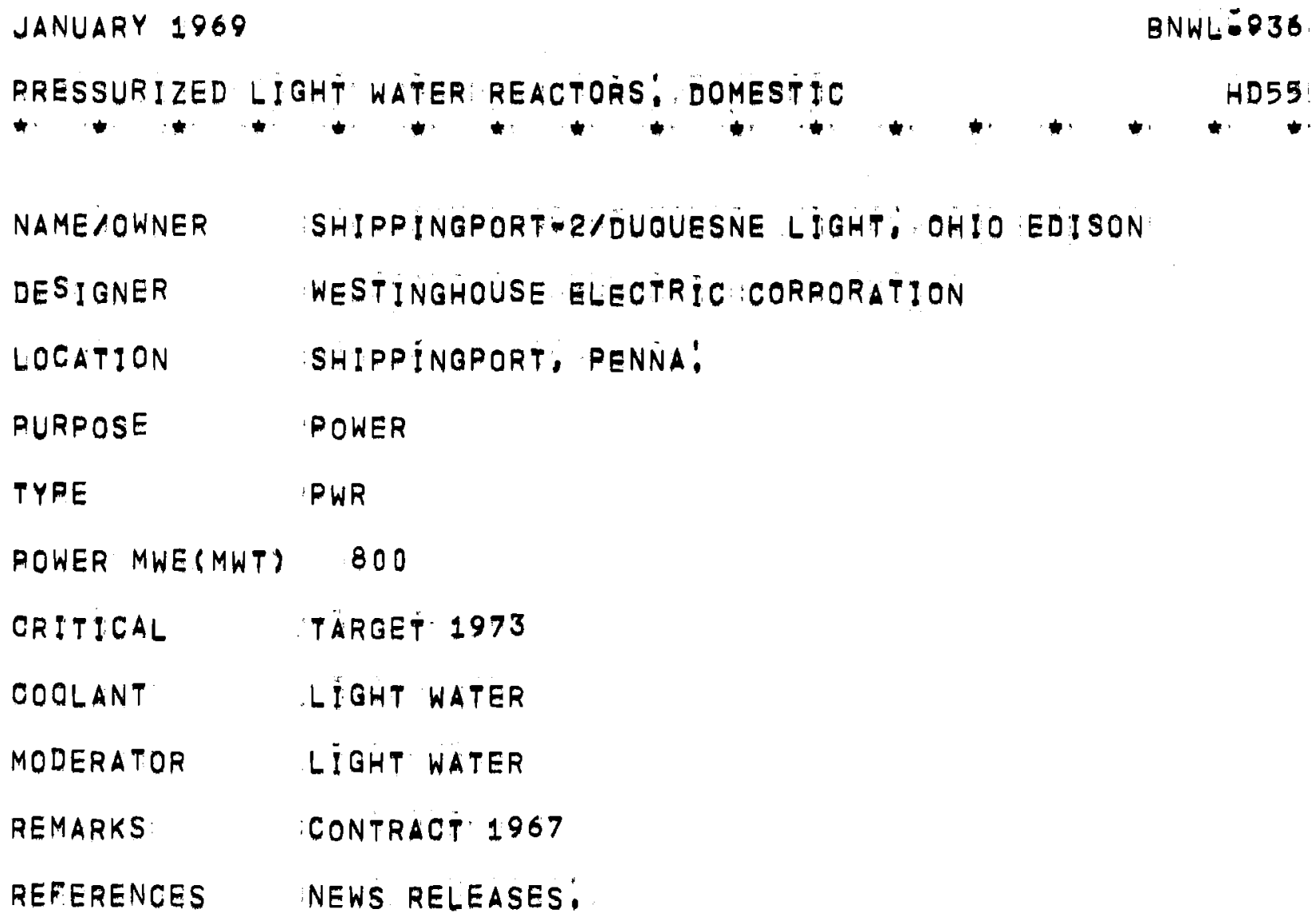




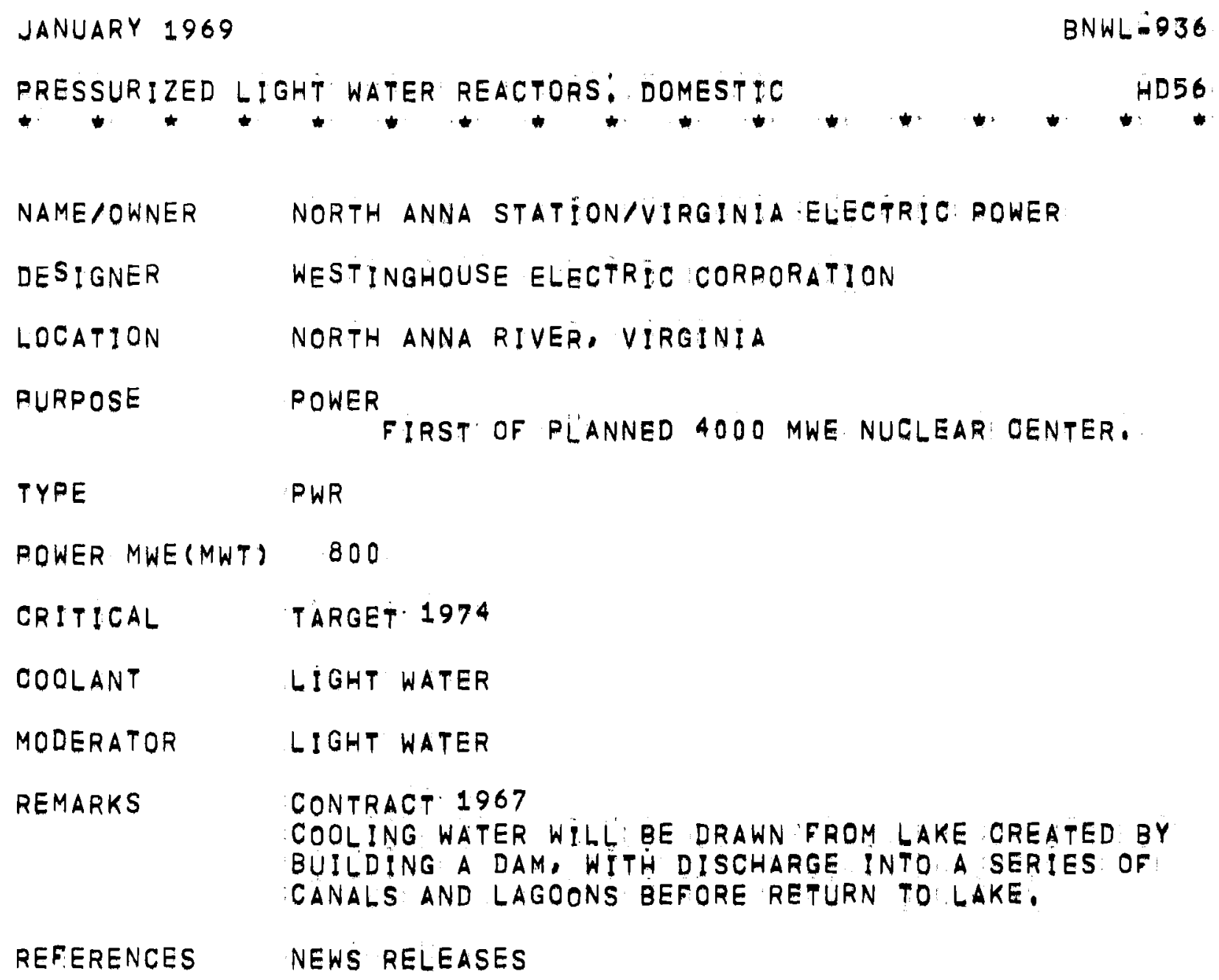




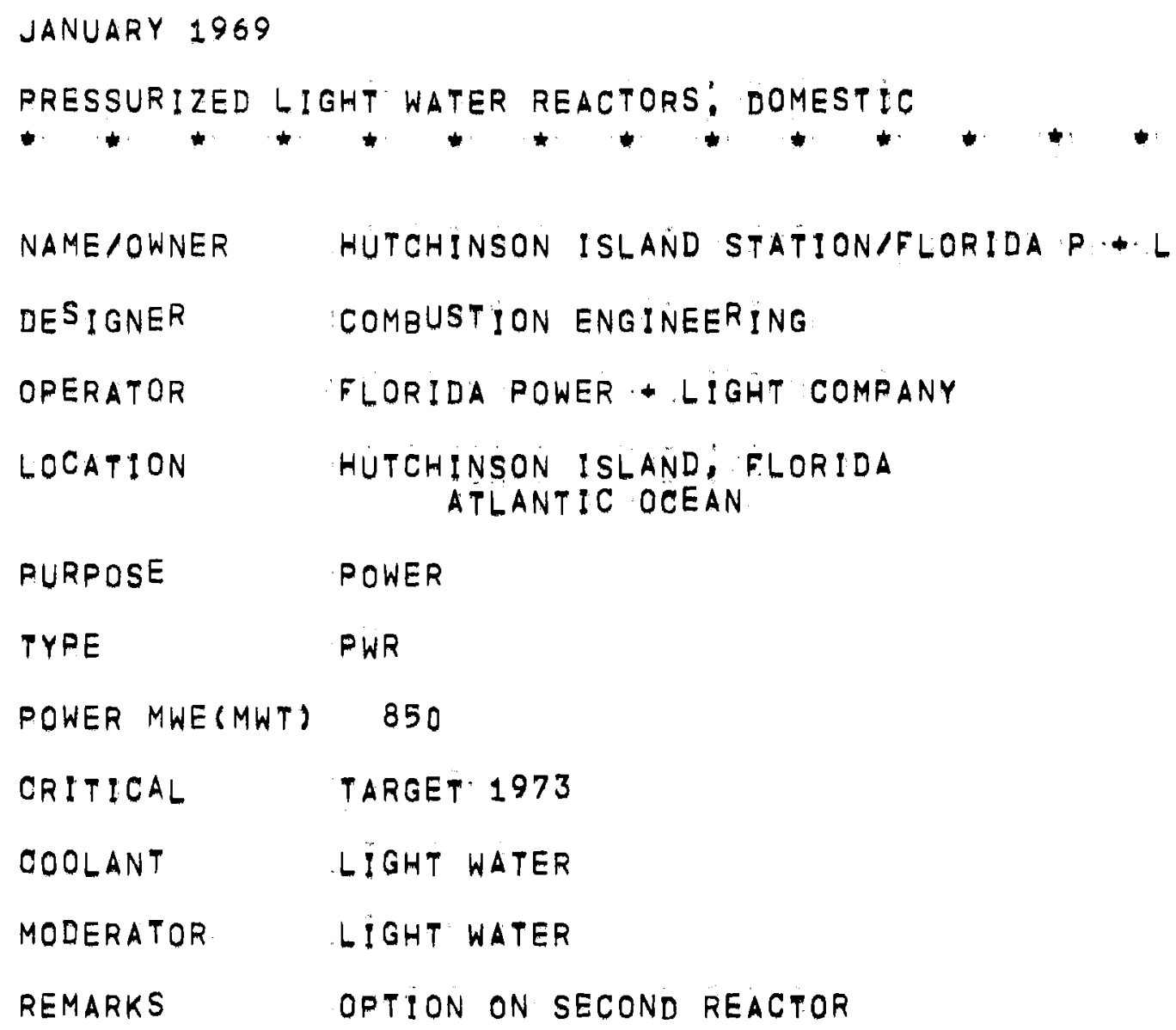


JANUARY 1969

BNWLEOS6

PRESSURIZED LIGHT WATER REACTOAS: DOMESTIC

HDSB

NAME / OWNER

MIDLAND STATIONACONSUMERS POWER: DOW OHEMICAL

DESIGNER

BABCOCK + WILCOX OOMPANY

LIOCATION

MIDLAND, MICHIGAN

PURPOSE

POWER AND PROCESS ISTEAM

TYPE

PWR: TWO-REACTOR STATION

ROWER MWE (MWT

No. $1: \quad 800$

2452

No, 2 ,

550

2452

QRITICAL

TARGET NO:1; 1974

NO.2, 1975

COQLANT

LIGHT WATER

MODERATOR

LIGHT WATER

REMARKS

CONTRACT' AWARD JULY 1960 
JANUARY 1969
RRESSURIZED LIGHT WATER REACTORS: DOMESTIC

NAMENOWNER SEQUOYA STATION/TVA

DESIGNER WESTINGHOUSE ELECTRIC CORPORATION

OPERATOR TENNESSEE VALLEY AUTHORITY

LOCATION ICHICKAMAUGA LAKE, TENNESSEE

RURPOSE POWER

TYPE PWR, TWO-REACTOR STATION

POWER MWE(MWT) 1175 P412 PER REACTOR

CRITICAL TARGET NO; 1,1973

NO:2, 1974

OOQLANT LIGHTWATER

MODERATOR LIGHTWATER

CONTAINMENT ICEICONDENSER CONTAINMENT ISYSTEM

REMARKS CONTRACT JULY 1968 


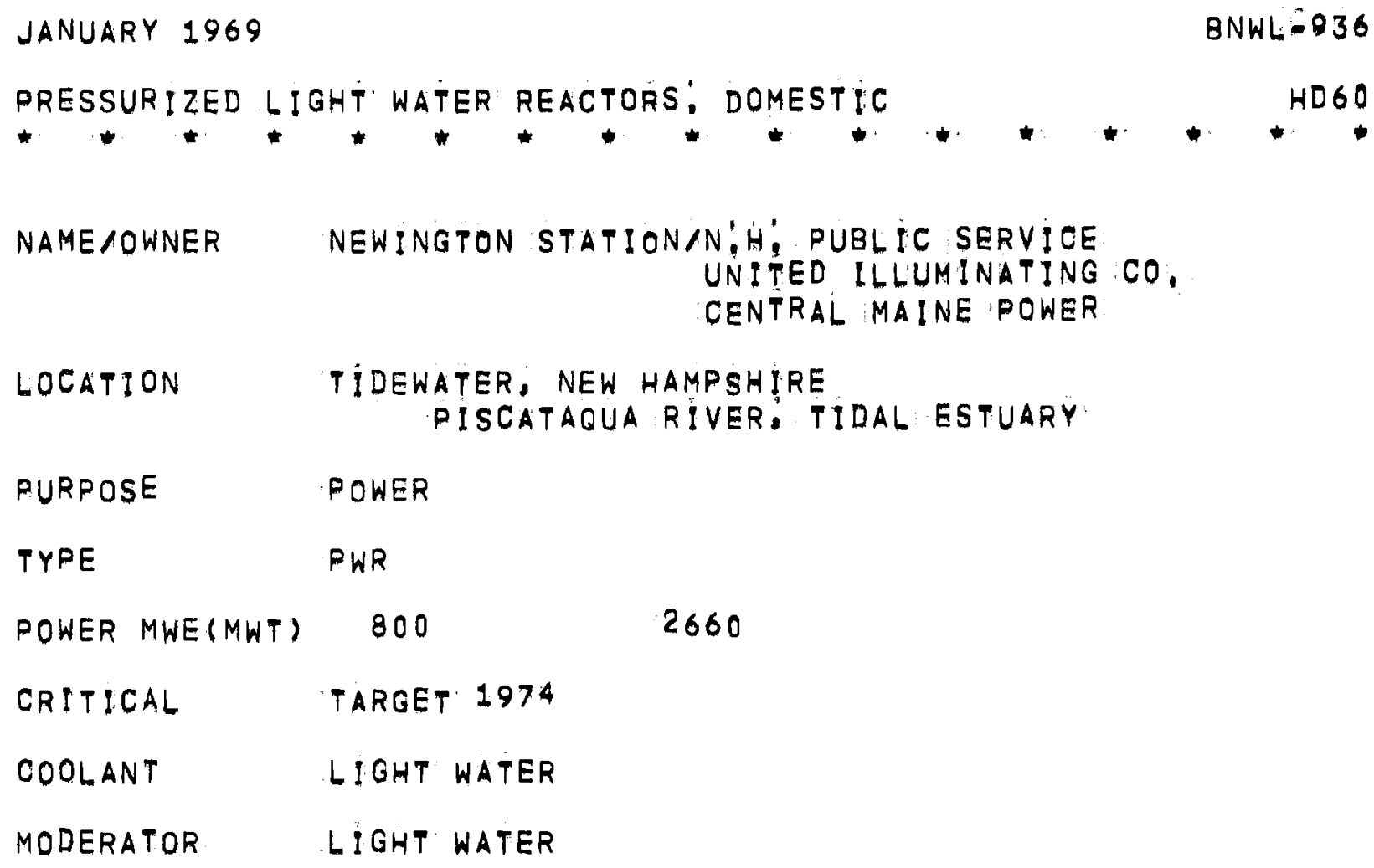


HD 61

JANUARY 1969

PRESSURIZED LIGHT WATER REACTORS: DOMESTIC

NAME / OWNER

DESIGNER

OPERATOR

LOCATION

PURPOSE

TYPE

PORTLAND GE

NEAR RAINIER, OREGON COLUMBIA RIVER

POWER

PW
TROJAN STATION/PORTLLAND GENERAL ELECTRIC CO,

WESTINGHOUSE ELECTRIC CORPORATION.

POWER MWE(MWT) 1100

CRITICAL

TARGET 1974

LIGHT WATER

LIGHT WATER

CONTRACT AWARD NOV, 1968
COOLANT

MODERATOR

REMARKS
BOWL: $=236$

HD 61

$$
\text { CONTRACT AWARD NOV, } 1968
$$


HD62

JANUARY 1969

BNWLEOSB

PRESSURIZED LIGHT WATER REACTORS: DOMESTIC

HD 62:

NAME1OWNER

DAVISAMESSE STATTON/TOLEDO EDISON COO. CLEVELAND ELECTRIC ILIUM, CO.,

DESIGNER

LOCATION

PURPOSE

TYPE

POWER AWE (NWT)

CRITICAL

COOLANT

MODERATOR

REMARKS
BABOOCK + WILCOX

NEAR OAK HARBOR: OHIO

LAKE ERIE

POWER

PHR

890

2650

TARGET 1974

LIGHT WATER

LIGHT WATER

CONTRACT AWARD NOV 1968 


\section{PRESSURIZED LIGHT WATER REACTORS \\ FOREIGN}


JANUARY 1969

BNWL $=936$

PRESSURIZED LIGHT' WATER REACTORS: FOREIGN HF O1:

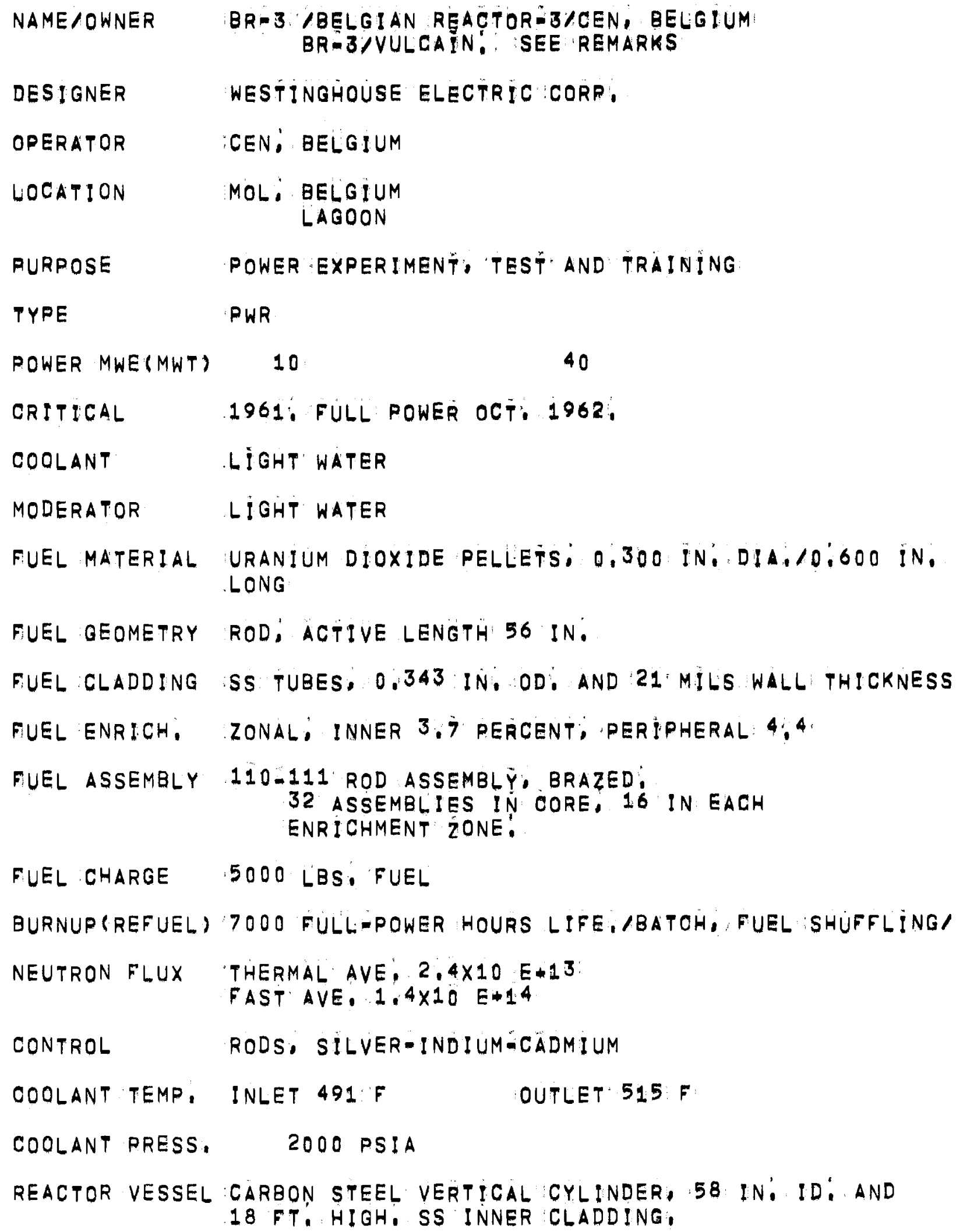


JANUARY 1969

BNWL $=936$

PRESSURIZED LIGHT WATER REACTORS: FOREIGN

HFO 1:

CONTAINMENT

STEEL VESSEL 54 FT: DIA: BY 107 FT: HIGH: INNER CONCRETE SHIELDING, $1 / 4$ BELOW GROUND LEVEL?

REMARKS

BR-3 WAS SHUT DOWN IN AUG 1963 AND RLACED AT THE DISPOSAL OF THE VULCAIN PROJECT: AFTER MODIFICATIONS: A VULCAIN CORE WAS INSTALLED IN MAY, 1966 AND CRITICAL TESTING DONE WITH BORATED WATER, FIRST CRITICALITY OF BR-3/VULCAIN WITH HEAVY WATER IN THE CIRCUITS WAS IN NOV: 1966 AND DESIGN POWER REACHED IN DEC: 1966: THE VULCAIN PROGRAM WILL BE ENDED IN 1970; WHEN A THIRD CORE CONTAINING PLUTONIUM WILL BE INSTALLED IN BR:3?

REFERENCES BR-3， GENERAL DESCRIPTION, DESIGN PHILOSOPHY AND PLANT CONSTRUCTION, CORE DESIGN AND TESTING: L MERGAN, OTHERS

NUGLEAR ENG, $5,337: 52$ /AUGUST $1960 /$

THE BR-3 REACTOR, WF DAVIS WESTINGHOUSE ENG, 20, 162:5 /NOVEMBER 1960/

VULCAIN PROJECTESTATUS REPORT: NUCLEAR ENG. 12; 34-6 /JAN, $1967 \%$

ANNUAL REPORT 1966:. CENTRE DUETUDE DE LEENERGIE NUCLEAIRE $N P-17126$ 
JANUARY 1969

$B N W L=936$

PRESSURIZED LIGHT WATER REACTORS: FOREIGN

NAME/OWNER FRANCOABELGE ARDENNES STATION/SENA, FRANCE-BELGIUM

DESIGNER WESTINGHOUSE ELECTRIC CORP:

OPERATOR SOCIETE D-ENERGIE NÜCLEAIRE FRANCOBBELGE DES

ARDENNES /SENÄ/: FRANCE

LOCATION CHOOZ; FRANCE /NEAR GIVET/ MEUSE RIVER

PURPDSE POWER, US-EURATOM PROJECT,

TYPE PWR

POWER MWE (MWT) $288 \quad 825$

SEE REMARKS

CRITICAL MARCH 1967

DOWN DUE TO MALFUNCTION IN COOLING SYSTEM

IN JAN, 1968: ON-LINE TARGET 1969:

COQLANT LIGHT WATER

MODERATOR LIGHT WATER

FUEL MATERIAL URANIUM DIOXIDE PELLETS 0:890 CM: DIAM.

FUEL GEOMETRY ROD; 2.68 M. ACTIVE LENGTH

FUEL CLADDING SS TUBES $0.978 \mathrm{CM}$. OD; WALL $0.038 . \mathrm{CM}$ :

FUEL ENRICH, 3:1 PER CENT U- 235

FUEL ASSEMBLY 209"ROD BUNDLE

CENTRAL POSITION VACANT.

EGG $=$ CRATE ASSEMBLY WITH SPRING CLIPS,

120. ASSEMBLIES/CORE

FUEL CHARGE 45000 KG URANIUM DIOXIDE

BURNUP(REFUEL) 14700 MWD/T BATCH, FUEL SHUFFLING:

NEUTRON FLUX THERMAL: 2.3 E\$13

OONTROL SS TUBES CONTAINING SILVER-INDIUM-CADMIUM ALLOY CHEMICAL SHIM

OOQLANT TEMP, INLET $267 \mathrm{C}$ OUTLET $300 \mathrm{C}$

OOQLANT PRESS: 2148 PSIA 
PRESSURIZED LIGHT WATER REACTORS: FOREIGN
$* \cdots+\cdots+\cdots+\cdots$

REACTOR VESSEL STEEL VERTICAL CYLINDER 39.4 FT: HIGH BY 10.5:FT: ID. SS INNER CLADDING

CONTA!NMENT UNDERGROUND CONSTRUCTION /CAVERN/

REMARKS

SECOND CORE WILL BE FABRICATED BY SOCIETE BELGE METALLURGIE ET MECANIQUE NUCLEAIRE.

REFERENCES THE SENA UNDERGROUND' PWR',

NUCLEAR POWER 7:79/FEBRUARY $1962 /$

THE ARDENNES NUCLLEAR STTATION,

PIERRE - A GRAU; PIERRE-A ERKES

INDUSTRICS ATOMIOUES NO. $1 / 2,1964, P ; 55.64$

ARDENNES NUCLEAR CENTRAL STATION: SPECIAL FEATURES AND IMPROVEMENTS IN COMPARISON WITH SIMILAR EXISTANT PLANTS:

P ERKES

3RD: U,N, INTL, CONF, PEAGEFUL USES OF ATOMIC ENERGY, GENEVA, 1964 ,

$A / C O N F, 28 / P / 514$

SENA- -BELGIAN-FRENCH ATOMIC POWER STATION

ATOMWIRTSCHAFT I1; 444-46 /AUGASEPT, 1966 /

ARDENNES

ENERGIE NUCLEAIRE 8; 520-34 /DEC; $1966 /$

THE NUCLEAR DESIGN OF SENA REACTOR:

FJ. FRANK

WCAP-2785 /MAY 1965 / 


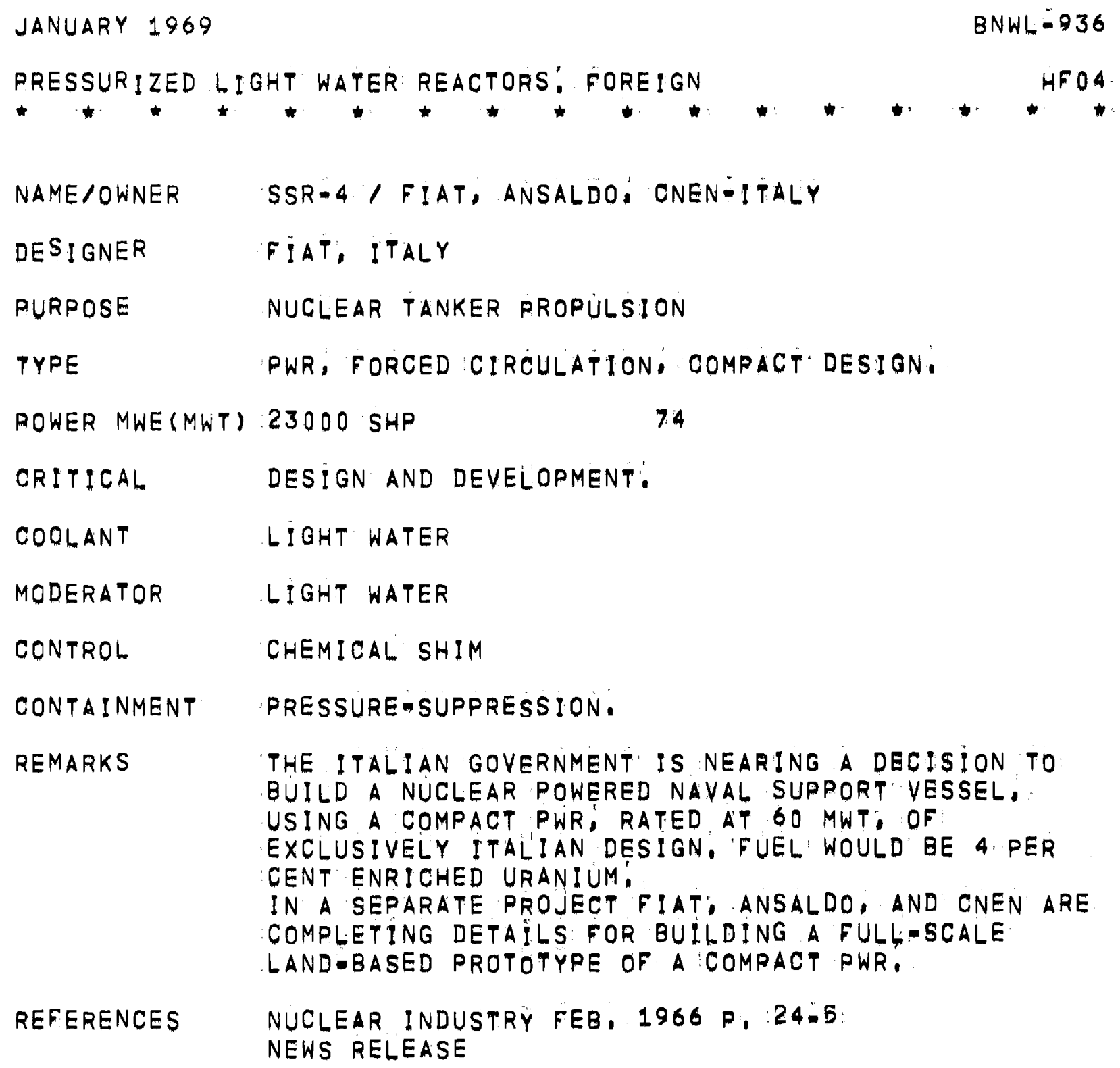


JANUARY 1969

BNWL: $=936$

RRESSURIZED LIGHT WATER REACTORS: FOREIGN

H. 05

NAME /OWNER

ENRICO FERM! NUCLEAR STATION/SELN! ITALYY

DES IGNER

WESTINGHOUSE ELECTRIC CORP,

OPERATOR

SOCIETA ELETTRONUCLEARE ITALIANA /SELNIJ

LOCATION

TRINO VERCELLESE $1 O N$ PO RIVER/, ITALY

PURPOSE

POWER

TYPE

PWR: CLOSED CYCLE

POWER MWE(MWT) 290

825

WITH CHEMICAL SHIM

CRITICAL

JUNE 1964: POWER QPERATION SEPT: 1964

OOQLANT

LIGHT WATER

MODERATOR

LIGHT WATER

FUEL MATERIAL URANIUM DIOXIDE PELLETS; 0,35 IN: DIA: SINTERED

FUEL GEOMETRY ROD; ACTIVE LENGTH 103:2 IN:

FUEL CLADDING SS TUBE; 15 MILS WALL THICKNESS

FUEL ENRICH, 2:,7 PER CENT U-235/3-REGION ENRIOHMENT/

FUEL ASSEMBLY 208-ROD ASSEMBLY, 15X15, OMISSIONS FOR: CONTROL RODS

SPRING-CLIP ASSEMBLY, BOX-TYPE

120 FUEL ASSEMBLIES

BURNUP (REFUEL)

FUEL SHUFFLING, BATOH

CONTROL

RODS, SILVER-INDIUM-CADMIUM; FUEL-ROD FOLLOWERS, CHEMICAL SHIM.

COOLANT TEMP, $\quad 539.5 \%$

COQLANT PRESS. 2200 PSIA

REACTOR VESSEL STEEL VESSEL 144 IN: OD: BY 465 IN, HIGH, 8 IN: THICK WALLS.

CONTAINMENT CONTAINMENT SHELL: ADJACENT ANNULUS PROVIDED BY SPLITTING THE SECONDARY B!OLOGICAL SHIELDING INTO TWO PORTIONS, THE ANNULUS CAN BE KEPT AT A

PRESSURE SLIGHTLY BELOW ATMOSPHERIC: 
JANUARY 1969

BNWL: $=936$

RRESSURIZED LIGHT WATER REACTOAS: FOREIGN

ḦFO5:

REMARKS

SELNI WILL PIONEER CHEMICAL SHIM CONTROL AND 3.REGION ENRICHMENT CORES! EVENTUAL OUTPUT WILL BE 290 MWE BY THE INSTALLATION OF A FURTHER TURBOGENERATOR.

REFERENCES

EUROPEAN POWER REACTYORS: ITALY': MECH, ENG: 81: 63\%NOVEMBER 1959/

ATOMIC ENERGY IN ITALY.

NUCLEAR POWER 7! 51-78/FEBRUARY $1962 /$ 


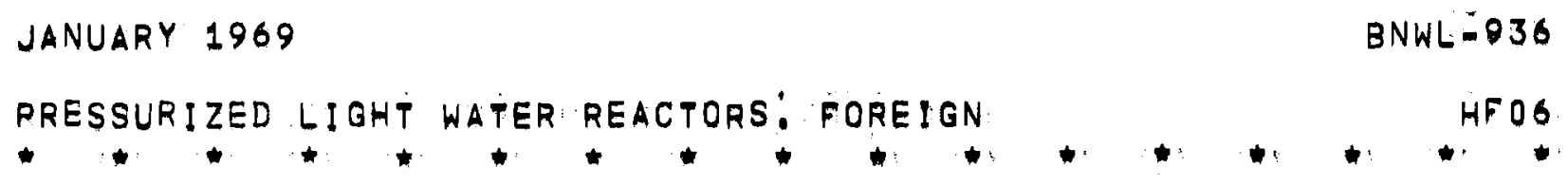

NAME IOWNER OCEANOGRAPHIC RESEARCH VESSELAJAPSD UJAPAN

DESIGNER

MITSUBISHI ATOMIC POWER INDUSTRY CO: JARAN

/MAPI/

RURPOSE SHIP PROPULSION

TYPE PWR

POWER MWE (MWT)

35

CRITICAL NO CURRENT INFORMATION

COQLANT LIGHT WATER

MODERATOR LIGHT WATER

FUEL MATERIAL URANIUM DIOXIDE

FUEL CLADDING SS

FUEL ENRICH, TWÖ-REGION OORE: LOW ENRIOHMENT.

BURNUP(REFUEL): $5860 \mathrm{MWD} / \mathrm{T}$

REMARKS JAPSD HAS WORKED OUT A SET OF BASIC CONDITIONS FOR PROPOSED CONSTRUCTION OF A 6,OOO TON OCEANIC. OBSERVATION VESSEL POWERED BY NUCLEAR ENERGY, SPECIFICATIONS WERE COMPLETED AND BIDS: REQUESTED IN FEBRUARY 1965.

REFERENCES NUCLEONICS WEEK JULY:21; 1966

NEWS RELEASE 
JANUARY 1969

$B N W L=936$

PRESSURIZED LIGHT WATER REACTORS: FOREIGN

HFO

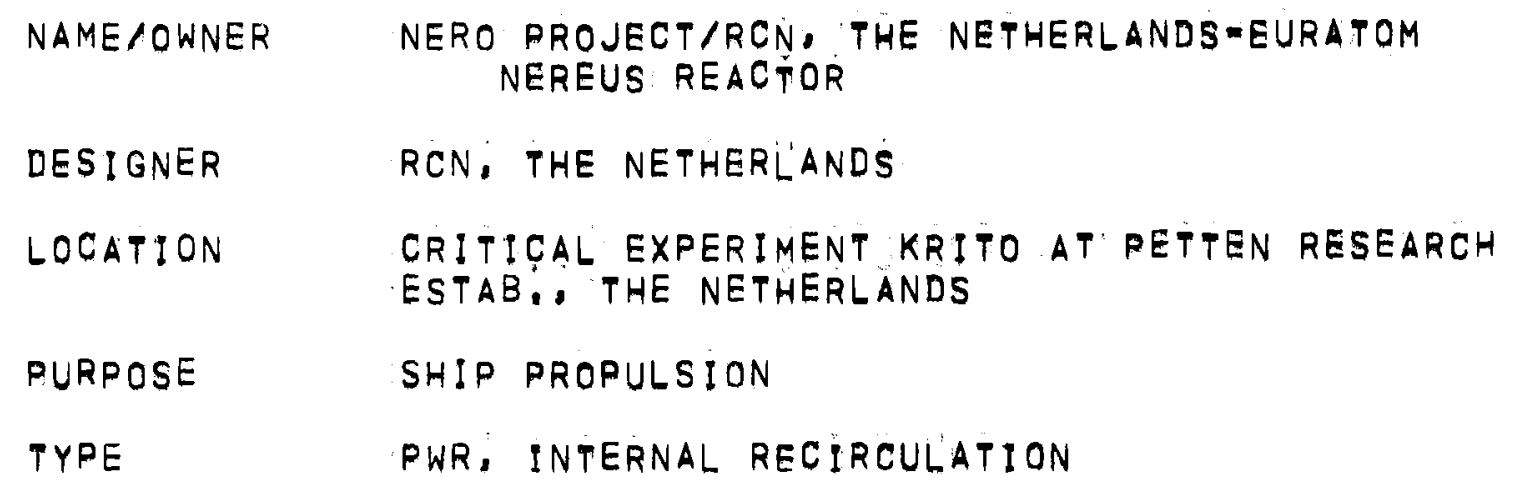

DESIGNER

RCN, THE NETHERLANDS

LOCATION

CRITICTAL EXPERIMENT KRITO AT PETTEN RESEARCH ESTAB:, THE NETHERLANDS

PURPOSE

SHIP PROPULSION

TYPE

PWR, INTERNAL RECIRCULIATION

POWER MWE(MWT) 20,000 SHP

CRITICAL

DESIGN, SEE REMARKS

COOLANT

LIGHT WATER

MODERATOR

LIGHT WATER

FUEL MATERIAL

URANIUM DIOXIDE PELLETS 10.03 MM: DIA: BORON

CARBIDE BURNABLE POISON

FUEL GEOMETRY ROD $10 \mathrm{MM}$. DIAM, ACTIVE LENGTH $1370 \mathrm{MM}$.

FUEL CLADDING ZIRCALOY TUBE 1:7 MM: THICK:

FUEL ENRICH, TWO-ZONE, 4.4 AND 4:8 PER CENT U:235

FUEL ASSEMBLY 30 FULL-SIZED ELEMENTS

12 ZIRCALOY-CLAD ELEMENTS, HEXAGONAL, EQUIPPED

WITH CLUSTER-TYPE CONTROL IRODS, 3300 FUEL RODS/CORE

FUEL CHARGE 3700 KG URANIUM DIOXIDE

BURNUP (REFUEL) 16,600 MWD/MT 4IYEAR PERIODS: URANIUM DIOXIDE

CONTROL ROD-CLUSTER CONTROL RODS WITH BORON CARBIDE BURNABLE POISON IN FUEL PELLETS

COOLANT TEMP, INLET $273 \mathrm{C}$ OUTLET $300 \mathrm{C}$ COQLANT PRESS. $150 \mathrm{KG} / \mathrm{SO}, \mathrm{CM}$.

CONTAINMENT

SPHERICAL STEEL VESSEL ENGLOSING COMPLETE PRIMARY SYSTEM. EQUIPPED WITH INTERNAL VENTILATING SYSTEM

REMARKS KRITO; CRITICAL IN MARCH 1963; IS FUELED WITH 3:1' 
PRESSURIZED LIGHT WATER REACTORS: FOREIGN

\begin{tabular}{|c|c|}
\hline & 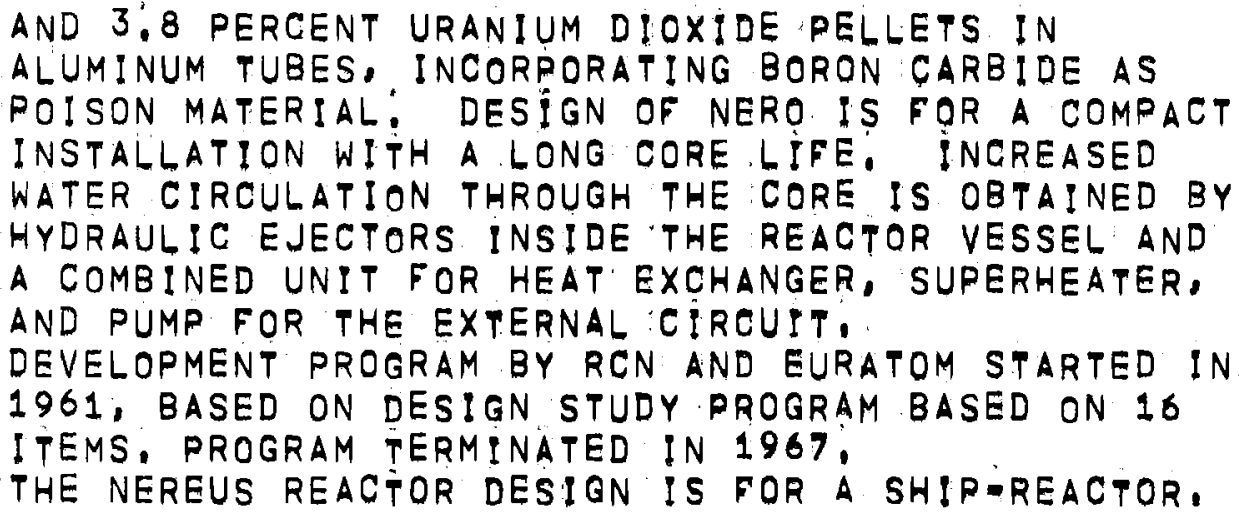 \\
\hline \multirow[t]{4}{*}{ REFERENCES } & $\begin{array}{l}\text { THE CRITICAL FACILITY KRITO } \\
\text { JG ACKERS, OTHERS } \\
\text { INTL, ATOMIC ENERGY AGENCY, } \\
\text { SYMP, OF EXPONENTIAL AND CRITICAL EXPERIMENTS } \\
\text { WITH CALCULATIONS, AMSTERDAM, SEPT, 1963, } \\
\text { EUR -A98E /1964/ }\end{array}$ \\
\hline & $\begin{array}{l}\text { THE NERO REACTOR FOR SHIP PRORULSION, } \\
\text { M BOGAART, M, MUYSKEN } \\
\text { BRDU, INTL: CONF:, PEACEFUL USES OF ATOMIC } \\
\text { ENERGY, GENEVA, } 1964 \text {. } \\
\text { A/CONF, } 28 / P / 588\end{array}$ \\
\hline & $\begin{array}{l}\text { NERO DEVELOPMENT PROGRAM. REPORT COVERING PERIOD } \\
\text { JULY } 1964 \text {-DECEMBER } 1965 \text {, } \\
\text { REACTOR CENTRUM NEDERLAND } \\
\text { EUR-3125.E /1966/ }\end{array}$ \\
\hline & $\begin{array}{l}\text { DUTCH NUCLEAR PROPULSION SHIP REACTOR } \\
\text { WHOFMAN } \\
\text { RCN=76 /1967, }\end{array}$ \\
\hline
\end{tabular}




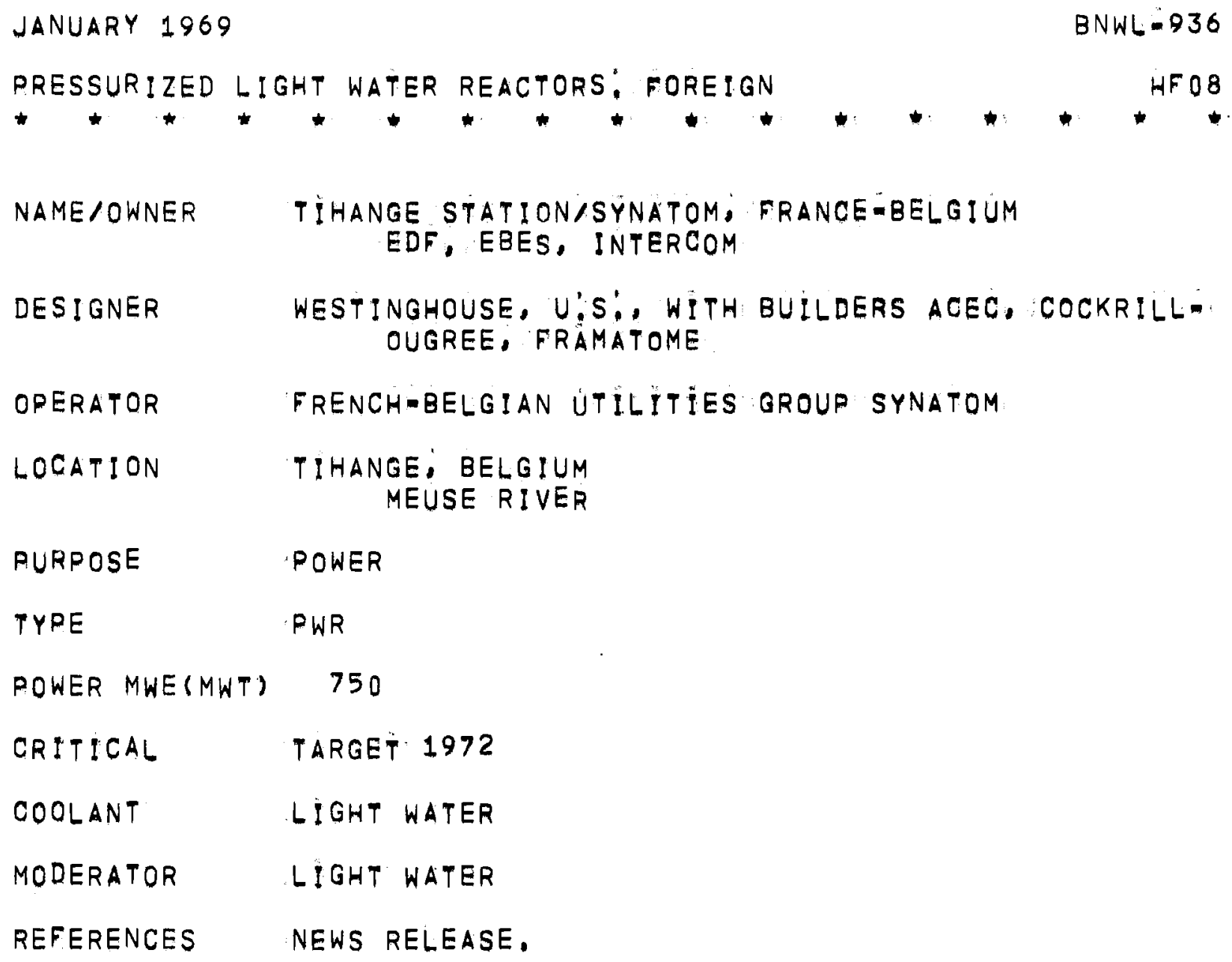




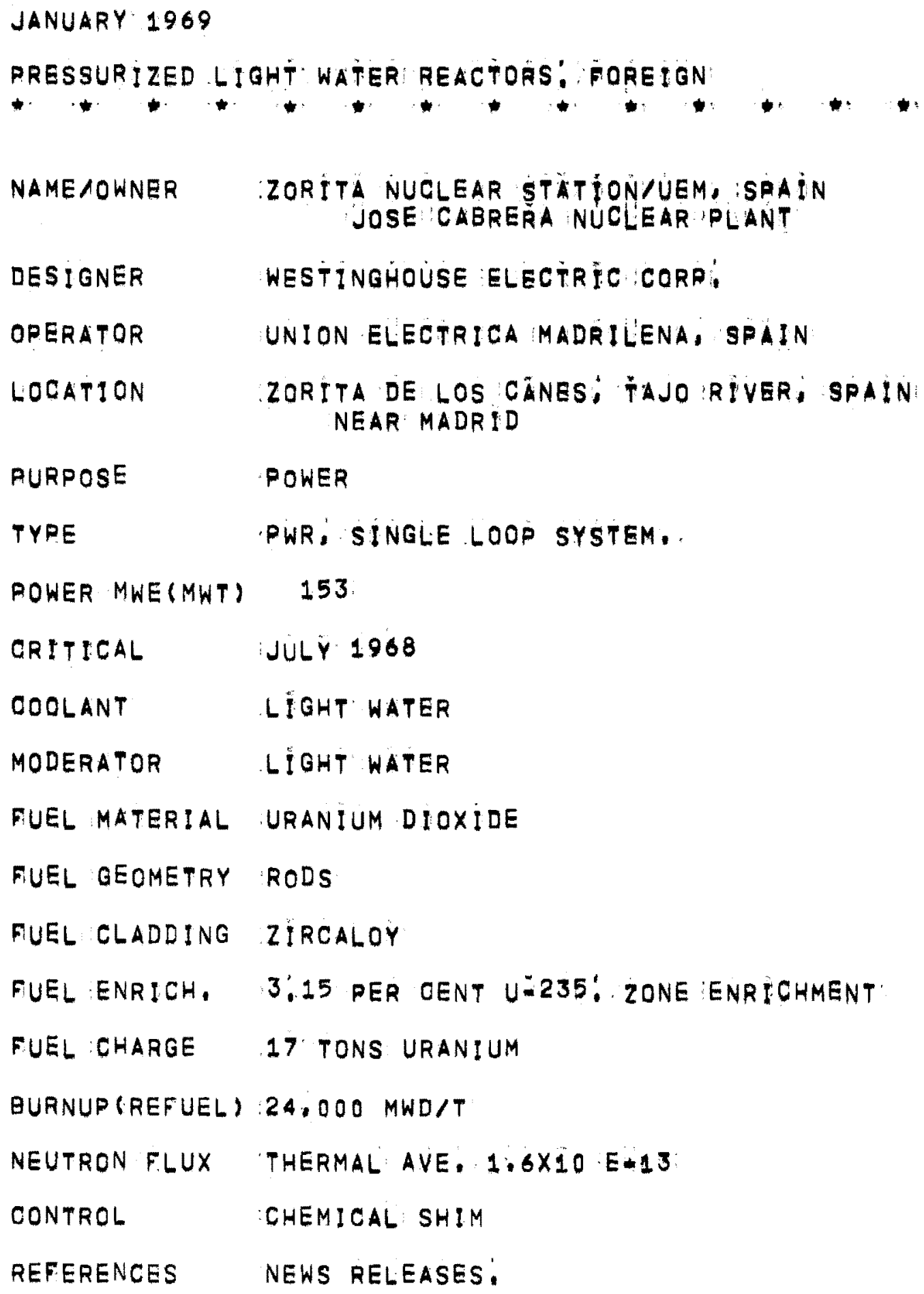


JANUARY 1969

BNWL:D36

RRESSURIZED LIGHT WATER REACTORS: FOREION

HF 10

NAME IOWNER GOTAVERKEN PROJECFYOOTAVERKENESWEDEN

DESIGNER GOTAVERKI: SWEDEN

RURPOSE TANKER RROPULSION

TYPE IPWR

POWER MWE (MWT) 30,000 SHP

CRITICAL IESIGN AND DEVELOPMENT:

COQLANT LIGHT WATER

MODERATOR IIGHT WATER

FUEL MATERIAL URANIUM: METAL

FUEL GEOMETRY SLUGS

FUEL ENRICH, 1 PER CENT U-235 OR SMALL ADDITION OF PLUTONIUM

FUEL ASSEMBLY ROTATING FUEL ELLEMENTS

FUEL CHARGE 24 TONS URANIUM

CONTROL NEUTRON ABSORPTION BY SHIELDS INCORPORATED IN THE FUEL CLADDING TUBE.

OONTAINMENT VERTICAL STEEL C̈YLINDER IN WHICH THE PRESSURE VESSEL IS SUSPENDDED

REMARKS

STUDY PROJECT; COOPERATION WITH BRITISH AND US

B + W. PRIMARY SYSTEM, VERTICAL STEEL CYLINDER

INSIDE OF WHICH THE PRESSURE VESSEL WOULD BE

SUSPENDED; CORE DESIGN FEATURES ROTATING FUEL

ELEMENTS WITH NEUTRON ABSORBENT SHIELDS COVERING A

SECTION OF THE CYLINDER SURFACE TO CONTROL REACTOR

OUTRUT. A 4-YEAR R AND D AGREEMENT ON A NUCLEAR

SHIP HAS BEEN SIGNED BY THE NORWEGIAN INST: FOR

ATOMIC ENERGY AND ATOMENERGI OF SWEDEN.

REFERENCES FIRST SCANDINAVIAN PROJECT FOR TANKER NUCLEAR PROPULSION.

FA ABADIE MMAUMERT

ENERGIE NUCLEAIRE 2:, 44-5/JANUARY-FEBRUARY 1960 / 
JANUARY 1969

BNWL $=936$

PRESSURIZED LIGHT WATER REACTORS: FOREIGN

HF 12

NAME IOWNER

DESIGNER

OPERATQR

LOCATION

RURPOSE

TYPE

POWER MWE(MWT)

QRITICAL

COOLANT

MODERATOR

FUEL MATERIAL

FUEL GEOMETRY TUBES 0:9 CM: 10.11:36 CM: OD:1170:CM: LONG

FUEL CLADDING SS INNER 0.4 MM: OUTER 0,2 MM THICK

FUEL ENRICH, 5 PER CENT U $=235$

FUEL ASSEMBLY 4 CYLINDERS, SQUARE ARRAY, AROUND CENTRAL SS PRESSURE TUBE, GRARH TE SUPPORT' STRUOTURAL MEMBER 128 FUEL CHANNELS.

FUEL CHARGE $550 \mathrm{KG}$ ENRICHED URANIUM

SPECIFIC POWER $54,5 \mathrm{KW} / \mathrm{KG}$ ENRICHED URANIUM

BURNUP(REFUEL) 20 PERCENT OF U $=235$

PARTIAL; ABOUT 2 MONTHS

NEUTRON FLUX THERMAL AVE, $5 \times 10: E 13$

CONTAOL

SHIM AND REGULATIING RODS

COOLANT TEMP, INLET 190 C

OUTLET $300 \mathrm{C}$

COOLANT PRESS. INLET $109 \mathrm{KG} / \mathrm{KO}: \mathrm{CM}$ OUTLET $100 \mathrm{KG} / \mathrm{SO}$ : CM.

REACTOR VESSEL CARBON STEEL CYLINDER 320 MM: DIA:, IS MM: WALL THICKNESS

CONTAINMENT REACTOR HALL ONLY 


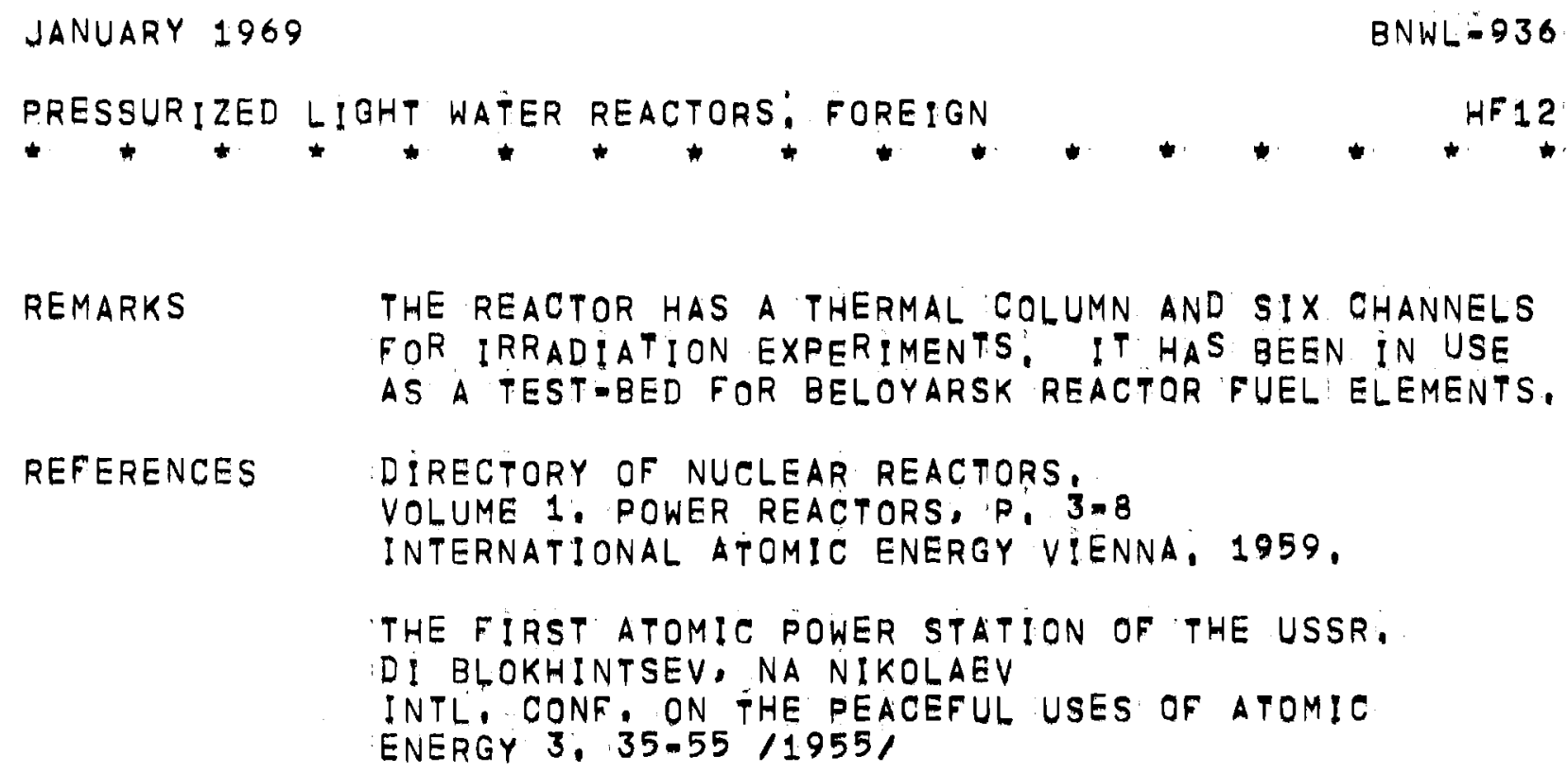




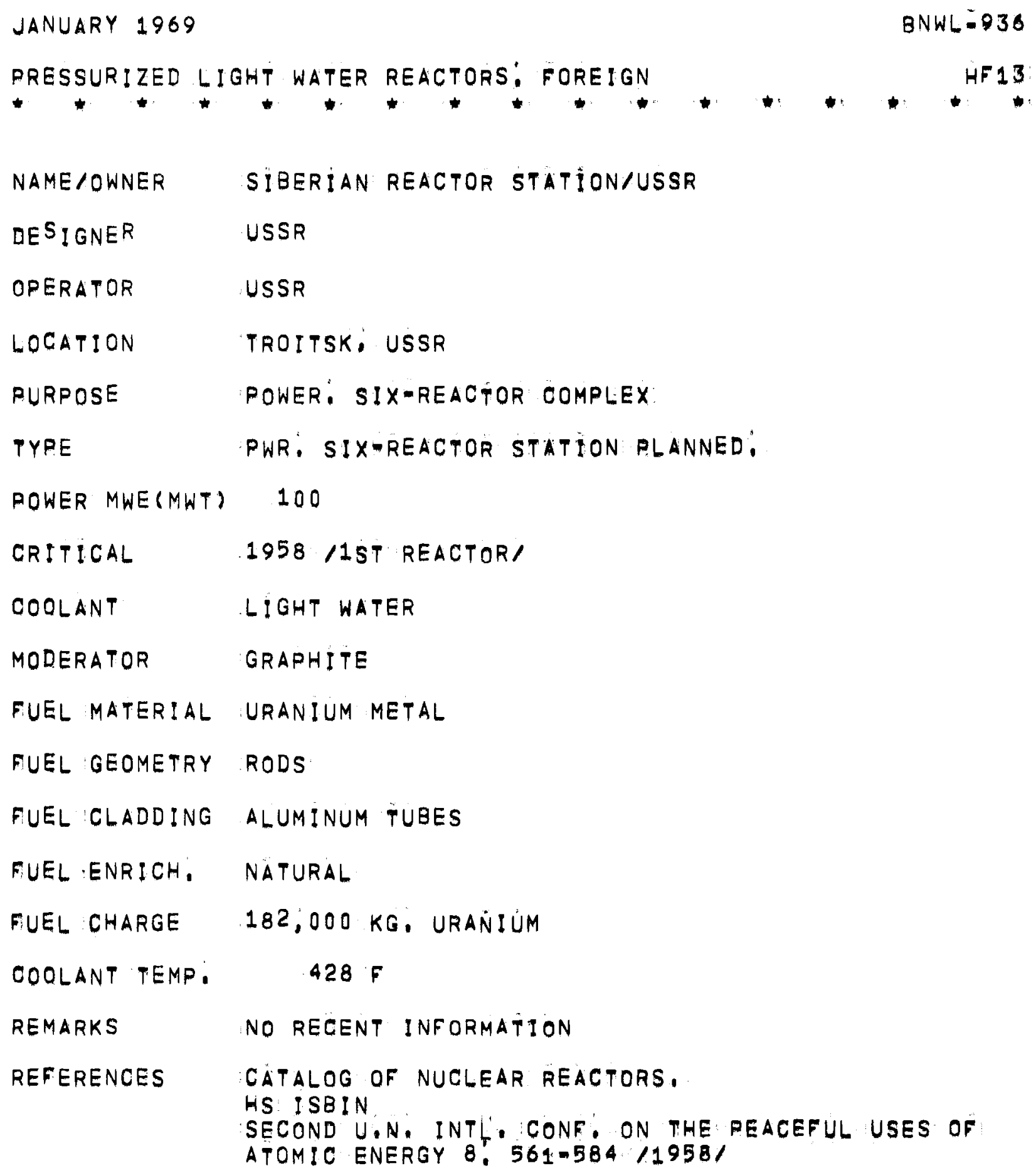




$$
\begin{aligned}
& \text { JANUARY } 1969 \text { BNWLEO36 } \\
& \text { RRESSURIZED LIGHT WATER REACTORS: FOREION HFIA. }
\end{aligned}
$$

NAME NOWNER NOVO-VORONEZH NULCLEAR STATIONAUSSR: WWER-1; WWER-2:

$\begin{array}{ll}\text { DESIGNER } & \text { USSR } \\ \text { OPERATOR } & \text { USSR } \\ \text { LOCATION } & \text { NOVO-VORONEZH; USSR } \\ & \text { DON RIVER } \\ \text { PURPOSE } & \text { POWER } \\ \text { TYPE } & \text { PWR: AIREACTOR STATION }\end{array}$

ROWER MWE (MWT) 760 LATER REACTORS UPARATED TO 360 MWE AND 1500 MWF PER REACTOR

CRITICAL

$$
\begin{aligned}
& \text { No.1: } 1963 \\
& \text { NO,2, CONSTRUCTION } 1964 \text {, TARGET } 1969
\end{aligned}
$$$$
\text { NO. 3. CONSTRUCT TON } 967 \text { TARGET } 1972
$$$$
\text { NO.4, CONSTRUCTION } 1967 \text { TAROET: } 1973
$$

$$
\text { COQLANT LIGHT WATER }
$$

MODERATOR LIGHT WATER

FUEL MATERIAL URANIUM DIOXIDE PELLEETS

FUEL GEOMETRY :ROD 1:02 CM: DIÄ. 250 CM: ACTIVE LENGTH

FUEL :CLADDING ZIRCONIUM-NIOBIUMM ALLOY SEE REMARKS

FUEL ENRICH, 1:5 PER CENT U-235 SEE REMARKS

FUEL ASSEMBLY 91-ROD ASSEMBLY IN ZIRCONIUM CANISTER 320 CM: LONG BY 16,5 CM. DIA: FUEL ELEMENTS SRAOED AROUND THE WALLS.

349 ASSEMBLIES/CORE

FUEL CHARGE 17 TONS URANIUM DIOXIDE

SPECIFIC POWER 19 KW/KG ENRICHED URANIUM

BURNUP(REFUEL) 200003000 MWD/T PÄTIAL OR TOTAL SEE REMARKS

NEUTRON FLUX THERMAL AVE' $1 \times 10$ E $\$ 13$ 


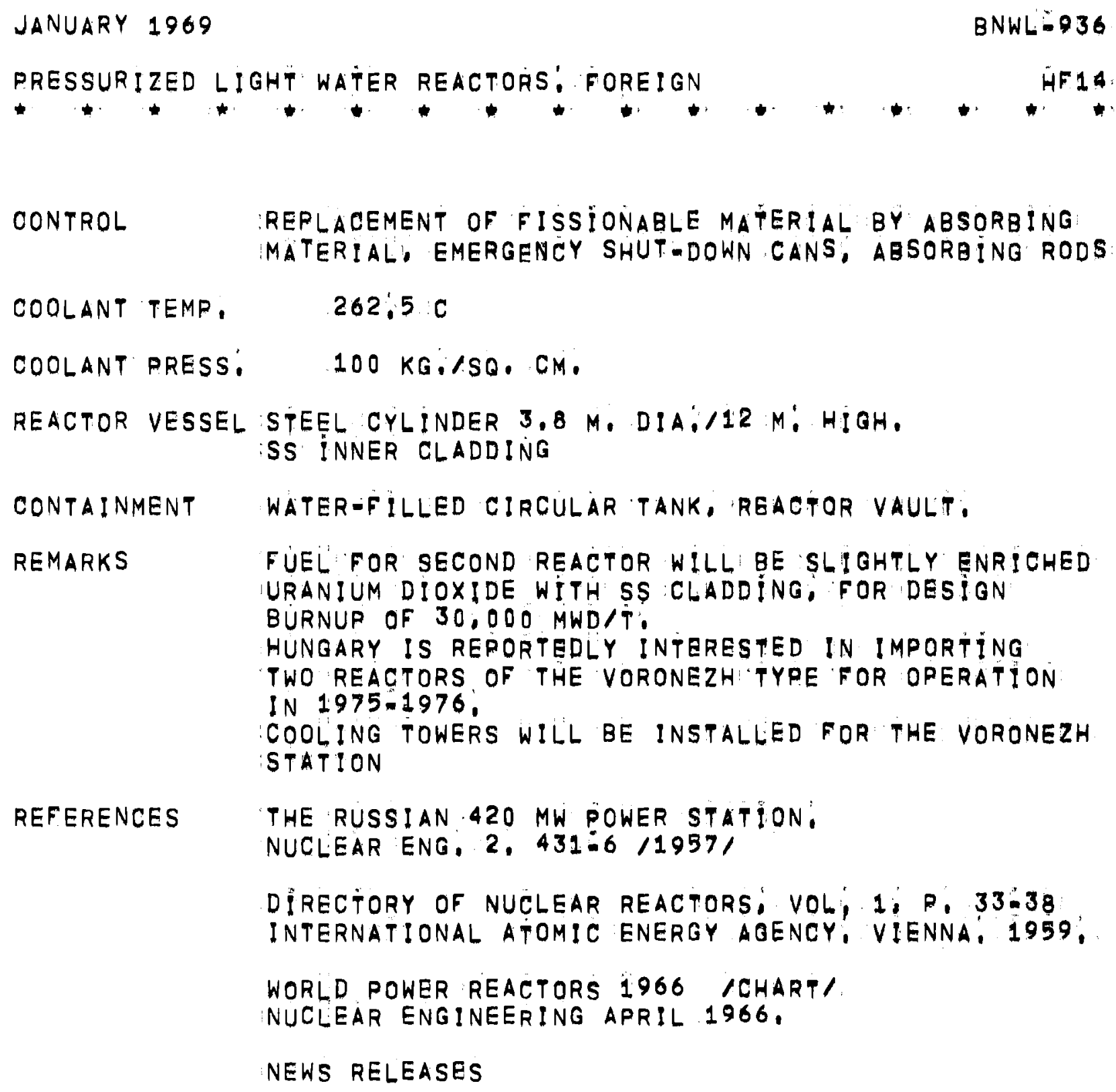



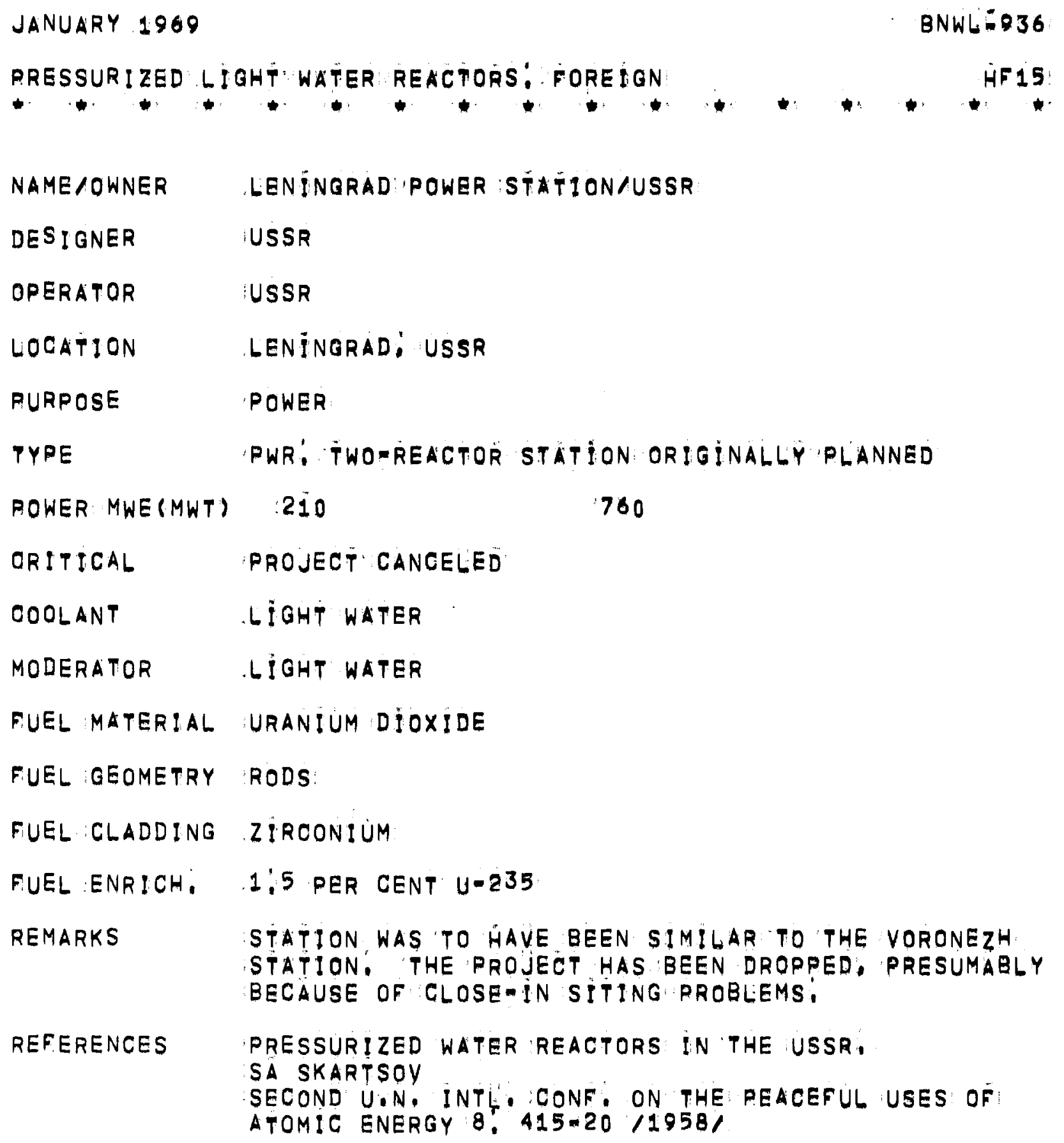


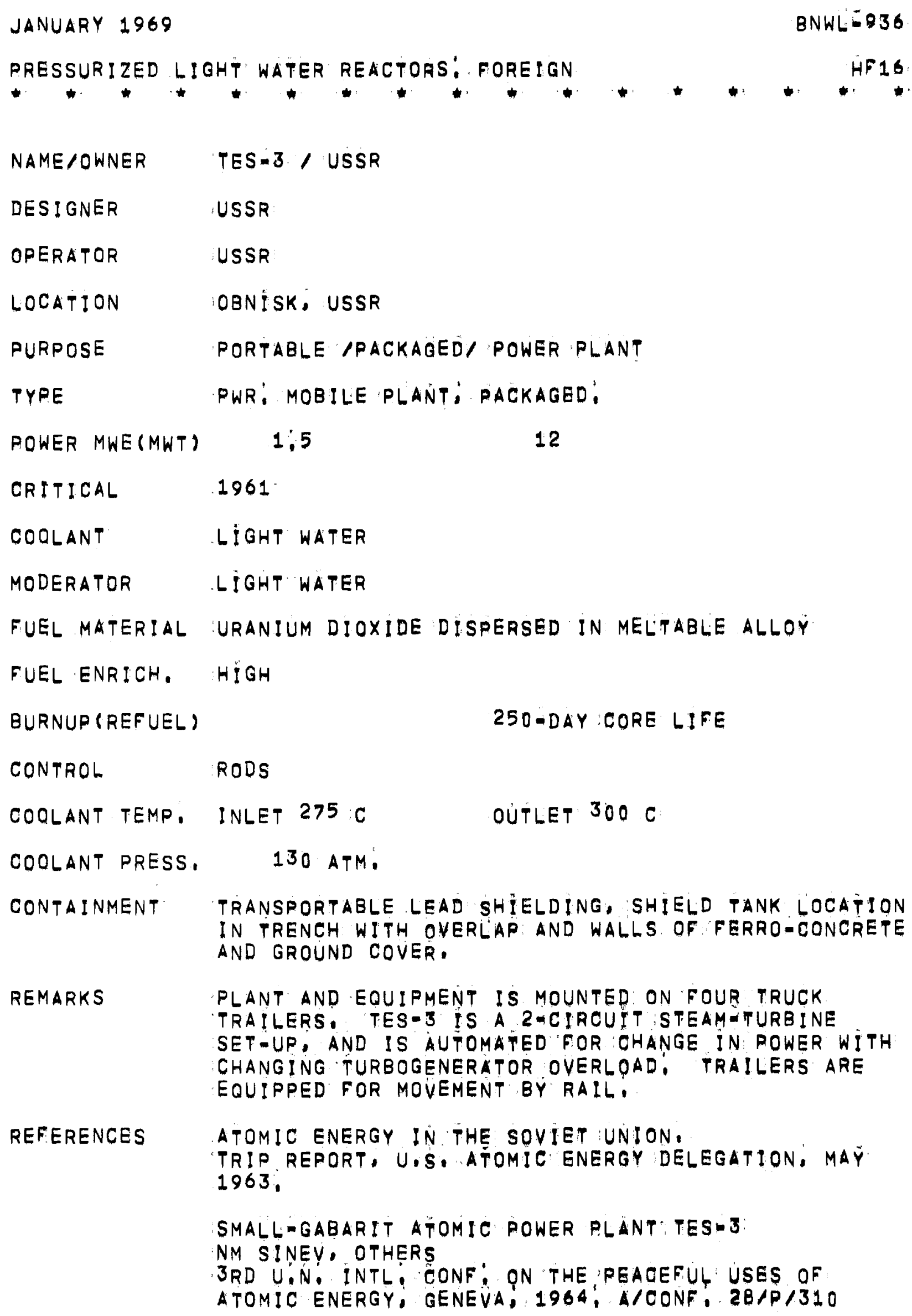




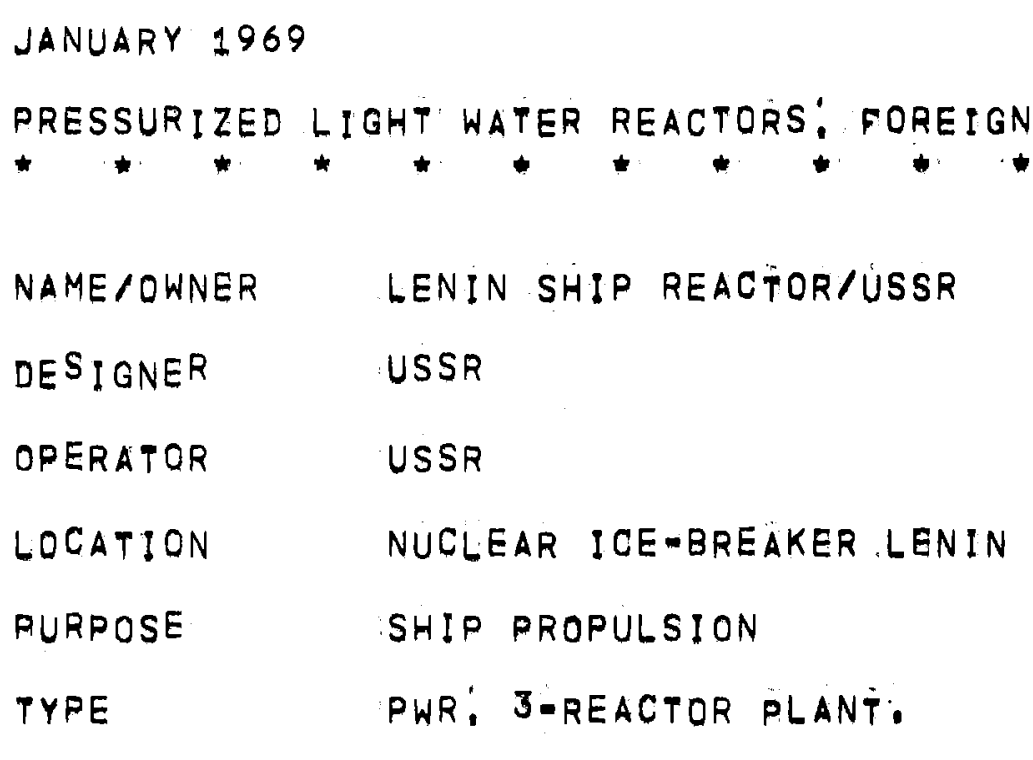

POWER MWE(MWT) SEE REMARKS 90

ORITICAL OPERATION 1959. REFÚELED IN SPRING, 1963;

COQLANT LIGHT WATER

MODERATOR LIGHT WATER

FUEL MATERIAL URANIUM DIOXIDE PELLETS

FUEL GEOMETRY ROD; 6.1 MM. DIA.:1:6 M: ACTIVE HEIGHT

FUEL CLADDING ZIRCONIUM ALLOY O:75 MM. THICK, SEE REMARKS

FUEL ENRICH, 5 PER CENT U-235

FUEL ASSEMBLY 36-ROD BUNDLE IN CIRCULLAR SHROUD, IN CONTAINMENT CHANNELS OF ZIRCONIUM ALLOY: 219 CHANNELS

FUEL CHARGE $80 \mathrm{KG}: \mathrm{U}-235$

BURNUP(REFUEL) $11000-13000$ MWD/T

CONTROL BURNABLE, POISON DISTRIBUTED IN CONTAINMENT CHANNELS, RODS.

COOLANT TEMP, INLET 261:C OUTLET $317 \mathrm{C}$

REACTOR VESSEL CYLINDRICAL, 2 M. OD. BY 5 M: HIGH, LOW-ALLOY STEEL WITH SS SHELL!

CONTAINMENT STEEL AND LIMONITE CONCRETE PLUS SHIELDING:

REMARKS WITH THREE REACTORS OPERATING; THE FULL POWER OF 40,000 SHP CAN BE OBTAINED, NORMALLY ONLY TWO REACTORS OPERATE, ON THE FIRST REFUELING, ONE REACTOR WAS CHARGED WITH STAINLESS: STEEL CLAD 


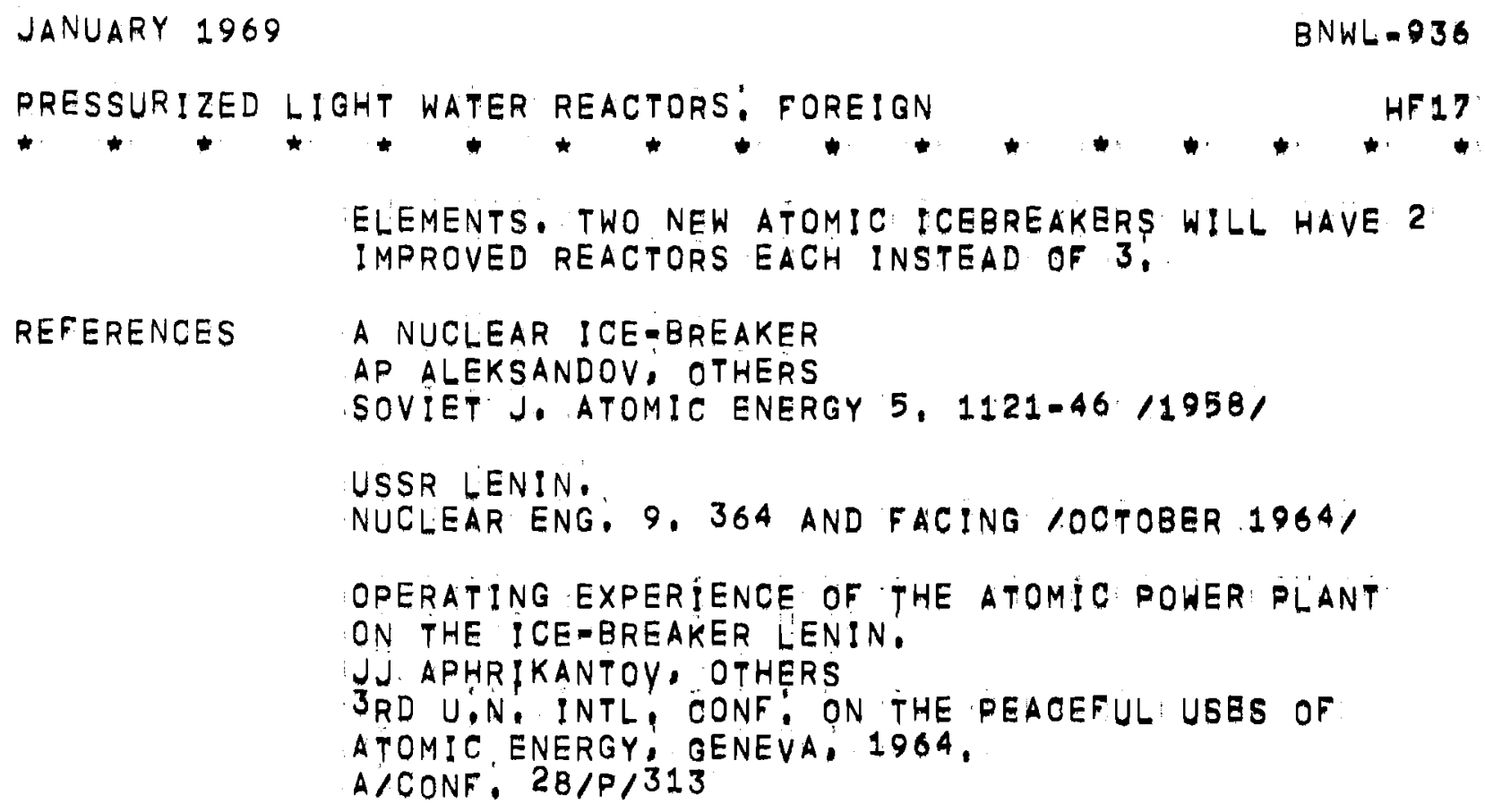


JANUARY 1969

$B N W L=036$

PRESSURIZED LIGHT WATER REACTORS: FOREIGN

HF 18

NAME/OWNER

SHIP REACTOR STUDIES /UK AEA

PURPOSE

SHIP PROPULSION

REMARKS

THE UK AEA HAS JOINED BELGONUCLEAIRE IN THE JOINT DEVELOPMENT OF THE VULCAIN CONCEPT FOR NUGLEAR SHIP PROPULSION, CURRENTLY ALL SHIP DESIGNS HAVE BEEN REPORTED AS DROPPED EXCEPT THE VULCAIN CONCEPT AND THE IBR /INTEGRAL BOILING REACTOR/: A PRESSURE TUBE SYSTEM. 

JANUARY 1969
PRESSURIZED LIGHT WATER REACTORS: FOREIGN

NAME/OWNER FDR /FORTSCHNITTLICHER DRUCKWASSERREAKTOR//GKSS OTTO HAHN

DESIGNER DEUTSCH BAW-INTERATOM: SEE REMARKS

OPERATOR GKSS-W GERMANY

RURPOSE SHIP PROPULSION; ORE CARRIER OTTO HAHN,

TYPE ADVANCED PWR, INTEGRAL DESIGN

POWER MWE(MWT) 10,000-11,000 SHP 38

CRITICAL FIRST CRUISE OF THE OTTO HAHN INTO THE BALTIC SEA IN NOVEMBER, 1968:

COQLANT LIGHT WATER

MODERATOR LIGHT WATER

FUEL MATERIAL URANIUM DIOXIDE PELLEETS

FUEL GEOMETRY ROD; 11:0 MM, DIA:, ACT: LENGTH 1120 MM,

FUEL CLADDING SS TUBES, WALL THICKNESS: 0,35 MM:

FUEL ENRICH, ZONE ENRICHMENT $2 ; 5 ; 2 ; 9 ; 3 ; 5$ AND $4: 3$ PER CENT

FUEL ASSEMBLY 226:ROD BUNDLES; $17 \times 17$ SQUARE; AND TRIANGULAR CORNER ASSEMBLIES:

16 FUEL ASSEMBLIES/OORE

FUEL CHARGE 2:95 TONS URANIUM DIOXIDE

BURNUP(REFUEL) $7200.14 ; 000$ MWDIT 3-YEAR CORE LIFE

NEUTRON FLUX THERMAL AVE, $1 \times 10$ E +13

FAST AVE. $7.5 \times 10$ E +13

CONTROL RODS, BORON CARBIDE-SS, AND BURNABLE POISON ZIRCONIUM BORIDE/ZIRCONIUM DIOXIDE

COOLANT TEMP, INLET $266 \mathrm{C}$ OUTLET $278 \mathrm{C}$

COQLANT PRESS. $\quad 63.5 \mathrm{KG} / \mathrm{SQ}$. CM.

REACTOR VESSEL VESSEL OF FORGED RINGS, 2300 MM: DIAI/ 8700 MM I HIGH, DIVIDED BY A CYLINDRICAL WALL INTO INNER AND OUTER SPACE. 
JANUARY 1969

BNWL:-036

PRESSURIZED LIGHT WATER REACTOAS: FOREIGN

CONTAINMENT VERTICAL CYLINDER 13:1:M:IHIGH, 9.5 M:DIA:, 30 MM WALL THICKNESS.

REMARKS

AWARD WAS TO DEUTSCH B $+W$ AND INTERATOM FOR THE U.S: B+W DEVELOPED CONSOL IDATED NUCLEAR STEAM GENERATOR: POWER PLANT WILL BE INSTALLED IN THE RESEARCH VESSEL OTTO HAHN, WHICH WAS LAUNCHED IN JUNE 1964:

REFERENCES GERMANY=S NUCLEAR SHIP:

NUCLEAR ENG. 9.8/JANUARY $1964 /$

ADVANCED PRESSURIZED-WATER REACTOR FOR FIRST GERMAN NUCLEAR ENERGY SHIP.

W KLIEFOTH

ATOMKERNENERGIE 8:, 458.60 /DEC, 1963 /

THE REACTOR PLANT OF THE RESEAROH SHIP OF GESELLSCHAFT FOR KERNENERGIEVERWERTUNG IN SCHIFFBAU U. SCHIFFAHRT MBH: W WIEBE; OTHERS

3RD U.N. INTL: CONF! PEACEFUL USES OF ATOMIC ENERGY, GENEVA, 1964,

$A / C O N F, 28 / P / 540$

NUCLEAR SHIP PROPULLSION

EURO-NUCLEAR 2/4/, 174-8 /APRIL $1965 /$

GERMANY-S NUCLEAR SHIP

C SALANDER, D UL'KEN /GKSS/

NUCLEAR ENG. 10:324-9 /SEPT: $1965 /$

EXPERIMENTAL ORE CARRIER, OTTO HAHN

ENGINEER 219,54-6 /JAN, 1, 1965/ 
JANUARY 1969

BNWL:-936

PRESSUR!ZED LIGHT WATER REACTORS: FOREIGN

HF 21

NAME / OWNER

OBRIGHEIM NUCLEAR STATION/KWO，W: GERMANY

DES I

SIEMENS-SCHUCKERTWERKE

OPERATOR

KERNKRAFTWERK OBRIGHEIM GMBH /KWO/, W. GERMANY

LOCATION OBRIGHEIM AM NECKAR: W: GERMANY NECKAR RIVER

PURPOSE

POWER

TYPE

PWR, CLOSED CYCLE; TWO-LOOP SYSTEM

POWER MWE (MNT)

283

$907: 5$

CRITICAL

TARGET FOR CORE LOADING 1968

COOLANT

LIGHT WATER

MODERATOR

LIGHT WATER

FUEL MATERIAL

URANIUM DIOXIDE

FUEL GEOMETRY ROD; ACTIVE LENGTH 2750 MM, 10.7 MM OD:

FUEL CLADDING ZIRCALOYM 4 TUBES

FUEL ENRICH, AVE; 3 PER CENT U-235; REGIONAL

FUEL ASSEMBLY 180-ROD BUNDLE $14 \times 14: 6$ SITES ARE OCCUPIED BY CONTROL-ROD GUIDE TUBES.

SPRING-CLIP ASSEMBL $\dot{Y}$

121 ELEMENTS/CORE

FUEL CHARGE $35,200 \mathrm{KG}$ URANIUM

BURNUP (REFUEL) 24,000 MWD/T

FUEL SHUFF, ING

$1 / 3$ CORE

CONTROL RODICLUSTER CONTROL

CHEMICAL SHIM

COOLANT TEMP. INLET $283 \mathrm{C}$

OUTLET 310:4 C

COOLANT PRESS.

$145 \mathrm{KG} / \mathrm{SQ}$; CM: $A B S$.

REACTOR VESSEL

C-STEEL CYLINDER $3,27 \mathrm{M}$, ID, $10 \mathrm{M}$, HIGH $S S=C L A D$ 


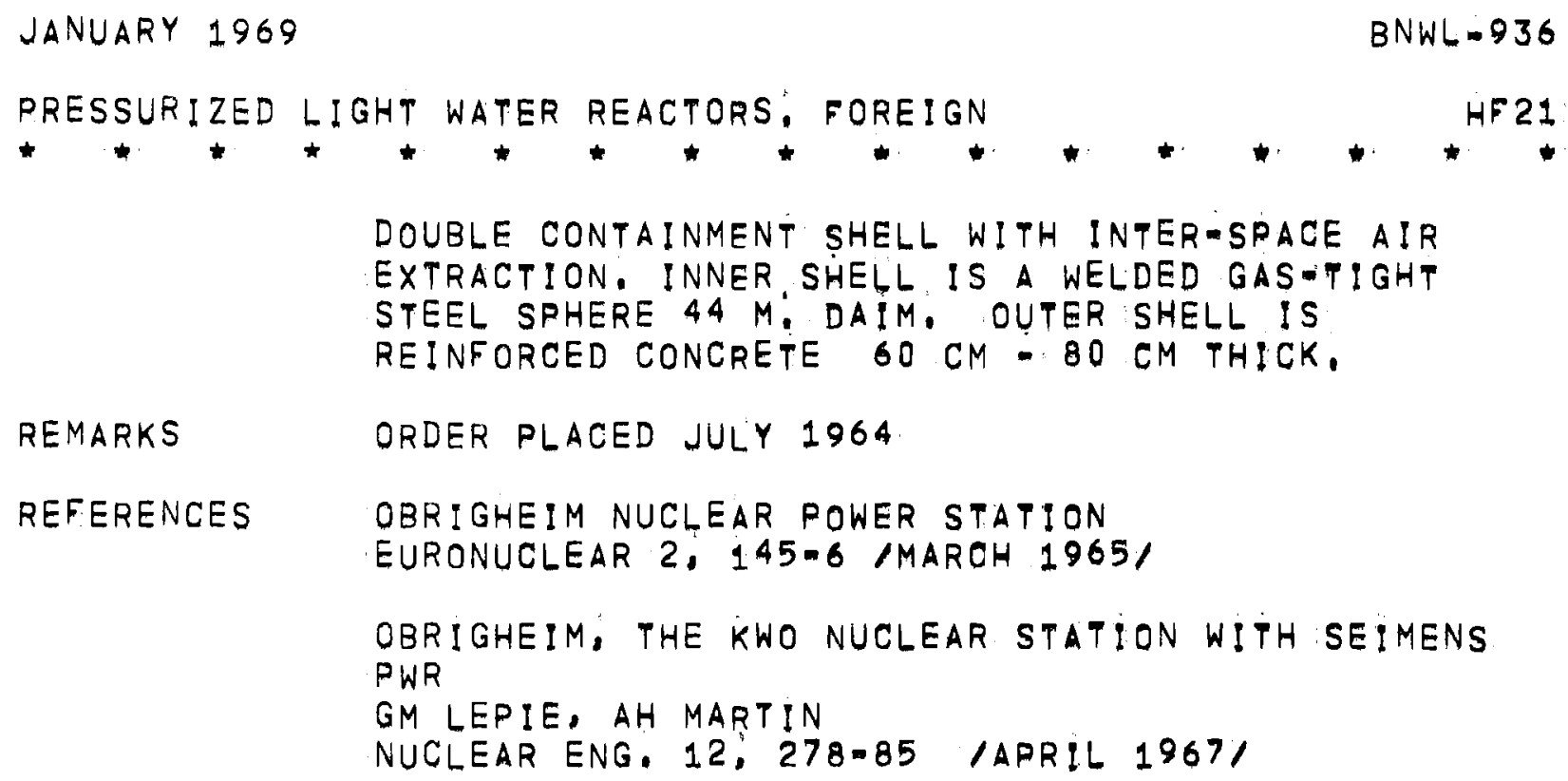

REMARKS

ORDER PLACED JULY 1964

REFERENCES

OBRIGHEIM NUCLEAR POWER STATION

EURONUCLEAR 2, 145-6/MARCH $1965 /$

OBRIGHEIM; THE KWO NUCLEAR STATION WITH SEIMENS PWR

GM LEPIE, AH MARTIN

NUCLEAR ENG, 12; 278-85 /APRIL $1967 /$ 
JANUARY 1969

BNWL $=936$

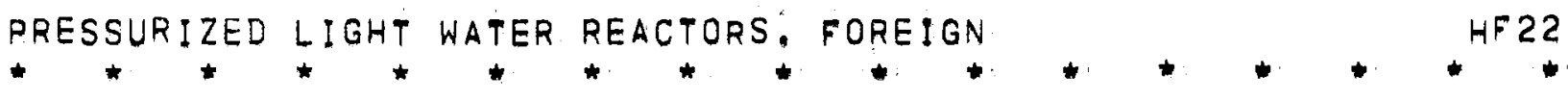

NAME/OWNER NOK POWER STATION/NOK: SWITZERLAND

BEZNAU NUCLEAR STATION

DESIGNER WESTINGHOUSE ELECTRIC CORP:

OPERATOR NOK /FORCES ELECTRIQUES DU NORD-EST DE LA SUISSE/

LOCATION BEZNAU-DOTTINGEN, SWITZERLAND

ON AAR RIVER

PURPOSE POWER

TYPE PWR, CLOSED CYCLE: TWOREACTOR STATION.

POWER MWE(MNT) $350 \quad 1000$ PER REACTOR

CRITICAL TARGET NO:1,1969

No.2, 1972

COQLANT LIGHT WATER

MODERATOR LIGHT WATER

FUEL MATERIAL URANIUM DIOXIDE PELLETS

FUEL GEOMETRY ROD

FUEL CLADDING ZIRCALOY

FUEL ENRICH, ZONE, AVE: 3 PER CENT U-235

FUEL ASSEMBLY ROD BUNDLE

120 ASSEMBLIES/CORE

SPRING-CLIP ASSEMBLY

FUEL CHARGE 40 TONS URANIUM

SPECIFIC POWER $28 \mathrm{KW} / K G$ URANIUM

CONTROL SILVER-INDIUM-CADMIUM RODS, SS CLAD CHEMICAL SHIM

COQLANT PRESS, $\quad 210,4$ PSIA

REACTOR VESSEL STEEL VESSEL 10.7 M, HIGH, $37 \mathrm{M}$. ID. WALL THICKNESS 16.6 CM:

CONTAINMENT DOUBLE=WALL ENCLOSURE CONSISTING OF A STEEL CYLINDER $61 \mathrm{M}$ : HIGH: $33 \mathrm{M}$. ID, SURROUNDED BY AIR-TIGHT CONCRETE CYLINDER $38 \mathrm{M}$. ID: ANNULAR 


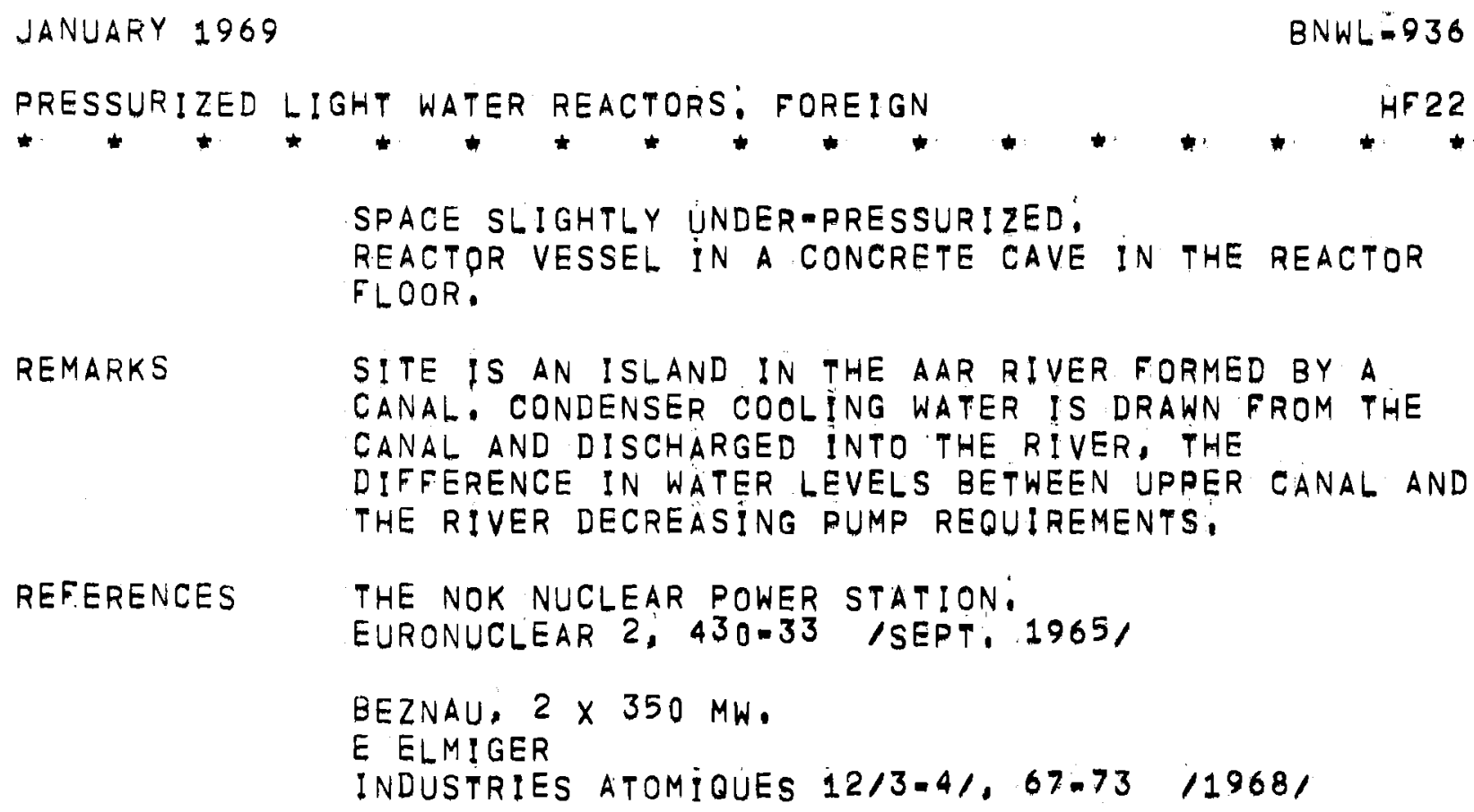




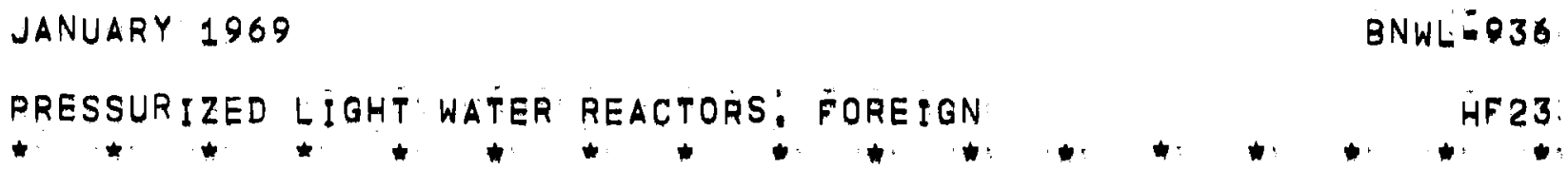




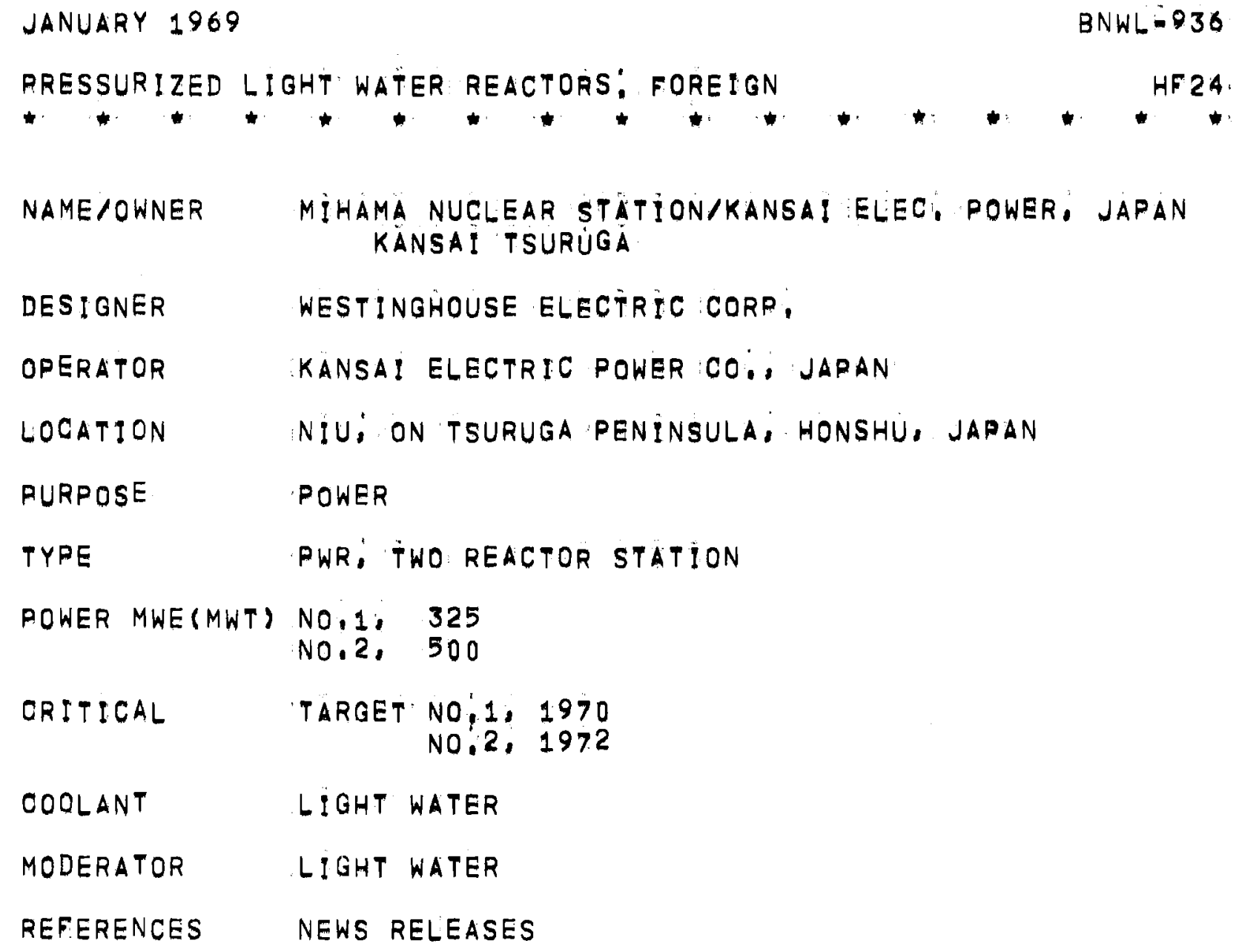




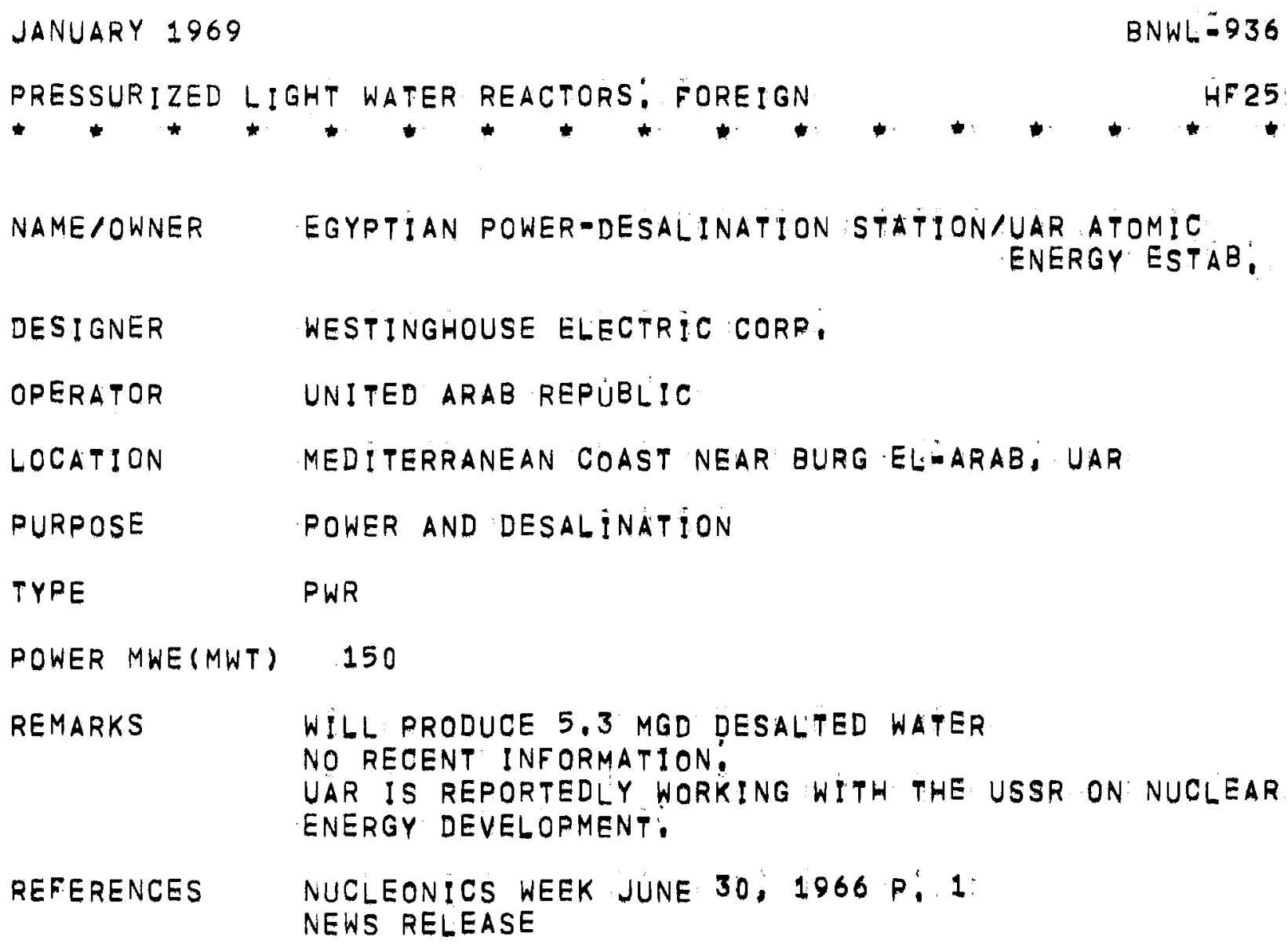




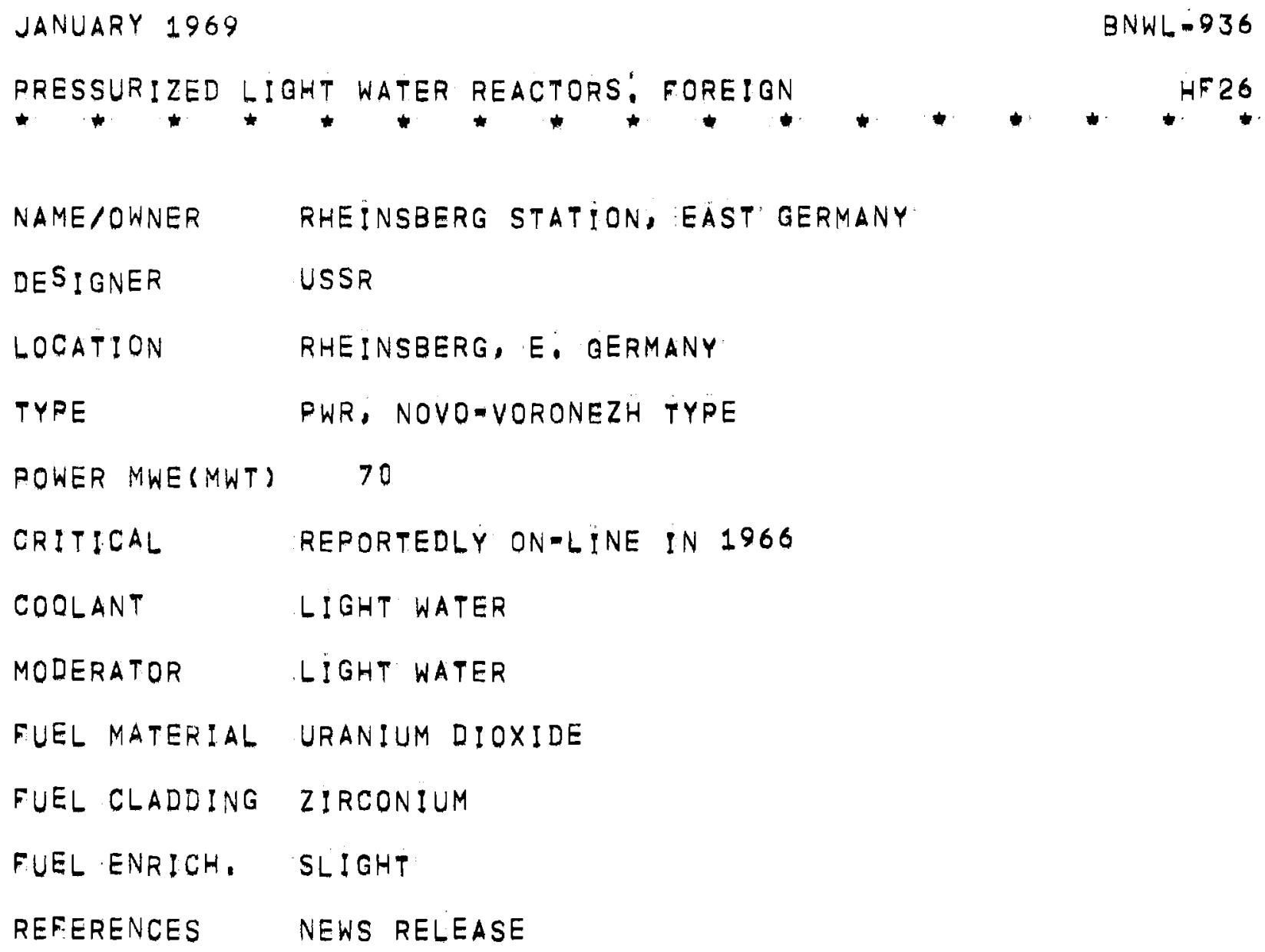


JANUARY 1969

$B N W L=936$

PRESSURIZED LIGHT WATER REACTORS: FOREION

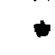

NAME / OWNER

DOEL STATION/EBES; BELGIUM FLANDERS STATION

DES!GNER

ATELIERS DE CONSTRUCTIONS ELECTRIOUES DE CHARLEROI IACEC/, BELGIUM

OPERATOR

TRACTION ET ELEETT:

LOCATION

DOELL: NEAR ANTWERP; BELGIUM

PURPOSE

POWER

TYPE

PWR: TWOMREACTOR STATION

ROWER MWE (MWT) 365

PER REACTOR

CRITICAL

TARGET NO:1, 1973
NO:2, 1975

COOLANT

LIGHT WATER

MODERATOR

LIGHT WATER

REMARKS

CONTRACT AWARD 1968

REF:ERENCES

NEWS RELEASES 


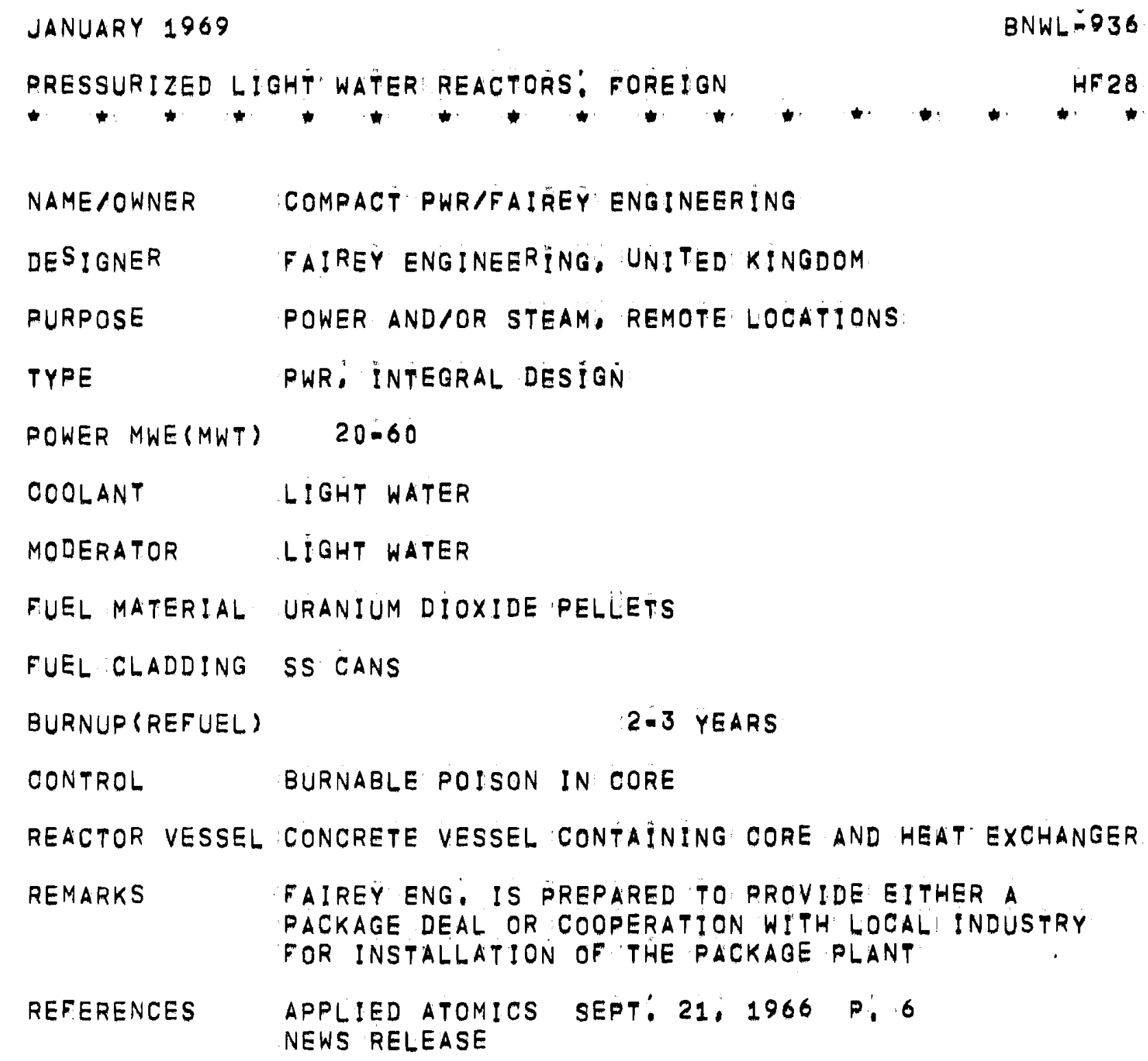




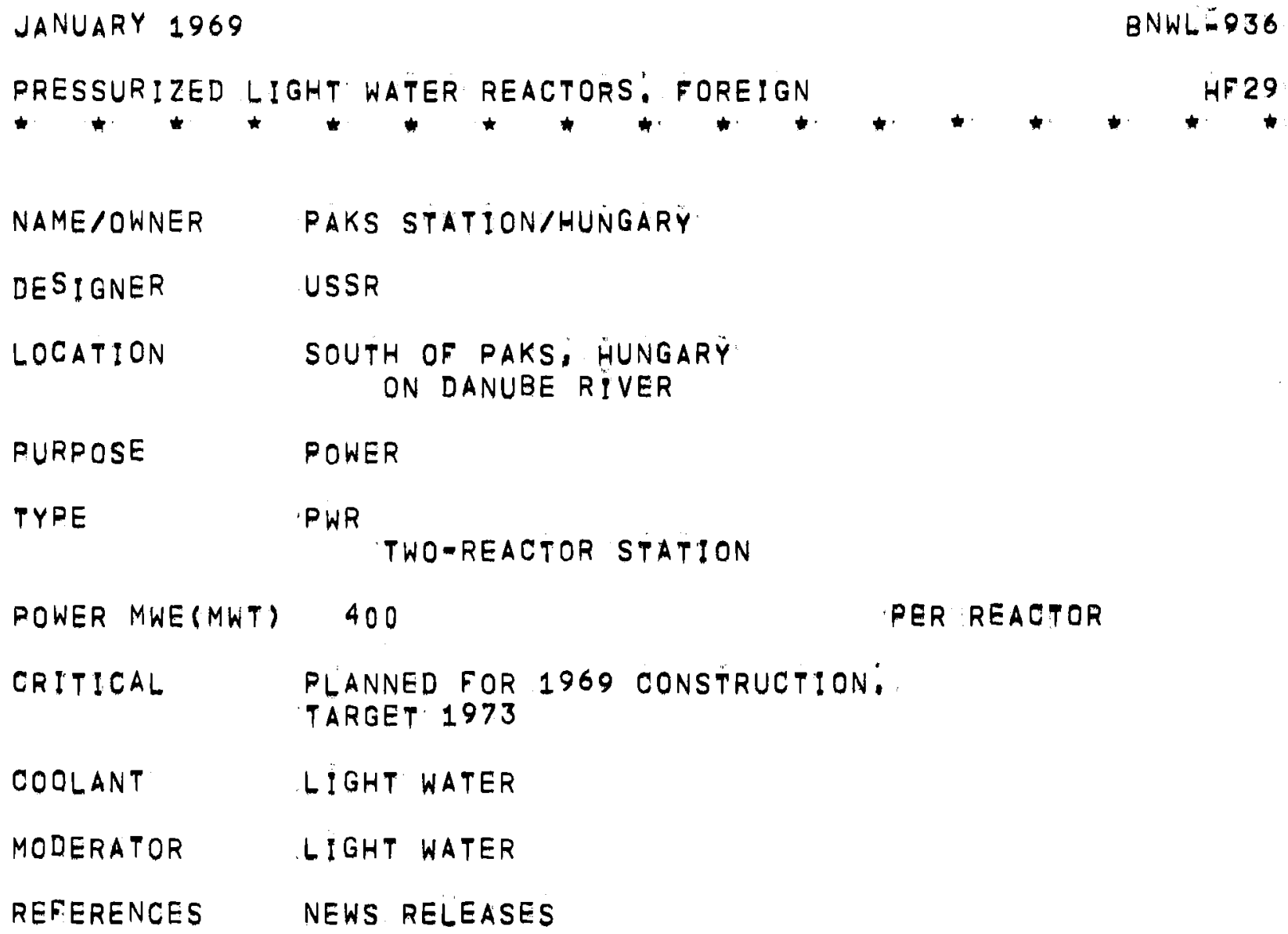




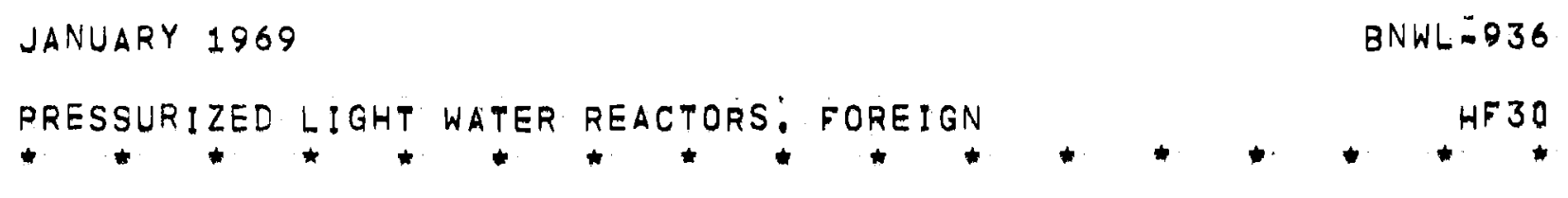

NAME/OWNER STADE STATION/NKW-HEW; W: GERMANY

DESIGNER SEIMENS-SCHUCKERTWERKE

OPERATOR NORDWESTDEUTSCHE KRAFTWERK AG INWK/ AND
HAMBURGISCHE ELETRIZITATSWERKE IHEW/:W, GERMANY:

LOCATION STADERSAND, AT STADE NEAR HAMBURG, W, GERMANY

ON ELBE RIVER

PURPOSE POWER

TYPE PWR, 4-LOOP SYSTEM

POWER MWE(MWT) $630 \quad 1900$

CRITICAL TARGET 1972

COQLANT LIGHT WATER

MODERATOR LIGHT WATER

FUEL MATERIAL URANIUM DIOXIDE

FUEL GEOMETRY PIN; ACTIVE LENGTH $3 \mathrm{M}$.

FUEL :CLADDING ZIRCALOYMA TUBES, $10,74 \mathrm{MM}$, OD, 0,72 MM, WALL

FUEL ENRICH, 2:5 PER CENT U-235, REGIONAL

FUEL ASSEMBLY 2O4-PIN BUNDLE
EACH BUNDLE CONTAINS A $20-R O D$ CONTROL CLUSTER 157 ASSEMBLIES/CORE

F.UEL CHARGE 56,2 TONS URANIUM

BURNUP(REFUEL) $23,000-31,000 \mathrm{MWD} / T$ ANNUAL, BATCH

CONTROL ROD-CLUSTER CONTROL

SOLUBLE ROISON /BORIC: ACID/ IN COOLANT

COQLANT TEMP, INLET $288: 5 \mathrm{C}$ OUTLET $316,3 \mathrm{C}$

COOLANT PRESS. 158 ATM.

REACTOR VESSEL PRESSURE VESSEL $4: 08$ M. DIAM.. $8.148 \mathrm{M}$. HIGH

REFERENCES STADE AND WURGASTSEN

NUCLEAR ENG. 12; 750-7, 10CT: $1967 \%$ 


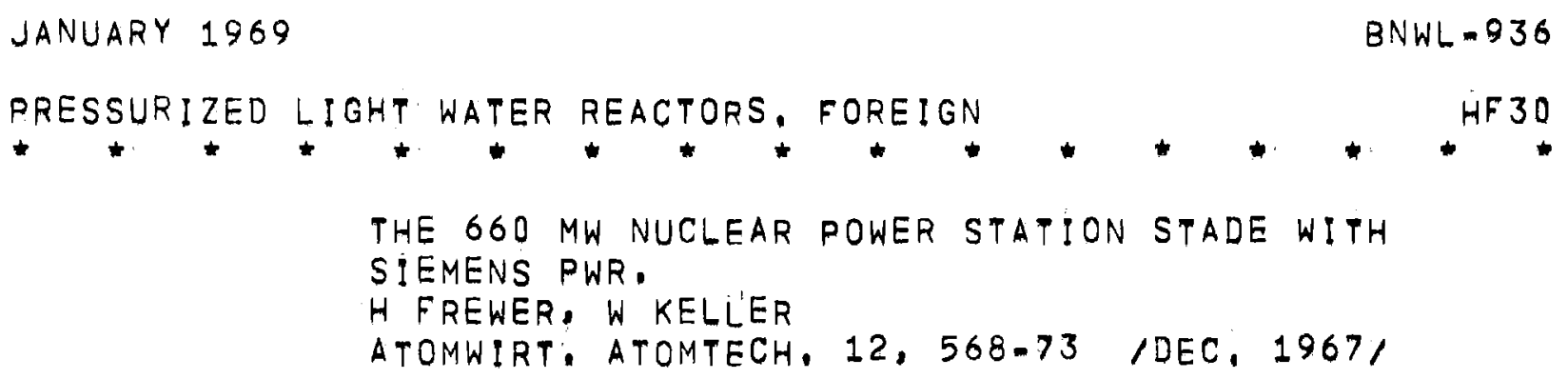


HF 31

JANUARY 1969

BNWLE:036

PRESSURIZED LIGHT WATER REACTORS: FOREIGN

HF 31:
NAME/OWNER

DESIGNER

LOCATION

TYPE
PURPOSE

BULGARIAN NUCLEAR STATION/GOVERNMENT OF BULGARIA

USSR

KOZLODUY; BULGARIA

POWER

PW

TWO-REACTOR STATION

ROWER AWE (NWT)

400

TARGET NO; 1; 1972

NO, 2, 1974

REFERENCES NEWS RELEASES

PER REACTOR 


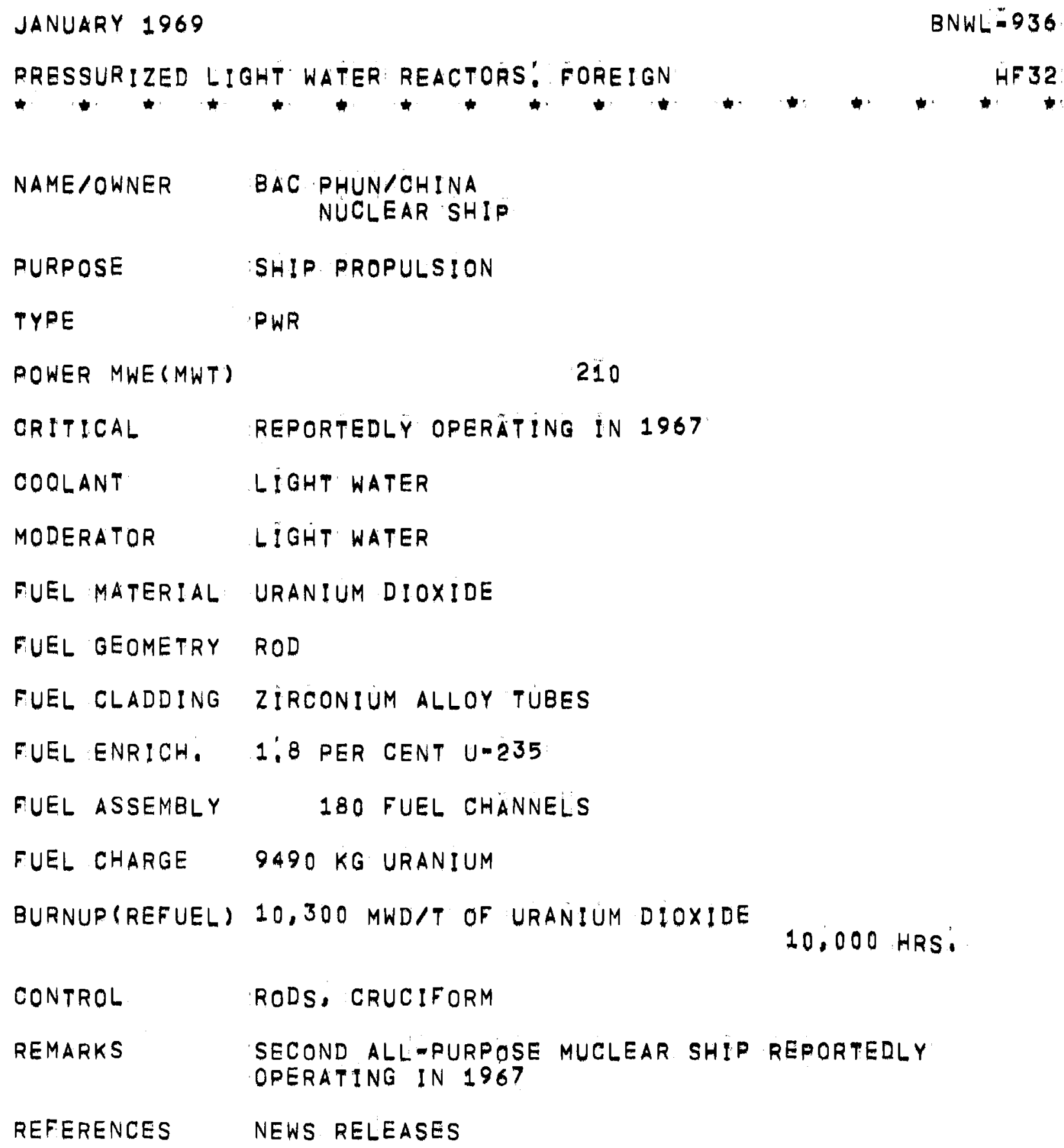



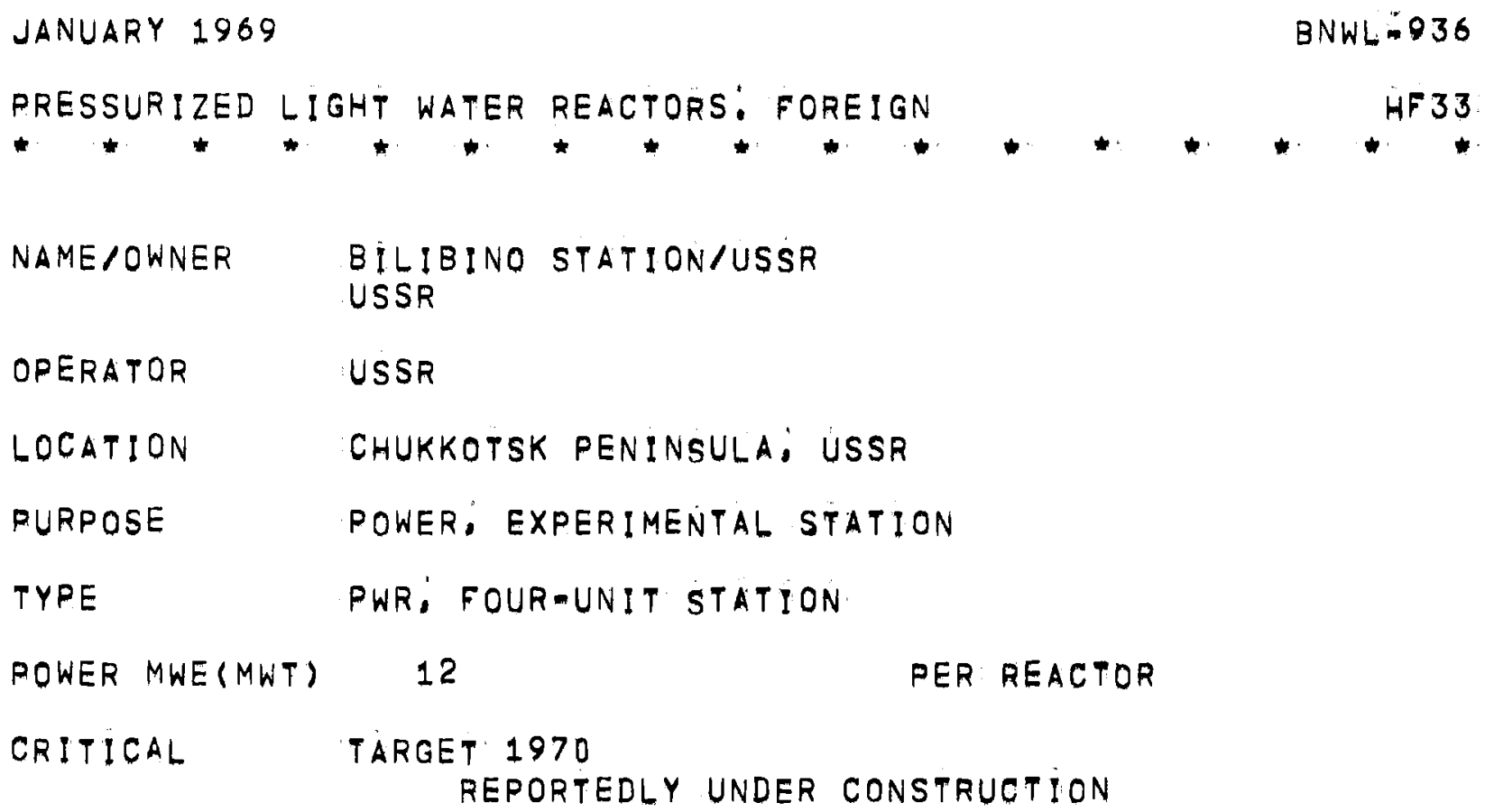


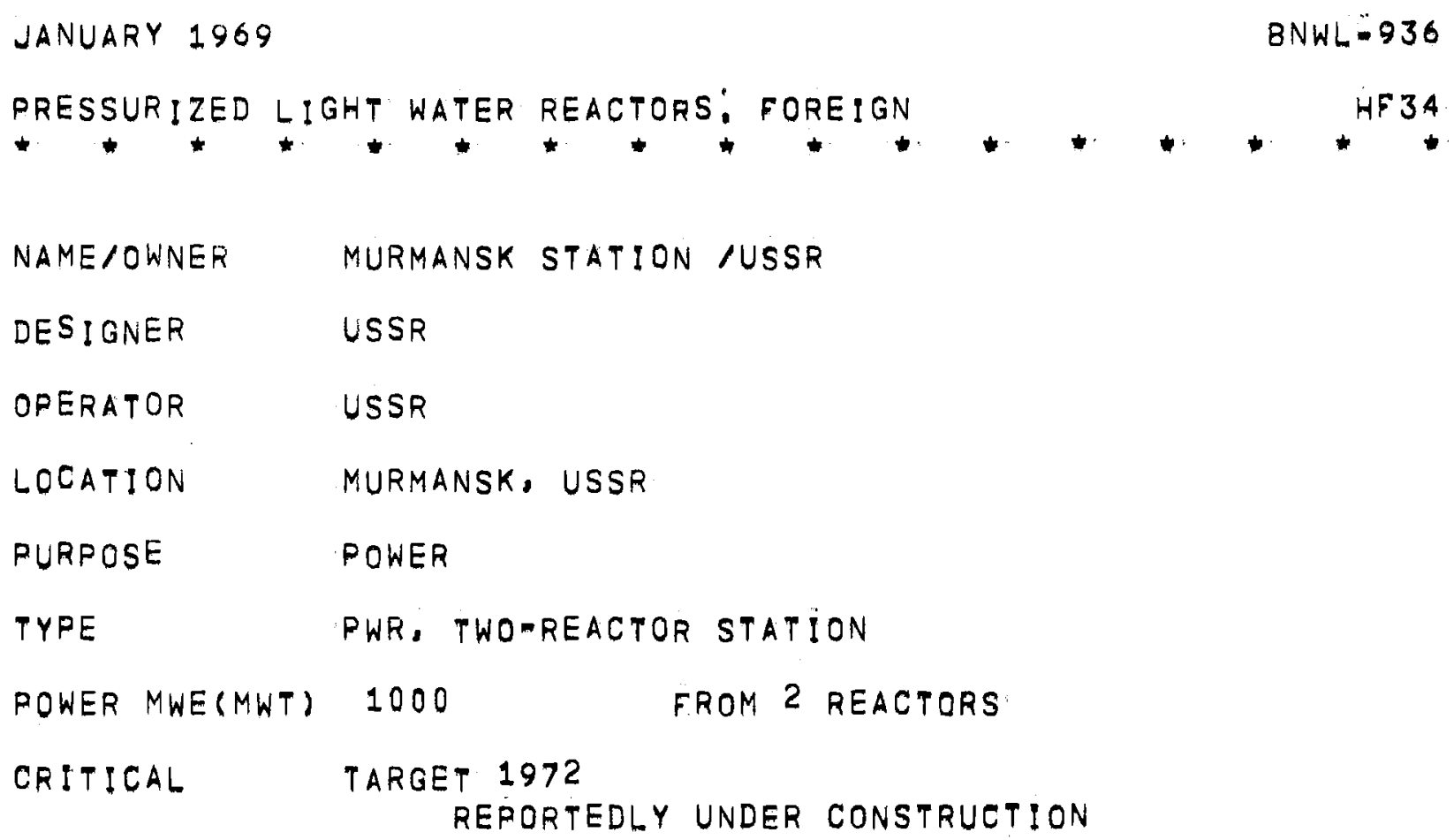


STEAM OR SUPERCRITICAL WATER COOLED REACTORS

DOMESTIC 
JANUARY 1969

BNWL"̈O36

STEAM OR SUPERCRIT: WATER COOLED REACTORS, DOMESTIO

NAME /OWNER

DES IGNER

PURPOSE

TYPE

POWER MWE (MWT)

CRITICAL

ODOLANT

MODERATOR

FUEL MATERIAL.

FUEL GEOMETRY

FUEL CLADDING

FUEL ENRICH.

FUEL ASSEMBLY
GBSR /GRAPHITE BOILING-SUPERHEAT REACTOR//A!

ATOMIOS INTERNATIONAL

POWER

BOILING-SUPERHEÄTING, PRESSURE TUBE:

760

CONCEPTUAL DESIGN

LIGHT WATER AND STEAM

GRAPHITE, GRAPHITE REFLECTOR

URANIUM CARBIDE IN GRAPHITE MATRIX.

SLUGS $4: 0$ IN: DIAI/3 FT: LONG

SILICON CARBIDE COATING

2:8 PER CENT U-235 AVERAGE

BOILING：480 FUEL CHANNELS

SUPERHEAT: 448 FUEL CHANNELS

REHEAT, 368 FUEL: CHANNELS

10 RODS/CHAÑNEL; STACKED, EXTRA FUEL CHANNELS ARE PROVIDED:

FUEL CHARGE 120,000 KG. URANIUM

SPECIFIC POWER 6:33 KW/KG URANIUM

BURNUP(REFUEL) 25,000 MWD/MTU CONTINUOUS

CONTROL

RODS, BORON CARBIDE ABSORBER

COOLANT TEMP.

$\begin{array}{lll}\text { INLET } & 613 & \mathrm{C} \\ \text { INLET } & 645 & \mathrm{C} \\ \text { INLET } & 660 & \mathrm{C}\end{array}$

OUTLET 645 C /BOILER/

OUTLET 1000 :C /SUPERHEATER/

OUTLET 1000 C /REHEATER/

COQLANT PRESS, BOILER 2200 PSIG

SUPERHEATER 2120

REHEATER 500

REACTOR VESSEL STEEL VESSEL 51:31 FT: ID:162:5 FT, HIGH: CONCRETE SHIELDING ON SIDES, WITH BURIED COOLING COILS: FIRE BRICK AGAINST INTERIOR SURFACE OF CONTAINMENT TANK. 
JANUARY 1969

$B N W L=036$

STEAM OR SUPERCRIT: WATER COOLED REACTORS, DOMESTIC

ID01:

CONTAINMENT

PRESSUREISUPPRESSION, CONVENTIONAL REINFORCED CONCRETE OR CARBON STEEL STRUCTURE,

REMARKS

EACH GRAPHITE BLOCK CONTAINS A VERTICAL FUEL CHANNELS, EACH SURROUNDED BY 4 COQLANT TUBES: CORE IS 3-REG!ON. CENTRAL BOILING, INNER ANNULAR SUPERHEATING, AND OUTER ANNULAR REHEAT' REGIONS:. WATER CIRCULATES IN THE CENTRAL SECTION THROUGH THE COOLANT TUBES: STEAM IS SEEPARATED IN, A STEAM DRUM AND RETURNS TO THE SUPERHEAT REGION, FROM THERE IT FLOWS TO A HIGH PRESSURE TURBINE THROTTLE RETURNING TO THE REACTYR TO BE REHEATED IN THE ANNULAR REHEAT REGION: BOILER REGION PIPING IS PH 15-7 MO OR ZIRCALOY-2, IN THE SUPERHEAT AND REHEAT REGIONS INCONEL $=X$.

REFERENCES STEAMECOOLED POWER REACTOR EVALUATION. GRAPHITE MODERATED, BOILING WATER, STEAM-SUPERHEAT REACTOR: ATOMICS INTERNATIONAL NAA-SR-6100 /SEPT. $1961 /$ 
JANUARY 1969

$B N W L=036$

STEAM OR SUPERCRIT: WATER COOLED REACTORS, DOMESTIC

i002

NAME IOWNER

DES \GNER

PURPOSE

TYPE

POWER MWE (MWT)

CRITICAL

COQLANT

MODERATOR

FUEL MATERIAL
CBSR /COUPLED BREEDING SUPERHEATING REACTOR//ANL ARGONNE NATIONAL' LABORATORY

POWER

PWR, STEAM COOLANT: PRESSURE TUBE, FASFITHERMAL BREEDER.

216

CONCEPTUAL DESIGN

THERMAL CORE LIGHT WATER

FAST CORE SUPERHEATED STEAM

LIGHT WATER

FAST

ZONE 1. PLUTONIUM DIOXIDE AND DEPLETED URANIUM DIOXIDE PELLETS, $0,287 \mathrm{CM}$, DIA:

ZONE 2,3A AND 3B /RADIAL BUFFER, UPPER AXIAL

FAST BLANKET, LOWER AXIAL

FAST BLANKET/, DEPLETED

URANIUM DIOXIDE PELLETS.

$0.856 \mathrm{CM}$, DIA.

THERMAL

ZONE 5. NATURAL URANIUM DIOXIDE PELLETS, 0:673 CM. Diá.

ZONE 6-7 /RÄDIAL THERMAL BLANKET\%: ZONE 6 , DERLETED URANIUM DIOXIDE PELLETS

0.673 CM: DIA, ZONE 7, DEPLETED URANIUM, 1.214:CM, DIA.

ZONE 8A-8B JUPPER AXIAL THERMAL BLANKET, LOWER AXIAL THERMAL BLANKET\%, DEPLETED URANIUM DIOXIDE PELLETS $0,673 \mathrm{CM}$.

FUEL GEOMETRY 'FAST

ZONE 1. ROD $0: 318 \mathrm{CM}$, DIA

ZONES 2-3A, 3B, ROD 0.952 CM: DIA.

THERMAL

ZONE 5-6. ROD; 0.785 CM: DIA:

ZONE 7. ROD: 1:407 CM, DIA,

ZONE BA-8B. ROD 0.785 CM. DIA.

FUEL CLADDING FAST, STAINLESS STEEL:

ZONE 1; 0.0127 CM. THICK

ZONE 2, 3A, 3B: 0.043 OM: THICK

THERMAL: ZIRCALOY=? ?

ZONE $5,6,8 \mathrm{~A} ; 8 \mathrm{~B}, 0,051$ CM: THICK 
JANUARY 1969

$B N W L=036$

STEAM OR SUPERCRIT: WATER COOLED REACTORS, DOMESTIC IDOZ

ZONE 7. $0.089 \mathrm{CM}$. THICK

FUEL ASSEMBLY FAST, HEXAGONAL BUNDLES /CANS/,

ZONE 1. 547\%RODS/BUNDLE

ZONES 2,3A,3B, 61-ROD BUNDLE

TOTAL RODS,

ZONE 1.26,256

ZONE 2. 793

ZONE $3 A: 3721$

ZONE 3B: 3721

THERMAL

ZONES $5,6,81,8 B^{\prime}: 61$ RODS/PRESSURE TUBE.

ZONE 5 T2 PRESSURE TUBES: ZONE 7, 246: ZONE 8A AND 8B; 66 EACH

ZONE 7. 19 RODS/PRESSURE TUBE, 246 PRESSURE TUBES.

FUEL CHARGE FAST CORE 242 KG, PLUTONIUM

BURNUP(REFUEL) FAST CORE 1 PER CENT

CONTROL RODS

COQLANT TEMP, INLET $308 \mathrm{C}$ OUTLET $453 \mathrm{C}$ aVERAGE

COOLANT PRESS, 95 ATM.

REACTOR VESSEL CARBON STEEL, SS CLAD; 228 CM, ID:1648 CM, LONG.

REMARKS

A HEAVY WATER SYSTEM WAS FIRST CONSIDERED; OTHER

CONCEPTS INCLUDED ONE IN WHICH ALL STEAM FOR

SUPERHEATING WAS PRODUCED IN THE THERMAL REGION

/THERMAL-FAST SŸSTEM/ WITH HEAVY WATER AS COOLANT,

AND ONE, IN WHICH NO STEAM IS GENERATED IN THE

REACTOR: THE THERMAL PORTION OF THE COUPLED

REACTOR ACTING AS A PRESSURIZED WATER PREHEATER

FOR EXTERNAL BOILERS /FAST-THERMAL SYSTEMI:

REFERENCES COUPLED FAST-THERMAL BREEDER,

R AVERY

NUCLEAR SCI, ENG, 3:129/1958/

CONCEPTUAL DESIGN OF A COUPLED BREEDING

SUPERHEATING REACTOR CBSR.

R AVERY; OTHERS

ANL -6286 /MARCH $1961 /$ 
JANUARY 1969

BNWL: $=936$

STEAM OR SUPERCRIT: WATER COOLED REACTORS: DOMESTIC IDO3

NAME/OWNER SCBR /STEAM COOLED BREEDER REACTOR//BAW-ECNG

DESIGNER BABCOCK AND WILCOX

PURPOSE POWER

TYPE SUPERCRITICAL STEAM COOLED FASTT BREEDER, DIRECT OR INDIRECT CYCLE, PRESSURE TUBE:

POWER MWE(MWT) $1000 \quad 2320$

CRITICAL CONCEPTUAL DESIGN

COQLANT STEAM: LIGHT/HEAYY WATER

CIRCULATING COOLANT HEAVY WATER

MODERATOR HEAVY WATER

FUEL MATERIAL URANIUM-PLUTONIUM OXIDE

BLANKET URANIUM DIOXIDE

FUEL GEOMETRY ROD, 0.23 IN, DIA:

FUEL CLADDING INCONEL, INCOLOY, HASTELLOY-X; 19-9 DL CONSIDERED,

FUEL ENRICH, BLANKET NATURAL URANIUM

FUEL ASSEMBLY 504-ROD BUNDLE WITH 1 BORON CONTROL ROD

108 ASSEMBLIES/CORE

CORE IS COMPLETELY SURROUNDED BY BLANKET

SPECIFIC POWER 0:91 MW/KG PLUTONIUM

BURNUP(REFUEL) 80.000 MWD/T

CONTROL ROD

COOLANT TEMP, INLET $750 F$ OUTLET $1000 \mathrm{~F}$

COOLANT PRESS, $\quad 3650$ PSI

REMARKS

CONCEPTUAL DESIGN OF A 1000 MWE DIRECT CYCLE REACTOR HAS BEEN COMPLETED, ECNG, FORMED BY BAW AND 14 UTILITIES, WILL DEVELOP A 3500 PSI STEAM COOLED BREEDER FOR THE PRODUCTION OF LOW-COST ELECTRICAL ENERGY: LONG-RANGE PROGRAM IS EXPECTED TO LEAD TO A 1000 MWE DEMONSTRATION REACTOR IN THE 1970.5 .

SEE ALSO NDA STUDY 1D-8

REFERENCES

STEAM COOLED REACTOR UNDER STUDY, 
JANUARY 1969

$B N W L=936$

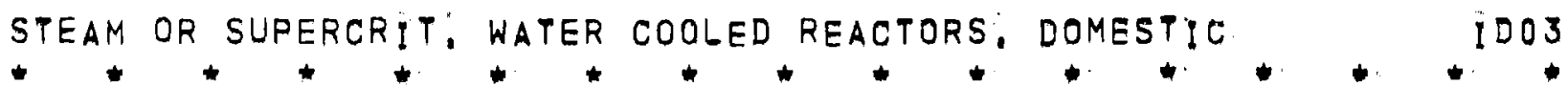

ELECTRICAL WORLD 162,40 /AUG, 24; $1964 /$

STEAM COOLED BREEDER REACTORS:

MC EDLUND; OTHERS

FAST REACTOR TECHNOLOGY. NATIONAL TOPICAL MEETING APRIL 26-28, 1965:

AMERICAN NUCLEAR SOC.

ANS $=100$ P. 85-88 


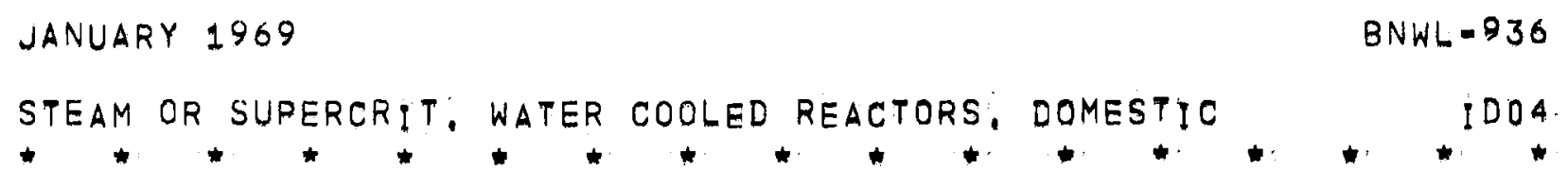

NAME/OWNER VESR /VALLECITOS EXPERIMENTAL SUPERHEAT REACTOR/ GE-ESADA

DESIGNER GENERAL ELECTRIC COMPANY

OPERATOR GENERAL ELECTRIC COMPANY

LOCATION VALLECITOS ATOMIC LABORATORY, CALIF.

PURPOSE EXPERIMENTAL AND TESTING, SUPERHEAT FUEL DEVELOPMENT.

TYPE

STEAM COOLED REÄCTOR, SEPARATE SUPERHEAT

POWER MWE (MWT)

12,5

CRITICAL

NOV: 1963: FULL POWER MAY 1964

COOLANT

SATURATED STEAM:' SEE REMARKS

MODERATOR

LIGHT WATER

FUEL MATERIAL URANIUM DIOXIDE HOLLOW PELLETS

FUEL GEOMETRY ANNULAR RODS

FUEL CLADDING SS INNER AND OUTER, CONCENTRIC TUBES,

FUEL ENRICH, $3: 6$ PER CENT U-235

FUEL ASSEMBLY 9-ROD BUNDLE, ENCLOSED IN PROCESS TUBE 32 BUNDLES/CORE

FUEL CHARGE 5300 POUNDS URANIUM DIOXIDE

BURNUP(REFUEL) 5000 MWD/T FIRST CORE

COOLANT TEMP, $\quad 545$ F

COOLANT PRESS: $\quad 1000$ PSI

REACTOR VESSEL STEEL VESSEL

CONTAINMENT CONVENTIONAL STEEL CONTAINMENT STRUCTURE INTEGRATED, LEAK= TIGHT VESSEL

REMARKS

THE SADE /SUPERHEAT ADVANCED DEMONSTRATION EXPERIMENT/ PROGRAM PRODUCED SUPERHEATED STEAM BY DIRECT NUCLEAR MEANS IN 1959: THE ESADA REACTOR IS DESIGNED TO TEST A LARGE NUMEER OF 
STEAM OR SUPERCRIT: WATER COOLED REACTORS, DOMESTIC $* * * *$ IDOA.

REFERENCES

EXPERIMENTAL FUEL ELEMENTS IN AN OPERATING REACTOR ENVIRONMENT, THE MARK 2 CORE DESIGN CONSISTS OF A 6 X6 ARRAY OF 32 FUEL BUNDLES, COMBINATION BOILING-SUPERHEATING ELEMENTS WERE ALSO STUDIED. 3OO AND 600 MWE CONCEPTS HAVE BEEN INVESTIGATED. THE 600 MWE REACTOR USING SOLID-ROD ELEMENTS. COOLING IS BY A COMBINATION OF MODERATOR BOILING AND FORCED CONVECTION COOLING WITH SATURATED STEAM SEE IDO5

THE REACTOR AND PLANT DESIGN FOR ESADA AND EVSR, $J$ BARNARD

ASME PREPR!NT 61-WA-223/1961/

PRELIMINARY HAZARDS SUMMARY REPORT FOR THE VALLECITOS SUPERHEAT REACTOR, GL MURRAY: ED: GEAP-3642 /FEB, $1961 /$

THE ESADA VALLECITOS EXPERIMENTAL SUPERHEAT REACTOR: DH IMHOFF

POWER REACTOR EXPERIMENTS VOL,2, P, $53-79$ INTERNATIONAL ATOMIC ENERGY AGENCY, VIENNA: 1962.

FINAL HAZARDS SUMMARY REPORT FOR THE ESADA VALLECITOS EXPERIMENTAL SUPERHEAT REACTOR, JL MURRAY: ED: APED-3958/OCT. 1; 1962/

DESIGN AND OPERÄTING EXPERIENCE OF THE ESADA VALLECITOS EXPERIMENTAL SUPERHEAT REACTOR: W IANN I: OTHERS APED-4784 /FEB: 1965,

PRELIMINARY HAZARDS SUMMARY REPORT FOR THE VALLECITOS SUPERHEAT REACTOR

JL MURRAY

GEAP.A643 /FEB: 1; $1961 /$ 
JANUARY 1969

BNWL: $=936$

STEAM OR SUPERCRIT: WATER COOLED REACTORS, DOMESTIC . IDOS:

NAME /OWNER

SSHR /SEPARATE SUPERHEAT REACTOR//GE

DES IGNER

GENERAL ELECTRIC COMPANY

PURPOSE

POWER

TYPE

BWR-SEPARATE SUPERHEAT' SYSTEM.

POWER MWE(MWT) 607

1202 BWR

421 SSHR

CRITICAL

STUDY

COQLANT

LIGHT WATER STEÄM

MODERATOR

LIGHT WATER

FUEL MATERIAL URANIUM DIOXIDE PELLETS 0:450 IN: DIA:

FUEL GEOMETRY RODS 106 IN. ACTIVE LENGTH

FUEL CLADDING SS OR INCONEL O:011 IN. THICK

FUEL ENRICH, 2:8 PER CENT U-235

FUEL ASSEMBLY SSHR:

7-ROD CLUSTER IN A SINGLE PROOESS TUBE, FUEL ASSEMBLY CONSISTS OF 9 PROCESS TURES, INLET AND OUTLET STEAM PLENUMS AND PIPINGS. 172 ASSEMBLIES.

SEE REMARKS

FUEL CHARGE 28,200 KG: URANIUM

BURNUP(REFUEL) 16,535 MWD/MTU BATCH; 20 PER CENT'

CONTROL RODS, INCONEL ABSORBER MATERIAL

COQLANT TEMP, INLET $553.5 \mathrm{~F}$ OUTLET $950 \mathrm{~F}$

COOLANT PRESS, INLET 1060 PSIA OUTLET 980 PSIA

REACTOR VESSEL SEPARATE STEEL VESSELS

CONTAINMENT SEPARATE LOWMPRESSURE VESSELS, PRESSURESUPPRESSION POOL.

REMARKS

THE BWR IS A FORCED CIRCULATION SINGLE CYCLE PRESSURE VESSEL REACTOR WITH INTERNAL STEAM SEPARATION, SUPERHEAT REACTOR IS A SINGLE-PASS 
JANUARY 1969 BNWL:-936

STEAM OR SUPERCRIT, WATER COOLED REACTORS, DOMESTYC 1005

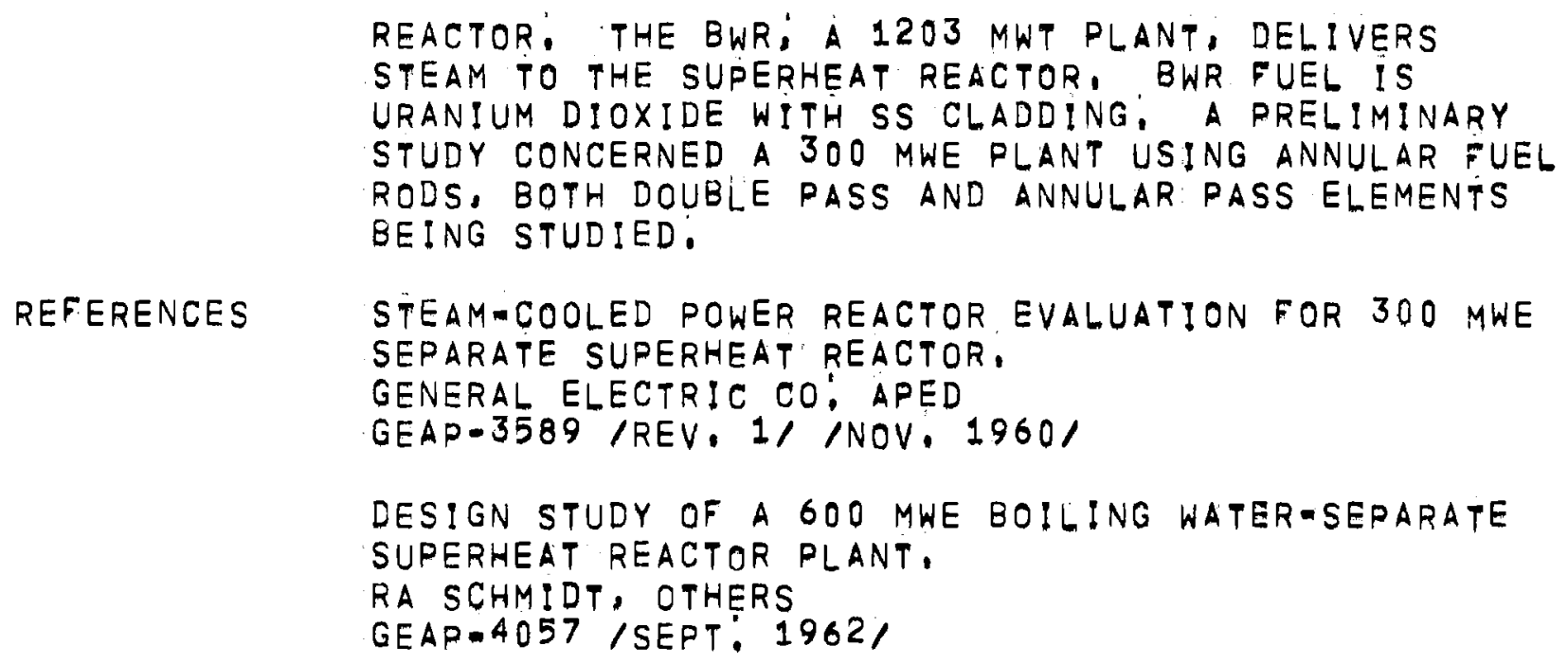


JANUARY 1969

$B N W L \div 936$

STEAM OR SUPERCRIT; WATER COOLED REACTORS: DOMESTIC

1006

NAME/OWNER

DES IGNER

PURPOSE

TYPE

POWER MWE (MWT)

CRITICAL

COOLANT

MODERATOR
FSPPR /FAST SURERCRITICAL PRESSURE POWER REACTOR/ GENERAL: ELECTRIC COMPANY

POWER

SUPERCRITICAL STEAM COOLED FAST REACTOR, DIRECT CYCLE:

677

CONCEPTUAL DESIGN

LIGHT WATER STEAM /SUPERCRITICAL/

YTTRIUM OR ZIRCONIUM HYDRIDE CORE SUBDIVIDERS; THE INNER CORE IS UNMODERATED.

FUEL MATERIAL DEPLETED URANIUM DIOXIDE-PLUTONIUM DIOXIDE

FUEL GEOMETRY MASSIVE FUEL ASSEMBLY PIERCED BY COOLANT TUBES, 5 FT, ACT, LENGTH:

FUEL CLADDING RENE-41; RENE-41 COOLANT: TUBES.

FUEL ENRICH, 3:8 PER CENT PLUTONIUM

FUEL ASSEMBLY MASSIVE CERAMIC ELEMENT, TRIANGLE OR TRAPEZOID;

PIERCED BY HIGH OPRESSURE COOLANT TUBES, EACH

ELEMENT INCORPORATES AXIAL BLANKET SECTIONS,

72 ELEMENTS/CORE,

42 ARE FUEL -BEARING, 30 ARE RADIAL BLANKET

ELEMENTS.

SEE REMARKS:

FUEL CHARGE DEPLETED URANIUM 30:895 KG.. PLUTONIUM 1559 KG. SPECIFIC POWER $50 \mathrm{KW/KG} \mathrm{URANIUM} \mathrm{+} \mathrm{PLUTONIUM}$

BURNUP(REFUEL) 82,700 MWD/T

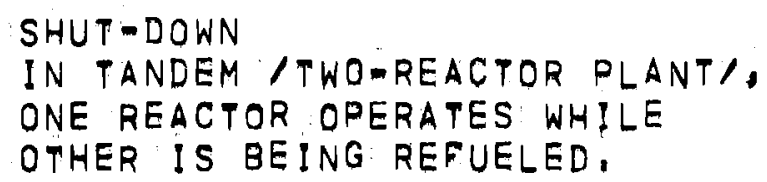

CONTROL

RODS, SS-BORON

COQLANT TEMP.

$1050 \mathrm{~F}$ INITIAL' TURBINE THROTTLE CONDITIONS,

COOLANT PRESS,

3515 PSIA INITIAL TURBINE THROTTLE CONDITIONS.

REACTOR VESSEL SS TANK CONTAINING THE SEGMENTED CORE: 
JANUARY 1969

BNWL $=936$

STEAM OR SUPERCRIT: WATER COOLED REACTORS, DOMESTIC IO IDOG

CONTAINMENT STEEL BUILDING, PRESSURE-SUPPRESSION,

REMARKS

THE REACTOR IS COMPOSED OF 7 RADIAL REGIONS, THE CORE IS 5 FT. HIGH 15,28 FT: DIA, SURROUNDED BY RADIAL AND AXIAL BLANKETS EACH O,6 FT: THICK, THIN LAYERS OF YYTTRIUM OR ZIRCONIUM HYDRIDE SUBDIVIDE THE SEGMENTS. WITH A LAYER OF U-238 AS ABSORBING MATERIAL ON EACH SIDE OF THE MODERATING ELEMENT TO FORM AN ANNULAR FLUUX TRAP. FOUR CONCENTRIC REGIONS ARE THE RADIAL BLANKET, FIRST CORE, AND SECOND CORE, SEPARATED BY THE MODERATOR=ABSORBER, AND THE UNMODERATED 3RD, OR CENTRAL CORE THERE ARE PROVISIONS FOR DUAL-REACTOR INSTALLATION AND REMOTE FUEL HANDLING. CONCEAT IS BASED ON THE SSPR DESIGN.

REFERENCES ECONOMIC EVALUATION OF A 300 MWE SUPERCRITICAL POWER REACTOR /SSPR/. H HARTY, OTHERS

HW-68420 /REV./ /JUNE 1961 /

SEGMENTED CORE DESIGN FOR A SUPERCRITICAL PRESSURE WATER-COOLED FAST REACTOR, RE PETERSON, SL STEWART

PROC, CONF, ON BREEDING, ECONOMICS AND SAFETY IN LARGE FAST POWER REACTORS, OCT, 7-10, 1963;

ANL $=6792$ P. 539.552

$H W=S A-3149$ /OCT: 3,1963/

ECONOMIC EVALUATION OF A 300 MWE FAST

SUPERCRITICAL PRESSURE POWER REACTOR,

DT AASE, OTHERS

$H W=78953$ /DEC? $9,1963 /$

FAST SUPERCRITICAL WATER REACTOR

POWER REACTOR TECHNOLOGY $8,186: 93$ /SUMMER $1965 /$ 
JANUARY 1969

$B N W L=936$

STEAM OR SUPERCRIT', WATER COOLED REACTORS, DOMESTIC

1007

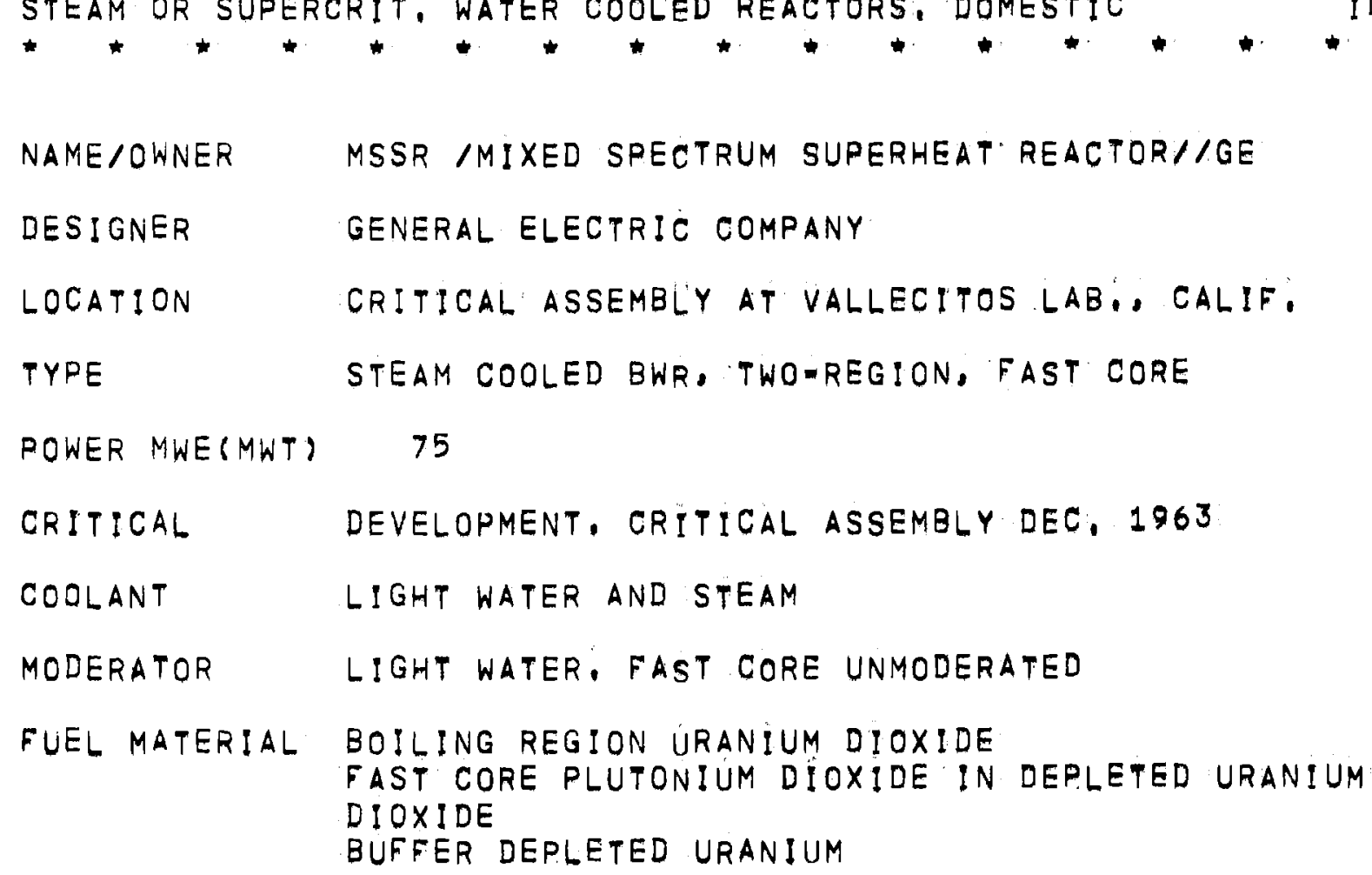

REFERENCES

SATURATED STEAM GENERATED IN THE BOILING REGION PASSES THROUGH AN INTERNAL STEAM SERARATING SYSTEM AND GOES THROUGH THE INNER SUPERHEATING REGION OF THE CORE, IN THE 75 MWE PROTOTYPE THE FAST CORE IS ROUND, THE FUEL HEXAGONAL, THE MIXED SPECTRUM CRITICAL ASSEMBLY /MSCA/ WAS STARTED UP IN DEC. 1963, AND THE FAST SPECTRUM SUPERHEATER LOADED IN MAY 1964, AT VALLECITOS. MSCA IS A SINGLE CORE REACTOR WITH TWO SEPARATE FUEL REGIONS:

STEAM COOLED POWER REACTOR EVALUATION: MIXED SPECTRUM SUPERHEATER, B WOLFE GEAP-3590/REV.1//NOV, $1960 /$

CONCEPTUAL DESIGN FOR A 75 MWE MIXED. SPECTRUM SUPERHEATING REACTOR POWER PLANT, GV BRYNSVOLD, OTHERS GEAP.4O16 /FEB, 25, 1962/ 
JANUARY 1969

BNWL $=936$

STEAM OR SUPERCRIT: WATER COOLED REACTORS, DOMESTIC IDOB

NAME /OWNER

DES IGNER

PURPOSE

TYPE
STEAM COOLED FAST REACTOR STUDY/NDA

NUCLEAR DEVELOPMENT CORPORATION OF AMERICA

POWER

STEAM COOLED FAST BREEDER: FORCED CIRCULATION, DIRECT CYCLE.

POWER MWE(MWT) $300 \quad 840$

CRITICAL

FEASIBILITY STUDY; CONCEPTUAL DESIGN

COOLANT

STEAM: LIGHT AND HEAVY WATER STUDIED,

MODERATOR NONE

FUEL MATERIAL URANIUMIPLUTONIUM DIOXIDE PELLETS. BLANKET DEPLETED URANIUM DIOXIDE

FUEL GEOMETRY RODS 0,25 IN. DIA:, 5 FT. ACT', LENGTH * BLANKET.

FUEL CLADDING INCONEL-X TUBES 0:015 IN, THICK: BLANKET SS

FUEL ENRICH, 15,4 PER CENT

BLANKET, DEPLETED URANIUM

FUEL ASSEMBLY CORE, 127-ROD CLUSTER

AXIAL AND RADIAL BLANKET; 37-ROD CLUSTER

326 CORE ASSEMBLIES

256 BLANKET ASSEMBLIES

SPECIFIC POWER 0:50 MWT/KG PLUTONIUM-239

BURNUP(REFUEL) 33,300 MWD/TT PARTIAL: FUEL SHIFT

CONTROL BORON CARBIDE RODS

COOLANT TEMP, $\quad 945 \mathrm{~F}$

COQLANT PRESS, 1400 PSIG

REACTOR VESSEL STEEL PLATE VESSEL', SS CLAD. $10.7 \mathrm{FT}$, ID./31 FT. HIGH.

REMARKS

URANIUM / THORIUM DIOXIDE FUEL WAS ALSO STUDIED;

REFERENCES

STEAM-COOLED POWER REACTOR EVALUATION: STEAM

COOLED FAST BREEDER REACTOR: 
JANUARY 1969

$B N W L=936$

STEAM OR SUPERCRIT: WATER COOLED REACTORS, DOMESTIC **** IDOO

G SOFER; OTHERS

NDA $=2148-4$ /APRIL 1961 ,

CONCEPTUAL DESIGN AND ECONOMIC EVALUATION OF A

STEAM-COOLED FAST BREEDER REACTOR.

$G$ SOFER, OTHERS

NDA-2148-5/NOV: 1961 ,

THE NDA STEAM-COOLED FAST REACTOR CONCEPT:

NUCLEAR POWER 6: 75 /OCT: $1961 \%$ 
JANUARY 1969

$B N W L=036$

STEAM OR SUPERCRIT: WATER COOLED REACTORS; DOMESTIC

¿Do9.

$\begin{array}{ll}\text { NAMEIOWNER } & \text { SWR /STEAM-WATER REACTOR//NDA } \\ \text { DESIGNER } & \text { NUCLEAR DEVELOPMENT CORPORATION OF AMERICA } \\ \text { PURPOSE } & \text { POWER } \\ \text { TYPE } & \text { STEAM COOLED; HEAVY WATER MODERATED: PRESSURE TUBE } \\ & \text { /CALANDRIAI }\end{array}$

POWER MWE(MWT) 200

530

CRITICAL

FEASIBILITY STUDY

COOLANT

STEAM /LIGHT WATER FOG/

MODERATOR

HEAVY WATER

FUEL MATERIAL

URANIUM DIOXIDE PELLETS 0:750 IN: DIA:10,750 IN. LONG

FUEL GEOMETRY ROD, 24 IN. ACTIVE LENGTH

FUEL CLADDING SS $0.010 \mathrm{IN}$. THICK

FUEL ENRICH, 1 PER CENT U-235

FUEL ASSEMBLY 19-ROD BUNDLE; 2 BUNDLES/ASSEMBLY

5 ASSEMBLIES/PRESSURE TUBE

BUNDLES INCLUDE THERMAL INSULATION

1260 BUNDLES/CORE

REF, DESIGN 272 PRESSURE TUBES.

FUEL CHARGE 9 METRIC TONS: URANIUM DIOXIDE

BURNUP (REFUEL) 5500 MWD/T

COOLANT TEMP, $\quad 1060 \mathrm{~F}$

COOLANT PRESS. 2015 PSIA

REACTOR VESSEL ALUMINUM CALANDRIA, ZIRCALOY PRESSURE TUBES,

CONTAINMENT CONCRETE BUILDING:

REMARKS

AN ALTERNATE DESIGN WAS STUDIED IN WHICH SATURATED WATER INSTEAD OF STEAM IS SUPPLIED, AND BOILING OCCURS IN THE CENTRAL REGION OF THE REACTOR=A BOILING-SUPERHEATING SWR, FUEL AND COOLANT ARE WITHIN THE PRESSURE TUBES WHICH PASS THROUGH THE CALANDRIA-CONTAINED HEAVY WATER MODERATOR. A 
STEAM OR SUPERCRIT: WATER COOLED REACTORS, DOMESTIO

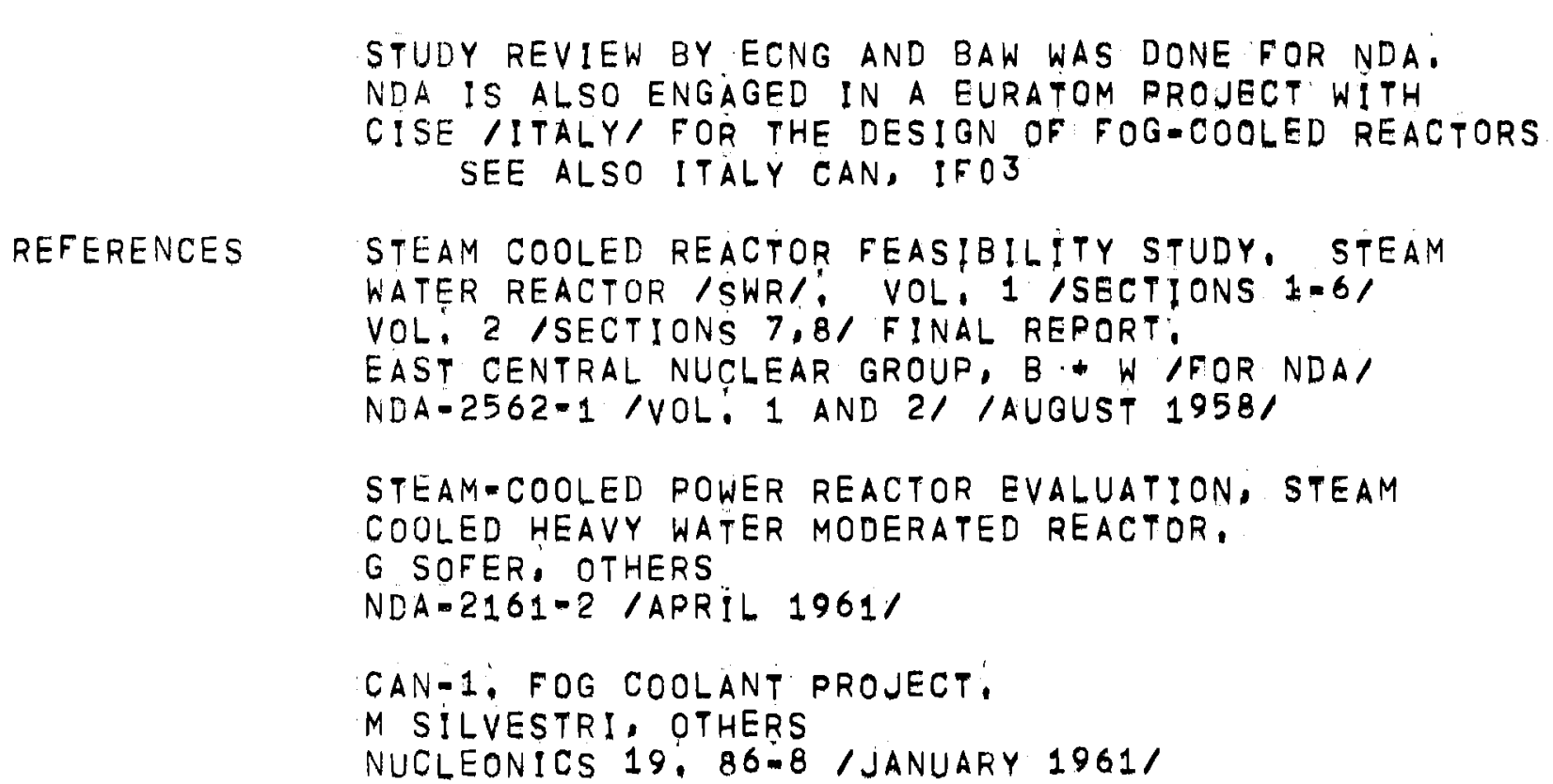


JANUARY 1969

BNWL -936

STEAM OR SUPERCRIT', WATER COOLED REACTORS, DOMESTIC

NAME/OWNER SWR /SUPERCRITICAL WATER REACTOR/

DESIGNER BATTELLE MEMORIÄL INSTITTUTE

PURPOSE UNDERSEA PROPULSION AND AUXILIARY POWER

POWER MWE(MWT) $250 \mathrm{KWE} \quad 1440 \mathrm{KWT}$

COOLANT SUPERCRITICAL LIGHT WATER

MODERATOR LIGHT WATER

FUEL MATERIAL URANIUM DIOXIDE

FUEL GEOMETAY FUEL COUPONS 40 MILS THICK IN SS PLATE

FUEL CLADDING SS 7 MILS THICK

FUEL ENRICH, FULL

FUEL ASSEMBLY 15-PLATE ELEMENT: SOUARE CAN

45-ELEMENT CORE

FUEL CHARGE 64,8 KG: U-235

CONTROL SSMLLAD BORON CARBIDE VANES IN REFLLECTOR

COOLANT TEMP: INLET $300 \mathrm{~F}$ OUTLET $1000 \mathrm{~F}$

COOLANT PRESS, $\quad 4340$ PSI

REMARKS

THE BMI STUDY WAS CONCERNED WITH APPLICATION FOR PROPULSION AND AUXILIARY POWER IN A DEEP. SUBMERGENCE VEHICLE, CONSIDERED FOR MISSIONS TO $10,000 \mathrm{FT}$.

REFERENCES A SUPERCRITICAL WATER REACTOR FOR PRORULSION AND AUXILIARY POWER IN A DEEP SUBMERSIBLE RESEARCH VEHICLE: JW RAY, JW CHASTAIN MARINE TECHNOLOGY SOC!ETY MEETING WASHINGTON, JUNE 1965 
JANUARY 1969

$B N W L=36$

STEAM OR SUPERCRIT", WATER COQLED REACTORS; DOMESTIC

ID11

NAME /OWNER

ESCR /EXPERIMENTAL STEAM-COQLED REACTOR/ECNG-GE

DES IGNER GENERAL ELECTRIC COMPANY

TYPE STEAM-COOLED EXPERIMENTAL REACTOR; DEMONSTRATION

POWER MWE (MWT) 50

CRITICAL

FEASIBILITY STUDY AND DES!GN

COQLANT

STEAM

REMARKS

GENERAL ELECTRIC AND ECNG HAVE TERMINATED THE STEAM-BREEDER EFFORT AS OF JULY 1968 BECAUSE OF FUEL ELEMENT PROBLEMS SUCH AS CREEP COLLAPSE AND STRAIN CYCLING OF THE CLADDING UNDER HIGH EXTERNAL STEAM PRESSURES:

REFERENCES

NEWS RELEASES 
STEAM OR SUPERCRITICAL WATER COOLED REACTORS FOREIGN 
JANUARY 1969

$B N W L=936$

STEAM OR SUPERCRIT. WATER COOLED REACTORS, FOREIGN IFOI

NAME/OWNER

HERMES/BELGONUCLEAIRE-BELGIUM

DESIGNER

BELGONUCLEAIRE, BELGIUM

PURPOSE

POWER

TYPE

STEAM COOLED, DIRECT CYCLE, FAST REACTOR. PRESSURE TUBE, MODULAR, LOEFFLER CYCLE,

POWER MWE(MWT) 300

870

CRITICAL

DESIGN CONCEPT

COQLANT

DRY STEAM

FUEL MATERIAL URANIUMIPLUTONIUM DIOXIDE

FUEL GEOMETRY PINS 5:8 MM: OD.

FUEL CLADDING INCONEL, INCOLOY, HASTELLOY, NIMONIC, AND RENEAAI STUDIED

FUEL ENRICH, 20 PER CENT PLUTONIUM-239

FUEL ASSEMBLY CLOSE=PACKED PRESSURE TUBES CONTAIN FUEL ELEMENTS, 151-PIN ASSEMBLY MODULUS, CHANNEL DIA, 100 MM., HEXAGONAL TUBE WITH CIRCULAR INNER CHANNEL. MODULI FIT TOGETHER, A BUNDLE OR HYPERMODULES MAKING UP THE CORE CONSISTS OF 7 CHANNELS SEPARATED BY 3 MM: THICK WALLS

SPECIFIC POWER 150 KWE/KG FISSIONABLE MATERIAL

BURNUP(REFUEL) 30.000 MWD/T /CORE/ AND 5000 MWD/T /BLANKET/ COQLANT TEMP, INLET 315C SUPERHEAT $540 \mathrm{C}$

REMARKS

BELGONUCLEAIRE, IN A EURATOM PROGRAM, HAS COMPLETED A DESIGN STUDY FOR A 1000 MWE STEAM-. COOLED FAST REACTOR IN WHICH CORE, CIRCULATORS, AND STEAM GENERATORS ARE CONTAINED IN A PRE-. STRESSED CONCRETE VESSEL:

REFERENCES

HERMES FAST NEUTRON SUPERHEATER REACTOR, $G$ TAVERNIER, OTHERS

3RD U. N. INTL, CONF, PEACEFUL USES OF ATOMIC ENERGY, GENEVA 1964: A/CONF, 28/P/516 
JANUARY 1969

$B N W L=936$

STEAM OR SUPERCRIT: WATER COOLED REACTORS, FOREIGN $* * * * * * * * *$

HERMES - A MODULAR STEAM COOLED FAST REACTOR CONCEPT G TAVERNIER, OTHERS

ANS-100P:99-103

1000 MWE STEAM COOLED BREEDER WITH PRESTRESSED CONCRETE VESSEL

$\checkmark$ CHERMANNE, J HENR!ETTE

EURFNR-522 /DEC, $1967 /$ 
JANUARY 1969

BNWLE: $=36$

STEAM OR SUPERCRIT: WATER COOLED REACTORS, FOREIGN

If 02

NAME/OWNER FOG COOLED REACTOR STUDY/AEGL; CANADA

DESIGNER ATOMIC ENERGY OF CANADA LTD; /AECLI

PURPOSE POWER

TYPE STEAM/SPRAY/ COOLED; DIRECT CYCLE, PRESSURE TUBE, HOT,

POWER MWE(MWT) 200

CRITICAL

ECONOMIC STUDY

COOLANT

LIGHT WATER STEAM OR SPRAY

MODERATOR HEAVY WATER

FUEL MATERIAL URANIUM DIOXIDE PELLETS

FUEL GEOMETRY RODS

FUEL CLADDING ZIRCALOY-2

FUEL ENRICH. NATURAL

FUEL ASSEMBLY 19-ROD BUNDLE

BURNUP(REFUEL) $8600 \mathrm{MWD/T}$

REACTOR VESSEL CALANDRIA, ZIRCONIUM ALLOY PRESSURE TUBES;

REMARKS

THE PRESSURE TUBE IS SURROUNDED BY AN INSULATING

GAS, IN TURN SURROUNDED BY A CALANDRIA TUBE?

REFERENCES

PARTIAL ECONOMIC STUDY OF STEAM COOLED HEAVY WATER MODERATED REACTORS. AECL, NUCLEAR ENG, BRANCH

AECL-1018 /APRIL 1963/

AFTER CANDU-FOG COOLED REACTORS:

GA PON

CANADIAN CHEM, PROCESSING 45; $83,92: 4$

IOCTOBER 1961/

PROSPECTIVE HEAVY WATER-MODERATED POWER REACTORS, GA PON, OTHERS

3RD U:N: INTL. CONF: PEACEFUL USES OF ATOMIC

ENERGY, GENEVA, 1964, A/CONF, 28/P/10

LIGHT WATER COOLED HEAVY WATER MODERATED NATURAL 
JANUARY 1969

BNWL $=936$

STEAM OR SUPERCRIT! WATER COQLED REACTORS: FOREIGN

IF02

$+$

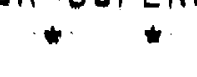

URANIUM POWER REACTORS.

GA PON

$A E C L=1807 / P ; 59=67 / 1963 /$ 
JANUARY 1969

$B N W L=936$

STEAM OR SUPERGRIT: WATER COOLED REACTORS, FOREIGN IFO3

NAME/OWNER CAN-ITALY EURATOM-US PROGRAM INVOLVING CISE /ITALY/,NDA

DESIGNER

ANSALDO IITALY/, NDA

TYPE

STEAM COOLED, heAVY WATER MOD., PRESSURE TUBE

CRITICAL $\quad R+D$

CDOLANT WET STEAM /SPRAYI

MODERATOR HEAVY WATER

FUEL MATERIAL URANIUM-ZIRCONIUM ALLOY

FUEL GEOMETRY HOLLOW RODS

FUEL CLADDING ZIRCALOY-2

FUEL ENRICH, NATURAL

FUEL ASSEMBLY CLUSTER AND CONCENTRIC ELEMENTS STUDIED. PREFERRED ELEMENT CONSISTS OF TWO CONEENTRIC ANNULI OF ZIRCALOY-2 CANNED FUEL WITH THREE ANNULAR COOLANT FLOW CHANNELS AND A SOLID CENTRAL ZIRCALOY ROD.

REMARKS

PRELIMINARY DESIGN AND DEVELOPMENT IN A STUDY CONTRACT UNDER THE UUS-EURATOM AGREEMENT. HEAT TRANSFER, HEAT FLUX, AND CORROSION INVESTIGATIONS /CAN-1; CAN-2, CAN-3/ HAVE BEEN COMPLETED. THE CIRENE PROGRAM /CISE REATTOREA NEBB!A/ AIMED AT THE DESIGN OF A 20 MWE, 100 MWT PROTOTYPE, IS TO BE COMPLETED IN 1971, SEE IFO4

REFERENCES CALCULATION METHODS FOR THE CRITICAL SIZE OF A HEAVY WATER MODERATED; WET STEAM COOLED REACTOR WITH A NATURAL URANIUM CLUSTER FUEL ELEMENT. $R$ BONALUME, OTHERS

CISE-67/1959/

ENERGIA NUCLEARE 7, $192-209$ /MARCH $1960 /$

PRELIMINARY DESIGN STUDIES ON FUEL ELEMENTS FOR A HEAVY WATER MODERATED? WET STEAM COOLED REACTOR: I CASAGRANDE, OTHERS CISE-68 /MAY $1959 /$

EVALUATION AND INTERPRETATION OF THE EXPERIMENTS ON ADIABATIC TWOMPHASE FLOW PERFORMED AT CISE UNDER THE CAN-1 PROGRAM. 
STEAM OR SUPERCRIT: WATER COOLED REACTORS, FOREIGN

CISE /ITALY/

EURAEQW445/1962/

ADVANCED RESEARCH PROGRAM ON THE APPLICATION OF STEAM-WATER SPRAY TO THE COOLING OF LIGHTHWATER REACTORS /CAN=2\%, CISE /ITALYI

EURAEO $=475 / 1962 /$

LARGE-SCALE AND IN-PILE TESTING OF LIGHT WATER STEAM MIXTURES IS REACTOR COOLANTS ICAN-3\%:

CISE /ITALY/

EURAEC-476/1962/

THE SPRAY COOLED REACTOR:

JG COLLIER, PMC LACEY

NUCLEAR POWER 5: 68:73 /AUGUST' $1960 /$

CAN-1: FOG COOLANT PROJECT: M SILVESTRI, OTHERS

NUCLEONICS 19,86.8/JANUARY $1961 /$

THE /CAN/ RESEARCH AND DEVELORMENT' PROGRAM ON THE APPLICATION OF STEAM-WATER MIXTURES TO THE REACTOR COOLING:

E VILLANI/CISE,

ENERGIA NUCLEARE/MILAN/ 9,84-96/FEBRUARY $1962 /$

INVESTIGATION OF WET STEAM AS A REACTOR COOLANT' ICAN-2\%. VOLUME 2: CONCEPTUAL DESIGN EVALUATION OF A FOG-COOLED LIGHT WATER REACTOR, FINAL REPORT $M$ RABER, OTHERS UNC-5008-2/AUGUST. 10:1962,

FINAL REPORT COVERING THE PERIOD OCTORER, 1959 TO APRIL 30.1960

$N D A-2132=5$

SEE ALSO NDA STEAM COOLED REACTOR STUDIES: 
JANUARY 1969

BNWL: :-936

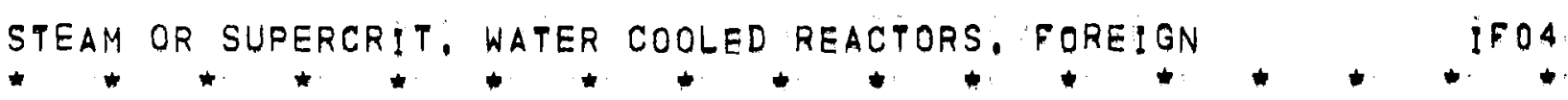

NAME/OWNER CIRENE/CISE, CNEN, ENEL, EURATOM

DESIGNER CISE /ITALY/ AND EURATOM

NUCLEAR CONTRACT FOR 35 MWE PROTOTYPE TO

ANSALDO MECCANICA NUCLEARE

LOCATION LATINA, ITALY

PURPOSE FOWER PROTOTYPE

TYPE HEAVY WATER MODERATED; LIGHT WATER FOG COOLED

PWR: VERTICAL PRESSURE TUBE:

POWER MWE(MWT) $35 \quad 100$

CRITICAL TARGET 1971

COQLANT LIGHT WATER FOG /STEAM/

MODERATOR HEAVY WATER=COLD, UNPRESSURIZED

FUEL MATERIAL URANIUM DIOXIDE PELLETS OR U METAL TUBES

FUEL GEOMETRY RODS OR TUBES

FUEL CLADDING ZIRCALOY=2

FUEL ENRICH, NATURAL

FUEL ASSEMBLY 19-ROD BUNDLES

8 BUNDLES/CHANNEL, STACKED

60 FUEL CHANNELS

BURNUP(REFUEL) $1000-1500$ MWD/T

CONTAINMENT SHIELDING BY CONCRETE WALLS,

REMARKS AN R D PROGRAM FOR A 20 MWE PROTOTYPE FOGIEOOLED

REACTOR INCLUDES CONSTRUCTION BY 1969;

A PROTOTYPE 100 MWT REACTOR WILL BE DESIGNED

UNDER A CNEN-EURATOM-CISE AGREEMENT, CISE WILL

REQUEST ENEL AND INDUSTRY SUPRORT FOR PROTOTYPE

DESIGN AND CONSTRUCTION. THE CONCEPT CONTEMPLATES

DIRECT EXTRACTION AND TRANSFER OF HEAT TO THE

TURBO-GENERATOR: ELIMINATING THE NEED FOR HEAT

EXCHANGERS.

REFERENCES EURO-NUCLEAR MARCH $1965 \mathrm{P}: 114.15$

NEWS RELEASE 


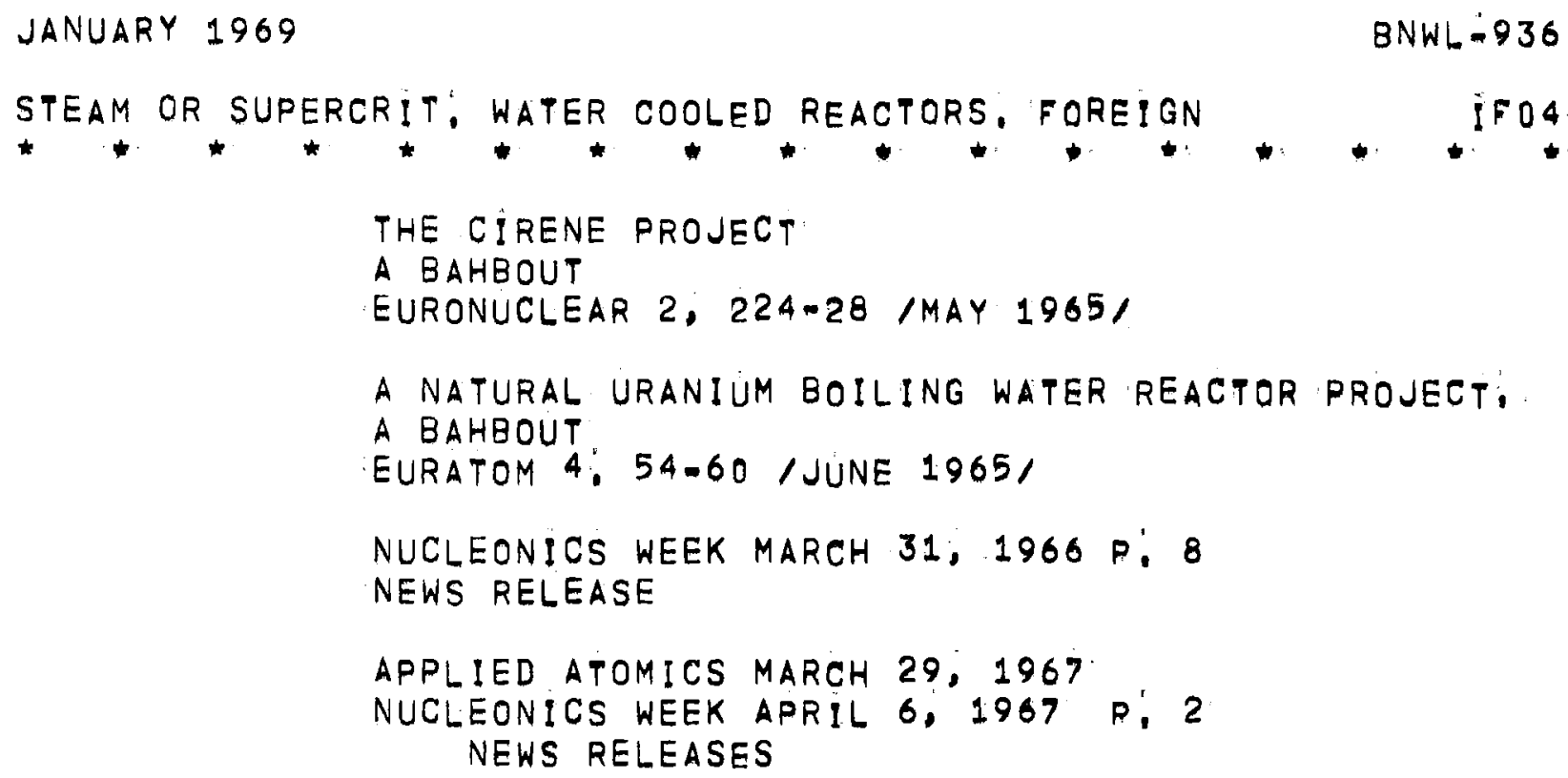


JANUARY 1969

BNWL-936

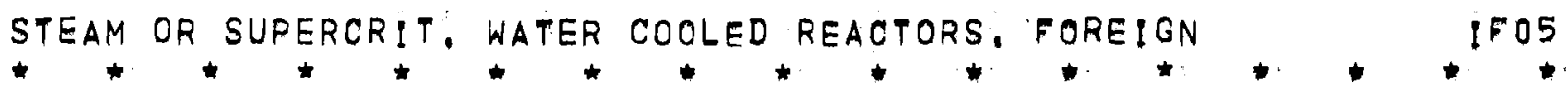

NAME/OWNER

DESIGNER

PURPOSE

TYPE

COQLANT

MODERATOR

FUEL MATEFIAL

FUEL GEOMETRY ROD, ACT: LENGTH 5 FT: ICONCENTRIC TUBES/

FUEL ASSEMBLY

CONTROL

COOLANT TEMP:

COOLANT PRESS:

REACTOR VESSEL

CONTAINMENT

REMARKS

REFEERENCES
SCLMR /STEAM COOLED LIGHT WATER MODERATED REACTOR/ MITCHELL, UNITED KINGDOM

MITCHELL-UK

SHIP PROPULSION:

STEAM COOLED, PRESSURE TUBE, INDIRECT: CYCLE,

LIGHT WATER STEAM; SUPERHEATED

LIGHT WATER

URANIUM DIOXIDE HOLLOW PELLETS 0:33 IN, ID:10,54

IN, OD.

116-ROD CLUSTER; 11X11: /MINUS 4 FOR HOLD-DOWN

BOLTS AND 1 FOR SAMPLING AND THERMOCOUPLE TUBE. 21 ASSEMBLIES/CORE

BORON=SS CRUCIFORM RODS

$850-900 \mathrm{~F}$

650 PSIA

CYLINDRICAL VESSEL CONTAINING MODERATOR AND CORE.

VESSEL 21 FT, DIA:/30,FT: HIGH.

MODERATOR VESSEL PARTIALLY SUBMERGED IN A WATER

SHIELD TANK AS AN INDIRECT VAPOR-SUPPRESSION

SYSTEM,

THE STEAM COOLANT FLOWS UPWARD THROUGH THE OUTER ANNULUS BETWEEN PRESSURE AND BAFFLE TUBES, BACK DOWN BETWEEN THE INNER AND OUTER FUEL CANS, OUTER PRESSURE TUBE OF EACH FUEL ELEMENT; MADE OF ZIRCONIUM: FORMS THE BOUNDARY BETWEEN THE PRIMARY STEAM COOLANT AND THE MODERATOR, WHICH FILLS THE SPACES BETWEEN THE FUEL RODS, THE HIGH TEMPERATURE STEÄM OUTLET PLENUM CHAMBER IS BENEATH THE CORE. THE UK CONSIDERED THE CONCERT IN ITS NUCLEAR SHIP PROGRAM.

MITCHELLOS SCLMR-DESIGN OF COMPETITIVE MARINE REACTOR.

HJ COLES

NUCLEAR ENG, 8,58.63 /FEBRUARY $1963 /$ 
JANUARY 1969

BNWL:036

STEAM OR SUPERCRIT: WATER COOLED REACTORS, FOREION IFOS

NUCLEAR PROPULSION BY MITCHELLEFAIRFIELD; NEW MARINE SYSTEM OF LONG LIFE, LOW WEIGHT: G JACKSON

IMPULSE NO: 24, 6.11/1964/ 


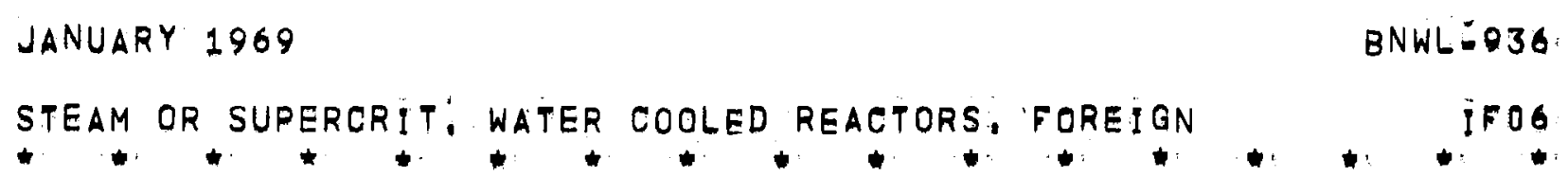

NAME/OWNER KFK STEAM-COOLED BREEDER/KFK, W: GERMANY Di 1 REACTOR

DESIGNER AEG; MAN, AND BELGIAN FIRMS

OPERATOR GESELLSCHAFT FUR KERNFORSCHUNG KARLSRUHE, W. GERM:

LOCATION KARLSRUHE; W. GERMANY

PURPOSE PROTOTYPE; FAST BREEDER PROGRAM

TYPE STEAMNOCOLED FASTT BREEDER, DIRECT CYCLE

PANCAKE CORE

POWER MWE(MWT) 300

CRITICAL REFERENCE DESIGN.

SEE REMARKS

COOLANT LIGHT WATER STEAM

FUEL MATERIAL URANIUM-PLUTONIUM DIOXIDE

FUEL GEOMETRY PIN: 7 MM: OD

FUEL CLADDING INCONEL-625, 0:4 MM, THICK, SPIRAL FINS.

FUEL ENRICH, AVE: 17 PER CENT FISSILE PLUTONIUM.

FUEL ASSEMBLY 39-PIN BUNDLE + CONTROL RODS, HEXAGONAL 180 ELEMENTS/CORE

BURNUP(REFUEL) DESİGN,75,000 MWD/T

CONTROL RODS, SUBASSEMBLY POSITIONS

COQLANT TEMP, INLET $365 \mathrm{C}$ OUTLET $540 \mathrm{C}$

COQLANT PRESS, $\quad 170 \mathrm{KG} / \mathrm{SQ}$. CM.

REACTOR VESSEL HEAVY STEEL CYLINDRICAL VESSEL

CONTAINMENT REINFORCED CONCRETE CELL, UPPER PART OF WHICH IS FILLED W! TH WATER AND SERV!NG AS A REFUELING POQL, SECOND GAS-TIGHT ENVELOPE IN WHICH SMALL NEGATIVE PRESSURE IS MAINTAINED.

REMARKS

A 25 MW HOT STEAM REACTOR AT GROSSWELZHEIM HAS BEEN TEST BED FOR THE STUDY PROJECT.

THE PROJECT MAY BE TERMINATED: SEE ID11. 
JANUARY 1969

BNWL $=036$

STEAM OR SUPERCRIT', WATER COOLED REACTORS FOREIGN IFOG

REFERENCES REFERENCE STUDY FOR THE 1000 MWE STEAM-OOOLED FAST BREEDER D - 1

A MUELLER; OTHERS

ORNL-TR-1541

DESIGN AND EVALUATIION OF A STEAM-COOLED FAST BREEDER REACTOR OF 1000 MWE

'RA MUELLER, OTHERS

FAST BREEDER REACTORS: PROC: LONDON CONF: BRITISH NUCLEAR ENERGY SOC:: 1966 P. 79:97.

PERGAMON, 1967

DESIGN CHARACTERISTICS OF A 300 MWE STEAM-COOLED

FAST REACTOR PROTOTYPE

F KILIAN, OTHERS

ANS TRANS: $11 / 1 / 275$ /JUNE, $1968 /$ 
JANUARY 1969

BNWL: $=936$

STEAM OR SUPERCRIT: WATER COOLED REACTORS FOREIGN

NAME/OWNER

DES IGNER

PURPOSE

TYPE

POWER MWE (MWT)

CR!TICAL

COOLANT

MODERATOR

FUEL GEOMETRY

FUEL CLADDING

FUEL ENRICH,

FUEL ASSEMBLY
FUEL MATERIAL

SWISS SCHWR/FED: INST: REACTOR RESEARCH, SWITZ;

SWISS FED: INST: REACTOR RESEARCH /EIR/

'POWER

STEAM COOLED, SUPERHEAT, DIRECT CYCLE HORIZONTAL PRESSURE-TUBE

$$
250
$$

$723: 7$

DESIGN STUDY. PROPOSED FOR CONSTRUCTION

LIGHT WATER STEAM

HEAVY WATER

URANIUM DIOXIDE PELLETS 13,6 MM: DIA:

ROD, ACT, LENGTH 660 MM:

ZIRCONIUM ALLOY $0: 8$ MM. TH!CK, RIBBED

$0: 99$ PER CENT U:235

18-ROD BUNDLE: RODS ARRANGED IN TWO CIRCLES WITH SP IDERS AND SPACERS: 6 . ELEMENTS/CHANNEL

552 COOLANT CHANNELS, ZIRCONIUM-NIOB!UM

PRESSURE TUBES, INTERNAL INSULATION,

FUEL CHARGE 50.5 TONS URANIUM

BURNUP (REFUEL) 12,930 MWD/TU

$O N=L O A D$

5 ELEMENTS/DAY

NEUTRON FLUX THERMAL MAX, $6.7 \times 10 . E \neq 13$

CONTROL RODS

CHEMICAL SHIM

COOLANT TEMP, INLET 324:3 C OUTLET 393,8 C

COOLANT PRESS, INLET 112. BARS

CONTAINMENT CYLINDRICAL BUILDING CONTAINING REACTOR, COLLECTORS, BOILERS AND BLOWEAS, REFUELING EQUIPMENT AND FUEL STORAGE POOLS

REMARKS 


$$
\begin{aligned}
& \text { JANUARY } 1969 \\
& \text { BNWL:-036 } \\
& \text { STEAM OR SUPERCRIT: WATER COOLED REACTORS, FOREIGN IFO? } \\
& \text { A LOEFFLER-TYPE CYCLE IS ADOPTED, WITH WATER } \\
& \text { EVAPORATED IN AN EXTERNAL HEAT EXCHANGER, } \\
& \text { THE REACTOR ACTS AS A SUPERHEATER, DELIVERING } \\
& \text { STEAM BOTH TO THE EVAPORATORS AND TO THE TURBINES: } \\
& \text { REFERENCES DESIGN OF A STEÁM COOLED NUCLEAR POWER PLANT: } \\
& \text { EIR. SWITZERLAND } \\
& \text { NT. INEUE TECHNIK/ FEB, } 1966 \text { P: 1:10 }
\end{aligned}
$$




\section{NUCLEAR AUXILIARY POWER SYSTEMS}

SNAP-TYPE REACTORS

DOMESTIC 


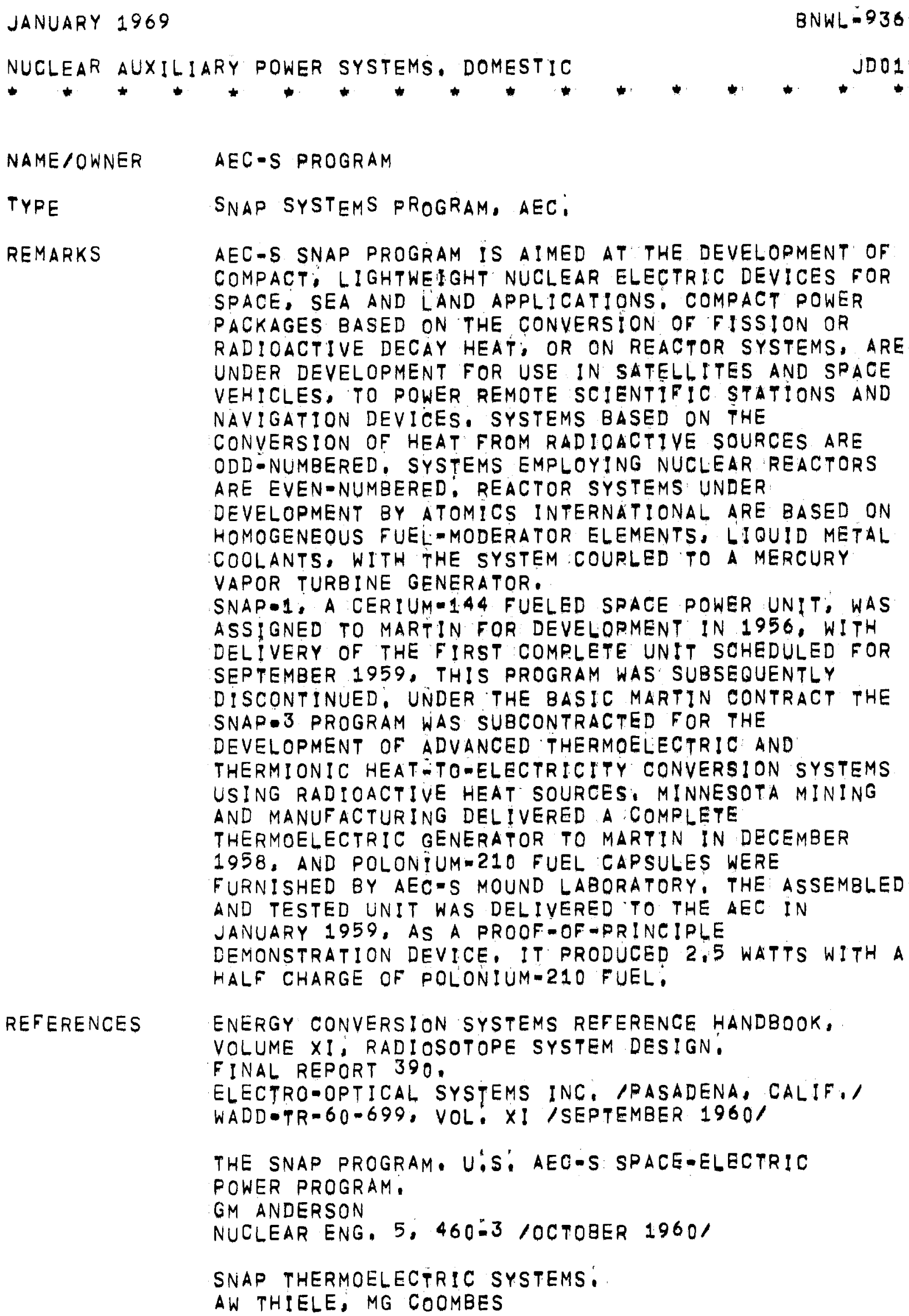




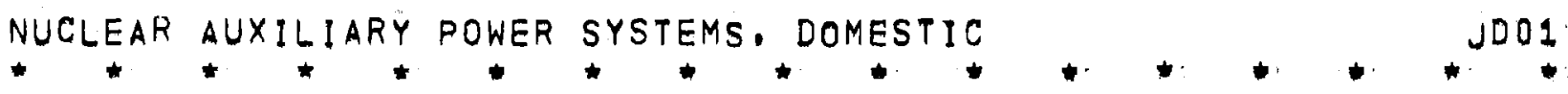

ARS SPACE POWER SYSTEM CONFERENCE, SANTA MONICA. CALIFORNIA, SEPTEMBER 27-30, 1960

PAPER $1330=60$

AMERICAN ROCKET SOC:, N.Y, $11960 \%$

NUCLEAR FRONTIERS - 1960

A FORUM REPORT /NQ. 32 I

PROC, ANNUAL CONF, FOR MEMBERS AND GUESTS,

SAN FRANCISCO, CALIFORNIA, DECEMBER 14-16,1960

-EA WIGGIN, ED.

ATOMIC INDUSTRIAL FORUM, N,Y, /1961/

SNAP FACT SHEET:

AEC NEWS RELEASE, JUNE 15, 1962 
NUCLEAR AUXILIARY POWER SYSTEMS, DOMESTIC

NAME/OWNER SNAP-2/AEC-AIR FORCE

DESIGNER

ATOMICS INTERNATIONAL

PURPOSE

SPACE AUXILIARY POWER

TYPE

REACTOR SYSTEM, AUXILIARY POWER: LIO, METAL COOLED THERMOELECTRIC CONVERSION

POWER MWE(MWT)

$3 \mathrm{KW}$

$55 \mathrm{KW}$

CRITICAL

DESIGN, DEVELOPMENT

COQLANT

SODIUMAPOTASSIUM NAK

MODERATOR

HYDROGEN IN FUEL-MODERATOR ELEMENTS

FUEL MATERIAL URANIUM-ZIRCONIUM HYDRIDE

FUEL GEOMETRY RODS $1: 25$ IN. DIA:

FUEL CLADDING SS

FUEL ENRICH, 93 PER CENT U $=235$

FUEL ASSEMBLY 37 RODS/CORE

SPECIFIC POWER O:O3 PER CENT

BURNUP(REFUEL) LIFETIME 1 YEAR

NEUTRON FLUX $3: 1 \times 10 \quad E+11$

CONTROL DRUMS

COOLANT TEMP, INLET 1000F OUTLET $1200 \%$

CONTAINMENT STAINLESS STEEL

REMARKS

POWER CONVERSION EQUIPMENT IS A MERCURY-RANKINE COMBINED ROTATING UNIT, SNAP-2 AS A SYSTEM HAS BEEN TERMINATED; BUT IS CONTINUING AS A BASIC RESEARCH PROGRAM ON ZIRCONIUM HYDRIDE REACTOR SYSTEMS: SEE JDOS.

REFERENCES THE SNAP=2 CONCEPT.

HM DIECKAMP

ARS SPACE POWER SYSTEMS CONFERENCE, SANTA MONICA, CALIFORNIA; SEPTEMBER 27-30; 1960

PAPER NO. $1324 \cdot 60$ 


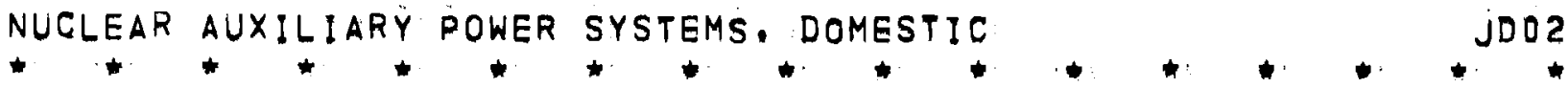

AMERICAN ROCKET SOC: N.Y: 11960/

AN ENRICHED UOZ-ZRH CRITICAL ASSEMBLY:

MV DAVIS, OTHERS

NAA:SR-5610/NOVEMBER $1960 /$

SNAP-2, NUCLEAR SPACE, POWER SYSTEM. JR WETCH, HM DIECKAMP: DJ COCKERAM /AII. ASTRONAUTICS 5 /12\%: 24-5 /DECEMBER 1960/

PRELIMINARY RESULTSS ON THE SNAP-2 EXPERIMENTAL REACTOR:

MW HULIN, J BEALL, ED:

NAA-SR-5991/ARRIL 1961/

SNAP-2 POWER CONVERSION STUDIES,

DL SOUTHAM

SPACE POWER SYSTEMS: R, 291

NW SNYDER; ED;

ACADEMIC PRESS, N:Y'

SYSTEMS ANALYSIS OF NUCLEAR ISNAPIZ/ LIQUID METAL CELL SPACE POWER SYSTEM.

GENERAL MOTORS CORP:

EDR-3113/DEC, 5, 1962/

NUCLEAR REACTOR SYSTEMS

GM ANDERSON /US AEC/

ASTRONAUTICS/AEROSPACE ENG, 1/4/, 27-36 MAY 1963 
JANUARY 1969

$B N W L=036$

NUCLEAR AUXILIARY POWER SYSTEMS, DOMESTIC

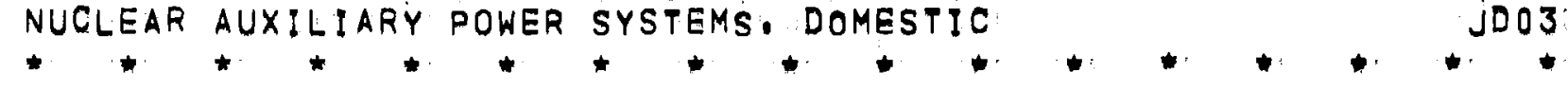

NAME /OWNER

SNAP-A/AEC-NAVY

DES IGNER

ATOMICS INTERNATIONAL

PURPOSE

UNATTENDED UNDERWATER OPERATION

TYPE

REACTOR SYSTEM, AUXILIARY POWER: BWR, THERMOELECTRIC CONVERSION

POWER MWE(MWT)

2

12

CRITICAL

DESIGN, DEVELOPMENT

COQLANT

LIGHT WATER

MODERATOR

HYDROGEN IN FUEL-MODERATOR ELEMENTS

FUEL MATERIAL URANIUM-ZIRCONIUM HŸDRIDE

FUEL GEOMETRY RODS

FUEL CLADDING SS

FUEL :ENRICH, 93 PER CENT U-235

BURNUP(REFUEL) OPERATING LIFETIME I-PLUS YEARS

COOLANT TEMP, INLET 540 F OUTLET 570 F

REMARKS

SNAPDA IS BEING PHASED OUT AS A SYSTEM BY THE AEC, IN-PILE, TESTING OF FUEL SAMPLES WILL BE FOLLOWED

THROUGH: AI WILL APPLY ACOUIRED TECHNOLOGY TO ITS COMPACT ICOMPACT MULTI-PURPOSE AUTOMATIC.

CONTROLLED TRANSPORTABLE/ REACTOR EFFORT.

REFERENCES SNAP FACT SHEET:

USAEC, NEWS RELEASE; JUNE 1962

HEARING BEFORE THE U.S: JOINT COMMITTEE ON ATOMIC ENERGY. CONGRESS OF THE UNITED STATES, 87TH CONGRESS, SECOND SECTION ON, PEACEFUL USES OF ATOMIC ENERGY APRIL 10.1962 /P: 140.143/ 


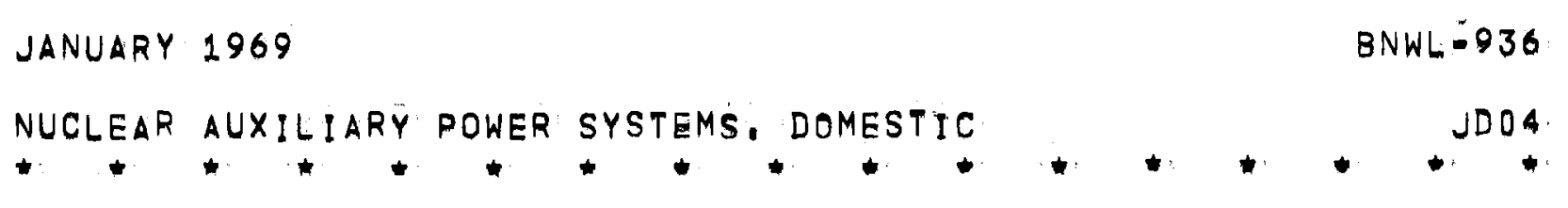

NAME/ OWNER

DES I GNER

LOCATION

PURPOSE

TYPE

POWER MWE (MWT)

CRITICAL

COOLANT

MODERATOR
$S N A P-B / A E C=N A S A$

ATOMICS INTERNATIONAL

S8ER AT SANTA SUSANA, CALIF!

SPACE POWER

REACTOR SYSTEM, AUXILIARY POWER: LIO. METAL COOLED THERMOELECTRIC CONVERSION

$450.600 \mathrm{KWT}$

SNAP-BER DEC: 1963

SNAP-BDR JULY 1968

SODIUM-POTASSIUM NAK

ZIRCONIUM HYDRIDE

BERYLLIUM REFLECTOR

FUEL MATERIAL URANIUM-ZIRCONIUM HYDRIDE

FUEL GEOMETRY ROD 0.56 2N: DIA./14.5 IN: LONG

FUEL CLADDING HASTELLOY: 0.010 IN. THICK

FUEL ENRICH, FULL

FUEL ASSEMBLY BUNDLE

211 ELEMENTS/CORE /S8ER/

FUEL CHARGE 6:56 KG:U-235

BURNUP(REFUEL) 10,000 HOUR OPERATING LIFE

NEUTRON FLUX THERMAL AVE, $5 \times 10$ E\$12

CONTROL 6 REFLECTOR CONTROL DRUMS

COOLANT TEMP, INLET $1100 \mathrm{~F}$ OUTLET $1300 \mathrm{~F}$

COQLANT PRESS.

40 PSIA

REACTOR VESSEL $9: 75$ IN: DIA./21 IN: HIGH VESSEL, SS

CONTAINMENT SHIELDING

REMARKS HIGH POWER, HIGH TEMPERATURE REACTOR DEVELOPMENT, MERCURY RANKINE CYCLE POWER CONVERSION SYSTEM. AS 
NUCLEAR AUXILIARY POWER SYSTEMS . DOMESTIO

OF APRIL 3, 1964, THE S8ER /SNAP-8 EXPERIMENTAL REACTOR/ PRODUCED AN INTEQRAL POWER OUTRUT OF OVER 1,000,000KWH/T/ DURING 48 DAYS OF OPERATION, AN ADVANCED TEST MODEL PROTOTYPE SNAP BDR REACHED DRY CRITICALITY IN GROUND TESTS IN JULY 1968; AS A CONTINUATION OF SNAP=1OA TECHNOLQGY. WET CRITICAL TESTING WAS WITH NAK. FULL POWER, $600 \mathrm{KWT}$.

REFERENCES NUCLEAR POWER AND SPACE: GT SEABORG

IRE TRANS: NUCLEAR SCI. NS=9, 1:8/JANUARY 1962 /

SNAP-8 REACTOR AND SHIELD

CE JOHNSON, CA GOETZ

AIAA J. 1/10/,2355-9/OCT: 1963/

NUCLEAR REACTOR SYSTEMS

GM ANDERSON /US AEC/

ASTRONAUTICS/AERSOPACE ENG, $1 / 4 \%, 27.36$ MAY 1963

SNAP-8 REACTOR ÄND SHIELD DESIGNS AND OPERATING EXPERIENCE.

CE JOHNSON, DG MASON

$J$ SPACECRAFT AND ROCKETS 3; 1099-1105/JULY 1966 
JANUARY 1969

BNWL-736

NUCLEAR AUXILIARY POWER SYSTEMS, DOMESTIC

J005

DES!GNER

LOCATION

RURPOSE

TYPE

POWER MWE (MWT)

CRITICAL

COQLANT

MODERATOR
NAME / OWNER

SNAP - 1OA/AEC - NASA

ATOMIOS INTERNATIONAL

SPACE

SPACE AUXILIARY POWER

REACTOR SYSTEM, AUXILIARY POWER: LIO, METAL COOLED THERMOELECTRIC CONVERSION

35 KWT

ORBITAL LAUNCH IN APRIL 1965

SODIUM-POTASSIUM NAK

ZIRCONIUM HYDRIDE

EXTERNAL BERYLLIUM REFLECTOR

FUEL MATERIAL URANIUM-ZIRCONIUM HYDRIDE

FUEL GEOMETRY ROD 1:25 IN, DIA./13 IN, LONG

FUEL CLADDING HASTELLOY-N 0.015 IN: THICK

FUEL ENRICH, 93 PER CENT U.235

FUEL ASSEMBLY 37 INDIVIDUAL ELEMENTS/CORE IN A CLOSE-PACKED TRIANGULAR MATRIX

FUEL CHARGE $\quad: 75 \mathrm{KG} \mathrm{U}=235$

BURNUP (REFUEL) 1:YEAR LIFETIME

NEUTRON FLUX 1;8X10 E*11

CONTROL ROTATING ELEMENTS OF REFLECTOR, RADIAL REGION

COOLANT TEMP, INLET $880 \mathrm{~F}$ OUTLET $990 \mathrm{~F}$

REACTOR VESSEL SS CYLINDER 8.94 IN: DIAM, 0:032 IN. WALL

CONTAINMENT SHIELDING

REMARKS

SNAP-1OA IS A TEMPERATURE AND, POWER DERATED

VERSION OF THE SNAP-2 CONOEPT: GROUND TESTING OF

THE 500-WATT SYSTEM WAS DONE IN JANUARY 1965, AND

COMPLETE SYSTEM LAUNCHED INTO PQLAR ORBIT ON 


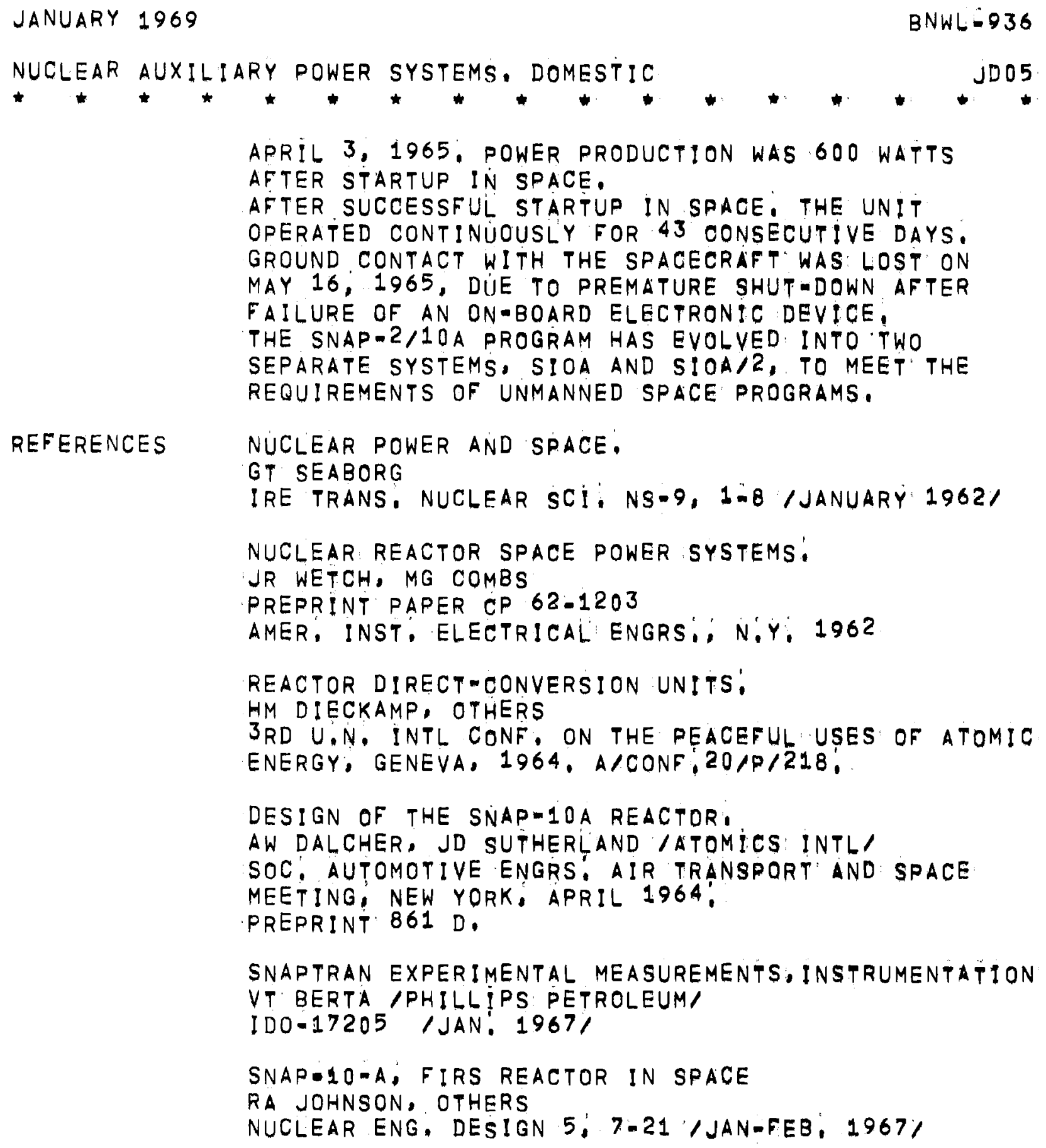


JANUARY 1969 $B N W L=936$

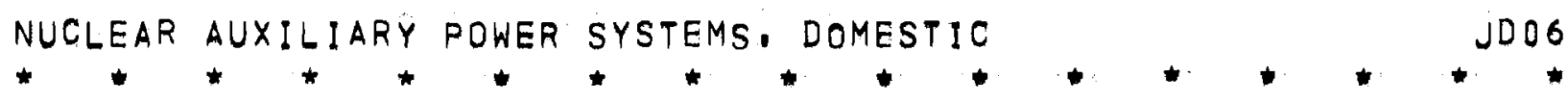

NAME/OWNER SNAP-15A/AEC

DESIGNER

GENERAL ATOMICS DIV: GENERAL DYNAMICS NOW GULF GENERAL ATOMIC

PURPOSE

AUXILIARY POWER TERRESTRIAL APPLICATION.

TYPE

ISOTOPE SYSTEM, POWER GENERATOR

POWER MWE(MWT)

4.10 WATTS

CRITICAL

OPERATION 1965 AS ACTIVATOR IN CONTROL DEVICE

FUEL MATERIAL

PLUTONIUM=238

REFERENCES

ISOTOPES AND RADIATION TEOHNOLOGY, VOL, A/2/, P, 178 /WINTER 1966-1967\% 


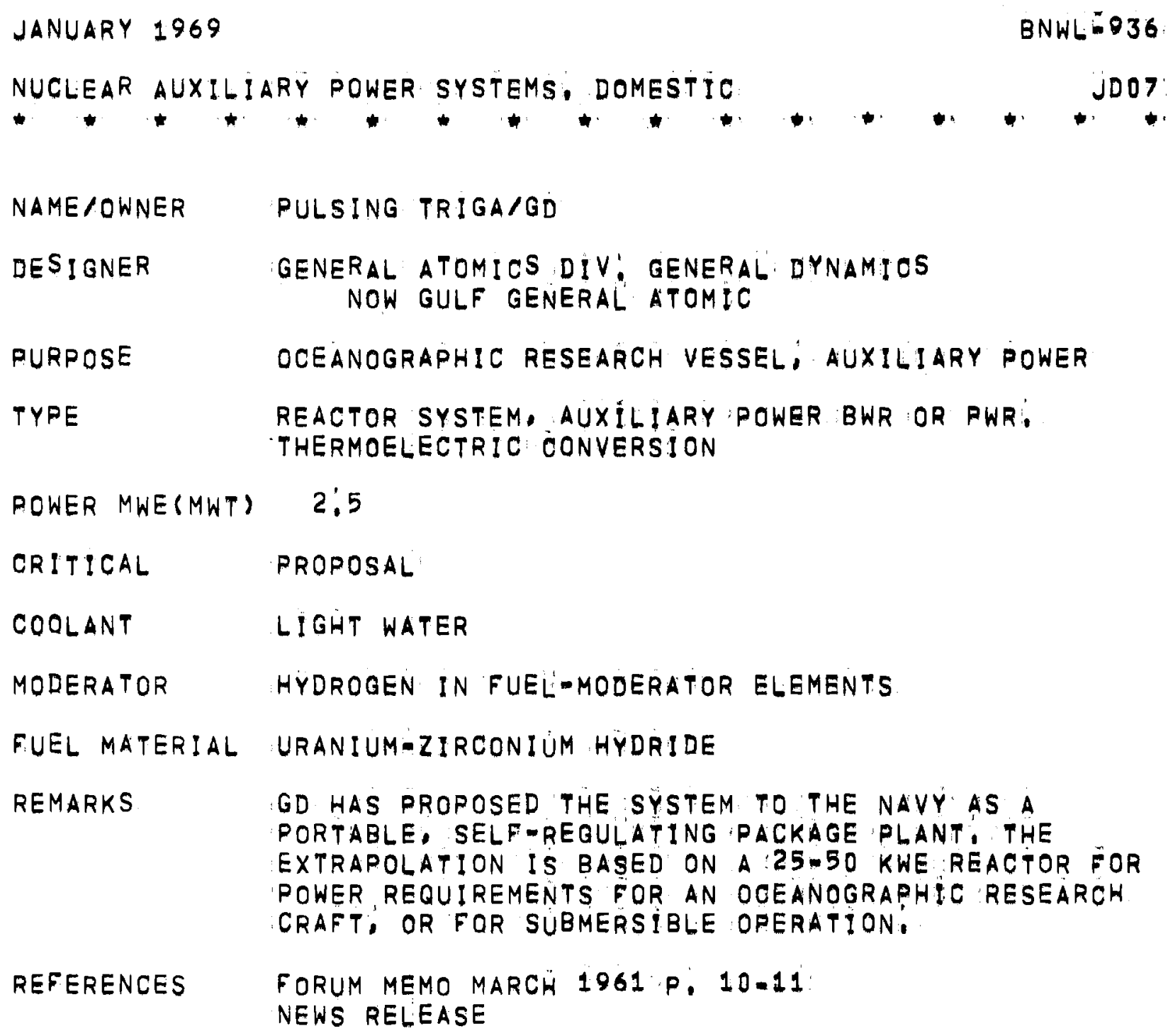


JANUARY 1969

BNWL $\div 36$

NUCLEAR AUXILIARY POWER SYSTEMS. DOMESTIC

JDOB

NAME/OWNER THERMIONIC REACTOR/GD

DESIGNER

GENERAL ATOMICS DIV: GENERAL DYNAMICS

NOW GULF GENERAL ATOMIC

PURPOSE

AUXILIARY POWER

TYPE

REACTOR SYSTEM, AUXILIARY POWER

THERMIONIC CONVERSION

POWER MWE (MWT)

1

10

CRITICAL

STUDY

COOLANT

LIGHT WATER

FUEL MATERIAL URANIUM-ZIRCONIUM CARBIDE

FUEL GEOMETRY CYLINDRICAL RODS

FUEL CLADDING SS SHROUD

FUEL :ENRICH, FULL

FUEL ASSEMBLY

35 SER IES-CONNECTED THERMIONIC ELEMENTS, CYLINDRICAL FUEL RODS SURROUNDED BY TUNGSTEN EMITTER AND NIOBIUM COLLECTOR, ALUMINA INSULLATION

FUEL CHARGE $56 \mathrm{KG} \mathrm{U-235}$

CONTROL BORON"SS BLADES

REMARKS DEVELOPMENT. STUDY

REFERENCES A 1-MWE THERMIONIC REACTOR CONCERTUAL DESIGN: CW SAYERY; OTHERS /GD/

TRANS. AMERICAN NUCLEAR SOC: 6111,93 /JUNE 1963/ 
JANUARY 1969

BNWL: 936

NUCLEAR AUXILIARY POWER SYSTEMS, DOMESTIC:

NAME/OWNER

DES IGNER

PURPOSE

TYPE

POWER MWE (MWT)

CRITICAL

COOLANT

MODERATOR

FUEL MATERIAL

FUEL CLADDING

FUEL ENRICH.

FUEL ASSEMBLY

CONTROL

REMARKS

REFERENCES
STAR /SPACE THERMIONIC AUXILIARY REACTOR//GE

GENERAL ELECTRIC COMPANY

SPACE AUXILIARY POWER

REACTOR SYSTEM, THERMIONIC CONVERSION

1

11

DEVELOPMENT

SODIUM

NONE

REFLECTOR; BERYLLIUM OXIDE

URANIUM DIOXIDE INDIVIDUAL: RINGS

TUNGSTEN, MOLYBDENUM, NIOBIUM: OR ALUMINA

FULL

HOLLOW CYCLINDER COMPOSED OF SEGMENTED RINGS; EACH RING CONSISTING OF INDIVIDUAL SEGMENTS OF REACTOR FUEL AND CESIUM PLASMA CONVERTER.

MOTION OF END CONVERTER.

DEVELOPMENT AT PLEASANTON, CALIF: LABORATORY: AN AIR FORCE CONTRACT FOR A STAR-R THERMIONIC REACTOR UNIT WAS AWARDED. 6IMONTH DESIGN ANALYSIS HAS BEEN COMPLETED: PHASE 2 CONTRACT FOR STAR-C, A

DEVELOPMENT OF A $100 \mathrm{KWE}$-1. MWE CONDUCTION-COOLED REACTOR: AEC CONTRACT IS FOR IN-PILE TESTING OF CONVERTERS.

DEVELOPMENT OF NUCLEAR THERMIONIC FUEL ELEMENT: ANNUAL SUMMARY AND QUARTERLY RROGRESS REPORT NO, 4; MARCH 1, 1963-MAY 30, 1963 GENERAL ELECTRIC CO:, VALLECITOS LAB,: PLEASANTON GESR-2017/ND/

LARGE TURBO-NUCL'EAR SPACE POWER SYSTEMS.

MA ZYSKIN /GENERAL ELECTRICI, PHILAII

$P: 201-59$ 3F ADVANCED PROPULSION CONCEPTS, VOL: 1 N.Y., GORDON AND BREACH, 1963.

THERMIONIC CONVERTERS FOR SPACE POWER

VC WILSON; RC HAMILTON /GEN, ELEC, CO, RES, LAB,/ ASTRONAUTICS/AEROSPACE ENG, 1/A\%, 62-67 MAY 1963 
JANUARY 1969
NUCLEAR AUXILIARY POWER SYSTEMS, DOMESTIC BNL-936
$*+\cdots+\cdots+\cdots$

NAME/OWNER

$D E S_{\text {IGNER }}$

LOCATION

PURPOSE

TYPE

POWER MWE(MWT) $0: 5$ WATT

CRITICAL

PROTOTYPE TESTING 1963

FUEL MATERIAL THULIUM-170

FUEL ASSEMBLY

PENCIL-SIZED THERMOCOUPLE MODULES OF

LEAD TELLURIDE THERMOELECTRIC ELEMENTS, WITH THE ENCAPSULATED FUEL IN THE MIDDLE? MODULES ARE CONNECTED IN SERIES:

FUEL CHARGE

REMARKS

REFERENCES

THERMOELECTRIC GENERATOR/AIR FORCE

GENERAL ELECTRIC COMPANY

PROTOTYPE AT GE INSTALLATION

POWER FOR ELECTRONIC EOUIPMENT AND SMALL SATELLITES.

ISOTOPE SYSTEM, THERMOELECTRIC CONVERSION.

10,000 CURIES THULIUMM-170

GE HAS DESIGNED; BUILT, AND TESTED A PROTOTYPE UNIT, LONG-TERM PERFORMANCE TESTS ARE UNDER WAY, OTHER APPLICATIONS WOULD BE FOR UNDERWATER

NAVIGATIONAL BEACONS AND OTHER REMOTE DEVICES.

FORUM MEMO OCT, 1963 P. 21. NEWS RELEASE 
JANUARY 1969

BNWL: $=036$

nuClear auxiliary power systems. DOMESTIC: JD11.

NAME/OWNER

DESIGNER

PURPOSE

TYPE

POWER MWE (MWT)

QRITICAL

FUEL MATERIAL
MFP IMIXED FISSION PRODUCTS GENERATOR/AEC

GENERAL INSTRUMENT' CORPORATION

AUXILIARY POWER; UNDERWATER ENVIRONMENT

ISOTOPE SYSTEM. THERMOELECTR!O CONVERSION

5-10 WATTS

DEVELOPMENT, CONCEPTUAL DESIGN,

MIXED FISSION PRODUCTS IPOTS FROM CALEINATION PROCESSES/

FUEL GEOMETRY POT /CYLINDER OR SPHERE/ OF HASTELLOY

FUEL ASSEMBLY SINGLE POT

BURNUP(REFUEL) S:YEAR LIFETIME

REACTOR VESSEL STAINLESS STEEL CYLINDRICAL CONTAINER FOR HEAT TRANSFER FLUID, SURROUNDING THE POT FUEL, AND OUTER PRESSURE VESSEL,

CONTAINMENT CONCRETE AND IRON SHIELDING:

REMARKS

REFERENCES
AN ELECTRICALLY HEATED PROOF - OF-PRINCIPLE 3-5 WATT MFP GENERATOR WAS OPERATED CONTINUOUSLY FOR OVER A YEAR AT 4 PER CENT OVER ALL EFFICIENCY, PROGRAM REORIENTATION WILL DELAY DELIVERY DATE, ORIGINALLY SET FOR EARLY 1964, A FUELED MFP GENERATOR MAY BE ELIMINATED, WITH CONTINUED EXPERIMENTATION TO BE DIRECTED TOWARD GENERATORS WITH HIGHER OUTPUTS: THE GENERATOR WOULD BE FUELED AT AN AEC

INSTALLATION, CONCEPTUAL DESIGNS FOR GENERATORS UP TO POWER LEVELS OF 1000 WATTS HAVE BEEN DONE.

DEVELOPMENT TECHNIQUES FOR POWER PRODUCTION FROM MIXED FISSION PRODUCTS. PHASE 2 MIDITERM REPORT. EJ LEMANSKI /GENERAL INSTRUMENT CORP/, NYO-9700 /MAY 21, 1962/

ECONOMIC FACTORS OF MFP THERMOELECTRIC GENERATORS, FINAL REPORT. EJ LEMANSK! /GENERAL INSTRUMENT CORP, / NYO-10462 /MAY 1963, 
JANUARY 1969

$B N W L=936$

NUCLEAR AUXILIARY POWER SYSTEMS, DOMESTIC

$j 012$

NAME/OWNER

SNAP-15B/AEC

DES I GNER

GENERAL INSTRUMENT CORPORATION

RURPOSE

AUXILIARY POWER: TERRESTRIAL: USE

TYPE

ISOTOPE SYSTEM

POWER MWE(MWT

MILLIWATT RANGE

CRITICAL

DEVELOPMENT

FUEL MATERIAL

PLUTONIUM-238

REMARKS

AEC DEVELOPMENT CONTRACT FOR FUELED GENERATORS IN THE MILLIWATT RANGE:

REFERENCES NEWS RELEASE 
JANUARY 1969

BNWL-936

NUCLEAR AUXILIARY POWER SYSTEMS, DOMESTIC

J013

NAME/OWNER

DES IGNER

PURPOSE

TYPE

CRITICAL

COOLANT

MODERATOR

FUEL MATERIAL

FUEL GEOMETRY

F.UEL ENRICH.

F.UEL ASSEMBLY

REMARKS

REFERENCES
GLOWWORM/LASL

LOS ALAMOS SCIENTIFIC LABORATORY

CONCEPTUAL STUDŸ, SATELLITE POWER, RESEARCH FACILITY.

REACTOR SYSTEM, RADIATION HEAT'EXCHANGE CONCEPT, CONCEPTUAL DESIGN

LIQUID OR GAS

GRAPHITE, BERYLLIUM OR GRAPHITE REFLECTOR

URANIUM/PLUTONIUM DIOXIDE OR CARBIDE IN

GRAPHITE /CERMETI:

CYLINDER OR SLEEVE, FUELED GRAPHITE

SLIGHT

SIMPLE CYLINDRICAL REACTOR, FUELED. GRAPHITE SLEEVE 3:REGION REACTOR, TWO ANNULAR FUELED GRAPHITE SLEEVES, INTERMEDIATE AND INNER, WITH HIGHER FUEL CONCENTRATION IN INNER CYLINDER, HOLLOW CORE

TUNGSTEN SLEEVES ARE ALSO CONSIDERED.

EXPLORATORY STUDY OF EARLIER CONCERT, LARRE ILOS ALAMOS RADIATION REACTOR EXPERIMENT\%, VERY HIGH FUEL TEMPERATURE IS REQUIRED FOR ENERGY EXTRACTION BY RADIATION. HEAT EXCHANGERS WOULD BE IN THE REFLECTORS, AS TUBES OR HOLES IN THE GRAPHITE THROUGH WHICH COOLANT WOULD CIRCULATE: THE CENTER CAVITY COULD BE A RESEARCH CAVITY OR CONTAIN HEAT EXCHANGE SYSTEM.

A RADIATION REACTOR CONCEPT /GLOWWORM/ LDP KING

LAMS-2427/NOV. 18, 1963/ 


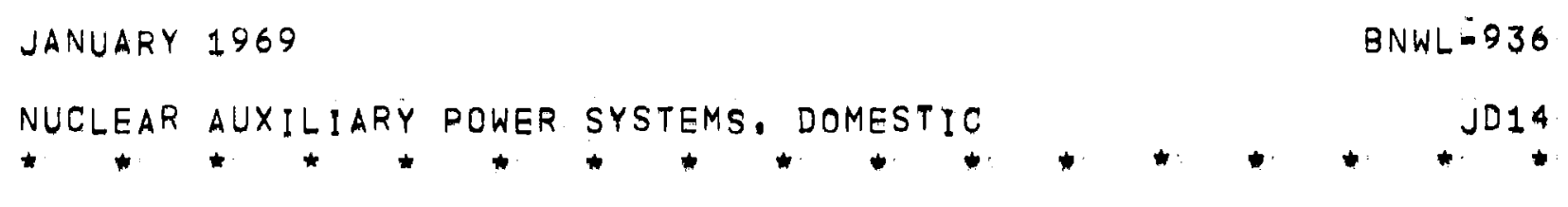

NAME/OWNER SNAP-1 AND SNAP-1A/AEC

DESIGNER MARTIN-MARIETTA CORPORATION

PURPOSE DEVELOPMENT

TYPE ISOTOPE SYSTEM. THERMOELECTRIC CONVERSION.

POWER MWE(MWT) 125 WATTS 6500 WATTS

CRITICAL DEVELOPMENT CONTRACT DRDPPED, SEE SNAR=3

FUEL MATERIAL CERIUM-144 OXIDE CERAMIC PELLETS

FUEL GEOMETRY CAPSULES

FUEL CLADDING TANTALUM CAPSULES

FUEL ASSEMBLY 7 CAPSULES/GENERATOR, IN CHANNELS DRILLED IN AN INCONEL CYLINDER.

FUEL CHARGE 0:88 MEGACURIES CERIUM-144

BURNUP(REFUEL) 1-YEAR LIFETIME

REACTOR VESSEL INCONEL =X CYLINDER /SOLID/ WITH CHANNELS FOR FUEL,

CONTAINMENT BIOLOGICAL SHIELD OF MERCURY FILLS INTERNAL YOLUME OF OUTER SHELL, AND IS DRAINED BEFORE LAUNCH. OUTER SHELL IS AN ALUMINA-INSULATION-SS SANDWICH, WITH LEAD TELLURIDE THERMOCOUPLE ELEMENTS INS!DE THE INSULATION,

REMARKS THE PROGRAM, ASSIGNED TO MARTIN IN 1965, WAS DISCONTINUED. THE SNAP-3 PROGRAM WAS SUBCONTRACTED UNDER THE BASIC MARTIN CONTRACT: SNAP=1 DESIGN USED MERCURY VAPOR AS HEAT CONVERSION WORKING FLUID. TASK 2 /SNAP-1A/ GENERATORS WERE DESIGNED FOR 1-YEAR LIFETIME IN A SATELLITE SYSTEM.

REFERENCES SNAP-1 RADIOISOTOPE-FUELED TURBOELECTRIC POWER CONVERSION SYSTEM SUMMARY, JANUARY 1957 TO JUNE 1959: PJ. DICK

MND-P-2350/JUNE $1960 /$

FINAL SAFETY ANALYSES REPORT, SNAP-1A RADIOISOTOPE FUELED THERMOELECTRIIC GENERATOR: MARTIN COMPANY /GP DIXI MND-P-2352/JUNE $1960 /$ 


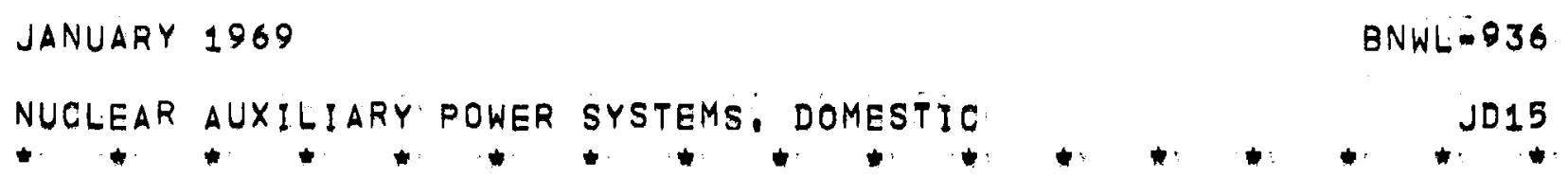

NAME/OWNER

DES I GNER

OPERATOR

LOCATION

PURPOSE

TYPE

POWER MWE (MWT)

CRITICAL

FUEL MATERIAL

FUEL GEOMETRY

FUEL CLADDING

FUEL ASSEMBLY

FUEL CHARGE

BURNUP (REFUEL)

REACTOR VESSEL HEAVY VESSEL OF HAYNES ALLOY TO HOLD GANISTER. CONTAINMENT

REMARKS

REFERENCES

SNAP - 3/AEC - NAVY

MARTIN-MARIETTA CORPORATION

NAVY

SPACE

AUXILIARY POWER; SPACECRAFT

ISOTOPE SYSTEM. THERMOELECTRIC OONVERSION

$$
2: 7 \text { WATT }
$$

69 WATT

DEMONSTRATION JANUARY 1959

TRANSIT OPERATION JUNE 1961

DEMONSTRATION; POLONIUM-210

TRANSIT; PLUTONIUM=238

CAPSULES

STAINNLESS STEEL CAPSULES

2 CAPSULES CONTAINING FUEL IN A STAINLESS STEEL CANISTER,

2200 CURIES POLONIUM-210

95 GRAMS PLUTON IUM- 238

SPHER ICAL COPPER SHELL CONTAINING THE GENERATOR, THERMOELECTRIC ELEMENTS PLACED AGAINST THE REACTOR VESSEL.

SNAP-3 SYSTEM DEVELOPMENT WITH PLUTONIUM-238 FUEL HAS RESULTED IN GENERATORS FOR THE TRANSIT /U.S. NAVY/ SATELLITES, TRANSIT-AB WITH PLUTONIUME. 238 GENERATOR WAS LAUNCHED IN JUNE 1961 AND A SECOND IN NOV, 1961! BOTH SATELLITES ARE STILL OPERATING:

HAZARDS SUMMARY REPORT FOR A 3-WATT POLONIUM-210

FUELED THERMOELECTRIC GENERATOR:, MARTIN COMPANY

MND-P-2047/JUNE 1959 DECLASSIFIED SEPTEMBER $1960 /$ 
JANUARY 1969

BNWL: 936

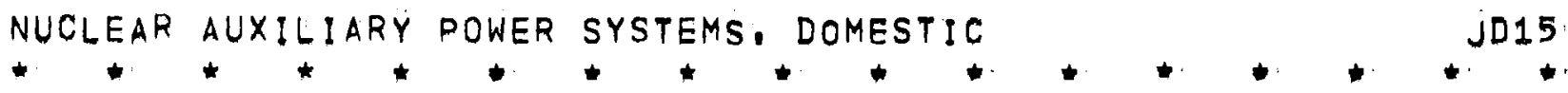

FINAL SAFETY ANALYSİS REPORT: SNAP-3

THERMOELECTRIC GENERATOR,

H HAGIS, GP DIX

MND-P-2364/JUNE $1960 /$

CONCEPTUAL DESIGN OF A SNAP-3 TYPE GENERATOR

FUELED WITH CERIUM=144,

RJ. WILSON

MND-P-2369/JUNE $1960 /$

NUCLEAR SAFETY ANALYSIS OF SNAP-3 FOR SPACE

MISSION,

W HAGIS, OTHERS

ARS J, 31L 1744:51. /DECEMBER $1961 /$

FROM SNAP-3 TO IMP

JG MORSE, CR FINK

IEEE TRANS. AEROSPACE 2;642,5 /APRIL:1964/ 


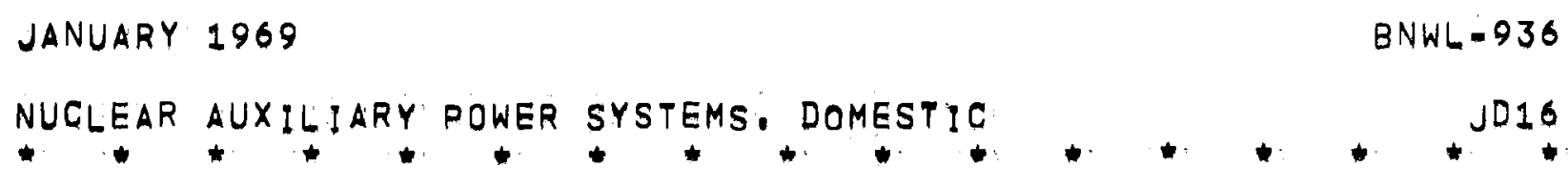

NAME/OWNER SNAP-7/AEC
LCG $=25$

DESIGNER MARTIN-MARIETTA CORPORATION

OPERATOR SEE REMARKS

LOCATION SEE REMARKS

PURPOSE AUXILIARY POWER; TERRESTRIAL APPLICATIONS

TYPE ISOTOPE SYSTEM. THERMOELECTRIC CONVERSION.

POWER MWE(MWT) 5:60 WATTS, SEE REMARKS:

CR!TICAL SEE REMARKS

FUEL MATERIAL STRONTIUM=90 TITANATE

FUEL GEOMETRY CYLINDER

FUEL ASSEMBLY FUEL BLOCK IN HASTELLOY CYLINDRICAL CONTAINER.

FUEL CHARGE ISEE REMARKS

REACTOR VESSEL FUEL BLOCK, HASTELLOY

CONTAINMENT DOUBLE-WALLED HASTELLOY VESSEL, DEPLETED URANIUM SHIELD WITHIN THE WALLS.

REMARKS SNAP-7 INSTALLED AT UNMANNED WEATHER STATION ON AXEL HEIBERG ISLAND IN THE ARCTIC, AUG, 1961:

5 WATTS. 41,000 CURIES STRONTIUM-90:

SNAP-7A INSTALLED IN FLOATING NAVIGATIONAL BUOY ICOAST GUARD/ IN DEC, 1961 AT ARUNDEL COVE, MD, 5 WATTS, 41.000 CURIES STRONTIUM-90: HAS EXPERIENCED STEADY POWER DEGRADATION,

SNAP-7B TRANSFERRED FROM CHESAPEAKE BAY NAVIGATION LIGHT TO OFF-ISHORE DRILLING BARGE IN GULF OF MEXICO, 30 WATTS, 41;000 CURIES STRONTIUM-90:

SNAP-7C INSTALLED AT REMOTE ANTARCTIC WEATHER STATION AT MCMURDO SOUND IN FEB: 1962 /NAVYI: 10-WATT: 41,000 CURIES STRONTIUM-90, OPERATION HAS BEEN SUCCESSFUL SINCE INSTALLATION,

SNAP-7D; BARGE-MOUNTED WEATHER STATION /NOMAD/ IN GULF OF MEXICO: MID-1963; SUCCESSFUL ORERATION. 


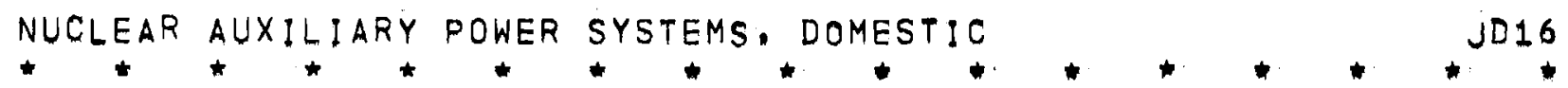

FIRST OF SERIES OF STATIONS RLANNED BY NAVY,

30 WATTS, 225,000 CURIES STRONTIUM-90.

SNAP-7E, AN 8-WATT GENERATOR, IN OPERATION IN JULY 1964 AS POWER SOURCE FOR NAVIGATIONAL AIDS, OFF JACKSONVILLE, FLORIDA: HAS RECENTLY COMPLETED 3RD YEAR OF OPERATION AT DEPTH OF 15,000 FT, AS POWER FOR SONAR BEACON.

SNAP-7F WAS PLACED ON AN OIL RIG IN THE GULF OF MEXICO FOR TRIAL USE IN 1964, 60 WATTS,

MARTIN WILL OFFER A COMMERCIAL GENERATOR

PATTERNED ON THE SNAP-7 SERIES, THE LOG-25

LOW COST GENERATOR WILL BE A 25-WATT STRONTIUM:OD

FUELED THERMOELECTRIC PACKAGE, WITH THE THERMO:

ELECTRIC GENERATOR MOVED OUTSIDE THE RADIATION

SHIELD, LATER VERSIONS MAY REACH 4O-WATTS, THE NAVAL ELECTRONICS LAB;, HAS BOUGHT A 25-WATT MODEL AND INSTALLED IT ON AN ISLAND IN BERING STRAIT OFF ALASKA TO POWER AN OOEANOGRAPH!C MEASURING STATION:

REFERENCES

HAZARDS SUMMARY REPORT FOR A 2-WATT STRONTIUM-9O

FUELED THERMOELECTRIC GENERATOR:

MARTIN COMPANY

MND-P-2048/JUNE 1959 DECLASSIFIED SEPTEMBER 1960/

STRONTIUM-90 FUELED THERMOELECTRIC GENERATOR POWER SOURCEF-5-WATT U:S: NAVY WEATHER STATION, FINAL REPORT.

MARTIN COMPANY, NUCLEAR DIV:

$M N D=P=2707 / N D /$

7A, STRONTIUM-90 FUELED THERMOELECTRIC GENERATOR FOR 5-WATT U.S, COAST GUARD LIGHT BUOY: FINAL REPORT. MARTIN COMPANY MND $=P-2720$ /FEBRUARY $1962 /$

7C FINAL SAFETY ANALYSIS, 10-WATT STRONTIUM-90 FUELED GENERATOR FOR AN UNAT TENDED METEOROLOGICAL STATION, SNAP $-7 C$. MART IN COMPANY MND=P-2614/FEBRUARY $1962 /$

7D, SNAP STRONTIUM-90 FUELED THERMOELECTRIC GENERATOR POWER SOURCE 3O-WATT U.S, NAVY FLOATING WEATHER STATION, FINAL REPORT, 
NUGLEAR AUXILIARY POWER SYSTEMS, DOMESTIC MARTIN COMPANY, NUCLEAR DIV, MND-P-2835 /MARCH 15, 1963/

7E, FINAL SAFETY EVALUATION OF A 10-WATT STRONTIUM-90 FUELED GENERATOR FOR A DEEP SEA APPLICATION-SNAP=7E:

HN BERKOW; VG KELLY

$M N D=P-2761 \cdot 1962 /$

RADIOISOTOPES AND RADIATION DEVELOPMENTS IN THE USA-1967, EE FOWLER ISOTOPES AND RADIATION TECH: 5/3/, 169-84/WINTER $1966-1967 /$ 
JANUARY 1969

BNWL $=936$

NUCLEAR aUXILIARY POWER SYSTEMS. DOMESTIC

jD17

NAME /OWNER

DES I GNER

LOCATION

PURPOSE

TYPE

POWER MWE(MWT)

CRITICAL

FUEL MATERIAL

FUEL GEOMETRY

FUEL CLADDING

FUEL ASSEMBLY

BURNUP (REFUEL)

REMARKS

REFERENCES
SNAP-9A/AEC-NAVY

MARTIN-MARIETTA CORPORATION

SPACE

AUXILIARY POWER; SPACE SYSTEMS,

ISOTOPE SYSTEM. THERMOELECTRIC CONVERSION.

20 WATTS

TRANSIT LAUNCH SEPT: 1963

PLUTONIUM-238 CARBIDE

CAPSULE

STAINLESS STEEL CAPSUL'E

FUEL CAPSULES IN CANISTER

5-YEAR LIFETIME

THE NAVY NAVIGATIONAL SATELLITE. WITH SNAP-9A

INSTALLED FAILED TO ACHIEVE ORBIT ON APRIL; 1964 LAUNCH. AEC REPORTED ALL FUEL BURNED UP COMPLETELY EARLIER LAUNCHES IN SEPTEMBER AND DECEMBER /TRANSIT/ WERE SUCCESSFUL AND THE GENERATORS ARE PROVIDING DESIGN POWER, PROJECTS IMP, NIMBUS, AND SURVEYOR WILL USE ADVANCED GENERATORS FOR HIGHER PERFORMANCE. SEE SNAP=19.

SNAPS 9A AND 19A STATUS REPORT: $J G$ MORSE, CR FINK MEETING MAY 18-19; 1964 ,

FROM SNAP 3 TO IMP

$J G$ MORSE, CR FINK

IEEE TRANS. AEROSPACE 2,642.5 /APRIL $1964 /$ 
JANUARY 1969

BNWL: $=36$

NUCLEAR AUXILIARY POWER SYSTEMS. DOMESTIC

JD18

NAME/OWNER

DES IGNER

OPERATOR

PURPOSE

TYPE

POWER MWE (MWT)

CRITICAL

FUEL MATER!A

BURNUP (REFUEL)

REMARKS

REFERENCES
SNAP-19/AEC-NASA

MARTIN-MARIETTA CORPORATION

NATIONAL AERONAUTICS AND SPACE ADMINISTRATION

AUXILIARY POWER

ISOTOPE SYSTEM, THERMOELECTRIC CONVERSION:

15 WATTS

TARGET' 1965

PLUTONIUM-238

5. YEAR LIFETIME

SNAP-19 IS A MULTI-PURPOSE FOLLOW-ON OF SNAPIOA: TAILORED TO NIMBUS-B REQUIREMENTS,

A NIMBUS-B WEATHER SATELLITE WAS SCUTTLED AFTER TAKE-OFF WHEN THE THORAD-AGENA-O LAUNCH VEHICLE VEERED OFF COURSE; DROPPING TWO SNAP-19 GENERATORS INTO THE PACIFIE /MAY 1968/. THE MISSION IS TO BE REPEATED IN THE SPRING OF 1969.

SNAPS-9A AND 19: A STATUS REPORT, $\checkmark G$ MORSE, CR FINK

ATOMIC INDUSTRIAL, FORUM, INDUSTRY INFORMATION MEETING MAY 18-19; 1964

NUCLEONICS WEEK MAY 23, 1968 P,4 NEWS RELEASE 
JANUARY 1969

BNWL -936

NUCLEAR AUXILIARY POWER SYSTEMS, DOMESTIC

JD19

NAME/OWNER

DES I GNER

PURPOSE

TYPE

POWER MWE (MWT)

CRITICAL

FUEL MATER!AL

FUEL GEOMETRY

FUEL CLADDING

FUEL ASSEMBLY

FUEL CHARGE

BURNUP (REFUEL)

CONTAINMENT

REMARKS

REFERENCES
$S N A P-11 / A E C-N A S A ̈$

MARTINGMARIETTA CORPORATION

AÚXILIARY POWER; SURVEYOR SPACECRAFT:

ISOTOPE SYSTEM, THERMOELECTRIC CONVERSION.

25 WATTS

DESIGN AND DEVELOPMENT

CURIUM-242, GOLD-CURIUM ALLOY

HOLLOW CYLINDER

TANTALUM, THIN INNER AND OUTER LINER,

THIN-WALLED TANTALUM CANISTERS

6:3 GRAM CURIUM 2242 FOR A 755-WATT' UNIT.

6-MONTH MISSION:

HASTELLOY OUTER VESSEL; TANTALUM INNER VESSEL, AN OUTER THIN ALUMINUM CYLINDER DISSIPATES HEAT' TO ENVIRONMENT.

SURVEYOR PRDJECT FOR SOFT-LANDING OF INSTRUMENTED PACKAGES ON THE MOON WILL USE CURIUM $=242$

GENERATORS, ALTERNATE TO SNAP=11 IS SNAP-13. CURIUM-242 TECHNOLOGY IS BEING APPLIED TO STUDIES OF CURIUM-244; A THERMAL MOCKUP OF SNAP-11. HAS BEEN TESTED. PAYLOAD INTEGRATION TESTING UNDER WAY

100-WATT CURIUM-242 FUELED THERMOELECTRIC GENERATOR CONCEPTUAL DESIGN, SNAP SUBTASK 5,7

FINAL REPORT,

JB WEDDELL, J BLOOM

MND $=P-2342$ /MAY $1960 /$

13-WATT CURIUM-FUELED THERMOELECTRIC GENERATOR FOR A SIX-MONTH SPACE MISSION, FINAL: REPORT, JL BLOOM

$M N D=P-2373 / J U L \ddot{Y} 1960 /$

SPACE ISOTOPIC POWER SYSTEMS, RT CARPENTER /USAF/ ASTRONAUTICS AEROSPACE ENG, $1 / 4 / 68-72$ MAY 1963 
JANUARY 1969

NUCLEAR AUXILIARY POWER SYSTEMS, DOMESTIC

FROM SNAP $=3$ TO IMP

JG MORSE, CR FINK

IEEE TRANS, AEROSPACE 2, 642-5 /APRIL 1964/
BNWLing 936

jD19. 


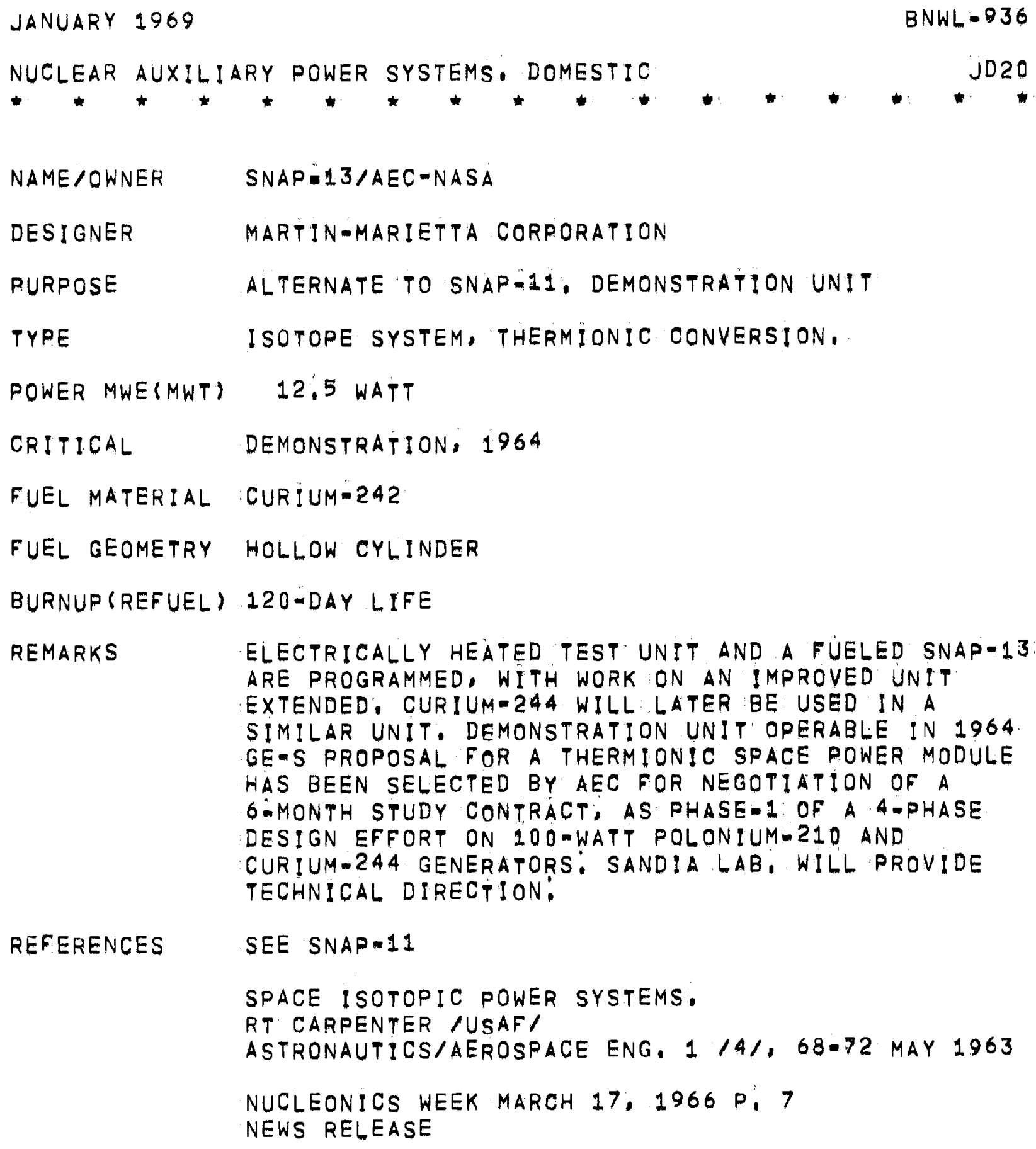


JANUARY 1969

BNWLing $=36$

NUCLEAR AUXILIARY POWER SYSTEMS. DOMESTIC

$J D 21$

NAME /OWNER DCR-1 /DIRECT CONVERSION REACTOR//MARTIN

DESIGNER MARTINMMARIETTA CORPORATION

RURPOSE AUXILIARY POWER

TYPE

REACTOR SYSTEM, AUXILIARY POWER: BWR THERMIONIC CONVERSION.

POWER MWE (MWT)

1

16

CRITICAL

DEVELOPMENT

COOLANT

LIGHT WATER

MODERATOR

ZIRCONIUM HYDRIDE

FUEL MATERIAL

URANIUM DIOXIDE-MOLYBDENUM CERMET, PELLETS.

FUEL GEOMETRY TUBES

FUEL CLADDING

MOLYBDENUM CLADDING ON PELLETS, ZIRCONIUM OXIDE SLEEVE, AND SS TUBE:

FUEL ASSEMBLY 5 -ELEMENT CLUSTER CORE CONSISTS OF 33 FUEL BUNDLES, CONTAINING 7 FUEL-TUBE CLUSTERS AND ZIRCONIUM HYDRIDE MODERATOR, THERMIONIC CONVERTER UNITS ARE INTEGRAL TO THE FUEL BUNDLE,

REMARKS

REACTOR SYSTEM IS A FORCED CIRCULATION, DIRECT CYCLE BWR, AN ALTERNATE FUEL SYSTEM CONSIDERED WAS THE URANIUM DIOXIDE-NIOBIUM SYSTEM, A CONCEPTUAL LIOUID METAL COOLED SYSTEM USED PHOSPHORUS SESQUISULFIDE OR NAK AS COOLANT, HOMOGENEOUS URAN IUM-ZIRCONIUM HYDRIDE FUEL-MODERATOR ELEMENTS, CONVERSION WOULD BE THERMOELECTRIC, ADVANCED APPROACHES INCLUDE NUCLEAR SUPERHEAT, A PRELIMINARY DESIGN FOR A 100 KWE REACTOR CORE HAS BEEN COMPLETED.

REFERENCES

A WATER-COOLED THERMIONIC PORTABLE POWER PLANT: GL REED

ANS TRANS: $6,318-19$ /NOV 1963/

FEASIBILITY STUDY OF A WATER-COQLED THERMIONIC NUCLEAR POWER PLANT: AEROSPACE DIVI, MARTIN-MARIETTA CORP, MND-2903, VOL. 2 /NOV. 1962/ 
JANUARY 1969

BNWL- $=36$

NuGlear aUXiliary power systems, dOMESTIC

NAME /OWNER

DESIGNER

PURPOSE

TYPE

POWER MWE(MWT)

CRITICAL

COOLANT

MODERATOR

FUEL MATERIAL

FUEL GEOMETRY

CONTROL

REMARKS

REFERENCES
TURPS /TERRESTRIAL UNATTENDED REACTOR POWER SYSTEM/MARTIN

MARTIN-MARIETTA CORPORATION

AUXILIARY POWER; REMOTE SITES OR UNDERSEA

REACTOR SYSTEM, AUXILIARY POWER! L10, METAL COOLED THERMOELECTRIC CONVERSION:

$100 \mathrm{KWE} \quad 1,56$

RESEARCH AND DEVELOPMENT

TETRAPHOSPHORUS TRISULIFIDE, BOILING

NATURAL CIRCULATION

ZIRCONIUM HYDRIDE

ZIRCONIUM-URANIUM HŸDRIDE

TWO-SEGMENT ELEMENT!

FUELED AND UNFUELED ZIRCONIUM HYDRIDE

A CONTROL CONCEPT TO BE EVALUATED IS BASED ON THE MIGRATION OF HYDROGEN IN THE ZIRCONIUM HYDRIDE UNDER TEMPERATURE CHANGE; AND THE MODERATING ACTION OF THE HYDROGEN AS IT MIGRATES,

A PLANT WOULD CONSIST OF SEVEN 20,000-POUND PACKAGES. FIRST PACKAGE WOULD BE THE REACTOR, GENERATOR; AND SHIELD TANK. PHOSPHDRUS

SULFIDE AS THE COOLANT WOULD BE BOILED IN THE REACTOR AND THE VAPOR TRANSPORTED TO THE THERMOELECTRIC GENERATOR BY NATURAL CIRCULATION. PLANT FEASIBILITY HAS BEEN DETERMINED! THE SYSTEM IS BEING SPONSORED BY THE AF SPECIAL WEAPONS CENTER, KIRTLAND AFB, UNDER A CONTRACT TO RUN UNTIL JANUARY 1966, AEC AND THE ARMY ARE EVALUATING THE CONCEPT. TURPS IS ALSO BEING EVALUATED AS AN UNDERSEA PLANT MARTIN HAS RECEIVED A 9-MONTH CONTRACT FROM THE $U$. S. ARMY CORPS OF ENGINEERS FOR A FEASIBILITY STUDY OF THE TURPS CONCEPT FOR A MOBILE NUCLEAR PLANT.

TETRAPHOSPHORUS TRISULFIDE COOLED THERMOELECTRIC REACTOR PLANT, CONCEPTUAL DES!GN, CE MURPHY 


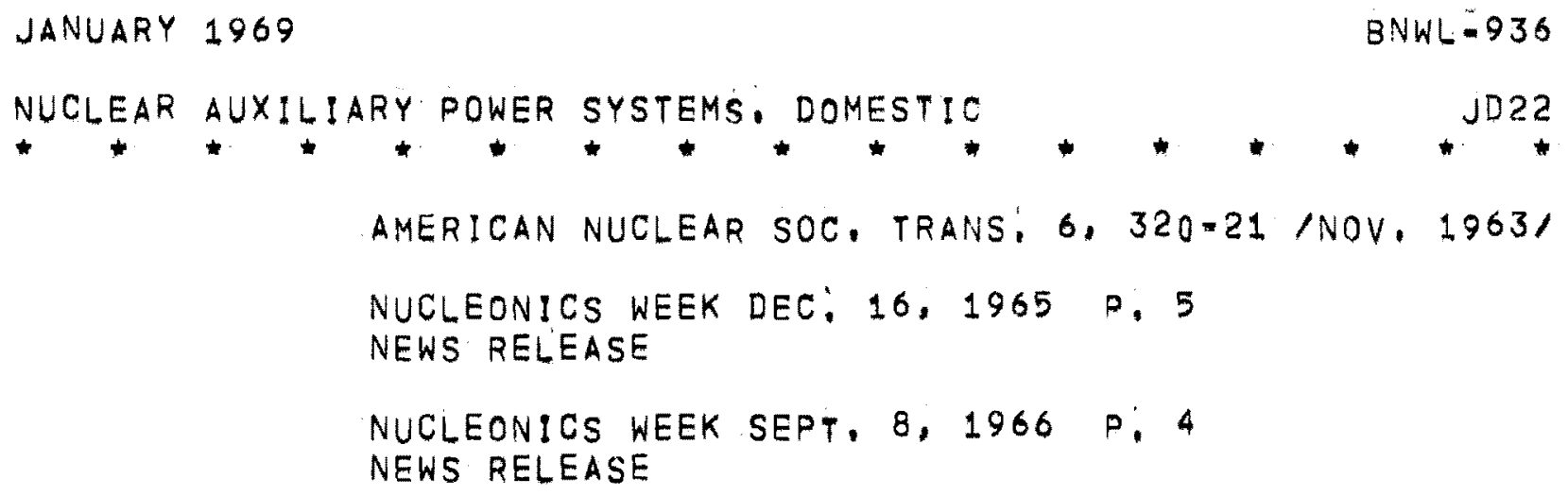


nUClear aUXiliary POWER SYSTEMS, DOMESTIC

NAME/OWNER UNDERWATER THERMOELECTRIC POWER PLANT/MARTIN

DESIGNER MARTIN-MARIETTA CORPORATION

PURPOSE ELECTRICAL POWER FOR UNDERSEA OPERATIONS,

TYPE REACTOR SYSTEM, AUXILIARY POWER, PWR

THERMOELECTRIC CONVERSION:

POWER MWE(MWT) $100 \mathrm{KWE}$

CRITICAL CONCEPTUAL DESIGN

COOLANT LIGHT WATER

MODERATOR LIGHT WATER

FUEL MATERIAL URANIUM DIOXIDE PELLETS

FUEL GEOMETRY TUBES

FUEL ENRICH. LOW

FUEL ASSEMBLY 104-TUBE BUNDLE

12 BUNDLES/CORE

CONTROL BURNABLE POISON: BORON, IN GLADDING.

REACTOR VESSEL SPHERICAL

CONTAINMENT THERMAL SHIELD, SEA-WATER SCREEN, AND SEA-WATER RETAINING SHELL:

REMARKS CONCEPTUAL DESIGN, A SELF-PRESSURIZED, NATURAL CIRCULATION REACTOR SYSTEM IPWR/. DESIGNED FOR REMOTE STARTUR AND COMPLETE UNATTENDED OPERATION, SYSTEM CONSISTS OF A LOWER PRESSURE VESSEL CONTAINING THE LOW-ENRICHMENT REACTOR CORE, A RISER, AN UPPER PLENUM, THREE SEVEN-PLATE ASSEMBLIES OF THERMOELECTRIC ELEMENTS. AND INTERCONNECTED PIPING:

REFERENCES AN 100 KWE UNDERWATER THERMOELECTRIC REACTOR PLANT CONCEPTUAL DESIGN: KH DUFRANE

AMERICAN NUCLEAR SOC, TRANS; 6, 319-20/NOV, 1963/ 


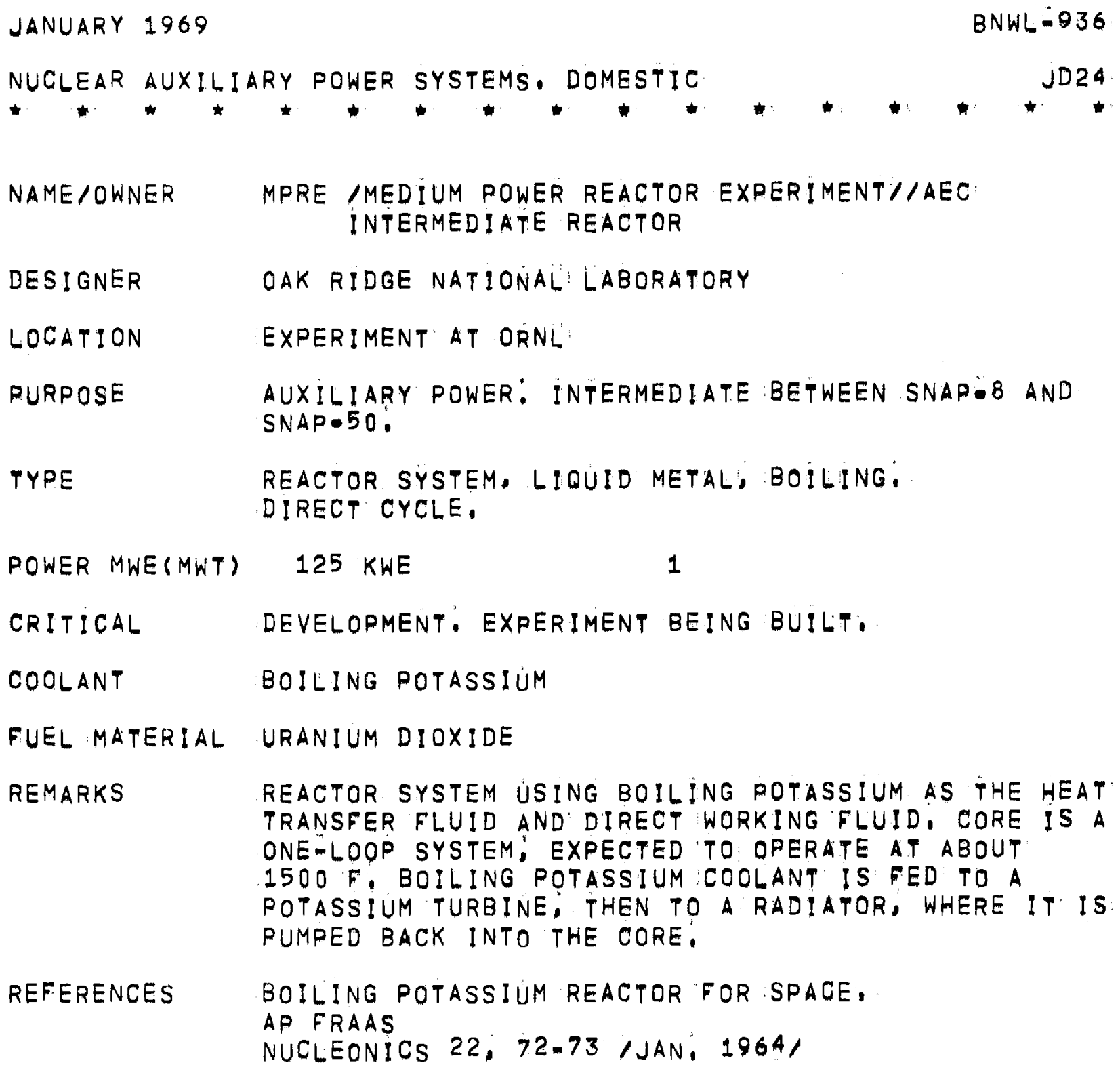




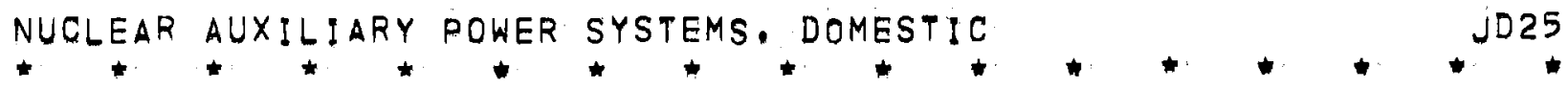

NAME /OWNER

DES IGNER

PURPOSE

TYPE

POWER MWE(MWT)

COOLANT

MODERATOR

FUEL MATERIAL

REMARKS

REFERENCES
$S N A P=50 / S N A P=50 / S P U R / / A E G=N A S A-D O D$

PRATT AND WHITNEY AIRCRAFT

AUXILIARY POWER; SPACE

REACTOR SYSTEM, LIQ: METAL COOLED, FAST.

$300-1000 \mathrm{KWE}$

LITHIUM

NONE

URANIUM/BERYLLIUM OXIDE.

CARBIDE AND NITRIDE STUDIED.

SYSTEM CONSISTS OF A HIGH TEMPERATURE COMPACT REACTOR WITH LITHIUM COOLANT AND POTASSIUM VAPOR TURBINE: THE LCRE ILITHIUM COOLED REACTOR EXPERIMENT/ WHICH WAS TO HAVE BEEN BUILT AT AN IDAHO FACILITY HAS BEEN CANCELED; THE PROGRAM WILL PROCEED WITH SNAP-5O DESIGN AND DEVELOPMENT; THE SNAP-5O PROGRAM HAS BEEN REDUOED TO THE LEVEL OF TECHNOLOGICAL DEVELOPMENT, AND WILL BE MOVED TO LAWRENCE RADIATION LABORATORY IN CALIFORNIA. THE PRATT AND WHITNEY OPERATED INSTALLATION /CANELI HAS BEEN CLOSED OUT

LRL WORK WILL BE DIRECTED AT TRYING TO DETERMINE HOW TO BUILD THE HIGHEST TEMPERATURE REACTOR. SYSTEM THAT COULD BE USED WITH RANKINE CYCLE, HIGH TEMPERATURE BRAYTON CYCLE, OR OUF=OF=CORE THERMIONIC SYSTEMS.

SNAP-5O/SPUR REACTOR EXPERIMENT, FD HAINES TRANS: AMERICAN NUCLEAR SOC, $6 / 1 /, 88 /$ JUNE 1963/ /ABSTRACTS/

THE SNAP-50/SPUR NUCLEAR ELECTRIC SPACE POWER PLANT PROGRAM

EM DOUTHETT, RI STROUGH /PAW/

CONF-650416-1, APRIL 28, 1965

NUCLEONICS WEEK DEC: 2, 1965 P, 4 NEWS RELEASE 


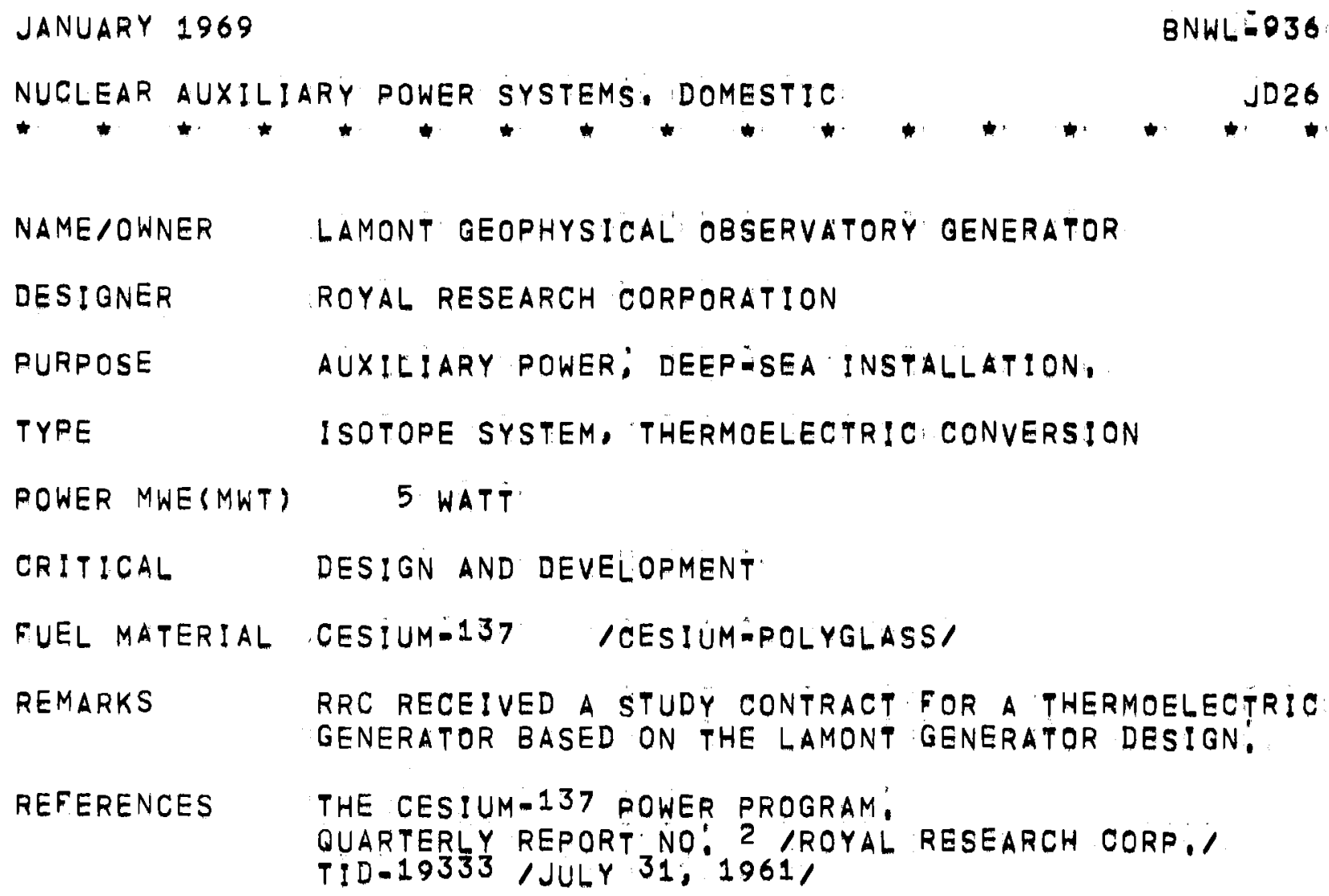


NUCLEAR AUXILIARY POWER SYSTEMS. DOMESTIC

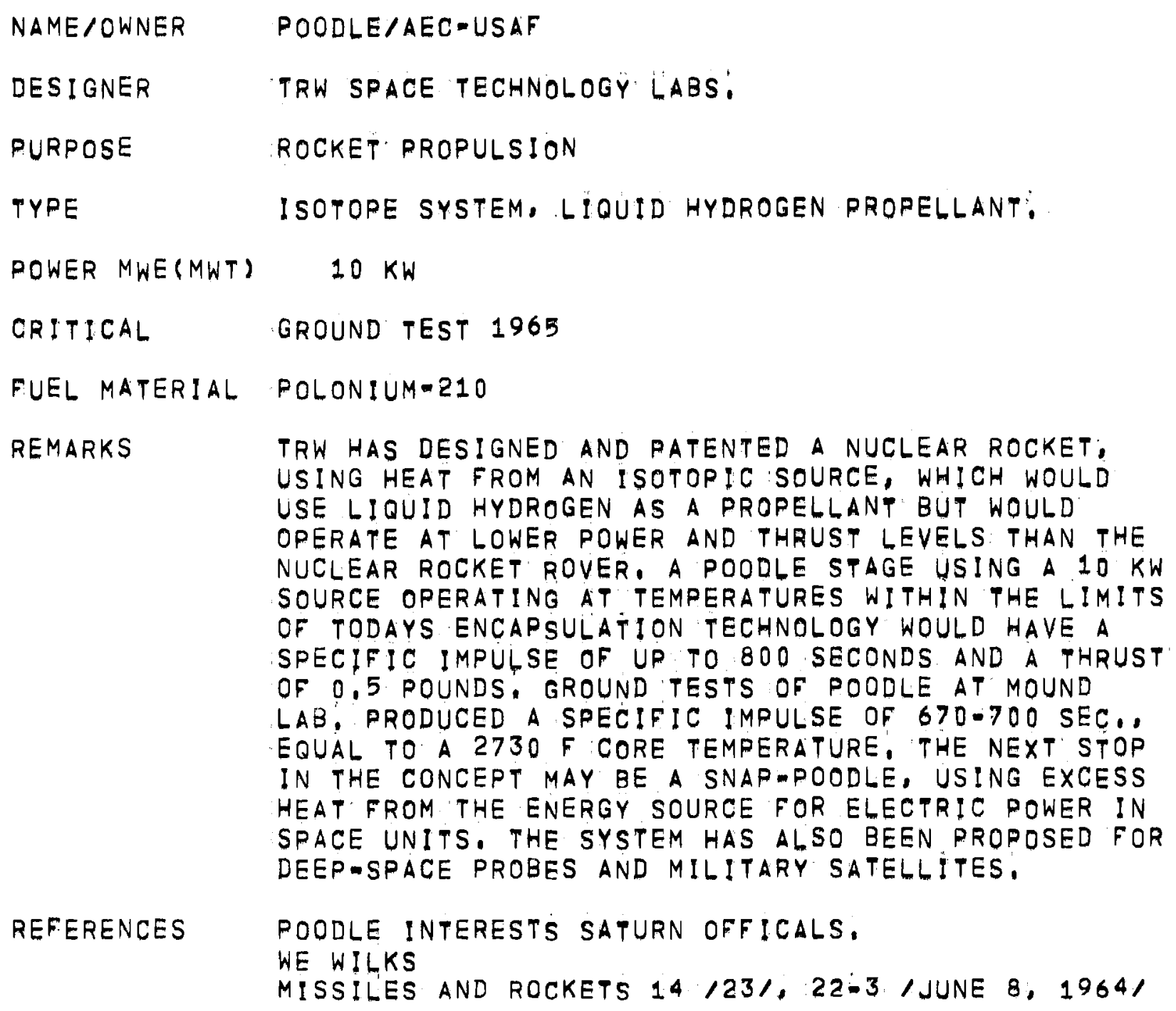

REFERENCES POODLE INTERESTS SATURN OFFICALS. WE WILKS

MISSILES AND ROCKETS $14 / 23 /, 22-3$ /JUNE 8, 1964/ 
JANUARY 1969

$B N W L=936$

NUCLEAR AUXILIARY POWER SYSTEMS, DOMESTIC

JD28

NAME /OWNER

DES I GNER

PURPOSE

TYPE

POWER MWE (MWT)

CRITICAL

COOLANT

MODERATOR

FUEL MATERIAL

FUEL GEOMETRY

FUEL ENRICH,

FUEL ASSEMBLY

BURNUP (REFUEL)

REMARKS

REFERENCES
WANL SUBMERGED REACTOR UNIT/WEST

WESTINGHOUSE ELECTRIC CORP. ASTRONUCLEAR LAB,

UNDERSEA POWER, UNATTENDED

REACTOR SYSTEM, AUXILIARY POWER, BWR. THERMOELECTRIC CONVERSION.

3

45

FEASIBILITY STUDY:

LIGHT WATER

BOILING SULFUR CONSIDERED FOR HIGH TEMPERATURE SYSTEM.

LIGHT WATER

URANIUM DIOXIDE-NIOBIUM CERMET; URANIUM=BERYLLIUM OXIDE, OR URANIUM DIOXIDE.

RODS, WITH CONCENTRIC REGIONS OF THERMOELECTRIC MATERIAL, CONDUCTORS, INSULATION, AND CLADDING,

LOW FOR URANIUM DIOXIDE, HIGH FOR CERMET FUELS,

32-ROD BUNDLE /URANIUM DIOXIDE-NIOBIUM FUEL/

64 BUNDLES/CORE

2 -3 YEAR LIFETIME

FEASIBILITY STUDY HAS BEEN COMPLETED, DESIGN AND DEVELOPMENT STATUS. THE BWR HAS NATURAL CONVICTION CIRCULATION.

PRELIMINARY CORE DESIGN OF A $500 \mathrm{KW/E/}$

THERMOELECTRIC REACTOR.

R MARKLEY; E SCHWARTZ

WCAP $=1737$ /MARCH $1961 /$

FEASIB!LITY STUDIES FOR MWE NUCLEAR

THERMOELECTRIC PLANT:

RA CLARK, OTHERS

WANL-PR/A/-OO2 /DECEMBER 1961/.

DIRECT CONVERSION THERMOELECTRIC REACTOR STUDIES, JC DANKO, OTHERS

PREPRINT PAPER NO'. 11: ENGINEERS JOINT COUNCIL, 


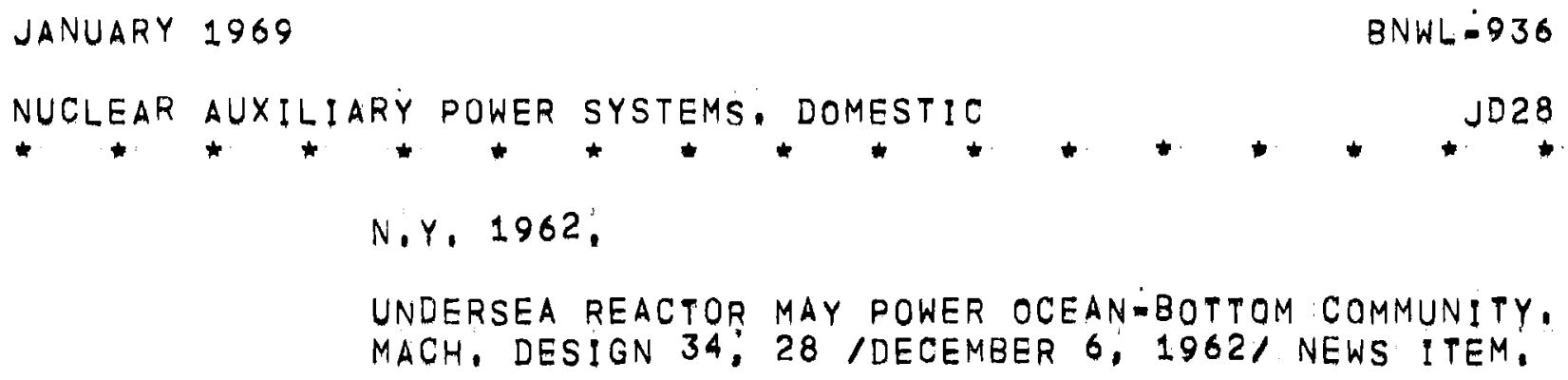


JANUARY 1969

NUCLEAR AUXILIARY POWER SYSTEMS, DOMESTIC

NAME / OWNER

DES IGNER

PURPOSE

TYPE

POWER MWE (MWT

CR!TICAL

COQLANT

MODERATOR

FUEL MATERIAL

FUEL CHARGE

REMARKS

REFERENCES

AUXILIARY POWER

THERMIONIC CONVERSION

$$
100 \mathrm{KWE}
$$

DES IGN

SODIUM-POTASSIUM NAK

URANIUM/YTTRIUM HYDRIDE

$145 \mathrm{KG}, U-235$

PRELIMINARY DESIGN
THERMIONIC CONVERSION REACTOR/MARTIN

MARTIN-MARIETTA CORPORATION

REACTOR SYSTEM. LIOUID METAL COOLED.

YTTRIUM HYDRIDE", BERYLLIA REFLECTOR

PRELIMINARY DESIGN OF A $100 \mathrm{KWE}$ THERMIONIC CONVERSION REACTOR CORE,

FA RUSSO /MARTIN/

TRANS, AMERICAN NUCLEAR SOC, 7; 378 /NOV, 1964, 


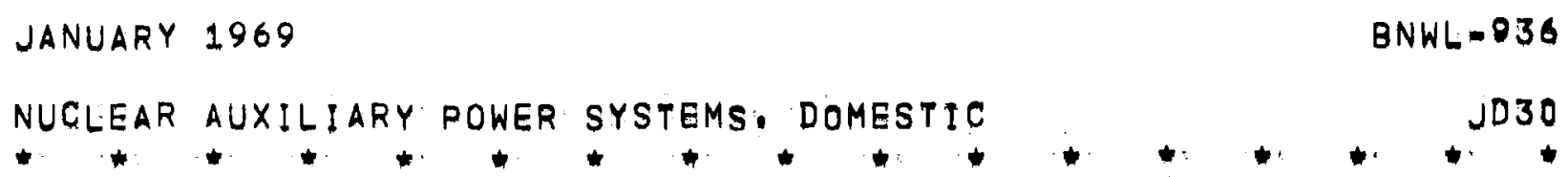

FUEL GEOMETRY CYLINDRICAL SPHERICAL SHAPE STUUDIED

FUEL CLADDING PLATINUM JACKET ON FUEL PELLET FUEL CAPSULE OF HASTELLOY $-E$

FUEL ASSEMBLY HASTELLOY-C CAPSULE 2.89 IN. ID/3.89 IN, 00/4.06 IN. LONG

HASTELLOY-C LINER

FUEL CHARGE 28 KILOCURIES STRONTIUM-90

BURNUR (REFUEL) :5: YEAR MINIMUM LIFETIME

REACTOR VESSEL BERYLLIUM-COPPER ALLOY, FORGED VESSEL. INSULATION ENVELOPE COMPOSED OF LAYERED FOILS INTERSPERSED WITH NON-CONDUCTIVE SPACER MATERIAL,

CONTAINMENT BIOLOGICAL SHIELD: DEPLETED URANIUM-MOLYBDENUM ALLOY, BARRIER MATERIAL BETWEEN BIOLOGICAL SHIELD, SPIDER, AND INNER LINER IS SPRAYED ALUMINUM OXIDE. URANIUM-MOL YBDENUM BIOLOGICAL SHIELD?

REMARKS

$3 M$ IS DEVELOPING THE SYSTEM FOR OCEANOGRAPHIC :RESEARCH AND UNDERWATER NAVIGATIONAL: AIDS, A 10-WATT POWER SUPPLY SYSTEM WAS CONSTRUCTED AND TESTED FROM JULY 1966 TO DEC, 1967 , PHASE 2 IS A TECHNOLOGY PROGRAM LEADING TO THE CONSTRUCTION OF A FULLY-QUALIFIED AND TESTED 10-: AND 20-WATT SYSTEM: 
NUGLEAR AUXILIARY POWER SYSTEMS. DOMESTIC

PHASE 1. Final REPORT: FEASIBILITY STUDY. MARTIN CO.

MND-3180-1N JJAN: 1965 /

DIRECT ENERGY CONVERSION DIGEST JULY 1966

NEWS RELEASE

SNAP-21 PROGRAM: PHASE 3

QUARTERLY REPORT NO: 3

MMM-3601-17

SNAP-21 PROGRAM:

QUARTERLY REPORT NO: 6

MMM-3691-26 /JAN: 1968/

SUMMARY REPORT; PHASE 1:

SNAP-21A TEN-WATT DEEP-SEA ISOTOPE POWER SUPPLY,

VOL: 1

MND-3322-25 /JUNE 1965/ 
JANUARY 1969 BNWL $=936$

NUCLEAR AUXILIARY POWER SYSTEMS, DOMESTIC JD31:

NAME/OWNER

DES IGNER

PURPOSE

TYPE

POWER MWE (MWT)

CRITICAL

REMARKS

REFERENCES
SNAP-25/AEC

LOCKHEED MISSILES/SPACE CO: NUMEC

SPACE POWER

ISOTDPE SYSTEM, CONVERSION BY THERMOCOUPLES

75 WATTS

DEVELOPMENT

SEE REMARKS

WORK ON SNAP-25 HAS BEEN STOPPED, APPARENTLY BECAUSE CONFIGURATION AND POTENTIAL USE OVERLAP THE 50-WATT SNAP-27 GENERATOR

NEWS RELEASES 


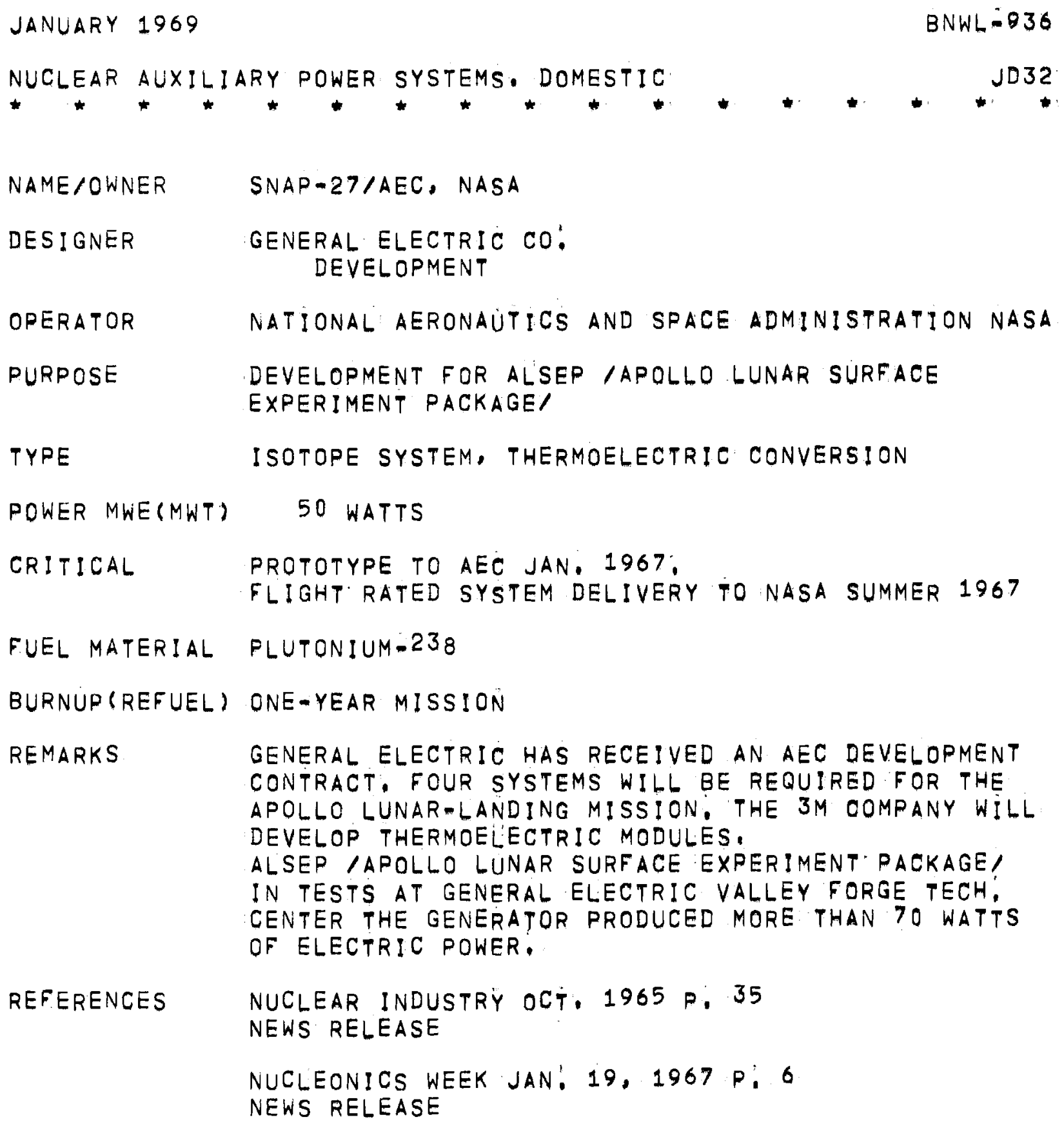




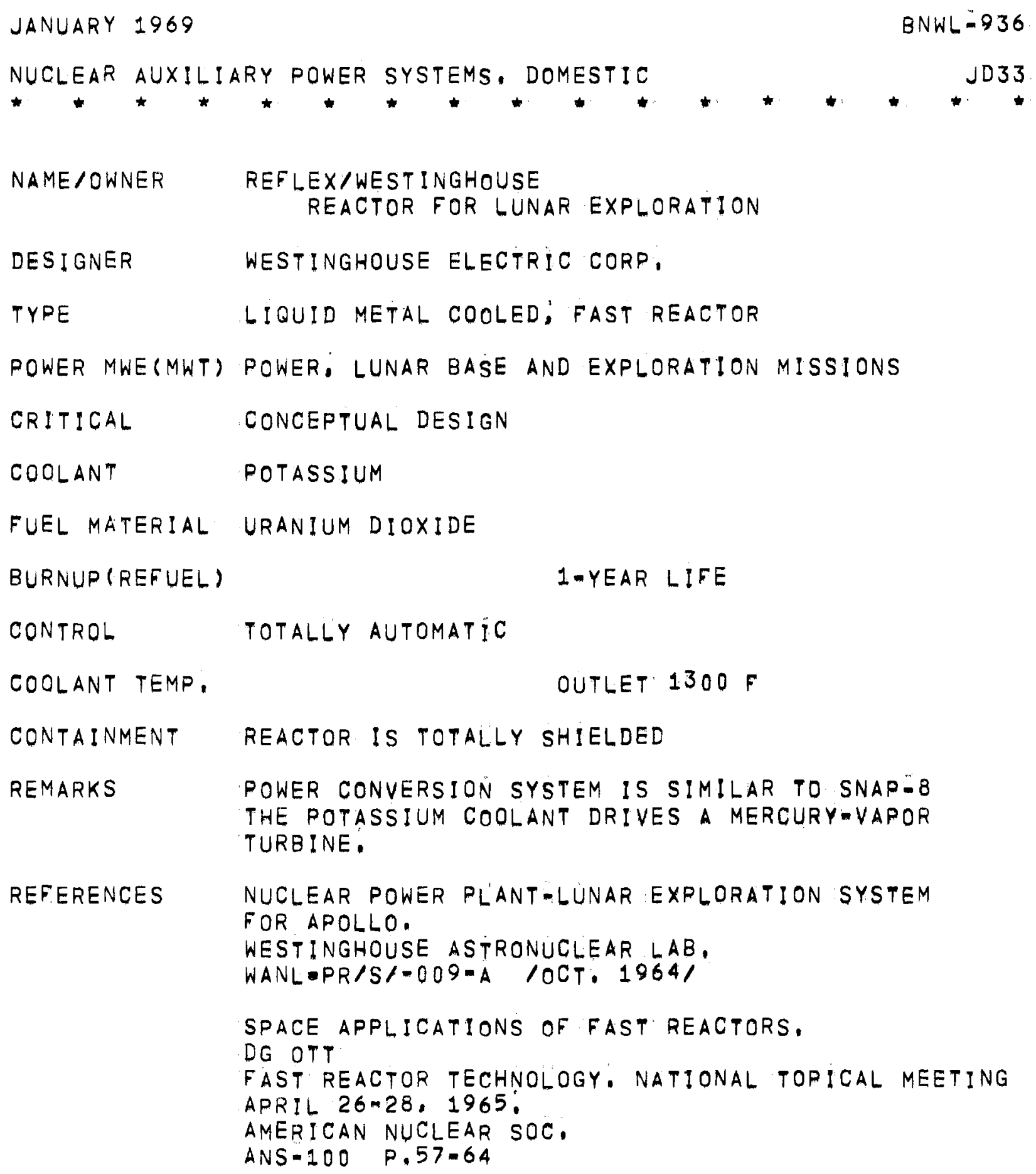


JANUARY 1969 BNWL -936

nuglear auxiliary power systems, domestic * * * * * **3a

NAME/OWNER THERMIONIC CONVERTER PROPULSION SYSTEMIDOUGLAS

DESIGNER DOUGLAS AIRCRAFT

PURPOSE SPACE PROPULSION

TYPE REACTOR SYSTEM, THERMIONIC OONYERSION

POWER MWE (MWT) 5

CRITICAL CONCEPTUAL DESIGN

FUEL MATERIAL URANIUM DIOXIDE-FUNGSTEN CERMET

COOLANT TEMP, $1595 \mathrm{~F}$

REMARKS PROPULSION PLANT INTEGRATED WITH PAYLOAD

REQUIREMENTS FOR MANNED MARS MISSION. LAUNCH

VEHIQLE IS AN AUGMENTED SATURN G-S

REFERENCES CONCEPTUAL DESIGN ANALYSIS OF A 5 MWE

THERMIONIC REACTOR SPACE PROPULSION PLANT.

FA ROSS, TF PLUNKETT

NP-14985 /FEB. 1965/ 


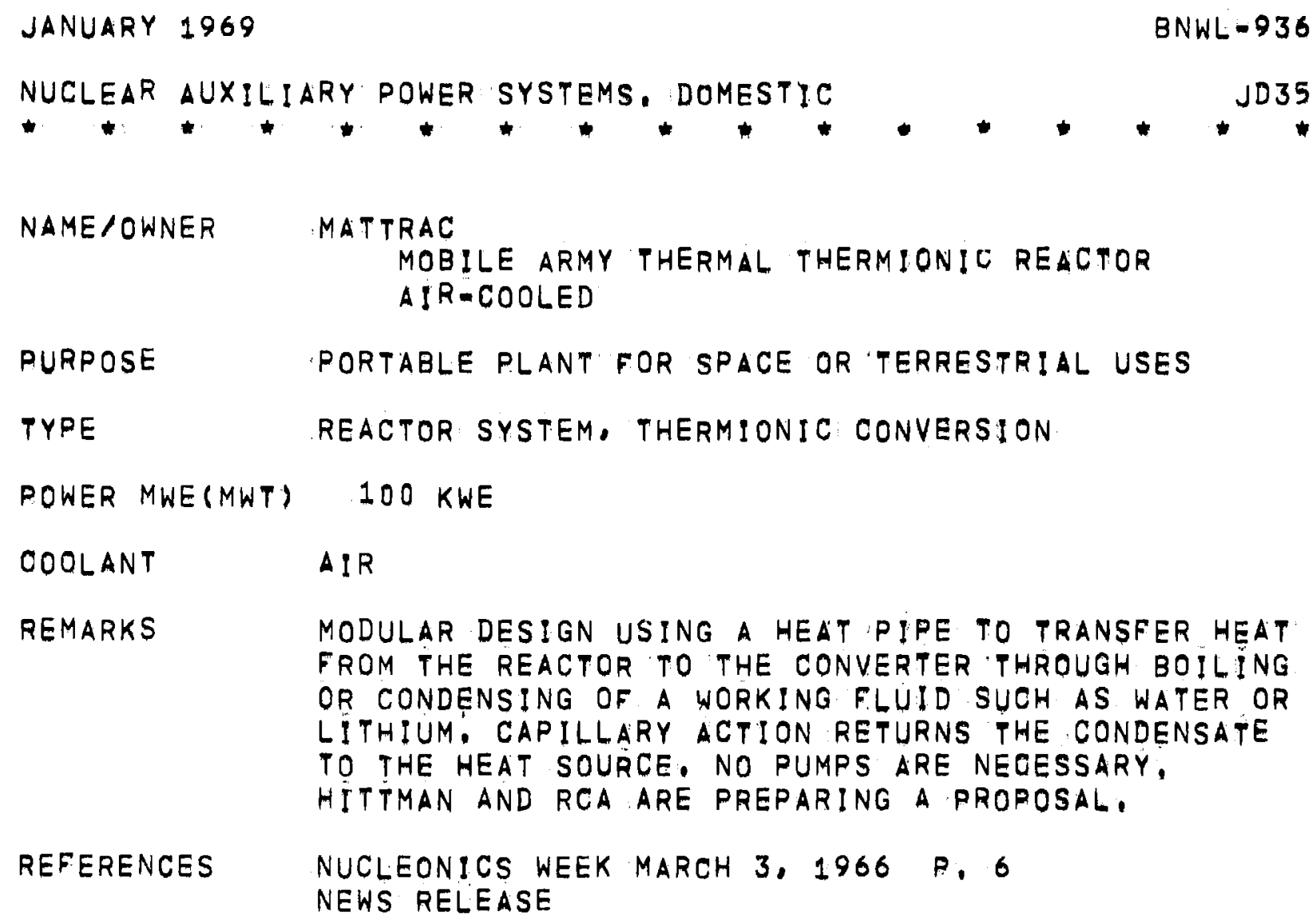




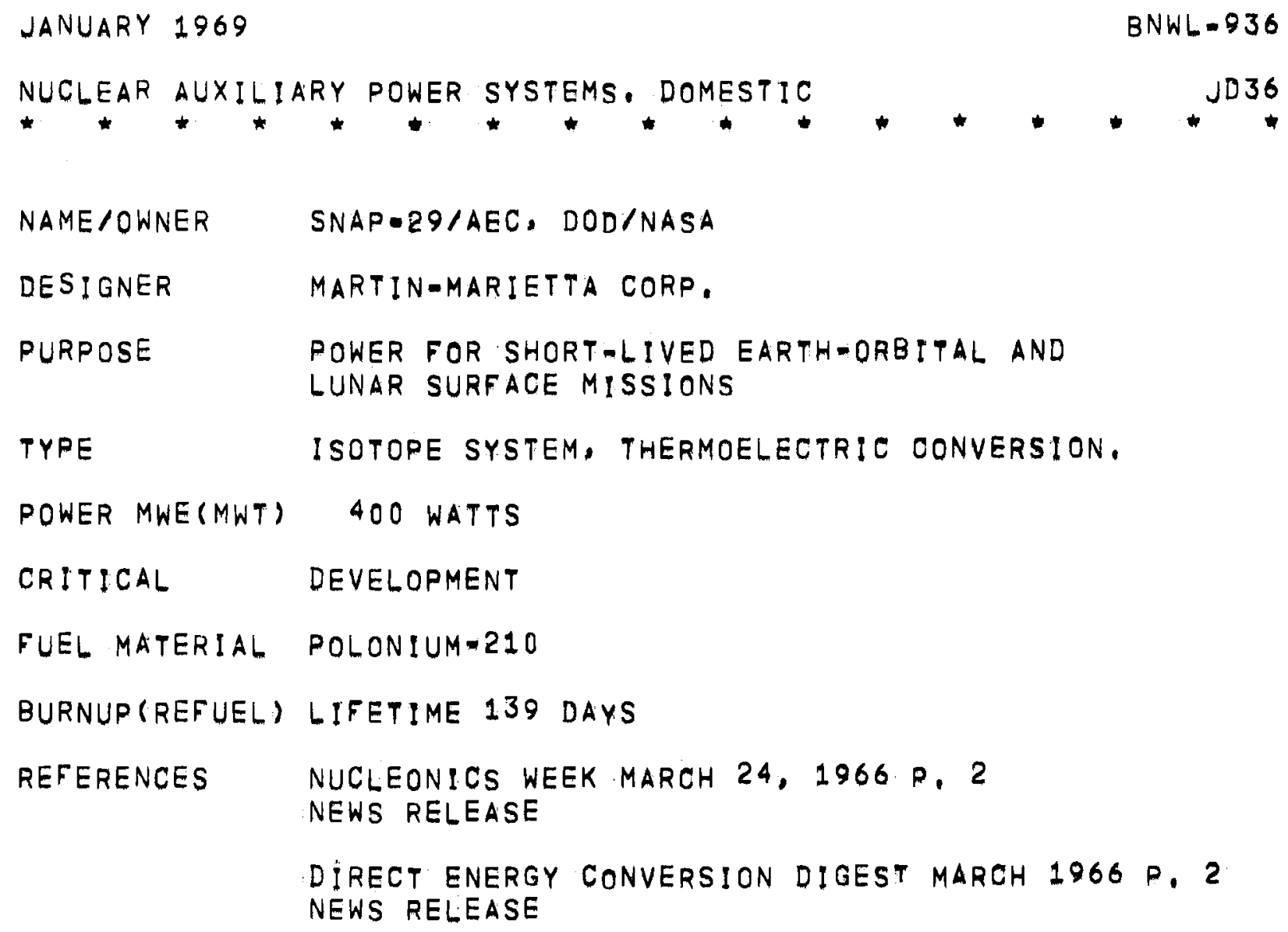




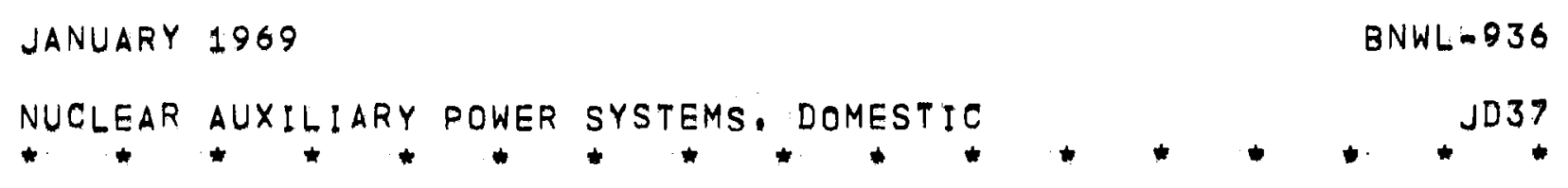

NAME / OWNER

RISE /RADIOISOTOPE SUBMERSIBLE ENGINE/AGN

DES \GNER

AEROJET-GENERAL NUCLEONICS

PURPOSE

UNDERSEA PROPULSION OR POWER FOR

MANNED PLATFORMS.

TYPE

ISOTOPE SYSTEM

POWER MWE (MWT)

$70 \mathrm{~kW}$

CRITICAL

CONCEPTUAL DESIGN

COOLANT

WATER=STEAM. SEE REMARKS

FUEL MATERIAL CERIUM-144 AS CERIUM OXIDE

FUEL GEOMETRY PINS 1.495 IN. DIA., ACT, LENGTH 7,86 IN.

FUEL CLADDING HASTELLOYMC 0.050 IN. THICK

FUEL ASSEMBLY HEXAGONAL CLOSE-PACKED ASSEMBLY

TOTAL 19. PINS

SPECIFIC POWER

$1 / 3$ CORE IN 6 MONTHS

COQLANT TEMP, INLET $141 \mathrm{~F}$

OUTLET $567 \%$

COQLANT PRESS, INLET 1200 PSIA

OUTLET APPROX, 1200 PSIA

REACTOR VESSEL VESSEL $8-25$ IN, OD, 114,5 IN, LONG/0,2 IN, THICK

CONTAINMENT SHIELDING IS NATURAL OR DEPLETED URANIUM.

REMARKS

AGN HAS PROPOSED AN ORGANIC-COOLED RISE TO THE

AEC AS AN UNDERSEA PROPULSION SYSTEM, USING THE

RANKINE CYCLE:

REFERENCES EVALUATION OF RADIOISOTOPE APPLICATIONS IN HYDROSPACE. FINAL REPORT.

SE BUCK, OTHERS

$A G N=8135$ /MARCH $1965 /$ 


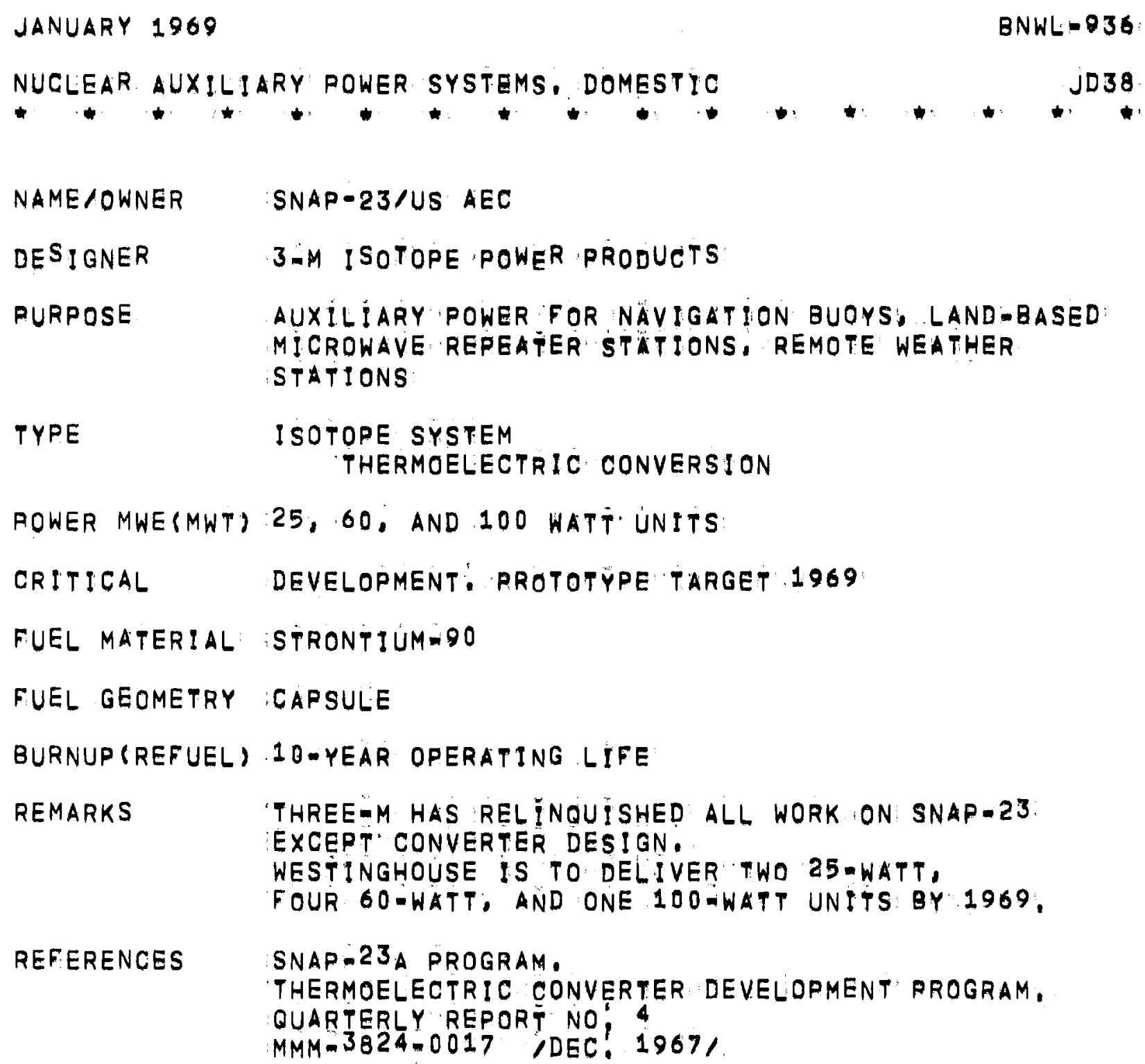


JANUARY 1969

BNWL- -036

NUGLEAR AUXILIARY POWER SYSTEMS. DOMESTIC

JD39

NAME/OWNER

URIPS /UNDERSEA RADIO ISOTOPE POWER SYSTEM/ AGN

DESIGNER

AEROJET-GENERAL NUCLEONICS

LOCATION

COMMERCIALLY AYAILABLE

PURPOSE

AUXILIARY POWER, UNDERWATER

TYPE

ISOTOPE SYSTEM, THERMOELECTR!C: CONVERTER

POWER MWE(MWT)

1 WATT

FUEL MATERIAL STRONTIUM=90

BURNUP (REFUEL)

10-YEAR LIFETIME

CONTAINMENT

LEAD OR DEPLETED URANIUM SHIELDING

REMARKS

SOLID-STATE THERMOELECTRIC GENERATOR FOR POWERING UNDERWATER 3ENCH MARKS, WELLIMHEAD MARKERS? REMOTE DATA LINES $\cong T C$.

AVA ILABLE POWER IS 3-28 VOLTS,

DEVICE IS DESIGNED FOR CONTINUOUS 10-YEAR USE TO DEPTHS OF 2000 FT:

THE NAVY IS MAKING an EVALUATION OF THE SYSTEM AT

THE NAVAL CIVIL ENG, LAB, at POINT HUENEME, CALIF.

REFERENCES

NUCLEONICS WEEK MARCH $2,1967 \quad P, 4$

NUCLEAR INDUSTRY MARCH 1967 P. 34

NEWS RELEASES 
JANUARY 1969 BNWL -936

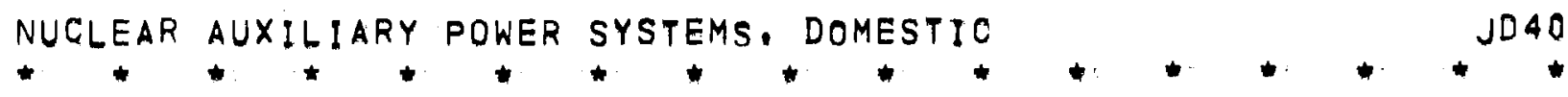

NAME/OWNER ISOMITE/ISOTOPE MINIATURENTHERMIONIC ELECTRIC/DWD

DESIGNER DONALD W. DOUGLAS LABORATORY

PURPOSE LOW-LEVEL COMPACT POWER SOURCE

TYPE

ISOTOPE SYSTEM. THERMIONIC GENERATOR

POWER MWE(MWT) 10 MICROWATTS TO 10 MILLIWATTS

CRITICAL DEVELOPMENT

FUEL MATERIAL PROMETHIUM-147 AS PROMETHIUM OXIDE /POWDER/

REFERENCES A MINIATURE ATOMIC BATTERY BASED ON THERMIONIC CONVERSION.

NS RASOR, OTHERS

PRESENTED TO SECOND INTERNATIONAL CONF, ON THERMIONIC POWER GENERATION, STRESA, ITALY, MAY 27-31; 1968:

$D P=4947$ 
JANUARY 1969

$B N W L=936$

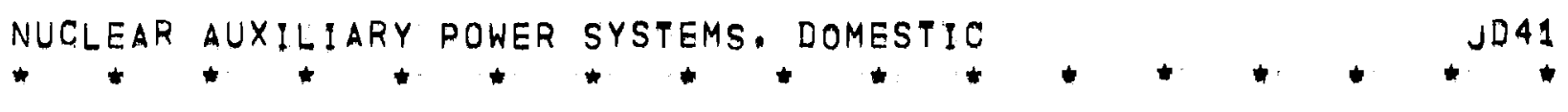

NAME/OWNER SPACE REACTOR/LAWRENCE RADIATION LAB,

DESIGNER LAWRENCE RADIATION LABORATORY

TYPE L10, METAL COOLED, COMPACT REACTOR, HEAT PIPE

POWER MWE(MWT) 10

CRITICAL STUDY

COOLANT LITHIUM

FUEL MATERIAL URANIUM NITRIDE

COOLANT TEMP, HEATEPIPE WASTE HEAT REJECTION AT $1100 \mathrm{~K}$

REMARKS OPERATING TEMPERATURE $1650 \mathrm{~K}$.

STRUCTURAL MATERIAL TUNGSTEN-RHENIUM ALLOY

REFERENCES CONCEPTUAL DESIGN OF A 10 MWE NUCLEAR RANKINE SYSTEM FOR SPACE POWER

JH PITTS, CE WALTER

UCRL.50382 /JAN, 30, 1968/ 
NUCLEAR AUXILIARY POWER SYSTEMS

SNAP-TYPE REACTORS

FOREIGN 
JANUARY 1969

$B N W L=936$

NUCLEAR AUXILIARY POWER SYSTEMS, FOREIGN

JF 01

NAME /OWNER

FRENCH AUXILIARY POWER DEVELOPMENT PROGRAM:

DES I GNER

CEA; FRANCE

PURPOSE

RESEARCH AND DEVELOPMENT

POWER MWE (MWT)

12:5 WATTS

FUEL MATERIAL

STRONTIUM TITANÁTE

REMARKS

FRANCE WILL DEVELOP NUCLEAR UNITS SIMILAR TO U.S', SNAP GENERATORS: A PILOT PLANT AT SACLAY WILL SEPARATE CESIUM-137 AND STRONTIUM-90. FISSION REACTORS WILL ALSO BE STUDIED: TWO STRONTIUM-FUELED UNITS ARE BEING DEVELOPED FOR USE IN THE NATIONAL SATELLITE PROGRAM. AN R+D CONTRACT HAS BEEN AWARDED BY THE CENTRE NATIONALE D-ETUDES SPATIAL'ES TO THE ELLECTRONICS FIRMS CFS AND SNECMA. HISPANO-SUIZA; FRANCE; HAS A DEVELOPMENT CONTRACT FROM EURATOM FOR A STRONTIUM TITANATE FUELED THERMOELECTRIC SYSTEM: SIRENE-302, A THERMIONIC CONVERTER IN-PILE EXPERIMENT AT SACLAY, IS FUELED WITH MOLYBDENUM-. CLAD URANIUM DIOXIDE AND COOLED BY NA=K.

REFERENCES NEWS RELEASES 


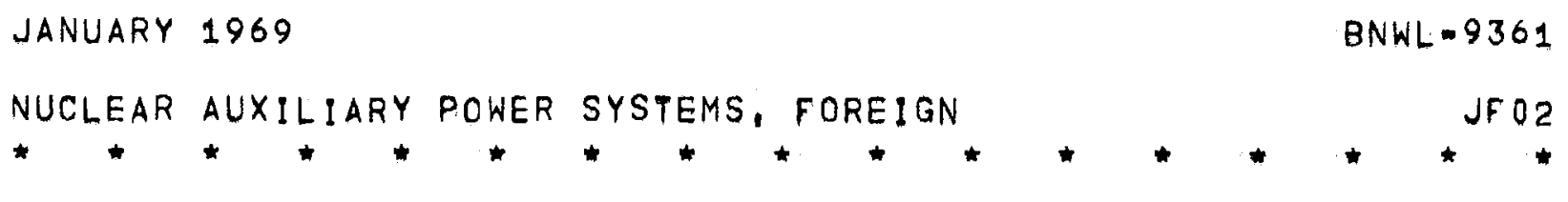

NAME/OWNER RIPPLE/AERE, UNITED KINGDOM

RADIOACTIVE ISOTOPE:POWERED PROLONGED-LIFE

DESIGNER AERE, UNITED KINGDOM

OPERATOR AERE, UNITED KINGDOM

LOCATION DEMONSTRATION AT HARWELL ATOMIC ENERGY RES, ESTAE, , U,K.

PURPOSE REMOTE LOCATIONS, UNATTENDED OPERATIÓN.

TYPE ISOTCPE SYSTEM, THERMOELECTRIC CONVERSION.

POWER MWE(MWT) RIPPLE-1, 75 MILLIWATTS /DEMONSTRATION/

RIPPLE=2, 90 MILLIWATTS /DEMONSTRATION/

RIPPLE $=3,750$ MILLIWATTS /EXPERIMENT/

RIPPLE-4, 2,5 WATTS /DESIGN/

RIPPLE-5, 1 WATT

RIPPLE $=6,1$ WATT

RIPPLE-7, 1 WATT

CRITICAL RIPPLE-1 IDEMONSTRATION/ MARCH 1965

RIPPLE-2 /DEMONSTRATION/ MARCH 1965

RIPPLEM 3 /EXPERIMENTAL/ MARCH 1966

RIPPLE - 4 /SUEMARINE CABLE REPEATER/ DESIGN

RIFPLEWK /NAVIGATION LIGHT/ JULY 1966, IN LAB,

RIPPLE-6 /NAVIGATION LIGHT.IN.SERVICE, AUG, 1966

RIPPLE-7/NAVIGATION LIGHT-IN-SERVICE/ AUG, 1966

FUEL MATERIAL STRONTIUM-90 TITANATE PELLETS

FUFL CLADDING THIN SS CAPSULE

FUEL CHARGE 700 CUFIES STRONTIUM-90

BURNUP(REFUEL) 28-YEAR HALF-LIFE, 10-YEAR LIFETIME

CONTAINMENT RIPPLE 1, LEAD SHIELDING,

RIPPLE 2 AND 3 , TUNGSTEN ALLDY SHIELDING

RIPPLE 5 TO 8, DEPLETED URANIUM SHIELDING

REMARKS BISMUTH TELLURIDE THERMOELECTRIC ELEMENTS IN MODULE LOCATED ON ONE END OF CYLINDRICAL ISOTOPE CAN.

RIPPLE-1 HAS BEEN EXHIEITED AT THE LONDON RADIOELECTRONICS CONFERENCE, AND RIPPLEm2 AT THE ROME INTERNATIONAL CONFERENCE ON LIGHTHOUSES AND AIDS TO NAVIGATION, 
JANUARY 1969

NUCLEAR AUXILIARY POWER SYSTEMS, FOREIGN
BNWL $=9361$

JF 02

RIPFLE-3 HAS BEEN UNDER TEST AT THE NAVAL CIVIL ENEINEERING LAB, AT PT, HUENEME, CALIF. RIPPLE-6 HAS BEEN INSTALLED ON A SMALL ISLAND NEAR STOCKHCLN TO POWER A NAVIGATION BEACON. RIPFLE. 7 IS ON LOAN TO DANISH LIGHTHOUSE AUTHORITY FOR IN SERVICE EVALUATION

REFFRENCES

INTERIM REPORT ON THE FIRST BRITISH RADIOACTIVE ISOTOPE FOWERED THERMOELECTRIC GENERATORS RIPPLE- 1 ANE RIPPLE-2, PD DUNN, FW YEATS $A E F E-M-1594 \quad / 1965 /$

NEKS RELEASES 
JANUARY 1969

BNWL"̈. 936

NUCLEAR AUXILIARY POWER SYSTEMS. FOREIGN

$J F 03$

$\downarrow$

NAME/OWNER

ROMASHKA/USSR

DES IGNER

USSR

LOCATION

KURCHATOV ATOMIC ENERGY INST,, MOSCOW, USSR,

PURPOSE

AUXILIARY POWER

TYPE

REACTOR SYSTEM, THERMOELECTRIC CONVERSION

CONDUCTION COOLED; FAST REACTOR

POWER MWE (MWT)

500 WATTS

$40 \mathrm{KW}$

CRITICAL

OPERATION AUG. 1964

COOLANT

COOLING IS BY CONDUCTION.

MODERATOR

GRAPHITE

BERYLLIUM REFLECTOR

FUEL MATERIAL URANIUM CARBIDE, PIE-SHAPED SEGMENTS

FUEL GEOMETRY GRAPHITE BOXES OR DISHES LOADED WITH

URANIUM CARBIDE: 20 CM. DIA.

FUEL CLADDING FUEL IS NOT COATED, NOT BONDED TO BOXES OR DISCS,

FUEL ENRICH. 90 PER CENT U.235

FUEL ASSEMBLY STACKED-DISH ELEMENTS IN A GRAPHITE LINER, FORMING A CYLINDER APPROX: 2 IN. HIGH:

11 BOXES OR DISHES/CORE

FUEL CHARGE $49 \mathrm{KG}$. ENRICHED URANIUM

NEUTRON FLUX TOTAL, CORE CENTER $1 \times 10 E+13$

TOTAL, CORE BOUNDARY $7 \times 10 \cdot E+12$

CONTROL BORON NEUTRON REFLECTOR RODS

CONTAINMENT LEAK-TIGHT VESSEL CONTAINING HELIUM ATMOSPHERE, COVER IS WATER-COOLED

REMARKS

THERMOELECTRIC CONVERSION IS BY MEANS OF SILICONGERMANIUM THERMOCOUPLES CONNECTED TO THE REFLECTOR IN SERIES AND IN PARALLEL, HEAT DISSIPATION IS BY COPPER COOLING FINS AT THE COLD JUNCTIONS OF THE CONVERTERS, OPERATING TEMPERATURES: ARE 3 A52 F IN THE FUEL AND $1650 \mathrm{~F}$ AT THE CONVERTER, REACTOR HAS 


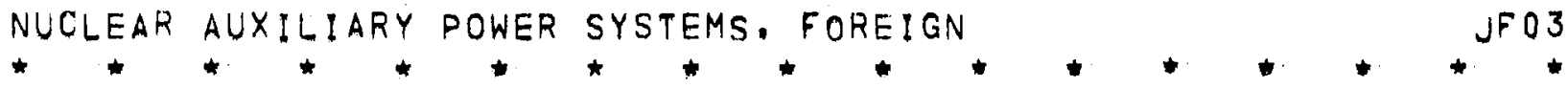

NO MOVING COOLANT AND NO MOVABLE PARTS, ROMASHKA REPORTEDLY HAS OPERATED AS A COMPLETE SYSTEM FOR 500 OR MORE HOURS:

REFERENCES ROMASHKA IN PERSPECTIVE. SD STRAUSS

NUCLEONICS $22 / 12 /, 68-70 / D E C, 1964 /$

HIGH TEMPERATURE DIRECT CONVERSION REACTOR ROMASHKA. MD MILLIONSHCHIKOV, OTHERS

3RD, UN INTL. CONF PEACEFUL USES ATOMIC ENERGY, GENEVA, 1964, A /CONF:28/P/873.

NUCLEAR REACTORS IN THE SOVIET UNION, REPORT OF RECIPROCAL EXCHANGE TRIP TO USSR BY US ATOMIC SCIENTISTS, DEC, 11.22, 1964 WASH-1060

HIGH TEMPERATURE REACTOR = CONVERTER ROMASHKA MD MILLIONSHCHIKOV. OTHERS SOVIET ATOMIC ENERGY 17, 1071.77/NOV: $1964 /$ 


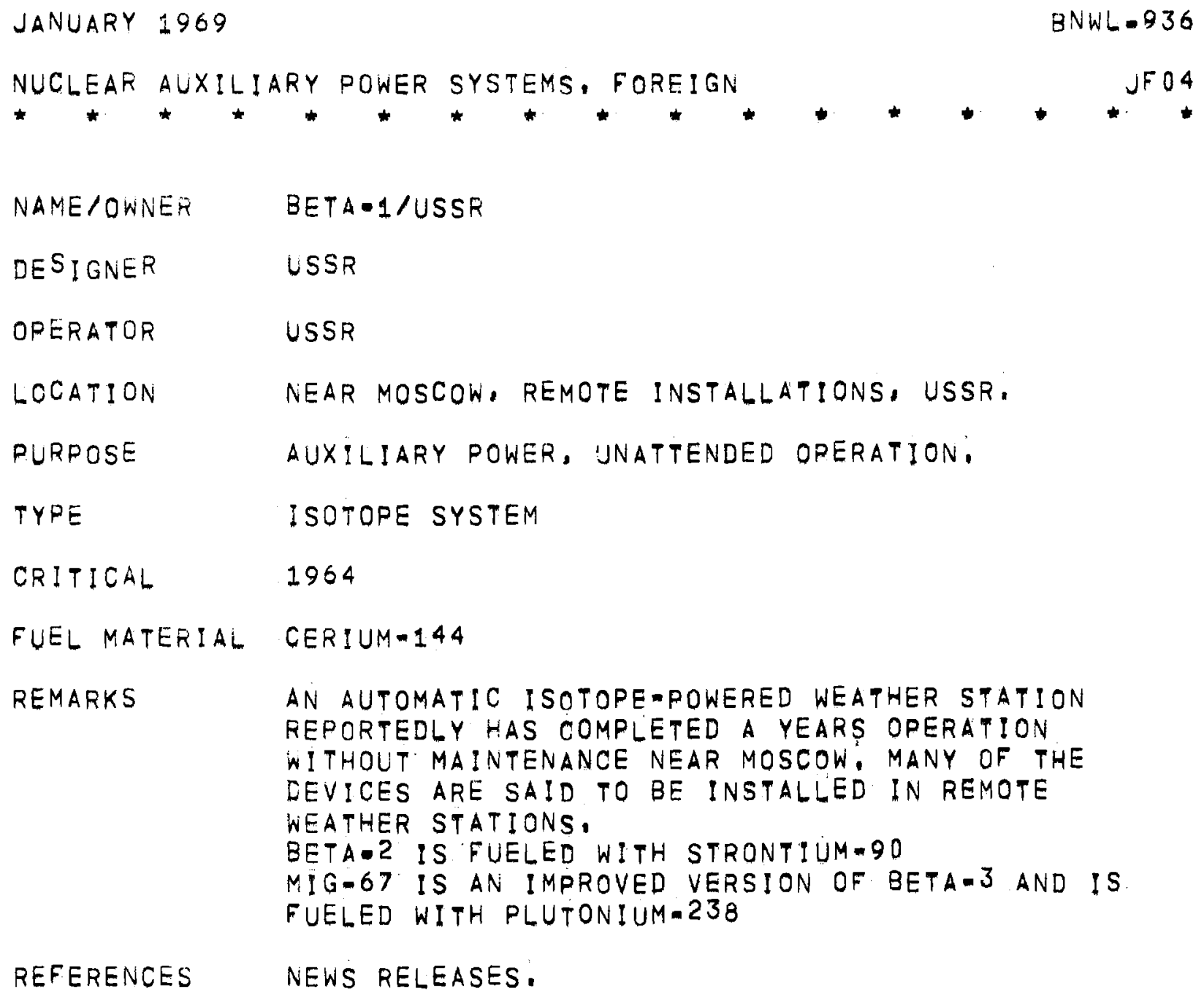


JANUARY 1969

$B N W L=936$

NUCLEAR AUXILIARÝ POWER SYSTEMS. FOREIGN

NAMEIOWNER ITR /IN-CORE THERMIONIC REACTOR/ BUNDEMINISTERIUM FUR WISSENSCHAFTLICHE

DESIGNER INTERATOM; BROWN=BOVERI: SIEMENS IW, GERMANY' FORSCHUNG ABMWF/; W. GERMANY

RURPOSE POWER SOURCE, POSSIBLY TV AND COMMUNICATION SATELLITIES

TYPE

LIOUID METAL COOLED REACTOR; SNAPNTYPE COMPACT SYSTEM

POWER MWE $(M W T) \quad 20 \mathrm{KW}$

CRITICAL

DES IGN

TEST INSTALLATION AT JUELICH

PROTOTYPE CONSTRUCTION PLANNED

COOLANT

SODIUM

MODERATOR

URANIUM AND ZIRCOONIUM HYDRIDE CONSIDERED

FUEL MATERIAL URANIUM METAL HYDRIDE

FUEL GEOMETRY RODS

FUEL CLADDING MOLYBDEN TUBES COATED WITH. TUNGSTEN

FUEL ENRICH, HIGH

FUEL ASSEMBLY 19 FUEL RODS/CORE

RODS WHICH PRODUCE CURRENT ARE PACKED AROUND: THE OUTSIDE

CORE IS 45 CM, LONG, 35 CM: DIAM.

EACH FUEL TUBE SURROUNDED BY INSULATED

CYLINDER COOLED BY LIOUID SODIUM TO $650 \mathrm{c}$.

CONTROL

ADJUSTMENT OF BERYLLIUM REFLECTOR ELEMENTS:

COOLANT TEMP: INLET $610 \mathrm{C}$ OUTLET $650: \mathrm{C}$

REFERENCES SECOND INTERNATIONAL CONFERENEE ON THERMIONIC

ELECTRIC POWER GENERATION, STRESA, ITALY, MAY 1968 REVIEW OF PAPERS .

NUCLEAR ENG, 13, 677-8/AUG: $1968 /$ 


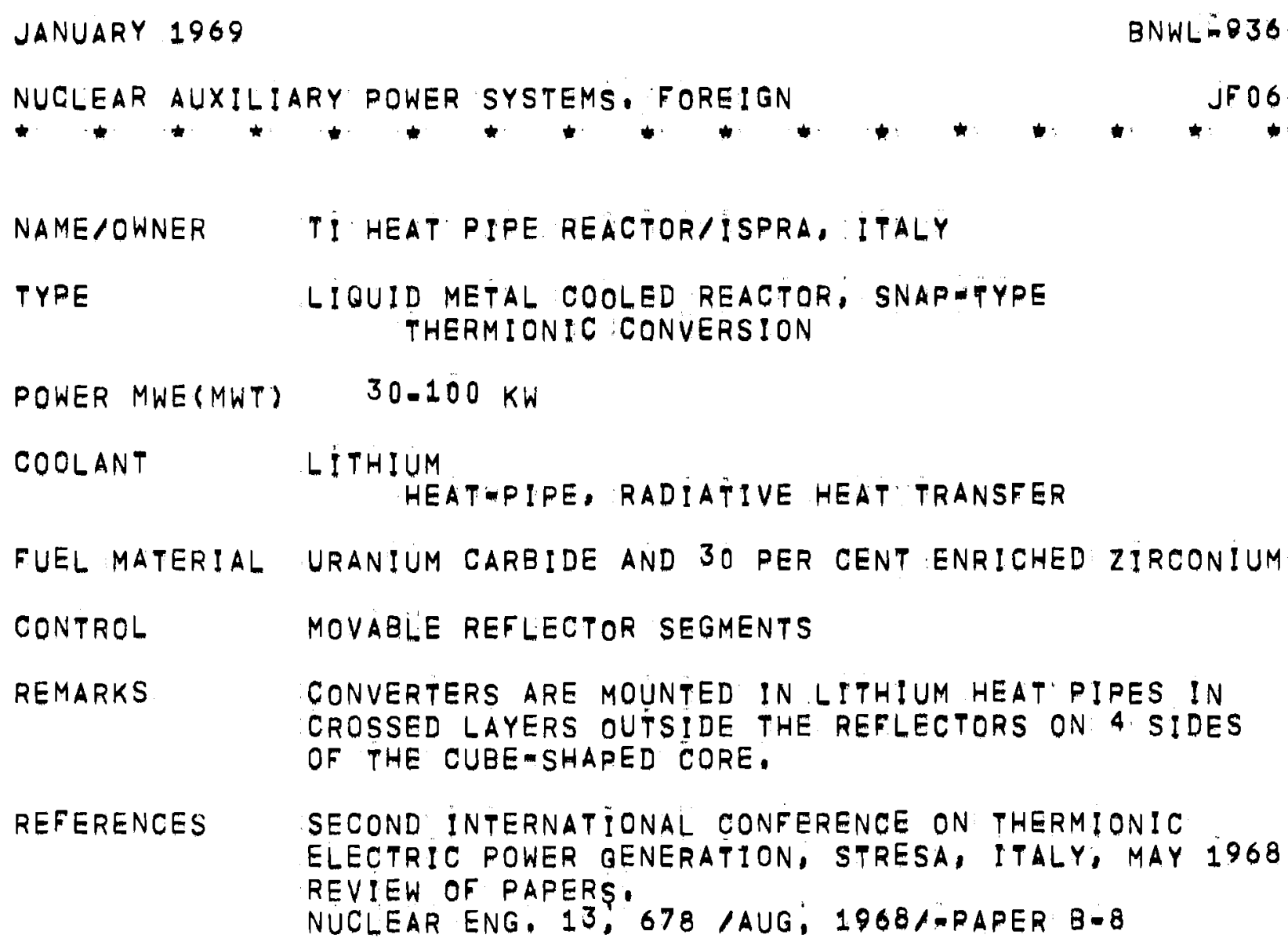


PAGINATION IS BY THE ALPHA-NUMERIC CODE ASSIGNED TO EACH REACTOR DESIGN. THE CODE APPEARS AT THE UPPER RIGHT CORNER OF EACH PAGE. TABS ARE PROVIDED FOR LOCATING THE SECTIONS. 
JANUARY 1969

INDEX

ADVANCED SHIP REACTOR/CE

AEC-S PROGRAM

AETR/ADVANCED EPITHERMAL THORIÜM BREEDER//AEC,SAEA

AGESTA REACTOR STATION/STATE POWER; AB-ATOMENERG!,

AGR /UK/ SEE SEATON-CAREW

AGR /UK/, SEE HINKLEY POINT-B, HUNTERSTON-B

AGR IUK/, SEE WINDSCALE agR, DUNGENESS=B

AIRCRAFT PROPULSION, BRENDA /FRANCE/

DF 09

AIRCRAFT PROPULSION, GCR, TORY: UCRL

AIRCRAFT PROPULSION, HTRE=3, GE

AIRCRAFT PROPULSION, LIO; METAL COOLED, PWAR-6 PWA

AKB /ATOMKRAFT-BAYERN/ SEE KNK

ALPR /ARGONNE LOW POWER REACTOR//AEC-ARMY

AMERICAN ELECTRIC POWER, SEE DONALD C COOK STATION

ANGLESEY STATION; GCR, /UK-WALES/. HAS BEEN DROPPED

APQLLO MISSION, SEE REFLEX, SNAP-27

$\triangle P P R=1, S E E S M-1$

APPR-1A, SEE SM-1A

$A P R R=18$, SEE $S M=2$

APPR, SKID-MOUNTED： SEE PM-2A

APS-1 /SOVIET ATOMIC POWER STATION-1//USSR

AQUEOUS HOMOGENEQUS REACTOR DESIGN /BAW

AQUEOUS HOMOGENEOUS REACTOR. BAW

AQUEOUS HOMOGENEOUS REACTOR, DAVID /SWEDEN/

AQUEOUS HOMOGENEOUS REACTOR, HRE: ORNL
HF 12

$A D 06$

$\triangle D 06$

HD 12

J00 1

FD07

EF 13

$D D 2 A$

DD12

FD26

BD12

AFO4

$A D 03$ 
JANUARY 1969

INDEX

AQUEOUS HOMOGENEOUS REACTOR, HYDRAZINE PROD, AGN AQUEOUS HOMOGENEOUS REACTOR, KSTR /NETHERLANDS/ AQUEOUS HOMOGENEOUS REACTOR, LAPRE: AGN AQUEOUS HOMOGENEOUS REACTOR, PAR: WEST AQUEQUS HOMOGENEOUS REACTOR, PHOEBUS /FRANCE/ AQUEOUS HOMOGENEOUS REACTOR, SUS-POP /NETHERLANDS/ ARBUS /ARCTIC MODULAR PLANT//USSR ARGENTINE MZFR/ARGENTINE AEC ARGONNE TUNGSTEN REACTOR/AEC ARKANSAS $P+L$, SEE RUSSELLVILLE STATION ARMY GCR PROGRAM: SEE GCRE, HDMR; ML-1 ARTIFICIAL ISLAND, SEE PELICAN POINT; SALEM ATLANTIC CITY ELECTRIC: SEE PEACH BOTTOM-2 AND.-3 ATOMIC + SPACE DEVELOP: AUTH., N:Y: SEE SURFSIDE ATUCHA STAION, SEE ARGENTINE MZFR AUXILIARY POWER DEVELOPMENT PROGRAM /FRANCE/ AUXILIARY POWER DEVELOPMENT PROGRAM, AEC AUXILIARY POWER, SEE ALSO SNAP: DIRECT CONVERSION AUXILIARY POWER, ISOTOPE SYSTEM, GE AUXILIARY POWER, ISOTOPE SYSTEM, BETA IUSSR/ AUXILIARY POWER, ISOTOPE SYSTEM, ISOMITE, DWD AUXILIARY POWER, ISOTOPE SYSTEM, LAMONT GENERATOR, RRC AUXILIARY POWER, ISOTOPE SYSTEM, RIPPLE IUK/ AUXILIARY POWER, LUNAR BASE, FAST REACTOR, REFLEX, WEST AUXILIARY POWER, OCEAN RESEARCH VESSEL, TRIGA, GD
INDEX

$A D 01$

$A F 03$

$\triangle D 02$

$\triangle D 05$

AF 01

AF 02

GFO2

EF24

D005

JF 01

JDO 1

JD10

JFO

JD 40

JD26

JF 02

$J D 33$

JDOP 
JANUARY 1969

BNWL -936

INDEX

AUXILIARY POWER, REACTOR SYSTEM /W: GERM:

AUXILIARY POWER, REACTOR SYSTEM, GD

JDO8

AUXILIARY POWER, REACTOR SYSTEM. MARTIN

JD29

AUXILIARY POWER, REACTOR SYSTEM, DCR: MARTIN

$J 021$

AUXILIARY POWER, REACTOR SYSTEM, GLOWWORM, LASL

$J D 13$

AUXILIARY POWER, REACTOR SYSTEM, GLOWWORM, LASL

JD13

AUXILIARY POWER, REACTOR SYSTEM, ITR /W, GERMANYI

JFO5

AUXILIARY POWER, REACTOR SYSTEM, LRL SPACE REACTOR

JD41

AUXILIARY POWER, REACTOR SYSTEM, MPRE, ORNL

JD24

AUXILIARY POWER, REACTOR SYSTEM, ROMASHKA IUSSR/

$\mathrm{J} F \mathbf{0 3}$

AUXILIARY POWER, REACTOR SYSTEM, STAR, GE

JDOQ

AUXILIARY POWER, REACTOR SYSTEM, TI SYSTEM /ITALY,

$J F 06$

AUXILIARY POWER, SPACE OR TERRESTRIAL, MATTRAC: RCA

J035

AUXILIARY POWER, UNDERSEA PRQPULSION; SWR, BMI

1010

AUXILIARY POWER, UNDERSEA, ISOTOPE SYSTEM, AGN

JD39

AUXILIARY POWER, UNDERSEA, ISOTOPE SYSTEM, GE

JD10

AUXILIARY POWER, UNDERSEA, ISOTOPE SYSTEM, GIC

JD11

AUXILIARY POWER, UNDERSEA, ISOTORE SYSTEM, RRC

JD26

AUXILIARY POWER, UNDERSEA, REACTOR SYSTI, TURPS, MARTIN

JD22

AUXILIARY POWER, UNDERSEA, REACTOR SYSTEM, MARTIN

$J D 23$

AUXILIARY POWER, UNDERSEA, REACTOR SYSTEM, WEST

$J D 28$

AUXILIARY POWER, UNDERSEA, REACTOR SYSTEM, SNAP-4, AI

JDO3

AUXILIARY POWER, UNDERSEA, URIPS; AGN

J039

AVR PEBBLE BED REACTOR/AVR, W, GERMANY

DF 37

BAC PHUN/CHINA

HF 32 
JANUARY 1969

$B N W L=936$

INDEX

BAC PHUN/CHINA

HF 32

BAILEY POINT, SEE MAINE YANKEE

BAILEY STATION. SEE BURNS HARBOR

BALTIMORE G+E, SEE CALVERT CLIFFS, GPU-A!

BASHFUL, SEE MARVIKEN

BAXLEY, GEORGIA. SEE EDWIN I HATCH STATION

BAYERNWERK-AG, SEE AKB

BECANCOUR, CANADA. SEE CANDU-BiW

BEERSHEBA STATION/ISRAEL

DF 17

BELL STATION/N,Y: STATE ELECTRIC + GAS CO.

$B D 41$

BELOYARSK STATION/MINISTRY OF POWER, USSR:

BF 09

BERKELEY/CENTRAL ELECTRIC GENERATING BOARD, U,K.

DF 20

BERNESE POWER CO: SEE BKW

BETA-1/USSR

JFOA

BETA/DANATOM, DENMARK

DFO4

BEZNAU, SWITZERLAND, SEE NOK

BHWR STUDY/GNEC-AEPSC

ED10

BHWR / CZECHOSLOVAKIA

EF 25

BIG ROCK POINT STATION/CONSUMERS POWER CO,

$B D 20$

BILIBINO STATION/USSR

HF 33

BKW, SEE MUEHLBERG

$B N-1000 / U S S R$

FF 11

$B N-350 / U S S R$

FFO9

$B N=50, U S S R$

FF 08

$B N-600 / U S S R$

FF 15 
JANUARY 1969

INDEX

BODEGA BAY ATOMIC PLANT/PACIFIC G*E
BNWL-936

INDEX

BD22

$B D 25$

8D10

BD11

HFO 1

$B R=3 / V U L C A I N$, SEE BR-3

BR-5 /SOVIET BREEDER REACTOR//USSR

FF 07

$B R=60 / U S S R$

FF10

DF 34

BRADWELL/CENTRAL ELECTRIC GENERATING BOARD, U.K. BRATTLEBORO, VERMONT, SEE VERMONT YANKEE

BRAZILIAN POWER REACTOR/CEN, BRAZIL'

DFO2

BRENDA/SNECMA AND CEA, FRANCE

DFO9

BRIDGMAN, MICHIGAN: SEE DONALD C COOK STATION

BRITTANY REACTOR: SEE EL $=4$

BROOKWOOD NUCLEAR STATION/ROCHESTER GAS/ELECTRIC

HD25

BROWNS FERRY NUCLEAR STATION/TVA

BD34

BROWNVILLE, NEBRASKA. SEE COOPER NÚCLEAR STATION

BRUNSWICK STATION/CAROLINA POWER - LIGHT CO.

$B D 42$ 
INDEX

BUGEY, FRANCE, SEE EDF $=5$

BULGAR!AN NUCLEAR STATION/GOVERNMENT OF BULGARIA

HF 31

BURG EL-ARAB, UAR, SEE EGYPTIAN STATION

BURGOS, SPAIN: SEE SANTA MARIA DE GARONA

BURNS HARBOR STATION/NIPSCO

BD39

BWR DUAL PURPOSE STUDY/AMF=MITCHELL'

$B D 07$

BWR STUDY/AEG, DEUTSCH WERFT, W. GERMANY

BF 12

BWR-PWR STUDY/AMF

BDO 8

BWR-PWR, NUCLEAR SUPERHEAT, AMF

$B D 08$

BWR, AUXILIARY POWER; UNDERSEA: WEST

$J D 28$

BWR, BELL STATION, GE

BD 41

BWR, BELOYARSK STATION /USSR/

BF 09

BWR, BIG ROCK POINT. GE

BD19

BWR, BIG ROCK POINT: GE

BD20

BWR, BODEGA BAY, GE

$B 022$

BWR, BODEGA BAY, GE

$\mathrm{BD} 22$

BWR, BORAX $=4$, ANL

$B D 10$

BWR, BROWNS FERRY, GE

BD34

BWR, BURNS HARBOR, GE

BD39

BWR, CAPE FEAR STATION: GE

$B D 42$

BWR, CHOGOKU /JAPAN/

$8 F 20$

BWR, CON-ED STATION-5, GE

$B D 37$

BWR, COOPER NUCLEAR STATION, GE

$B D 43$

BWR, DESALINATION, DEEP POOL REACTOR: BECHTEL

BD29

BWR, DODEWAARD, GE /NETHERLANDS/

BF 05 
INDEX

BWR, DRESDEN-1, GE

$B D 16$

BWR, DRESDEN-2, GE

BD17

BWR, DUAL PURPOSE, AMF-MITCHELL'

$B D 07$

BWR, DUAL PURPOSE, ISRAELI STUDY: KAISER

$B D 31$

BWR, DUANE ARNOLD STATION, GE

BD 44

BWR, EASTON STATION, GE

$B D 38$

BWR, EBWR, ANL

BD13.

BWR, EDWIN! HATCH STATION, GE

$B D 45$

$B W R$, ELK RIVER, AC

$B D 05$

BWR, ENRICO FERMI-2, GE

$B D 48$

BWR, FUKUSH!MA, GE /JAPAN/

BF 16

BWR, GAMMA PROJECT /DENMARK/

$B F 01$

BWR, GARIGLIANO, GE /ITALY/

$B F 03$

BWR, GARONA, GE /SPAIN/

$B F 17$

BWR, HEAVY WATER MOD:, CANDU-BLW /CANADA/

$B F 14$

BWR, HEAVY WATER MOD: P PRESSURE TUBE; ORNL

ED11

BWR, HIGH POWER DENSITY, GE

$B D 40$

BWR, HITACH! /JARAN/

$B F 20$

BWR, HUMBOLDT, GE

$B D 21$

BWR, HYDRIDE MODERATED; DCR, MARTIN

$J 021$

BWR, HYDRIDE MODERATOR; SNAP-4: A I

$J 003$

BWR, INTEGRAL BOILING REACTOR. IUK AEA'

BF 07

BWR, INTEGRAL BOILING SUPERHEAT, IBSHR: WEST

8D46

BWR, JAPCO TSURUGA: GE /JAPAN/

$B F 15$

BWR, JPDR, GE /JAPAN/

$B F O 4$ 
INDEX

BWR, KAHL EXP: HIGH TEMP: REACTOR, GE/AEQ/W, GERMANY/

$B F 10$

BWR, KRB /W: GERMANY/

$B F 11$

BWR, LA CROSSE, $\triangle C$

BDO2

BWR, LINGEN/W, GERMANY/

BF 13

BWR, MILLSTONE FOINT: GE

BD30

BWR, MONTICELLO. GE

BD33

BWR, MUEHLBERG /SWITZERLAND/

BF 18

BWR, MULTI-PURPOSE; SURFSIDE; ASDA

BD28

BWR, NIAGARA MOHAWK. GE

BD23

BWR, NINE MILE POINT: GE

$B D 23$

BWR, NUCLEAR SUPERHEAT: AC

8003

BWR, NUCLEAR SUPERHEAT, AC

BDO4

BWR, NUCLEAR SUPERHEAT: AI

8006

BWR, NUCLEAR SUPERHEAT; BELOYARSK IUSSR/

BF 09

BWR, NUCLEAR SUPERHEAT; BONUS, GNEC

BD25

BWR, NUCLEAR SUPERHEAT, BORAX-5, ANL

$B D 11$

BWR, NUCLEAR SUPERHEAT; IBSHR, WEST

$B D 46$

BWR, NUCLEAR SUPERHEAT; NUSU, GNEC-CE

BD26

BWR, NUCLEAR SUPERHEAT; PATHFINDER: GE

BDO1

BWR, OYSTER CREEK-1: GE

BD24

BWR, PACKAGE POWER: CE

BD14

BWR, PACKAGE POWER, ALPR: ANL

BD12

BWR, PATHFINDER, AC

BDO1

BWR, PEACH BOTTOM=2 AND =3, GE

BD36

BWR, PHILADELPHIA ELECTRIC STATION: GE

BD 47 


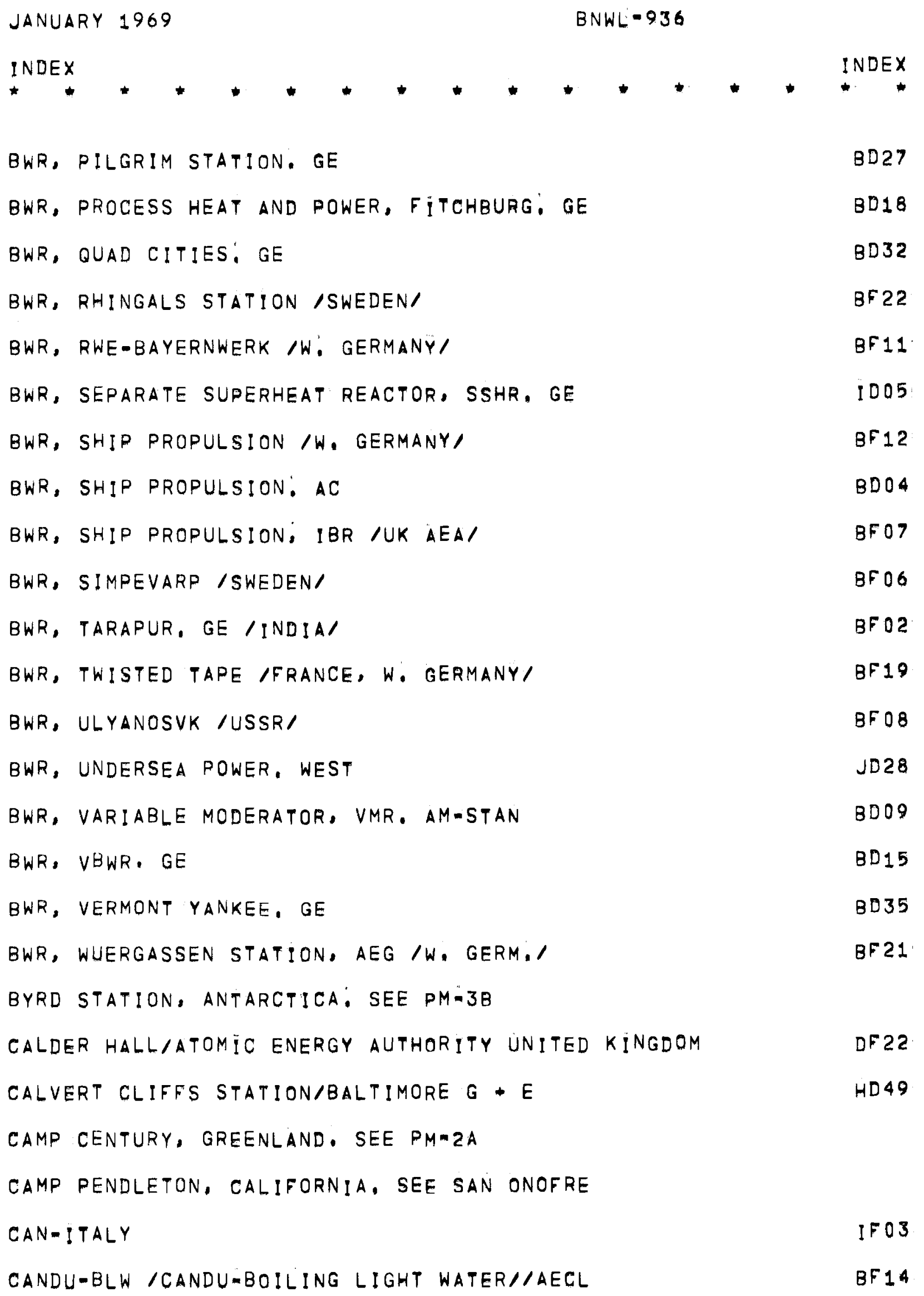


INDEX

-

....

CANDU/AECL, CANADA

EFO3

CARBON DIOXIDE CRACKING, GCR, BNL

DDO6

CAROL INA POWER + LIGHT: SEE CAPE FEAR

CAROLINA POWER + LIGHT, SEE HB ROBINSON

CAROLINAS-VIRGINIA TUBE REACTOR, SEE PARR SHOALS.

CATALONIA STATION, SPAIN: SEE VANDELLOOS

CAVITY REACTOR, SEE GAS CORE REACTOR

CAYUGA LAKE. SEE BELL STATION

CBSR /COUPLED BREEDING SUPERHEATING REACTOR//ANL

I 02

CENTRAL MAINE POWER: SEE NEWINGTON

CENTRALES NUCLEARES DEL NORTE, SEE NUCLENOR

CHAPEL CROSS/ATOMIC ENERGY AUTHORITY: U:K.

DF 23

CHARLEVOIX, MICHIGGAN, SEE BIG ROCK POINT

CHEMICAL SHIM, PWR, RUSSELLVILLE; BAW

HD54

CHEMICAL SHIM, PWR; CNSG, BAW

HDO9

CHEMICAL SHIM, PWR, CONNECTICUT YANKEE; WEST.

HD 31

CHEMICAL SHIM, PWR, CRYSTAL RIVER, BAW

HD 45

CHEMICAL SHIM, PWR, DIABLO CANYON, WEST

HD 44

CHEMICAL SHIM, PWR, ENRICO FERMI/ITALY/

HF 05

CHEMICAL SHIM, PWR, FT, CALHOUN, CE

HD 42

CHEMICAL SHIM, PWR, INDIAN POINT:2: WEST

HD26

CHEMICAL SHIM, PWR; MALIBU, WEST

HD32

CHEMICAL SHIM, PWR; OBRIGHEIM /W: GERM:,

$H F 21$

CHEMICAL SHIM, PWR, OCONEE, BAW

HD39

CHEMICAL SHIM, PWR; PALISADES PARK: CE

HD10 
INDEX

CHEMICAL SHIM, PWR, SAN ONOFRE: WEST

HD33

CHEMICAL SHIM, PWR, SAXTON, WEST

HD 30

CHEMICAL SHIM, PWR, SURRY, WEST

HD 41

CHEMICAL SHIM, PWR, THREE MILE ISLAND, BAW

$H D 43$

CHEMICAL SHIM, PWR, TURKEY POINT: WEST

HD38

CHEMICAL SHIM, PWR, YANKEE, WEST

HD29

CHEMICAL SHIM, PWR, ZION STATION: WEST

HD53

CHEMICAL SHIM, PWR, ZORITA /SPAIN/

HFO9

CHEMONUCLEAR REACTOR STUDY-1/BNL

DD06

CHEMONUCLEAR REACTOR STUDY-2/BNL

0007

CHEMONUCLEAR REACTOR STUDY=3/BNL

DD31

CHEMONUCLEAR REACTOR, AQ: HOMOGENEOUS. AGN

$A D 01$

CHEMONUCLEAR REACTOR, GCR, BN

DD31

CHEMONUCLEAR REACTOR, GCR, BNL

DD06

CHEMONUCLEAR REACTOR, GCR, BNL

DDO 7

CHINON REACTORS, SEE EDF-1, EDF=2, EDF=3

CHOGOKU ELECTRIC STATION/CHOGOKU ELECTRIC POWER CO

$B F 20$

CHOOZ, FRANCE. SEE FRANCO-BELGIUM ARDENNES

CHUKKOTSK PENINSULA, USSR. SEE BILIBINO

CIRENE/CISE, CNEN, ENEL, EURATOM

IF 04

CLEVELAND ELECTRIC ILLUM. SEE DAVIS-BESSE

CNSG /CONSOLIDATED NUCLEAR STEAM GENERATOR//BAW

HDOO

COAL GASIFICATION, GCR, USBM

DD25

COLORADO HTGR. SEE FT, ST, VRAIN

COMMONWEALTH ASSOCIATES. SEE WEST FAST BREEDER 
JANUARY 1969

INDEX

COMMONWEALTH EDISON. SEE DRESDEN

COMMONWEALTH EDISON, SEE QUAD CITIES

COMMONWEALTH EDISON, SEE ZION STATION, WEST FAST BREEDER

COMPACT PWR/FAIREY ENGINEERING

COMPACT UNDERWATER PWR/CE

COMPACT, SEE SNAP=4

CONDUCTION COOLED, FAST REACTOR, ROMASHKA IUSSR,

CONNECTICUT LIGHT + POWER. SEE MILLSTONE POINT

CONNECTICUT YANKEE STATION/CYAPC

CONSOLIDATED EDISON CO: SEE INDIAN POINT-1 AND -2

CONSUMERS POWER CO: SEE BIG ROCK POINT, haLlaM

CONSUMERS POWER CO: SEE MIDLAND STATION

CONSUMERS POWER CO: SEE PALISADES PARK

CONSUMERS PUBLIC POWER DISTRICT. SEE COOPER NUCLEAR STATION

CONTROL, ROD CLUSTER, SURRY STATION, WEST

CONTROL, ROD-CLUSTER, BROOKWOOD. WEST

CONTROL, ROD-CLUSTER; CALVERT CLIFFS: CE

CONTROL, ROD-CLUSTER; CONNECTICUT YANKEE， WEST

CONTROL, ROD-CLUSTER; DIABLO CANYON. WEST

CONTROL, ROD-CLUSTER, DONALD C COOK STATION, WEST

CONTROL, ROD-CLUSTER, HB ROBINSON, WEST

CONTROL, ROD-CLUSTER, INDIAN POINT-2, WEST

CONTROL, ROD-CLUSTER; MAINE yANKEE: CE

CONTROL, ROD-CLUSTER, MALIBU, WEST

CONTROL, ROD-CLUSTER; NEREUS /REN-EURATOM,
HF 28
HD 15

HF 28
HD 15

$J 503$

HD 31

INDEX

HD 41

HD25

HD 49

HD 31

HD 44

HD51

HD37

HD26

HD 46

HD32

HFO 
INDEX *

CONTROL, ROD-CLUSTER; OBRIGHEIM /SIEMENS-W, GERM, ,

HF 21

CONTROL, ROD-CLUSTER; OCONEE, BAW

HD39

CONTROL, ROD=CLUSTER, POINT BEACH. WEST

HD36

CONTROL, ROD-CLUSTER, SALEM. WEST

$H D 4 O$

CONTROL, ROD-CLUSTER, SAN ONOFRE: WEST

HD 33

CONTROL, ROD-CLUSTER, STADE STATION /W. GERM.I

HF 30

CONTROL, ROD-CLUSTER; THREE MILE ISLAND; BAW

$H D 43$

CONTROL, ROD-CLUSTER, TURKEY POINT: WEST

HD38

CONTROL, ROD=CLUSTER, ZION STATION: WEST

HDS3

CONTROLLED VENTILATION CONTAINMENT; BWR: BECHTEL

BD29

COOLING TOWERS, CLOSED CIRCULATION; DUANE ARNOLD STATION

$B D 44$

COOLING TOWERS, CLOSED CIRCULATION; threE MILE ISLAND

$H D 43$

COOLING TOWERS, EDWIN I HATCH STATION

$B D 45$

COOLING TOWERS, FORCED DRAFT, FT: ST: VRAIN, GGA

DD10

COOLING TOWERS, FORCED DRAFT; SGHWR IUK/

EF18

COOLING TOWERS, HYPERBOLIC, CALDER HALL IUKI

DF 22

COOLING TOWERS, INDUCED DRAFT, CROSS-FLOW, PATHFINDER

BDO1

COOLING TOWERS, INDUCED DRAFT, MONTICELLO

$B D 33$

COOLING TOWERS, INVESTIGATION, VERMONT YANKEE

$B D 35$

COOLING TOWERS, NOVO-VORONEZH IUSSR,

$H F 14$

COOLING TOWERS, PRAIRIE ISLAND

HDSO

COOLING TOWERS, RANCHO SECO

HD52

COOPER NUCLEAR STATION/CONSUMERS PUBLIC POWER,

$B D 43$

CORAL-1/SPAIN

FF17

CORDOVA STATION. SEE OUAD CITIES 
CORRAL CANYON, CALIFORNIA. SEE MALIBU

CROSS-FLOW CORE DESIGN, PHWR /SWEDEN/

EF 15

CRYSTAL RIVER STATION/FLORIDA POWER

HD 45

CVNA REACTOR/WEST-CAROLINAS-VIRGINIA NUCLEAR ASSOC

ED13

CVTR: SEE PARR SHOALS

CZECHOSLOVAKIA ATOMIC POWER STATION/CZECHISOC,REP.

DFO3

D-1 REACTOR, SEE KFK STEAM COOLED BREEDER

DA!RYLAND POWER, SEE LA CROSSE BWR

DANATOM, DENMARK: SEE GAMMA PROJECT, BETA PROJECT

DANIELS, FARRINGTON, SEE PBRE, AVR

DAVID POWER REACTOR /ASEA. SWEDEN

AFOA

DAVIDS ISLAND STA, /CONSOLIDATED EDISON CO,

BD37

DAVIS-BESSE STATION/TOLEDO EDISON CO.

HD62

DCDR /DIRECT CYCLE DIPHENYL REACTOR//MARQUARDT

GDO6

DCR-1 /DIRECT CONVERSION REACTOR//MARTIN

JD21

DCR/DIRECT CONTACT REACTOR//LASL

FD24

DEEP SEA GENERATOR: SEE SNAP-21

DEEP-POOL REACTOR/AEC

BD29

DELAWARE VALLEY UTILITIES. SEE PEACH BOTTOM-2 AND - 3

DENMARK, SEE BETA PROJECT

DESALINATION AND POWER: SEE ALSO MH-1A

DESALINATION AND POWER; BWR, AMF-MITCHELL

BDO 7

DESALINATION AND POWER; BWR-RWR, AMF

BDO 8

DESALINATION AND POWER; BWR, ISRAELI STUDY; KAISER

BD 31

DESALINATION AND POWER; BWR, SURFSIDE; ASDA

$\mathrm{BD} 28$ 
INDEX $+*+*+*+*+*+*+*+*^{+}$

DESALINATION AND POWER; GCR, KSK/W. GERMANY/

DF 43

DESALINATION AND POWER; HEAVY WATER MOD; ORNL' ED11

DESALINATION AND POWER; LIO. METAL: FAST, ORNL FD29

DESALINATION AND POWER; LIQ, METAL; FAST, BN-350 /USSR/ FFO9

DESALINATION AND POWER; PM-3A HD21

DESALINATION AND POWER; PWR, EGYPT /UAR/ HF25

DESALINATION AND POWER, PWR, ISRAEL STUDY, KAISER HDI8

DESALINATION AND POWER, PWR, PELICAN POINT, BECHTEL HD16

DESALINATION AND POWER, PWR, PM-3A: MARTIN HD22

DESALINATION, BWR, DEEP POOL REACTOR, BECHTEL. BD29

DESALINATION, HEAVY WATER MOD. DU PONT EDOS

DESALINATION, HEAVY WATER MOD. DU PONT EDO6

DESALINATION, LIO. METAL COOLED, FAST REACTOR, LASL FD25

DESALINATION, LIO, METAL COOLED, FAST, UMBR, ORNL FD33

DESALINATION, OMR, ROVI IITALY, GFOA

DESALINATION, PWR, ELPHR: AC HD14

DETROIT EDISON, SEE PALISADES,

DETROIT EDISON, SEE WEST FAST BREEDER

DFR /DOUNREAY FAST REACTOR/ AEA, UNITED KINGDOM FFO3

DIABLO CANYON/PACIFIC G AND E HDA4

DIRECT CONVERSION, BETA IUSSR/ JFO4

DIRECT CONVERSION, LRL SPACE REACTOR JDA1

DIRECT CONVERSION, SNAP=15A, GD JDO6

DIRECT CONVERSION, SNAP-15B, GIC JD12

DIRECT CONVERSION, THERMIONIC IW: GERM:I JFO5 
INDEX

.

\section{DIRECT CONVERSION, THERMIONIC. MARTIN}

JD29

DIRECT CONVERSION, THERMIONIC, DCR: MARTIN

jD21

DIRECT CONVERSION, THERMIONIC, ISOMITE. DWD

JD 40

DIRECT CONVERSION. THERMIONIC, ITR /W. GERMANY,

JFO5

DIRECT CONVERSION, THERMIONIC, MATTRAC: RCA

JD35

DIRECT CONVERSION, THERMIONIE, REACTOR SYSTEM, GD

JDOB

DIRECT CONVERSION. THERMIONIC, SNAP=50. PWA

jD25

DIRECT CONVERSION, THERMIONIC, SPACE PROP, DOUGLAS

J034

DiRECT CONVERSION, THERMION!E, STAR, GE

JDOQ

DIRECT CONVERSION, THERMIONIC, TI SYSTEM /ITALY/

JFO6

DIRECT CONVERSION, THERMOELECTRIC. SNAP-25: LOCKHEED

$J D 31$

DIRECT CONVERSION, THERMOELECTRIC, ISOTOPE SYSTEM, GE

JD10

DIRECT CONVERSION, THERMOELECTRIC, LAMONT GENERATOR, RRC

JD26

DIRECT CONVERSION, THERMOELECTRIC, MFP, GIC

JD11

DIRECT CONVERSION, THERMOELECTRIC, RIPPLE IUKJ

$\mathrm{JFO2}$

DIRECT CONVERSION, THERMOELECTRIC, ROMASHKa IUSSR,

JFO3

DIRECT CONVERSION, THERMOELECTRIC, SNAP-1: MARTIN

JD 14

DiRECT CONVERSION, THERMOELECTRIC, SNAP-10A, al

$J 005$

DIRECT CONVERSION. THERMOELECTRIC, SNAP-11: MARTIN

JD19

DIRECT CONVERSION, THERMOELECTRIC, SNAP-19: BMARTIN

JD18

DIRECT CONVERSION, THERMOELECTRIC, SNAP-2, AI

$J 002$

DIRECT CONVERSION. THERMOELECTRIC, SNAP-23; 3M

$J D 38$

DIRECT CONVERSION, THERMOELECTRIC, SNAP-27; GE

$J D 32$

DIRECT CONVERSION. THERMOELECTRIC, SNAP-29: MARTIN

$J 036$

DIRECT CONVERSION, THERMOELECTRIC, SNAP-3, MARTIN 
INDEX

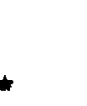

DIRECT CONVERSION, THERMOELECTRIC, SNAP-A, AI

$J 003$

DIRECT CONVERSION, THERMOELECTRIC, SNAP=7, MARTIN

4016

DIRECT CONVERSION, THERMOELECTRIC, SNAP-8, A!

$J D 04$

DIRECT CONVERSION, THERMOELECTRIC, SNAPAgA. MARTIN

$J \square 17$

DIRECT CONVERSION, THERMOELECTRIC, TURPS, MARTIN

JD22

DIRECT CONVERSION, THERMOELECTRIC, UNDERSEA PWR, MARTIN

$J D 23$

DIRECT CONVERSION, THERMOELECTRIC, UNDERSEA, RRE

JD26

DIRECT CONVERSION, THERMOELECTRIC, UNDERSEA, SNAR-21, 3M

$J D 30$

DIRECT CONVERSION, THERMOELECTRIC, UNDERSEA. WEST

j028

DIRECT CONVERSION, THERMOELECTRIC, URIPS, AGN

JD39

DIRECT CONVERSION, UNDERSEA, RISE, AGN

JD37

DODEWAARD NUGLEAR STATION/GKN

BF 05

DOEL STATION/EBES, BELGIUM

HF27

DON / JEN, CENUSA-SPAIN

EF 12

DONALD C. COOK NUCLEAR PLANT/INDIANA-MICHIGAN

$H D 51$

DORTMUND-EMS CANAL: SEE LINGEN BWR

DOUgLas rOINT REACTOR. SEE CANDU

DOW CHEMICAL CO. SEE MIDLAND STATION

DRAGON/ATOMIC ENERGY AUTHORITY; U.K, AND

DF27

DRESDEN-1/COMMONWEALTH EDISON

$B D 16$

DRESDEN-2/COMMONWEALTH EDISON

$B D 17$

DUANE ARNOLD STATION/IOWA ELECTRIC LIGHT + POWER

$B D 44$

DUKE POWER CO, SEE OCONEE, WEST FAST BREEDER, GPU-AI

DUNE ACRES. SEE BURNS HARBOR

DUNGENESS-A/CEGB; UNITED KINGDOM

DF 32 
JANUARY 1969
BNWL $=936$

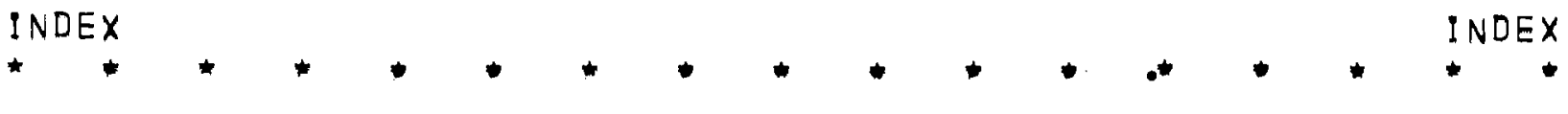

DUNGENESS-B/CEGB; UNITED KINGDOM

DF 25

DUQUESNE LIGHT CO. SEE SHIPPINGPORT

EAST CENTRAL NUCLEAR GROUP, SEE DD27

EAST COAST FLORIDA WEST COAST. SEE GCHWR

EASTON STATION HAS BEEN DROPPED. SEE PASNY

EBES, SEE FLANDERS STATION

EBOR /EXPERIMENTAL BERYLLIUM OXIDE REACTOR//AEC

DDO8

EBR-1/EXP, BREEDER REACTOR-1//AEC

FDO9

EBR=2/EXP, BREEDER REACTOR-2//AEC

FD10

EBWR/EXPERIMENTAL BOILING WATER REACTOR//AEC

$B D 13$

EDEYRN STATION/CEGB, UNITED KINGDOM

DF 21

EDF-RWE STATION

DF 16

EDF-1/ELECTRICITE DE FRANCE

DF 10

EDF-2/ELECTRICITE DE FRANCE

DF 11

EDF.3/ELECTRICITE DE FRANCE

DF 12

EDF-4/ELECTRICITE DE FRANCE

DF 13

EDF-5/EDF-CEA, FRANCE

DF 14

EDWIN I. HATCH NUCLEAR PLANT/GEORGIA POWER CO.

$B D 45$

EFCR, SEE SEFOR

EGCR /EXPERIMENTAL GAS COOLED REACTOR//AEC

DD16

EGYPTIAN POWER-DESALINATION STATION/UAR ATOMIC

HF 25

$E L=4$ /EAU LOURDE/ CEA-FRANCE

$D F O B$

ELK RIVER REACTOR/AEC

BDO5

ELPHR /EXPERIMENTAL LOWATEMPERATURE PROCESS HEAT

HD 14

ENERGY DEPOT, SEE MCR 
INDEX

ENRICO FERMI ATOMIC POWER PLANT/PRDC

FD13

ENR!CO FERMI NUCLEAR STATION/SELN!; ITALY

HFO 05

ENRICO FERMI-2/DETROIT EDISON

BD 48

EOCR /EXP. ORGANIC COOLED REACTOR//AEC

GDO 4

EPITHERMAL BREEDER, L10. METAL COOLED, AETR, SAEA

FDO 7

ERR, SEE ELK RIVER REACTOR

ESCR /EXPERIMENTAL STEAM-COOLED REACTOR/ECNG-GE

ID11

ESSOR /ESSAI ORGEL//EURATOM

EFO5

EUREKA, CALIFORNIA: SEE HUMBOLDT BAY

EXPERIMENTAL BERYLLIUM OXIDE REACTOR: SEE EBOR

EXPERIMENTAL POWER PLANT LUCENS/SNA, SWITZERLAND

EF 17

EXPO /EXPONENTIAL EXPERIMENT/. SEE ORGEL

FARET /ARGONNE FAST REACTOR TEST FACILITY//AEC

FD12

FAST BREEDER DEMONSTRATION/WESTINGHOUSE, DUKE

FD28

FAST BREEDER DEMONSTRATION, GE-UTILITIES-SAEA,

FD20

FAST BREEDER DEMONSTRATION, WESTIUTILITIES

$F D 28$

FAST BREEDER DEVELOPMENT /W, GERM. -BELG, -NETH, /

FFO5

FAST BREEDER DEVELOPMENT; AI-ESADA

FD31

FAST BREEDER DEVELOPMENT; AI-GPU

FD33

FAST BREEDER EXP:, KALPAKKAM /INDIA/

$F F 18$

FAST BREEDER EXPERIMENT, CORAL-1 /SPAIN/

FF 17

FAST BREEDER PROGRAM / W' GERMANY

FF 05

FAST BREEDER PROGRAM /ITALY/

FF 16

FAST BREEDER PROGRAM /W, GERMANY /

FFO5

FAST BREEDER PROGRAM, AEC

FDO1 
FAST BREEDER REACTOR DESIGN/JAERI, JAPAN FF 13

FAST BREEDER, SEE ALSO FAST REACTOR:

FAST OXIDE REACTOR STUDY/LASL

FAST OXIDE REACTOR: SEE SEFOR

FAST REACTOR, CONDUCTION COOLED, ROMASHKA IUSSR/ JFO3

FAST REACTOR, GCR, GGA DD27

FAST REACTOR, GCR, ORNL DD29

FAST REACTOR, GCR, ORNL D030

FAST REACTOR, LIQ. METAL COOLED /JAPAN/ FF 13

FAST REACTOR, LIO. METAL COOLED. AC FDO2

FAST REACTOR, LIO. METAL COOLED. ANL FD32

FAST REACTOR, LIO. METAL COOLED. ANL-WEST FD 34

FAST REACTOR, LIO. METAL COOLED, BN=1000 /USSR/

FF11

FAST REACTOR, LIO. METAL COOLED, BN-350 /USSR/

FFO9

FAST REACTOR, LIQ. METAL COOLED, BN=50 /USSR/

FFO8

FAST REACTOR, LIQ. METAL COOLED, BN=600 /USSR/

FF 15

FAST REACTOR, LIQ. METAL COOLED, BR=5 IUSSR/ FFO 07

FAST REACTOR, LIQ. METAL COOLED, BR-60 IUSSRI

FF10

FAST REACTOR, LIO. METAL COOLED, CORAL-1 /SPAIN/

FF17

FAST REACTOR, LIO. METAL COOLED, DCR: LASL

FD24

FAST REACTOR, LIQ. METAL COOLED, DESALINATION, ORNL

FD29

FAST REACTOR, LIQ. METAL COOLED, DOUNREAY IUK/

FFO3

FAST REACTOR, LIQ. METAL COOLED, EBR-1, ANL

FDO9

FAST REACTOR, LIO, METAL COOLED, EBR-2, ANL

FD10 
FAST REACTOR, LIO, METAL COOLED, FAST OXIDE, LASL FD25

FAST REACTOR, LIO, METAL COOLED, FERMI: APDA FD 13

FAST REACTOR, LIO. METAL COOLED, FFTF, BMI-PNWL FD30

FAST REACTOR, LIO, METAL COOLED, FRCTF; LASL FD23

FAST REACTOR, LIO. METAL COOLED, KALPAKKAM /INDIA/

FF 18

FAST REACTOR, LIQ. METAL COOLED, KNK /W, GERM,I

FFO6

FAST REACTOR, LIQ, METAL COOLED, LAMPRE: LASL

FD22

FAST REACTOR, LIQ, METAL COOLED, LMFBR, ANL

FD32

FAST REACTOR, LIO. METAL COOLED, LMFBR, BAW

FD35

cast Reactor, lio. metal cooled. LMFBR; oE

FD 17

FAST REACTOR, LIO, METAL COOLED, LMFBR, GE

FD20

FAST REACTOR, LIO, METAL COOLED, LMTBR: BAW

FD15

FAST REACTOR, LIO. METAL COOLED, LUNAR PLANT: ANL

F011

FAST REACTOR, LIO. METAL COOLED, LUNAR POWER, WEST

$J D 33$

FAST REACTOR, LIO. METAL COOLED, MCR: QM

FD21

FAST REACTOR, LIO. METAL COOLED, MEREURY /POLAND/

$\triangle F 07$

FAST REACTOR, LIQ. METAL COOLED, MODULAR, A!

FD31

FAST REACTOR, LIO. METAL COOLED. MODULLAR: WEST

$F D 28$

FAST REACTOR, LIO. METAL COOLED, NA=2 /W, GERMi,

FF 14

FAST REACTOR, LIQ. METAL COOLED, OBFR, BNL

FD 36

FAST REACTOR, LIO. METAL COOLED, PASTE FUEL, AI

FDOB

FAST REACTOR, LIO. METAL COOLED. PASTE FUEL, APDA

FD14

FAST REACTOR, LIO. METAL COOLED, PEC /ITALY/

FF 16

FAST REACTOR, LIO. METAL COOLED, PFR /UK/

FF 04 
INDEX

INDEX

FAST REACTOR, LIO. METAL COOLED, PHENIX /FRANCE,

$F F 12$

FAST REACTOR, LIO. METAL COOLED, RAPSODIE /FRANCE,

FFO1

FAST REACTOR, LIQ, METAL COOLED, RAPTUS /ITALY,

$F F_{02}$

FAST REACTOR, L10. METAL COOLED. SEFOR, GE

FD19

FAST REACTOR, LIQ, METAL COOLED, SETTLED BED, SBR, BNL

FD16

FAST REACTOR, LIO. METAL COOLED, SNAP=50; PWA

JD25

FAST REACTOR, LIQ. METAL COOLED, SPUR: AGN

FDO3

FAST REACTOR, LIO. METAL COOLED, SPUR, aGN

$F D 03$

FAST REACTOR, LIO, METAL COOLED, UMBR, ORNL

FD 33

FAST REACTOR, MOLTEN SALT HOMOGENEOUS, SAWA /POLAND/

AFOO

FAST REACTOR, MOLTEN SALT HOMOGENEOUS, WARS /POLAND,

AF07

FAST REACTOR, STEAM COOLED, NDA

IDOS

FAST REACTOR, STEAM COOLED, ESCR: GE-ECNG

ID11

FAST REACTOR, STEAM COOLED, HERMES /BELGIUM/

IF 01

FAST REACTOR, STEAM COOLED, KFK /W: GERM:'

if 06

FAST REACTOR, STEAM COOLED, SCBR: BAW

1003

FAST REACTOR, SUPERCRITICAL WATER COOLED, FSPPR, GE

IDO6

FAST-THERMAL REACTOR; STEAM COOLED; CBSR; ANL

$\square 02$

FAST-THERMAL REACTOR; STEAM COOLED; MSSR; GE : 007

FAYETTEVILLE, ARKANSAS: SEE SEFOR

FBR /FLUIDIZED BED REACTOR//AEC

HD2 3

FDR /FORTSCHNITTLICHER DRUCKWASSERREAKTOR/IGKSS

HF 20

FESSENHEIM STATION/EDF, FRANGE

DF 41

FFTF /FAST FLUX TEST FACILITY/AEC

FD 30

FITCHBURG GAS AND ELECTRIC PLANT/FITCHBURG G*E,

BD18 
INDEX

FLANDERS STATION: SEE DOEL STATION

FLORIDA POWER * LIGHT. SEE HUTCHINSON ISLAND

FLORIDA POWER * LIGHT. SEE TURKEY POINTT

FLORIDA POWER CO: SEE CRYSTAL RIVER

FLUIDIZED-BED REACTOR, SEE PEBBLE-BED REACTOR

FOG COOLED REACTOR STUDY/AECL, CANADA

IF 02

FOG COOLED REACTOR: SEE STEAM COOLED

FOIL GEOMETRY, FUEL, GCR: BNL

DD06

FOIL GEOMETRY, FUEL, GCR: BNL

DDO 7

FOIL GEOMETRY, FUEL, GCR: BNL DD31

FORT ST, VRAIN NUCLEAR STATION/PSC; COLORADO

DD10

FRANCO-BELGE ARDENNES STATION/SENA: FRANCE-BELGIUM

HF 03

FRCTF /FAST REACTOR CORE TEST FACILITTY//AEC

FD23

FRENCH AUXILIARY POWER DEVELOPMENT PROGRAM:

$J F 01$

FRUSS/POLAND

DF 39

FSPPR /FAST SUPERCRITICAL PRESSURE POWER REACTOR/

1006

FT. BELVOIR, VIRGINIA: SEE SM=1

FT. CALHOUN STATION/OMAHA PUBLİC POWER

HD 42

FT. GREELY, aLASKA: SEE SM-1A

FUKUSHIMA NUCLEAR STATION/TOKYO ELECTRIC POWER CO.

$B F 16$

G-1 /GRAPHITE-REACTOR 1/CEA, FRANCE

DFO6

G-2, G-3/CEA-ELECTRICITE DE FRANCE

DFO

GAFRE, SEE DD27

GAMMA PROJECT/DANATOM. DENMARK

BF 01

GARIGLIANO NUCLEAR STATION/SENN, ITALYY 
INDEX

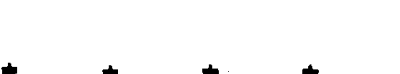
INDEX

GARONA. SEE SANTA MARIA DE GARONA

GARY, INDIANA: SEE BURNS HARBOR

GAS COOLED REACTOR: SEE GCR

GAS CORE REACTOR: BURNS/ROE

CDO1

GAS CORE REACTOR: GE

CDO2

GAS CORE REACTOR: LASL

$C D O 3$

GAS CORE REACTOR: NASA

CDOA

GAS CORE REACTOR: NUCLEAR LIGHT BULB; NASA-UAC

CDOS

GAS CORE REACTOR; AGN

CDOB

gas core reactor; central station. purdue

CDO6

GAS CORE REACTOR, EXPERIMENT, USSR

CFO1

gas cORE REACTOR, GLOW PLUG, DOUGLAS

CDO7

GAS-CORE NUCLEAR ROCKET/AEROJET-GENERAL

CDO8

GASEOUS CORE REACTOR EXPERIMENT/USSR

CF 01

GASEOUS CORE REACTOR POWER STATION STUDYIPURDUE CDOG

GASEOUS CORE REACTOR STUDY-2/LEWIS RESEARCH CENTER

CDOA

GASEOUS CORE REACTOR STUDY/GE

CDO2

GASEOUS CORE REACTOR STUDY/LASL'

CDO3

GASEOUS FUEL REACTOR STUDY/BURNS AND ROE

$C D 01$

GBSR /GRAPHITE BOILING-SUPERHEAT REACTOR//A!

1001

GCFR /GAS-COOLED FAST REACTOR/ UK AEA

DF 42

GCHWR /GAS COOLED HEAVY WATER-MODERATED REACTOR/

DD15

GCR FAST REACTOR STUDY-1/ORNL

DD29

GCR FAST REACTOR STUDY-2/ORNL

DD 30

GCR FAST REACTOR STUDYIGGA 
INDEX $+*+*+*+*+*+*+*+*$

GCR STUDY/FAPIG, JAERI: JAPAN

DF 20

GCR STUDY, PROCESS HEAT/US BU-MINES

$\mathrm{DD} 25$

GCR STUDY, SPACE PROPULSION/THIOKOL

DD21

GCR-MHD/WESTINGHOUSE

DD20

GCR-3/GAS COOLED REACTOR-3/

$D D 22$

GCR, AIR COOLED, G-1/FRANCE/

DF 06

GCR, AIR COOLED, HTRE-3, GE

0012

GCR, AIR COOLED, TORY, UGRL

DD24

GCR, AIR OR STEAM COOLED, MMPPR, AGN

$D D 28$

GCR, AIR, DIRECT CONVERSION, MATTRAC: RCA

$J 035$

GCR, AIRCRAFT PROPULSION: BRENDA /FRANCE/

DFO9

GCR, CARBON DIOXIDE COOLED, AKB /W: GERMANY/

DF 38

GCR, CARBON DIOXIDE COOLED, GEERSHEBA /ISRAEL/

DF 17

GCR, CARBON DIOXIDE COOLED, BERKELEY /UK/

DF 26

GCR, CARBON DIOXIDE COOLED, BETA /DENMARK/

DFO4

GCR, CARBON DIOXIDE COOLED, BRADWELL /UK/

DF 34

GCR, CARBON DIOXIDE COOLED, CALDER /UK/

DF 22

GCR, CARBON DIOXIDE COOLED, CHAPEL CROSS /UK/

DF 23

GCR, CARBON DIOXIDE COOLED, CHEMONUCLEAR, BNL

DDO6

GCR, CARBON DIOXIDE COOLED, CZECH, USSR/CZECH/

DFO3

GCR, CARBON DIOXIDE COOLED, DUNGENESS-A IUK/

DF 32

GCR, CARBON DIOXIDE COOLED, DUNGENESS-B /UK/

DF 25

GCR, CARBON DIOXIDE COOLED, EDEYRN/UK/

DF 21

GCR, CARBON DIOXIDE COOLED, EDF-1 /FRANCE,

DF 10

GCR, CARBON DIOXIDE COOLED, EDF=2 /FRANCE/

DF 11 
INDEX

GCR, CARBON DIOXIDE COOLED, EDF=3 /FRANCEI

DF 12

GCR, CARBON DIOXIDE COOLED, EDF=4 /FRANCE/

DF 13

GCR, CARBON DIOXIDE COOLED, EDF=5 /FRANCE/

DF 14

GCR, CARBON DIOXIDE COOLED, EL-4 /FRANCE/

DFO

GCR, CARBON DIOXIDE COOLED, FESSENHEIM /FRANCE,

DF 41

GCR, CARBON DIOXIDE COOLED, G-2 IFRANCE,

DFO 7

GCR, CARBON DIOXIDE COOLED, GCHWR, GNEC

DD15

GCR, CARBON DIOXIDE COOLED, HINKLEY POINT=B /UK/

DF 40

GCR, CARBON DIOXIDE COOLED, HINKLEY POINT-I IUK,

DF 29

GCR, CARBON DIOXIDE COOLED, HTGR /AUSTRALIA/

DFO1

GCR, CARBON DIOXIDE COOLED, HUNTERSTON-A IUK/

DF 31

GCR, CARBON DIOXIDE COOLED, LATINA: UK/ITALY/

DF 18

GCR, CARBON DIOXIDE COOLED, NEUBRANDENBERG /E, GERM,।

DFOS

GCR, CARBON DIOXIDE COOLED, OLDBURY IUK/

DF 33

GCR, CARBON DIOXIDE COOLED, PEBBLE-BED, hTGR: aUSTRALIA

DF 01

GCR, CARBON DIOXIDE COOLED, SIZEWELL IUK/

DF 30

GCR, CARBON DIOXIDE COOLED, TOKAI, UK/JARAN/

DF19

GCR, CARBON DIOXIDE COOLED, TRAWSFYNYDD /UK/

DF 28

GCR, CARBON DIOXIDE COOLED, VANDELLOS /SPAIN/

DF 15

GCR, CARBON DIOXIDE COOLED, WINDSCALE AGR IUK/

DF 24

GCR, CARBON DIOXIDE COOLED, WYLFA IUKI

DF 35

GCR, EDF=RWE, FRANCE/W. GERMANY

$D F 16$

GCR, FRUSS /POLAND,

DF 39

GCR, HDMR, AGN

DDO4

GCR, HELIUM COOLED STUDY IJAPAN'

DF 20 
JANUARY 1969
BNWL-936

INDEX $+*+*+*+*+*+*+*^{+}+$INDEX $^{+}$

GCR, HELIUM COOLED, DRAGON /UK/ENEA/ DF27

GCR, HELIUM COOLED; EBOR: GD DDO8

GCR, HELIUM COOLED, EGCR; KE/ACF DD15

GCR, HELIUM COOLED; FAST REACTOR: GGA DD27

GCR, HELIUM COOLED, FAST REACTOR: ORNL DD29

GCR, HELIUM COOLED, FAST REACTOR; GCFR JUK, DFA2

GCR, HELIUM COOLED; GCR-3; ORNL/TVA/CE/WEST DD22

GCR, HELIUM COOLED, HIGH TEMPERATURE /W, GERM./ DF43

GCR, HELIUM COOLED, HIGH TEMPERATURE; GGA DDOQ

GCR, HELIUM COOLED; HIGH TEMPERATURE; FT; ST; VRAIN, GGA DO10

GCR, HELIUM COOLED, MHD PLANT, WEST OD26

GCR, HELIUM COOLED, PEBBLE-BED: SAND-PORTER DD23

GCR, HELIUM COOLED, PEBBLE-BED; PBRE: ORNL DD2O

GCR, HELIUM COOLED; PROCESS HEAT: USBM DD25

GCR, HELIUM COOLED, TARGET, GGÄ DD11

GCR, HELIUM COOLED; THTR/W. GERMANY/ DF 44

GCR, HELIUM COOLED, UHTREX. LASL DD17

GCR, HELIUM COOLED, 630-A, GE DD13

GCR, HELIUM OR NITROGEN, NGE, ORNL DD19

GCR, HELIUM-NEON COOLED, PEBBLE=BED, AVR /W. GERM,/ DF37

GCR, HUNTERSTON-B IUK/ OFA6

GCR, HYDROGEN COOLED. ARGONNE TUNGSTEN REACTOR, ANL DDOS

GCR, HYDROGEN COOLED; KIWI-ROVER: AEC-NASA DD18

GCR, HYDROGEN COOLED; PEBBLE-BED; THIOKOL DD21

GCR, NITROGEN COOLED; GCRE-1: AGN DDO1 
INDEX

GCR, NITROGEN COOLED, GCRE-2, AGN

$D 002$

GCR, NITROGEN COOLED; ML-1, AGN

DDO3

GCR, NITROGEN-OXYGEN COOLED, BNL

DD31

GCR, OXYGEN COOLED; CHEMONUCLEAR: BNL

DDO 7

GCR, PROPOSAL, BRAZIL

DF 02

GCR, SEATON-CAREW /UK/

DF 45

GCR, SULFUR DIOXIDE COOLED, FAST REACTOR, ORNL

$D D 30$

GCR, WEISMOOR /W: GERMANY/

DF 36

GCRE-1/GAS COOLED REACTOR EXPERIMENT-1//AEC

DDO 1

GCRE-2 /GAS COOLED REACTOR EXPERIMENT-2//AEC

DDO2

GEESTHACHT HTGR, SEE KSH REACTOR

GENERAL PUBLIC UTILITIES; SEE GPU-AI FAST BREEDER

GENOA, WISCONSIN: SEE LA CROSSE BWR

GENTILLY NUCLEAR STATION: SEE CANDU-BLW

GEORGIA POWER, SEE EDWIN I HATCH STATION

GEOTHERMAL-NUCLEAR PLANT. AMF-MITCHELL

$B D 07$

GINNA STATION. SEE BROOKWOOD

GLOW PLUG REACTOR

CDO 7

GLOWWORM/LASL

JD13

GOLDBORO, PENNA. SEE THREE MILE ISLAND

GOLDEN VALLEY, aLASKA BWR. HAS BEEN DROPPED

GOTAVERKEN PROJECT/GOTAVERKEN - SWEDEN

HF 10

GPU-AI FAST BREEDER STUDY

FD31

GUNDREMMINGEN STATION, SEE KRB

HADDAM NECK, CONNECTUCUT; SEE CONNECTICUT YANKEE 
HALDEN REACTOR/JENER; NORWAY AND OEEC EF 10 HALLAM NUCLEAR POWER FACILITY/AEC,CONSUMERS POWER FD06 HARTFORD ELECTRIC, SEE MILLSTONE POINT HARTLEPOOL STATION: SEE SEATON CAREW HARTSVILLE NUCLEAR STATION. SEE HB ROBINSON HB ROBINSON UNIT $2 / C A R O L I N A P+L^{\prime}$ HD37 HDMR /HIGH DENSITY MODERATOR REACTOR/AEC-ARMY DDOA

HEAT AND POWER. SEE also PACKAGE POWER REACTORS

HEAT AND POWER, HEAVY WATER MOD.: AGESTA /SWEDEN/

EF 13

HEAT AND POWER, HEAVY WATER MODI: MARVIKEN /SWEDEN/

EF 14

HEAT AND POWER, HEAVY WATER MOD,; SULZER /SWITZ/

EF 16

HEAT AND POWER, OMR, DCDR, MAROUARDT

GDO6

HEAT PIPE, LRL SPACE REACTOR JD41

HEAT PIPE, TI SYSTEM /ITALY/ $J F 06$

HEAVY WATER MOD, REACTOR: ORGANIC COOLED, HWOCR, AI-OE GDQ8 HEAVY WATER MOD. REACTOR: AGESTA /SWEDEN/ EF 13 HEAVY WATER MOD. REACTOR: AO. HOMOGENEOUS /NETH/ AFO3 HEAVY WATER MOD, REACTOR, AQ: HOMOGENEOUS /SWEDEN/ AFOA HEAVY WATER MOD, REACTOR: AQ: HOMOGENEOUS, WEST $A D 05$ HEAVY WATER MOD. REACTOR, ARGENTINE MZFR /ARGENTINA/ EF 24 HEAVY WATER MOD, REACTOR, BWR, /CZECH/ EF 25

HEAVY WATER MOD. REACTOR: BWR. CANDU-BLW/CANADA/ BF 14 HEAVY WATER MOD, REACTOR: BWR, HALDEN /NORWAY/ EF 10 HEAVY WATER MOD. REACTOR; BWR, MARVIKEN /SWEDEN/ EF14 HEAVY WATER MOD. REACTOR; BWR, YUGOSLAVIA 
I NDEX

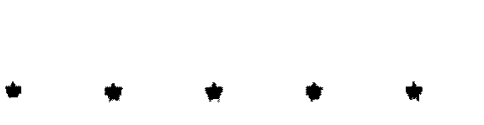

HEAVY WATER MOD, REACTOR; GCR, BEERSHEBA /ISRAEL'

DF 17

HEAVY WATER MOD, REACTOR; GCR, CZECH: USSR/CZECH'

DFO3

HEAVY WATER MOD, REACTOR; GCR, EL-A /FRANCE/

DF 08

HEAVY WATER MOD, REACTOR: GCR, GCHWR: QNEC

DD14

HEAVY WATER MOD, REACTOR; GCR, GCHWR: GNEC

DD15

HEAVY WATER MOD, REACTOR; GCR, KNK /W: GERMANY/

DF 38

HEAVY WATER MOD, REACTOR; GCR, NEUBRANDENBURG /E. GERMANY, DFOS HEAVY WATER MOD, REACTOR; HWCTR, DU PONT

EDO3

HEAVY WATER MOD. REACTOR: LIQ. METAL COOLED /S: AFRICA/ FFI9

HEAVY WATER MOD. REACTOR: LT: WATER COOLED, ORNL ED11

HEAVY WATER MOD, REACTOR, LT, WATER COOLED, SGHWR /UK/ EF 18

HEAVY WATER MOD. REACTOR: MZFR /W. GERMANY, EF 20

HEAVY WATER MOD. REACTOR; ORGANIC COOLED, DU PONT EDOG

HEAVY WATER MOD, REACTOR; ORGANIC COOLED, DON /SPAIN/E EFI2

HEAVY WATER MOD, REACTOR; ORGANIC COOLED; OHWR: CE-BAW EDO2

HEAVY WATER MOD, REACTOR; ORGANIC COOLED, ORGEL /EURATOM, EFOG

HEAVY WATER MOD. REACTOR; ORGANIC COOLED, R-1 /USSA/ EF19

HEAVY WATER MOD, REACTOR; ORGANIC COOLED, WR-1 /CANADA/ EFOA

HEAVY WATER MOD. REACTOR: PHWR /SWEDEN/ EF 15

HEAVY WATER MOD, REACTOR; PRESSURE TUBE; DU PONT EDO 7

HEAVY WATER MOD. REACTOR; PRESSURE TUBE, DU PONT EDOA

HEAVY WATER MOD, REACTOR: PRESSURE TUBE; DU PONT EDO5

HEAVY WATER MOD, REACTOR; PRESSURE TUBE, DU PONT EDO 7

HEAVY WATER MOD, REACTOR: PRESSURE TUBE. GNEC EDOQ

HEAVY WATER MOD. REACTOR, PRESSURE TUBE: ORNL ED 11 
JANUARY 1969

INDEX
BNWL $=936$

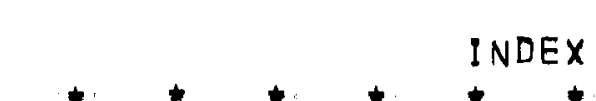

HEAVY WATER MOD, REACTOR; PRESSURE TUBE; BHWR. GNEC ED10

HEAVY WATER MOD. REACTOR; PRESSURE TUBE, CANDU /CANADA/ EFOJ

HEAVY WATER MOD, REACTOR; PRESSURE TUBE, CVNA, WEST ED13

HEAVY WATER MOD, REACTOR; PRESSURE TUBE, DON /SPAIN/ EF12

HEAVY WATER MOD, REACTOR; PRESSURE TUBE; ESSOR /EURATOM/ EFOS

HEAVY WATER MOD, REACTOR, PRESSURE TUBE, KALPAKKAM /INDIA/ EFOQ

HEAVY WATER MOD, REACTOR; PRESSURE TUBE; KANUPP /PAKISTAN/ EF11

hEAVY hATER MOD, REACTOR: PRESSURE TUBE; LUCENS /SWITZ/ EF17

HEAVY WATER MOD, REACTOR; PRESSURE TUBE; M!TSUB!SH! /JAPAN/ EF 21

HEAVY WATER MOD, REACTOR; PRESSURE TUBE; NPD-2 /CANADA/ EFO1

HEAVY WATER MOD; REACTOR; PRESSURE TUBE; ONTARIO /CANADA/ EFOZ

HEAVY WATER MOD, REACTOR; PRESSURE TUBE, PARR SHOALS, WEST ED12

HEAVY WATER MOD, REACTOR; PRESSURE TUBE; PRTR, GE EDOB

HEAVY WATER MOD, REACTOR; PRESSURE TUBE; RAPP/INDIA/, EFO8

HEAVY WATER MOD, REACTOR; PRESSURE TUBE; SGHWR /UK/ EF 18

HEAVY WATER MOD, REACTOR; PRESSURE TUBE, SULZER /SWITZ/ EF16

HEAVY WATER MOD, REACTOR; PRESSURE TUBE, VENTURE/CANADA/ EF 23

HEAVY WATER MOD, REACTOR; PWR, ATUEHA /ARGENTINA/ EF24

HEAVY WATER MOD, REACTOR; STEAM COOLED, CAN /ITALY/ IFO2

HEAVY WATER MOD, REACTOR; STEAM COOLED, CIRENE IITALY/ IFO2

HEAVY WATER MOD, REACTOR; STEAM COOLED, SCHWR /SWITZ/ IFO7

HEAVY WATER MOD. REACTOR; STEAM COOLED, SWR. NDA IDOQ

HEAVY WATER REACTOR STUDY-1/DU PONT EDOA

HEAVY WATER REACTOR STUDY-2/DU PONT EDO5

HEAVY WATER REACTOR STUDY-3/DU PONT $\quad$ EDOG 


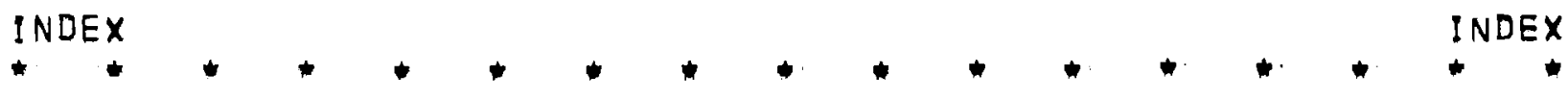
$\begin{array}{ll}\text { HEAVY WATER REACTOR STUDY-4/DU PONT EDO7 } & \text { ENT }\end{array}$ HEAVY WATER REACTOR STUDY/ORNL ED11. HEAVY WATER-LIGHT WATER MOD,; SSCR: BAW EDO1 HEAVY WATER-LIGHT WATER MOD,; VULCAIN /UK-BELGIUM/ EFO7 HEAVY WATER-LIGHT WATER, SPECTRAL SHIFT; VULCAIN/BELG-UK/ EFO7 HERMES/BELGONUCLEAIRE-BELGIUM IF 01 HERO EXPERIMENT, SEE WINDSCALE AQR HEW, SEE STADE STATION HIFRENSA, SEE VANDELLOS. HIGH POWER DENSITY BWR BD 40 HIGH POWER DENSITY BWR: GE $\quad$ BD4O HIGH POWER DENSITY BWR: JAPCO TSURUGA /JAPAN/ BF 15 HIGH POWER DENSITY BWR; COOPER NUCLEAR STATION: GE BD43 HIGH POWER DENSITY BWR; TWISTED TAPE: /FRANCE, W, GERMANY/ BF19 HIGH POWER DENSITY MSRE. ORNL $A D 04$ HINKLEY POINT-B/CEGB; GT: BRITAIN DF 40 HINKLEY POINT-1/CENTRAL ELECTRIC GEN: BOARD, U,K. DF 29 HIROSHIMA, JAPAN: SEE CHOGOKU ELECTRIC STATION HOG ISLAND. SEE SURRY STATION HOXTRA, W. GERMANY: SEE WUERGASSEN STATION HRE /HOMOGENEOUS REACTOR EXPERIMENT//AEC $A D 03$ HTGR /HIGH TEMP, GAS-COOLED REACTOR/AAEC,AUSTRALIA DFO1 HTGR /HIGH TEMP, GCR/PHILADELPHIA ELECTRIC CO, DDO9 HTGR, SEE PEACH BOTTOM: FORT ST, VRAIN HTRE-3 /HEAT TRANSFER REACTOR EXPERIMENT-3//AEC- 
INDEX

HUMBOLDT BAY PLANTIPACIFIC G+E

8021

HUNTERSTON-A/S, SCOTLAND ELECTRICITY BOARD

DF 31

HUNTERSTON-B/S, SCOTLAND ELECTRICITY BOARD

DF 46

HUTCHINSON ISLAND STATION/FLORIDA $P$ * L

HD57

HWCTR/HEAVY WATER COMPONENTS TEST REACTOR/AEC.

EDO3

HWOCR /HEAVY WATER ORGANIC COOLED REACTOR,

EDO2

HYDRAZINE PRODUCTION; AQ: HOMOGENOUS REACTOR; AGN

$A D 01$

IBR IINTEGRAL BOILING REACTOR/AEA, UNITED KINGDOM.

BF 07

IBSHR /INTEGRAL BOILING SUPERHEATING REACTOR/WEST

$B D 46$

ICE-CONDENSER CONTAINMENT, PWR: DONAL'D C COOK STATION, WEST HDSI

ICE-CONDENSER CONTAINMENT, PWR; SEQUOYA STATION: WEST HOSO

INDIA, SEE TARAPUR

INDIAN POINT 2 AND 3/CONSOLIDATED EDISON

HD26

INDIAN POINT-1/CONSOLIDATED EDISON

HDOT

INDIANA + MICHIGAN ELECTRIC CO: SEE DONALD C COOK STATION

IOWA ELECTRIC LIGHT + POWER, SEE DUANE ARNOLD STATION

IOWA LIGHT + POWER: SEE COOPER NUCLEAR STATION

IOWA-ILLINOIS GAS + ELECTRIC. SEE QUAD CITIES

ISOMITE/ISOTOPE MINIATURE-THERMIONIC ELECTRIC/DWD

JD 40

ISOTOPE PRODUCTION, BWR, SURFSIDE, ASDA

$B D 28$

ISOTOPE PRODUCTION, COBALT-60, BIG ROCK POINT, GE

$B D 20$

ISOTOPE PRODUCTION, COBALT=60, CANDU /CANADA/

EFO3

ISRAELI BWR STUDY/US-ISRAEL JOINT BOARD

BD31

ISRAELI PLANT STUDY/US-ISRAEL JOINT BOARD

HD18

ITR IIN-CORE THERMIONIC REACTOR/ 
INDEX

JAMES A, FITZPATRICK STA,/POWER AUTHORITY STATE

BD 38

JAPAN ATOMIC POWER CO. SEE JAPCO TSURUGA STATION

JAPAN ATOMIC POWER CO. SEE TOKAI

JAPCO TSURUGA STATION/JAPAN ATOMIC POWER CO:

$B F 15$

JERSEY CENTRAL POWER + LIGHT, SEE GPU-AI

JERSEY CENTRAL POWER + LIGHT. SEE OYSTER CREEK-1

JERSEY CENTRAL POWER * LIGHT, SEE OYSTER CREEK-2

JOINT NETHERLANDS NUCLEAR STATION. SEE DODEWAABD

JOSE CABRERA NUCLEAR PLANT. SEE ZORITA

JPDR /JAPAN POWER DEMONSTRATION REACTOR//JAERI

BF 04

KAHL EXP, HIGH TEMPERATURE REACTOR/RWE AND BAYERN,

BF 10

KALPAKKAM FAST BREEDER/INDIA

FF 18

KALPAKKAM STATION, INDIA

EFO9

KANSAI ELECTRIC POWER CO. SEE MIHAMA STATION

KANUPP/KARACH! NUCLEAR POWER PROJ.IPAKISTAN AEC

EF 11

KARACHI, PAKISTAN, SEE KANUPP

KARLSRUHE FAST REACTOR NA-2/W, GERMANY

FFIA

KBWP PROJECT/KBWP, W: GERMANY

GF03

KEOWEE DAM, SEE OCONEE STATION

KEOWEE DAM, SEE OCONEE STATION

KEWAUNEE STATION/WISCONSIN UTILITY GROUP

HD 47

KFK STEAM-COQLED BREEDER/KFK; W. GERMANY

1506

KIWI. SEE PROJECT ROVER

KNK /KOMPAKTES NATRIUM KERNKRAFTWERK//W', GERMANY

FFOO

KNK NUCLEAR STATION/ATOMKRAFT-BAYERN; W', GERMANY

DF 38 
INDEX

KOZLODUY, BULGARIA: SEE BULGARIAN STATION

KRB NUGLEAR STATION/KRB, W, GERMANY

BF 11

KRITO, SEE NERO PROJECT

KSH REACTOR/KSH, W: GERMANY

DF 43

KSTR /KEMA SUSPENSION TEST REACTOR, KEMA-RCNmEUR,

$A F 03$

KURCHATOV NUCLEAR STATION, SEE BELOYARSK

KWO STATION, SEE OBRIGHEIM

LA CROSSE BWR/DAIRYLAND POWER

BDO2

LAMONT GEOPHYSICAL OBSERVATORY GENERATOR

JD26

LAMPRE /LOS ALAMOS MOLTEN PLUTONIUM REACTOR//AEC

FD22

LAMPRE-2, SEE FRCTF

LAPRE /LOS ALAMOS POWER REACTOR EXPERIMENT//AEC

$\triangle D 02$

LARGE FAST BREEDER/ANL; WESTINGHOUSE

FD34

LARGE FAST REACTOR STUDY/AEC

FDO2

LATINA, SEE ALSO CIRENE

LATINA/SIMEA, ITALY

DF 18

LCG-25, SEE SNAP:7

LCRE /LITHIUM COOLED REACTOR EXP:, PWAC

FD27

LEMONT, ILLINOIS: SEE EBWR

LENIN SHIP REACTOR/USSR

$H F 17$

LENINGRAD POWER STATION/USSR

HF 15

LIMB /LIOUID METÄL BREEDER//DOUGLAS AIRCRAFT

FD18

LINGEN BWR/KERNKRAFTWERK LINGEN GMBH

$B F 13$

L10, METAL COOLED, BISMUTH, FAST REACTOR, LMTBR: BAW

FD15

LIQ. METAL CDOLED. LEADMGRAPHITE; LIMB. DOUGLAS

FD18 
JANUARY 1969

INDEX
$B N W L=936$

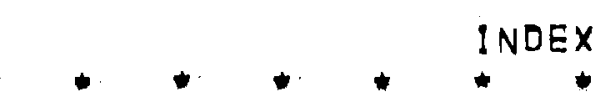

L10. METAL COOLED, LITHIUM, FAST REACTOR, LCRE, PWA

FD27

L10. METAL COOLED, LITHIUM, FAST REACTOR, SNAP-50, PWA JD25

L10. METAL COOLED, LITHIUM, FAST REACTOR, SPUR: AGN FDO3

LIO. METAL COOLED, LITHIUM, LCRE: PWA $\quad$ FD27

LIO. METAL COOLED, LITHIUM, SPACE REACTOR-LRL- JD41

LIQ. METAL COOLED, LITHIUM, TI SYSTEM/ITALY/ JF06

L10, METAL COOLED, MERCURY, FAST REACTOR /POLAND/ AFO7

LIO. METAL COOLED, MERCURY, fAST REACTOR, LUNAR PLANT, ANL FD11

LIQ. METAL COOLED, PHOSPhORUS TRISULFIDE, AUX, POWER, MARTIN jDZZ

LIQ. METAL COOLED. POTASSIUM, FAST; MCR: GM FD21

LIO. METAL COOLED, POTASSIUM, FAST: REFLEX: WEST JD33

LIQ. METAL COOLED, POTASSIUM: MPRE: ORNL JD24

LIO. METAL COOLED, SODIUM, /S, AFRICA/ FF19

LO, METAL COOLED, SODIUM-GRAPHITE: HALLAM: A! FDO6

LIO, METAL COOLED, SODIUM-GRAPHITE; SGR-1000, AI FDO5

LIQ. METAL COOLED, SODIUM-GRAPHITE; SRE: AI FDO4

LIQ. METAL COOLED, SODIUM-POTASSIUM, AUX, POWER. MARTIN JD29

LIQ, METAL COOLED, SODIUM-POTASSIUM, AUXILIARY POWER, MARTIN JD29

LIQ, METAL COOLED, SODIUM-POTASSIUM, FAST, EBR-1. ANL FDO9

LIQ. METAL CDOLED, SODIUM-POTASSIUM, PWAR-6, PWA FD26

LIQ, METAL COOLED, SODIUM-POTASSIUM, SNAPIIOA, A! JDO5

LIO, METAL COOLED, SODIUM-POTASSIUM, SNAF-2, A! JDO2

L10. METAL COOLED, SODIUM, AUXILIARY POWER /W. GERMANY/ JFO5

L1Q. METAL COOLED. SODIUM, AUXILIARY POWER, ITR/W, GeRMANY, jFO5

L10. METAL COOLED, SODIUM, AUXiLiARY POWER; STAR, GE JDO9 
L10, METAL COOLED, SODIUM, EPITHERMAL', AETR, SAEA FDO 7 L10. METAL COOLED, SODIUM, FAST REACTOR /JAFAN, FF 13 LIO. METAL COOLED, SODIUM, FAST REACTOR, aC $F D 02:$ L10. METAL COOLED, SODIUM, FAST REACTOR: AI FD31. LIO. METAL COOLED, SODIUM, FAST REACTOR, ANL FD32 L10. METAL COOLED, SODIUM, FAST REACTOR: ANLEWEST. FD34. LIQ. METAL COOLED, SODIUM, FAST REACTOR: BAW FD35. L10. METAL COOLED, SODIUM, FAST REACTOR: LASL FD25 LIO. METAL COOLED, SODIUM, FAST REACTOR: ORNL FD29 LIO. METAL COOLED, SODIUM, FAST REACTOR: WEST $F D 28$ L10. METAL COOLED, SODIUM, FAST REACTOR, BN-1000 /USSR/

$F F 11$ L10. METAL COOLED, SODIUM, FAST REACTOR, BN-350 /USSR/

FFOQ L10. METAL COOLED, SODIUM, FAST REACTOR, BN-50 /USSR/ FFOB L10. METAL COOLED, SODIUM, FAST REACTOR; BN-600 IUSSR/ FF15 LIQ. METAL COOLED, SODIUM, FAST REACTOR, BR-5 IUSSR/ FF 07 LIO. METAL COOLED, SODIUM, FAST REACTOR, BR=60 /USSR/ FF10 LIO, METAL COOLED, SODIUM, FAST REACTOR, DCR: LASL FD24 L10. METAL CDOLED, SODIUM, FAST REACTOR, DESALINATION, ORNL FD29 LIO. METAL COOLED, SODIUM, FAST REACTOR, DOUNREAY IUK/ FFO3 L10. METAL COOLED, SODIUM, FAST REACTOR, EBR-2: ANL FD10 LIQ, METAL CDOLED, SODIUM, FAST REACTOR, FARET: ANL FD12 L10. METAL COOLED, SODIUM, FAST REACTOR, FERMI: APDA FDI3 10. METAL COOLED, SODIUM, FAST REACTOR, FFTF, BMI=PNWL FD3O LIO. METAL COOLED, SODIUM, FAST REACTOR, FRCTF, LASL FDZ3. - LiO. METAL COOLEQ, SODIUM, fast REACTOR, GPU-Ai 
JANUARY 1969

INDEX

L10, METAL COOLED, SODIUM, FAST REACTOR, KALPAKKAM /INDIA, FF18 L1Q. METAL COOLED̈, SODIUM, FAST̈ REACTOR, KNK /W. GERM.I GFOG LIO, METAL COOLED, SODIUM, FAST REACTOR, LAMPRE, LASL LIO, METAL COOLED, SODIUM, FAST REACTOR, LMFBR: GE LIO, METAL COOLED, SODIUM, FAST REACTOR, LMFBR: GE LIO, METAL CDOLED, SODIUM, FAST REACTOR, Na-2/W. GERM./ LIQ. METAL COOLEÖ, SODIUM, FAST REACTOR, OBFR, BNL L10. METAL COOLED, SODIUM, FAST REACTOR, PASTE FUEL, A! L10. METAL COOLED, SODIUM, fAST REACTOR, PASTE FUEL, APdA LIO, METAL COOLED, SODIUM, FAST REACTOR, PEC /ITALYI LIQ. METAL COOLED, SODIUM, FAST REACTOR, PFR /UK/ LIO, METAL COOLED, SODIUM, FAST REACTOR, PHEN!X /FRANCE, L10. METAL COOLED, SODIUM, FAST REACTOR, PROGRAM. AEC L10. METAL COOLED, SOdIUM, faSt REACTOR, RAPSODIE IFRANCE, fFOI LIO, METAL COOLED, SODIUM, FAST REACTOR, RAPTUS IITALY, L10. METAL COOLED, SODIUM, FAST REACTOR, SBR, BNL L10. METAL COOLED, SODIUM, FAST' REACTOR, SEFOR: GE L10. METAL COOLED, SODIUM, FAST REACTOR, UMBR, ORNL L10. METAL FUELED, Fast REACTOR, DCR: LASL LIO. METAL FUELED, FAST REACTOR, FRCFF: LASL L10. METAL FUELED, FAST REACTOR, LAMPRE: LASL LIOUID METAL COOLED FAST BREEDER/ORNL' LIOUID METAL COOLED REACTOR/S. AFRICA LMFBR /L10, METAL FAST BREEDER REACTOR/CE LMFBR /LIO, METAL FAST BREEDER REACTOR/GE
INDEX

$$
\text { FD22 }
$$

F017

$F D 20$

FF14.

FD36

FDOB

FD14

FF16

FFOA

$F F 12$

FDO 1

FFO2

FD16

FD19

FD 33

FD 24

F023

FD22

FD29

FF 19

FD17

FD 20 
INDEX

LMFBR PROGRAM, ARGONNE NATIONAL': L'ABORATORY

FD32

LMFBR/L!Q. METAL FAST BREEDER REACTOR/BAW, AEC

FD35

LMTBR/LIO. METAL THORIUM BREEDER REACTOR/

FD15

LOIRE-ET=CHER, NORMANDY, SEE EDF: 4

LOS ALAMOS TURRET REACTOR, SEE UHTREX

LOS ANGELES DEPT: WATER AND POWER, SEE MALIBU

LOW-PRESSURE CONTAINMENT; BWR, BONUS: GNEC

$B D 25$

LSBR /LARGE SEED:BLANKET REACTOR/ WEST.

HD28

LUCENS REACTOR, SEE EXPERIMENTAL POWER PLANT LUCENS

LUNAR POWER PLANT

$F D 11$

MADRAS, INDIA; SEE KALPAKKAM

MAGNETOHYDRODYNAMIC POWER, SEE MHD POWER

MAINE YANKEE/MAINE YANKEE ATOMIC POWER CO:

HDA6

MAL!BU PLANT, LADWP

HD32

MAPP /MADRAS ATOMIC POWER PROJECTI: SEE KALPAKKAM

MARITIME GAS COOLED REACTOR PROGRAM. SEE EBOR

MARVIKEN POWER STATION/STATE POWER BOARD; SWEDEN

EF 14

MASURCA: SEE RAPSODIE

MATTRAC

$J \square 35$

MCMURDO SOUND, ANTARCTICA. SEE PM=3A

MCR /MILITARY COMPACT REACTOR/IDOD-ARMY

FD21

MELEKESS REACTOR; SEE ULYANOSVSK

MELEKESS, USSR, SEE BR=60, ARBUS

MERCHANT SHIP REACTOR, SEE MSR

METAL VAPOR COOLED, SODIUM, AUX, POWER /W, GERM.

$J F 05$ 
JANUARY 1969

INDEX

METROPOLITAN EDISON. SEE GPU-AI

METROPQL!TAN EDISON, PENNA, SEE THREE MILE ISLAND

MFP /MIXED FISSION PRODUCTS GENERATOR/AEC

JD11

MH-1A /MOBILE HIGH-POWER 1A//DOD HD24.

MHD POWER, GCR-MHD; WEST DD26

MIDLAND STATION/CONSUMERS POWER, DOW CHEMICAL HDSB

$M ! G-67$. SEE BETA-1

MIHAMA NUCLEAR STATION/KANSAI ELEC: POWER, JAPAN HF24

MILLSTONE POINT STATION/CONNECTICUT L' * R, BD3O

MITSUBISHI HEAVY WATER REACTOR/MITSUBISHI ATOMIC EF21.

ML-1/MOBILE LOW-POWER-1//AEC,ARMY DDO3

MMPPR /MULTIPURPOSE MILITARY PORTABLE POWER D DD28

MOBILE REACTOR, SEE GCRE; ML-1; HDMR

MOB!LE REACTOR, GCR, MMPPR, $\triangle G N$ D D

MOBILE REACTOR, LIQ: METAL COOLED, FAST, MCR; GM FD21.

MOBILE REACTQR, OMR, ARBUS /USSR/ GFO2

MOBILE REACTOR, PWR, MH=1A. MARTIN HD24

MOLTEN SALT HOMOQENEOUS REACTOR, MOSEL /W, GERM,/, AFOS

MOLTEN SALT HOMOQENEOUS REACTOR, MSBR, ORNL ADO7

MOLTEN SALT HOMOGENEOUS REACTOR, MSRE, ORNL ADOA

MOLTEN SALT HOMOGENEOUS REACTOR; SAWÄ /POLAND/ AFO6

MOLTEN SALT HOMOGENEOUS REACTOR, WARS /POLAND/ AFO 7

MOLTEN SALT REACTOR, LIO: METAL COOLED, PWAR-6: PWA FDZG

MONROE, MICHIGAN: SEE ENRICO FERM!

MONT D-ARREE POWER STATION, SEE EL-A 
JANUARY 1969

INDEX

MONTICELLO NUCLEAR STATION/NORTHERN STATES POWER

MORRIS, ILLINOIS; SEE DRESDEN-1 AND -2

MOSEL /MOLTEN SALT EPITHERMAL REACTOR//W GERMANY

MPRE /MEDIUM POWER REACTOR EXPERIMENT//AEC

MSBR /MOLTEN SALT BREEDER REACTOR/ ORNL

MSR /MERCHANT SHIP REACTOR//AECNMA

MSRE /MOLTEN SALT REACTOR EXPERIMENT//AEC

MSSR /MIXED SPECTRUM SUPERHEAT REACTOR//GE

MUEHLBERG STATION/BKW，SWITZERLAND

MURMANSK STATION /USSR

MZFR, SEE ALSO ARGENTINE MZFR

MZFR/MEHR-ZWECK-FORSCHUNGS REAKTOR//MINISTRY

N-REACTOR/AEC, WPPSS

NEREUS REACTOR, SEE NERO

NERO PROJECT/RCN; THE NETHERLANDS-EURATOM

NERVA. SEE PROJECT ROVER

NEUBRANDENBERG STATION/EAST GERMANY

NEW hAMPSHIRE PUBLIC SERVICE, SEE NEWINGTON

NEW JERSEY POWER + LIGHT, SEE GPU-AI

NEW YORK STATE ELECTRIC - GAS, SEE BELL STATION

NEWINGTON STATION/NIH, PUBLIC SERVICE

NGE/NUCLEAR GAS ENGINE//AEC

niagara moHaWk nuClear PLANT /NINE MILE POINT/

NIAGARA MOHAWK, SEE NINE MILE POINT

NIEDERAICHBACH, W, GERMANY, SEE AKB
INDEX

$B D 33$

AFO5

$J D 24$

$A D 07$

HDOB

$A D O A$

I D O 7

$B F 18$

HF 34

EF20

HD19

HFO 7

DF 05

HD6O

DD19

BD23 
INDEX

NIMBUS SATELLITE', SEE SNAP=19

NIPSCO, SEE BURNS HARBOR

NITROGEN FIXATION, GCR: BNL

$D D 31$

NIU, JAPAN, SEE MIHAMA STATION

NOK POWER STATION/NOK, SWITZERLAND

HF 22

NORMANDY REACTOR: SEE EDF=4

NORTH ANNA STATION/VIRGINIA ELECTRIC POWER

HD56

NORTHERN STATES POWER. SEE PRAIRIE ISLAND

NORTHERN STATES POWER, SEE MONTICELLO

NORTHERN STATES POWER, SEE PATHFINDER

NOVO-VORONEZH NUCLEAR STATION/USSR

HF 14

NPD.2/AECL, ONTARIO HYDRO, CANADA

EF 01

NUCLEAR HYDRAZINE PRODUCTION REACTOR /AGN

$\triangle D O 1$

NUCLEAR LIGHT BULB/UAC; NASA

CDOS

NUCLEAR LIGHT BULB, GAS CORE REACTOR; NASA-UAC

$\operatorname{CDO5}$

NUCLEAR SUPERHEAT, BWR, AC

BDO3

NUCLEAR SUPERHEAT, BWR: $\triangle C$

8004

NUCLEAR SUPERHEAT, BWR. AI

BDO6

NUCLEAR SUPERHEAT, BWR. WEST

8046

NUCLEAR SUPERHEAT, BWR-PWR, AMF

$8 D 08$

NUCLEAR SUPERHEAT, BWR; BELOYARSK /USSR,

BF 09

NUCLEAR SUPERHEAT, BWR, BONUS, GNEC

BD25

NUCLEAR SUPERHEAT, BWR, BORAX-5, ANL

8D11

NUCLEAR SUPERHEAT, BWR, NUSU, GNEC-CE

$B D 26$

NUCLEAR SUPERHEAT, BWR; PATHFINDER: AC

BDO1 
JANUARY 1969

INDEX

NUCLEAR SUPERHEAT, HEAVY WATER MOD', MARVIKEN /SWEDEN/

NUCLEAR TANKER DESIGN STUDY/CE:AEC

NUCLENOR, SEE SANTA MARIA DE GÁRONA

NUSU /NUCLEAR SUPERHEAT/

NWK, SEE STADE STATION

OBFR /ORDERED BED FAST REACTOR/BNL

OBNINSK, USSR. SEE APS-1; TES-1

OBRIGHEIM NUCLEAR STATION/KWO, W: GERMANY

OCDRE. SEE WR-1

OCEANOGRAPHIC RESEARCH VESSEL/JAPSD-JAPAN

OCONEE STATION/DUKE POWER

OHIO EDISON, SEE SHIPPINGPORT-2

OLDBURY/CENTRAL ELECTRIC GENERATING BOARD, U:K:

omaha public poWer: see fi. calHoun

OMFBR /ORGANIC MOD; FLUIDIZED BED REACTOR//WEST

OMR-SHIP PROPULSION STUDY / A!

OMRE /ORGANIC MODERATED REACTOR EXP.//AEC-A!

ON=LOAD REFUELING, BWR; CANDU-BLW /CANADA/

ON-LOAD REFUELING, GCR HEAVY WATER MOD., KNK /W. GERMANY/

ON-LOAD REFUELING, GCR PEBBLE BED, AVR /W, geRMANY/

ON=LOAD REFUELING, GCR PEBBLE-BED, hTGR /AUSTRALIA/

ON=LOAD REFUELING, GCR PEBBLE=BED, THTR:, IWEST GERMANYI

ON=LOAD REFUELING, GCR-MHD. WEST

ON-LOAD REFUELING, GCR, BERKELEY /UK/

ON=LOAD REFUELING, GCR, DUNGENESS=A /UK/
$B D 26$

HF21

HFO6

G007

GDO5

GDO2

I NDEX

FD36

HD39

BF 14

DF 38

DF 37

DFO 1

DF 44

DD26

DF 26

DF 32 
JANUARY 1969

BNWL- -936

INDEX

ON-LOAD REFUELING, GCR, DUNGENESS-B /UK/

DF 25

ON-LOAD REFUELING, GCR, EL-4 /FRANCE,

$D F O B$

ON-LOAD REFUELING, GCR; G-2 AND G-3 /FRANCE/

DF 07

ON-LOAD REFUELING, GCR: HINKLEY POINT-B /UK/

DF 40

ON-LOAD REFUELING, GCR; HUNTERSTON-A /UK/

DF 31

ON-LOAD REFUELING, GER; HUNTERSTON-B/UK/

DF 46

ON-LOAD REFUELING, GCR, SIZEWELL /UK/

DF 30

ON=LOAD REFUELING, GCR, TRAWSFYNYDD/UK/

DF 28

ON-LDAD REFUELING, GCR; UHTREX: LASL

$D D 17$

ON-LOAD REFUELING, GCR; WINDSCALE /UK/

DF 24

ON-LOAD REFUELING, GCR; WYLFA /UK/

DF 35

ON-LOAD REFUELING, HEAVY WATER MOD: BWR /CZECH/

EF 25

ON-LOAD REFUELING, HEAVY WATER MOD: DU PONT

ED06

ON-LOAD REFUELING, HEAVY WATER MOD:' GNEC

ED09

ON=LOAD REFUELING, HEAVY WATER MOD:, ARG, MZFR /ARGENTINA'

EF 24

ON-LOAD REFUELING, HEAVY WATER MOD: BOHUNICE /CZECH/

DFOS

ON-LOAD REFUELING, heAVY WATER MOD:, CANDU /CANADA/

EF 03

ON-LOAD REFUELING, HEAVY WATER MOD:. KANUPP, /PAKISTAN/

EF 11

ON-LOAD REFUELING, HEAVY WATER MOD:. MZFR /W, GERMI/

EF 20

ON-LOAD REFUELING, HEAVY WATER MOD:, VENTURE /CANADA/

EF 23

ON-SITE FUEL PROCESSING, LIO. METAL COOLED, EBR-2, ANL

FD10

ON=SITE FUEL PROCESSING, LIO. METAL COOLED, LIMB, DOUGLAS

FD18

ON-SITE FUEL PROCESSING, LIQ. METAL COOLED, LMTBR, BAW

F015

ON-SITE FUEL PROCESSING, MOLTEN SALT: MSCR: ORNL

$A D 07$

ON=SITE FUEL PROCESSING, RAPTUS/ITALY,

FFO2 
JANUARY 1969

INDEX

ONCE-THROUGH PWR/BAW
$B N W L=936$

ONTARIO HYDRO/ONTARIO HYDRO, AECL, CANADA

HD17

ORANGE + ROCKLAND UTILITIES. SEE DAVIDS ISLAND

ORGANIC COOLED REACTOR: ARBUS IUSSR/

GFO2

ORGANIC COOLED REACTOR; DCDR; MARQUARDT

GD06

ORGANIC COOLED REACTOR: EOCR: A!

GDO4

ORGANIC COOLED REACTOR; FLUIDIZED BED, OMFBR; WEST

GDO?

ORGANIC COOLED REACTOR; HEAVY WATER MOD; DU PONT

EDO6

ORGANIC COOLED REACTOR; HEAVY WATER MOD:, DON /SPAIN/

EF12

ORGANIC COOLED REACTOR; HEAVY WATER MOD., HWOCR. Al-CE

GD08

ORGANIC COOLED REACTOR: HEAVY WATER MOD:, OHWR: CEMBW

EDO2

ORGANIC COOLED REACTOR; HEAVY WATER MOD:, ORGEL: /EURATOM/ EFOG

ORGANIC COQLED REACTOR, HEAVY WATER MOD:; R-1 IUSSR/

EF19

ORGANIC COOLED REACTOR; HEAVY WATER MOD;, WR-1 /CANADA,

EFO4

ORGANIC COOLED REACTOR; KBWP/W. GERM.I

GFO3

ORGANIC COOLED REACTOR; OMRE. AI

GDO2

ORGANIC COOLED REACTOR: PIOUA. AI

GDOS

ORGANIC COOLED REACTOR, POPR, II

GDO1

ORGANIC COOLED REACTOR; PRO IITALYY

GFO 1

ORGANIC COOLED REACTOR; ROVI /ITALY/

GFO4

ORGANIC COOLED REACTOR; SHIP PROPULSION, AI

GDO5

ORgEL /ORGaniQUe EAU LOURDE//EURATOM

ORGEL PROJECT: SEE ALSO EL-A; ESSOR

OSKARSHAMN NUCLEAR PLANT, SEE SIMPEVARP

OSWEGO, NEW YORK： SEE NIAGARA MOHAWK 
INDEX

INDEX

OTTO HAHN NUCLEAR SHIP: SEE FDR

OYSTER CREEK STATION-1/JERSEY CENTRAL' P $+L$

BD24

OYSTER CREEK UN!T $2 /$ JERSEY CENTRAL P + L.

$H D 48$

OZONE GENERATION, GCR. BNL

D007

PACIFIC GAS + ELECTRIC: SEE BODEGA BÄY, DIABLO CANYON

PACIFIC GAS + ELECTRIC: SEE HUMBOLDT; VBWR

PACKAGE POWER REACTOR, BWR, ALPR: ANL

$B D 12$

PACKAGE POWER REACTOR, BWR, PL: CE

$B D 14$

PACKAGE POWER REACTOR, GCR, MMPPR, AGN

DD28

PACKAGE POWER REACTOR, ORGANIC COOLED, ARBUS IUSSR/

GFO2

PACKAGE POWER REACTOR, PWR /FAIREY; UK/

$H F 28$

PACKAGE POWER REACTOR, PWR, PL-3: ALCO

HDO5

PACKAGE POWER REACTOR, PWR, PM-1

HD21

PACKAGE POWER REACTOR, PWR, PM-2A. ALCO

HDO 3

PACKAGE POWER REACTOR, PWR, PM-3A

HD21

PACKAGE POWER REACTOR, PWR, PME3B, AC

HDO6

PACKAGE POWER REACTOR, PWR, SM-1: ALCO

HDOI

PACKAGE POWER REACTOR, PWR, SM-1A. ALCO

HDO2

PACKAGE POWER REACTOR, PWR, SM:2: ALCO

HOO

PAKISTAN REACTOR: SEE KANUPP

PAKS STATION/HUNGARY

$H F 29$

PALISADES PARK NUCLEAR STATION/CONSUMERS POWER

HD10

PALO, IOWA, SEE DUANE ARNOLD STATION

PAR /PENNSYLVANII ADVANCED REACTOR//WEST 
JANUARY 1969

INDEX
$B N W L=936$

INDEX

FDO 8

FD 14

$F D 08$

FFO2

$B D 01$

DD23

0020

BD36

PEACH BOTTOM, SEE ALSO HTGR

PEBBLE-BED REACTOR, GCR, SANDERSON-PORTER

DD23

PEBBLE-BED REACTOR; GCR. THIOKOL

DD21

PEBBLE-BED REACTOR, GCR, AVR /W. GERMANY/

DF 37

PEBBLE-BED REACTOR, GCR, FAST, GCFR /UK/

DF 42

PEBBLE-BED REACTOR, GCR, HTGR, AUSTRALIA

DF O1

PEBBLE-BED REACTOR; GCR, PRBE, ORNL'

$D D 20$

PEBBLE-BED REACTOR, GCR, PROCESS HEAT, USBM

DD25

PEBBLE-BED REACTOR, GCR, THTR /W: GERMANY/

DF 44

PEBBLE-BED REACTOR; L10, METAL COOLED, FAST, OBFR, BNL

FD36

PEBBLE-BED REACTOR, LIQ, METAL COOLED, FAST; SBR. BNL

FD16

PEBBLE-BED REACTOR; LIO. METAL COOLED, HEAVY WATER/S,AFRICA/ FF19

PEBBLE-BED REACTOR; ORGANIC COOLED; OMFBR, WEST

G007

PEBBLE=BED REACTOR; PWR, FBR, MARTIN

HD23

PEC /PROVA ELEMENT! DI COMBUSTIBILI/CNEN, ITALY

FF 16

PEEWEE, SEE PROJECT ROVER

PELICAN POINT REACTOR/AEC, MWD AND S: CALIF, ED. 
JANUARY 1969

BNWL-936

INDEX

PENNSYLVANIA ELECTRIC. SEE GPU:AI

PFR /PRDTOTYPE FAST REACTOR/ UK ATOMIC ENERGY AUTH

FFO4

PHENIX/CEA, FRANCE

$F F 12$

PHILADELPHIA ELECTRIC STA. /PHILADELPHIA ELECTRIC

BD47

PHILADELPHIA ELECTRIC. SEE PEACH BOTTOM

PHILADELPHIA ELECTRIC. SEE PEACH BOTTOM-2 AND -3

PHOEQUS REACTOR. SEE PROJECT ROVER

PHOEBUS/CEA, FRANCE

AF 01

RHWR /PRESSURIZED HEAVY WATER REACTOR/

$E F 15$

PHWR STUDY/GNEC-AEPSC

EDOP

PICKERING STATION, SEE ONTARIOL̈HYDRO

PILGRIM STATION/BOSTON-ED.

PIQUA NUCLEAR POWER FACILITYIAEC-CITY OF PIQUA

PL /PORTABLE LOW-POWER//AEC-U.S. ARMY

PL=3 /PORTABLE LOW-POWER-3//AEC,DOD

HDO 5

PLASMA REACTOR, SEE GAS CORE REACTOR

PLATTEVILLE, COLORADO, SEE FT, ST, VRAIN

PLEASANTON, CALIF, SEE VBWR

PLYMOUTH, MASSACHUSETTS, SEE PILGRIM STATION

PM-1 /PORTABLE MEDIUM-POWER-1/DOD

HD21

PM-2A /PORTABLE MEDIUM POWER 2A/ DOD

HDOS

$P M=3 A / U S$ NAVY

HD22

$P M=3 B / P O R T A B L E$ MEDIUM-POWER-3B//AEC-DOD

HDO6

POINT BEACH NUCLEAR STATION/WMPCO

HD36

POINTE-AUX-ROCHES, QUEBEC, SEE CANDU-BLW 
JANUARY 1969

INDEX

POODLE/AEC - USAF

POPR /PROTOTYPE ORGANIC POWER REACTOR//AEC

BNWL -936

PORTABLE POWER REACTOR: SEE PACKAGE POWER REACTOR

PORTALND GENERAL ELECTRIC, SEE TROJAN STATION

POWER REACTOR DEVELOPMENT CO, SEE ENRICO FERM!

PRAIRIE ISLAND NUCLEAR STATION/NSP

HD50

PRESSURE RELEASE SYSTEM, PWR, N=REACTOR, GE

HD19

PRESSURE RELIEF CONTAINMENT, ONTARIOAHYDRO /CANADA/

EFO2

PRESSURE SUPPRESSION SYSTEM, BELL STATION, GE

$B 041$

PRESSURE SUPPRESSION SYSTEM, BWR: GE

$B D 18$

PRESSURE SUPPRESSION SYSTEM, BWR: GE /NETHERLANDS/

$B F O 4$

PRESSURE SUPPRESSION SYSTEM, BWR: WEST

$B D 46$

PRESSURE SUPPRESSION SYSTEM, BWR; BODEGA; GE

BD22

PRESSURE SUPPRESSION SYSTEM, BWR: BROWNS FERRY

$B 034$

PRESSURE SUPRRESSION SYSTEM, BWR; DODEWAARD

BF 05

PRESSURE SUPPRESSION SYSTEM, BWR; DRESDEN=2, GE

BD17

PRESSURE SUPPRESSION SYSTEM. BWR: GAMMA IDENMARK/

BF 01

PRESSURE SUPPRESSION SYSTEM, BWR; HUMBOLDT: GE

BD21

PRESSURE SUPPRESSION SYSTEM, BWR; ISRAELI STUDY. KAISER

$B D 31$

PRESSURE SUPPRESSION SYSTEM, BWR, NIAGARA MOHAWK, GE

$B D 23$

PRESSURE SUPPRESSION SYSTEM, BWR; NUSU, GNEC-CE

$B D 26$

PRESSURE SUPPRESSION SYSTEM, BWR; OYSTER CREEK, GE

8024

PRESSURE SUPPRESSION SYSTEM, BWR; RHINGALS /SWEDEN/

BF 22

PRESSURE SUPPRESSION SYSTEM, BWR; SIMPEVARP

BFO6

PRESSURE SUPPRESSION SYSTEM, BWR; TARAPUR

BF 02 
INDEX

PRESSURE SUPPRESSION SYSTEM, BWR; WUERGASSEN /W. GERMANY/ PRESSURE SUPPRESSION SYSTEM, COOPER STATION, GE BD43 PRESSURE SUPPRESSION SYSTEM, DOUBLE, PILGRIM STATION. GE BD27 PRESSURE SUPPRESSION SYSTEM, EDWIN I HATCH STATION, GE BD45 PRESSURE SUPPRESSION SYSTEM, GBSR, AI IDOI PRESSURE SUPPRESSION SYSTEM, HEAVY WATER, MARVIKEN /SWEDEN/ EFI4 PRESSURE SUPPRESSION SYSTEM, MILLSTONE POINT, GE BD3O PRESSURE SUPPRESSION SYSTEM, PEACH BOTTOM-2 AND-3. GE BD36 PRESSURE SUPPRESSION SYSTEM, PWR; CNSG. BAW HDOQ PRESSURE SUPPRESSION SYSTEM, PWR; SSR-4 HFO4 PRESSURE SUPPRESSION SYSTEM, QUAD CITIES', GE BD32 PRESSURE SUPRRESSION SYSTEM, SIMPEVARP /SWEDEN/ BF06 PRESSURE SUPPRESSION SYSTEM, STEAM COOLED, FAST, FSPPR, GE IDO6 PRESSURE TUBE /CALANDRIA/ VMR. AM-STAN BOO9 PRESSURE TUBE /CALANDRIa/, CANDUmblW /CANADa/ bF14 PRESSURE TUBE /CALLANDRIa/, ger: heaVy WATER MOD. ICZECH/ DFO3 PRESSURE TUBE /CALANDRIA/, gCR: heAVY WATER MOD, IE, GERMaNY DFO5 PRESSURE TUBE /CALANDRIA/, GCR: 630-A. GE DD13 PRESSURE TUBE /CALANDRIa/, heAvy WATER, DU PONT EDOG PRESSURE TUBE /CALANDRIA/, HEAVY WATER. KANUPP /PAKISTAN/ EFI1

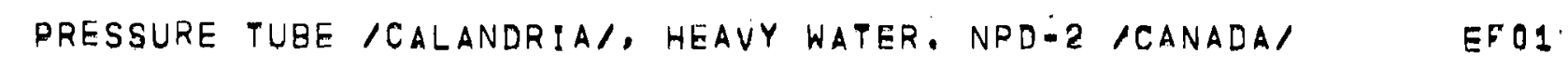
pressure tube /CALANDRIa/, heavy Water, orgel /euratom/ efo6 PRESSURE TUBE /CALANDRIA/, heAVY WATER, ORNL ED11 PRESSURE TUBE /CALANDRIa/, heavy Water. Rapp /INDIa/ EFOB pressure tube /Calandria/, heavy water, candu /Canada/ efo3. 
JANUARY 1969

INDEX

PRESSURE TUBE /CALANDRIA/, heAvY WATER, ESSOR /EURATOM/

PRESSURE TUBE /CALANDRIA/, HEAVY WATER, KALPAKKAM/INDIA/

PRESSURE TUBE /CALANDRIA/, hEAVY WATER, LT; WATER;, ORNL

PRESSURE TUBE /CALANDR!A/, heAUY WATER, LUCENS /SWITZ/

PRESSURE TUBE /CALANDRIA/, HEAVY WATER, ONTARIO /CANADA/

PRESSURE TUBE /CALANDRIA/, HEAVY WATER, PRTR， GE

PRESSURE TUBE /CALANDRIA/, HEAVY WATER, SGHWR /UK/

PRESSURE TUBE /CALANDRIA/, heAVY WATER, VENTURE/CANADA/

PRESSURE TUBE /CALANDRIA/, HEAUY WATER; WR-1/CANADA/

PRESSURE TUBE /CALANDRIA/, LIO: METAL', SGR-1000: AI

PRESSURE TUBE /CALANDRIA/, ORGANIC COOLED, DON /SPAIN/

PRESSURE TUBE /CALANDRIA/, STEAM COOLED/CANADA/

PRESSURE TUBE /CALANDRIA/, STEAM COOLED, SWR; NDA

PRESSURE TUBE /CALANDRIA/, VMR: AMSTAN

FRESSURE TUBE REACTOR, BOILING-SUPERHEAT, GBSR, Al

PRESSURE TUBE REACTOR, BWR, BELOYARSK /USSR/

PRESSURE TUBE REACTOR, BWR, BONUS, GNEC

PRESSURE TUBE REÄCTOR, BWR, IBR /UK $\triangle E A /$

PRESSURE TUBE REACTOR, BWR, IBSHR, WEST

PRESSURE TUBE REACTOR, BWR, SURFSIDE, ASDA

PRESSURE TUBE REACTOR, GCR HEAVY WATER MOD,, EL-A IFRANCE/

PRESSURE TUBE REACTOR, GCR, FRUSS /POLAND/

PRESSURE TUBE REACTOR, GCR, KNK /W: GERMANY/

PRESSURE TUBE REACTOR, GCR, MMPPR, AGN

PRESSURE TUBE REACTOR, GCR, 630 A. GE
I NDEX

EF 05

EFOO

ED11

EF 17

EFO2

EDO 8

EF 18

EF 23

EFOA

FDO5

EF 12

IF 02

I $D 09$

BDO9

I D 01

BFO9

BD25

BF 07

BD 46

BD28

DF0 8

DF 39

DF 38

DD28

DD13 
INDEX

PRESSURE TUBE REACTOR, HEAVY WATER MOD: CVNA. WEST

ED13

PRESSURE TUBE REACTOR, HEAVY WATER MOD, DU PONT

EDO4

PRESSURE TUBE REACTOR, HEAVY WATER MOD, DU PONT

EDO5

PRESSURE TUBE REACTOR, HEAVY WATER MOD. DU PONT

EDO7

PRESSURE tUBE REACTOR, heAVY WATER MOD. GNEC

EDOO

PRESSURE TUBE REACTOR, HEAVY WATER MOD. MITSUBISHI/JAPAN/ EF21

PRESSURE TUBE REACTOR, HEAVY WATER MOD, PARR SHOALS, WEST ED 12

PRESSURE TUBE REACTOR, HEAVY WATER MOD. R-1 IUSSR/

EF19

PRESSURE TUBE REACTOR, HEAVY WATER MOD. SULZER /SWITZ/

EF 16

PRESSURE TUBE REACTOR, heAVY WATER MOD., ORGANiC COOLED.

EDO2

PRESSURE TUBE REACTOR, PWR, $A P S-1$ /USSR/

HF 12

PRESSURE TUBE REACTOR, PWR, N-REACTOR, GE

HD19

PRESSURE TUEE REACTOR, PWR, SCOTTIR, WEST

HD34

PRESSURE TUBE REACTOR, StEAM COOLED FAST-THERMAL, CBSR, ANL IDO2

PRESSURE TUBE REACTOR, Steam COOLED, CAN / ITALy/

IF 03

PRESSURE TUBE REACTOR, Steam COOLED, CIRENE/ITALY/

IFO3

PRESSURE TUBE REACTOR, STEAM COOLED, FAST, SCBR, BAW

I D03

PRESSURE TUBE REACTOR, STEAM COOLED, heRMES/BELgiUm/

IF 01

PRESSURE TUBE REACTOR, STEAM COOLED, SCHWR /SWITZ/

IFO 07

PRESSURE TUBE REACTOR, STEAM COOLED, SCLMR /UK/

IF03

PRESSURIZED LIGHT WATER REACTOR, SEE PWR

PRESTRESSED CONCRETE CONTAINMENT; BHWR, MARVIKEN ISWEDEN/ EF14 PRESTRESSED CONCRETE CONTAINMENT; BRW. WEST BDA6

PRESTRESSED CONCRETE CONTAINMENT; BWR, SIMPEVARP /SWEDEN/ BF06

PRESTRESSED CONCRETE CONTAINMENT; HEAVY WATER, KANUPP /PAK/ EF11 
JANUARY 1969

INDEX
BNWLI-936

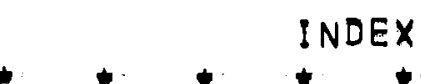

PRESTRESSED CONCRETE CONTAINMENT; HEAVY WATER, VENTURE /CAN/ EF23 PRESTRESSED CONCRETE CONTAINMENT; PWR, CALVERT CLIFFS, CE HD48 PRESTRESSED CONCRETE CONTAINMENT; OWR, CRYSTAL RIVER; BAW HD45 PRESTRESSED CONCRETE CONTAINMENT: PWR, MAINE YANKEE, CE HD46 PRESTRESSED CONCRETE CONTAINMENT; PWR, OCONEE, BAW HDZ9 PRESTRESSED CONCRETE CONTAINMENT; PWR, OYSTER GREEK-2, BAW HDAB PRESTRESSED CONCRETE CONTAINMENT; PWR, PALISADES PARK HDIO PRESTRESSED CONCRETE CONTAINMENT; PWR, POINT BEACH: WEST HD36 PRESTRESSED CONCRETE CONTAINMENT; PWR, TURKEY POINT HDZ8 PRESTRESSED CONCRETE CONTAINMENT; PWR, ZION; WEST HDS3 PRESTRESSED CONCRETE VESSEL, BWR /CZECH/ EF 25 PRESTRESSED CONCRETE VESSEL, GCR PEBBLE-BED, FAST'/UK/ DF42 PRESTRESSED CONCRETE VESSEL, GCR PEBBLE-BED, THTR/W， GERM/ DF44 PRESTRESSED CONCRETE VESSEL, GCR; AVR /W: GERMANY/ DF37 PRESTRESSED CONCRETE VESSEL, GCR; CATALONIA /SRAIN/ DF15 PRESTRESSED CONCRETE VESSEL, GCR; DUNGENESS-B /UK/ DF25 PRESTRESSED CONCRETE VESSEL，GCR: EDF=RWE DF16 PRESTRESSED CONCRETE VESSEL, GCR; EDF-3 /FRANCE/ DF 12 PRESTRESSED CONCRETE VESSEL, GCR: EDF-4 /FRANCE/ DF13 PRESTRESSED CONCRETE VESSEL, GCR: EDF-5/FRANCE/ DF14 PRESTRESSED CONCRETE VESSEL, GCR; EL-4/FRANCE/ DF08 PRESTRESSED CONCRETE VESSEL, GCR; FAST REACTOR, GGA DDZ7 PRESTRESSED CONCRETE VESSEL, GCR; FAST REACTOR: ORNL DD29 PRESTRESSED CONCRETE VESSEL, GCR; FESSENHEIM /FRANCE/ DF41 PRESTRESSED CONCRETE VESSEL, GCR; G-2/FRANCE/ DFO7 
INDEX

PRESTRESSED CONCRETE VESSEL, GCR: HINKLEY PT: B /UK/

DF 40

PRESTRESSED CONCRETE VESSEL, GCR; HUNTERSTON B /UK/

DF 46

PRESTRESSED CONCRETE VESSEL, GCR; OLDBURY IUK/

DF 33

PRESTRESSED CONCRETE VESSEL, GCR; TARGET; GGa

DD11

PRESTRESSED CONCRETE VESSEL, GCR; VANDELLOS /SPAIN/

DF 15

PRESTRESSED CONCRETE VESSEL, GCR; WYLFA /UK/

DF 35

PRESTRESSED CONCRETE VESSEL, HTGR, FT. ST, VRAIN, GGA

DD10

PREUSSEN-ELEKTRA: SEE WUERGASSEN

PRO IPROGRAMMA REATTORE ORGANICO/ CNEN, ITALY

GFO1

PROCESS HEAT, SEE ALSO DESALINATION

PROCESS HEAT, GCR, USBM

DD25

PROCESS STEAM AND POWER, BWR, GE

BD18

PROCESS STEAM AND POWER, ORGANIC COOLED, DCDR. MARQUARDT

GDOS

PROCESS STEAM AND POWER, PWR, MIDLAND; BAW

HDSB

PROCESS STEAM REACTOR/MARTIN

HD20

PROCESS STEAM, HEAVY WATER MOD: HALDEN /NORWAY/

EF 10

PROCESS STEAM, PWR: MARTIN

HD2O

PROCESS STEAM, PWR, ELPHR, AC

HD 14

PROJECT PLUTO. SEE TORY

PROJECT ROVER/NUCLEAR ROCKET//AEC-NASA

DD18

PROJECT-1, SEE BELOYARSK

PRTR /PLUTONIUM RECYCLE TEST REACTOR/ /AEC

PUBLIC SERVICE CO, COLORADO, SEE FORT ST, VRAIN

PUBLIC SERVICE ELECTRIC + Gas, N:J: SEE SALEM

PUERTO RICO WATER RESOURCES AUTH: SEE BONUS 
INDEX

...

PULSING TRIGA/GD

JDO 7

PUNTA FIUME, ITALY; SEE GaRIGLiano

PUNTA HIGUERA, PUERTO RICO: SEE BONUS

PWAR-6 /PRATT + WHITNEY AIRCRAFT REACTOR-6//PWAC

FD26

PWR, DESALINATION AND POWER, PELICAN POINT: BECHTEL

$H D 16$

PWR, BAC PHUN /SHIP PROP:-CHINAI

HF 32

PWR, BARGE-MOUNTED; MH-1A, MARTIIN

HD24

PWR, BILIBINO /USSRI

HF 33

PWR, BR-3 /BELGIUM/

HFO 1

PWR, BRDOKWOOD, WEST

HD25

PWR, BULGARIAN STATION

HF 31

PWR, CALVERT CLIFFS, CE

HD 49

PWR, CONNECTICUT YANKEE, WEST

HD31

PWR, CRYSTAL RIVER: BAW

HD 45

PWR, DAVIS-BESSE STATION: BAW

HD62

PWR, DESALINATION AND POWER, ISRAEL STUDY, KAISER

$H D 18$

PWR, DESALINATION, ELPHR: AC

HD14

PWR, DIABLO CANYON: WEST

HD 44

PWR, DOEL STATION /BELGIUM/

HF27

PWR, DONALD C COOK STATION. WEST

HD51

PWR, EGYPTIAN STATION IUAR/

HF 25

PWR, ENRICO FERMI IITALY

HFO5

PWR, FLUIDIZED BED, FBR. MARTIN

HD23

PWR, FRANCO-BELGIAN ARDENNES /FRANCEI

HFO3

PWR, FT, CALHOUN STATION: CE

HD 42 
PWR, HOOK=ON, SAXTON: WEST

HD30

PWR, HUTCHINSON ISLAND: CE

HD57

PWR, INDIAN POINT, BAW

HDO

PWR, INDIAN POINT-2; WEST

HD26

PWR, KEWAUNEE STATIION: WEST

HD 47

PWR, LENINGRAD /USSR/

HF 15

PWR, maine yankeE, CE HD46

PWR, MALIBU, WEST HD32

PWR, MIDLAND STATION: BAW HD58

PWR, MIHAMA /JAPAN/ HF 24

PWR, MURMANSK IUSSR/ HF 34

PWR, NEWINGTON STATION: WEST HDGO

PWR, NOK /SWITZ/ HF 22

PWR, NORTH ANNA STATION. WEST HD56

PWR, NOVO-VORONEZH IUSSR/ HF 14

PWR, OBRIGHEIM /W, GERM. I HF 21

PWR, OCONEE STATION, BAW HD39

PWR, ONCE-THROUGH SYSTEM: BAW HD17

PWR, OYSTER CREEK-2; BAW HDA 8

PWR, PACKAGE POWER: FAIREY ENG: IUK/ HF28

PWR, PACKAGE POWER, PL-3: ALCO HDO5 PWR, PACKAGE POWER, PM-1: MART IN HD21 PWR, PACKAGE POWER; PM-2A. ALCO HDO 3 PWR, PACKAGE POWER, PM=3A. MARTIN HD22 
PWR, PACKAGE POWER; PM-3B, AC

HDO6

PWR, PACKAGE POWER; SM-1: ALCO

HDO 1

PWR, PACKAGE POWER; SM-1A; ALCO

HDOZ

PWR, PACKAGE POWER, SM-2: ALCO

HDOA

PWR, PACKAGE POWER: TES-3 /USSR/

$H F 16$

PWR, PAKS STATION/HUNGARY

HF 29

PWR, PALISADES PARK, CE

HD10

PWR, POINT BEACH: WEST

HD36

PWR, PRAIRIE ISLAND. WEST

HDSO

PWR, PRESSURE TUBE, APS=1 /USSR/

$H F 12$

PWR, PRESSURE TUBE; N-REACTOR, GE

HD19

PWR, PRESSURE TUBE, SCOTT-R, WEST

HD34

PWR, PROCESS STEAM. MARTIN

$H D 20$

PWR, RANCHO SECO: BAW

HD52

PWR, RAVENSWOOD. WEST

HD35

PWR, RHEINSBERG /E: GERM:'

HF 26

PWR, RUSSELLVILLE STATION. BAW

HD54

PWR, SALEM STATION: WEST

HD 40

PWR, SAN ONOFRE, WEST

$H D 33$

PWR, SEED AND BLANKET, LSBR, WEST

HD28

PWR, SEED AND BLANKET, SHIPPINGPORT, WEST

HD27

PWR, SEQUOYA STATION. WEST

HD59

PWR, SHIP PROPULSION /CHINA/

HF 32

PWR, SHIP PROPULSION /JAPAN/

HFO6

PWR, SHIP PROPULSION; CNSG. BAW 
INDEX

PWR, SHIP PROPULSION, FDR /W. GERM:I

HF 20

PWR, SHIP PROPULSION; GOTAVERKEN /SWEDEN/

HF 10

PWR, SHIP PROPULSION, LENIN IUSSR/

HF 17

PWR, SHIP PROPULSION; MSR, BAW

HDO 8

PWR, SHIP PROPULSION, NERO /NETHERLANDS/

HFO

PWR, SHIP PROPULSION; NUCLEAR TANKER; CE

HD11

PWR, SHIF PROPULSION, NUCLEAR TANKER, CE

HD 12

PWR, SHIP PROPULSION, SSR-4/ITALY,

HFOA

PWR, SHIP PROPULSION, UMP, CE

HO13

PWR, SHIP PROPULSION; VULCAIN/BELGIUM-UK/

EF 07

PWR, SHIP PROPULSION, XAN THAN /CHINA/

$H F 23$

PWR, SHIPPINGPORT-2; WEST

HD5

PWR, SIBERIAN STATION IUSSR/

$H F 13$

PWR, SPECTRAL SHIFT REACTOR, SSCR, BAW

EDO1

PWR, SPECTRAL SHIFT REACTOR, VULCAIN /UK-BELGIUM/

EFO

PWR, STADE STATION/W, GERM,/

HF3O

PWR, STEAM COOLED, FAST-THERMAL BREEDER, CBSR, ANL

1002

PWR, SURRY STATION: WEST

HD 41

PWR, THREE MILE ISLAND. BAW

HD 43

PWR, TIHANGE STATION /FRANCE-BELGIUM,

HFO 8

PWR, TROJAN STATION: WEST

HD61

PWR, TURKEY POINT, WEST

HD 38

PWR, UNDERSEA POWER: MARTIN

$J D 23$

PWR, UNDERSEA PROPULSION, CE

HD15

PWR, YANKEE, WEST

HD29 
$\begin{array}{ll}\text { JANUARY } 1969 & \text { BNWL-936 }\end{array}$

INDEX INDEX

PWR, ZION STATION, WEST HD53

PWR, ZORITA /SPAIN/ HFOQ

QUAD CITIES NUCLEAR STATION/IOWA-ILLINOIS E*G BD32

$R=1$ AND R-2, USSR EF19

$R / 3=A D A M$. SEE AGESTA

RADIATION REACTOR, SEE GLOWWORM

RAJASTHAN, INDIA: SEE RAPP

RANCHO SECO STATION/SMUD HDSZ

RAPP/RAJASTHAN ATOMIC POWER PROJECT//INDIA: EFOB

RAPSODIEICEA， FRANCE FFO1

RAPTUS/RAPID THORIUM-URANIUM-SODIUM/CNEN, ITALY FFDZ

RAVENSWOQD NUCLEAR UNIT/CONSOLIDATED EDISON HD35

RCC, SEE CONTROL, ROD-CLUSTER

REDWING, MINNESOTA: SEE PRAIRIE ISL'AND

REFLEX/WEST INGHOUSE JD33

RHEINSBERG STATION, EAST GERMANY HF26

RHINGALS BWR/SWEDISH STATE POWER BOARD

RICKOVER REACTOR: SEE LSBR

RISE /RADIOISOTOPE SUBMERSIBLE ENGINE/AGN JD37

ROCHESTER GAS AND ELECTRIC CO. SEE BROOKWOOD

$\begin{array}{ll}\text { ROCKET PROPULSION, GAS CORE, AGN CDOB } & \text { COS }\end{array}$

ROCKET PROPULSION, GAS CORE, NASA CDOA

ROCKET PROPULSION, GAS CORE, NASA-UAC CD05

ROCKET PROPULSION, GCR: $\triangle$ RGONNE TUNGSTEN REACTOR, ANL DDO5

ROCKET PROPULSION, ISOTOPE SYSTEM, POODLE, TRW JDZ7 
INDEX

ROCKET PROPULSION, KIWIAROVER.

DD18

ROMASHKA IUSSR

$J F 03$

ROSPO. SEE PRO

ROVER. SEE PROJECT ROVER

ROVI PROJECT/CNEN, ITALY

GF 04

ROWE, MASSACHUSETTS, SEE yANKEE

RURAL COOP, POWER ASSOC, SEE ELK RIVER REACTOR

RUSSELLVILLE STATION/ARKANSAS P + L

HD54

RWE-BAYERNWERK, SEE KAHL EXP, HIGH TEMP: REACTOR

RWE.BAYERNWERK, SEE KRB

RWE-1. SEE KAHL EXP. HIGH TEMP: REACTOR

S. SCOTLAND AGR, SEE HUNTERSTON-B

SACRAMENTO MUNICIPAL UTILITY DIST. SEE RANCHO SECO

SADE, SEE VESR

SAEA; SEE ALSO SEFOR

SAINT LAURENT DES EAUX: SEE EDF=4

SALEM NUCLEAR STATION/DELAWARE VALLEY UTILITIES

HD 40

SAN CLEMENTE, CALIFORNIA: SEE SAN ONOFRE

SAN LUIS OBISPO, CALIF: SEE DIABLO CANYON

SAN qNOFRE NUCLEAR PLANT/S,CAL: ED:, S. DIEGO G+E

HD33

SANTA MARIA DE GARONA STATION/NUCLENOR, SPAIN

BF 17

SAVANNAH NUCLEAR SHIP, SEE MSR

SAWA/INST, NUCLEAR RESEARCH, POLAND

SAXTON EXPERIMENTAL POWER REACTOR/SNEC

HD30

SCBR /STEAM COOLED BREEDER REACTOR//BAW-ECNG 
JANUARY 1969

INDEX

SCHEVCHENKO, USSR, SEE BN-350

SCLMR /STEAM COOLED LIGHT WATER MODERATED REACTOR,

SCOTT-R /SUPERCRIT ONCE THROUGH TUBE REACTOR/ WEST

SEATON-CAREW STATION/CEGB, GT, BRITAINN

SEFOR /SOUTHWEST EXPERIMENTAL FAST OXIDE REACTOR/

SELNI. SEE ENRICO FERMI/ITALY,

SENA: SEE FRANCO-BELGIAN ARDENNES

SENN REACTOR, SEE GARIGLIANO

SEP BWR, SEE DODEWAARD

SEQUQYA STATION/TVA

SETTLED BED FAST REACTOR/BNL

SGHWR /STEAM GENERATING HWR/AEA, UNITED KINGDOM

$S G R=1000 / A$ !

SHIP PROPULSION PROGRAM IUKI

SHIP PROPULSION, BWR /W. GERMANY/

SHIP PROPULSION, GCR, NGE, ORNL'

SHIP PROPULSION, SURFACE, BWR JUK AEA'

SHIP PROPULSION, SURFACE; BWR. AC

SHIP PROPULSION, SURFACE; GCR, 630-A: GE

SHIP PROPULSION, SURFACE; LENIN IUSSR/

SHIP PROPULSION, SURFACE; ORGANIC COOLED REACTOR. AI

SHIP PROPULS:ON, SURFACE; PWR IJAPAN/

SHIP PROPULSION, SURFACE; PWR, BAC PHUN/CHINAI

SHIP PROPULSION, SURFACE; PWR, CNSG, BAW

SHIP PRORULSION, SURFACE; PWR, FDR /W, GERMII
HD59

INDEX

1505

HD34

DF 45

FD19

FD16

EF18

$F D 05$

HF 18

BF 12

DDI9

BF 07

BDO4

DD13

HF 17

GDOS

HFO

HF 32

HDO9

HF 20 
INDEX

SHIP PROPULSION, SURFACE: PWR, GOTAVERK /SWEDEN/

$H F 10$

SHIP PROPULSION, SURFACE; PWR, LENIN /USSR/

$H F 17$

SHIP PROPULSION, SURFACE; PWR, MSR: BAW

HDOB

SHIP PROPULSION, SURFACE; PWR, NERO /NETHERLANDS/

HF 07

SHIP PROPULSION, SURFACE; PWR, NÚCLEAR TANKER, CE

$H D 11$

SHIP PROPULSION, SURFACE; PWR, NUCLEAR TANKER, CE

HO12

SHIP PROPULSION, SURFACE; PWR, SSR-4/ITALY/

HFO4

SHIP PRORULSION, SURFACE; PWR, UMP: CE

HD13

SHIP PROPULSION, SURFACE; PWR, XAN THAN /CHINA/

HF 23

SHIP PROPULSION, SURFACE; STEAM COOLED REACTOR; SCLMR IUK/ IFO3

SHIP PROPULS!ON, UNDERSEA, STEAM COOLED REACTOR, SWR, BMI IDIO

SHIP REACTOR STUDIES IUK AEA

HF 18

SHIPPINGPORT-1/AEC, DUQUESNE

HD27

SHIPPINGPORT-2/DUQUESNE LIGHT, OHIO EDISON

HD55

SHOREHAM NUCLEAR STATION/LONG ISLAND LIGHTING CO.

$B D 19$

SIBERIAN REACTOR STATIONIUSSR

$H F 13$

SIMEA STATION. SEE LATINA

SIMPEVARP REACTOR/OKAB, SWEDEN

BF 06

SIOUX FALLS. SEE PATHFINDER

SIRENE, SEE FRENCH AUXILIARY POWER DEVELOPMENT PROGRAM

S:ZEWELL/CENTRAL ELECTRIC GENERATING BOARD, U.K.

DF 30

SM=1 /STATIONARY MEDIUM POWER-1/ DOD

HDO 1

SM-1A /STATIONARY MEDIUM POWER-1A/ DOD

HDO2

SM-2 /STATIONARY MEDIUM POWER-2/ AEC-DOD

HOO 4

SNAP PROGRAM, AEC

$J D 01$ 
JANUARY $1969 \quad$ BNWL=936

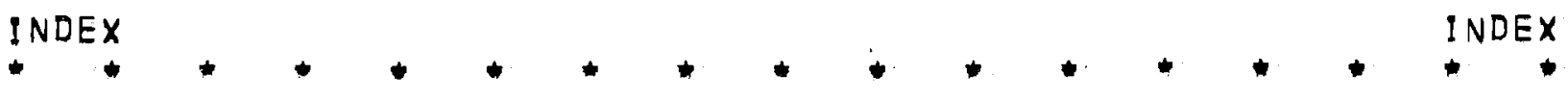

SNAPFPOODLE. SEE POODLE

SNAP-1 AND SNAP-1A/AEC JD14

SNAP-10A/AEC-NASA JDO5

SNAP-11/AEC-NASA JD19

SNAP-13/AEC-NASA JD20

SNAP-15A/AEC JDO6

SNAP-15B/AEC $\quad$ JD12

SNAP-19/AEC-NASA JD18

SNAP-2/AEC-AIR FORCE JDO2

SNAP-21A/AEC JD30

$\begin{array}{ll}S N A P=23 / U S \text { AEC } & \text { JD38 }\end{array}$

SNAP-25/AEC JD31

SNAP-27/AEC, NASA JD32

SNAP $=29 / A E C$, DOD/NASA JD36

SNAP-3/AEC-NAVY JD15

SNAP-4/AEC-NAVY JDO3

SNAP-50/SNAP-50/SPUR//AEC-NASA-DOD JD25

$\begin{array}{ll}\text { SNAP }-7 / A E C & \text { JD16 }\end{array}$

SNAP-8/AEC-NASA JDO4

$\begin{array}{ll}S N A P-9 A / A E C-N A V Y & \text { JD17 }\end{array}$

SNEAK, SEE FAST REACTOR PROGRAM, W: GERMANY

SOUTH CAROLINA GAS + ELECTRIC. SEE GRU-A!

SOUTHERN CALIFORNIA EDISON, SEE PELICAN POINT

SOUTHERN CALIFORNIA EDISON, SEE SAN ONOFRE, SRE. SOUTHWEST ATOMIC ENERGY ASSOC. SEE SEFOR, AETR 
INDEX

SPACE AUXILIARY POWER, SEE AUXILIARY POWER, SNAP

SPACE HEAT AND POWER; AQ; HOMOGENEOUS; BAW

ADO6

SPACE POWER, LIO: METAL COQLED; LUNAR PLANT, aNL

FD11

SPACE POWER, LIO: METAL COQLED: MPRE:' ORNL

$J D 24$

SPACE POWER, L10: METAL COOLED; SNAP-50:. PWA

$J D 25$

SPACE PRQPULSION, GAS CORE: BURNS/ROE

$\operatorname{CDO1}$

SPACE PROPULSION, GAS CORE, GE

$\mathrm{CDO} 2$

SPACE PROPULSION; GAS CORE. USSR

CF 01

SPACE PROPULSION: GAS CORE, GLOW PLUG: DQUGLAS

CDO 7

SPACE PROPULSION: GCR, PEBBLE-BED. THIOKOL

DD21

SPACE PROPULSION; ISOTOPE SYSTEM; POODLE; TRW

$J 027$

SPACE PROPULSION; LIO: METAL COOLED /W, GERM:I

JFO6

SPACE PROPULSION, LIO. METAL COOLED, LCRE, PWA

F $\square 27$

SPACE PROPULSION: L1O. METAL COOLED, SPUR, AGN

$F 003$

SPACE PROPULSION, REACTOR SYSTEM: DOUGLAS

JD34

SPACE REACTOR/LAWRENCE RADIATION LAB:

JDA 1

SPECTRAL SHIFT CONTROL; SEE SSCR; VULCAIN

SPLIT-CORE DESIGN, FFTF

FD 30

SPUR/SPACE POWER UNIT REACTOR//AGN-USAF

5003

SRE/SODIUM REACTOR EXPERIMENT//AEC;AI,S,CAL, ED.

FDOA

SSCR /SPECTRAL SHIFT CONTROLLED REACTOR//AEC-BAW

EDO1

SSEC, SEE HUNSTERSTON

SSHR /SEPARATE SUPERHEAT REACTOR//GE

ID05

SSR-A / FIAT, ANSALDO, CNEN-ITALY

HFO4

STADE STATION/NKW-HEW, W: GERMANY 


\section{INDEX}

INDEX

STAR /SPACE THERMIONIC AUXILIARY REACTOR//GE

JDO9 STARK, SEE FAST REACTOR PROGRAM, W: GERMANY STEAM COOLED FAST REACTOR STUDŸINDA

STEAM COOLED REACTOR, BOILING-SUPERHEAT, GBSR, A! IDO STEAM COOLED REACTOR; FAST BREEDER; ESCR, GE-ECNG ID11 STEAM COOLED REACTOR; FAST BREEDER; FSPPR, GE 1006 STEAM COOLED REACTOR, FAST BREEDER: HERMES /BELGIUM, IF 01 STEAM COOLED REACTOR; FAST BREEDER; KFK; /W. GERMANY, IF06 STEAM COOLED REACTOR; FAST BREEDER; NDA $1 D 08$ STEAM COOLED REACTOR, FAST BREEDER; SCBR, BAW 1003 STEAM COOLED REACTOR: FAST-THERMAL BREEDER, CBSR, ANL 1002 STEAM COOLED REACTOR; FAST-THERMAL BREEDER, MSSR, GE 1007 STEAM COOLED REACTOR; PRESSURE TUBE /CANADA/ IFO2 STEAM COOLED REACTOR, PRESSURE TUBE, CAN IITALY, IF03 STEAM COOLED REACTOR, PRESSURE TUBE, CBSR, ANL IDO2 STEAM COOLED REACTOR; PRESSURE TUBE, CIRENE /ITALY/ IFO3 STEAM COOLED REACTOR, PRESSURE TUBE, SCHWR /SWITZ, IFO7 STEAM COOLED REACTOR; PRESSURE TUBE, SCLMR IUK/ IFO3 STEAM COOLED REACTOR; PRESSURE TUBE, SWR, NDA IDO9 STEAM COOLED REACTOR, SSHR, GE IDOS STEAM COOLED REACTOR, VESR. GE IDOA STOCKHOLM, SWEDEN, SEE AGESTA STURGIS, BARGE-MOUNTED REACTOR: SEE MH-1A SUAK. SEE FAST REACTOR PROGRAM; W. GERMANY SULZER PROJECT/FEDERAL INST. TECH.: SWITZERLAND 
INDEX

SUNDANCE, WYOMING. SEE PM=1

SUPERCRITICAL WATER COOLED, FAST, FSPPR, GE

SUPERCRITICAL WATER COOLED, SHIP PROPULSION, SWR, BMI

ID10

SUPERHEAT REACTOR FOR PROPULSION/AC

$B D O 4$

SUPERHEAT REACTOR STUDY /AC

$B D O 3$

SURFS I DE/ASDA, N:Y: BD28

SURRY STATION/VIRGINIA ELECTRIC POWER $H D 41$

SURVEYOR SPACECRÄFT, SEE SNAP-I1

SUS-POP /SUSPENSION POWER ONLY PILE/ /RCN-THE

SWEDISH STATE POWER BOARD. SEE RHINGALS STATION

SWISS SCHWR/FED. INST, REACTOR RESEARCH, SWITZ.

SWR /STEAM-WATER REACTOR//NDA

SWR ISUPERCRITICAL WATER REACTOR/

SYSTEMS FOR NUCLEAR AUXILIARY POWER, SEE SNAP

TAMPA ELECTRIC CO. SEE GCHWR

TANDEM REACTORS, FSPPR: GE

TARAPUR STATION/INDIA

TARGET/THERMAL ADVANCED REACTOR GAS-COOLED

DD11

TARRAGONA, SEE VANDELLOS.

TENNESSEe VALLEy AuTHORITY, SEe tVA

TES.3 / USSR

THERMal BREeder, LiO, metal COOLED; hallam; a! FDO6

THERMAL BREEDER, LIO. METAL COOLED: LIMB FD18

THERMAL BREEDER, LIO. METAL COOLED; SGR-1000; A! 
JANUARY 1969

BNWL-936

INDEX

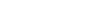$$
\text { . }
$$

INDEX

THERMAL BREEDER, MOLTEN SALT, MSBR: ORNL

ADQ7

THERMIONIC CONVERSION REACTOR/MARTIN

$J 029$

THERMIONIC CONVERTER PRORULSION SYSTEMIDOUGLAS

JD34

THERMIONIC REACTOR/GD

$J 008$

THERMOELECTRIC GENERATOR/AIR FORCE

JD10

THREE MILE ISLAND STATION/METROPOLITAN EDISON

HD 43

THTR /THORIUM HIGH TEMP, REACTOR, HKG, W, GERMANY

DF 44

THTR /THORIUM HIGH TEMPERATURE REACTOR/ SEE ALSO AVR

TI HEAT PIPE REACTOR/ISPRA, ITALY

$J 506$

TIDEWATER, NEW HAMPSHIRE: SEE NEWINGTON

TIHANGE STATION/SYNATOM, FRANCE BELGIUM

HFOB

TOKAI ATOMIC STATION/JAPCO, JAPAN

DF 19

TOKAI MURA, JAFAN, SEE JPDR

TOKYO ELECTRIC CO. SEE FUKUSHIMA STATION

TOLEDO EDISON CO: SEE DAVIS-BESSE

TORY/AEC

DD24

TRANSIT SATELLITES: SEE SNAP-3; SNAP-9A

TRAWSFYNYDD/CENTRAL ELECTRIC GENERATING BOARD, U.K

DF 28

TR!NO VERCELLESE; ITALY, SEE ENRICO FERMI ITALYI

TROITSK, USSR, SEE SIBERIAN STATION

TROJAN STATION/PORTLAND GENERAL' ELECTRIC CO.

HD61

TSURUGA, JAPAN, SEE JAPCO TSURUGA STATION

TSURUGA, JAPAN, SEE MIHAMA STATION

TUBE-IN=SHELL FUEL ELEMENT, CANDU-BLW/CANADA/

BF 14

TUBE-IN-SHELL FUEL ELEMENT, FSPPR, GE 
INDEX

TURKEY POINT 3 AND 4/FLORIDA POWER + LIGHT

HD38

TURPS /TERRESTRIAL UNATTENDED REACTOR POWER

JD22

TURRET, SEE UHTREX

TVA, SEE BROWNS FERRY

TVA, SEE EGCR

TVA, SEE SEQUOYA STATION

TWISTED TAPE BWR/W; GERMANY, FRANCE

BF 19

TWO CREEKS, WISCONSIN, SEE POINT BEACH

U,S, AEC FAST REACTOR PROGRAM

FDO1

UHTREX/ULTRA HIGH TEMP, REACTOR EXP/AEC

DD17

ULYANOVSK ATOMIC POWER STATION/CENTRAL ATOMIC

$B F 08$

UMBR/UNCLAD METAL BREEDER REACTOR/ ORNL

FD33

UMP /UNIFIED MODULAR PLANT//CE

HD13

UNDERSEA PROPULSION AND POWER, SUPERCRITICAL WATER： BMI

1010

UNDERSEA PROPULSION OR AUX, POWER, RISE; $\triangle G N$

J037

UNDERSEA PRORULSION, PWR, CE

$H D 11$

UNDERWATER THERMOELECTRIC POWER PLANT/MARTIN

JD23

UNITED ARAB REPUBLIC. SEE EGYPTIAN STATION

UNITED ILLUMINATING, SEE NEWINGTON

URIPS IUNDERSEA RADIO-ISOTOPE POWER SYSTEM/ AGN

VAK STATION, SEE KAHL EXP. HIGH TEMP: REACTOR

VALLECITOS BWR, SEE VBWR

VANDELLOS NUCLEAR STATION/FRANCE-SPAIN

$D F 15$

VAPOR SUPPRESSION, SEE PRESSURE SUPPRESSION

VARIABLE MODERATOR REACTOR, AMISTAN 
INDEX

VBWR/VALLECITOS BOILING WATER REACTOR//GE-PACIFIC

$B D 15$ VENTURE/CANADIAN GENERAL ELECTRIC EF 23 VERMONT YANKEE/VERMONT YANKEE NUCLEAR POWER CORP. BD35 VERNON, VERMONT. SEE VERMONT YANKEE VESR /VALLECITOS EXPERIMENTAL SUPERHEAT REACTOR, IDO 4 VEW STATION. SEE LINGEN BWR VIRGINIA ELECTRIC POWER CO. SEE SURRY STATION VIRGINIA ELECTRIC POWER, SEE NORTH ANNA STATION VMR /VARIABLE MODERATOR REACTOR/AM-STANDARD BDO9 VORONEZH, USSR, SEE NOVO-VORONEZH VORTEX FLOW BWR. SEE TWISTED TAPE BWR VORTEX FLOW GAS CORE REACTOR: NASA CDO5

VULCAIN / BELGONUCLEAIREMUK AEÁ EFO

VULCAIN, SEE ALSO BR=3/VULCAIN WANL SUBMERGED REACTOR UNIT/WEST JD28 WARS/INST. NUCLEAR RESEARCH, POLAND AFO 7 WASHINGTON PUBLIC POWER SUPPLY SYSTEM: SEE N-REACTOR WATERFORD, CONNECTICUT: SEE MILLSTONE POINT WEISMOOR STATION/APK, W. GERMANY DF 36

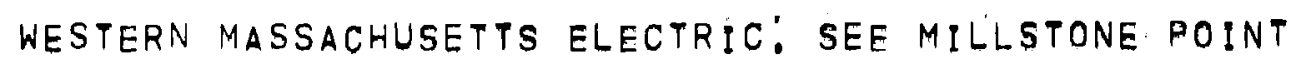
WHEEL FLOW GAS CORE REACTOR. LEWIS RESEARCH CDOA WINDSCALE AGR/ATOMIC ENERGY AUTHORITY, U.K. DF 24 WISCONSIN ELECTRIC POWER CO. SEE POINT BEACH WISCONSIN MICHIGAN POWER CO, SEE POINT BEACH WISCONSIN PUBLIC SERVICE; SEE kEWAUNeE 
JANUARY 1969

$B N W L=936$

INDEX

WR-1 /WHITESHELL REACTOR-1/AECL', CANADA

EFO4

WUERGASSEN STATION/PREAG; W.GERMANY

BF 21

WWER: SEE NOVO-VORONEZH

WYLFA/CENTRAL ELECTRIC GENERATING BOARD, U,K'.

DF 35

YANKEE ATOMIC ELECTRIC PLANT/YAEC

HD29

YUGOSLAV REACTOR

EF 22

ZAN THAN NUCLEAR SHIP/CHINA

HF 23

ZBSR /ZIRCONIUM BOILINO=SUPERHEATING REACTOR//AI

$B D 06$

ZION STATION/COMMONWEALTH EDISON

HD53

ZORITA NUCLEAR STATION/UEM, SPAIN

HFOQ

ZPR, SEE ARGONNE TUNGSTEN REACTOR

ZURICH REACTOR, SEE SULZER PROJECT

630-A NUCLEAR STEAM GENERATOR/GENERAL ELECTRIC CO.

DD13 


\section{DISTRIBUTION}

No. of

Copies

OFFSITE

265 Division of Technical Information Extension

1 Battelle Memorial Institute

R. J . Sprague

Special Projects Div.

2 Oak Ridge National Laboratory

Carlos Bell

W. E. Browning, Jr.

ONSITE

$5 \quad$ Richland Operations Office

B. Kirz

D. W. Mazur

R. L. Plum

R. K. Sharp

Technical Information Library

4 Atlantic Richfield Hanford Company

D. A. Hoover/N. Choate

H. H. Hopkins

R. D. Shimer

1 Computer Sciences Corporation

G. L. Ivy

1 Donald W. Douglas Laboratory

Shirley Gydesen

9 Douglas United Nuclear Inc.

T. W. Ambrose

W. J. Dowis

L. Grumme

W. K. Kratzer

M. Lewis

J. W. Riches

J. T. Stringer

W. K. Woods

$100-\mathrm{N}$ Library

6 Vitro Hanford Eng ineering Services

W. S. Graves/A. J. Hutzelman

E. E. Smith/G. Salzano/L. B. Ufkes

C. N. Zangar 
No. of

Copies

206
Batte 11 e-Northwest
F. W. Albaugh
E. R. Appleby
E. R. Astley
J.A. Ayres
W. J. Bailey
J. M. Batch
A. L. Bement
C. A. Bennett
A. G. Blasewitz
J. G. Bradley
S. H. Bush
J. J. Cadwe 11
T. D. Chikalla
J. A. Christensen
T. T. Claudson
D. L. Condotta
R. W. Dascenzo
F. G. Dawson
D. R. de Halas
R. L. Di11on
E. A. Eschbach
E. A. Evans
L. G. Faust
J. C. Fox
M. D. Freshley
S. Goldsmith
K. M. Harmon
H. Harty
R. E. Heineman
R. J. Hennig
P. L. Hofmann
R. L. Junkins
G. A. Last
W. R. Lewis
J. E. Minor
R. E. Nightingale
L. J. Nitteberg
D. E. Oleson
H. M. Parker
L. T. Pedersen
A. M. Platt
R. H. Purcell
W. E. Roake
C. A. Rohrmann

W. A. Snyder

R. J. Sorenson

D. H. Stewart

W. H. Swift

C. R. Tipton

C. J. Touhil1

F. W. Van Wormer

E. E. Voiland

O. J. Wick

R. D. Widrig

N. G. Wittenbrock

F. W. Woodfield

D. C. Worlton

Technical Information

Technical Publications 